University of Tennessee Health Science Center UTHSC Digital Commons

\title{
Cephalometric Evaluation of Bionator Therapy in the Early Treatment of Class II Malocclusions
}

Daniel C. Sawrie

University of Tennessee Health Science Center

Follow this and additional works at: https://dc.uthsc.edu/dissertations

Part of the Orthodontics and Orthodontology Commons

\section{Recommended Citation}

Sawrie, Daniel C. , "Cephalometric Evaluation of Bionator Therapy in the Early Treatment of Class II Malocclusions" (2008). Theses and Dissertations (ETD). Paper 233. http://dx.doi.org/10.21007/ etd.cghs.2008.0278.

This Thesis is brought to you for free and open access by the College of Graduate Health Sciences at UTHSC Digital Commons. It has been accepted for inclusion in Theses and Dissertations (ETD) by an authorized administrator of UTHSC Digital Commons. For more information, please contact jwelch30@uthsc.edu. 


\title{
Cephalometric Evaluation of Bionator Therapy in the Early Treatment of Class II Malocclusions
}

\begin{abstract}
Early or two-phase orthodontic treatment of Class II malocclusions is a debated topic in orthodontic circles primarily because the benefits of early intervention have not been consistently reproduced among researchers. The present study was a retrospective analysis of cephalograms from patients with Class II, division 1 malocclusions at the start of treatment. These were 50 consecutively treated youths who received phase 1 (early) treatment with a Bionator appliance and later treatment with full appliances (all treated by a single clinician). The comparison group consisted subjects treated in a single phase with fixed Edgewise appliances only. Importantly, subjects in the two samples were matched, on a one-to-one basis, for demographic and cephalometric variables (ANB, SNA, SNB, NAP, FMA) to ensure comparability in the nature and severity of the malocclusions. The key question was whether the cephalometric results at the end of treatment were comparable between groups. Two-phase subjects were treated on average 1.4 years longer than the one-phase group, though this measure of clock time may obscure the greater ease of treatment chair side. Neither of out two integumental variables ( $Z$ angle, $E$ plane) differed statistically, but two key skeletal variables did differ, namely ANB and NAP. ANB was significantly smaller ( $\left.=2.6^{\circ}\right)$ in the two-phase group than in the one-phase group $\left(=3.7^{\circ}\right)$, and NAP averaged $2.7^{\circ}$ in the twophase group compared to a mean of $4.8^{\circ}$ in the one-phase group. Consequently, the two-phase group was discernibly more orthognathic at the end of treatment, with most of the correction coming from mandibular growth rather than maxillary restriction. None of the dental variables differed statistically except the position of the maxillary incisors which were slightly more proclined in the two-phase group, and this was due to operator preference. The two-phase subjects had a lower rate of premolar extraction ( $12 \%$ vs $84 \%$ ) than the one-phase subjects. Overall, the two-phase group achieved a better bony facial profile, with the majority of the skeletal and dental correction occurring during the first phase of treatment. These favorable results contrast with published randomized clinical trials, possibly because of differences in patient selection and appliance design. Our results do support the value of early intervention, though it is not known whether the statistically significant improvements in the facial profile are large enough to be perceptible to the patient and parents.
\end{abstract}

\section{Document Type}

Thesis

\section{Degree Name}

Master of Dental Science (MDS)

\section{Program}

Orthodontics

\section{Research Advisor}

Edward Harris, Ph.D.

\section{Keywords}

Orthodontics, Class II malocclusion, Functional appliance, Mandibular retrognathia, Esthetics, Facial growth, Bionator

\section{Subject Categories}

Dentistry | Medicine and Health Sciences | Orthodontics and Orthodontology 


\title{
CEPHALOMETRIC EVALUATION OF BIONATOR THERAPY IN THE EARLY TREATMENT OF CLASS II MALOCCLUSIONS
}

\author{
A Thesis \\ Presented for \\ The Graduate Studies Council \\ The University of Tennessee \\ Health Science Center
}

In Partial Fulfillment

Of the Requirements for the Degree

Master of Dental Science

From The University of Tennessee

By

Daniel C. Sawrie, D.D.S.

May 2008 
Copyright $@ 2008$ by Daniel Corey Sawrie All rights reserved 


\section{ACKNOWLEDGEMENTS}

I would like to thank my wife, Lucy, for her unfailing love and support during my graduate program and during the writing of this thesis. I would also like to thank Dr. Edward Harris for his expertise, continued guidance and leadership. Without the help of Dr. Harris, this project would not be possible. I would also like to thank Dr. Marlin Grimes and Dr. Joe Wasson for serving on my committee. Their direction and support was invaluable. 


\begin{abstract}
Early or two-phase orthodontic treatment of Class II malocclusions is a debated topic in orthodontic circles primarily because the benefits of early intervention have not been consistently reproduced among researchers. The present study was a retrospective analysis of cephalograms from patients with Class II, division 1 malocclusions at the start of treatment. These were 50 consecutively treated youths who received phase 1 (early) treatment with a Bionator appliance and later treatment with full appliances (all treated by a single clinician). The comparison group consisted subjects treated in a single phase with fixed Edgewise appliances only. Importantly, subjects in the two samples were matched, on a one-to-one basis, for demographic and cephalometric variables (ANB, SNA, SNB, NAP, FMA) to ensure comparability in the nature and severity of the malocclusions. The key question was whether the cephalometric results at the end of treatment were comparable between groups. Two-phase subjects were treated on average 1.4 years longer than the one-phase group, though this measure of clock time may obscure the greater ease of treatment chair side. Neither of out two integumental variables ( $\mathrm{Z}$ angle, E plane) differed statistically, but two key skeletal variables did differ, namely ANB and NAP. ANB was significantly smaller $\left(\bar{x}=2.6^{\circ}\right)$ in the twophase group than in the one-phase group $\left(\bar{x}=3.7^{\circ}\right)$, and NAP averaged $2.7^{\circ}$ in
\end{abstract}


the two-phase group compared to a mean of $4.8^{\circ}$ in the one-phase group. Consequently, the two-phase group was discernibly more orthognathic at the end of treatment, with most of the correction coming from mandibular growth rather than maxillary restriction. None of the dental variables differed statistically except the position of the maxillary incisors which were slightly more proclined in the two-phase group, and this was due to operator preference. The two-phase subjects had a lower rate of premolar extraction ( $12 \%$ vs $84 \%$ ) than the one-phase subjects. Overall, the two-phase group achieved a better bony facial profile, with the majority of the skeletal and dental correction occurring during the first phase of treatment. These favorable results contrast with published randomized clinical trials, possibly because of differences in patient selection and appliance design. Our results do support the value of early intervention, though it is not known whether the statistically significant improvements in the facial profile are large enough to be perceptible to the patient and parents. 


\section{TABLE OF CONTENTS}

Chapter

I. INTRODUCTION 1

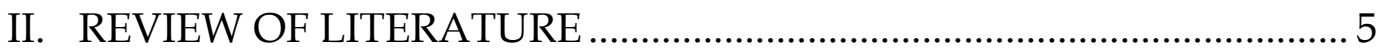

Removable Orthodontic Appliances ................................................ 5

Development of the Bionator......................................................... 6

Functional Appliances and Mandibular Growth .......................... 8

Condylar Cartilage and Mandibular Growth ................................. 9

Treatment Effects Produced by the Bionator Appliance............. 12

Characterizing the Class II Malocclusion ……………………...... 22

Occlusal Development in Untreated Class II Malocclusions..... 24

Comparison of One- and Two-Phase Treatments ....................... 27

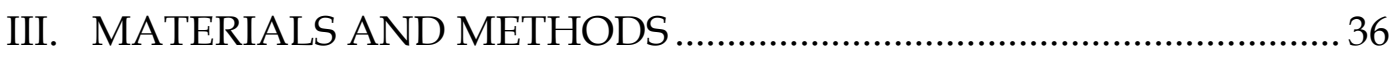

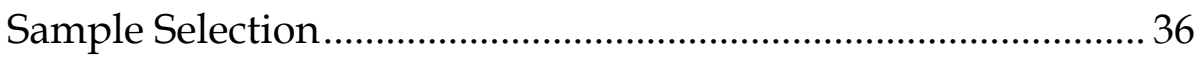

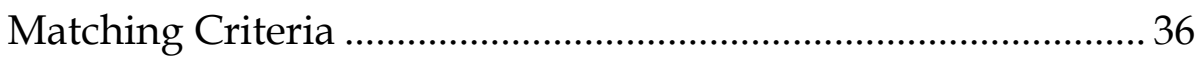

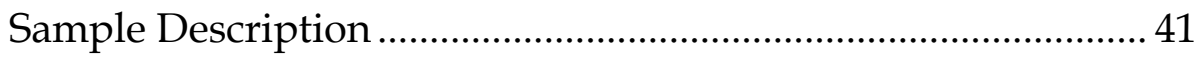

Class II Characteristics of the Sample ............................................ 42

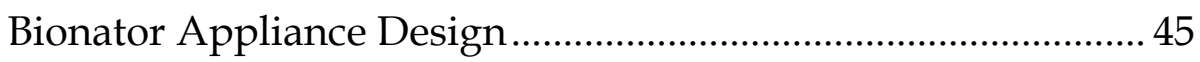

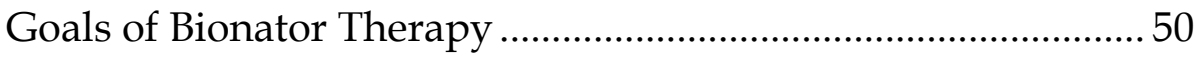

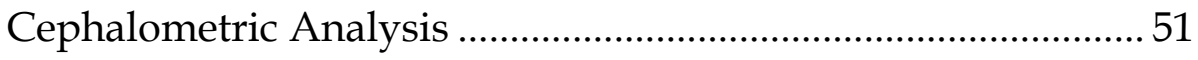


Data Entry 53

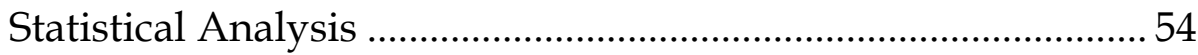

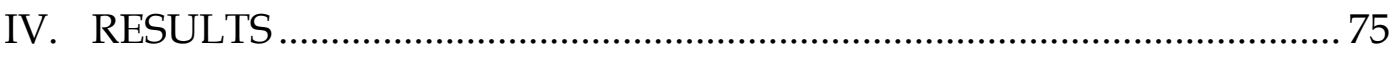

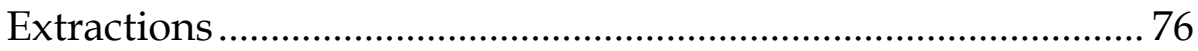

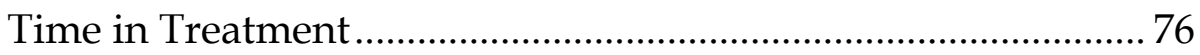

Changes between Phases ………………………………………... 77

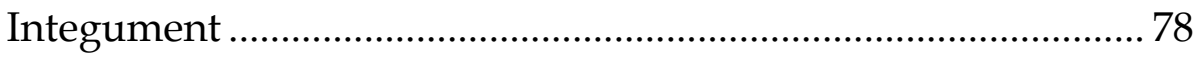

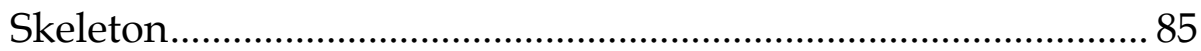

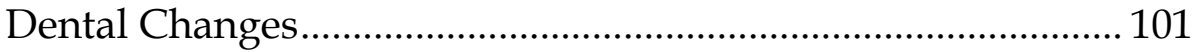

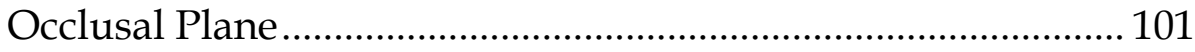

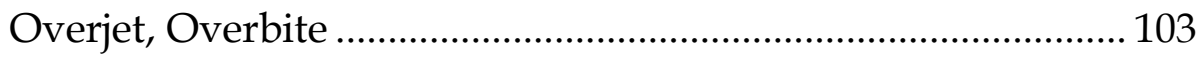

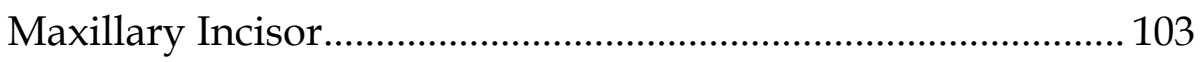

Timing of Orthodontic Correction.............................................. 117

End of Treatment...................................................................... 119

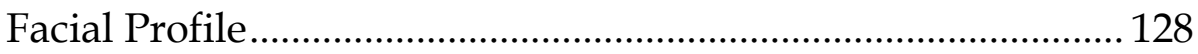

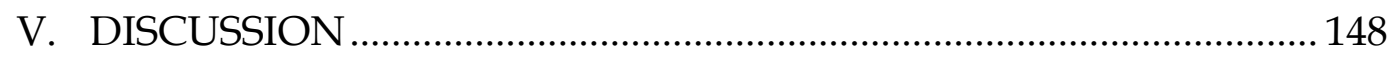

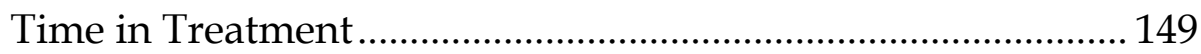

Extraction Treatment ………………………………………........ 151

Patterns of Skeletodental Change …………………………….... 153

Patterns of Dentoalveolar Change ................................................ 155

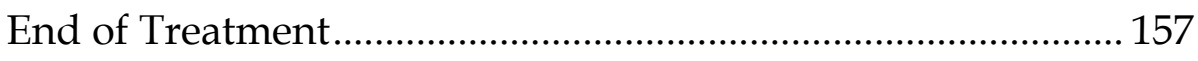

vii 


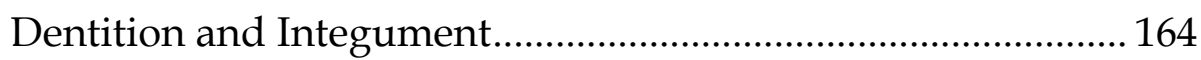

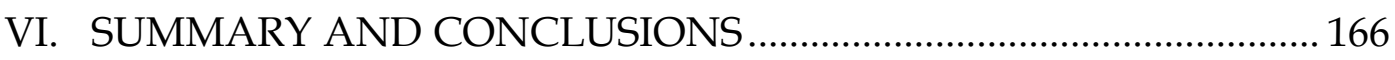

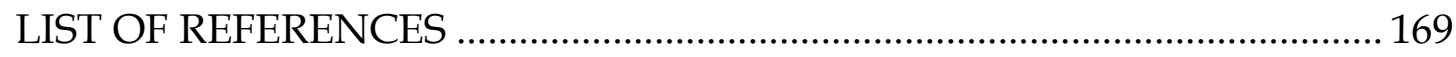

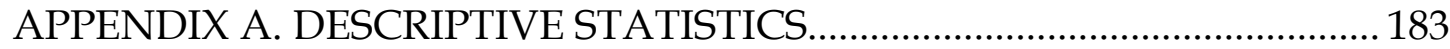

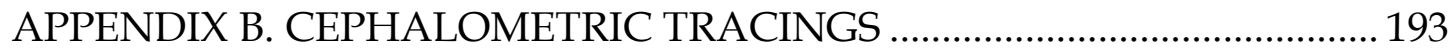

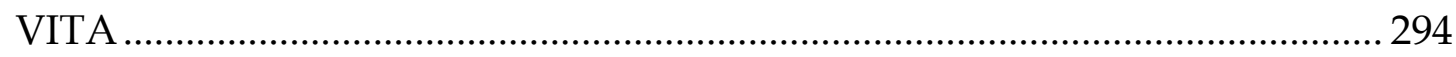




\section{LIST OF TABLES}

Table

1. Average changes in key cephalometric variables from 9 to 13 years of age.

2. Evaluation of the two samples at the start of treatment

3. Tests for group differences in the parasagittal measures of jaw relationships

4. Descriptive statistics of the examination dates, by group ......................77

5. Mean changes, by group (sexes pooled) and by sex within group.

6. Results of mixed-model ANOVA testing for differences in skeletodental changes between the two phases of treatment while controlling for sexual dimorphism

7. Sample means, by group (sexes pooled) and by sex within group

8. Results of mixed-model ANOVA testing for group differences at the end of treatment while controlling for sexual dimorphism 


\section{LIST OF FIGURES}

Figure $\quad$ Page

1. Occlusal view of Bionator appliance used in the study .............................46

2. Lateral view of Bionator appliance used in the study.............................47

3. Frontal view of Bionator appliance used in the study .............................48

4. Cephalometric diagram showing locations of the skeletodental landmarks to be used in this study .....................................56

5. Schematic tracing of a lateral cephalogram showing construction of the SNA angle.

6. Schematic tracing of a lateral cephalogram showing construction of the SNB angle

7. Schematic tracing of a lateral cephalogram showing construction of the ANB angle

8. Schematic tracing of a lateral cephalogram showing construction of the angle of convexity (NAP)

9. Schematic of the method of measuring the AOBO discrepancy.

10. Schematic tracing of a lateral cephalogram showing construction of FMA

11. Schematic tracing of a lateral cephalogram showing construction of the angle formed by the intersection of Frankfort Horizontal and the Se-NA line.

12. Schematic tracing of a lateral cephalogram showing construction of Sella-A Point linear distance measurement 64

13. Schematic tracing of a lateral cephalogram showing construction of Downs' facial angle 
14. Schematic tracing of a lateral cephalogram showing construction of the A Point to Nasion-Perpendicular (labeled D) and B Point to Nasion-Perpendicular (labeled D') measurements.

15. Schematic tracing of a lateral cephalogram showing construction of Sella-B Point linear distance measurement

16. Schematic tracing of a lateral cephalogram showing construction of the angle of the mandibular incisor and mandibular plane (IMPA) .68

17. Schematic tracing of a lateral cephalogram showing construction of FMIA measurement

18. Schematic tracing of a lateral cephalogram showing construction of the angle between the upper incisor with the lower incisor (i.e., interincisal angle)

19. Schematic tracing of overbite and overjet measurements

20. Schematic tracing of a lateral cephalogram showing construction of the $\mathrm{Y}$-axis angle 72

21. Schematic tracing of a lateral cephalogram showing construction of the Downs' occlusal plane angle. 73

22. Schematic tracing of a lateral cephalogram showing construction of Condylion to Gnathion .74

23. Mean changes in the $Z$ angle during phase one (1) and phase two (2) of compound orthodontic treatment, by sex

24. Mean changes in the E Plane during phase one (1) and phase two (2) of compound orthodontic treatment, by sex

25. Mean changes in the $\mathrm{Y}$-axis during phase one (1) and phase two (2) of compound orthodontic treatment, by sex

26. Mean changes in the NAP angle during phase one (1) and 
phase two (2) of compound orthodontic treatment, by sex .88

27. Mean changes in the SNA angle during phase one (1) and phase two (2) of compound orthodontic treatment, by sex

28. Mean changes in the SNB angle during phase one (1) and phase two (2) of compound orthodontic treatment, by sex

29. Mean changes in the ANB angle during phase one (1) and phase two (2) of compound orthodontic treatment, by sex

30. Mean changes in the AOBO discrepancy during phase one (1) and phase two (2) of compound orthodontic treatment, by sex

31. Mean changes in the facial angle during phase one (1) and phase two (2) of compound orthodontic treatment, by sex 94

32. Mean changes in the FMA during phase one (1) and phase two (2) of compound orthodontic treatment, by sex

33. Mean changes in the Mandibular Length during phase one (1) and phase two (2) of compound orthodontic treatment, by sex

34. Mean changes in the Sella-A Point length during phase one (1) and phase two (2) of compound orthodontic treatment, by sex

35. Mean changes in the Sella-B Point length during phase one (1) and phase two (2) of compound orthodontic treatment, by sex

36. Mean changes in the A Point to Na-Perp distance during phase one (1) and phase two (2) of compound orthodontic treatment, by sex

37. Mean changes in the B Point to Na-Perp distance during phase one (1) and phase two (2) of compound orthodontic treatment, by sex 
38. Mean changes in the Occlusal Plane to FH angle during phase one (1) and phase two (2) of compound orthodontic treatment, by sex.....

39. Mean changes in the incisor overjet during phase one (1) and phase two (2) of compound orthodontic treatment, by sex

40. Mean changes in the incisor overbite during phase one (1) and phase two (2) of compound orthodontic treatment, by sex

41. Mean changes in the U1 to Na Point angle during phase one (1) and phase two (2) of compound orthodontic treatment, by sex

42. Mean changes in the $\mathrm{U} 1$ to $\mathrm{Na}$ Point $(\mathrm{mm})$ during phase one (1) and phase two (2) of compound orthodontic treatment, by sex

43. Mean changes in the Interincisal angle during phase one (1) and phase two (2) of compound orthodontic treatment, by sex

44. Mean changes in the L1 to A Pg angle during phase one (1) and phase two (2) of compound orthodontic treatment, by sex

45. Mean changes in the $\mathrm{L} 1$ to $\mathrm{A} P \mathrm{Pg}(\mathrm{mm})$ during phase one (1) and phase two (2) of compound orthodontic treatment, by sex

46. Mean changes in the L1 to Na B Point angle during phase one (1) and phase two (2) of compound orthodontic treatment, by sex.

47. Mean changes in the L1 to Na B Point (mm) during phase one (1) and phase two (2) of compound orthodontic treatment, by sex

48. Mean changes in the IMPA during phase one (1) and 
phase two (2) of compound orthodontic treatment, by sex

49. Mean changes in the FMIA during phase one (1) and phase two (2) of compound orthodontic treatment, by sex

50. Changes during the two phases of treatment

51. Histogram of group averages for NAP angle evaluated at the end of treatment

52. Histogram of group averages for ANB angle evaluated at the end of treatment

53. Histogram of group averages for AOBO evaluated at the end of treatment

54. Histogram of group averages for A Point to NasionPerpendicular evaluated at the end of treatment

55. Histogram of group averages for B Point to NasionPerpendicular evaluated at the end of treatment

56. Histogram of group averages for E Plane evaluated at the end of treatment

57. Histogram of group averages for $\mathrm{Z}$ angle evaluated at the end of treatment

58. Histogram of group averages for SNA evaluated at the end of treatment

59. Histogram of group averages for SNB evaluated at the end of treatment

60. Histogram of group averages for U1 to Nasion-A Point angle evaluated at the end of treatment.

61. Histogram of group averages for U1 to Nasion-A Point ( $\mathrm{mm})$ evaluated at the end of treatment....

62. Histogram of group averages for IMPA evaluated 
at the end of treatment

63. Histogram of group averages for FMIA evaluated at the end of treatment

64. Histogram of group averages for Interincisal angle evaluated at the end of treatment

65. Histogram of group averages for L1 to A-Pogonion angle evaluated at the end of treatment

66. Histogram of group averages for L1 to A-Pogonion $(\mathrm{mm})$ evaluated at the end of treatment.

67. Histogram of group averages for L1 to Nasion-B Point angle evaluated at the end of treatment

68. Histogram of group averages for L1 to Nasion-B Point $(\mathrm{mm})$ evaluated at the end of treatment.

69. Histogram of group averages for incisor overjet evaluated at the end of treatment

70. Histogram of group averages for incisor overbite evaluated at the end of treatment

71. Histogram showing treatment times in years 153

72. Plot of mean NAP angle at the start and end of treatment in the two groups (sexes pooled)

73. Plot of mean AOBO discrepancy at the start and end of treatment in the two groups (sexes pooled)

74. Plot of mean SNA angle at the start and end of treatment in the two groups (sexes pooled)

75. Plot of mean SNB angle at the start and end of treatment in the two groups (sexes pooled).

76. Plot of mean ANB angle at the start and end of treatment 
in the two groups (sexes pooled) 


\section{CHAPTER I}

\section{INTRODUCTION}

Class II malocclusions are a prevalent type of skeletodental disharmony. Not only is the correction of these malocclusions challenging to the specialist, but the underlying etiological factors that produce the malocclusion are both variable and poorly understood. Insufficient mandibular growth rather than maxillary excess is a common issue with Class II malocclusions. One traditional form of fixed orthodontic treatment is extra-oral anchorage coupled with full appliances to retain maxillary position in the anticipation of favorable mandibular growth.

An alternative to conventional treatment in the early permanent dentition is the use of functional appliances in early or so-called phase 1 treatment in the mixed dentition. Functional appliances have been employed to promote the correction of skeletal Class II malocclusions by propulsing the mandible into a more desirable position to "encourage" mandibular growth and dental correction. True to its name, the intended goal of a functional appliance is to alter the functional environment in order to alter the form of the surrounding structures.

Since its introduction in the early 1960s, the Bionator has been a popular functional appliance for the early treatment of Class II malocclusions (Graber 
2005). The treatment value of functional appliances has been a topic of debate, and there has been considerable discussion in the orthodontic literature regarding the biological and clinical advantages and disadvantages of early orthodontic treatment. Some have argued that early treatment has no longterm advantage (Tulloch et al. 2004). Others argue that early treatment may reduce treatment time and reduce the complexity of the second phase of treatment (Dugoni 1998). Reported benefits of early treatment include the ability to intercept and/or reduce dentoalveolar, skeletal, and muscular abnormalities prior to the eruption of the permanent dentition (McNamara and Brudon 1998). Moyers (1998) suggests that early treatment of Class II malocclusions may harness normal growth to gain skeletal correction before the malocclusion becomes more severe, and he notes that there often is greater compliance in younger patients.

Other reported benefits include: reduced need for extraction, better treatment results, less potential for damage such as tooth fracture prior to treatment, and improved patient self esteem (Bishara et al. 1998). Opponents of two-phase treatment in Class II malocclusions contend that early treatment produces no reduction in the average time a child is in fixed appliances nor does it reduce the proportion of complex treatments involving extractions or orthognathic surgery (Tulloch et al. 2004). 
In cases of Class II malocclusions due to mandibular deficiencies, functional appliances often are used with the intent of stimulating mandibular growth. The notion that functional appliances can enhance mandibular growth has been controversial. Björk (1951) and Nelson et al. (1993) concluded that functional appliances contribute little to final mandibular size. In contrast, authors such as Bolmgreen and Mishiri (1986), Mills (1991), Ghafari and coworkers, (1998), and Illing et al. (1998) conclude that the use of functional appliances significantly enhances mandibular growth. Other research indicates that the treatment effects of functional appliances are restricted to dentoalveolar compensations (Chadwick et al. 2001).

Of principal concern is the benefit of early Class II treatment in the mixed dentition compared with treatment started in the early permanent dentition. What is gained with regards to treatment outcomes with a twophase treatment protocol compared to one-phase? A recent study on this question concluded that there is no difference between subjects treated in two phases compared to those treated in a single phase (Tulloch et al. 2004).

In a randomized clinical trial subjects are not allocated according to who would respond favorably to a certain treatment modality based on clinical evaluation; rather, they are randomly assigned to the treatment protocols. Although allocation bias is curtailed, the ability to choose treatment modalities based on clinical evaluation also is eliminated. Subjects who may respond 
favorably to early treatment may not be assigned to the treatment modality best suited to them.

The purpose of the present study is to gain substantive information through the analysis of cephalometric radiographs about the consequences of the Bionator during early treatment. Orthodontic cases were studied in which treatment was used coupled with subsequent fixed appliance treatment. By comparing the outcome of these cases to a matched group of cases treated with only fixed appliances in the early permanent dentition, the intent is to assess the relevant skeletodental benefits, if any, of the extra effort involved in twophase treatment. 


\section{CHAPTER II}

\section{REVIEW OF THE LITERATURE}

The use of removable appliances for the treatment of skeletodental discrepancies has been reported as early as the late nineteenth century. The term "functional jaw orthopedics" was originally employed to describe the form of treatment where these appliances were used to alter the patient's functional environment in hopes of improved dental and skeletal correction (Graber and Neumann 1984).

\section{$\underline{\text { Removable Orthodontic Appliances }}$}

Norman Kingsley in 1879 described a bite plate to change or "jump" the bite in patients who exhibited excessive overjet and retrognathic mandibles. The term "jumping the bite" originally described the concept of getting the mandible to "jump" into a favorable occlusal position and inducing it to stay there. This treatment was often utilized after expansion of the arches, but often did not prove successful due to the mandible's tendency to revert to its original position. In 1902 Pierre Robin described the monobloc appliance that he used for bimaxillary expansion. Watry (1910) later coupled use of the monobloc with muscular exercises for what he termed "functional reeducation." This would be a precursor to the activator appliance, but differed by having the 
mandible postured forward by the patient's voluntary muscle action only. Rogers (1918) recognized the influence of the facial musculature on orthodontic treatment and recommended exercises to develop these muscles. He was one of the first to emphasize the importance of the musculature on the growth and development of the oral environment. In 1908 Andresen made an appliance for his daughter meant for retention and to prevent mouth breathing. The device entailed a bite plate with extensions to cover the lingual surfaces of the mandibular teeth. After seeing the favorable results obtained on his daughter, Andresen recognized the potential of the new appliance. The development of the activator proved to be a milestone in the history of orthodontics and was a forerunner to the development of many other functional appliances (Graber and Neumann 1984).

\section{Development of the Bionator}

The Bionator is an activator-derived device that was introduced by Balters (1893-1973) in the early 1960s. Balters' treatment was holistic; the Bionator was used in conjunction with postural exercises, gymnastics, and dietary control. Balters' objective in treatment was to establish a muscular equilibrium between the tongue and the "outer neuromuscular envelope" (Eirew 1981). According to Balters, the equilibrium between the tongue and cheeks is paramount in the normal development of the dentition and of the 
dental arches, and providing the tongue with adequate functional space is of utmost importance (Graber and Neumann 1984). In Balters' perception, Class II malocclusions are a result of a backward (dorsal) position of the tongue that disturbs the cervical region and impedes respiratory function, causing faulty swallowing patterns and mouth breathing. Therefore, one of the main objectives of treatment in Class II division 1 malocclusions is to bring the tongue forward. In turn, continuously posturing the mandible forward also enlarges the respiratory pathways and enhances deglutition (Graber and Neumann 1984). According to Balters, the essential points for treatment are (1) to accomplish lip closure and bring the tongue back into contact with the soft palate, (2) to enlarge the oral space and to train its function, (3) to bring the incisors into an edge to edge relationship, (4) to elongate the mandible that will enlarge the oral space, and (5) to achieve an improved relationship of the jaws, tongue and dentition, as well as the surrounding soft tissues (Graber and Neumann 1984). The Bionator is indicated in the treatment of Class II, division 1 malocclusions when (1) the dental arches are originally well aligned, (2) the mandible is in a posterior position, (3) the skeletal discrepancy is not too severe, and (4) there is labial tipping of the upper incisor crowns (Eirew 1981). 


\section{$\underline{\text { Functional Appliances and Mandibular Growth }}$}

Enhanced mandibular growth with the use of functional appliances has been reported by many authors, but whether these findings indicate a true overall increase in mandibular length is a topic of debate. Functional protrusive appliances have been employed in laboratory animals to assess mandibular growth vectors and temporomandibular joint changes due to continued forward posturing. Oudet and others (1984) investigated the effects of a functional protrusive appliance on mandibular growth in rats. After 4 weeks of treatment, the experimental group showed an accelerated growth rate of the condylar cartilage, and the direction of growth became more backward oriented. McNamara et al. (1987) evaluated mandibular changes in rhesus monkeys that were fit with an adapted functional appliance. Cephalometric analysis of the 11 treated monkeys compared to controls $(n=12)$ revealed mandibular length increases of 5 to $6 \mathrm{~mm}$ over the 14 weeks of the experiment. Sampson et al. (2001) evaluated changes in mandibular condylar growth in sheep fitted with an experimental functional appliance. Four, 4-month old male sheep were fitted with the adapted appliance and were evaluated 15 weeks later. Fluorescence microscopy performed after sectioning of the temporomandibular joint revealed a range of 8.5 to $13.3 \mathrm{~mm}$ of condylar growth, with a mean of $11.4 \mathrm{~mm}$. The sheep in the control group exhibited a range of 8.8 to $11.9 \mathrm{~mm}$ of condylar growth with a mean of $10.6 \mathrm{~mm}$. Rabie $e t$ 
al. (2003) reported an increase in the concentration of Sox 9 (a regulator of chondrocyte differentiation) in a study involving 5-week-old female rats wearing adapted functional protrusive appliances.

\section{Condylar Cartilage and Mandibular Growth}

Correction of mandibular retrognathia by increased condylar growth due to hyperpropulsion of the mandible has been investigated. Studies vary in the manner in which the mandible was anteriorly positioned; some appliances were removable, while others were fixed. The histological composition of the temporomandibular joint has been shown, both in laboratory and clinical studies, to be susceptible to local extrinsic factors.

Condylar cartilage of the mandible is unique in several respects. Whereas the primary effectors of growth of the epiphyseal cartilages of long bones, nasal cartilage, and cranial base synchondroses are growth hormone and somatomedin, condylar cartilage is to a great degree regulated by extrinsic local factors (particularly tension of the lateral pterygoid muscle), as well as growth hormone (Petrovic et al. 1974; Koski 1977). Structurally, condylar cartilage consists of three basic zones. The articular zone is fibrous connective tissue and is located at the most superior aspect of the condyle. Internal to the connective tissue layer is the proliferative zone where appositional growth occurs by differentiation of mesenchymal cells into prechondroblasts, 
chondroblasts, and chondrocytes. The third, deepest zone is the hypertrophic zone, where chondrocytes are enlarged and bone formation occurs (Durkin 1972; McNamara et al. 1975).

Any type of appliance that causes chronic contraction of the lateral pterygoid muscle, reduction of the serial sacromeres, and, thus, actual shortening of the muscle has been shown by Petrovic (1975) to induce cellular proliferation in the prechondroblastic zone of the condylar cartilage. The "servosystem" theory is valuable here by way of interpretation (Petrovic et al. 1981). Petrovic and coworkers stated that the maxilla, in normal function, is under considerable genetic control and is a constantly changing reference point that the mandible must occlude with and react to. The optimal occlusal and cuspal relationship is a Class I molar relationship, whereas Class II and Class III relationships fall short of this. However, so long as a given occlusal relationship is not interrupted, skeletal and dental growth continues. Introduction of an appliance interrupts the occlusal relationship, and, consequently, initiates a postural response. The lateral pterygoid contracts, and protrudes the mandible in search of a comfortable intercuspal relationship from which dentoskeletal growth can continue. This action of the lateral pterygoid appears to be a prime cause of condylar cartilage proliferation where chronic protrusion induces endochondral bone growth (Petrovic et al. 1975). It is generally understood that the condyle is not a "pacemaker" for growth of the 
mandible. Instead, it possesses a capacity for growth and remodeling in selective response to varied mandibular displacement and rotation. Very simply, it provides regional adaptive growth (Enlow 1968).

Numerous studies have investigated the temporomandibular joint's response to functional appliance therapy, principally focusing on the condyle and glenoid fossa. Charlier and Petrovic (1969) described changes in the amount and rate of condylar cartilage growth in rats after mandibular hyperpropulsion. Stöckli and Willert (1973) performed a histological study of adaptations in the temporomandibular joint during induced protrusive function in monkeys. These authors suggested that the adaptive tissue changes seen in the temporomandibular joint could be induced by mechanical stimuli, and that these changes were resistant to relapse after the removal of the appliances. McNamara (1975), in a histological evaluation involving rhesus monkeys fitted with appliances to induce protrusive function, reported similar findings and identified the age of the subject as a principal factor for response to function alterations. He stated that, "a stimulus which may lead to adaptive changes at one particular time may be ineffective at a later stage of development " (McNamara 1975:133). Zhao et al. (1999) reported new bone formation through analysis of histological sections from the posterior aspect of the glenoid fossa in rats fitted with a bite-jumping appliance. Ruf and Pancherz (1999) examined magnetic resonance images of the 
temporomandibular joint of 25 adolescent and 14 young adult patients treated with the Herbst appliance for treatment of Class II malocclusions. In all of the adolescents, condylar remodeling occurred in the posterior aspect as well as remodeling in the posterior aspect of the glenoid fossa. In contrast, Katsavrias (2003) studied lateral tomograms of 35 patients (18 boys, 17 girls) treated with activator appliances and found no posttreatment changes in any portion of the articular eminence.

\section{Treatment Effects Produced by the Bionator Appliance}

Treatment effects produced by the Bionator appliance can be divided into two types, namely the effects on dentoalveolar development and the effects on skeletal development. Janson (1977) evaluated 134 patients treated with the Bionator for 12 to 18 months, and compared them to 73 untreated individuals. Each individual presented with a Class II malocclusion. Lateral cephalometric radiographs and hand-wrist films were taken at the start and end of treatment. Treatment was deemed complete when a Class I molar relationship was achieved along with an average of $2 \mathrm{~mm}$ of overjet. Skeletal maturity was assessed for both time points using hand-wrist films, and 14 cephalometric variables were used to evaluate the dentoalveolar and skeletal changes. Both dentoalveolar and skeletal changes occurred in the treatment group, but the dentoalveolar changes were greater as shown by an increase in 
protrusion of the lower incisors and retrusion of the upper incisors. Although there were marked skeletal changes in some individuals in the treatment group, especially those who began treatment just prior to the pubertal growth spurt, the authors found no significant difference between the two groups regarding the skeletal measurements. They ascertained that the Class II to Class I correction was achieved mostly through dentoalveolar adaptation in combination with a reduction in ANB through the natural pattern of mandibular growth.

Janson (1987) conducted another study using the same sample of 134 patients treated only with the Bionator. Of the 134 patients, 99 required fixed orthodontic appliances after Bionator therapy while the other 35 individuals showed a stable Class I occlusion with adequate alignment of the teeth following Bionator therapy alone. Five years after treatment, 28 of the 35 patients treated with the Bionator alone were compared to 31 of the 99 patients who underwent Bionator therapy and later fixed appliances. All patients were treated nonextraction to a Class I molar relationship. Full orthodontic records were taken on each patient, and cephalometric data were compared at four different time points: (1) at the start of treatment, (2) after the establishment of a Class I molar relationship, (3) at the completion of treatment with either the Bionator or fixed appliances, and (4) three to five years out of treatment. The individuals treated only with the Bionator exhibited a greater mandibular 
response as shown by an increase in mandibular length (Cd-Gn) and a subsequent reduction in ANB. In the other group, the reduction in ANB was accomplished mainly through the restriction of maxillary growth.

Bolmgren and Moshiri (1986) investigated the treatment effects of the Bionator on Class II division 1 patients in a cephalometric evaluation of 12 males and 8 females who were selected for Bionator treatment. The average age at the start of treatment was 11.8 years for the boys and 11.1 years for the girls. The duration of the Bionator therapy ranged from 8 to 20 months with a mean interval of 12.7 months. The second phase of treatment lasted on average of 15.9 months. The control groups consisted of cephalometric data from untreated males and females of comparable ages as reported by Riolo et al. (1974), and patients treated with only fixed appliances with the cephalometric data obtained from the records of the Rocky Mountain Data Systems in California. In the Bionator group, lateral cephalometric radiographs were taken at three time points: (1) at the start of treatment, (2) after Bionator treatment, and (3) after fixed appliances. The Bionator produced some maxillary incisor retraction and uprighting and protrusion of the lower incisors. The mandibular plane was minimally increased. There was an increase in mandibular length (Cd-Gn) of $5.0 \mathrm{~mm}$ in the Bionator group, 4.4 $\mathrm{mm}$ in the fixed appliance group, and $2.5 \mathrm{~mm}$ in the untreated group. There was a minor increase in anterior facial height in the Bionator group. The 
authors concluded that the orthodontic and orthopedic changes that occurred in the group treated with the Bionator and later fixed appliances were nearly identical to those that occurred in the group treated with fixed appliances alone.

Heij and others (1989) evaluated the influence of variable degrees of protrusion built into the Bionator on treatment effects in a group of patients with Class II division 1 malocclusion. Patients $(\mathrm{n}=27)$ matched for age, sex, and type of malocclusion were divided into two groups based on the varying amounts of protrusion built into the construction bite used for the construction of the Bionator. Group one consisted of six females and eight males with an average age of 10.3 years. The Bionator for this group was fabricated using a construction bite taken with the incisors in an end-to-end position with 2 to 4 mm of freeway space. The patients were instructed to wear the Bionator 22 hours per day. Group 2 consisted of 7 females and 6 males with an average age of 10.3 years. The Bionator for this group was fabricated from a construction bite with the maximum possible propulsion of the mandible in which the patient felt comfortable (usually the lower incisors were $2 \mathrm{~mm}$ in front of the upper incisors). The patients wore the appliance 12 to 14 hours per day. Lateral cephalometric radiographs were taken on each patient before and after treatment. As expected, in both groups there was a decrease in ANB and SNA angles along with a decrease in overjet and overbite. An increase in the 
amount of protrusion beyond edge to edge did not result in an increase in ramal growth. However, an increase in protrusion past edge to edge did improve the mandibular sagittal relationship, possibly due to the downward displacement of the condyle. The "headgear effect" was more pronounced in the edge-to-edge group. The results suggested that changes in ramal height, condyle displacement, and the "headgear effect" seem to be related to the amount of protrusion built into the construction bite.

Mammandras and Allen (1990) conducted a cephalometric analysis of 40 Class II division 1 patients treated with the Bionator. They were divided into two groups based on the horizontal change of Pogonion from pretreatment to posttreatment. Group one deemed the "small advancement group" consisted of individuals with a mean age of 12.4 months and who exhibited $3 \mathrm{~mm}$ or less of anterior movement of skeletal Pogonion. Group two, or the "large advancement group," consisted of subjects with a mean age of 13.2 months exhibiting at least $3.5 \mathrm{~mm}$ of anterior movement of Pogonion. Despite being slightly younger, the small advancement group exhibited mandibular dimensions that were similar or greater than the other group. Compared to published growth standards (Michigan Growth Study), males in the large advancement group had mandibular lengths (Cd-Gn) that were significantly smaller. The females exhibited this same trend. The pretreatment mandibular lengths of the individuals in the small advancement group did not differ 
significantly. The posttreatment mandibular lengths of the individuals in the small advancement group did not differ significantly from untreated controls. However, in the large advancement group, posttreatment comparisons revealed that the males experienced a $2.6 \mathrm{~mm}$ increase in mandibular length and females underwent a $4.3 \mathrm{~mm}$ increase. These results indicated that individuals with smaller mandibles, as compared to published growth standards, may benefit more from functional appliance therapy. In these individuals, the Bionator may serve to create an optimal environment for increased mandibular growth.

Lange and others (1995) conducted a study to determine the effects of the Bionator on the skeletal and dental tissues. Their sample consisted of 60 individuals with Class II division 1 malocclusions. Of the 60, 30 were treated with a Bionator appliance while the other 30 did not receive any form of orthodontic treatment. The four inclusion criteria consisted of the following: ANB of $5^{\circ}$ or more, FMA of $20^{\circ}$ to $29^{\circ}$, overjet of 6 to $10 \mathrm{~mm}$, and a positive overbite. Lateral cephalograms were taken before and after treatment, with treatment time averaging 18.7 months. SNB increased $1.0^{\circ}$ more in the Bionator group than in the control group. Mandibular length (Ar-Gn) increased $6.4 \mathrm{~mm}$ in the treated group compared to $2.8 \mathrm{~mm}$ in the untreated group. SNA decreased $0.5^{\circ}$ in the Bionator group and increased $0.4^{\circ}$ in the control group. In the Bionator group, there was a reduction in ANB of $2.0^{\circ}$. 
The maxillary incisors were retroclined by $3.9^{\circ}$, and the mandibular incisor angulation did not change. These results indicate a significant skeletal and dentoalveolar change in the treated group; however, the patients in the sample were chosen based on a successful treatment outcome with the Bionator and not chosen at random.

Illing et al. (1998) randomly allocated 47 adolescent patients to three different functional appliance groups for treatment with the Bionator, Twinblock, or Bass appliance. After roughly 9 months of treatment, the groups were compared to each other and to an untreated control group using pretreatment and posttreatment cephalograms. A significant increase in mandibular length (3.9 $\mathrm{mm}$ as measured from Cd-Gn) was observed in the Bionator group as well as a $3.7 \mathrm{~mm}$ increase in the Twin-block group. The Bionator group also demonstrated a significant increase in lower face height (ANS-Me). There was a reduction in the proclination of the upper incisors in all of the treated groups, and the Bionator group exhibited the most proclination of the lower incisors $\left(4.0^{\circ}\right)$. Of the three appliances, the Twin-block and the Bionator seemed to be the most effective in producing sagittal and vertical changes.

Ahn, Kim and Nahm (2001) defined some cephalometric predictors for good treatment results when using the Bionator in the treatment of Class II division 1 malocclusions. Their sample consisted of 40 patients, all of whom were in the mixed dentition and exhibited Class II, division 1 malocclusions. 
They were all treated with the Bionator for an average of 20 months. The 40 individuals were divided into two groups based on their treatment result. The criteria for a good treatment result consisted of: (1) Class I molar, (2) overjet and overbite within the normal range of 1-3 mm, (3) clinically acceptable profile as defined by the upper and lower lips within 1 standard deviation of Rickett's esthetic line, and (4) little relapse of the anterior and posterior relationships when assessed 2 to 5 years after Bionator therapy. All of these criteria had to be met for a case to be placed in the good treatment result group, which consisted of 20 patients with an average age of 10.4 years. Group 2 consisted of 20 patients, with an average age of 10.3 years, who did not meet the criteria for a good treatment result. Lateral cephalometric radiographs were taken before and after treatment, and they were used to assess orthodontic and orthopedic changes during treatment. A horizontal growth pattern, close to normal anteroposterior relationship between the maxilla and mandible, retrusive lower incisors, and a retrusive lower lip were all identified as possible predictors for a good treatment result with the Bionator.

Almeida and coauthors (2002) compared the dentoalveolar and skeletal cephalometric changes produced by the Fränkel and Bionator appliances in patients with Class II malocclusions. The sample consisted of 66 patients divided into 3 groups of 22. The first group consisted of 22 children (11 males, 11 females with a mean age of 9 years 10 months) who were treated for an 
average of 17 months with the Fränkel appliance. The second group consisted of 22 patients (11 males, 11 females with an average age of 10 years 8 months) treated with the Bionator for an average of 16 months. The control group consisted of 22 Class II children (mean age of 8 years 7 months) who were untreated but observed for 13 months. Cephalometric radiographs were taken at the beginning and end of treatment. No significant change in maxillary growth was observed during the observation period in either group. However, a statistically significant increase in mandibular length (Cd-Gn) of $2.0 \mathrm{~mm}$ occurred in the Bionator group. Both groups showed a slight downward rotation of the palatal plane, indicating no difference in craniofacial growth direction. The Bionator appliance produced a labial tipping of the lower incisors $\left(+2.6^{\circ}\right)$ and a lingual inclination of the upper incisors $\left(-5.5^{\circ}\right)$. In conclusion, the authors stated, "The major treatment effects of Bionator and FRII appliances were dentoalveolar, with a smaller, but significant, skeletal effects" (Almeida et al. 2002:464).

Faltin et al. (2003) studied the long-term effects and optimal treatment timing for Class II treatment with the Bionator. The cephalometric records of 23 patients consecutively treated with the Bionator were evaluated and compared to an untreated control group. The individuals whose treatment time included the pubertal growth spurt showed a significant reduction of overjet $(-4.2 \mathrm{~mm})$ and an increase in mandibular length (Cd-Gn) of $5.1 \mathrm{~mm}$. 
Their findings indicate that when treatment with the Bionator is coincident with the pubertal growth spurt, significant skeletal and dentoalveolar changes can be achieved.

Araujo and coauthors (2004) looked at mandibular response to Bionator therapy in 25 patients ( 15 males, 10 females). Using implants as immobile landmarks, each patient's pretreatment and posttreatment radiographs were superimposed. A horizontal and vertical reference line were determined by the implants and used to assess changes in specific mandibular landmarks (Me, $\mathrm{Gn}, \mathrm{Pg}, \mathrm{Ar}$ ). The functional appliance therapy changed the direction of condylar growth to a more posterior direction as measured from Condylion and Articulare. However, this redirection of growth did not translate into an increased total amount of mandibular growth.

Jena and others (2006) further investigated the dentoalveolar and skeletal effects of the Twin-block and Bionator appliances in the treatment of Class II malocclusions. Girls $(\mathrm{n}=55)$ with the same CVM status were selected on the following criteria: (1) Class II division 1 malocclusion, (2) stage 3 CVM, (3) full-cusp Class II molar relationship, (4) FMA of at least 25ㅇ, (5) little or no crowding, and (6) overjet of 6 to $10 \mathrm{~mm}$. The control group consisted of 10 of the 55 females who were left untreated and observed until the end of the study. The remaining 45 patients were divided into two groups with 25 receiving treatment with the Twin-block appliance and 20 receiving treatment with the 
Bionator appliance. Lateral cephalometric radiographs were taken at the beginning and end of treatment, and these were used to assess the skeletal and dentoalveolar changes that occurred during treatment. Neither appliance significantly restricted maxillary growth. However, the authors observed a 1.6 and $1.0 \mathrm{~mm}$ increase in mandibular length (Cd-Gn) with the Twin-block and Bionator groups, respectively. The maxillary molars were effectively restricted in both treatment groups. The upper incisors were retroclined $0.6 \mathrm{~mm}$ in the Bionator group while the lower incisors were proclined $1.5 \mathrm{~mm}$. Both appliances were effective in attaining molar correction and reducing overjet, but the changes during treatment stemmed mostly from dentoalveolar compensations rather than skeletal adaptations.

\section{Characterizing the Class II Malocclusion}

In a sample of Class II individuals, Elsasser and Wylie (1943) observed that maxillary protrusion occurred in males while the maxilla was in a relatively neutral position in females. No difference was noted in maxillary molar positioning compared to a Class I control group. In addition, these investigators found the mandibular length to be within normal limits for males, while it was less than normal in females.

Renfroe (1948) studied facial patterns in Class II malocclusions and observed that the maxilla was generally in a retrusive position in both sexes 
with maxillary incisor protrusion and molar retrusion relative to a Class I sample. He noted, as did Henry (1957), that while some Class II individuals have a deficiency in mandibular size, others have well formed mandibles of normal size. However, these normal mandibles are in a retruded position due to the posterior position of the glenoid fossae. Renfroe concluded that the mandibles of Class II individuals are retrognathic relative to other craniofacial structures.

Through an investigation of Class II individuals, Riedel (1952) determined that the maxillary skeletal base was normally positioned in both sexes but with incisor protrusion. He also noted that the mandible was retrusive relative to the averages of Class I individuals.

Henry (1957) developed a classification of Class II division 1 malocclusions. He selected his sample according to angle's classification system, and categorized four groups for this malocclusion: (1) maxillary alveolar protrusion; (2) maxillary basal protrusion; (3) micromandible; and (4) mandibular retrusion. From cephalometric evaluation, Henry noted an increased mandibular plane angle in Class II cases compared to Class I norms, suggesting an increase in lower facial height.

In assessing a Class II sample, Hunter (1967) found the maxilla to be in a relatively neutral position, but with incisor protrusion. The mandibular 
skeletal position was retrognathic while the mandibular incisors were retruded. He also observed a slight increase in anterior facial height.

McNamara (1981) examined a series of Class II patients to determine the nature and frequency of specific contributing components. The study was a cross-sectional evaluation of the lateral cephalograms of 277 children ages 8 to 10 years. From these records, he assessed maxillary and mandibular skeletal and dental relationships. The most common findings were an excessive lower facial height and mandibular retrusion.

Moyers et al. (1980) in a study of 697 North American white children divided Class II individuals into six horizontal and five vertical groups. Using a procedure of aborization, 15 subtypes of Class II malocclusions were established. Analysis of these subtypes revealed persistent skeletal characteristics for each group during intervals of growth. He concluded that $20 \%$ had a maxillary protrusion while over $50 \%$ had a mandibular retrusion with little, if any, maxillary protrusion.

\section{Occlusal Development in Untreated Class II Malocclusions}

An indication of how to treat a malocclusion may be gained by observing how it changes with time. White (1983) examined 34 patients who began with an end-to-end molar relationship in the early mixed dentition. Following these until the end of the mixed dentition, he observed that 24 
developed a Class I molar relationship, while the other 10 developed Class II relationships. The maxillary molars moved mesially an average of $2.3 \mathrm{~mm}$, and the average mandibular increment was $1 \mathrm{~mm}$ in a mesial direction from the initial observation. These tooth movements produced a worsening of the molar relationships. White (1983) concluded that differential jaw growth was a more important factor in the process of molar adjustment than mesial drift of the molars.

Whitney (1983) evaluated an untreated longitudinal Class II sample and recognized eight groups within this type of malocclusion. The groups displayed an array of skeletal variations and severities of protrusiveness and retrusiveness of the skeletal base. A majority of the cases were mandibular retrusive. He noted a tendency for maxillary protrusion with a maxillary bony arch that was consistently longer than the mandibular corpus. The differential between the two arches increased with age, resulting in a progressive worsening of the Class II relationship. Behrents (1985) conducted a follow-up study of the same sample and found that, while growth continues into adulthood, existing maxillomandibular relationships are maintained in a fairly uniform manner with only small variations.

Ngan, Byczeck, and Scheick (1997) compared skeletal growth changes between Class II, division 1 and Class I females between the ages of 7 and 14 years. Lateral cephalometric radiographs had been taken annually from age 5 
through 17. They observed that the maxilla was no more protrusive in the Class II sample when compared with the Class I sample. In fact, there was a decrease in maxillary prognathism in Class II subjects during the pubertal growth period. The maxillomandibular skeletal difference (ANB) was significantly greater in the Class II sample at age 7 and did not improve with age; consequently, skeletal differences maintained a greater degree of facial convexity in the Class II subjects. The authors state that, "results suggest that the Class II skeletal growth pattern is established early and maintained throughout puberty unless altered by orthodontic intervention" (Ngan et al. 1997).

Bacetti and others (1997) observed occlusal features of the Class II malocclusion during the transition from the deciduous to the mixed dentition in untreated subjects. During the observation period, cephalometric changes consisted of significantly greater maxillary growth increments and smaller mandibular increments in the Class II sample. In addition, they observed a downward and backward rotation of the mandible over time with a subsequent decrease in the Gonial angle for the Class II subjects. They concluded, "all occlusal Class II features were maintained or became exaggerated during the transition to the mixed dentition" (Bacetti et al. 1997). These findings are similar to those found by Fröhlich (1961) who reported that no improvement of Class II occlusal relationship occurs from 5 to 12 years of 
ages and Arya and coworkers (1973) who observed that all patients presenting with a distal step relationship of the second deciduous molars exhibit a Class II relationship in the permanent dentition.

Bishara (1988) conducted a cross-sectional and a longitudinal evaluation of the changes in mandibular length and maxillary-mandibular relationships in untreated Class II subjects from the deciduous to permanent dentition. These Class II samples were compared with matched "normal," untreated individuals. Longitudinal comparisons of growth profiles indicated that the growth trends were similar between the untreated Class II, division 1 subjects and normal subjects. There was no "self correction" with growth in the untreated Class II sample.

The aforementioned literature suggests that Class II dental malocclusions do not "self correct" or improve with time; if anything, they worsen with time. This would permit subjects with similar pretreatment Class II malocclusions to be compared with one another regardless of the age at the start of treatment.

\section{Comparison of One- and Two-Phase Treatments}

A goal of "early" orthodontic treatment is to correct existing or developing skeletal, dentoalveolar, and muscular imbalances to improve the orofacial environment before the eruption of the permanent teeth is complete 
(McNamara and Brudon 1993). Anticipation is that early intervention may reduce the overall need for complex orthodontic treatment that may include extractions of permanent teeth or orthognathic surgery. On the surface, this seems reasonable; it appears more logical to prevent an abnormality from occurring rather than waiting until it has developed fully. In a recent survey of the 159 Diplomates of the American Board of Orthodontics, participants were asked what they perceived to be the benefits of early treatment (Bishara et al. 1998). The most common responses were: (1) greater ability to modify growth, (2) improved patient self-esteem and parental satisfaction, (3) better and more stable results, (4) less-extensive therapy required later, and (5) reduced potential for tooth damage such as trauma, root resorption and decalcification. Responses of this survey were supported by a study by King and coworkers (1999) in which orthodontists perceived that subjects who had received phase 1 treatment had less complex malocclusions and lower treatment priority than subjects in an untreated control.

Proponents of two-phase orthodontic treatment often contend that treatment in the late mixed dentition gives the clinician only one chance at correction, and if cooperation is poor the results may be unsatisfactory (Dugoni 1998). In addition, by delaying treatment, many female patients may have passed the peak velocity of their skeletal growth and strategies aimed at growth modification may have reduced effectiveness. According to Dugoni 
(1998) benefits of treating Class II malocclusions in the early mixed dentition include the following: (1) reduced incidence of premolar extraction, (2) reduced need for surgical orthodontics, (3) better patient cooperation, (4) reduced incidence of root resorption, and (5) reduced incidence of ectopic cuspid eruptions. The Department of Orthodontics at the University of the Pacific is conducting a randomized retrospective study to evaluate treatment changes during early mixed dentition treatment (Dugoni 2006). The study sample was restricted to patients who originally were evaluated in the early and middle mixed dentition yielding three study groups: (1) delayed treatment (i.e., no treatment), (2) phase 1 treatment only, and (3) two-phase treatment. All subjects were treated by the same orthodontist. Preliminary analysis of the study indicates that approximately $42 \%$ of patients who received early treatment did not require a second phase of treatment. Subjects requiring full and only phase- 1 treatment had fewer visits, shorter treatment times, and lower fees. In addition, $82 \%$ of subjects in the early treatment group did not require extraction in the permanent dentition.

Another proposed benefit of early orthodontic intervention in Class II malocclusions is improved self-esteem. O'Brien and coworkers (2003) conducted a multicenter, randomized, controlled trial providing early functional appliance treatment for children aged 8 to 10 who presented with Class II, division 1 malocclusions. Comparisons were made to age and sex 
"matched" samples that were untreated. The second phase of the study examined the psychosocial impact of early orthodontic treatment. A total of 89 subjects were allocated to a Twin-block group and 87 to an untreated control group. Data were collected at baseline and 15 months later, at which time no subject was still wearing the Twin-block appliance. A series of questionnaires was used to gather data concerning psychosocial effects of early orthodontic intervention. Results indicated that children who had received early treatment reported higher self-concepts and more positive childhood experiences than the untreated controls.

However, not all clinicians agree, many preferring to wait until all the permanent teeth have erupted (excluding third molars) to start treatment. Opponents of two-phase treatment argue that there are few, if any, benefits that are unique to and dependent on earlier treatment. Gianelly (1995) contends that at least $90 \%$ of all growing patients can be treated successfully in only one phase by starting treatment in the late mixed dentition. Gianelly (1995) proposed that the other $10 \%$ of patients could benefit from immediate resolution of the problem such as those presenting with crossbites or Class III malocclusions. Opponents of two-phase treatment contend that patients only have a limited capacity to cooperate, and treatments that require two phases of compliance and retention may be more than patients can handle (Keeling et al. 1995; Berg 1979). 
Opponents also contend that there is no benefit concerning self-esteem and early Class II treatment. Dann and coworkers (1995) conducted research on children with Class II malocclusions concerning early treatment and effect on self-concept. The study consisted of 209 subjects whose overjet was greater than $4.5 \mathrm{~mm}$ and who were younger than 15 . Self-concept was measured using the Piers-Harris children's self concept scale, which is an 80 item "forced" choice self-report designed to quantitatively assess how children feel about themselves. Untreated controls were used for comparison. The results indicated that there was no change in mean self concept score in treated subjects, nor was there any association between reduction of Class II malocclusion features and improved self-concept. These findings suggest that children with Class II malocclusions do not generally present for treatment with low self-concept and, on average, self-concept does not improve during early orthodontic treatment.

Tulloch and coauthors (2004) conducted a randomized clinical trial of preadolescent (early) versus adolescent (later) treatment of children with severe Class II malocclusions. Severe malocclusions were those having an overjet greater than $7 \mathrm{~mm}$. A total of 166 preadolescent children in the mixed dentition with Class II division 1 malocclusions were randomly assigned to one of three groups: (1) headgear, (2) Bionator, or (3) no treatment (i.e., observation). After 15 months, records were taken and the groups pooled together and each child 
was randomly assigned to one of four orthodontists for treatment with traditional fixed appliances. Cephalometric radiographs were used to assess skeletal changes. The peer assessment rating (PAR) was used to rate alignment and occlusion. In the evaluation of Phase I treatment, statistically significant differences were observed between the treatment and observation groups although response was widely variable. The change in jaw relationship (annualized reduction in ANB angle) was favorable in $76 \%$ of the headgear group, in $83 \%$ of the functional appliance, and in $31 \%$ of control (observation only) group. Evaluation of Phase II treatment evaluated whether these changes represented long term changes. Results from Phase II of the study indicate that the initial correction becomes overshadowed with time. Differences among groups with respect to skeletal relationship and PAR score were not statistically significant at the end of Phase II treatment. In addition, two-phase treatment appeared to be inefficient in that it did not reduce the average time a child spent in fixed appliances nor did it reduce the complexity of later treatment (need for extraction or orthognathic surgery). "During phase 2 of the trial, the advantage created during phase 1 of treatment in the two early treatment groups was lost, and, by the end of fixed appliance treatment, there was no significant difference between any of the three groups" (Tulloch et al. 2004:660). 
O'Brien and coauthors (2003) evaluated the effectiveness of early orthodontic treatment with a Twin-block functional appliance in a multicenter, randomized clinical trial. In comparison to the study conducted by Tulloch and coauthors (2004), this study used 14 hospital-based orthodontic specialists in the United Kingdom. Importantly, treatment was provided by many operators outside of a controlled university setting. In summary, the authors wanted to see how effective early Class II treatment is in the "real world" of orthodontic practice outside dental schools. Children $(n=174)$, aged 8 to 10 years with Class II, division 1 malocclusions were randomly allocated to receive treatment with the Twin-block appliance or to an untreated control group. Data were collected at the start of the study and 15 months later. The results showed that treatment with the Twin-block appliance reduced overjets, corrected molar relationships, and reduced the severity of the malocclusions. The majority of the correction was attributed to dentoalveolar changes and small amounts of favorable skeletal change. The study continued until the children had completed phase 2 treatment. Operators treated the children according to their normal treatment protocols. An aim of the study was to learn whether early treatment resulted in a reduced need for phase 2 treatment, and if differences in skeletal pattern or final dental occlusion were evident. At the end of phase 2 treatment, there was no difference between the patients who had early treatment and those who did not for any variable evaluated, and 
most treated subjects required a second phase of treatment. In conclusion, these findings agree with the those of Tulloch and coauthors (2004) in that it appears that early orthodontic treatment for Class II malocclusions does not confer any advantage over a later single-phase treatment.

Breman and Pancherz (2002) studied the efficiency of early and late Class II division 1 treatment. Efficiency was defined as a better result in a shorter time. Pretreatment and posttreatment dental casts of 204 patients in the early mixed $(n=54)$, late mixed $(n=104)$, and permanent $(n=40)$ dentition were evaluated according to the peer assessment rating (PAR). Results showed that treatment time and PAR scores decreased with increasing dental development, indicating that early Class II division 1 treatment is less efficient and less successful than a later one-phase treatment.

Livieratos and Johnston (1995) conducted a study comparing one-stage and two-stage nonextraction alternatives in Class II samples. For all subjects, the clinician's initial intention was non-extraction therapy, either in a one-stage full appliance or two-stage Bionater and full appliance treatment. The study sample consisted of 47 one-stage patients and 49 two-stage patients, all treated by a single orthodontist. Comparisons between the two groups were conducted by examining pretreatment and posttreatment lateral cephalograms. Both groups exhibited similar patterns of skeletal change that could not be distinguished from each other. Skeletal changes, in both groups, were largely 
responsible for molar and overjet corrections. The magnitude of differential jaw growth was greater in the two-phase group, presumably because treatment started earlier and finished later.

Dolce et al. (2005) conducted a similar comparison and observed an early mandibular response in patients treated with a Bionator. The data revealed that the sagittal jaw relationship improved significantly in both Phase 1 treatment groups compared with the observation group. However, this initial mandibular response was not evident after both groups had received full appliance therapy. This study differed from previous studies in that it used centrographic analysis. The centrographic analysis is a visual analysis with no angles to measure or normative values to compare. Landmark position relative to an established reference plane allows the tracking of landmarks in horizontal and vertical planes. 


\title{
CHAPTER III
}

\section{MATERIALS AND METHODS}

\author{
$\underline{\text { Sample Selection }}$
}

This present study is a retrospective analysis of cephalograms from patients with Class II, division 1 malocclusions at the start of treatment. There were 50 consecutively treated patients who received phase 1 (early) treatment with a Bionator appliance and later treatment with full fixed appliances. Cephalometric radiographs were available at $\mathrm{T} 0$ (start of treatment), $\mathrm{T} 1$ (end of phase 1 treatment), and T2 (end of treatment) for these subjects. Records were obtained from the office of Dr. Joe L. Wasson, Memphis, Tennessee, who had treated all of these cases. The control group consisted of a sample of subjects with the same Class II, division 1 malocclusions who were treated at the University of Tennessee, Department of Orthodontics, with fixed appliances only (Standard Edgewise).

\section{$\underline{\text { Matching Criteria }}$}

The author devoted considerable time collecting data to provide a casecontrol matched sample of cases treated orthodontically. As noted above, the principal focus of the study was to evaluate the treatment outcomes of two samples, (1) a series first treated in the mixed dentition with the Bionator then 
transitioned into fixed Edgewise treatment and (2) a series treated in just one phase using conventional Edgewise mechanisms (Sandusky and Gramling 1988) in the early permanent dentition.

The commonly encountered design is to use a group comparison design, where the average characteristics of the two groups are argued to be comparable. This is expedient, but statistically much less efficient than comparing matched samples. In contrast, we first evaluated the two-phase sample and matched an Edgewise case to each based on what we deemed key characteristics of the skeletal malocclusion.

Necessarily, the chronological ages are different in the two groups at the start of treatment, and the issue here is that cephalometric measures of size and shape change as a child grows and develops. We matched the 50 two-phase cases to 50 conventional Edgewise cases using five cephalometric criteria reflective of the bony facial profile, namely (1) the SNA angle, (2) the SNB angle, (3) the ANB angle, (4) the NAP angle, and (5) the FMA angle using the following protocol: The average age at the start of Bionator treatment was 9 years, while the average age at the start of Edgewise treatment in this sample was 13 years. We used a simple, pattern extension method (Johnston 1975) to extrapolate from the 13-year-old status back to 9 years of age when Bionator treatment was initiated, a difference, obviously, of 4 years. As shown from the data on normal untreated children published by Riolo et al. (1974), there are 
only minor age changes in the angular variables of interest here (Table 1). That is, while there is appreciable linear growth during this interval, the shape (angular) changes in these variables are small, with the largest changes occurring in NAP as mandibular growth slightly exceeds maxillary forward growth, so the typical profile becomes less retrognathic with age.

For each Bionator case (at about 9 years of age), the pool of Edgewisetreated cases was reviewed to provide a close match for the five variables. Operationally, we felt there was adequate precision if all the five variables "matched" within 1 or 2 degrees of the 9-year-old Bionator case accounting for the characteristic sex-specific age changes listed in Table 1. Pointedly, we made no special effort to match for dental characteristics of the malocclusion, though all cases began treatment as Class II, division 1 malocclusions.

Table 2 lists the sample means for the two series along with the results of pairing design t-tests (Woolf 1968). This table is expanded to 10 variables, which is more than the 5 actually used for the matching process, but the results are confirmatory that our efforts to match the Edgewise cases with cephalometrically quite-similar two-phase cases was successful. All 5 of the cephalometric variables thought to be most critical (SNA, SNB, ANB, NAP, and FMA) fulfilled expectations of being comparable after accounting for the chronological age difference. Indeed, of the 10 variables compared in Table 2 the Edgewise sample was more aberrant, which is in keeping with these 
Table 1. Average changes of key cephalometric variables from 9 to 13 years of age. ${ }^{1}$

\begin{tabular}{cccccccc}
\hline & \multicolumn{3}{c}{ Boys } & & \multicolumn{3}{c}{ Girls } \\
\cline { 2 - 4 } \cline { 6 - 7 } Variable & $9 \mathrm{yr}$ & $13 \mathrm{yr}$ & Change & & $9 \mathrm{yr}$ & $13 \mathrm{yr}$ & Change \\
\hline SNA & $80.6^{\circ}$ & $81.2^{\circ}$ & $+0.6^{\circ}$ & & $80.5^{\circ}$ & $81.0^{\circ}$ & $+0.5^{\circ}$ \\
SNB & $76.4^{\circ}$ & $77.5^{\circ}$ & $+1.1^{\circ}$ & & $76.5^{\circ}$ & $77.5^{\circ}$ & $-1.0^{\circ}$ \\
ANB & $4.2^{\circ}$ & $3.7^{\circ}$ & $-0.5^{\circ}$ & & $4.0^{\circ}$ & $3.5^{\circ}$ & $-0.5^{\circ}$ \\
NAP & $8.0^{\circ}$ & $6.1^{\circ}$ & $-1.9^{\circ}$ & & $7.6^{\circ}$ & $5.4^{\circ}$ & $-2.2^{\circ}$ \\
FMA & $29.5^{\circ}$ & $20.0^{\circ}$ & $-0.5^{\circ}$ & & $28.4^{\circ}$ & $26.0^{\circ}$ & $-2.4^{\circ}$ \\
\hline
\end{tabular}

${ }^{1}$ Data from Riolo et al. (1974).

Riolo ML, Moyers RE, McNamara JA Jr, Hunter WS. An atlas of craniofacial growth: cephalometric standards from the University School Growth Study, the University of Michigan. Monograph 2, Craniofacial Growth Series. Ann Arbor: Center for Human Growth and Development, University of Michigan; 1974. 
Table 2. Evaluation of the two samples at the start of treatment. ${ }^{1}$

\begin{tabular}{lcccccc}
\hline Variable & $\begin{array}{c}\text { Bionator } \\
\text { Mean }\end{array}$ & $\begin{array}{c}\text { Edgewise } \\
\text { Mean }\end{array}$ & $\begin{array}{c}\text { Mean } \\
\text { Difference }\end{array}$ & $\mathrm{n}$ & t-test & P-Value \\
\hline NAP & 9.84 & 9.70 & -0.14 & 50 & -0.25 & 0.8012 \\
SNA & 81.86 & 82.08 & 0.21 & 50 & 0.33 & 0.7454 \\
SNB & 76.23 & 76.61 & 0.38 & 50 & 0.58 & 0.5652 \\
ANB & 5.64 & 5.47 & -0.17 & 50 & -1.14 & 0.2601 \\
AOBO & 3.48 & 4.85 & 1.36 & 50 & 3.35 & 0.0016 \\
FMA & 26.02 & 26.71 & 0.69 & 50 & 1.46 & 0.1507 \\
IMPA & 94.46 & 94.99 & 0.53 & 50 & 0.38 & 0.7023 \\
FMIA & 59.52 & 58.29 & -1.24 & 50 & -0.96 & 0.3432 \\
Overbite & 3.67 & 2.99 & -0.68 & 50 & -1.53 & 0.1323 \\
Overjet & 6.43 & 6.94 & 0.51 & 50 & 1.04 & 0.3041 \\
\hline
\end{tabular}

1Sample means at the start of treatment; t-tests are pairing design $\mathrm{t}$-tests. 
children being 4 years older, with more exacerbated malocclusions.

We pointedly did not worry about matching cases on dental variables because the avowed merits of two-phase treatment are to improve the basal bony relationships and facial profile (McNamara and Brudon 1993). Expectation was that the fixed phase of treatment could comparably correct dental malocclusions, whether in one phase or during the fixed phase of compound treatment.

\section{Sample Description}

Dr. Joe L. Wasson, Memphis, Tennessee employs a three-tier treatment strategy for Class II malocclusion treatment. The patients that fall under the first tier tend to be in the early mixed dentition ( 7 to 10 years of age), with high mandibular plane angles, openbite tendencies, and deleterious oral habits (e.g., tongue thrusts). In addition, these patients tend to have large overjets and constricted or underdeveloped dental arches. Treatment of these patients typically occurs in a two-phase regimen. The first phase consists of Fränkel II wear for approximately 22 hours a day for 1 year followed by a retention phase, wearing the appliance for 8 to 12 hours a day. A second phase of fixed appliances is started once all of the permanent teeth are present.

The second tier of the treatment triage is geared to patients who have Class II division 1 malocclusions. These patients have low mandibular plane 
angles and deepbite tendencies. These patients are somewhat older than the Fränkel patients but are still in the mixed dentition ( 8 to 11 years of age). Again, a two-phase regimen is employed for these patients. The first phase involves full-time Bionator wear for at least 9 to 12 months with 6 to 8 week appointment intervals to assess progress. After initial correction, the appliance is worn at night for retention while waiting on the rest of the permanent dentition. The second phase involves fixed appliances in order to detail and finish the case.

The third treatment strategy is aimed at Class II patients in the early permanent dentition (11 to 14 years of age). This group of patients often is treated with fixed functional appliances such as the Herbst appliance and MARA (i.e., mandibular advancement repositioning appliance).

\section{Class II Characteristics of the Sample}

There are several "sorts" of Class II malocclusions depending on whether the problem is dental or skeletal and depending on which jaw is more deviant (i.e., maxillary excess, mandibular retrognathia, or some combination of the two). The purpose here is to test whether the one- and two-phase samples compared here differed as to the offending skeletal problem. Three key angular variables were compared at the start of treatment, namely SNA, $\mathrm{SNB}$, and ANB. 
Starting ages for the two- and one- phase treatments are years apart (9.6 and 13.1 years, respectively), but the normative angular relationships change very little across this age span (e.g., Riolo et al. 1974).

These three cephalometric measures of parasagittal jaw relationships (SNA, SNB, ANB) do not differ statistically between groups (Table 3). Means for SNA are $82^{\circ}$ in both groups $(\mathrm{F}=0.1 ; \mathrm{P}=0.7613)$. There is just one case in each sample with an SNA exceeding $90^{\circ}\left(\right.$ i.e., $\left.91^{\circ}\right)$. None of the cases in the two-phase group has an SNA below $75^{\circ}$, but 3 of the one-phase cases are in the $70^{\circ}$ to $75^{\circ}$ range.

Data from Riolo et al. (1974) shows that normative SNA is very close to $81^{\circ}\left(\mathrm{sd}=3.5^{\circ}\right)$ throughout this age span, so just four of the 100 cases are beyond 2 sd (about $\left.88^{\circ}\right) ; 3$ of these are 2-phase cases and 1 case in the one-phase sample.

Means for $\mathrm{SNB}$ average $76^{\circ}$ in the two-phase group and $77^{\circ}$ in the onephase group, which is indistinguishable statistically $(\mathrm{F}=0.3 ; \mathrm{P}=0.5626)$. All cases have SNB values between $70^{\circ}$ and $85^{\circ}$. Normative standards for SNB in this age interval are close to $78^{\circ}\left(\mathrm{sd}=3.5^{\circ}\right)$, indicating that 6 cases from among the 100 here have SNBs between -2.0 and -2.5 standard deviations of the mean (and none below -2.5 sds).

The range of ANB angles are all within $1^{\circ}$ and $9^{\circ}$, with no statistical difference between the two samples $(\mathrm{F}=0.3 ; \mathrm{P}=0.5883)$. Mean ANB is the 
Table 3. Tests for group differences in the parsagittal measures of jaw relationships.

\begin{tabular}{|c|c|c|c|c|c|c|c|c|}
\hline \multirow[b]{2}{*}{ Variable } & \multicolumn{3}{|c|}{ Two Phase } & \multicolumn{3}{|c|}{ One Phase } & \multicolumn{2}{|c|}{ ANOVA } \\
\hline & $\mathrm{n}$ & mean & sem & $\mathrm{n}$ & mean & sem & F Ratio & $P$ value \\
\hline SNA & 50 & 81.86 & 0.49 & 50 & 82.08 & 0.49 & 0.09 & 0.7613 \\
\hline SNB & 50 & 76.23 & 0.47 & 50 & 76.61 & 0.47 & 0.34 & 0.5626 \\
\hline ANB & 50 & 5.64 & 0.22 & 50 & 5.47 & 0.22 & 0.29 & 0.5930 \\
\hline
\end{tabular}


same, $5.5^{\circ}$, in both groups, which is not far above the normative standard of about $4^{\circ}\left(\mathrm{sd}\right.$ about $\left.2.5^{\circ}\right)$ in this age interval. None of the cases in either groups here has an ANB beyond 2 standard deviations, which is about $9^{\circ}$.

\section{Bionator Appliance Design}

The following description presents an outline of features and functions of the Bionator appliance. The reader is referred to the texts by Graber and Neumann (1984) and McNamara and Brudon (1993) for actual construction of the appliance. Varying configurations of the Bionator are utilized to open, close, or maintain the bite in conjunction with posturing the mandible forward into a more desirable position. For the purposes of this study, the Bionator that is used to open the bite was employed during the initial phase of treatment, and this is shown in Figures 1 through 3. The following items are a list of the components that make up the appliance:

- Upper Labial Wire: This wire $\left(0.040^{\prime \prime}\right)$ is bent in a Hawley configuration with adjustment loops the same width as the canines and extending upward 7 to $8 \mathrm{~mm}$ above the gingival margin. The labial bow lies along the anterior teeth with roughly $0.5 \mathrm{~mm}$ of clearance to avoid lingual tipping of the upper incisors.

- Upper Lingual Wire: This wire (0.036") provides support for the appliance by using the lingual surfaces of the upper anterior teeth for anchorage. It is 


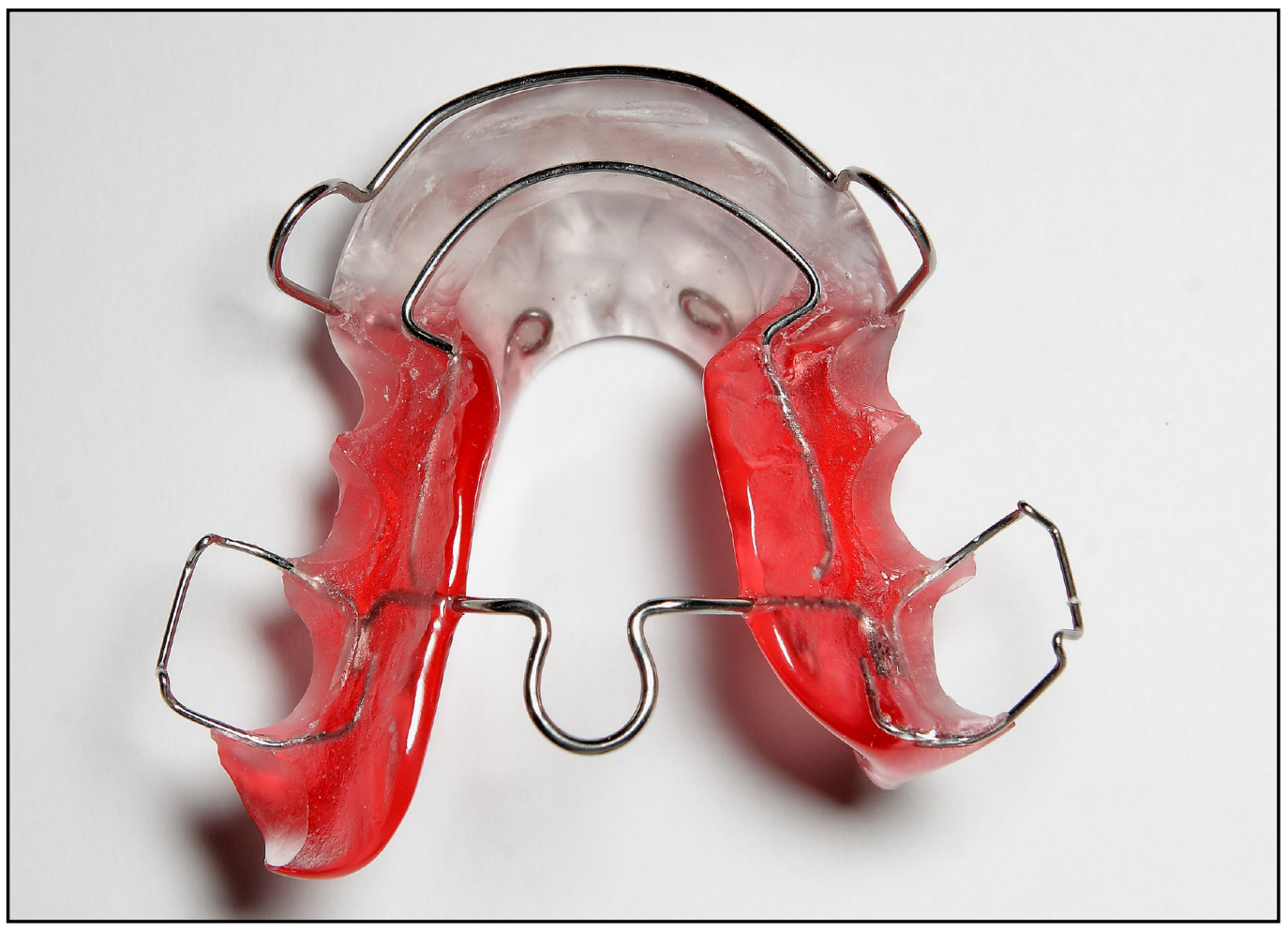

Figure 1. Occlusal view of Bionator appliance used in the study. The palatal bar acts to stabilize the appliance and also gives the tongue a place to rest. The Adams clasps fit to the maxillary first molars for retention. Behind the labial bow is a wire that can be cut and activated if needed to keep the upper incisors from tipping lingually. 


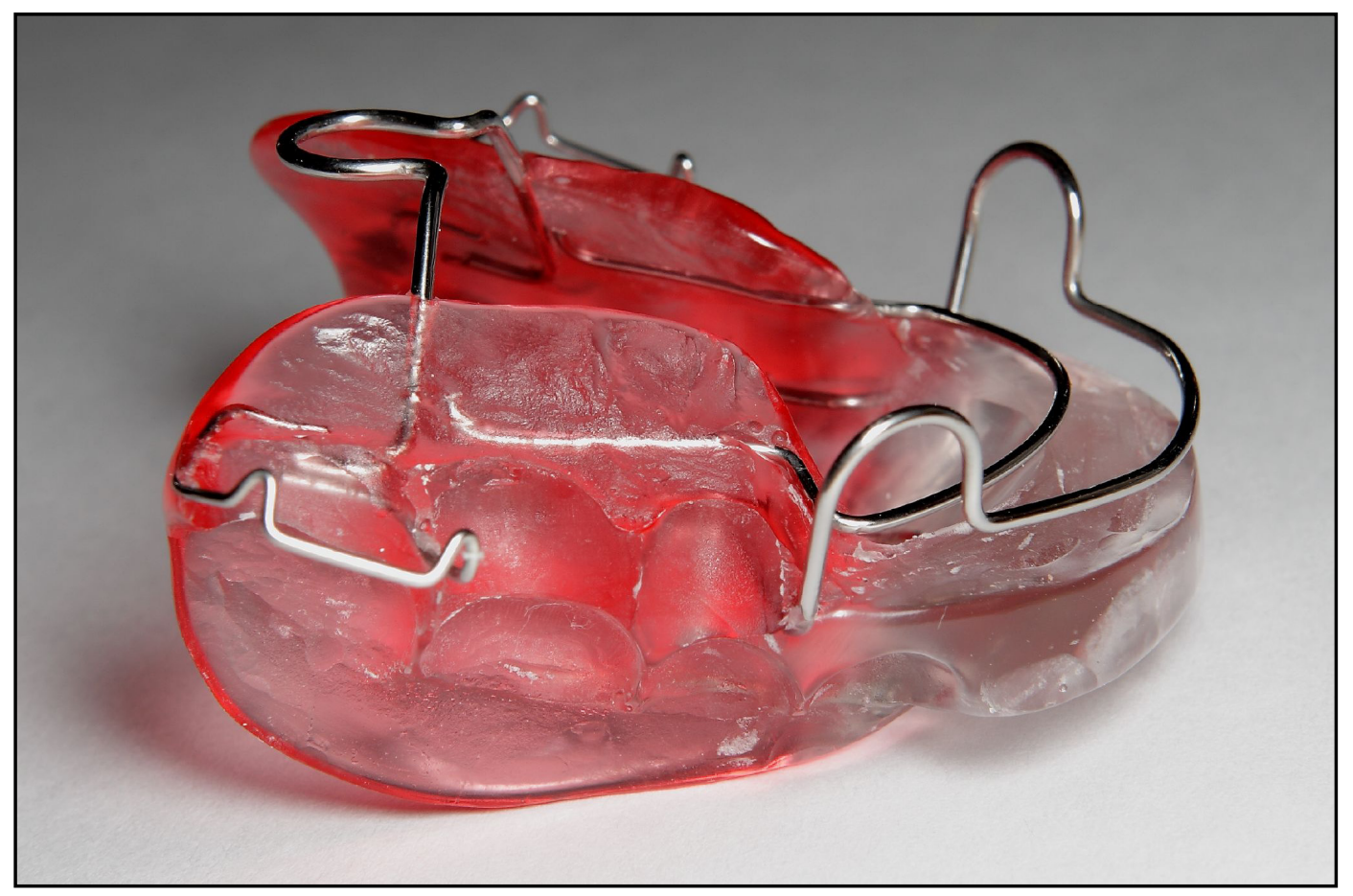

Figure 2. Lateral view of Bionator appliance used in the study. Here the facets can be seen that permit eruption of the posterior teeth to help open the bite. The incisors are fully covered with acrylic to prevent unwanted flaring. 


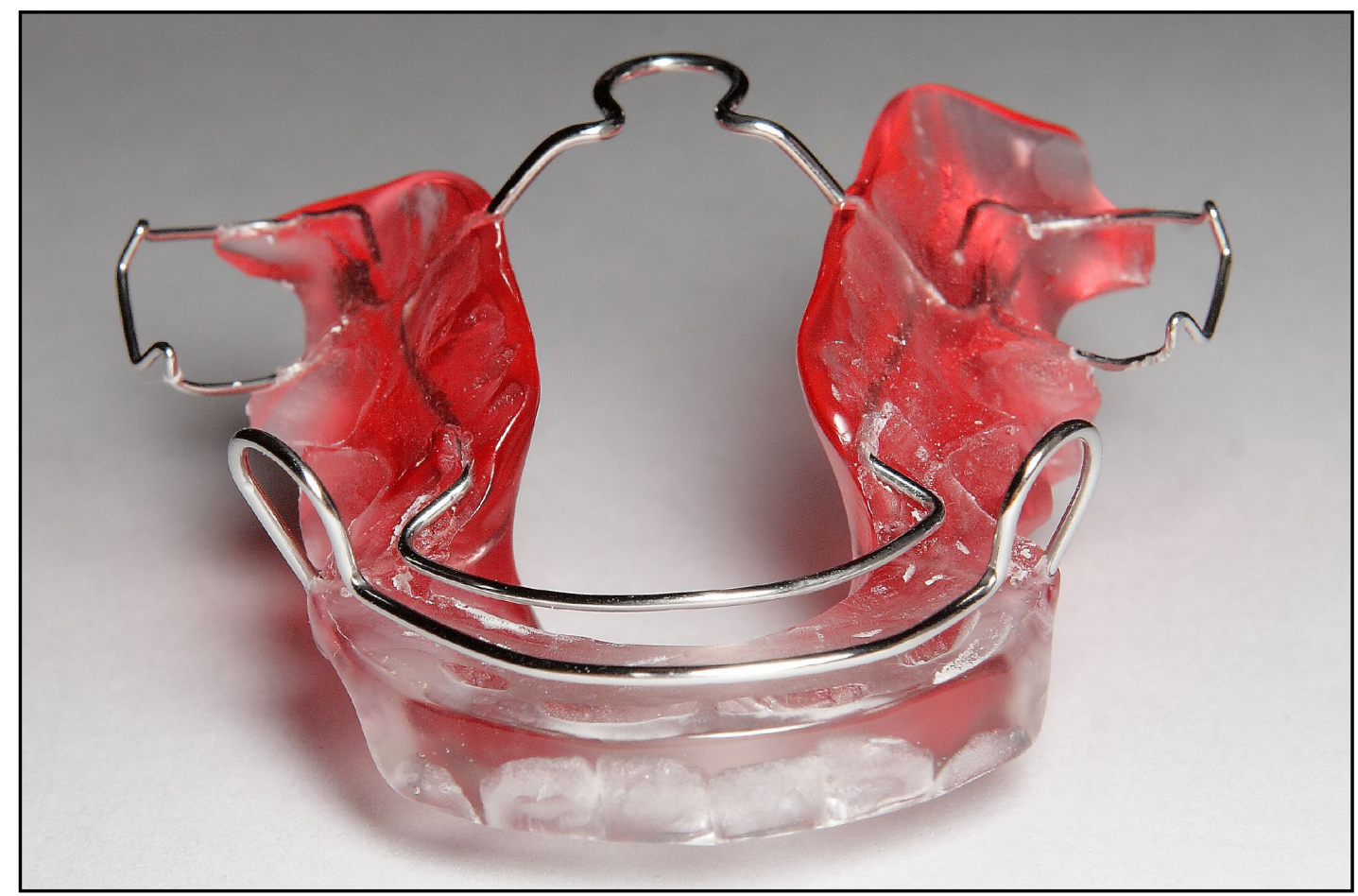

Figure 3. Frontal view of Bionator appliance used in the study. This view shows the appliance in full with the tongue space and palatal bar, labial bow, Adams clasps for retention, and the full coverage of acrylic for the lower incisors. 
constructed in an ideal arch form and lies at the level of the cingula. It can also be cut between the central incisors and activated if the incisors have been tipped lingually.

- Palatal Wire: This wire $\left(0.045^{\prime \prime}\right)$ or "Coffin spring" is constructed to lie 2 to $3 \mathrm{~mm}$ off the palatal tissue for comfort and can be activated for lateral arch development if needed.

- Eruption Facets: These can me made from the interocclusal acrylic to facilitate the eruption of both the upper and lower posterior teeth if desired. These pathways or channels are oriented so that the posterior teeth erupt either vertically or laterally.

- Occlusal Table: One method employed for Class II correction is the differential eruption of teeth. An occlusal table can be fabricated so that the upper posterior teeth are in contact with the acrylic, while the lower posterior teeth are allowed to erupt.

- Interocclusal Acrylic: If no eruption of the posterior teeth is desired, the interocclusal acrylic is designed so that the posterior teeth touch the acrylic evenly.

- Lower Incisor Coverage: The occlusal coverage prevents vertical eruption or labial tipping of the lower incisors, and it also stabilizes the appliance in the mouth. 
The appliance design is less bulky than the original activator, therefore speech is not affected, and it can be worn continuously (McNamara and Brudon 1993).

\section{Goals of Bionator Therapy}

The intended goal of functional appliance therapy is to create an environment whereby an individual's full growth potential can be realized and utilized to help correct the malocclusion. Eirew (1981), summarizing Balters' original treatment objectives, lists the goals of Bionator therapy as follows: (1) the elimination of a lip trap and abnormal relationship between the lips and incisor teeth; (2) the elimination of any mucosal damage due to an impinging overbite; (3) the correction of mandibular retrusion or retrognathia and faulty tongue positioning; and (4) the correction of the occlusal plane (Graber and Neumann 1984). In the present study, the Bionator was used to: (1) allow for continued eruption of the posterior teeth to open the bite and increase lower facial height; (2) propulse the mandible forward in hopes of maximizing the patient's growth potential; (3) achieve a Class I molar relationship; (4) gain some early skeletal correction (achieve a more orthognathic bony profile); (5) reduce the existing overjet; and (6) reduce the complexity and treatment time of the subsequent fixed appliance phase of treatment. 


\section{Cephalometric Analysis}

Lateral cephalometric radiographs from three time points were used for the group being studied: (1) the pretreatment examination; (2) at completion of phase 1 treatment; and (3) at completion of full appliance treatment.

Descriptive statistics for each cephalometric examination are listed in Appendix A. Since the control group was treated with fixed appliances in a single phase, only pretreatment and posttreatment radiographs were available for that group. A total of 28 angular and linear variables were used in this analysis. The following alphabetical listing provides definitions of the cephalometric landmarks used in the study:

A Point A (Subspinale): the most posterior point on the exterior ventral curve of the maxilla between the anterior nasal spine and Supradentale.

ANS Anterior Nasal Spine: the spinous process of the maxilla forming the most anterior projection of the floor of the nasal cavity.

B Supramentale: the most posterior point on the bony curvature of the mandible between Infradentale and Pogonion.

Ba Basion: the most inferior-posterior point in the midsagittal plane on the anterior margin of the foramen magnum at the base of the clivus.

Cd Condylion: the most superior and posterior point on the curvature of the capitulum of the condyle. 
DOP Downs' Occlusal Plane: the line that vertically bisects incisal overbite and the most anterior occlusal contact of the maxillary and mandibular first molars (Downs 1948).

Gn Gnathion (anatomic): the most anterior-inferior point of the mandibular symphysis.

Go Gonion (anatomic): the most posterior-inferior point on the Gonial angle of the mandible.

Ii Incision inferius: the incisal tip of the most anterior mandibular central incisor.

Is Incision superius: the incisal tip of the most anterior maxillary central incisor.

LIA Apex of mandibular central incisor: the apical end of the same mandibular central incisor used to locate Ii.

L6C L6 cusp: the mesial cusp tip of the mandibular first molar.

Me Menton: the most inferior point on the symphysis of the mandible.

$\mathrm{Na}$ Nasion: the anterior point of the intersection between the nasal and frontal bones.

Or Orbitale: the most inferior point on the lower margin of the bony orbit.

Pg Pogonion: the most anterior point on the anterior contour of the bony chin below B point and above Gnathion. 
PNS Posterior Nasal Spine: the most posterior point at the midsagittal plane on the bony hard palate.

Po Porion: the midpoint on the superior aspect of the rim of the external auditory meatus.

Pt Pterygomaxillary fissure: the most superior-posterior point on the radiographic outlines of end the pterygomaxillary fissure.

Se Sella turcica: the center of the hypophyseal fossa, determined by inspection.

U1A Apex of the maxillary central incisor: the apical of the most anterior maxillary central incisor of the same tooth used to locate Is.

U6C U6 cusp: the mesial cusp tip of the maxillary first molar.

\section{Data Entry}

The cephalometric radiographs from all subjects were scanned using a UMAX Powerlook III flatbed scanner at $300 \mathrm{dpi}$ and 256 gray scale, and the scans were saved as TIFF files. The radiographs in TIFF format were imported into Dolphin Imaging ${ }^{\circledR} 10.0$ and traced using the program's digital cephalometric tracing package. Prints of the cephalometric tracings of all the subjects used in the present study can be found in Appendix B. A custom analysis was created with the "custom analysis builder" function of Dolphin Imaging for measurement of the 28 skelotodental angular and linear variables 
used in the present study. The measurements were exported from Dolphin Imaging into Microsoft Excel ${ }^{\circledR} 2003$ and then into JMP ${ }^{\circledR}$ 5.0.2 (SAS Institute Inc. Cary, NC) for statistical analysis.

\section{Statistical Analysis}

Data were collated into an Excel spreadsheet (Microsoft $\left.{ }^{\circledR}\right)$ then transferred to the JMP statistical package (SAS Institute Inc., Cary, NC). Exploratory data analysis (Tukey 1977) was performed, searching for outliers; those due to technical errors were corrected. Conventional descriptive statistics (e.g., Sokal and Rohlf 1995) were calculated; these (and their abbreviations) are sample size (n, taken as counts of individuals, not sides), the arithmetic mean $(\bar{x})$, the standard deviation $(\mathrm{sd})$, and the standard error of the mean (sem, calculated as sd $/ \sqrt{ } \mathrm{n}$ ). The conventional alpha level of 0.05 was used throughout, and all of the tests were two-tail. No correction was made for multiple comparisons. Salient results of the analysis were graphed using Delta $\mathrm{Graph}^{\circledR} 4.0 .5$ for the PC.

The data were used to compare treatments with regard to (1) whether changes occurred during the first phase treated with a functional appliance, (2) whether changes occurred during the second phase treated with a fixed appliance, and (3) whether the amounts of change differed between the two phases for the experimental group. The differences were calculated at the start 
of treatment and the progress record, then between the progress record and the end of treatment. One sample t-tests (two tail) were used to evaluate changes in variables across time as a function of the sample variability. Repeated measures analysis of variance (Winer et al. 1991) was used to determine if the change from phase 1 to progress differed significantly from the change observed in phase 2 . The skeletodental landmarks and angular measurements used in this study are illustrated in Figures 4 through 22.

Statistical descriptions for the one-phase treatment group were calculated by obtaining the descriptive statistics for the sample at the two examinations, and then separately using pairing-design t-tests to assess whether the treatment change differed significantly from the null hypothesis of no change (two tail). To assess how comparable the skeletodental variables were at the end of treatment, we calculated the difference in the arithmetic means of the one- and two-phase groups. In addition, a paired t-test was calculated to determine if the two groups differed statistically. 


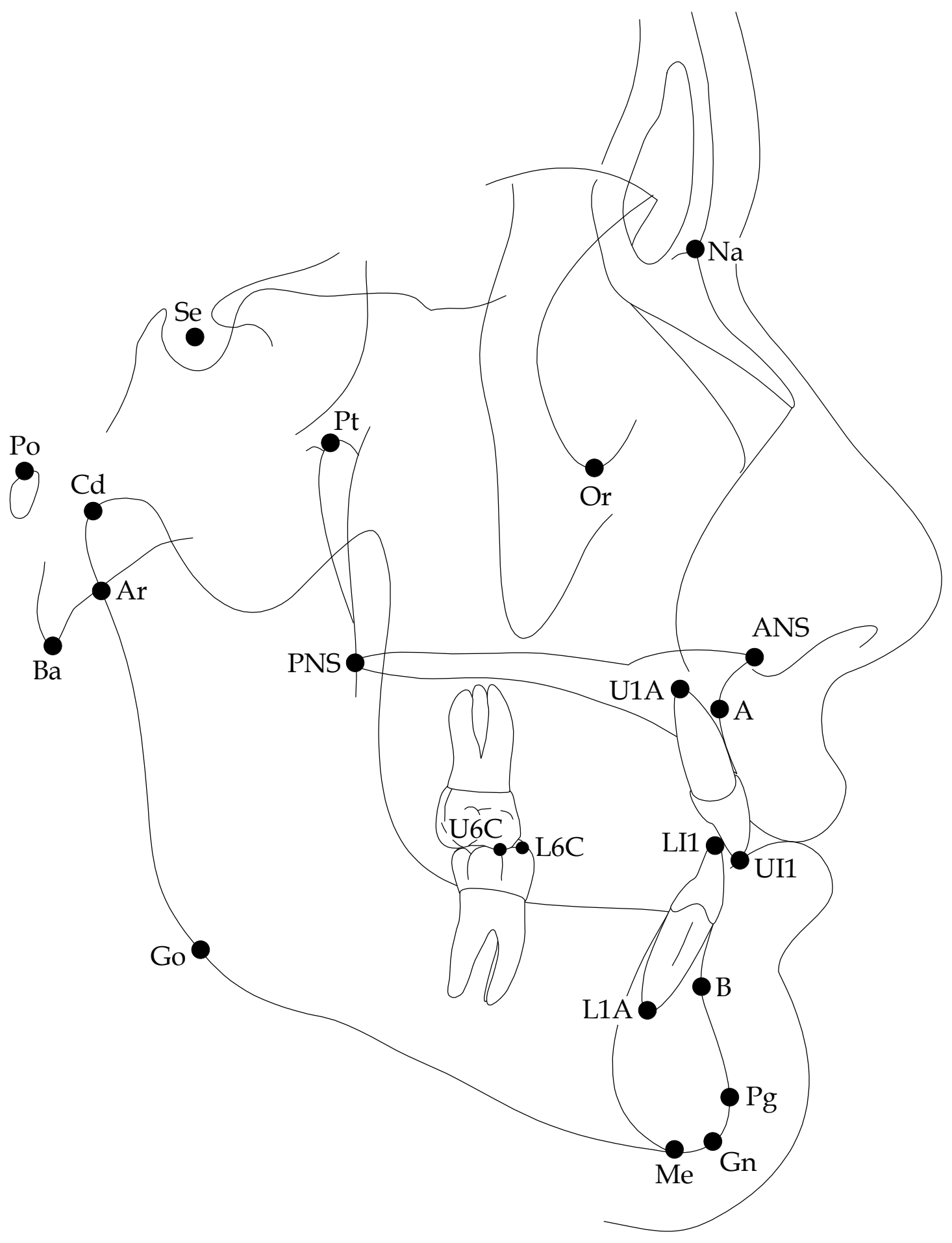

Figure 4. Cephalometric diagram showing locations of the skeletodental landmarks to be used in this study. 


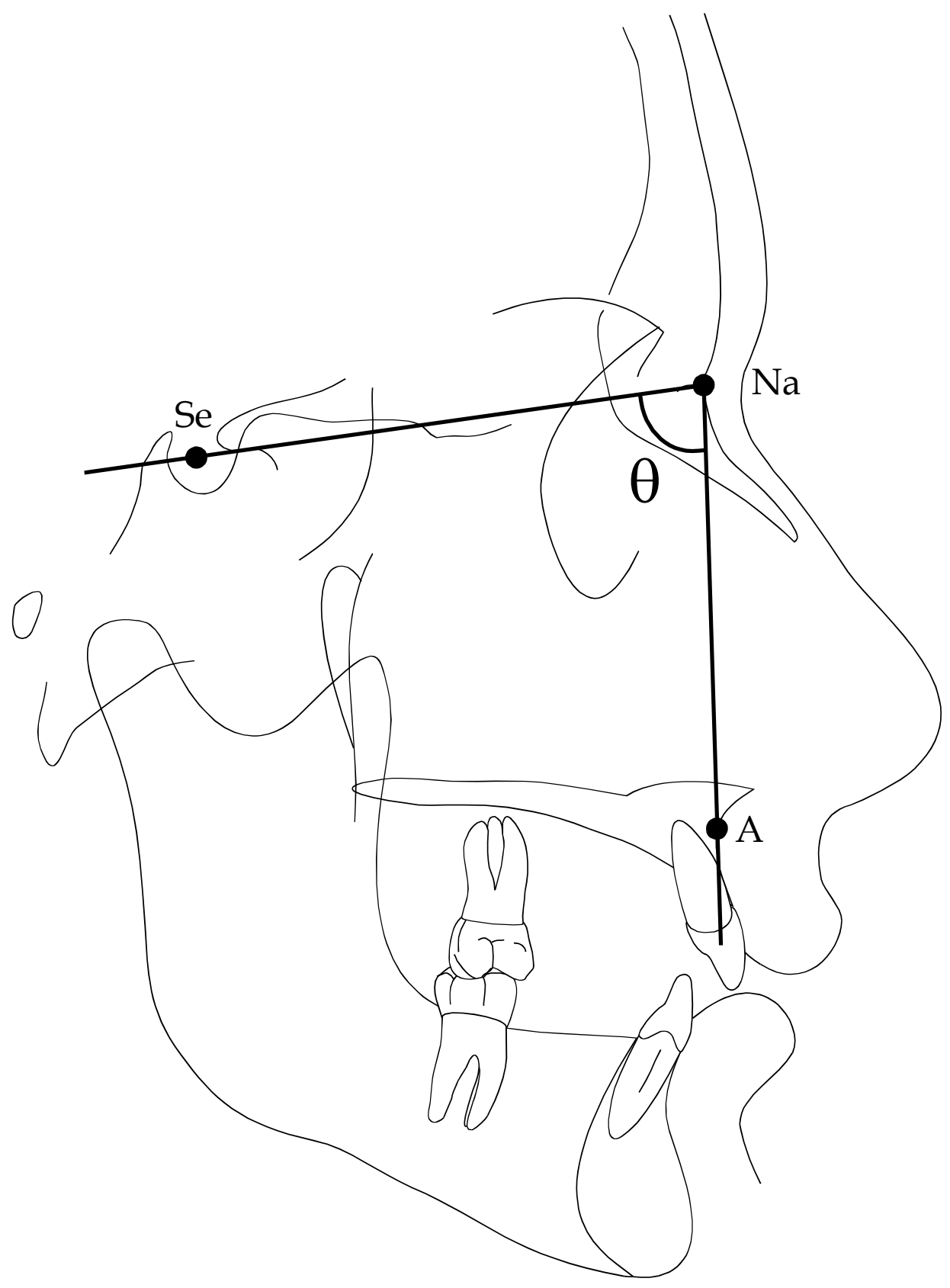

Figure 5. Schematic tracing of a lateral cephalogram showing construction of the SNA angle. 


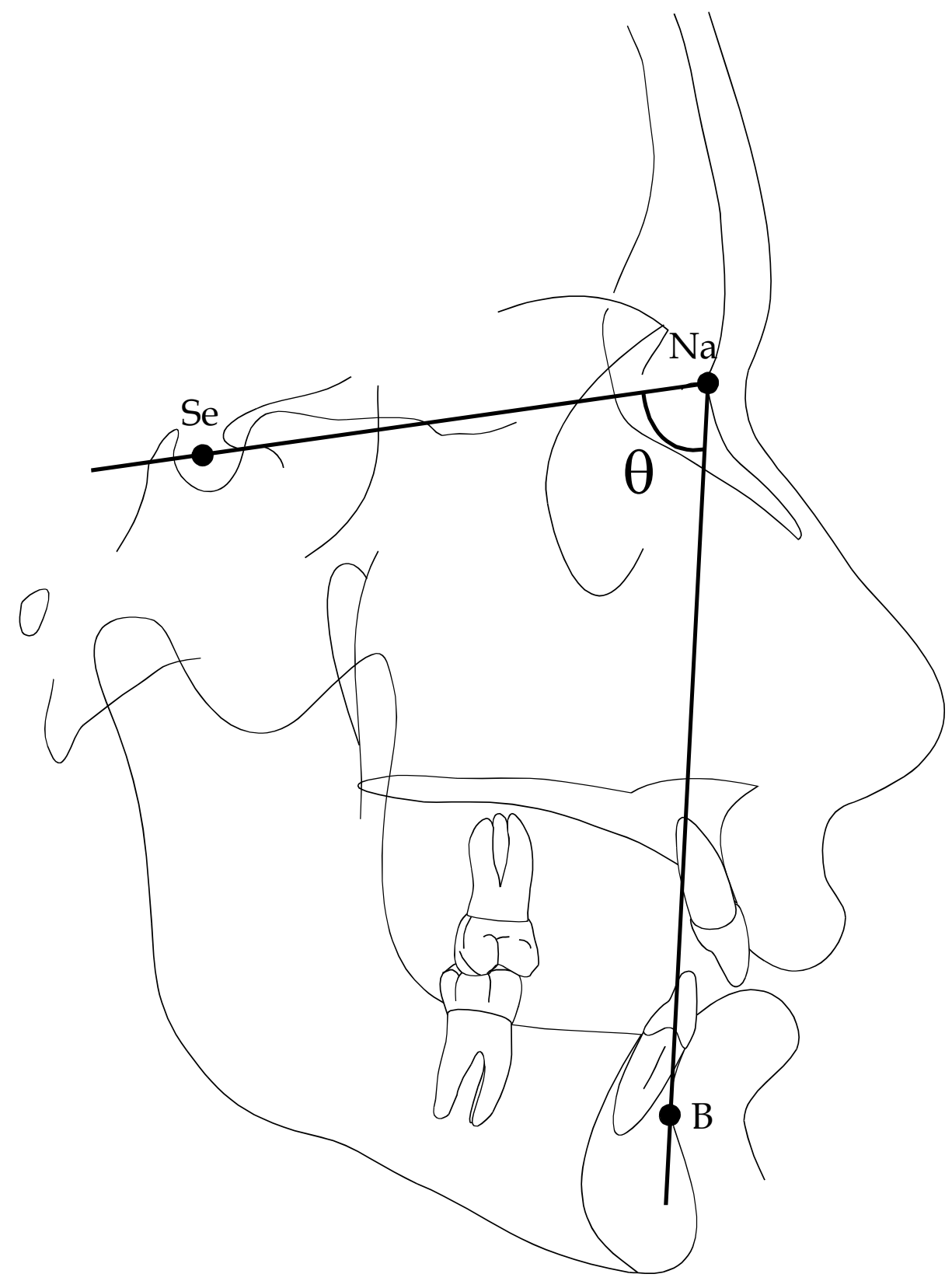

Figure 6. Schematic tracing of a lateral cephalogram showing construction of the SNB angle. 


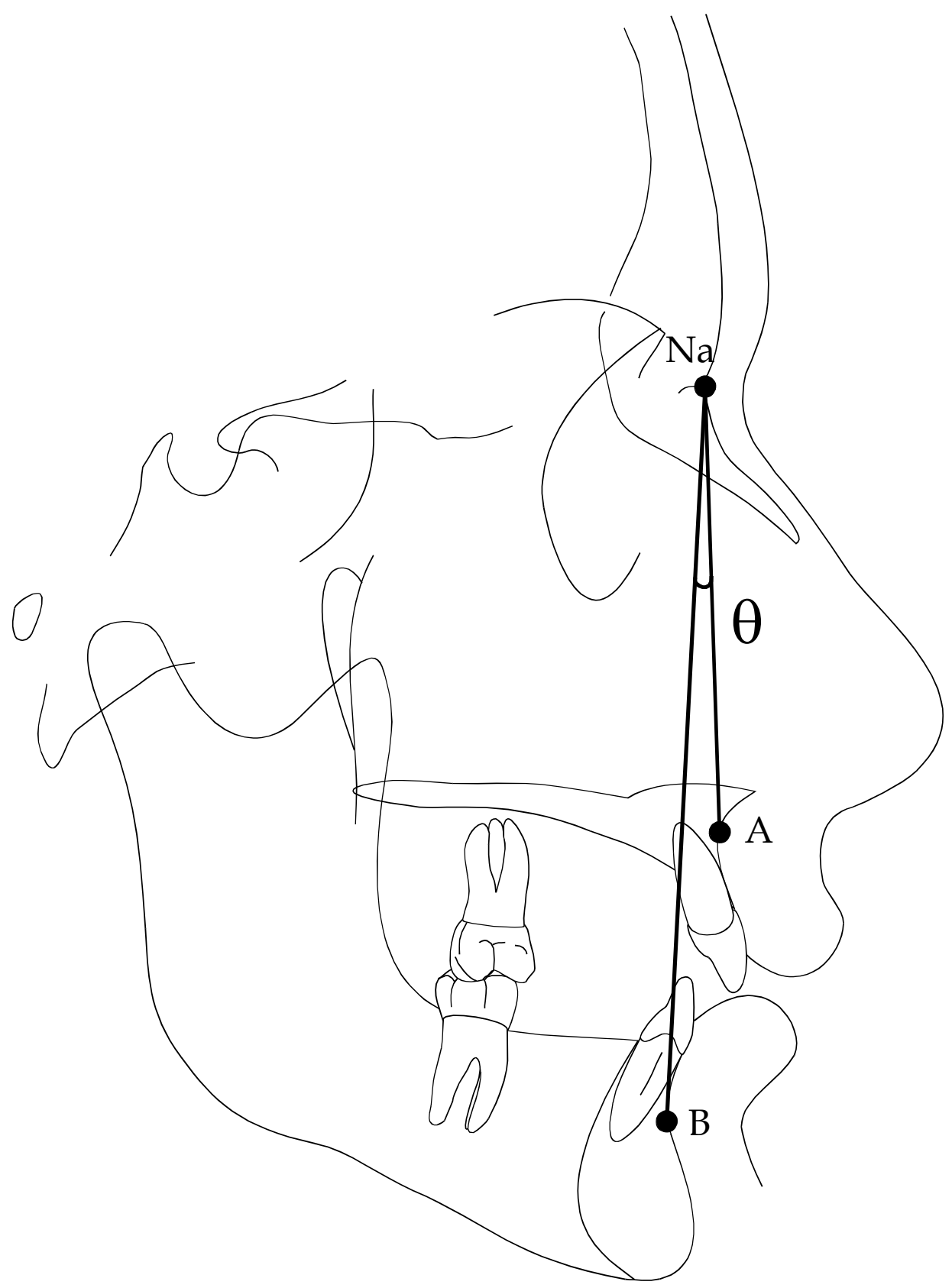

Figure 7. Schematic tracing of a lateral cephalogram showing construction of the ANB angle. 


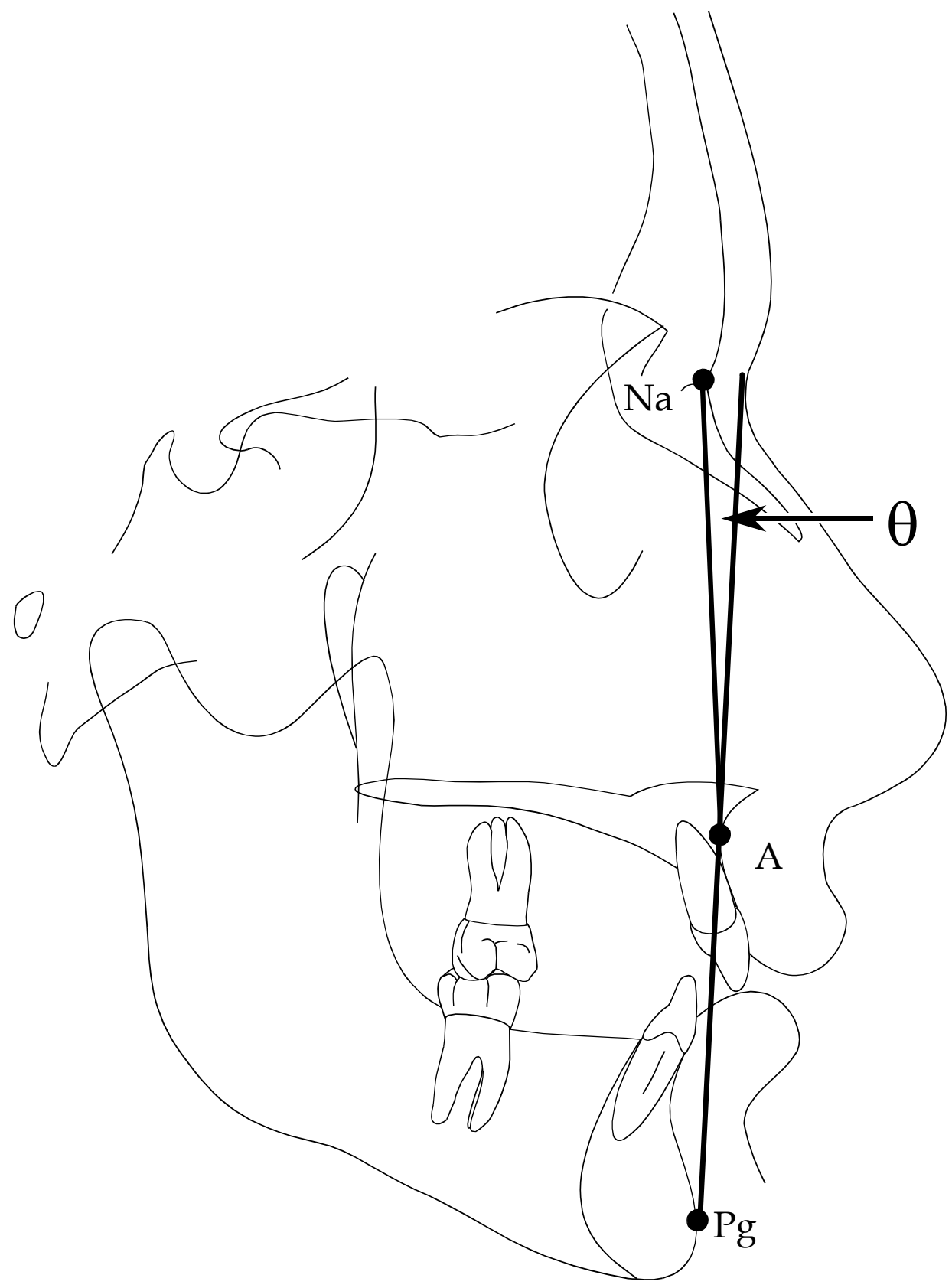

Figure 8. Schematic tracing of a lateral cephalogram showing construction of the angle of convexity (NAP). This is the superior-anterior angle at the intersection of the Nasion-A and the A-Pogonion lines. 


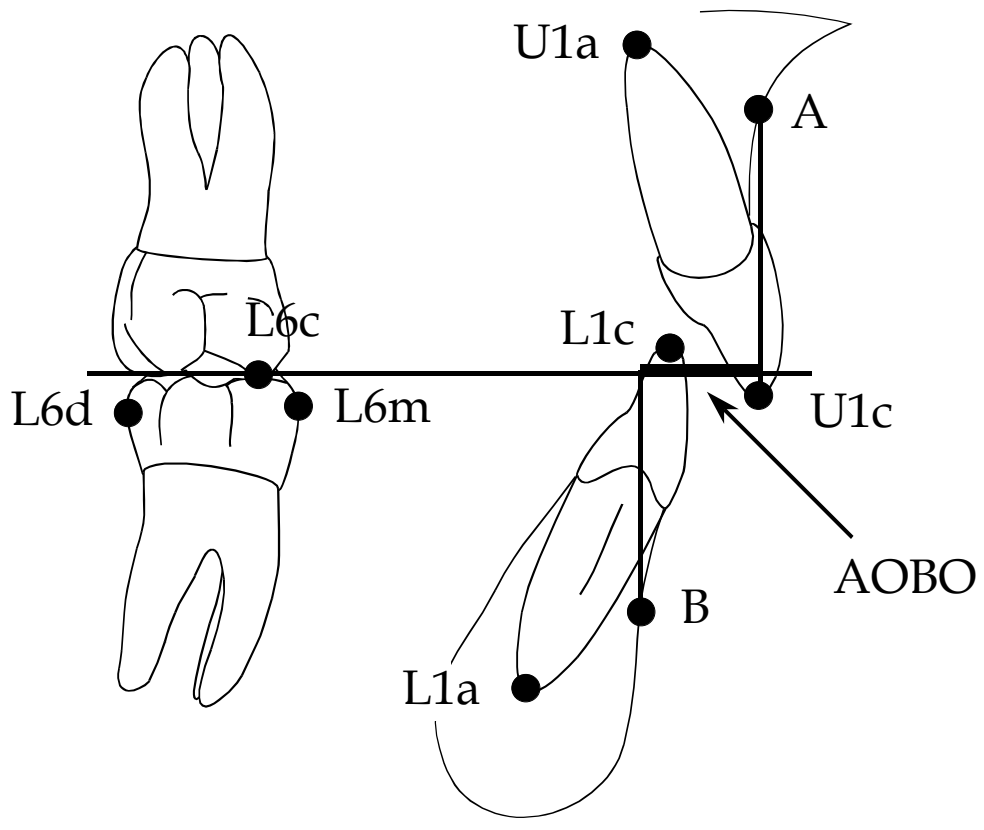

Figure 9. Schematic of the method of measuring the AOBO discrepancy. Point $A$ and Point $B$ are projected orthogonally onto the functional occlusal plane. $\mathrm{AOBO}$ is the millimetric distance between the projected line segment along the occlusal plane. 


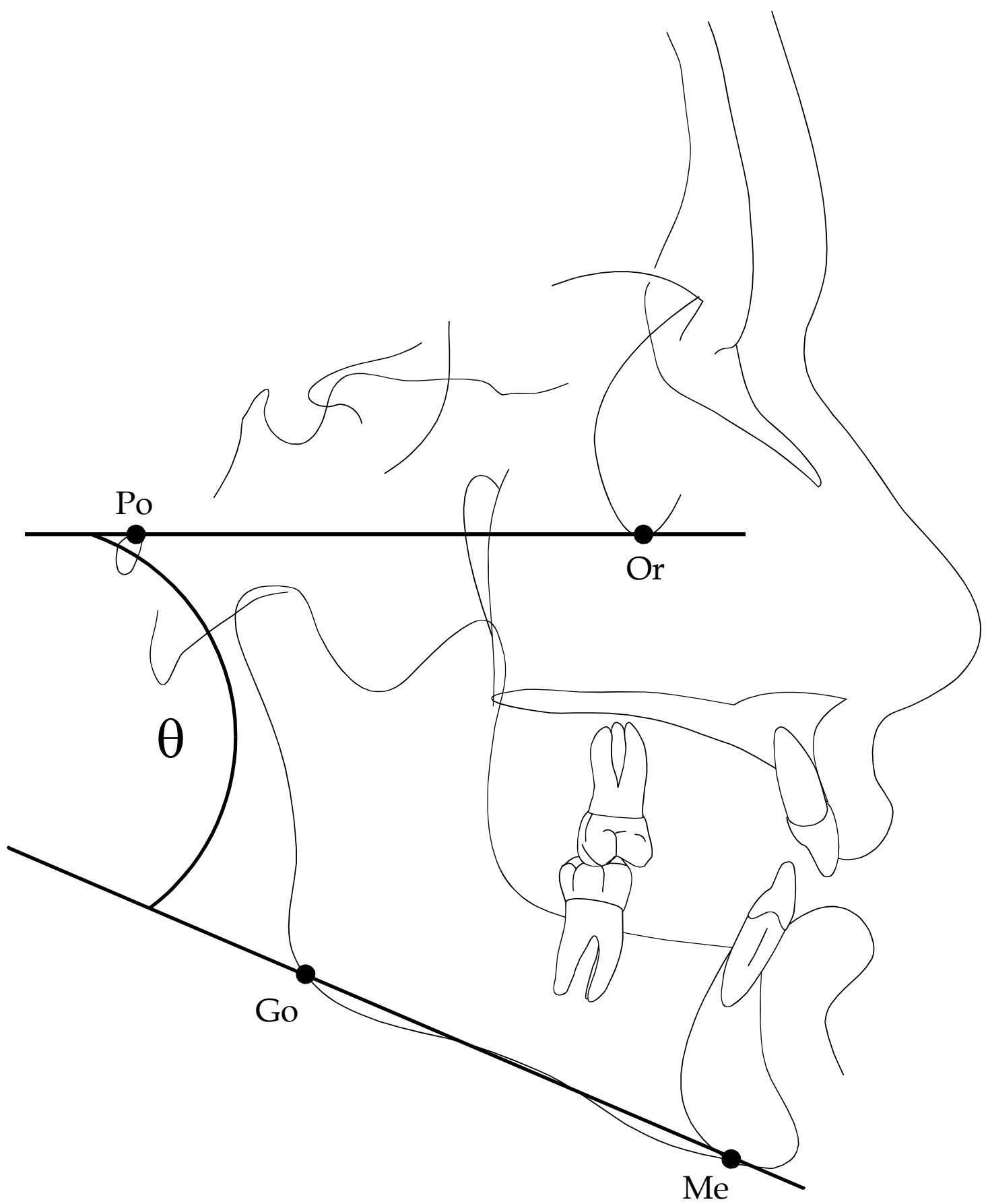

Figure 10. Schematic tracing of a lateral cephalogram showing construction of FMA. This is the angle formed by the intersection of Frankfort Horizontal and the line through anatomic Gonion and Menton. 


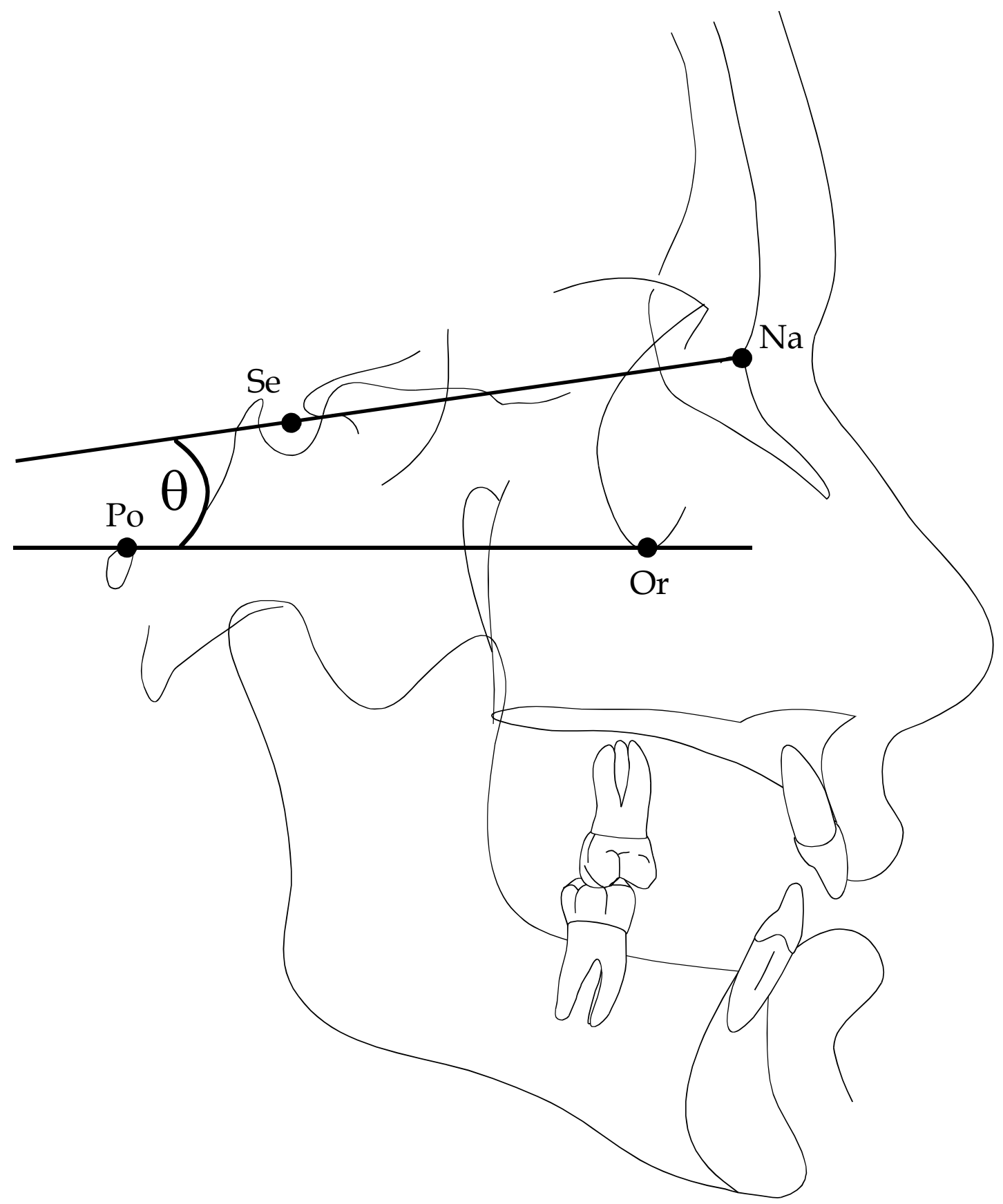

Figure 11. Schematic tracing of a lateral cephalogram showing construction of the angle formed by the intersection of Frankfort Horizontal and the Se-Na line. 


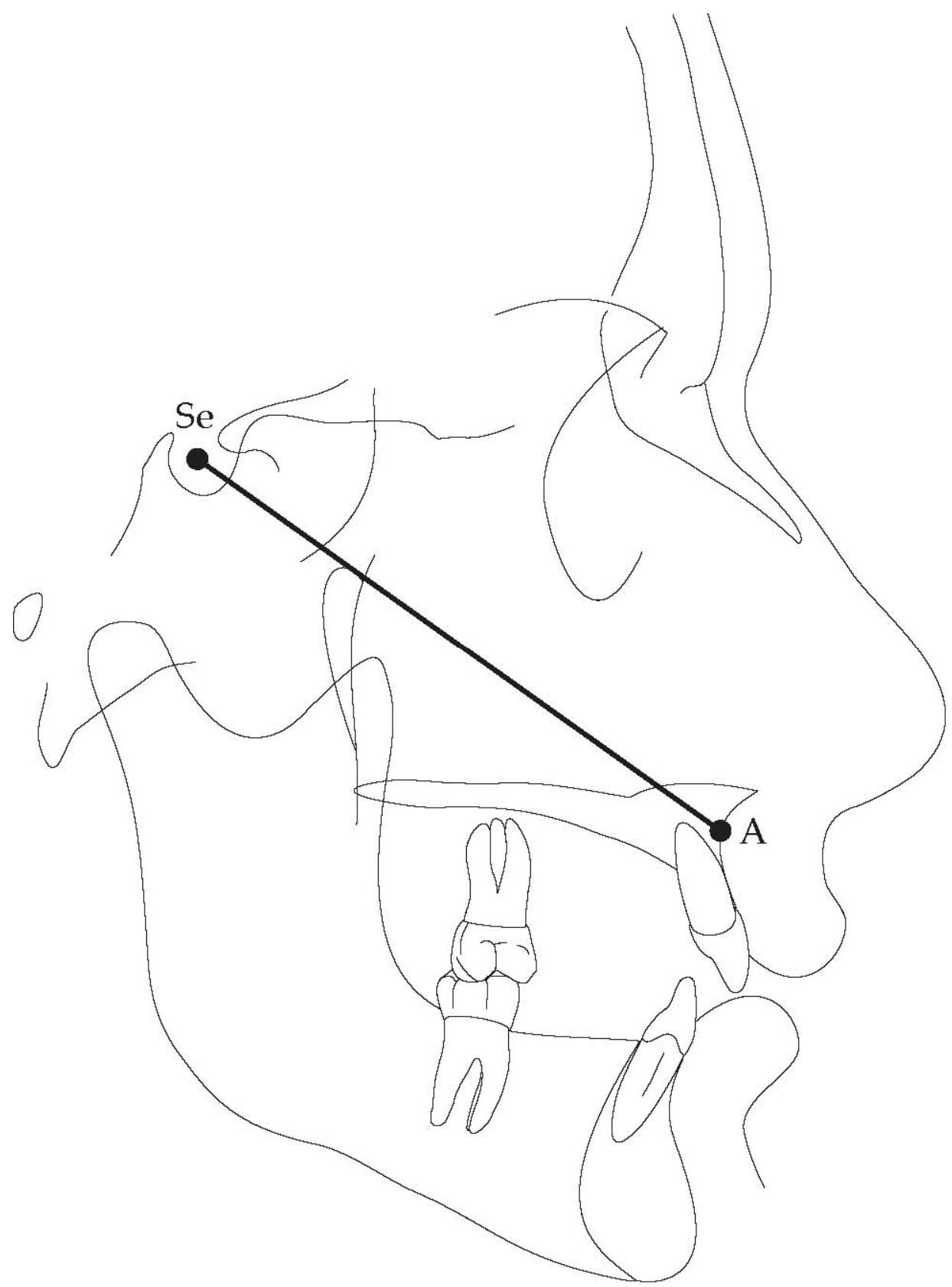

Figure 12. Schematic tracing of a lateral cephalogram showing construction of Sella-A Point linear distance measurement. The distance between the two points is measured in millimeters. 


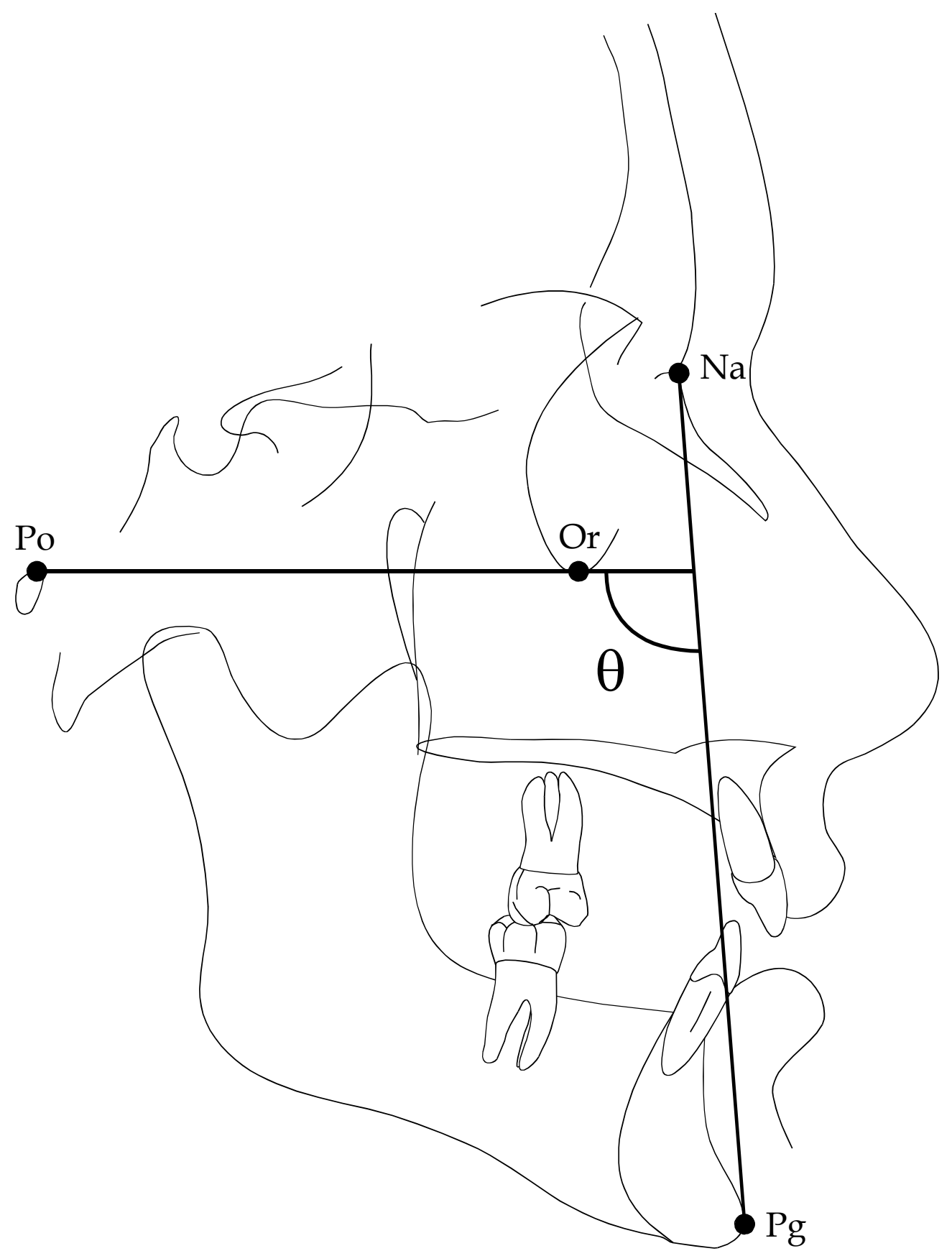

Figure 13. Schematic tracing of a lateral cephalogram showing construction of Downs' facial angle. This is the angle formed by the posterior-inferior intersection of Frankfort Horizontal and Nasion-Pogonion lines. 


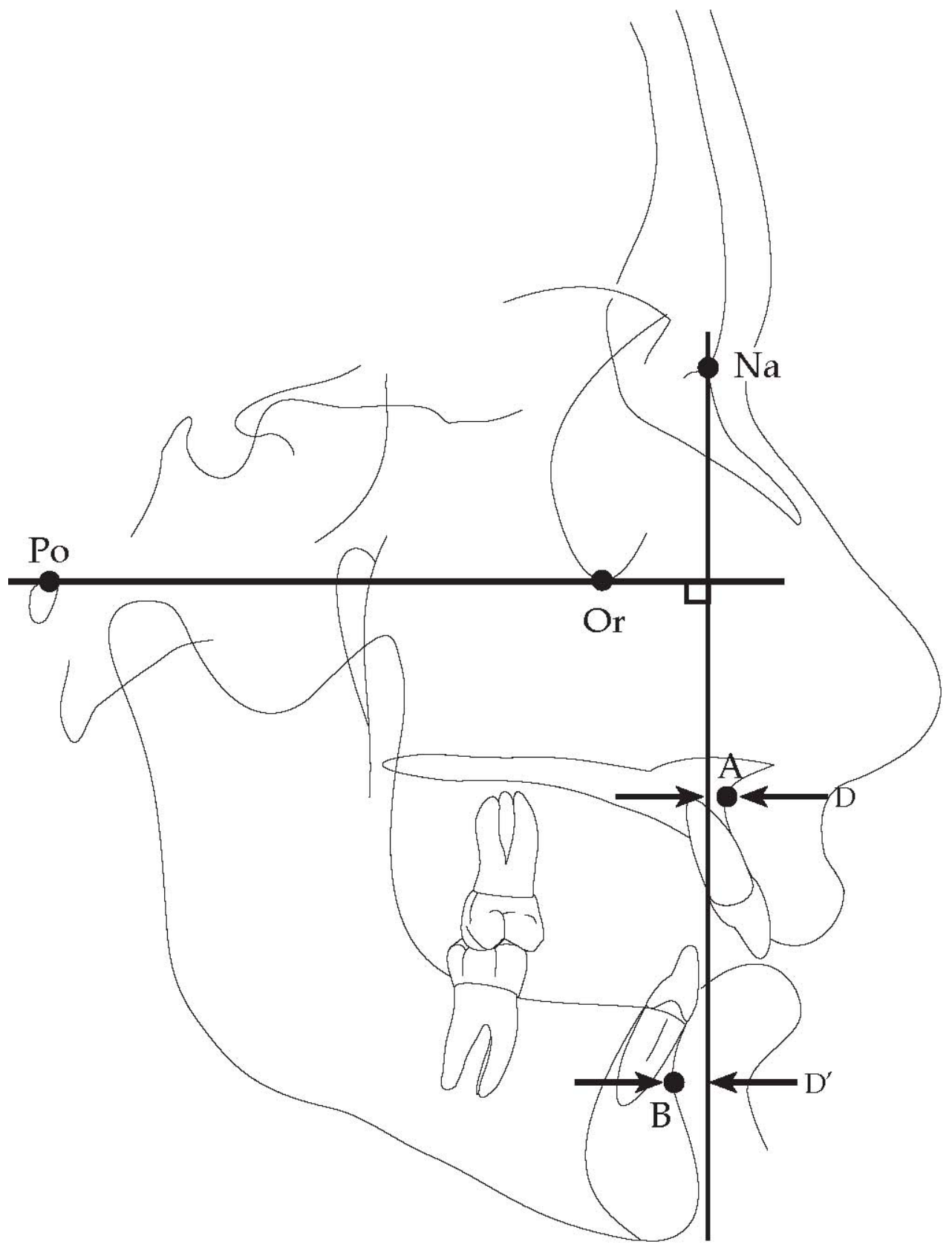

Figure 14. Schematic tracing of a lateral cephalogram showing construction of the A Point to Nasion-Perpendicular (labeled D) and B Point to NasionPerpendicular (labeled $\mathrm{D}^{\prime}$ ) measurements. Both distances are measured parallel with Frankfort horizontal in millimeters. 


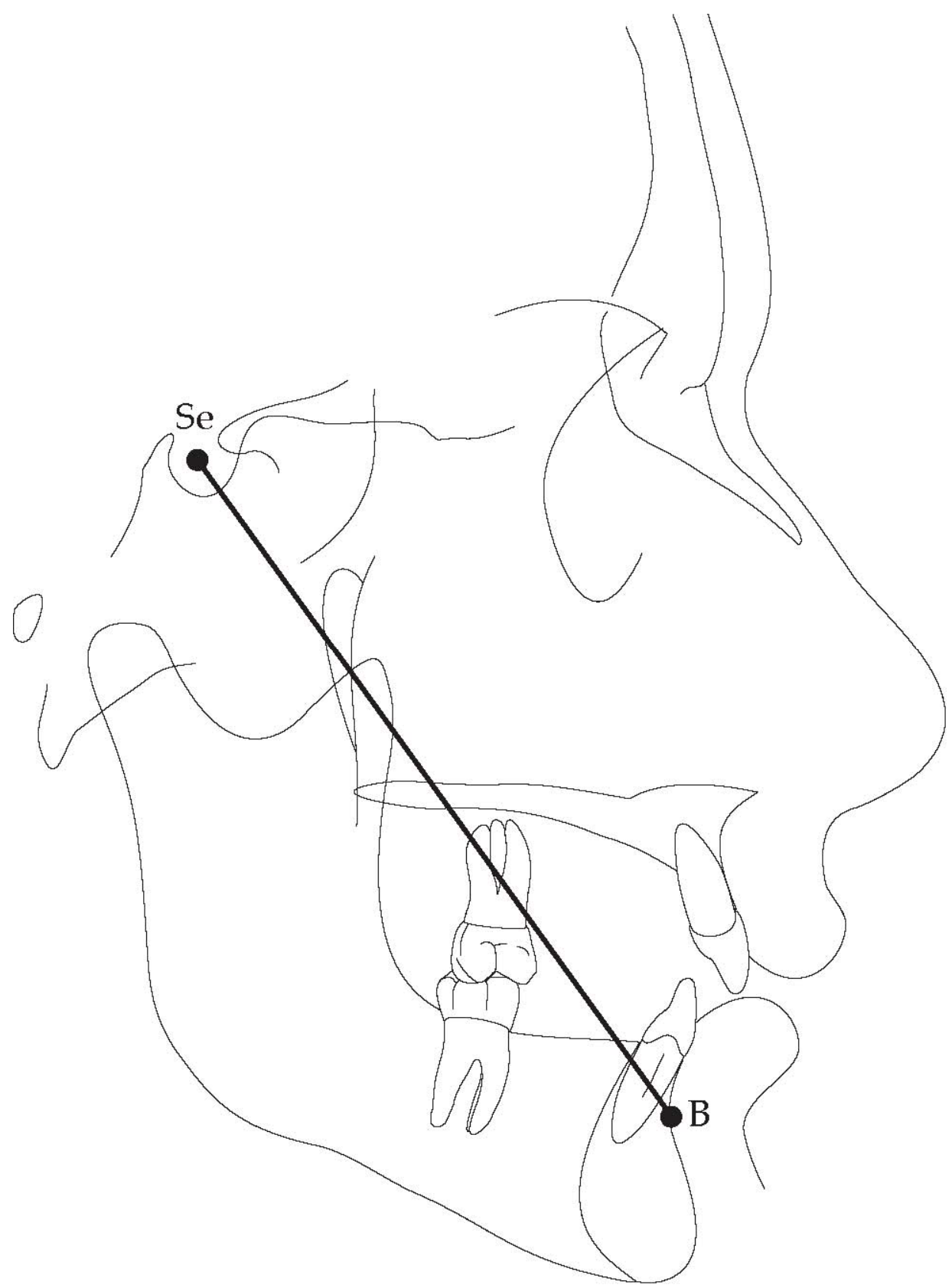

Figure 15. Schematic tracing of a lateral cephalogram showing construction of Sella-B Point linear distance measurement. The distance between the two points is measured in millimeters. 


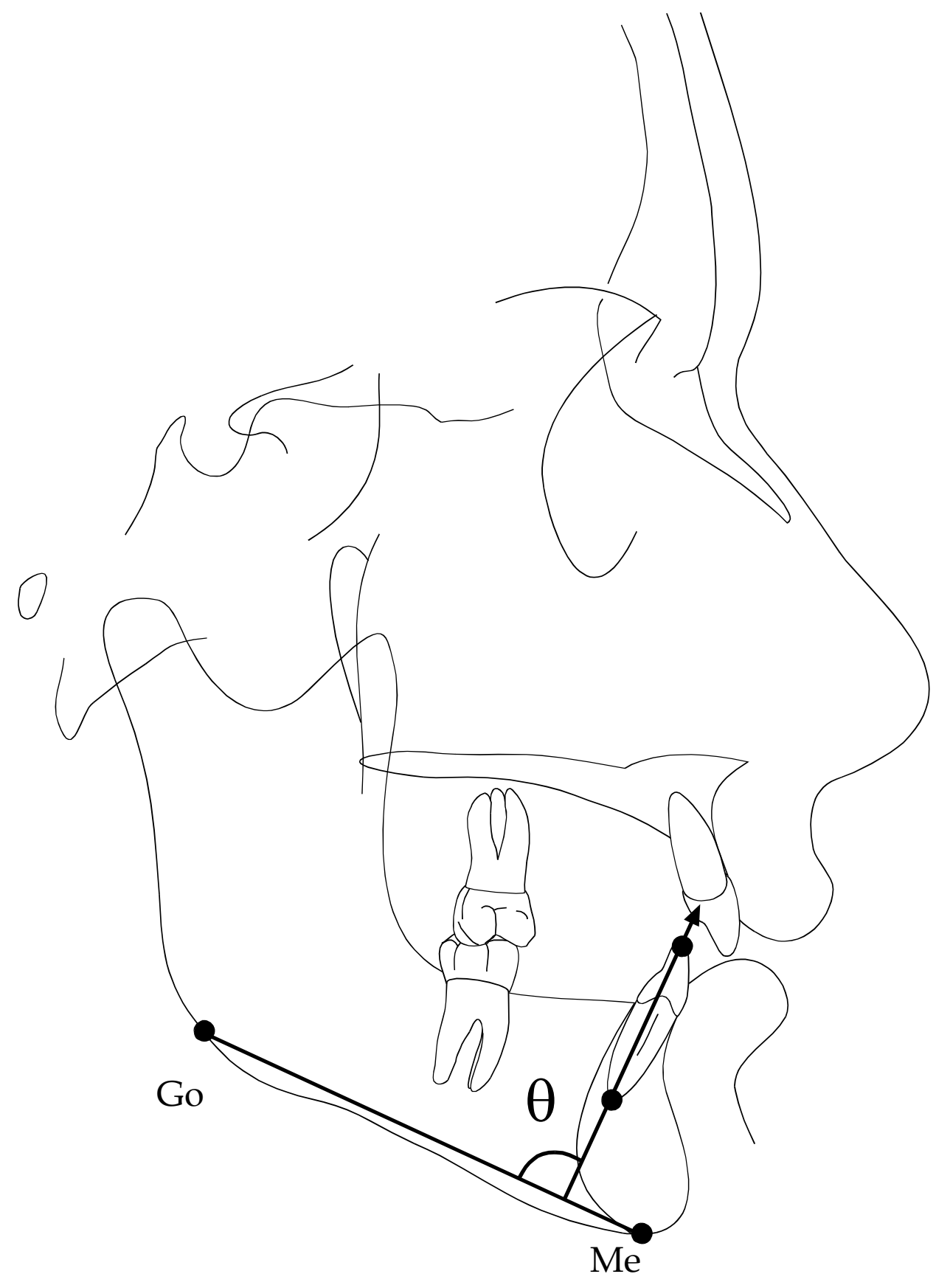

Figure 16. Schematic tracing of a lateral cephalogram showing construction of the angle of the mandibular incisor and the mandibular plane (IMPA). 


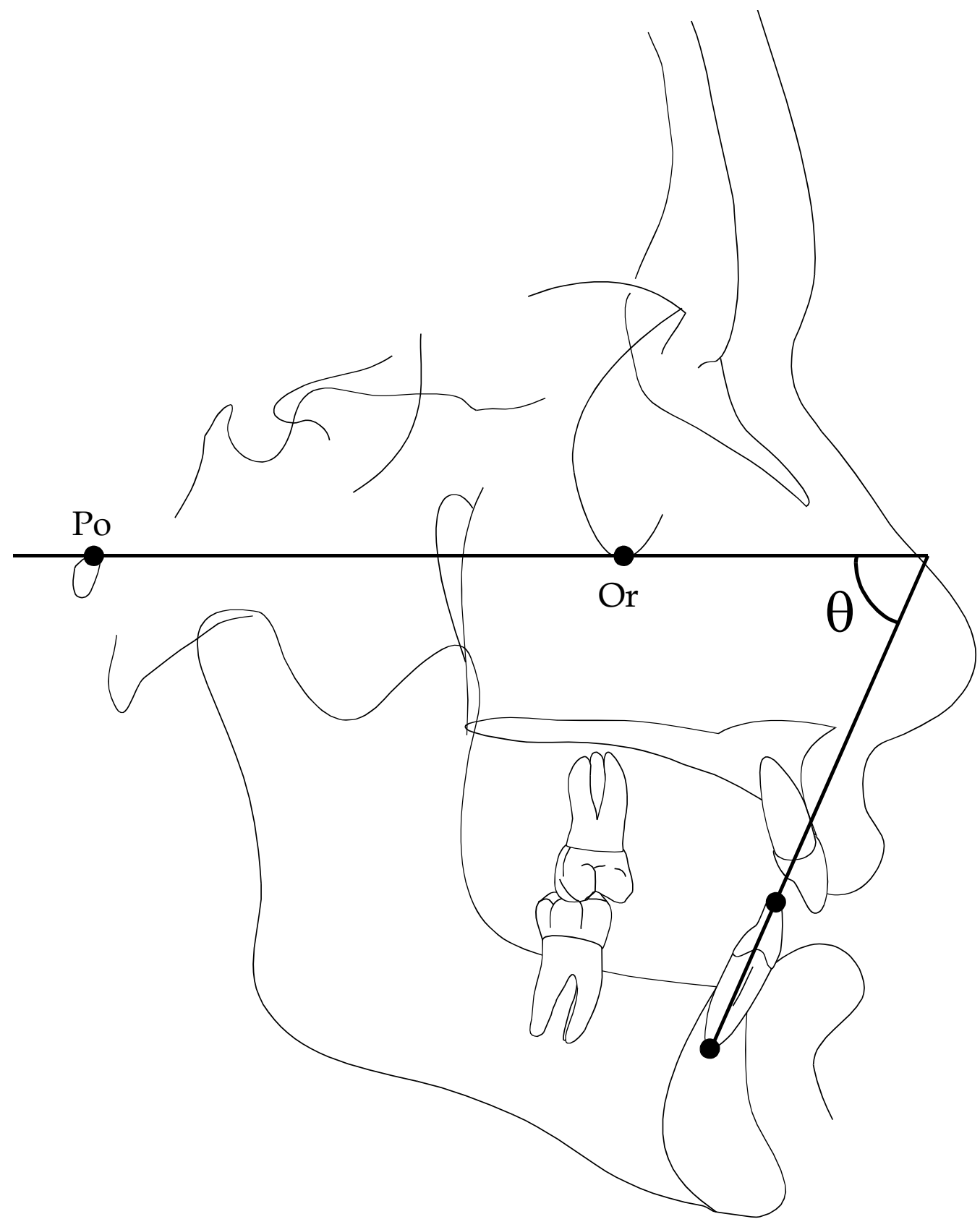

Figure 17. Schematic tracing of a lateral cephalogram showing construction of FMIA measurement. This is formed by the intersection of Frankfort Horizontal and the long axis of the mandibular incisor (L1 apex and L1 incisal edge). 


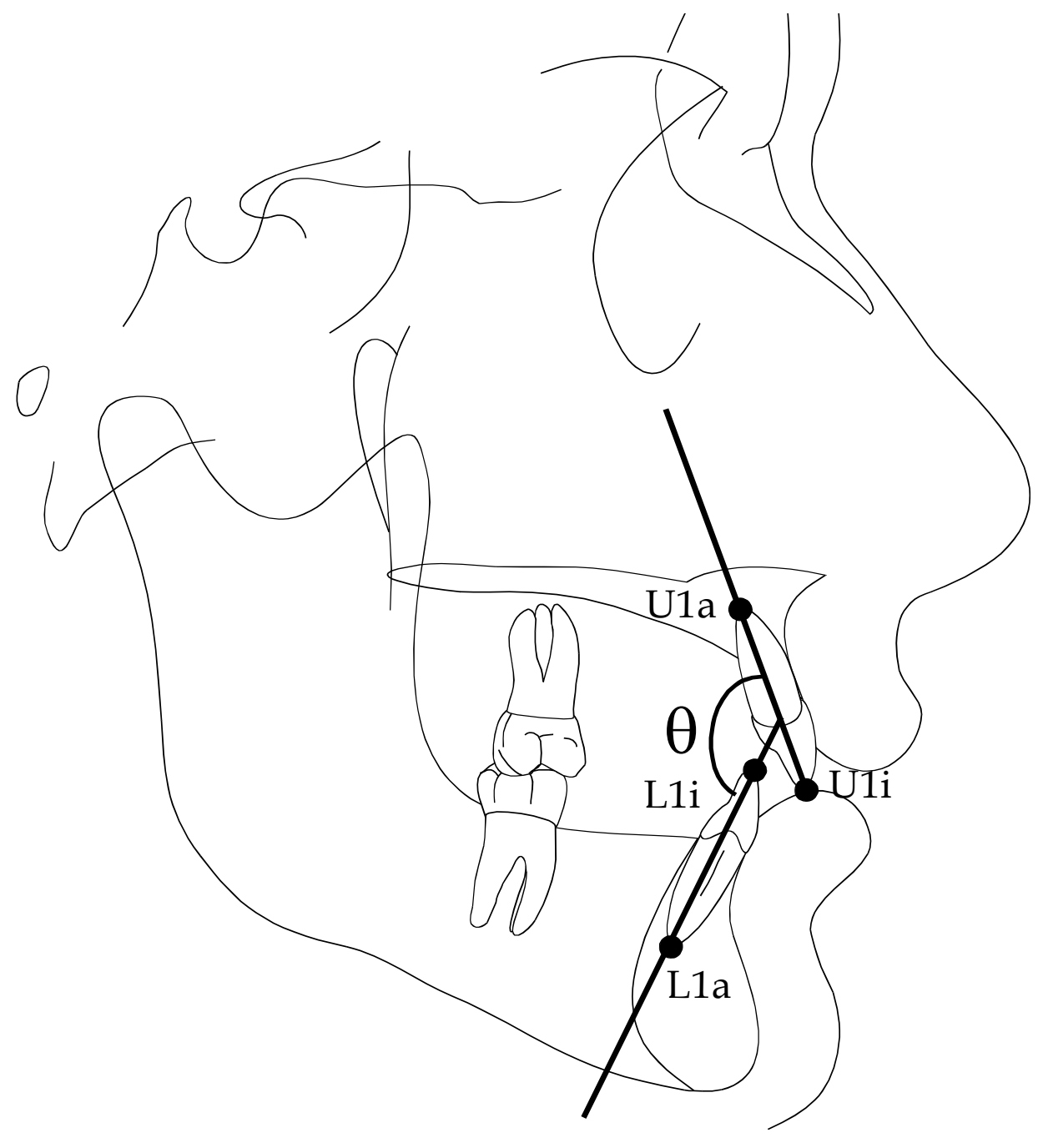

Figure 18. Schematic tracing of a lateral cephalogram showing construction of the angle between the upper incisor with the lower incisor (i.e., interincisal angle). 

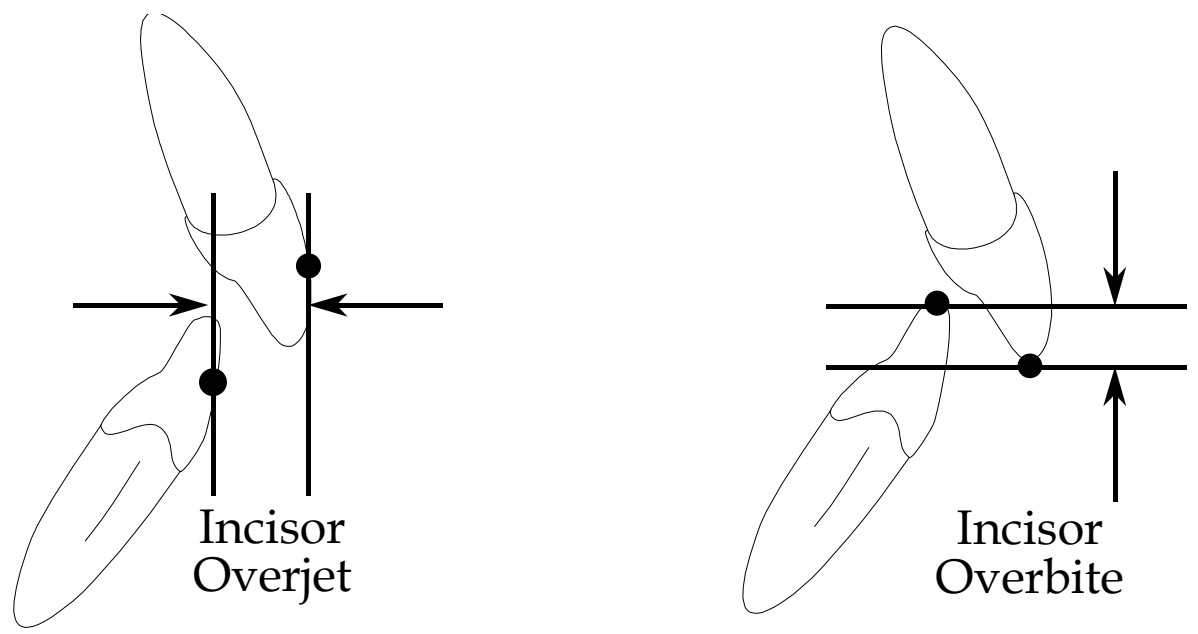

Figure 19. Schematic tracing of overbite and overjet measurements. 


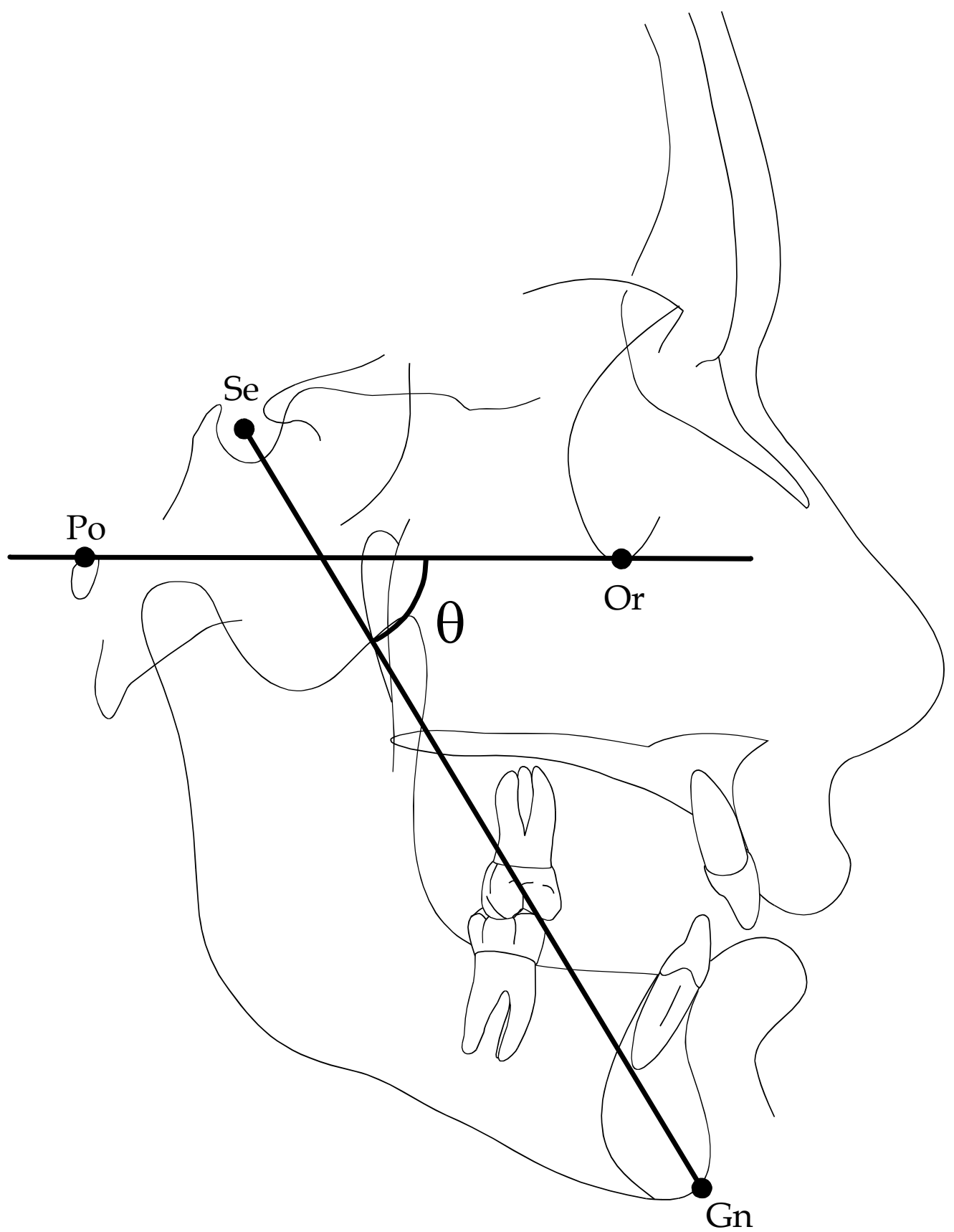

Figure 20. Schematic tracing of a lateral cephalogram showing construction of the $\mathrm{Y}$-axis angle. This is the anterior-inferior angle at the intersection of Frankfort Horizontal and the Sella-Gnathion line. 


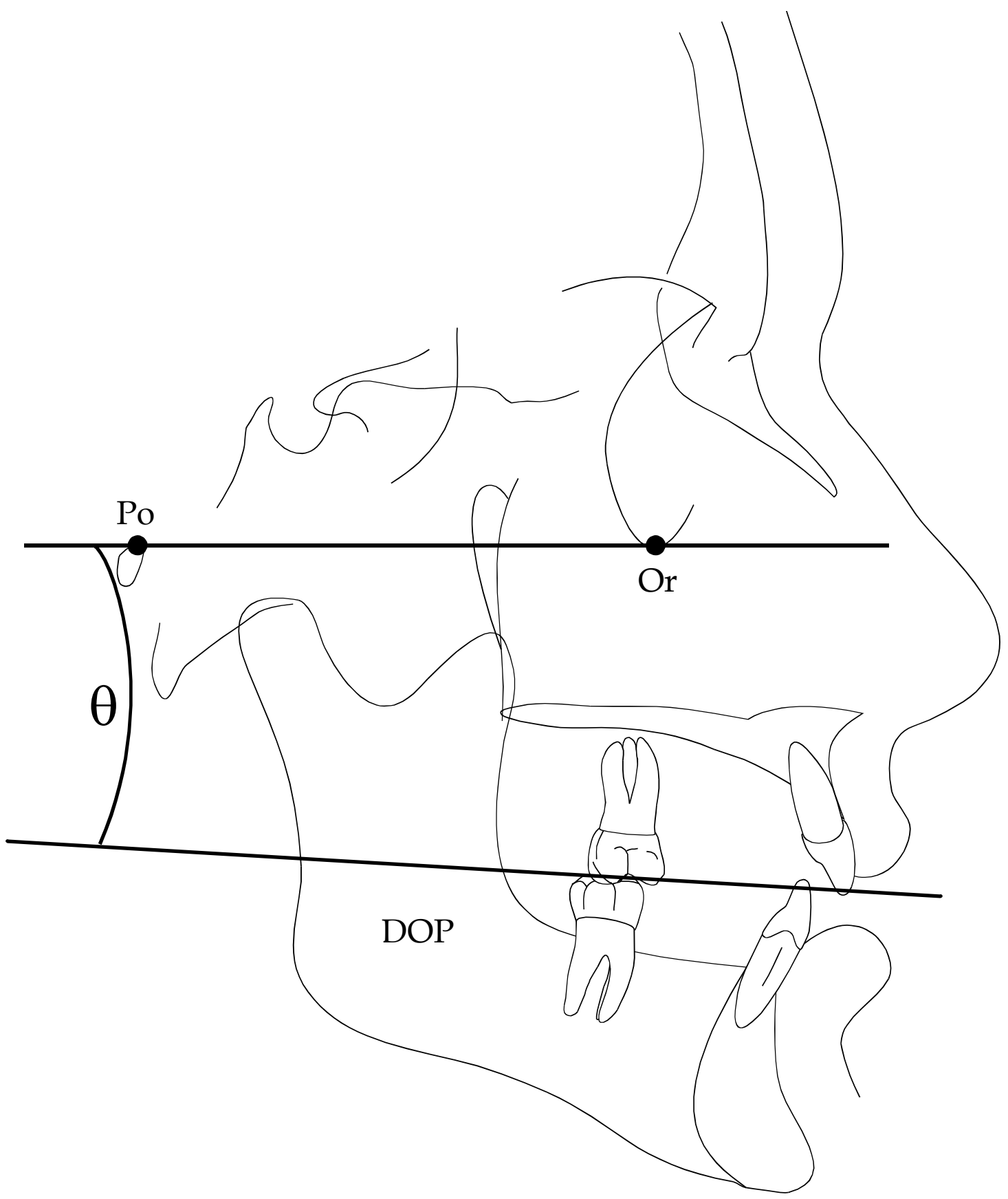

Figure 21. Schematic tracing of a lateral cephalogram showing construction of the Downs' occlusal plane angle. This is the angle formed by the intersection of Frankfort Horizontal and Downs' occlusal plane. 


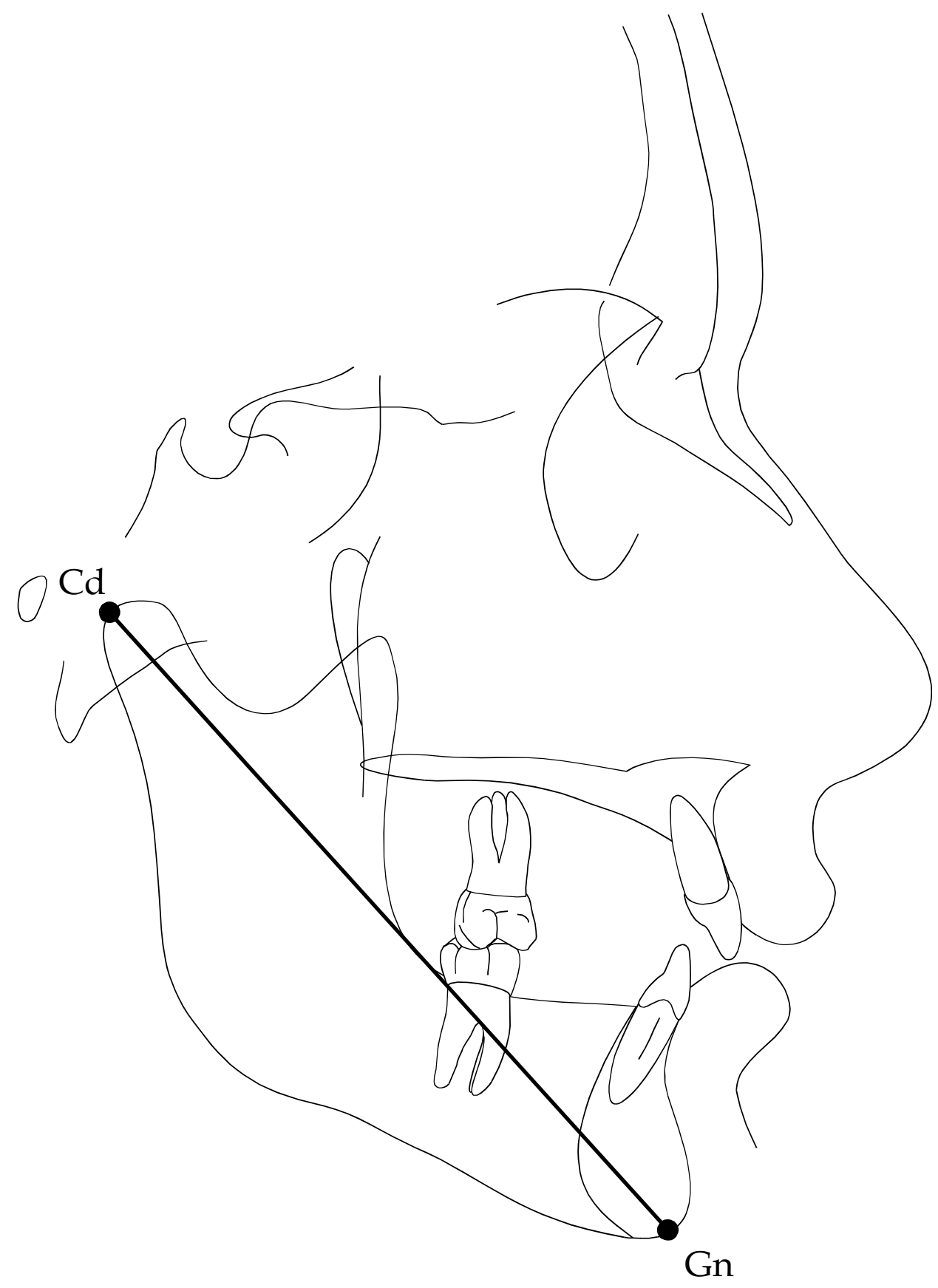

Figure 22. Schematic tracing of a lateral cephalogram showing construction of the Condylion to Gnathion. 


\section{CHAPTER IV}

\section{RESULTS}

\section{Extractions}

The manifest reason to extract teeth as part of orthodontic treatment is to alleviate tooth-size arch-size discrepancies (TSASD), but, in practice, many additional considerations come into play. In this study, extractions as part of orthodontic therapy were far more common in the one-phase group. Overall, just premolars were extracted, and the frequency was $12 \%$ (6/50 cases) in the two-phase series compared to $84 \%(42 / 50)$ in the one-phase series. This difference is highly significant statistically by chi-square analysis.

\section{$\underline{\text { Time in Treatment }}$}

A common criticism of two-phase treatment (by those averse to it) is that is takes longer. A longer interval is evident in the present study (Table 4). Children in the Bionator group began treatment at an average of 10.0 years, with this phase lasting an average of 1.4 years. This was followed by fixed treatment that began at an average age of 12.0 years, lasting 2.2 years. There was, on average, a gap of 0.5 years between the Bionator and the fixed phase. Overall treatment lasted from about 10 to 14 years of age, a duration of 4.2 
Table 4. Descriptive statistics of the examination dates, by group.

\begin{tabular}{lrrrrrr}
\hline Examination & $\bar{x}$ & sd & sem & $\mathrm{L}_{1}$ & $\mathrm{~L}_{2}$ & $\mathrm{n}$ \\
\hline \multicolumn{7}{c}{ Edgewise Treatment } \\
Starting Age & 13.15 & 1.39 & 0.20 & 12.76 & 13.55 & 50 \\
Age at End & 15.98 & 1.84 & 0.26 & 15.45 & 16.50 & 50 \\
Treatment Time & 2.82 & 1.31 & 0.18 & 2.45 & 3.19 & 50 \\
& \multicolumn{7}{c}{ Bionator + Edgewise Treatment } & & \\
Starting Age & 10.05 & 1.40 & 0.20 & 9.66 & 10.45 & 50 \\
End Phase 1 & 11.45 & 1.44 & 0.20 & 11.04 & 11.86 & 50 \\
Time in Phase 1 & 1.40 & 1.15 & 0.16 & 1.07 & 1.72 & 50 \\
Start Phase 2 & 12.05 & 1.31 & 0.19 & 11.68 & 12.43 & 49 \\
End Phase 2 & 14.31 & 1.53 & 0.22 & 13.87 & 14.74 & 50 \\
Time in Phase 2 & 2.24 & 0.88 & 0.13 & 1.99 & 2.49 & 49 \\
Treatment Time & 4.25 & 1.33 & 0.19 & 3.88 & 4.63 & 50 \\
Treatment Time* & 3.65 & 1.41 & 0.20 & 3.24 & 4.05 & 49 \\
\hline
\end{tabular}

*Time in treatment omitting the interval between phases. 
years. If the interval between the two phases is omitted, overall duration reduces to 3.6 years.

In contrast, single-phase treatment commenced at an average of 13.1 years, with an average duration of 2.8 years. Consequently, overall treatment time was longer in the two-phase sample (by an average of 1.4 years, or 17 months). The fixed phase was, as often-noted in prior studies, somewhat shorter in the two-phase groups (2.2 versus 2.8 years, a difference of 6 months). Ages at termination of treatment also were different: Patients treated in two phases were completed at 14 years of age compared to 16 years of age in the single-phase series. This means that the average Bionator patient would be debonded when a freshman in high school versus a junior.

\section{Changes between Phases}

Goals of the Bionator phase of treatment are fundamentally different from those of later fixed treatment. Cephalometric changes during these two phases are contrasted in this section. Because a cephalogram was taken at the end of the Bionator phase, we could quantitatively compare the changes in each variable between these two, successive phases. A mixed-model ANOVA design was used, since identically the same patients were followed across the two phases, and sex of the subjects was included in the model since boys' and girls' craniofacial growth vectors are apparently different (van der Linden 1986; 
Harris 2001). The mean changes during the first and second phases of treatment are listed in Table 5. Notice that there are two sets of figures here; the first two columns of numbers are the means during the two phases having pooled males and females, while the next four columns provide data for boys and girls separately. Table 6 lists the results of the mixed model ANOVA. There are three tests for each variable (each row): The first F-ratio tests whether boys and girls responded differently during treatment; the second Fratio tests whether the amount of change differed between Bionator and Edgewise phases; and the third F-ratio tests whether there was a significant sex-by-phase interaction effect. These comparisons only pertain to when the changes occurred in the two-phase group; no comparison is made here to the conventional Edgewise sample.

The duration of the two phases of treatment were fairly equal, namely an average of 2.1 years in Bionator treatment and an average of 2.3 years in fixed appliances thereafter.

\section{$\underline{\text { Integument }}$}

Of the two variables assessed here ( $\mathrm{Z}$ angle and $\mathrm{E}$ plane), neither differed in magnitude of change between the two phases (Figures 23, 24). The E plane changed less in boys than girls (Figure 24), but the clinical significance of this minor sex difference (if any) is not apparent. 


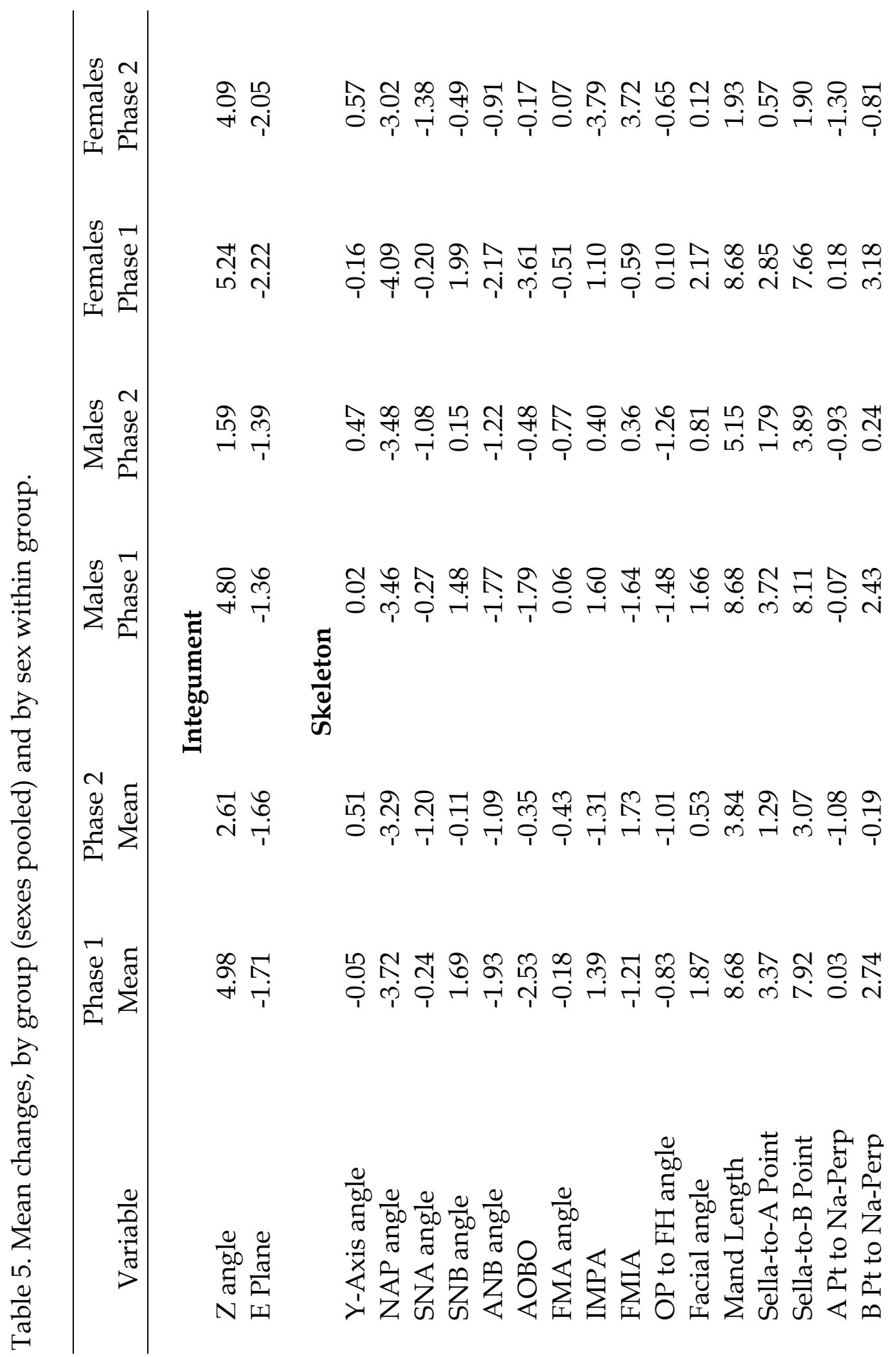




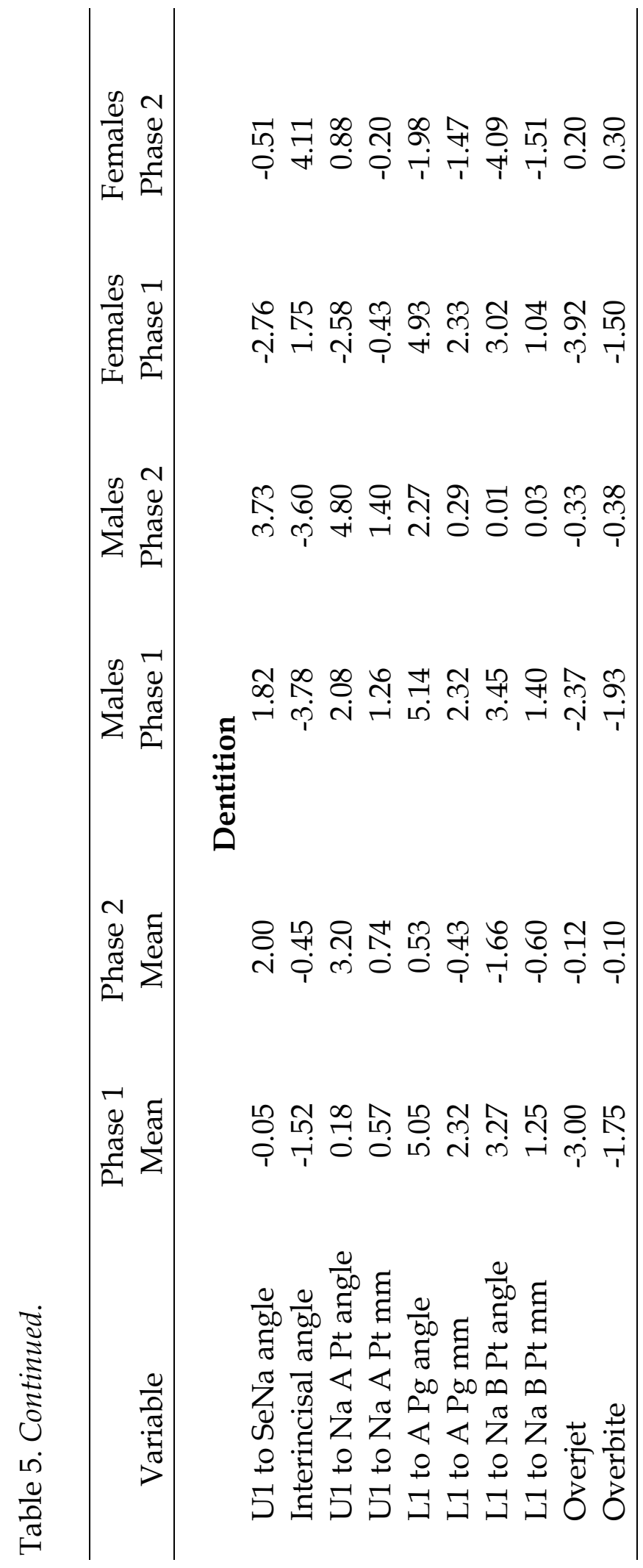




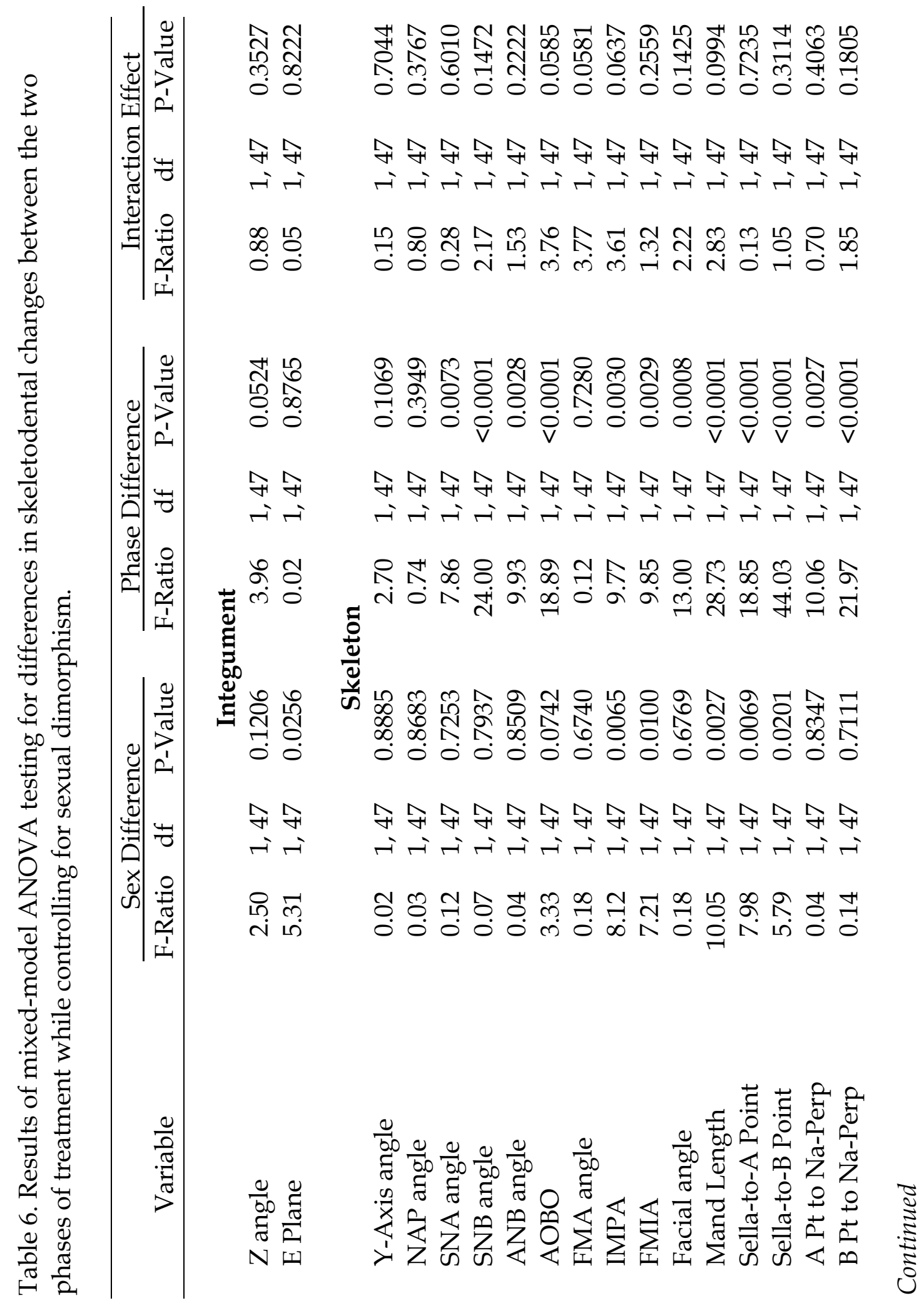




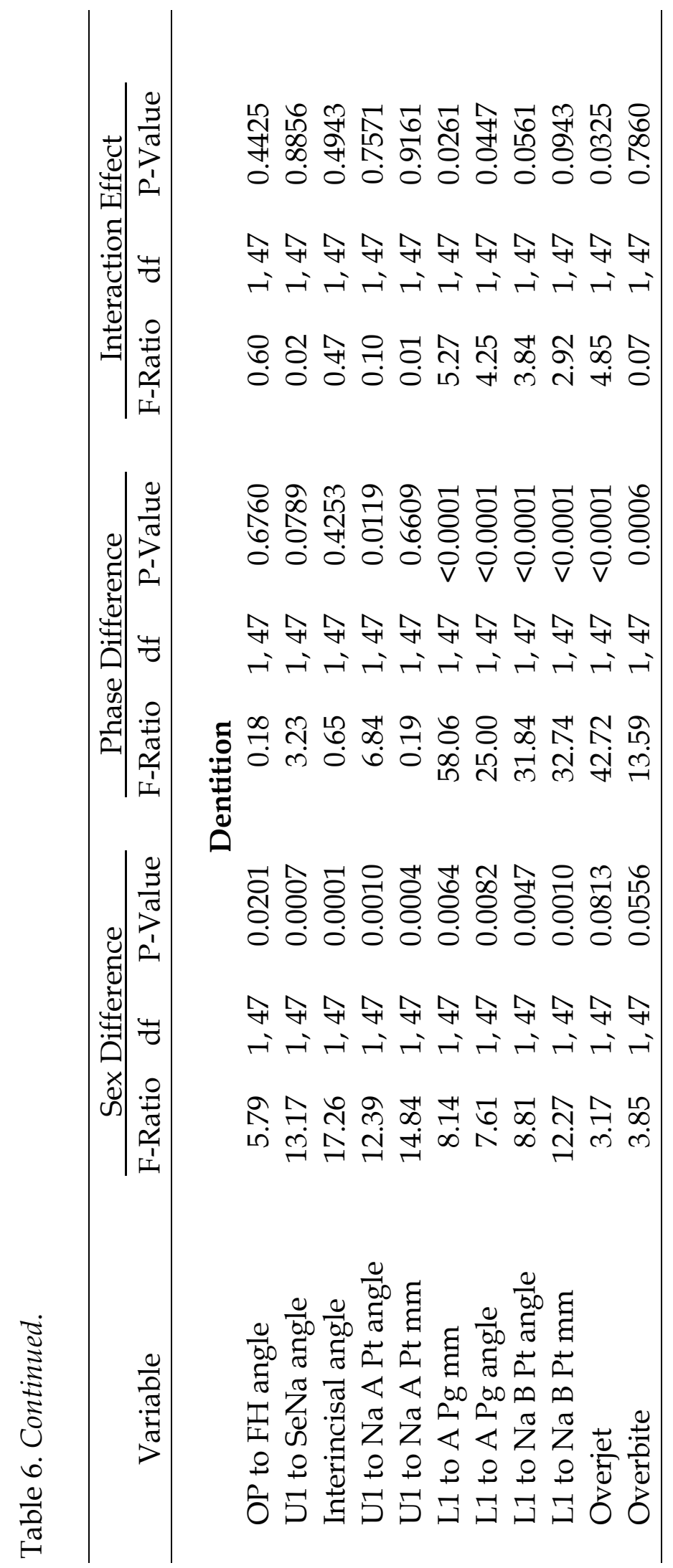




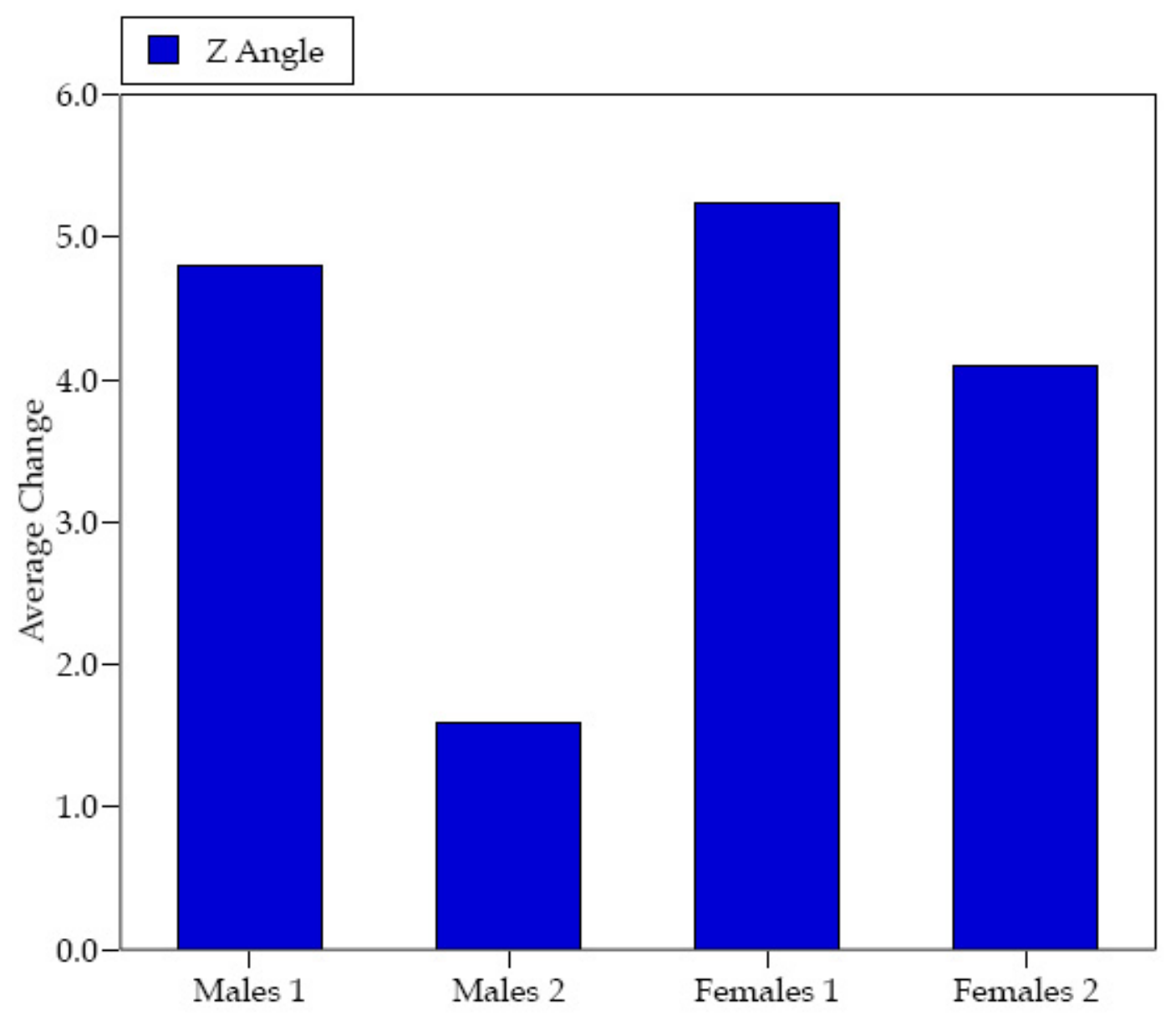

Figure 23. Mean changes in the $\mathrm{Z}$ angle during phase one (1) and phase two (2) of compound orthodontic treatment, by sex. 


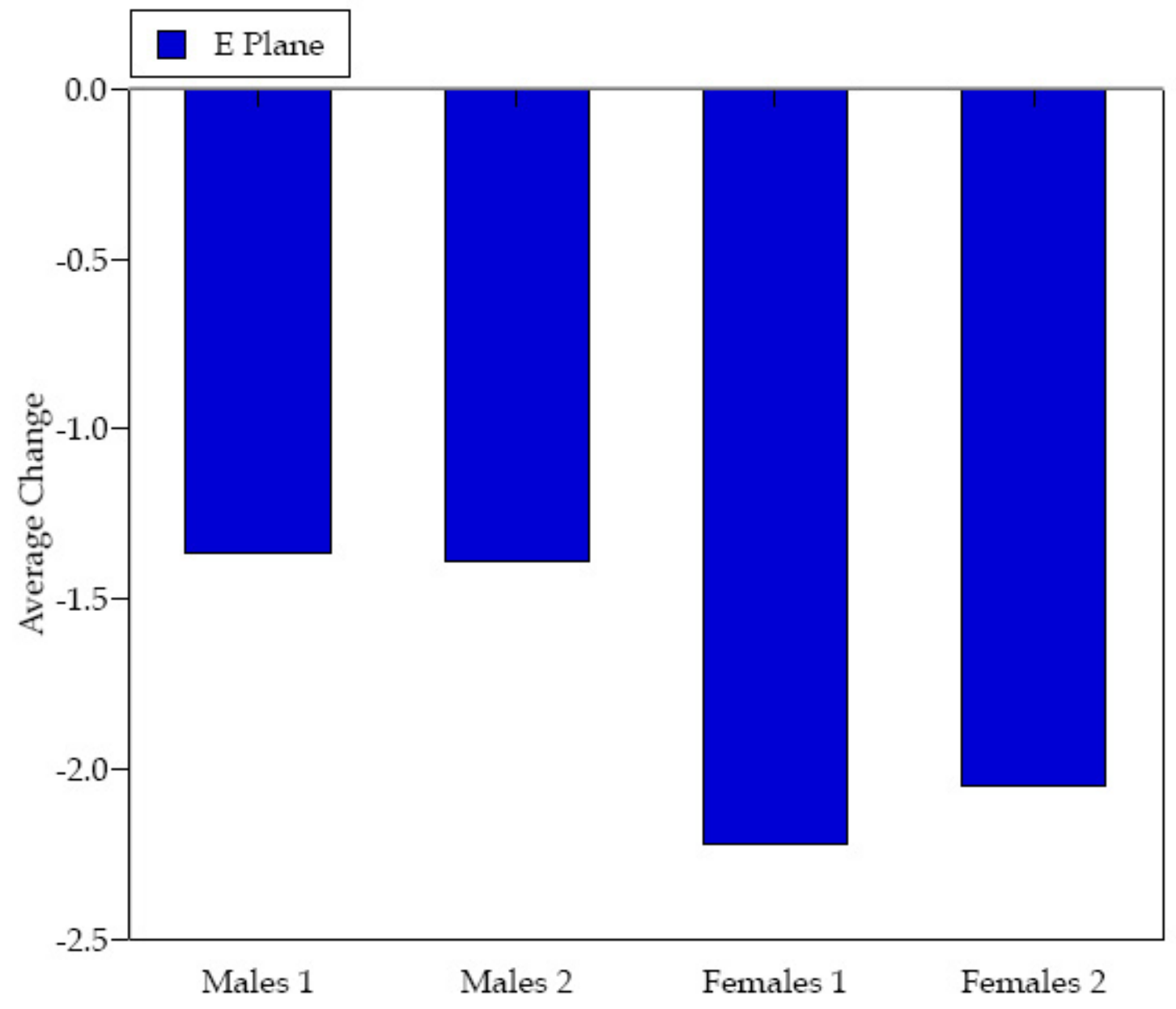

Figure 24. Mean changes in the E Plane during phase one (1) and phase two (2) of compound orthodontic treatment, by sex. 


\section{$\underline{\text { Skeleton }}$}

Changes did not differ between the two groups for the Y-axis (Figure 25) or for the NAP angle (Figure 26). However, the three variables that depend on Nasion (i.e., SNA, SNB, and ANB) show significantly more change during the fixed phase than during the Bionator phase (Figures 27-29). None of these three changes was sexually dimorphic. SNA changed slightly $\left(\bar{x}=0.2^{\circ}\right)$ during Bionator phase, but then decreased significantly $\left(\bar{x}=-1.7^{\circ}\right)$ during the Bionator treatment but almost not at all during the fixed phase $\left(\bar{x}=-0.1^{\circ}\right)$. The net effect is that ANB, a measure of maxillomandibular discrepancy, improved (decreased) significantly more in phase $1\left(\bar{x}=-1.9^{\circ}\right)$ than phase $2\left(\bar{x}=-1.1^{\circ}\right)$.

This overall decrease in ANB $\left(\bar{x}=3.1^{\circ}\right)$ is readily assuaged with the statement above that the mean change in NAP was not significant (Table 5). The ANOVA tests in Table 6 evaluate whether there is a difference in the amounts of change between the two phases, not whether the changes are statistically different from zero (i.e., no systematic change). Consequently, the test for NAP in Table C2 merely means that the change during the Bionator change $\left(\bar{x}=-3.7^{\circ}\right)$ was not different statistically from that during the later fixed phase $\left(\bar{x}=3.3^{\circ}\right)$.

SNA and SNB both changed significantly more during one phase than the other, but in complementary fashions (Figures 26, 27). SNA decreased 


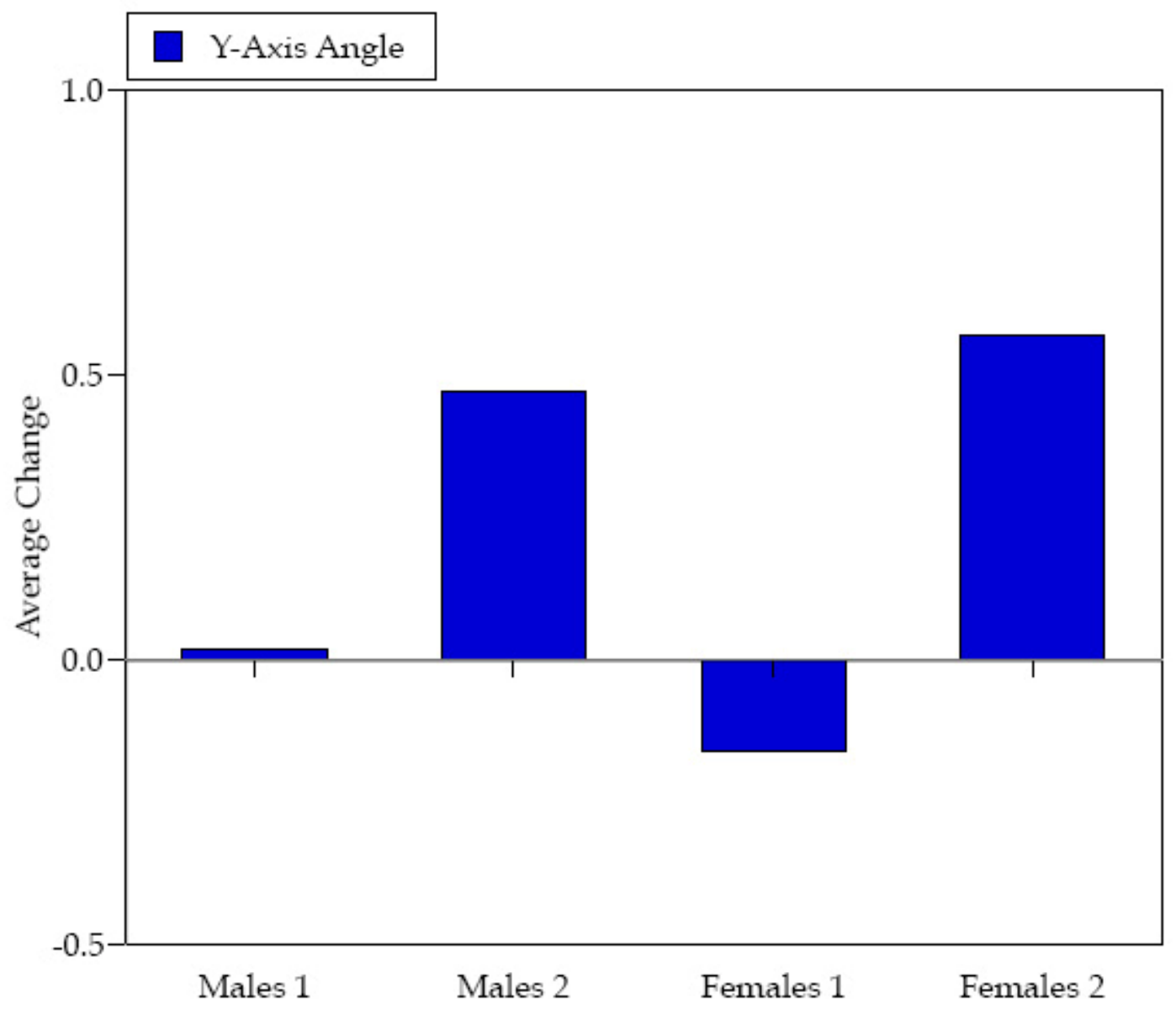

Figure 25. Mean changes in the Y-axis angle during phase one (1) and phase two (2) of compound orthodontic treatment, by sex. 


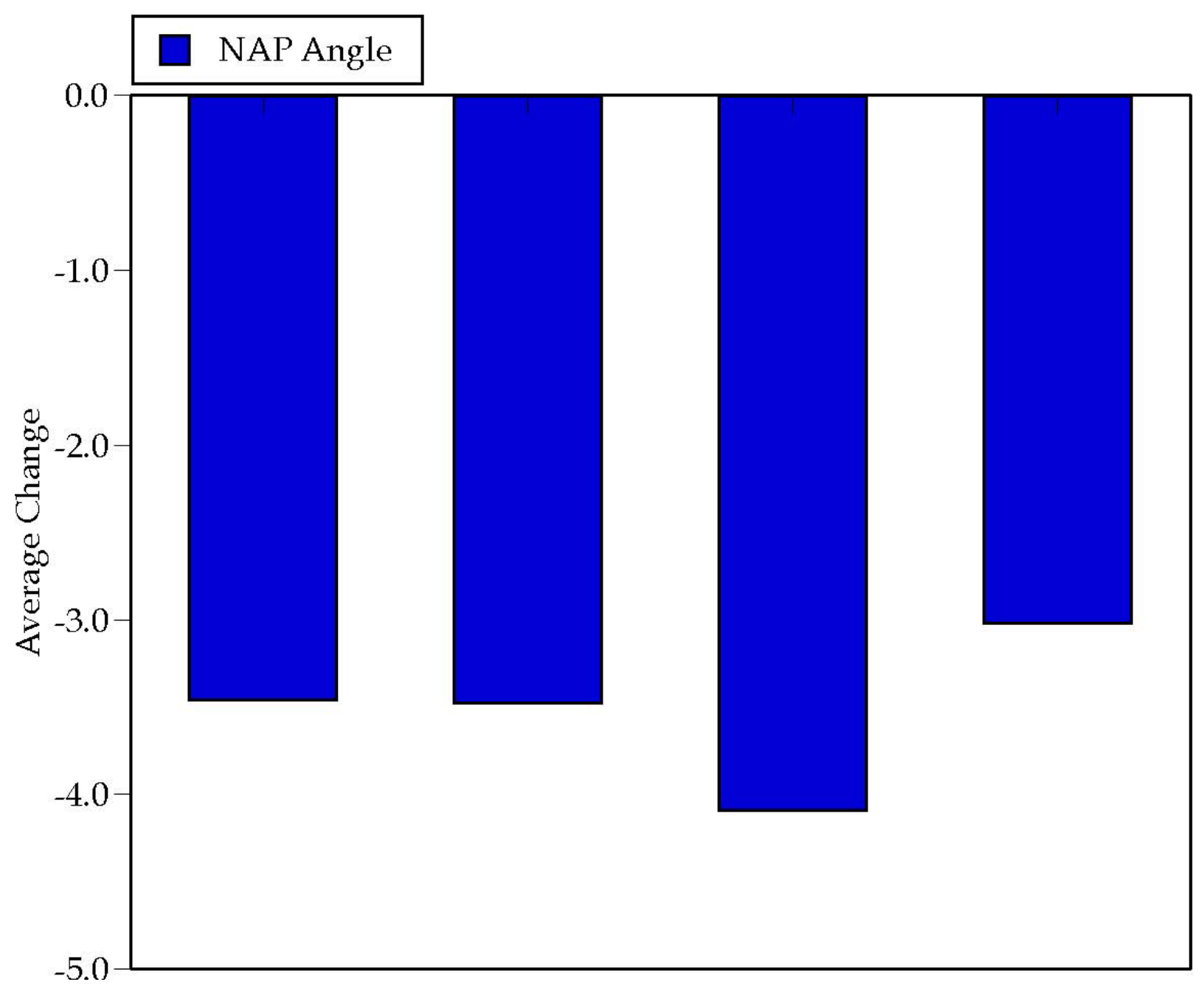

Figure 26. Mean changes in the NAP angle during phase one (1) and phase two (2) of compound orthodontic treatment, by sex. 


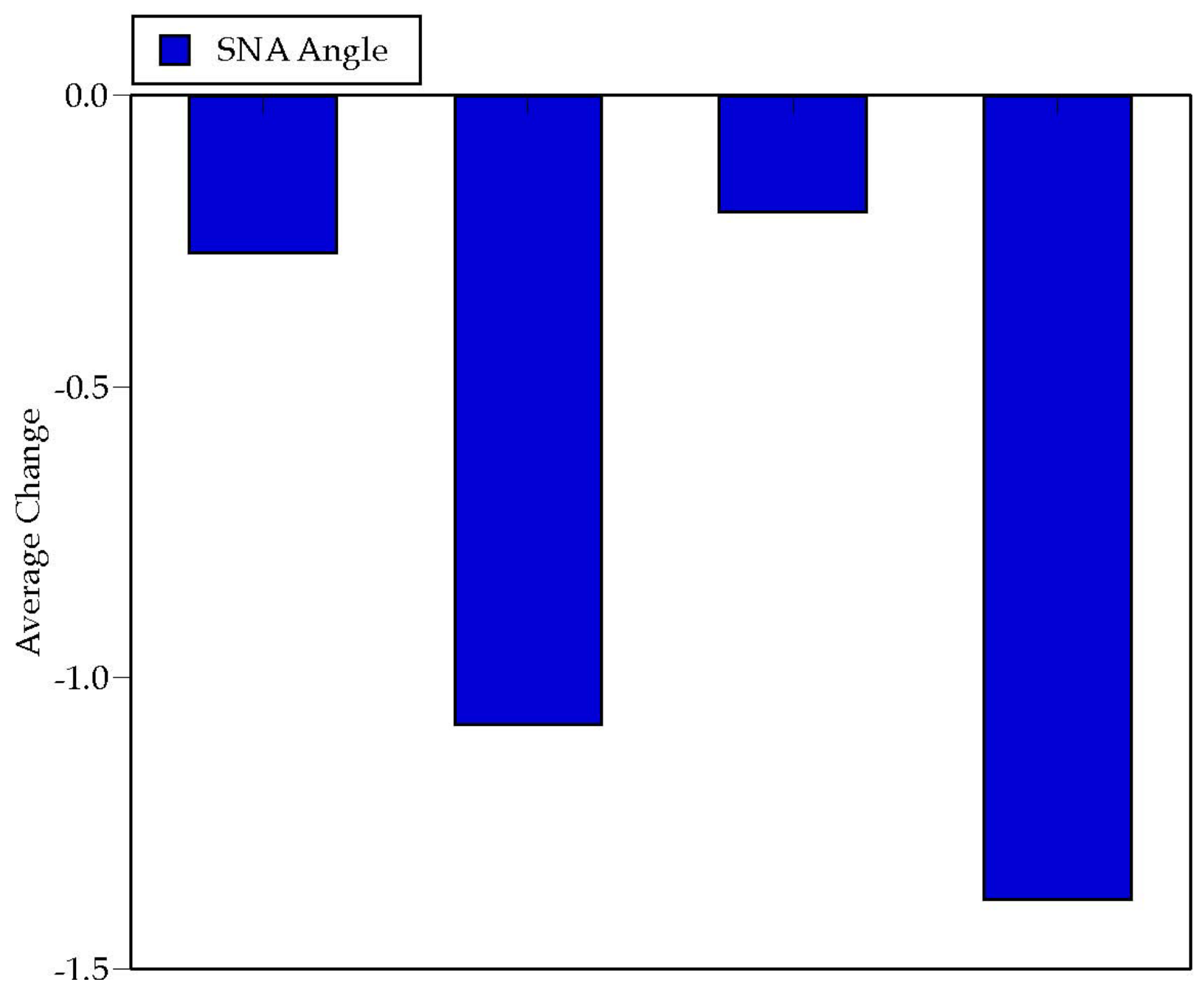

Figure 27. Mean changes in the SNA angle during phase one (1) and phase two (2) of compound orthodontic treatment, by sex. 


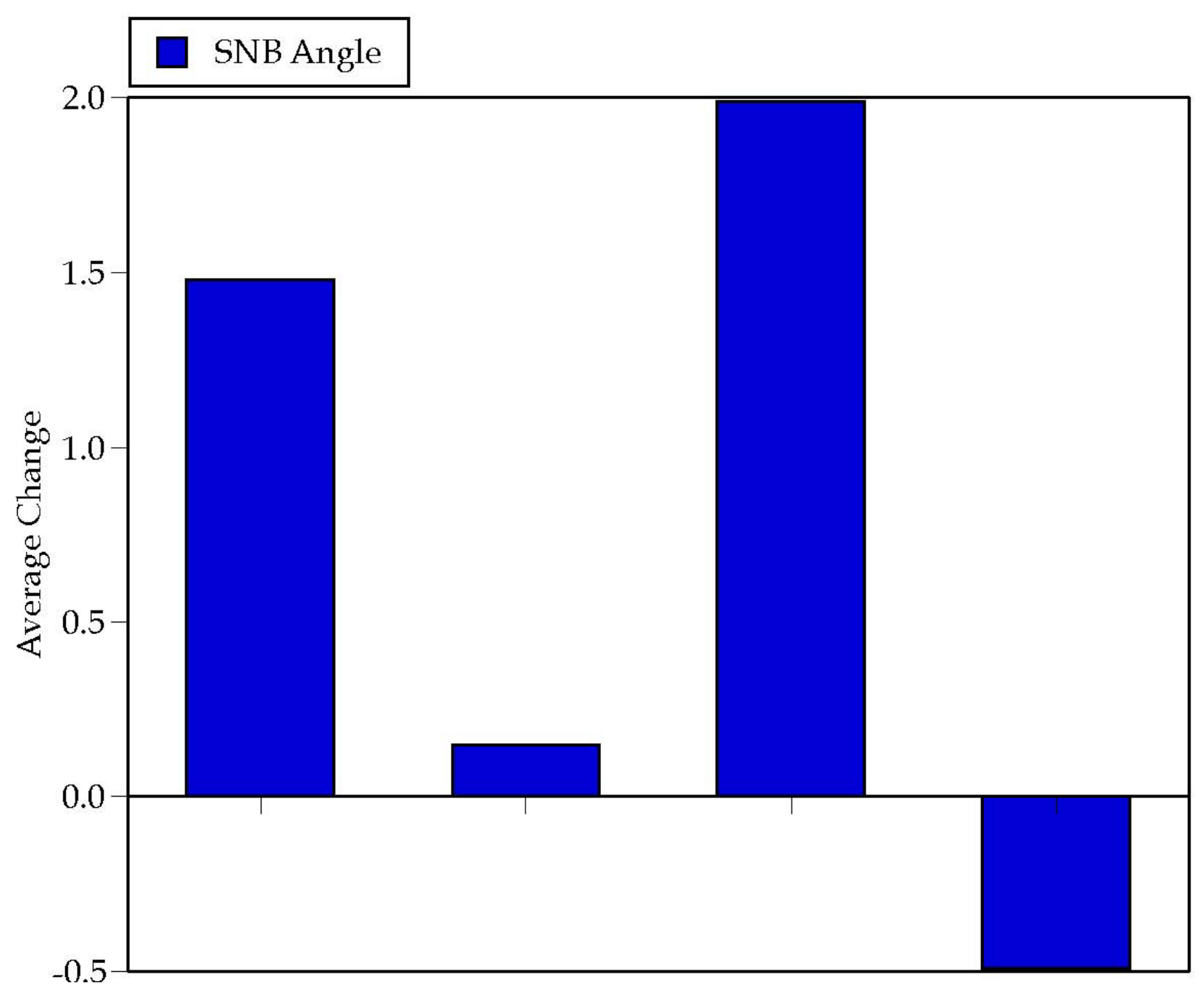

Figure 28. Mean changes in the SNB angle during phase one (1) and phase two (2) of compound orthodontic treatment, by sex. 


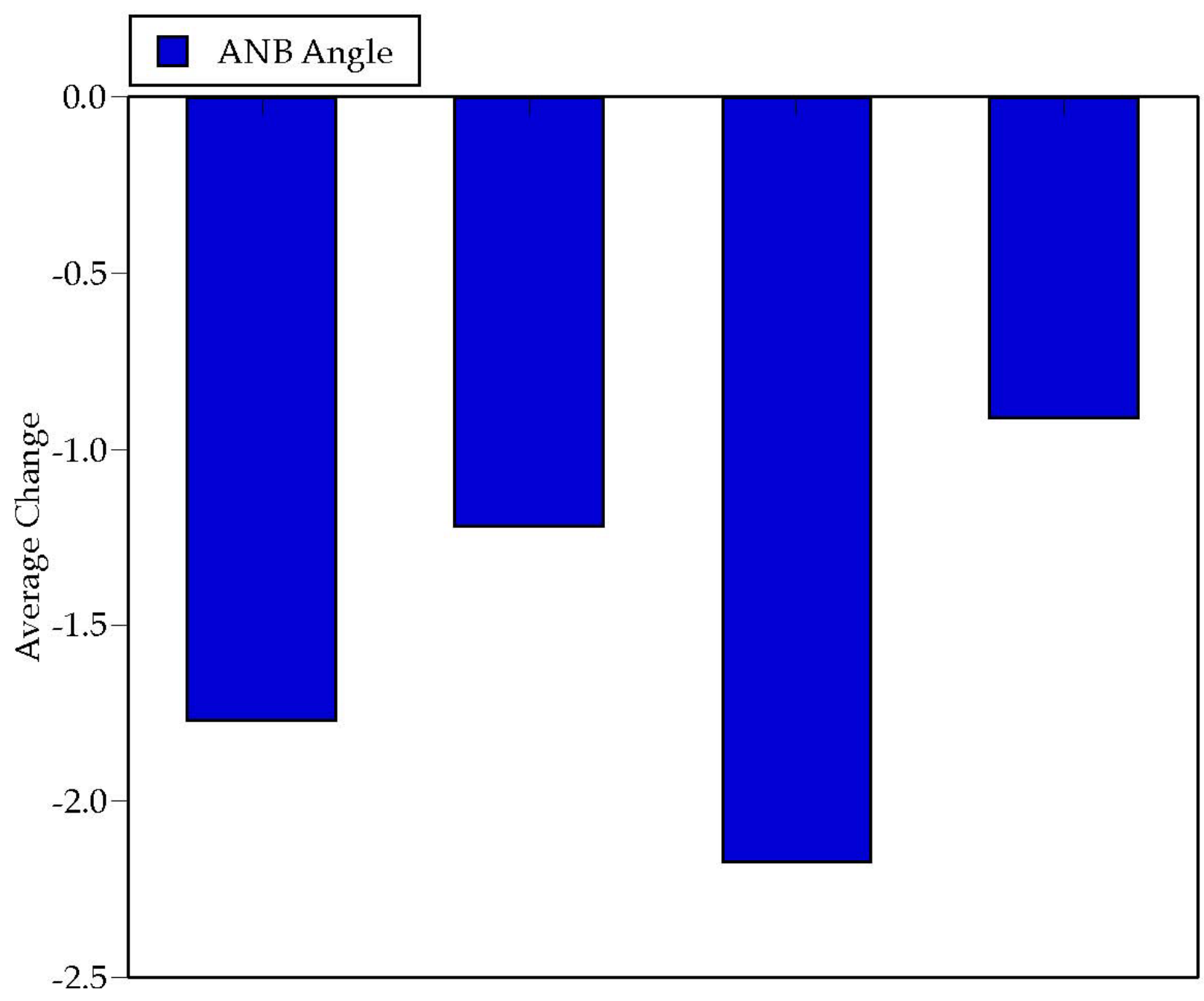

Figure 29. Mean changes in the ANB angle during phase one (1) and phase two (2) of compound orthodontic treatment, by sex. 
significantly more during the fixed phase, while SNB increased significantly more during the Bionator phase.

The composite change in the two jaws (i.e., ANB angle) was a decrease that averaged $-3.1^{\circ}$. Figure 29 shows that most of the change in ANB occurred during the Bionator phase of treatment, probably because propelling the mandible forward is a specific aim of the Bionator device. Indeed, the change was significantly greater during the Bionator phase $(P<0.003)$.

$\mathrm{AOBO}$ (the Wits discrepancy) is another measure of maxillomandibular harmony. Considerably more of the overall improvement of $2.9 \mathrm{~mm}$ occurred during the Bionator phase (Figure 30). The AOBO discrepancy averaged 3.5 $\mathrm{mm}$ at the start of treatment in the two-phase sample. The average reduction of $2.9 \mathrm{~mm}$, mostly achieved with Bionator treatment, left the group with a mean AOBO of $0.6 \mathrm{~mm}$, which is within desired limits (Jacobson 1976, 1988).

In this same vein (i.e., $\mathrm{SNA}, \mathrm{SNB}, \mathrm{ANB}$, and $\mathrm{AOBO}$ changes), the facial angle changed (increased) significantly more during the Bionator phase of treatment, a difference of $1.9^{\circ}$ versus $0.5^{\circ}$ during the fixed phase (Figure 31). These measures of inter-arch relationships show that there was appreciable improvement with the compound treatment, and most of the skeletal correction was achieved during the earlier, Bionator phase.

The FMA changed little overall, and the difference in change between the two phases was not significant (Figure 32). This consistency argues in 


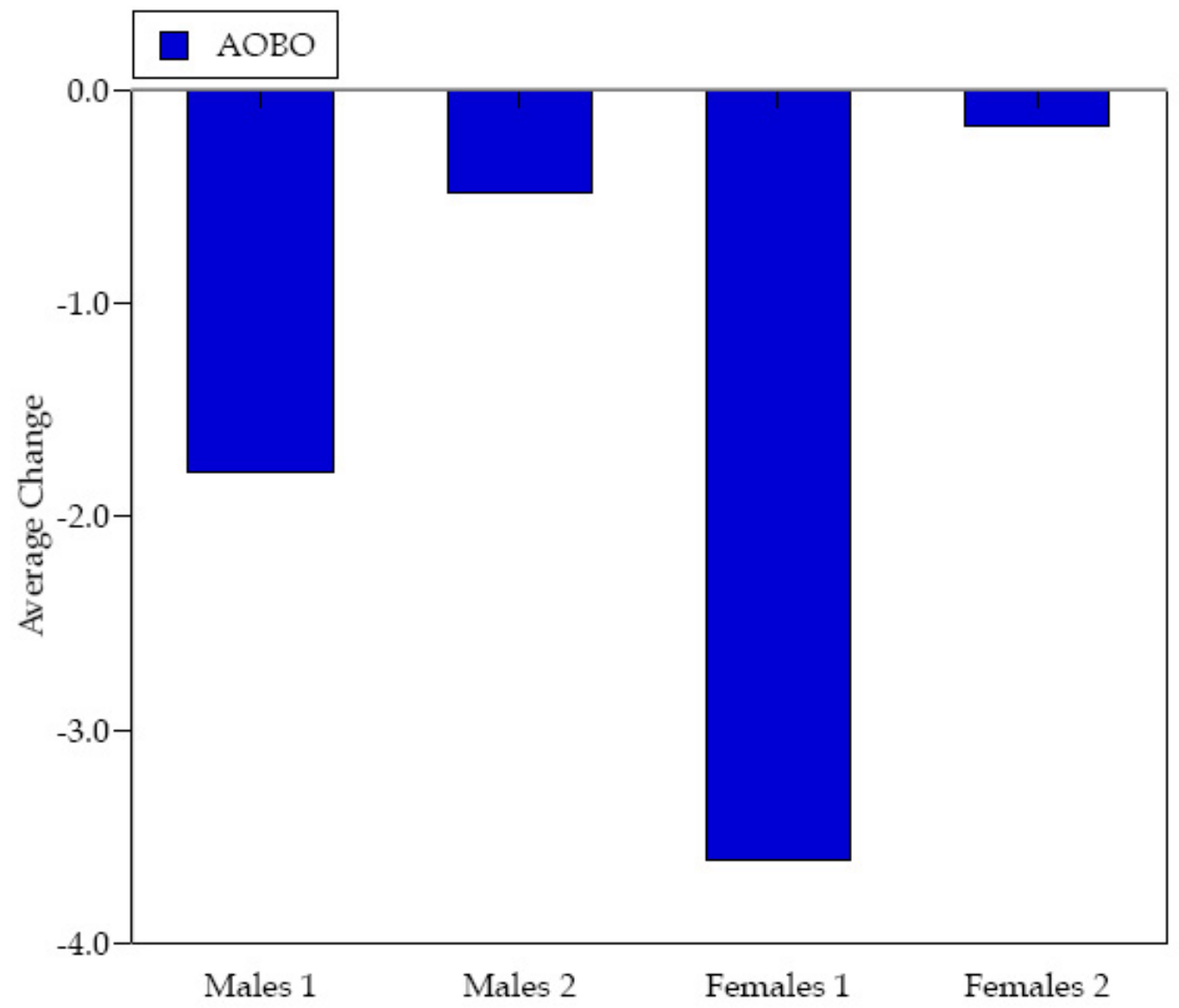

Figure 30. Mean changes in the AOBO Discrepancy during phase one (1) and phase two (2) of compound orthodontic treatment, by sex. 


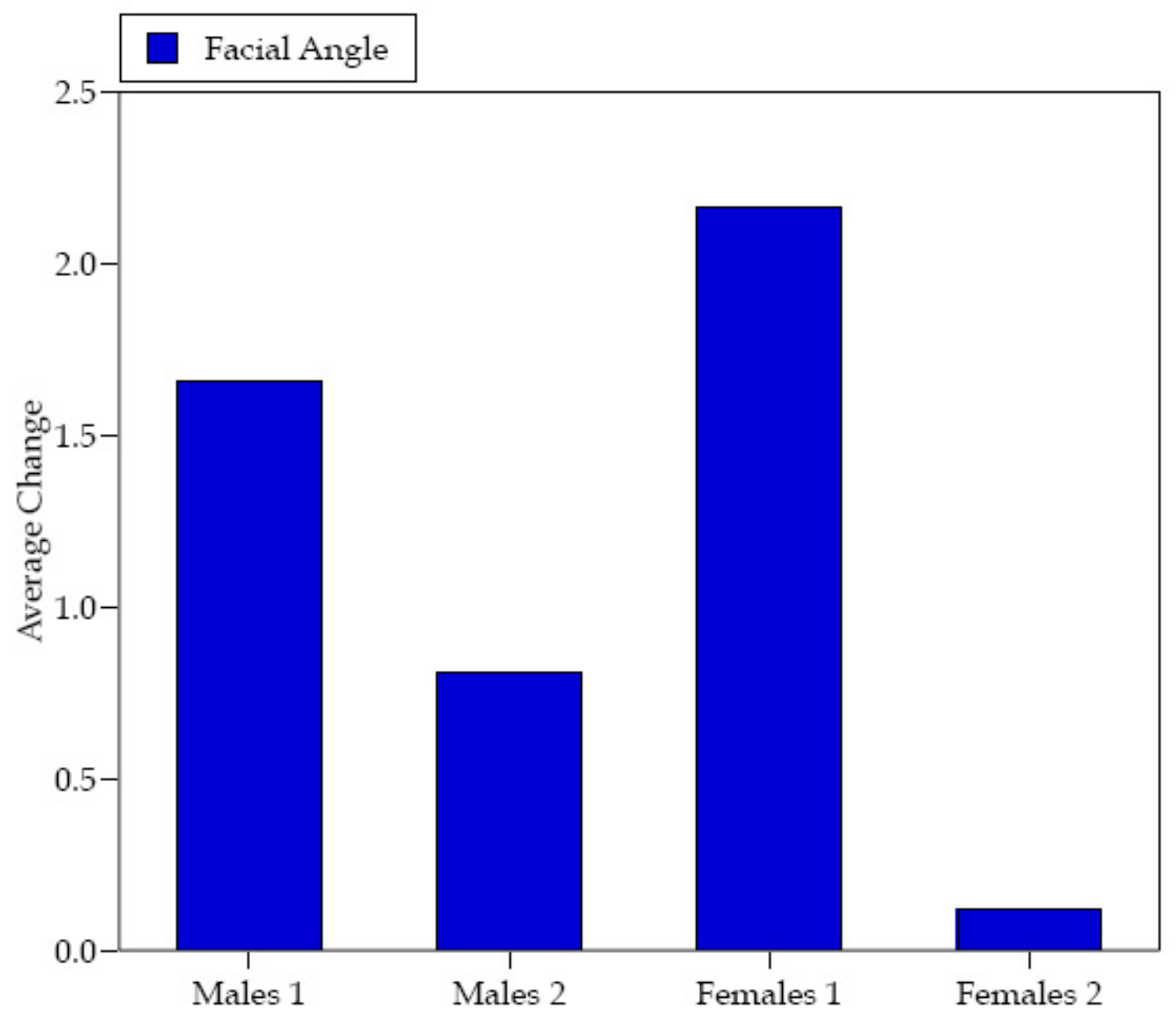

Figure 31. Mean changes in the facial angle during phase one (1) and phase two (2) of compound orthodontic treatment, by sex. 


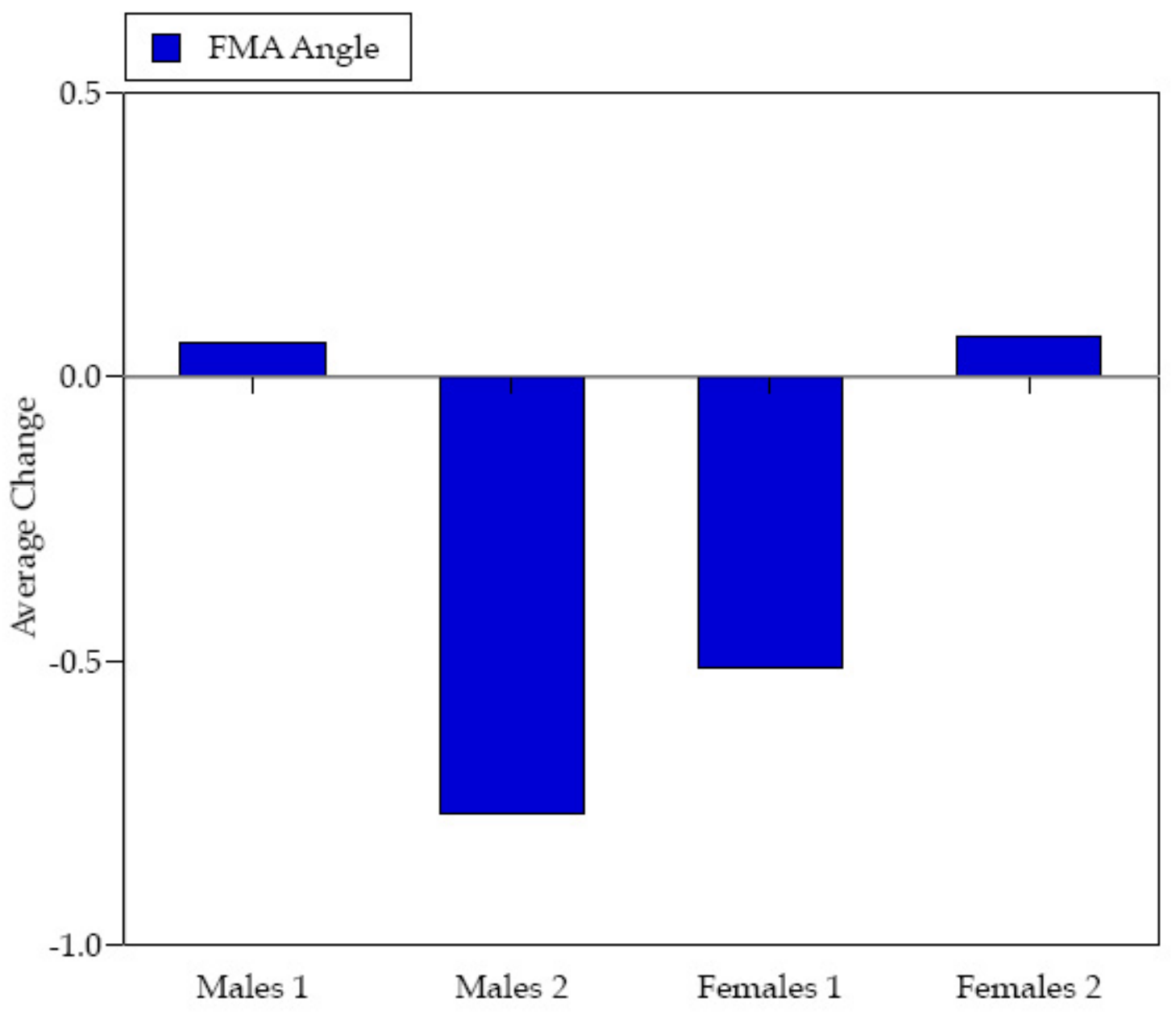

Figure 32. Mean changes in the FMA during phase one (1) and phase two (2) of compound orthodontic treatment, by sex. 
favor that proper orthodontic mechanics were maintained so there was no loss of vertical control.

The three measures of linear change in facial depth, namely (1) mandibular length (Figure 33), (2) Sella-A Point length (Figure 34), and (3) Sella-B Point length (Figure 35), all exhibit significantly more growth during the Bionator phase of treatment than during the later fixed phase of treatment. Of note, these greater rates of growth during the Bionator phase occurred during an average of 9.5 and 11.5 years of age, which, especially in boys, precedes the adolescent growth spurt (Tanner 1962). Growth of these three dimensions also is obvious during the fixed phase, but at significantly slower rates (Table 5).

Two variables were specifically included to evaluate changes in the bony facial profile. These are the distances to A Point and to B Point, each measured relative to Nasion-Perpendicular (Figures 36, 37). Both of these dimensions changed to significantly different extents during the two phases of treatment, but in complementary fashions. A Point changed almost not at all during Bionator treatment, but then dropped back $(\bar{x}=1.1 \mathrm{~mm})$ farther from NasionPerpendicular during the fixed phase. In contrast, B Point grew forward an average of $2.7 \mathrm{~mm}$ during the first phase, but then likewise dropped back a bit $(\bar{x}=0.2 \mathrm{~mm})$ during fixed treatment. The discrepancy between points $A$ and $B$ decreased to an average of $6.8 \mathrm{~mm}$ at the end of the Bionator phase. This 


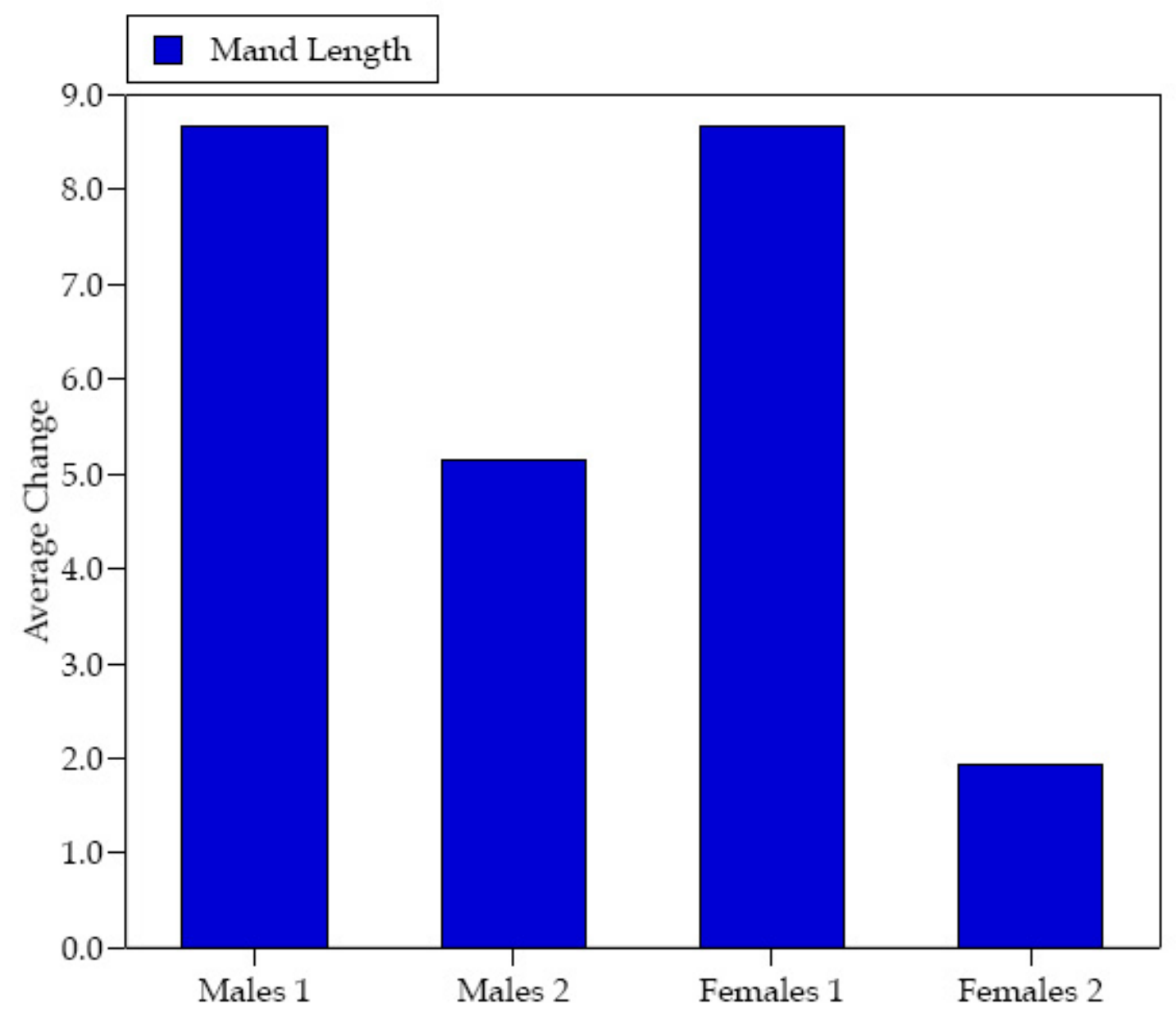

Figure 33. Mean changes in the Mandibular Length during phase one (1) and phase two (2) of compound orthodontic treatment, by sex. 


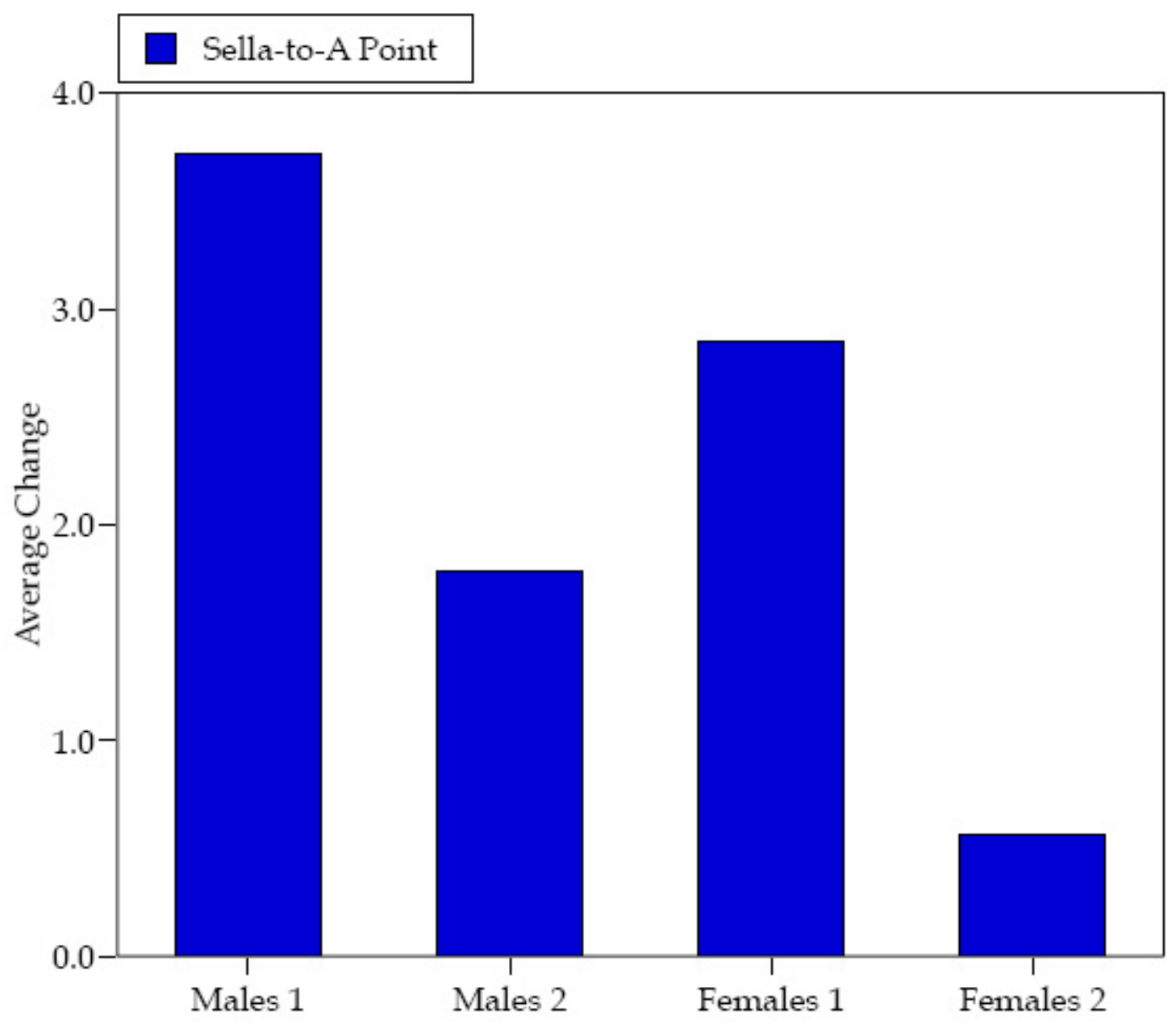

Figure 34. Mean changes in the Sella-A Point Length during phase one (1) and phase two (2) of compound orthodontic treatment, by sex. 


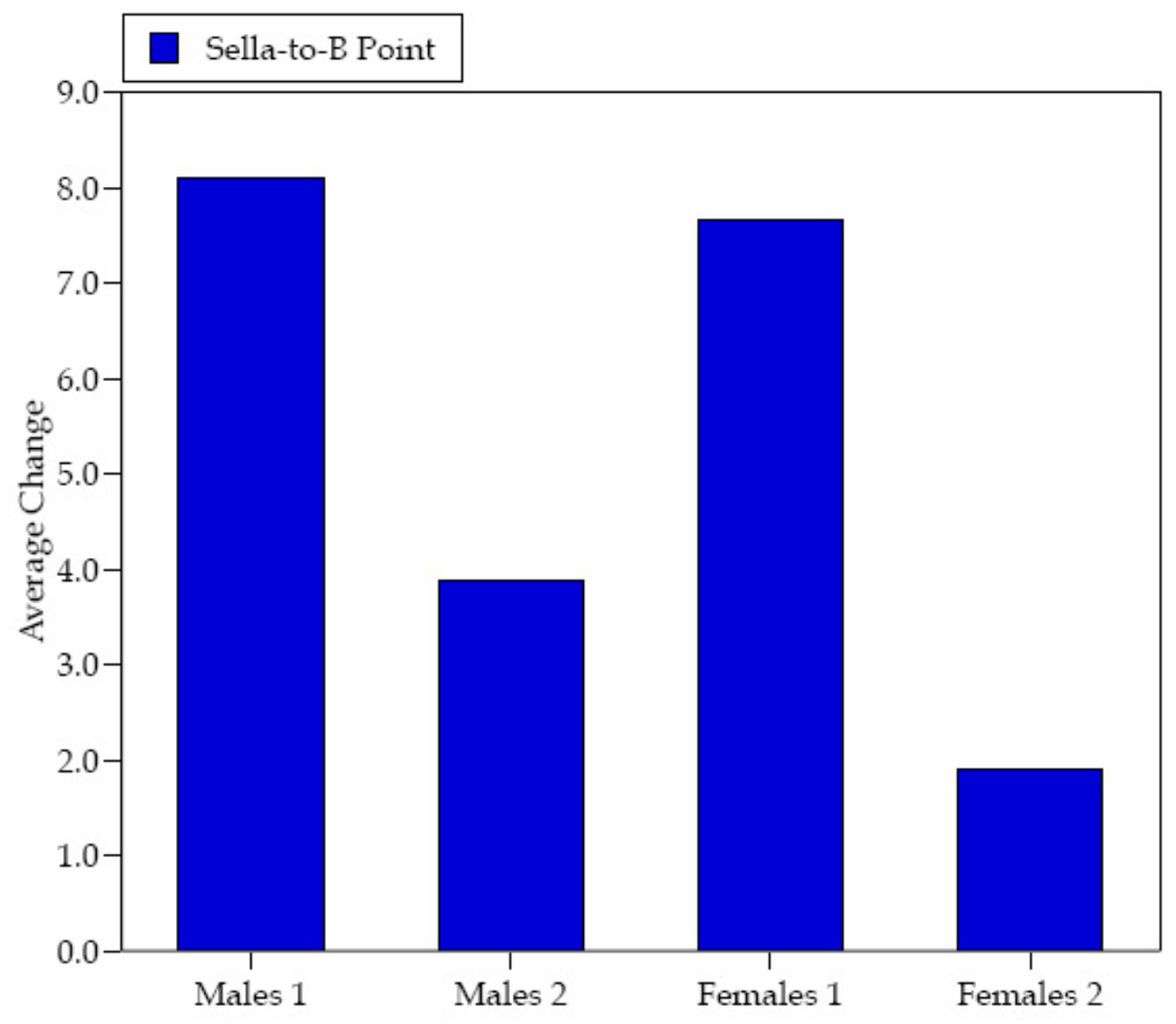

Figure 35. Mean changes in the Sella-B Point Length during phase one (1) and phase two (2) of compound orthodontic treatment, by sex. 


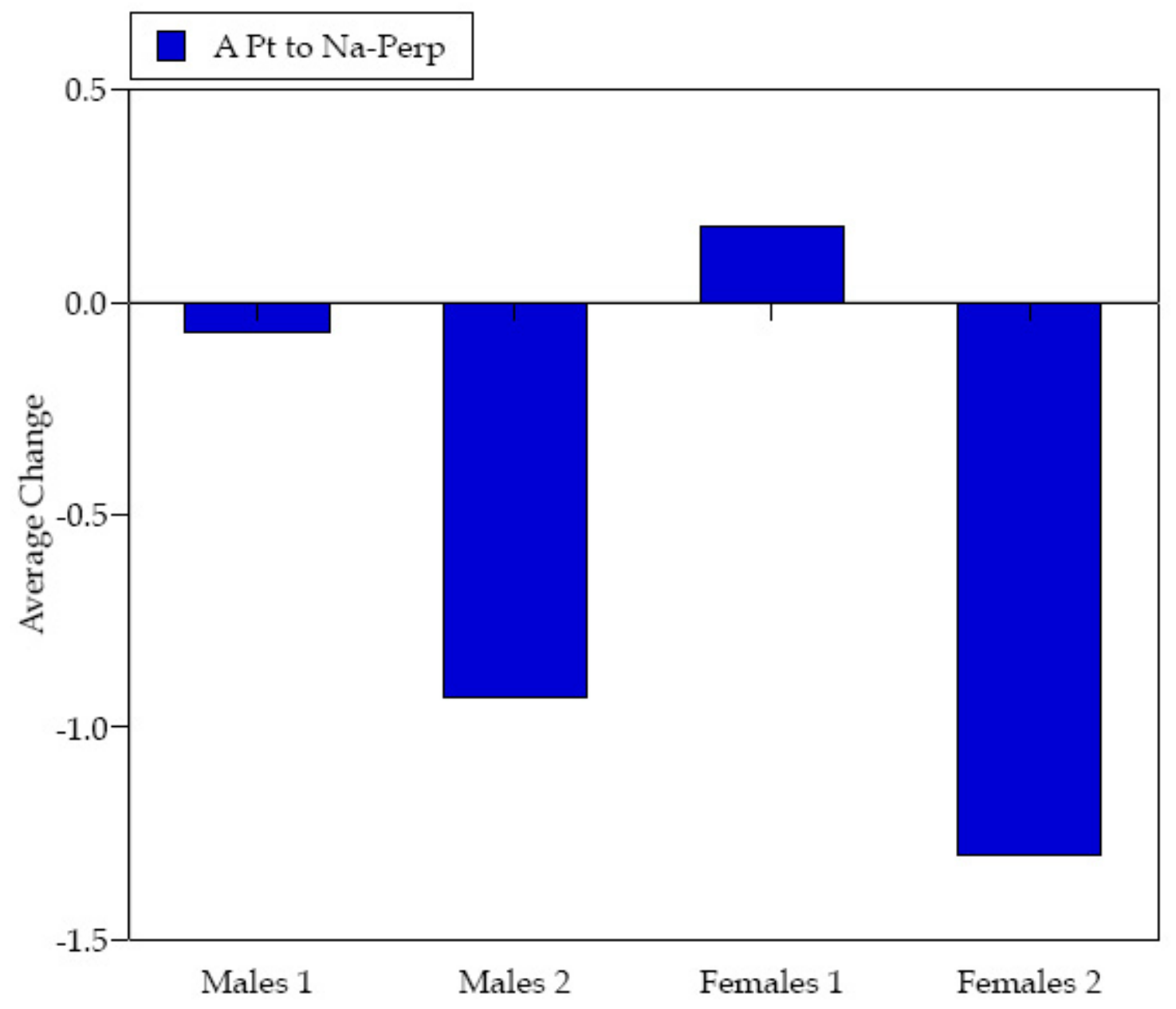

Figure 36. Mean changes in A Point to Na-Perp Distance during phase one (1) and phase two (2) of compound orthodontic treatment, by sex. 


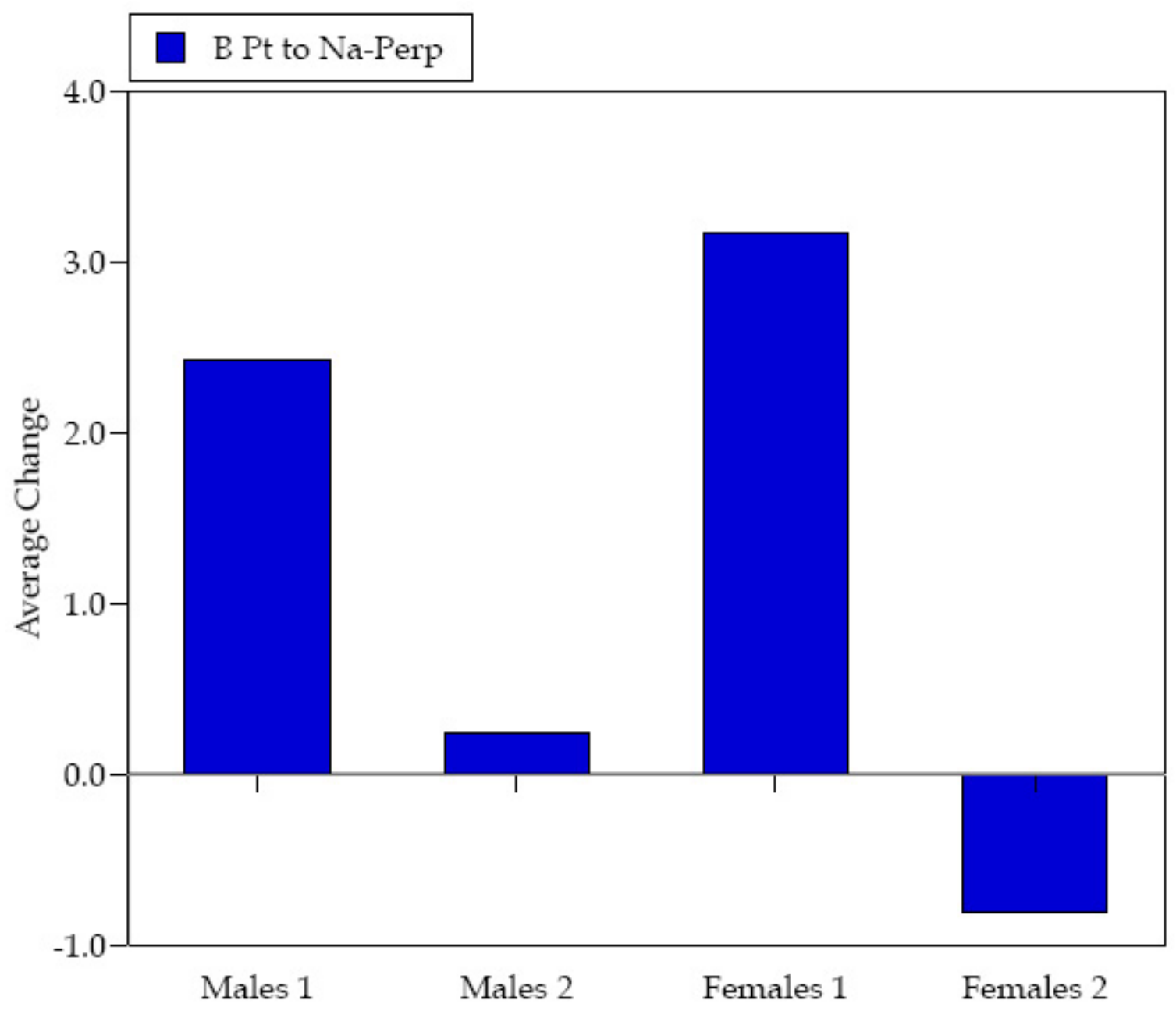

Figure 37. Mean changes in the B Point to Na-Perp Distance during phase one (1) and phase two (2) of compound orthodontic treatment, by sex. 
difference also decreased slightly during the fixed phase, to an average of 6.0 $\mathrm{mm}$ at the end of treatment. The Bionator effect, then, was to normalize the facial profile by advancing B Point more than inhibiting maxillary growth.

\section{Dental Changes}

Numerous, redundant measures of dental changes were calculated (Table 5) because the basic cephalometric analysis was borrowed from the Department's clinical analysis. Of some note, most of these 10 dental variables changed significantly more during the first, Bionator interval of treatment even though one supposes that there is greater control over tooth movement during the full-bonded appliance phase.

\section{Occlusal Plane}

Downs' occlusal plane (measured to FH) did not change much during either phase of treatment (Figure 38), though there was an unanticipated sex difference (Table 6), with boys exhibiting a greater decrease (counterclockwise rotation) than girls. On the other hand, the absolute differences are less than $2^{\circ}$ in all groups. 


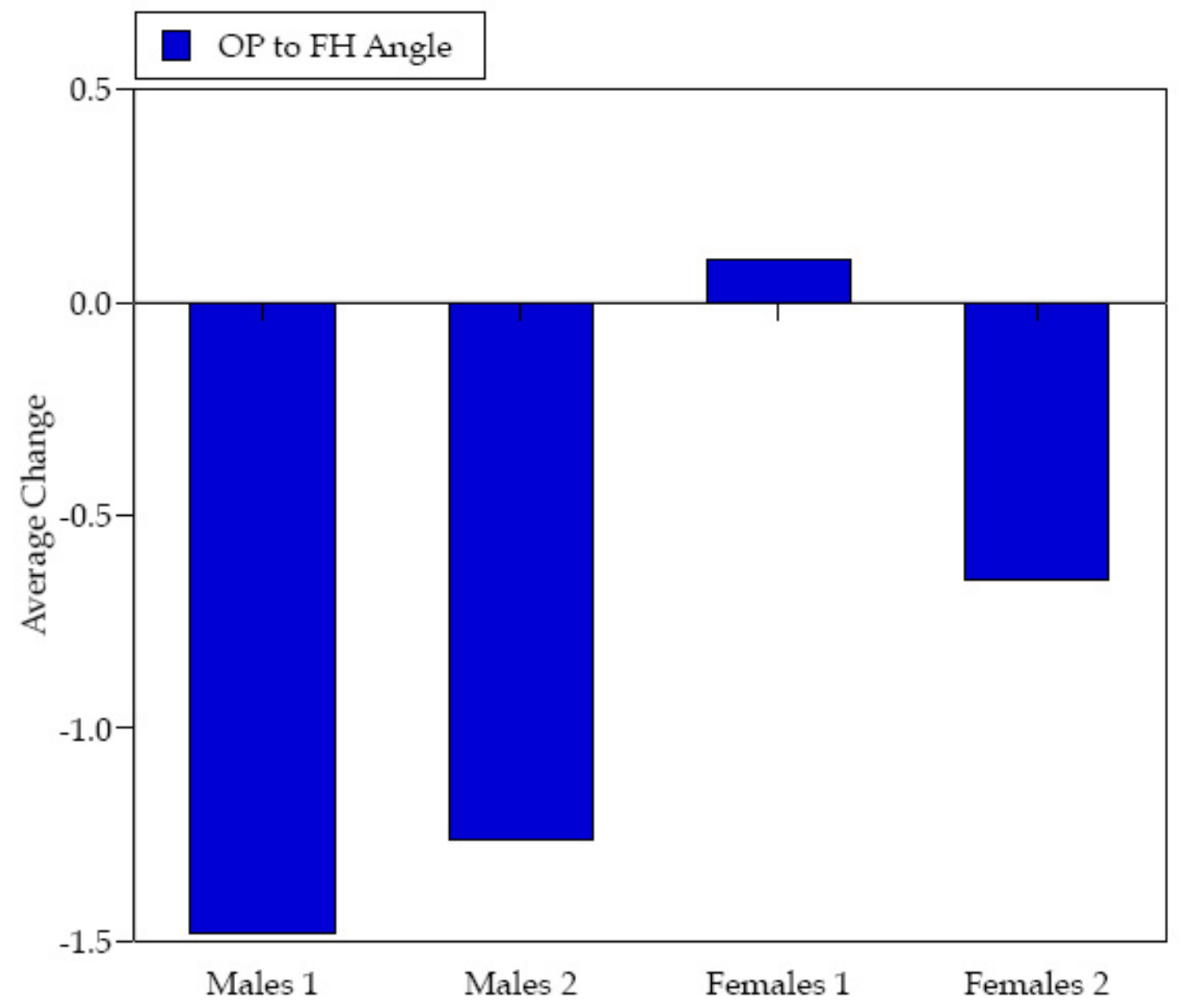

Figure 38. Mean changes in the occlusal plane to FH angle during phase one (1) and phase two (2) of compound orthodontic treatment, by sex. 


\section{Overjet, Overbite}

The expressions of overjet and overbite depend on both tooth and supporting bone positions, but are dealt with here because fixed treatment deals principally with repositioning the teeth themselves. Overjet improved (diminished) an average of $3.1 \mathrm{~mm}$ in these retrognathic cases over the course of treatment, but most of the correction $(3.0 \mathrm{~mm}$, or $97 \%)$ occurred during the Bionator phase (Figure 39). Overbite moved from a mean of $3.7 \mathrm{~mm}$ to $1.7 \mathrm{~mm}$ at the end of treatment, an overall change (reduction) in overbite of $1.9 \mathrm{~mm}$. Most of this change (95\%) occurred during the Bionator phase of treatment (Figure 40). Statistically, overjet and overbite both improved far more during the earlier phase. For those children with unaesthetic incisor relationships, these corrections years earlier than with conventional treatment should be seen as beneficial.

\section{$\underline{\text { Maxillary Incisor }}$}

The upper incisor was proclined, from a mean of $23^{\circ}$ to $27^{\circ}$ during treatment (i.e., U1 to Nasion-A Point angle). Statistically, there was no difference in the amount of change between the two phases. There is no obvious explanation for the observed sex difference (Figure 41), but the typical boy underwent appreciably - and statistically significantly - more U1 


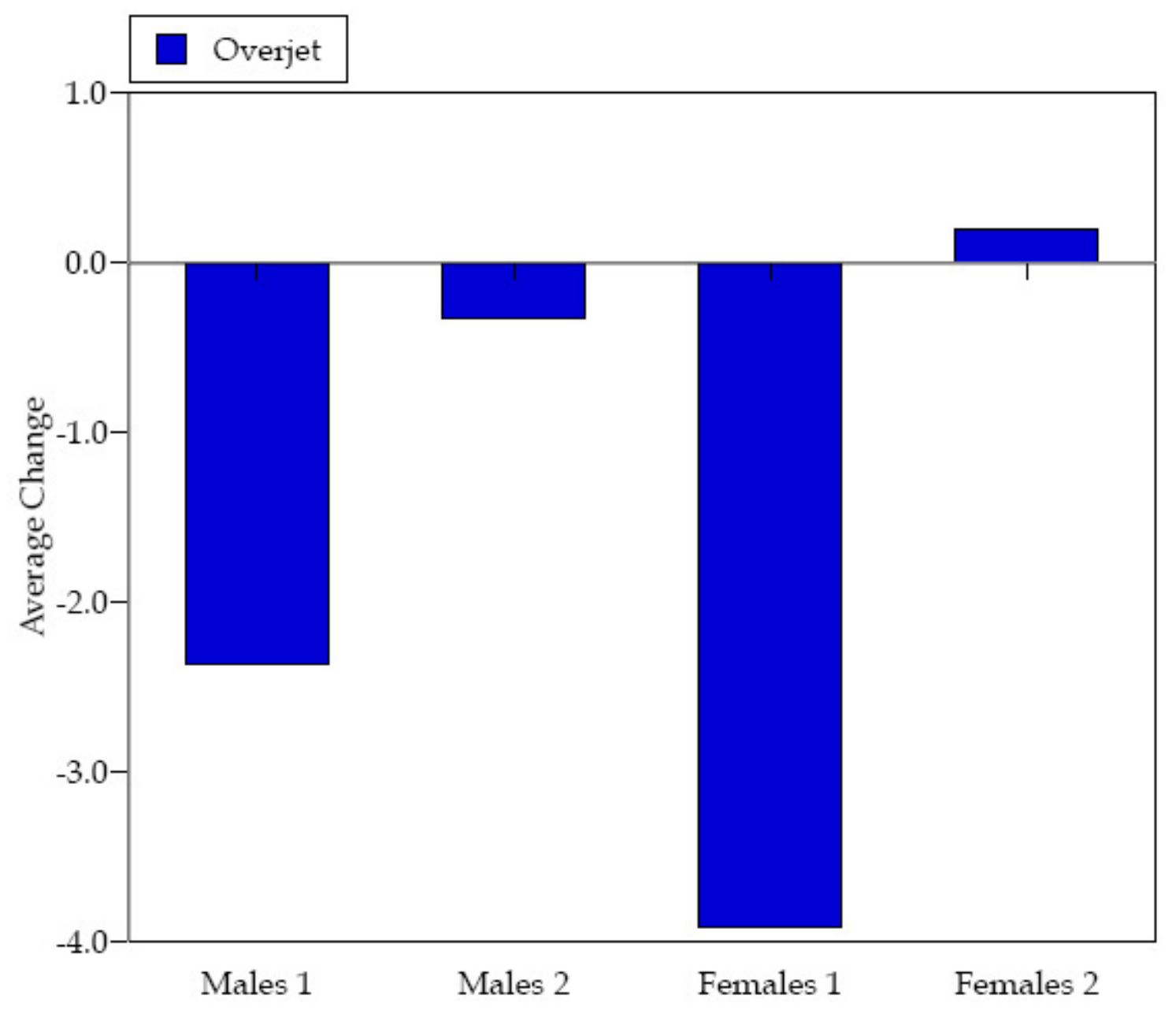

Figure 39. Mean changes in incisor overjet during phase one (1) and phase two (2) of compound orthodontic treatment, by sex. 


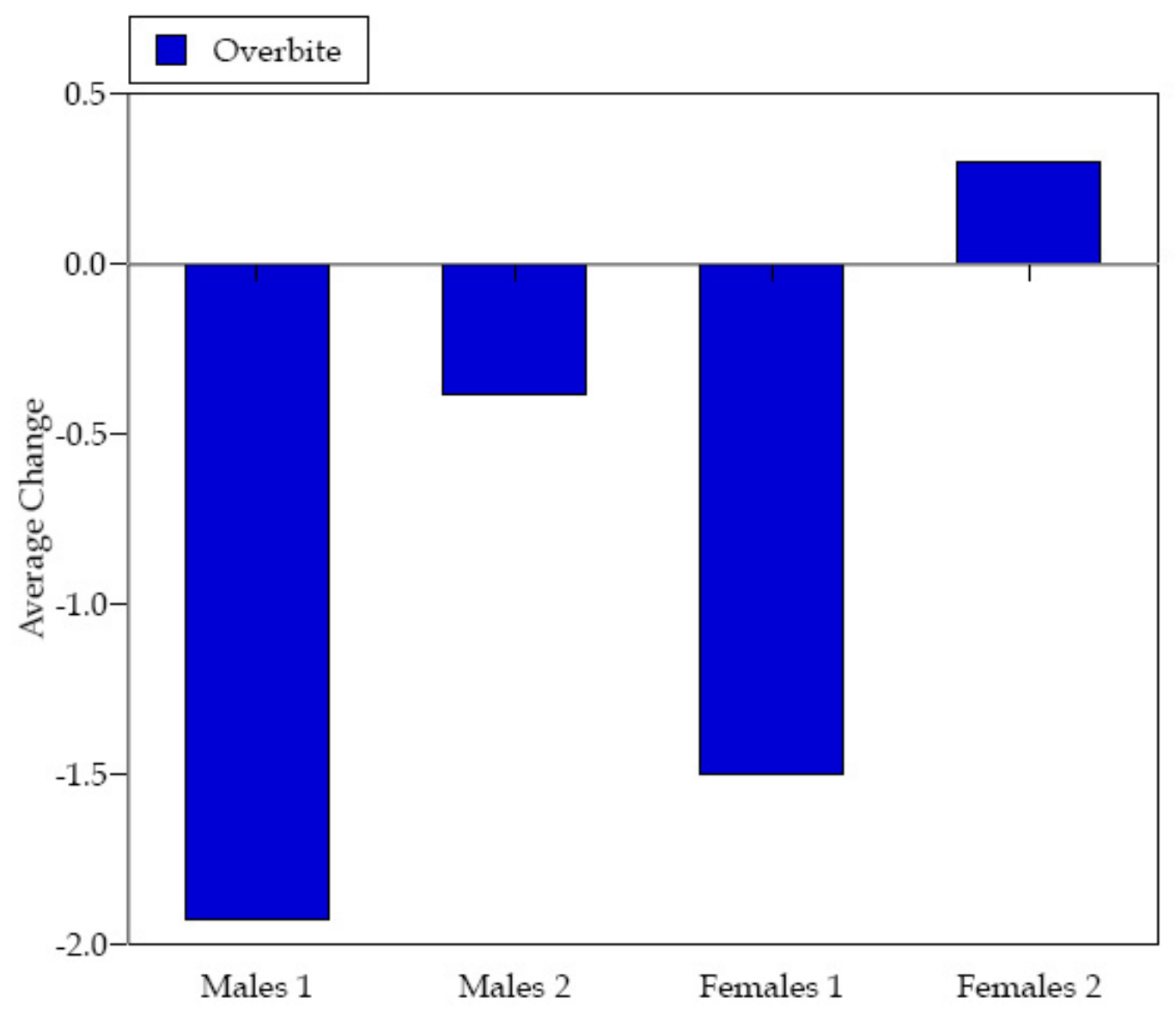

Figure 40. Mean changes in Incisor Overbite during phase one (1) and phase two (2) of compound orthodontic treatment, by sex. 


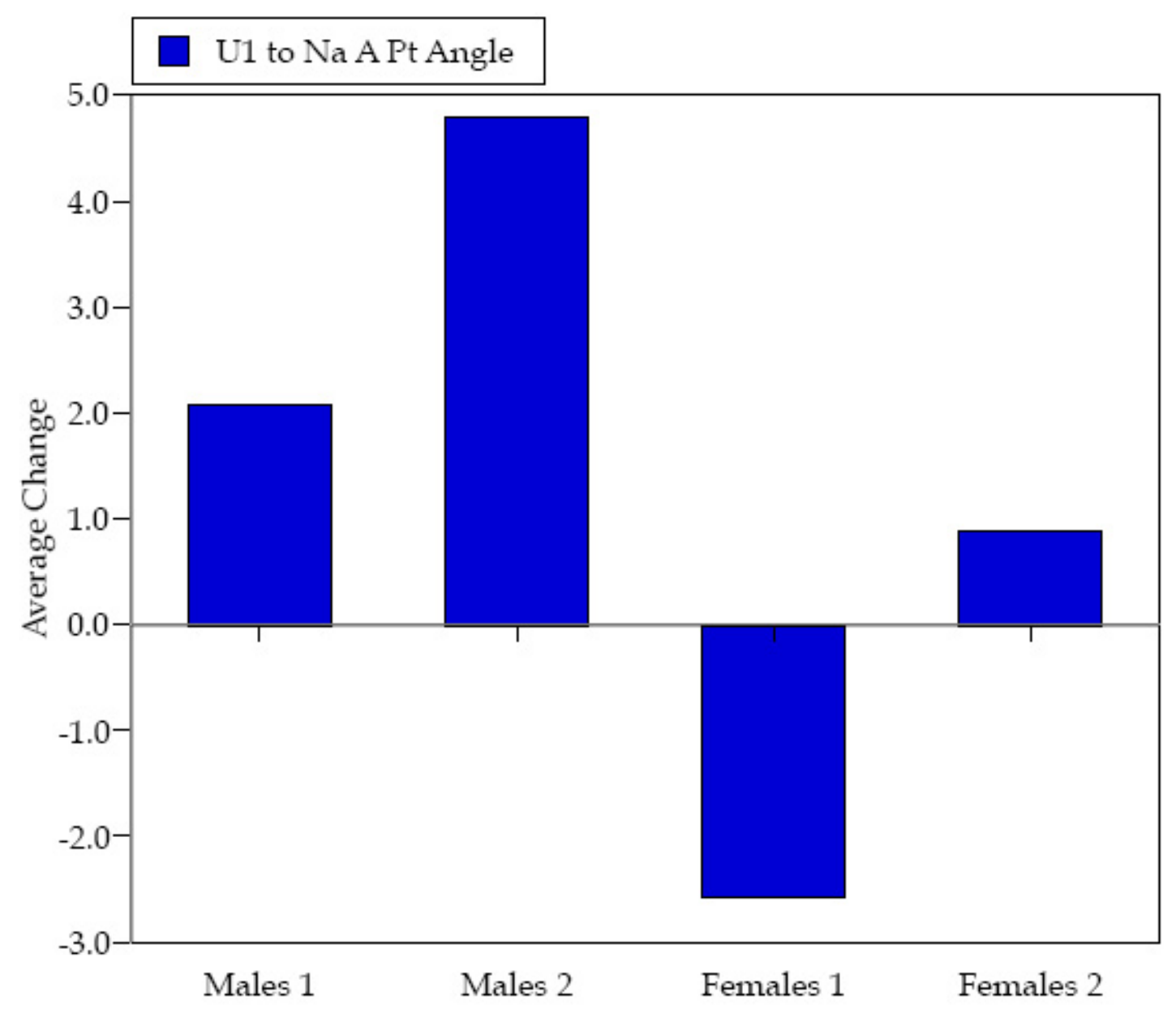

Figure 41. Mean changes in the U1 to Nasion A Point angle during phase one (1) and phase two (2) of compound orthodontic treatment, by sex. 
proclination during treatment compared to the girls who characteristically underwent U1 uprighting as measured from Nasion-to-A Point.

The maxillary central incisor also was measured relative to the Nasion-A Point line, both angularly and millimetrically. Angularly, U1 changed more during the Edgewise phase, but the changes are made complex by the significant sexual dimorphism (Figure 41; Table 5). Boys typically experienced incisor proclination; girls typically experienced incisor uprighting. These sex differences were evident during both phases of treatment (Figure 42). For the total two-phase sample, the incisal edge of U1 started $3.0 \mathrm{~mm}$ from the NasionA Point line, and treatment was completed with U1 $4.4 \mathrm{~mm}$ from this line.

Interincisal angle exhibited no difference in the amounts of change between phases (Table 5), but there was a highly significant sex difference (Figure 43). The angle decreased on the order of $3^{\circ}$ to $4^{\circ}$ during each phase in boys; this angle increased about $3^{\circ}$ during each phase in girls.

The mandibular incisor angle to the A-Pogonion line increased during treatment (Figure 44), but the amount of dental change is affected by uprighting of the A-Pogonion line as the retrognathia was alleviated. Still (as with comparison to IMPA), it is evident that the lower incisor experienced some proclination. Statistically, this angular change (L1 proclination) was significantly greater during the Bionator phase of treatment. Assessing the L1 


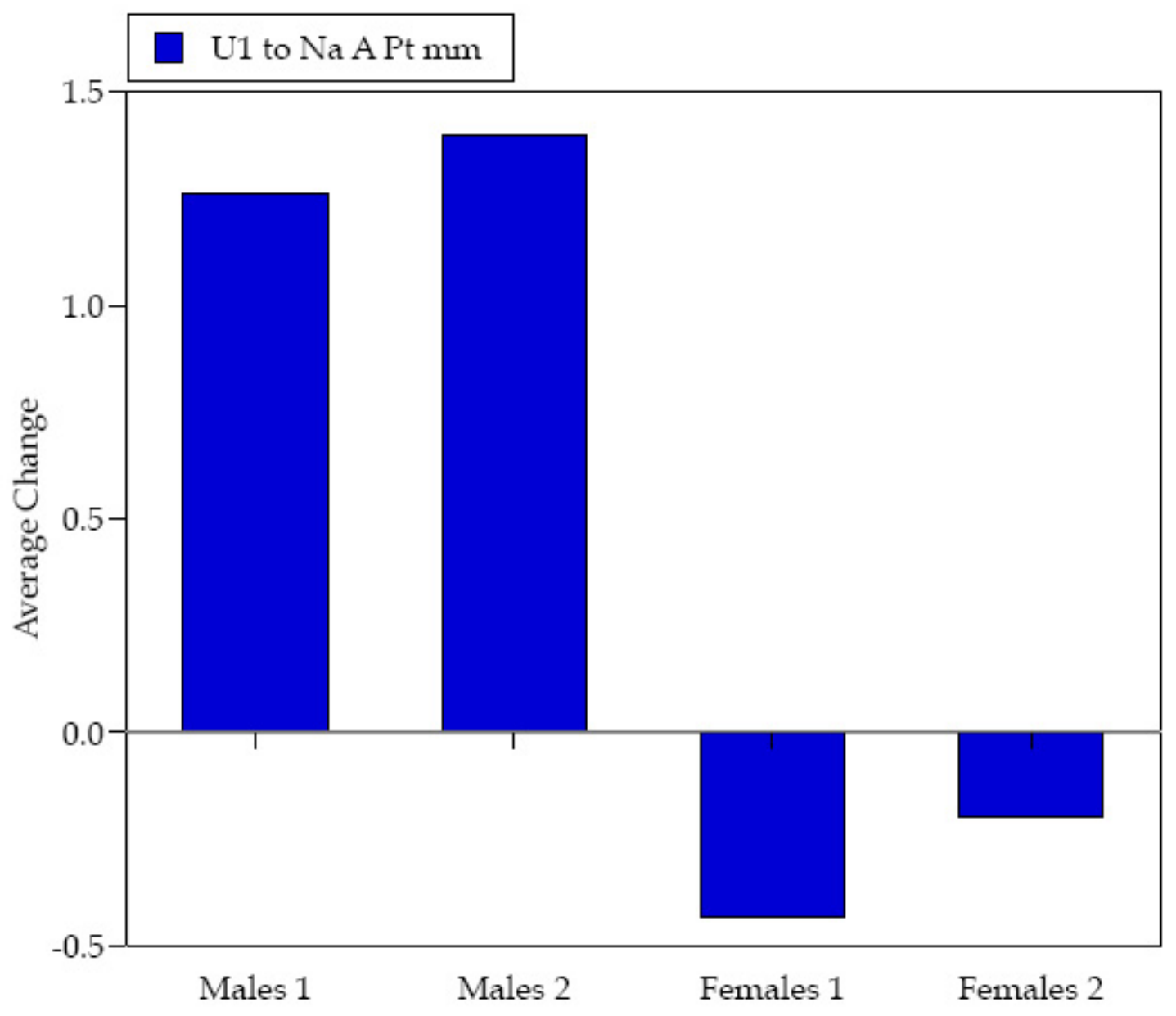

Figure 42. Mean changes in the U1 to Na A Point (mm) during phase one (1) and phase two (2) of compound orthodontic treatment, by sex. 


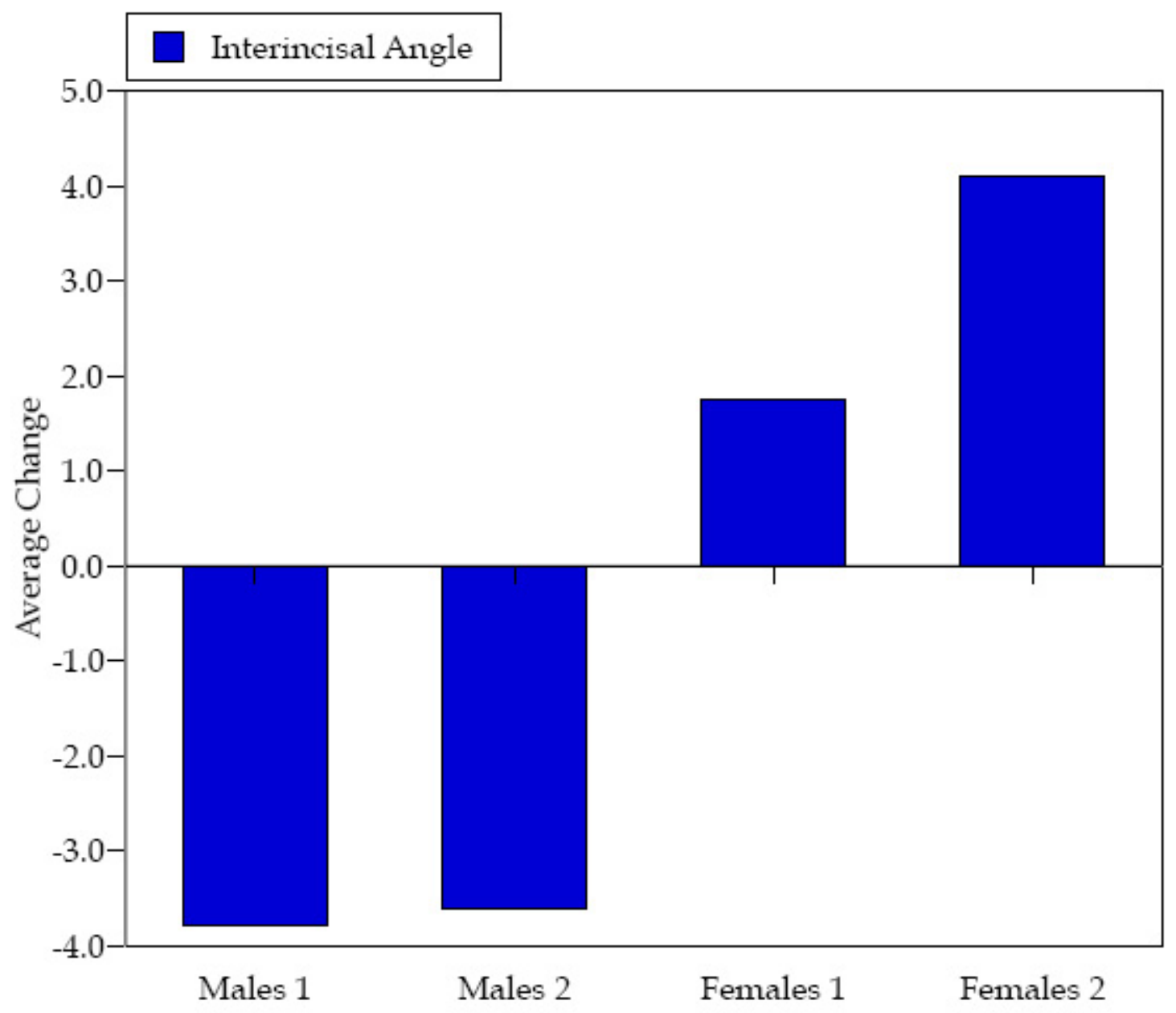

Figure 43. Mean changes in the interincisal angle during phase one (1) and phase two (2) of compound orthodontic treatment, by sex. 


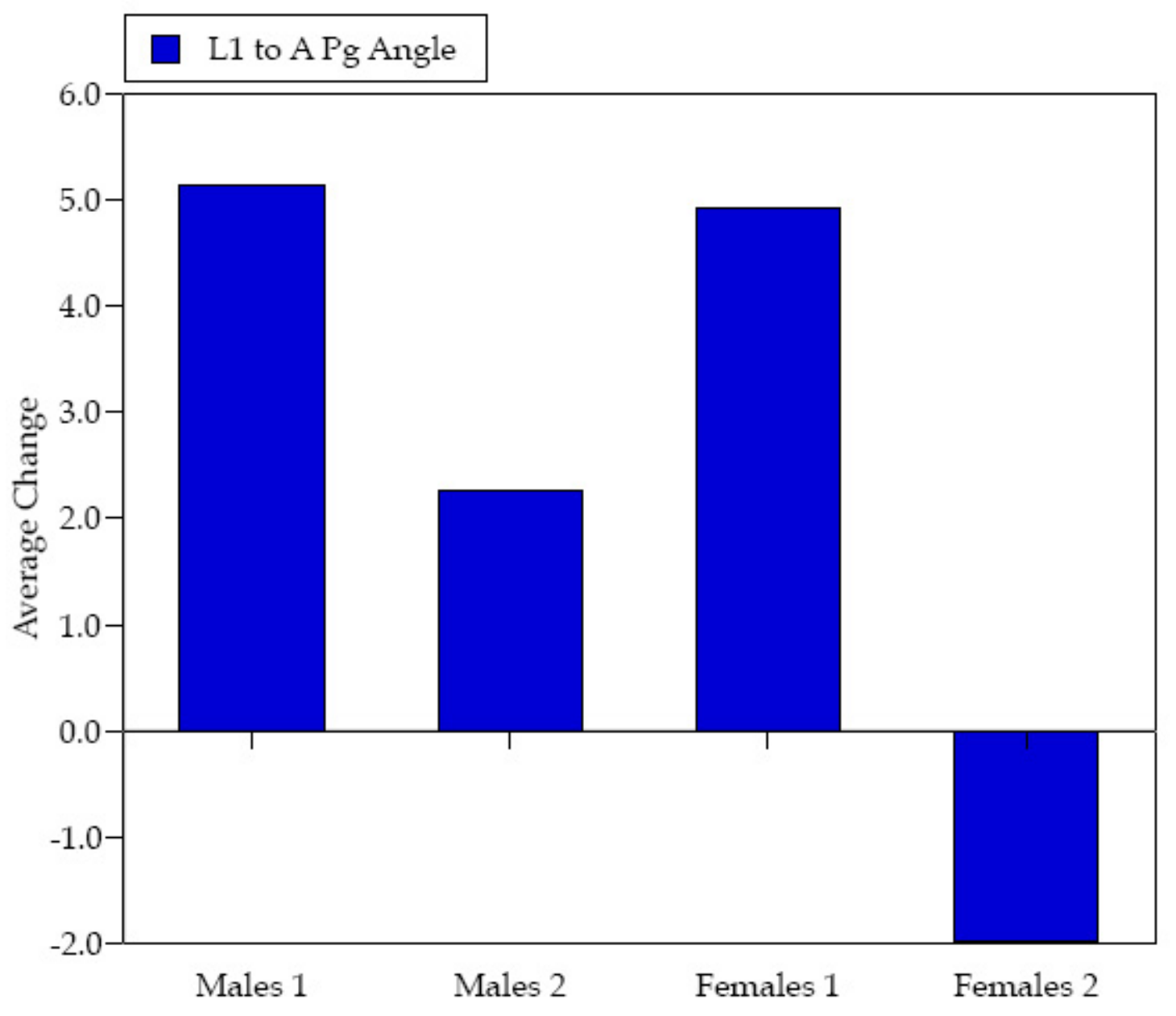

Figure 44. Mean changes in the L1 to A Pogonian angle during phase one (1) and phase two (2) of compound orthodontic treatment, by sex. 
to A-Pogonion line millimetrically (Figure 45) also disclosed a sex differencegreater L1 proclination in boys - as well as the greater change during the first, Bionator phase of treatment.

The L1 to Nasion-B Point changes (Tables 5, 6) also need to be viewed with an appreciation for the alleviation of mandibular retrognathia (and, thereby, uprighting of the Nasion-B Point line). Still, the improvement during treatment - and the sex differences seen in the prior variables - were evident here as well (Figures 46, 47). There was a net uprighting of L1 with its incisal edge being moved back (distally) relative to the Nasion-B Point line.

IMPA often is scrutinized with some care, largely because of claims that L1 is more stable when upright over basal bone (Riedel 1960; Joondeph and Riedel 1994). In this sample, IMPA changed to different extents during the two phases and also differently between boys and girls (Table 6). Overall, IMPA began at a mean of $94^{\circ}$, increased to a mean of $96^{\circ}$ at the end of Bionator treatment, and then decreased slightly to an average of $95^{\circ}$ at the end of treatment. Figure 48 shows that little change $\left(c a .1^{\circ}\right)$ was observed on the average in either phase in the boys; girls also changed little during phase 1 ( $c$ a. $\left.1^{\circ}\right)$, but then uprighted almost $4^{\circ}$ during the Edgewise phase.

FMIA - a key measure of change in the maxillary central incisor comparably exhibited little change is boys (Figure 49), but there was about $4^{\circ}$ of labial crown tipping during the Edgewise phase in girls. 


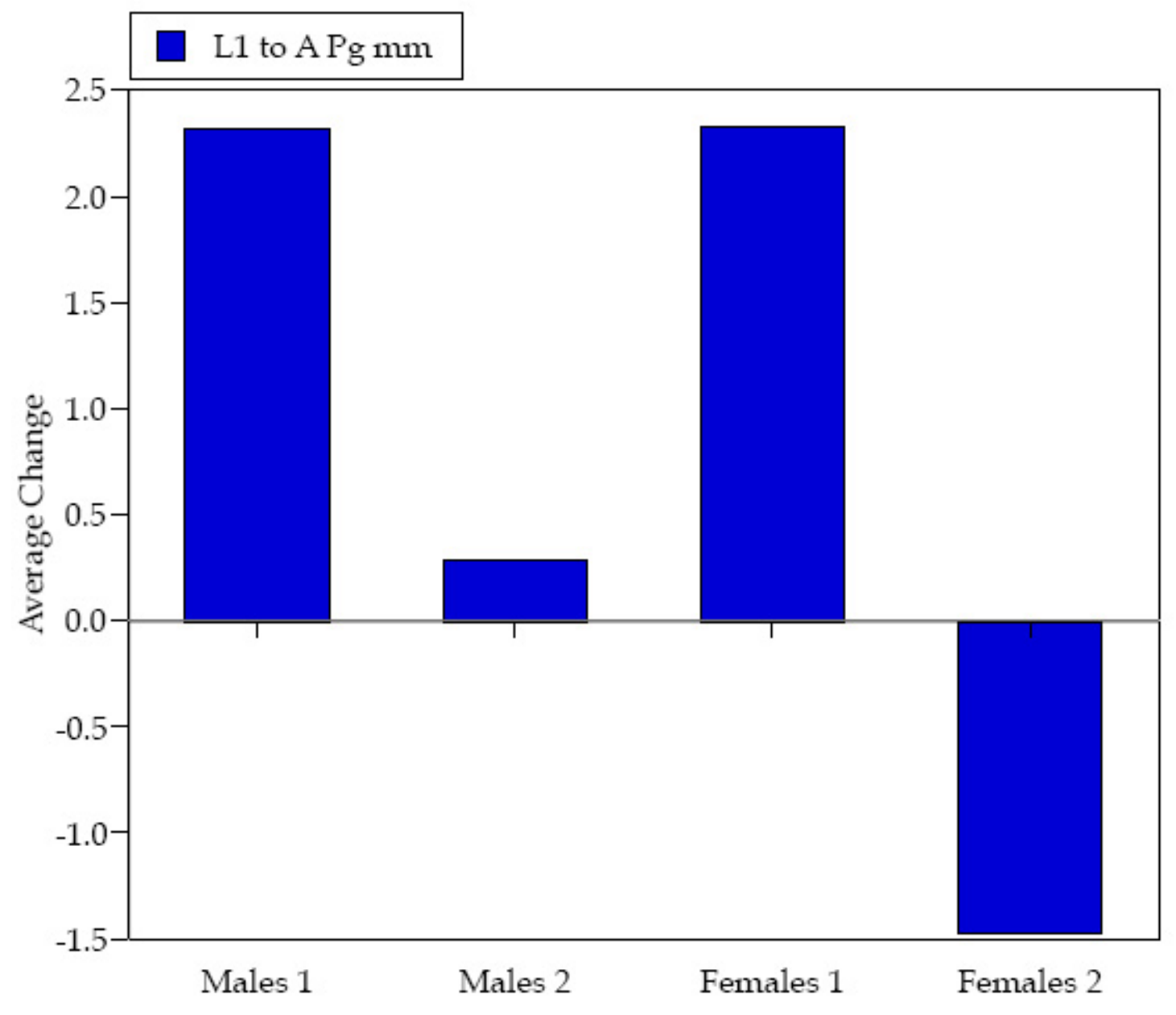

Figure 45. Mean changes in the L1 to A Pg ( $\mathrm{mm}$ ) during phase one (1) and phase two (2) of compound orthodontic treatment, by sex. 


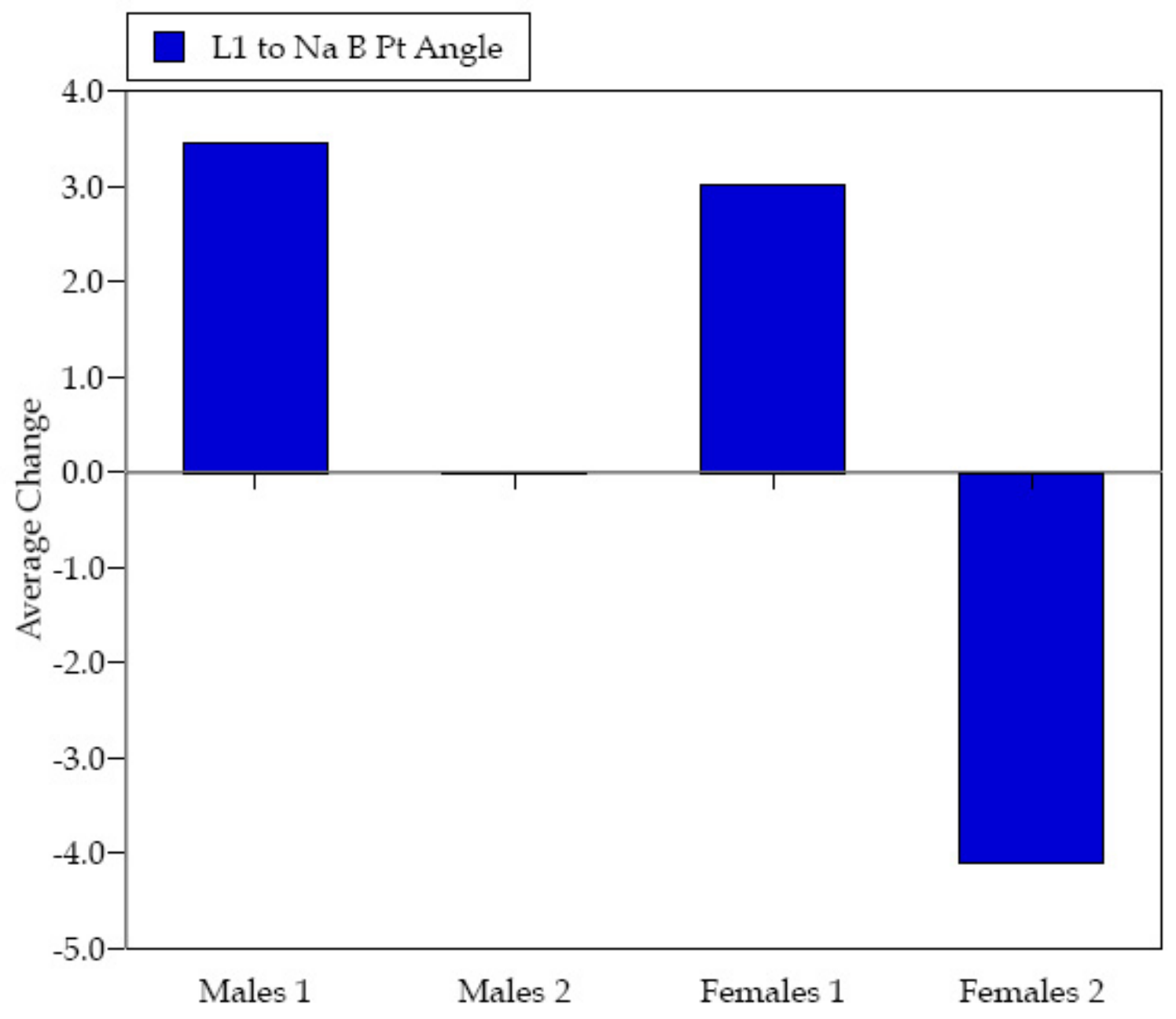

Figure 46. Mean changes in the L1 to Nasion B Point angle during phase one (1) and phase two (2) of compound orthodontic treatment, by sex. 


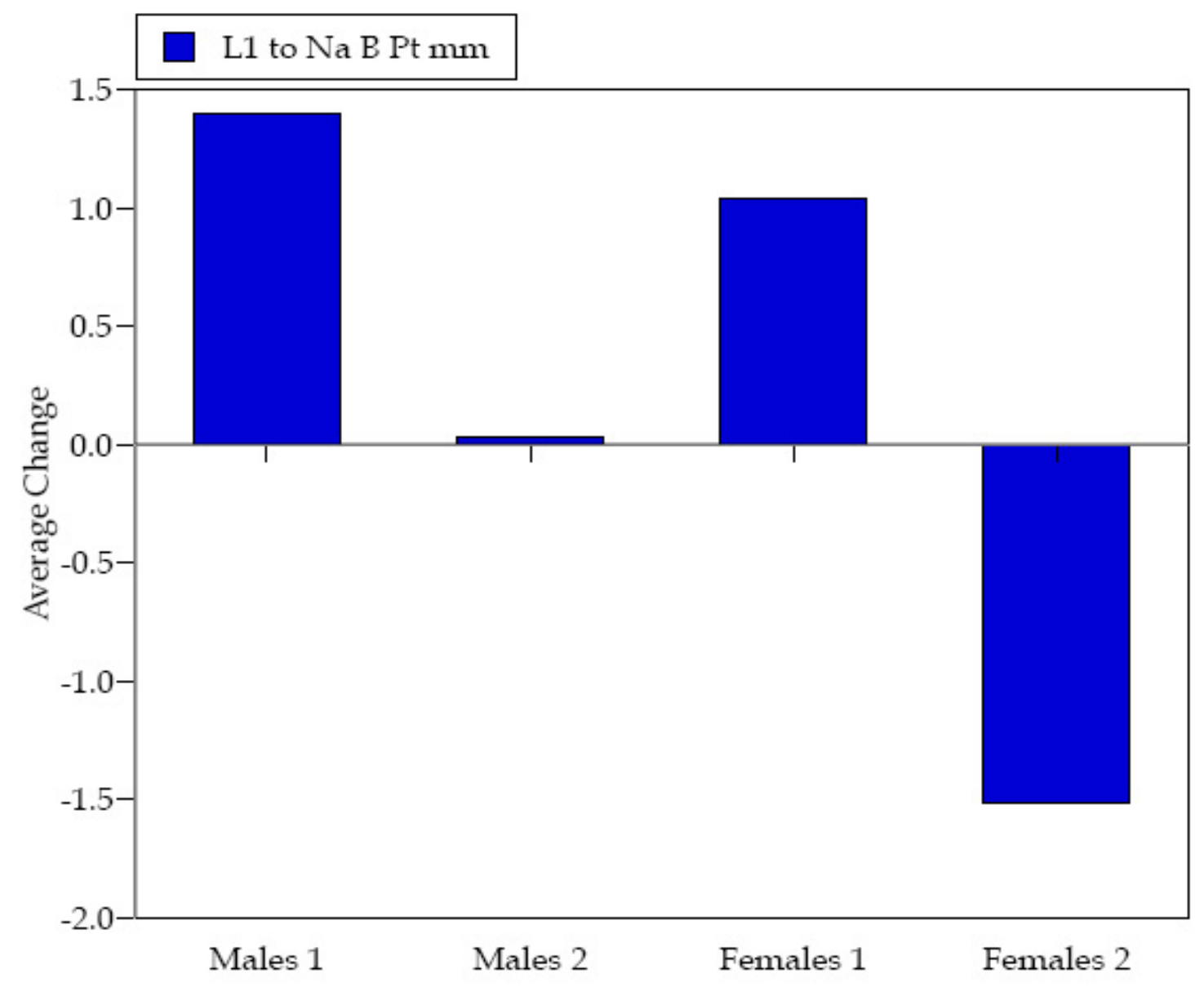

Figure 47. Mean changes in the L1 to Na B Point (mm) during phase one (1) and phase two (2) of compound orthodontic treatment, by sex. 


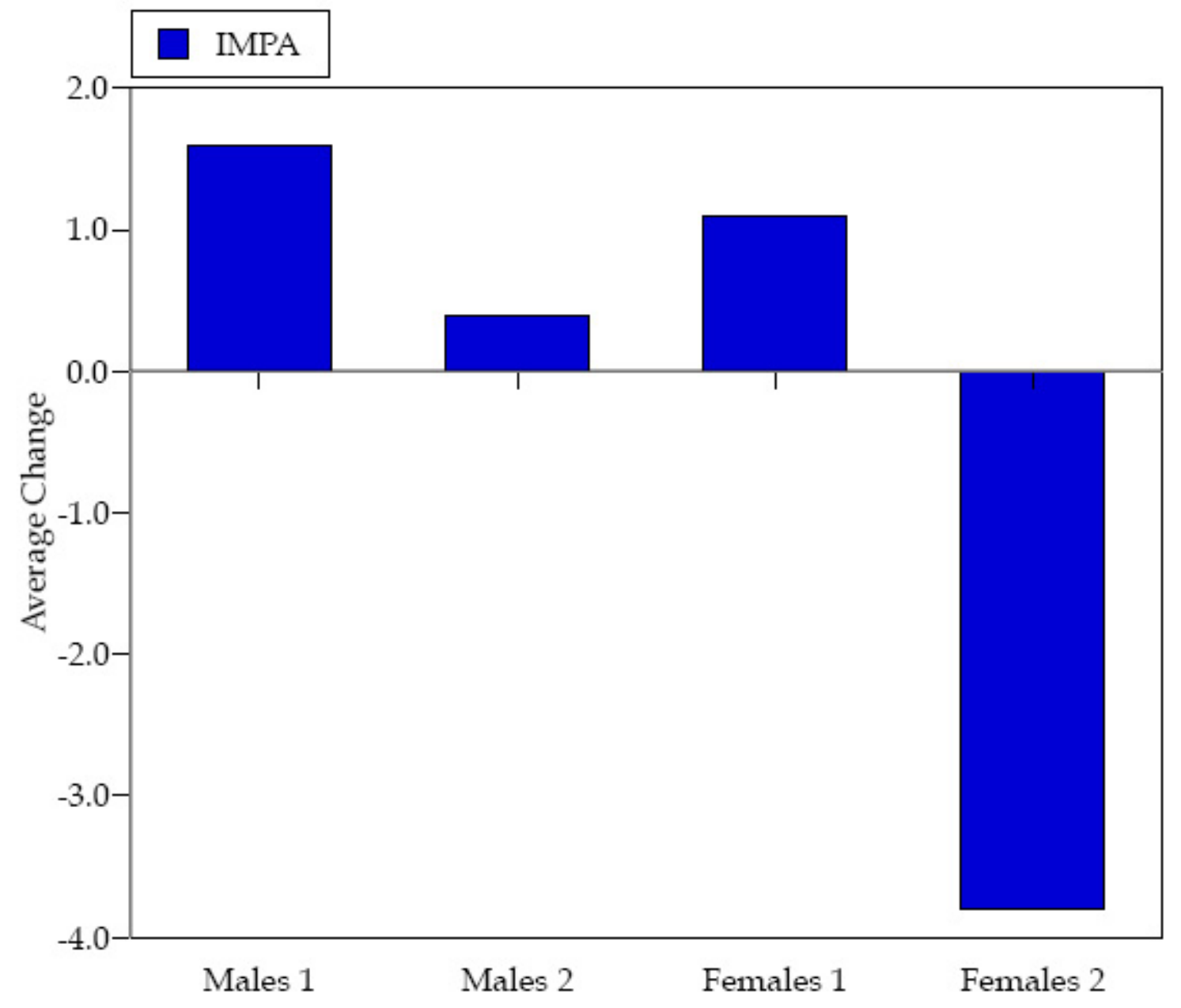

Figure 48. Mean changes in the IMPA during phase one (1) and phase two (2) of compound orthodontic treatment, by sex. 


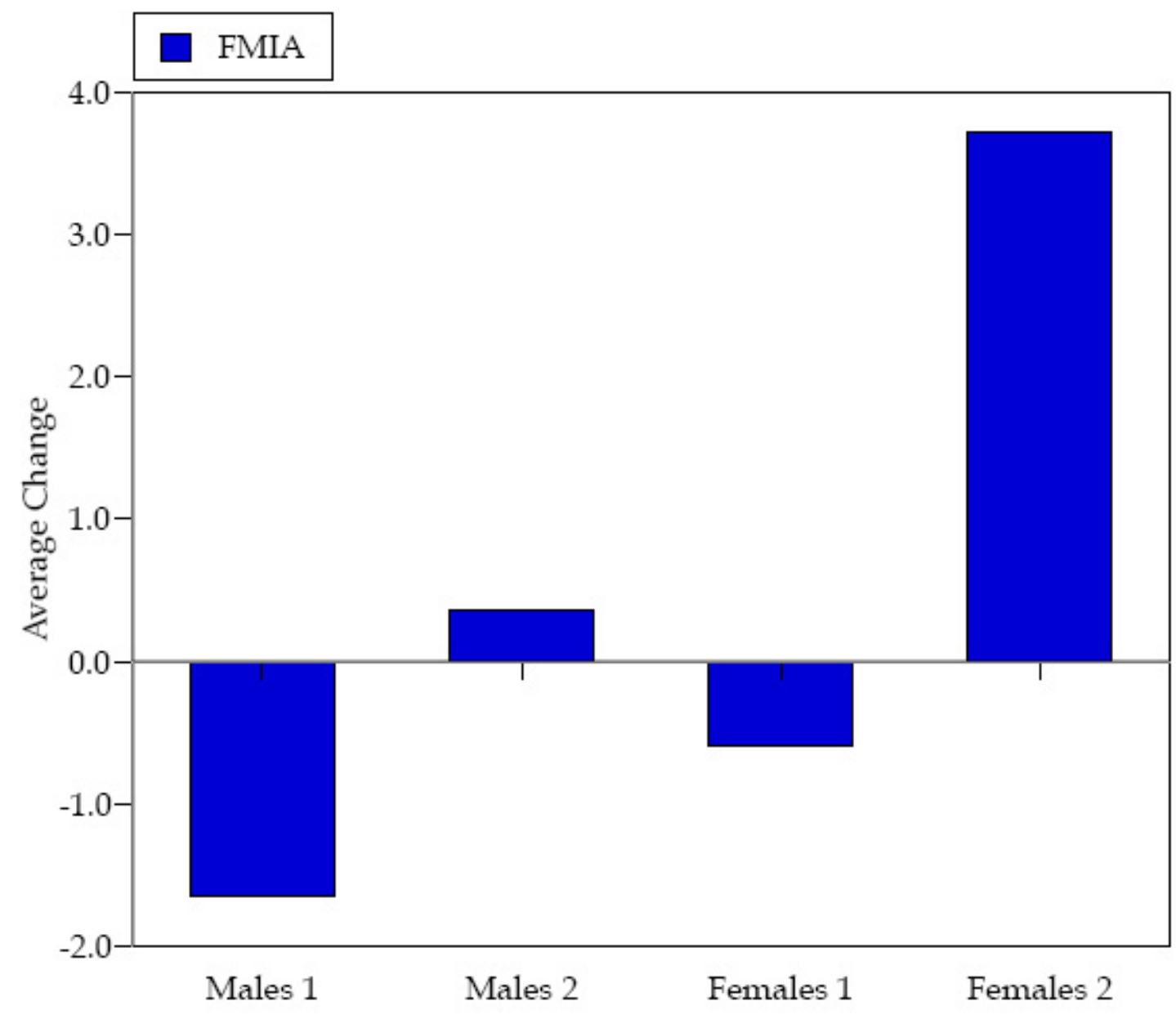

Figure 49. Mean changes in the FMIA during phase one (1) and phase two (2) of compound orthodontic treatment, by sex. 


\section{$\underline{\text { Timing of Orthodontic Correction }}$}

While a bit redundant, we can summarize the changes during the two phases of treatment in Figure 50. One expected advantage to early intervention of a developing malocclusion is that the correction is achieved at an earlier age, so some or most of the esthetic improvement occurs earlier. Consequently, the merit of this approach depends on how much of the overall skeletodental improvement occurs in the earlier, functional phase of treatment. This was evaluated here by calculating the change occurring during the Bionator versus the later fixed phase of treatment.

Roughly half of the correction in lower facial profile ( $Z$ angle, E plane) occurred during each phase of treatment. Also, about half the decrease in NAP occurred in each phase. Almost all of the correction in the lower facial profile (i.e., increase in $\mathrm{SNB}$, decrease in $\mathrm{ANB}$, decrease in $\mathrm{AOBO}$, increase in facial angle, and forward growth of B Point) occurred during the Bionator phase of treatment.

Dentally, most of the overall correction also was achieved prior to treatment with fixed appliances. This is obvious (Figure 50) for the changes in interincisal angle, U1 to A Point distance, overjet, and overbite.

Of note, the maxillary skeletal correction (a decrease in SNA averaging $1.5^{\circ}$ and a decrease in A Point to Na-perpendicular averaging $1.1 \mathrm{~mm}$ ) occurred almost wholly during the later, Edgewise phase, but these overall changes are 


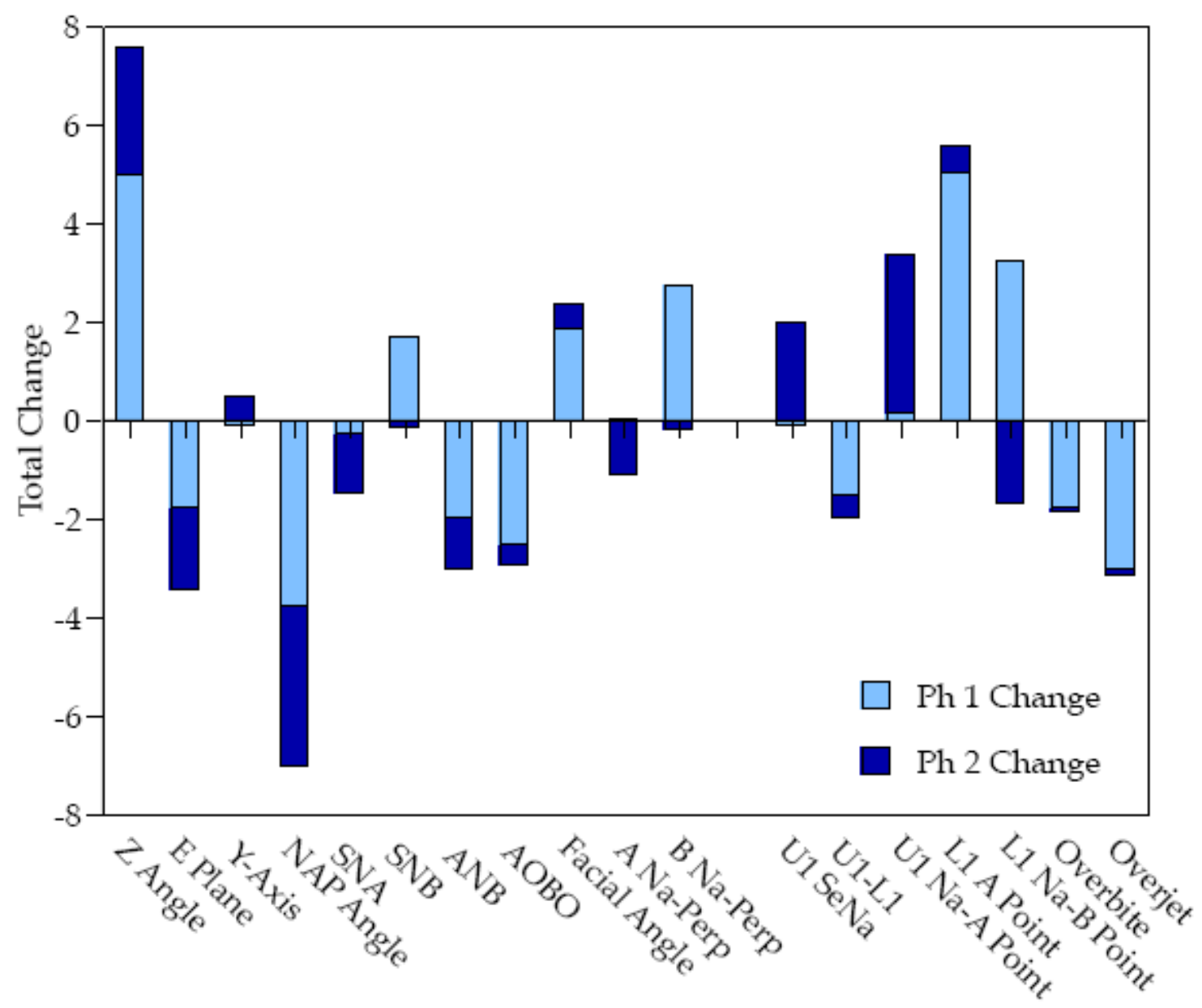

Figure 50. Changes during the two phases of treatment. 
averaging $1.6^{\circ}$ and an increase in B Point to Na- perpendicular averaging 2.6 $\mathrm{mm})$ were products of the Bionator phase of treatment.

Net treatment depends on both phases, and none of these cases was treated without conventional fixed appliances, but these results suggest that several aspects of the malocclusion are addressed at a younger age in the twophase modality, which may be pertinent as regards to (A) the child's self perception of his dental esthetics and (B) reducing the severity of the occlusal issues that need to be corrected with fixed appliances.

\section{End of Treatment}

The most informative aspect of this study, certainly from the clinician's perspective, is the comparison between the cephalometric results of the two treated methods at the end of treatment. It can be argued that the merit of any treatment method needs to be judged by how satisfactory the end result is.

Two tables show the end result of treatment. Table 7 lists the sample means (primarily so they are at hand here), and Table 8 lists the results of mixed model ANOVAs testing for between-group differences while controlling for any sexual dimorphism in size of the variable (Winer et al. 1991).

Table 8 shows that eight of the cephalometric variables are frankly significant statistically $(\mathrm{P}<0.05)$, while some others are suggestive $(0.10>\mathrm{P}>$ $0.05)$. 


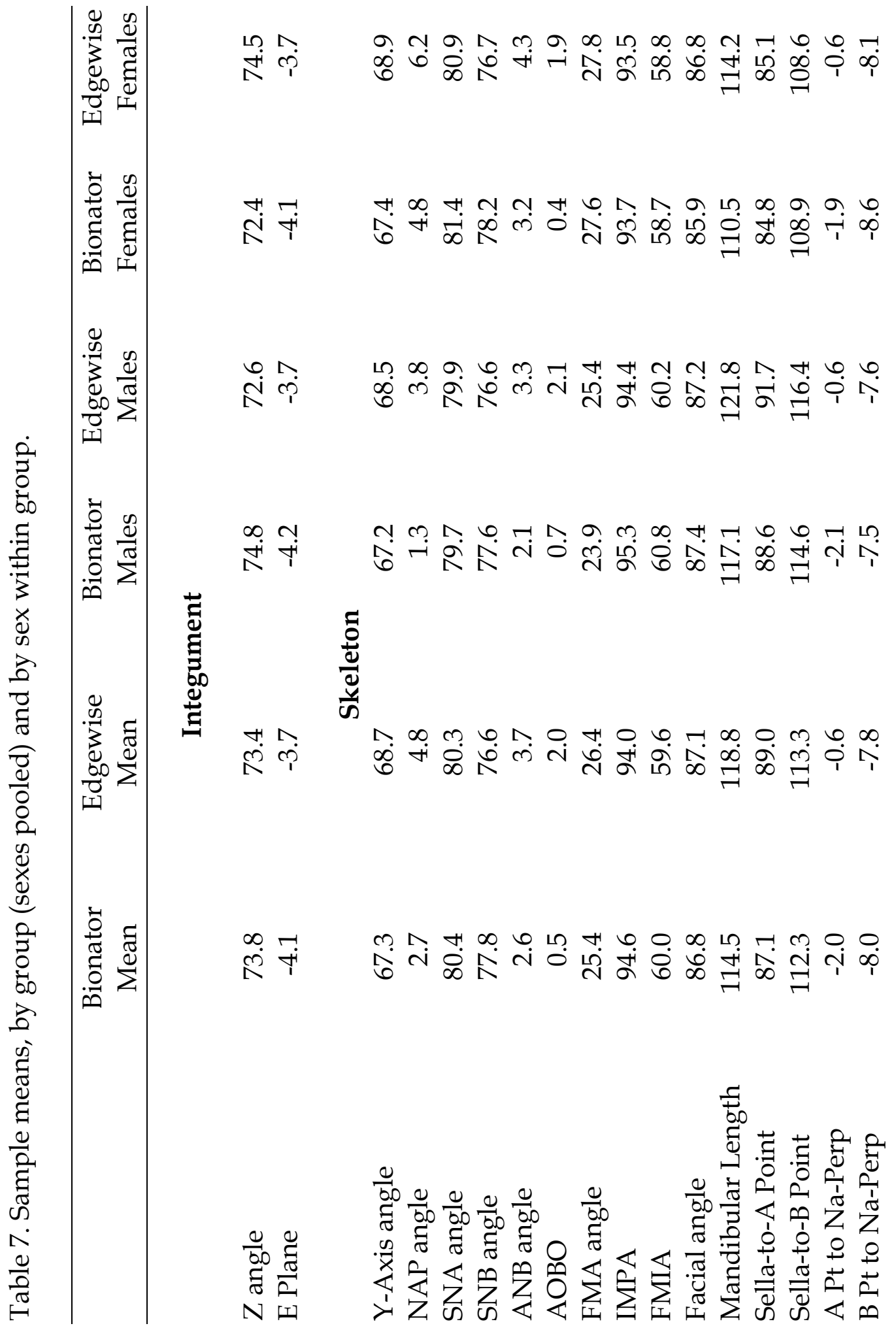




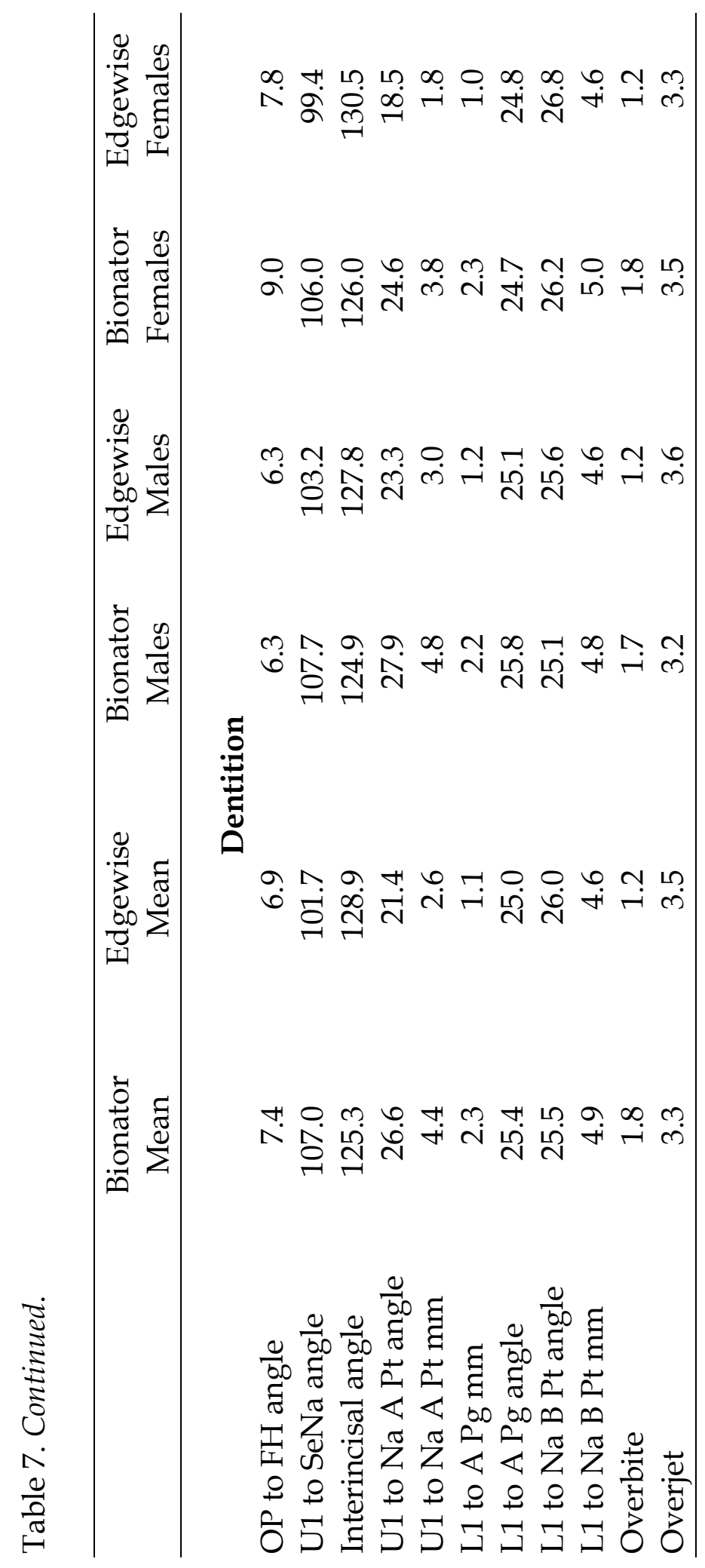




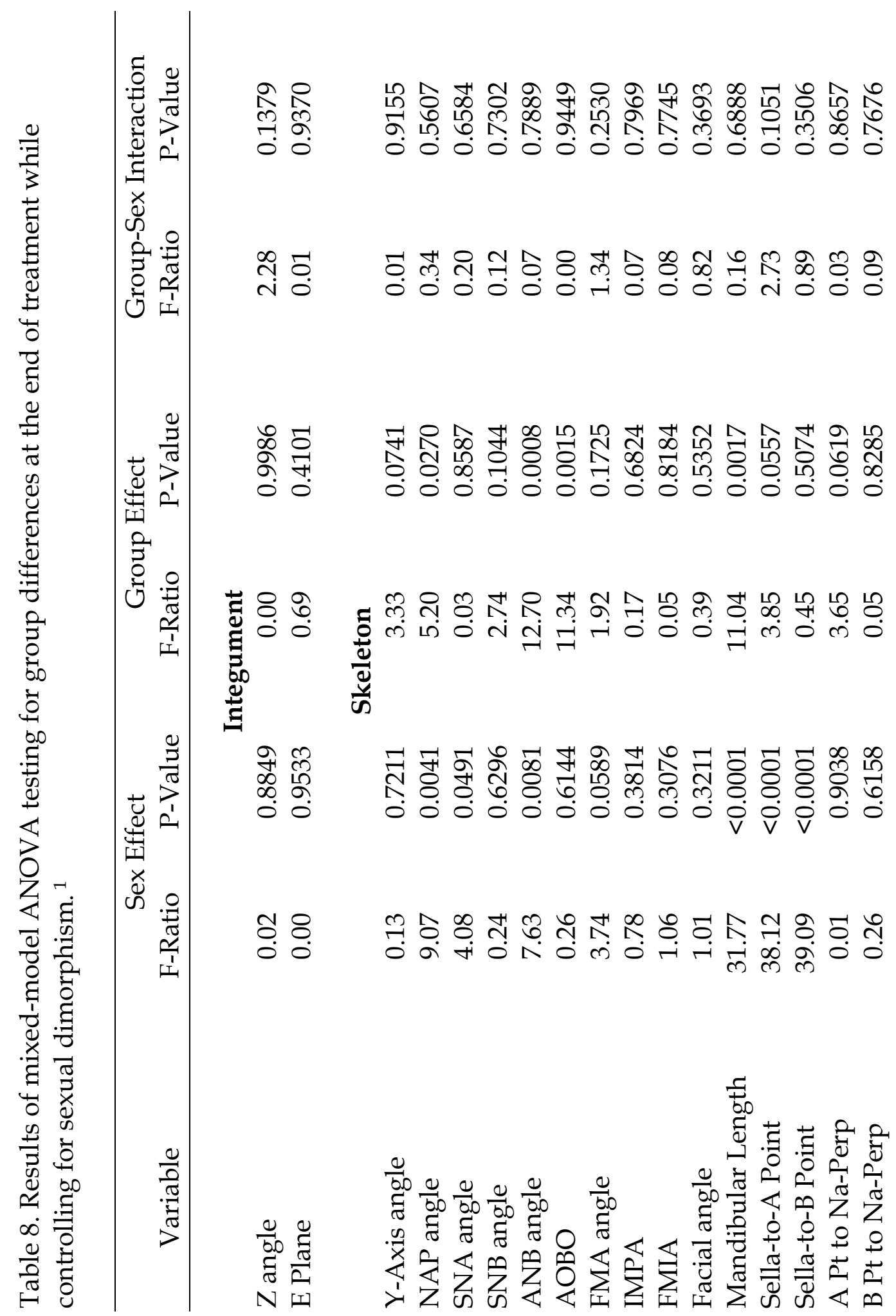




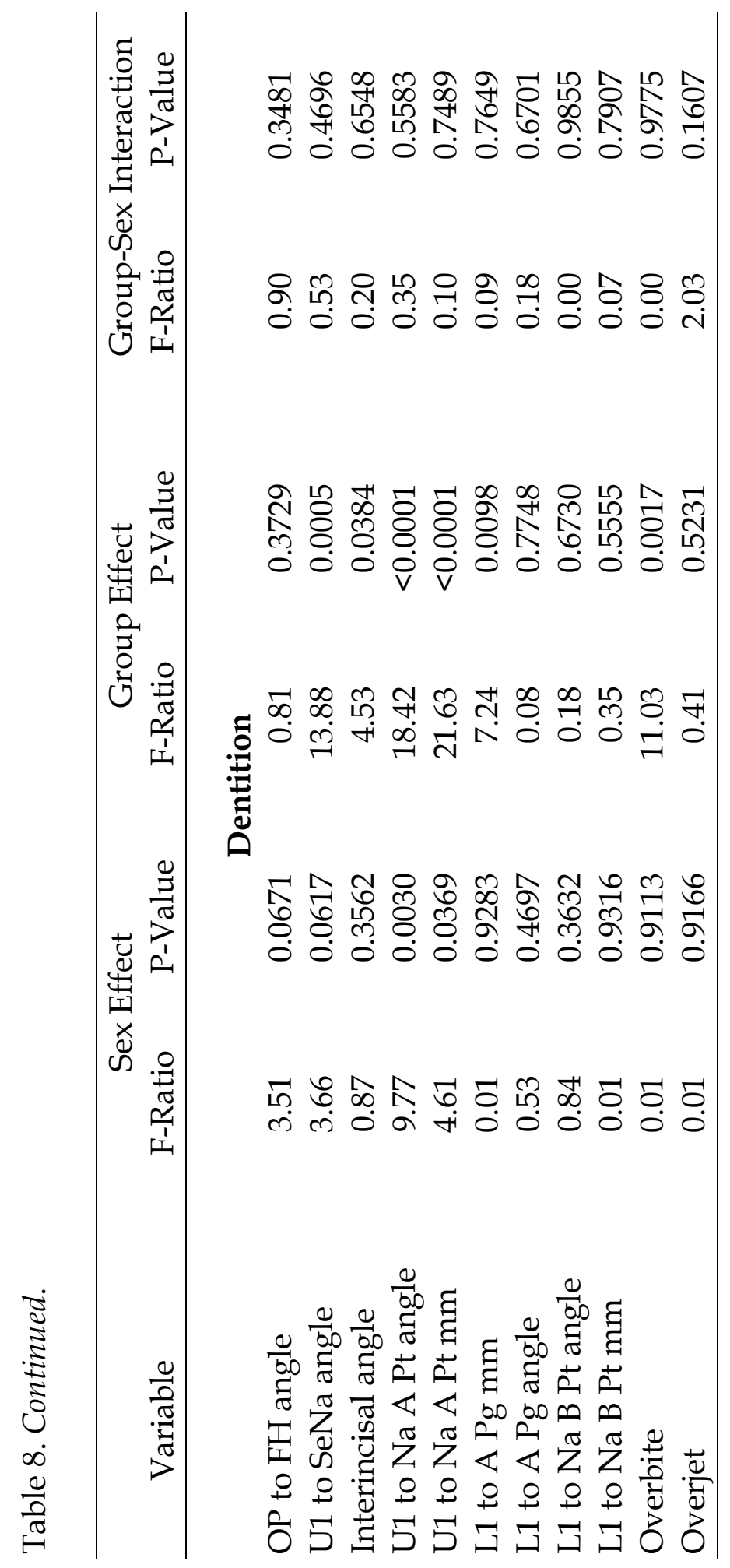


We can begin with the NAP angle (Downs' angle of convexity). This angular measure of facial convexity was significantly better (i.e., smaller) in the two-phase group. The angle averaged $2.7^{\circ}$ in the two-phase group compared to a mean of $4.8^{\circ}$ in the single-phase group. That is, the two-phase group, on the average, achieved a more orthognathic facial profile (Figure 51). Table 8 shows that there is also a significant sex difference in the terminal NAP relationship, with males having a straighter profile (i.e., a smaller mean angle) than the girls.

ANB, a complementary measure of maxillomandibular relationship (parasagittally), also exhibited a highly significant difference at the end of treatment (Table 8; Figure 52). ANB was significantly smaller $\left(\bar{x}=2.6^{\circ}\right)$ in the two-phase group than in the one-phase group $\left(\bar{x}=3.7^{\circ}\right)$. This is of interest because the mean ANB was quite comparable between groups at the start of treatment, even when adjusting for the differences in the starting ages. Both samples had a mean ANB jaw discrepancy of about $5.5^{\circ}$ at the start of treatment.

The AOBO discrepancy is another key measure of parasagittal jaw relationship. This comparison is made complex by different starting conditions (Figure 53). This was not a variable we matched the cases on, and the average $\mathrm{AOBO}$ was greater in the conventional Edgewise sample (mean of 2.0 vs. 0.5 


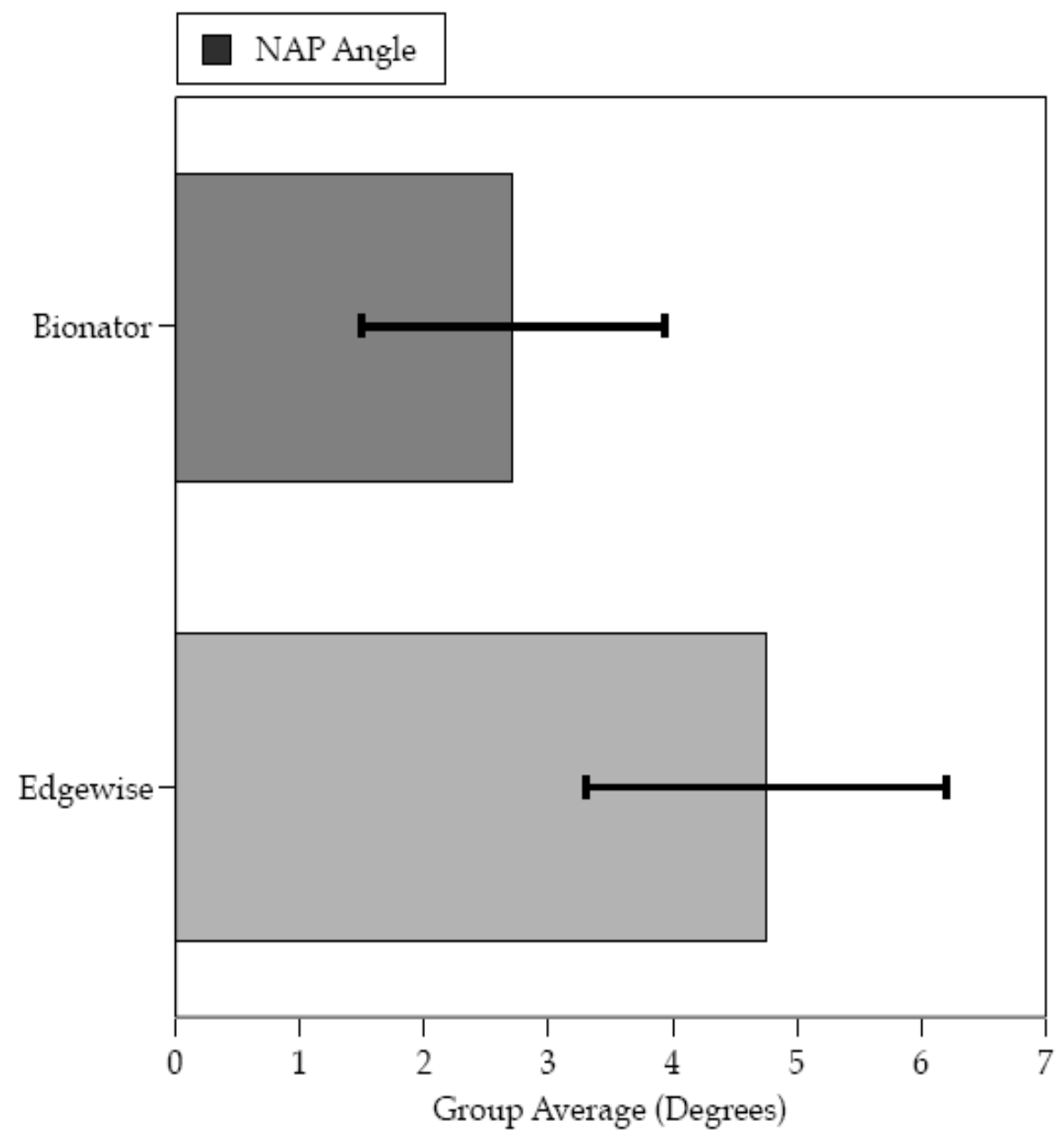

Figure 51. Histogram of group averages for NAP angle evaluated at the end of treatment. Error bars are 95\% confidence limits. 


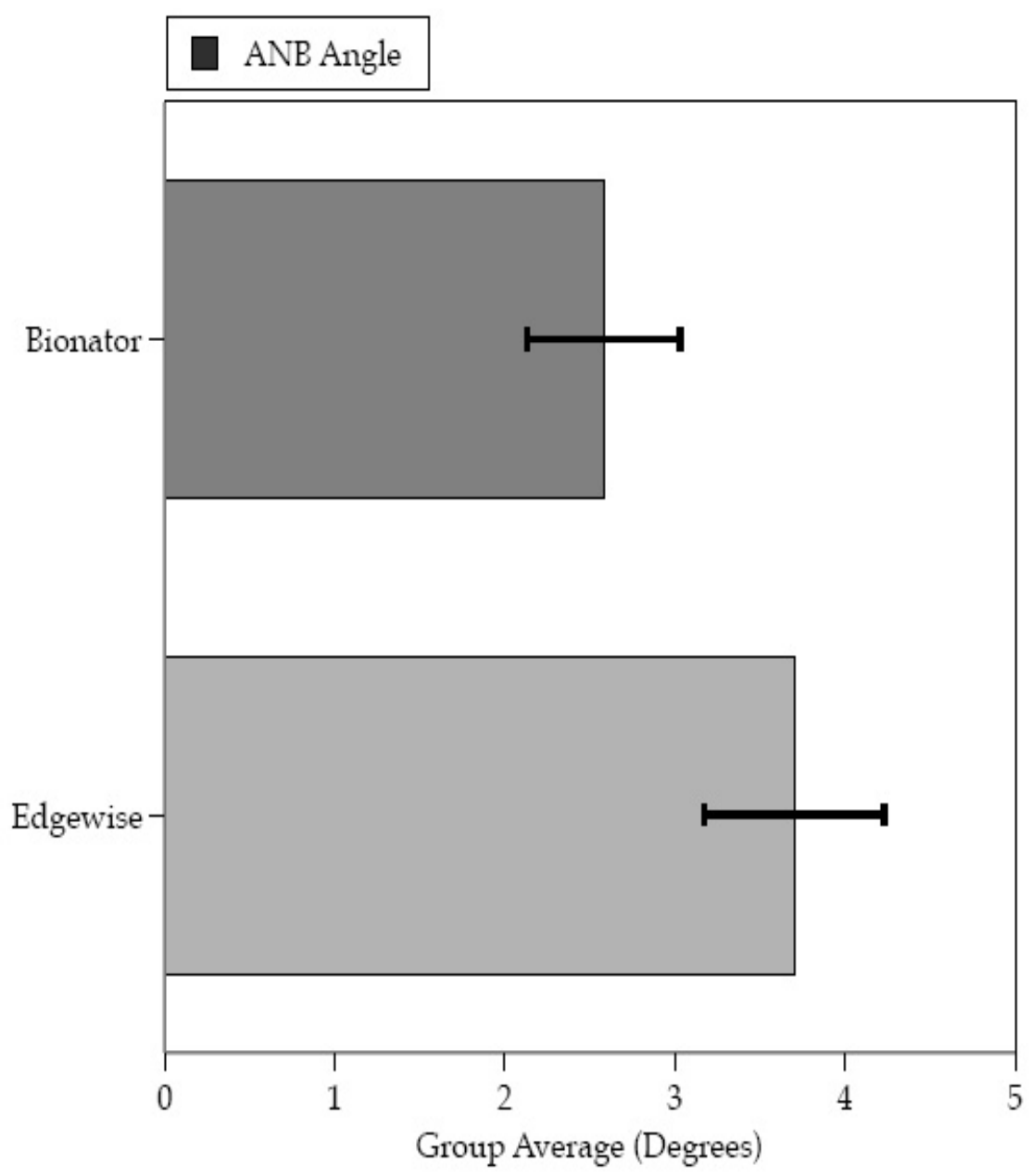

Figure 52. Histogram of group averages for ANB angle evaluated at the end of treatment. Error bars are 95\% confidence limits. 


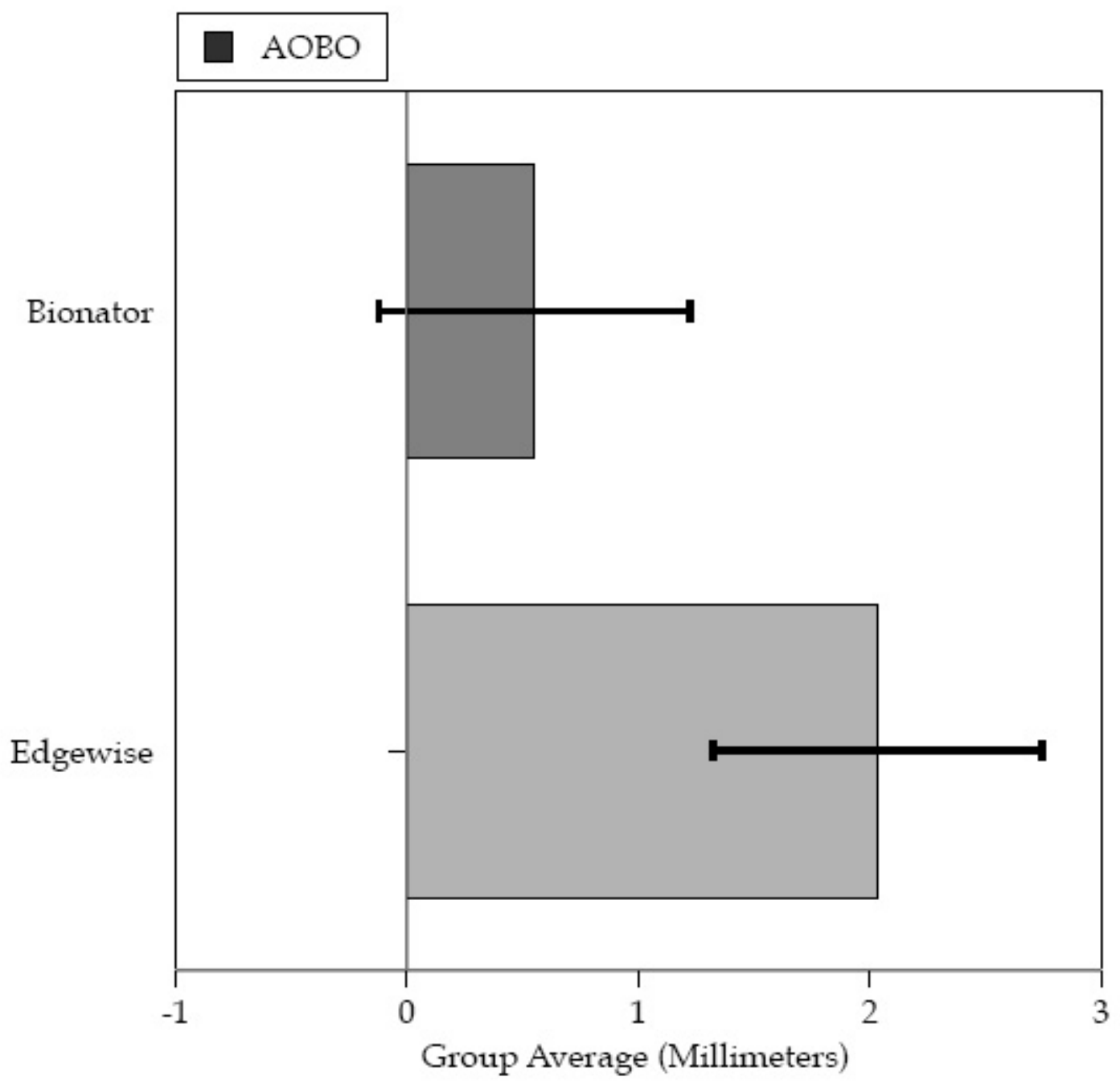

Figure 53. Histogram of group averages for $\mathrm{AOBO}$ evaluated at the end of treatment. Error bars are 95\% confidence limits. 
$\mathrm{mm})$, perhaps because the one-phase sample was treated at an older age and the malocclusion had gotten worse. The AOBO discrepancy was significantly worse at the end of treatment (Table $8 ; \mathrm{P}<0.0001)$ in the one-phase sample, but, again, it likewise was significantly worse at the start of treatment.

These comparisons merit reflection: The two measures of jaw relationships based on the basal bone relationships (NAP, ANB) show that mandibular growth was enhanced in the two-phase sample, whereas AOBOwhich depends more on dentoalveolar relationships - is not as fully resolved with the one-phase strategy (Table 8), perhaps because the discrepancy had gotten too entrenched at the older age of treatment.

\section{Facial Profile}

One simple perspective found in the specialty is that two-phase treatment resolves the inter-jaw discrepancy by moving B Point (i.e., the mandible) forward, while conventional one-phase treatment achieves the correction primarily by restraining A Point (i.e., the maxilla). This was investigated here by looking at the distance of A and B Points, respectively, measured to Nasion-Perpendicular (Table 8). Supposing that these are indeed discriminating variables, there was no statistical difference in either distance at the end of treatment (Figures 54,55). The descriptive statistics in Table 7 show that A and B Points are slightly better aligned vertically in the two-phase 


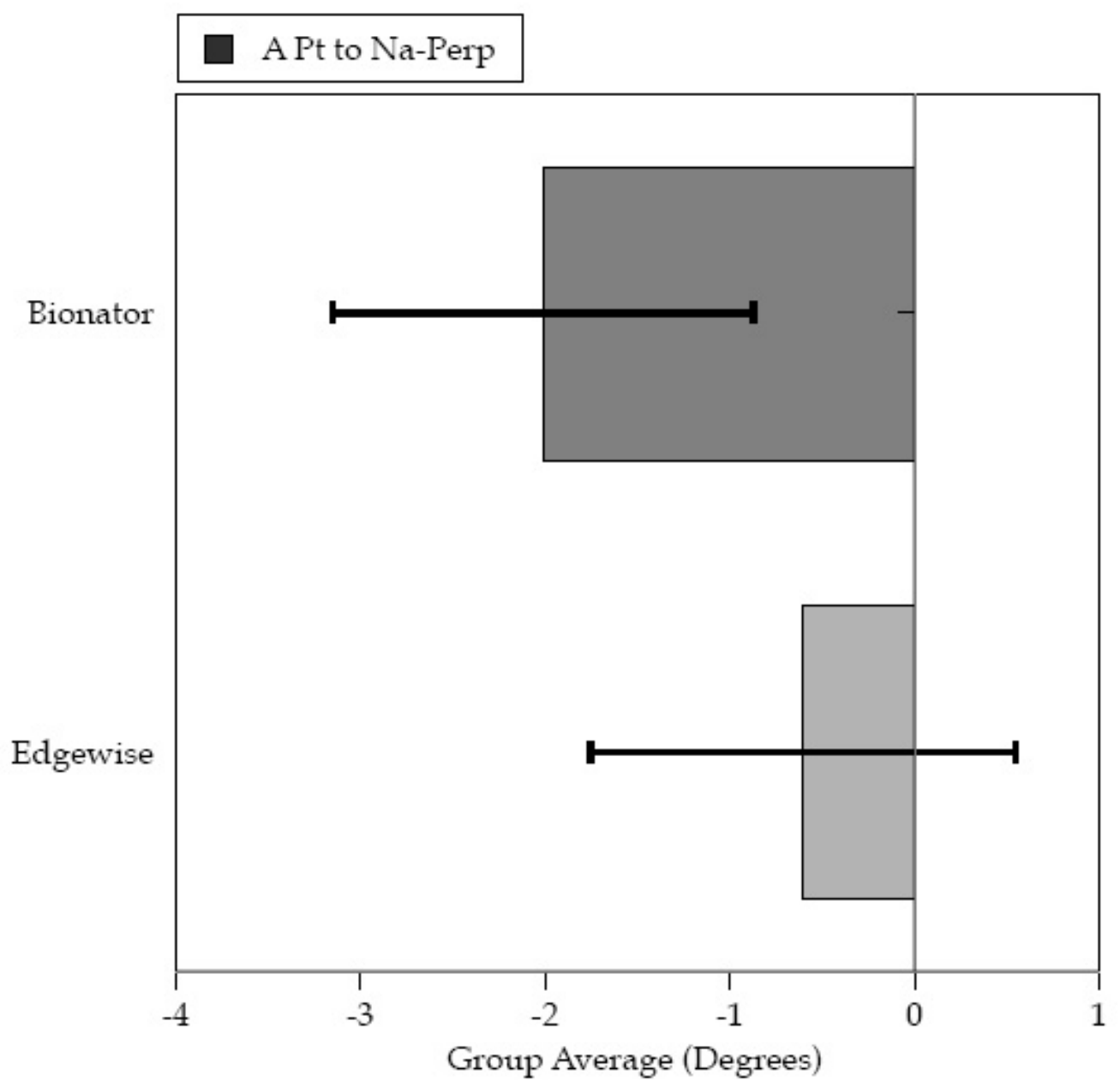

Figure 54. Histogram of group averages for A Point to Nasion-Perpendicular evaluated at the end of treatment. Error bars are 95\% confidence limits. 


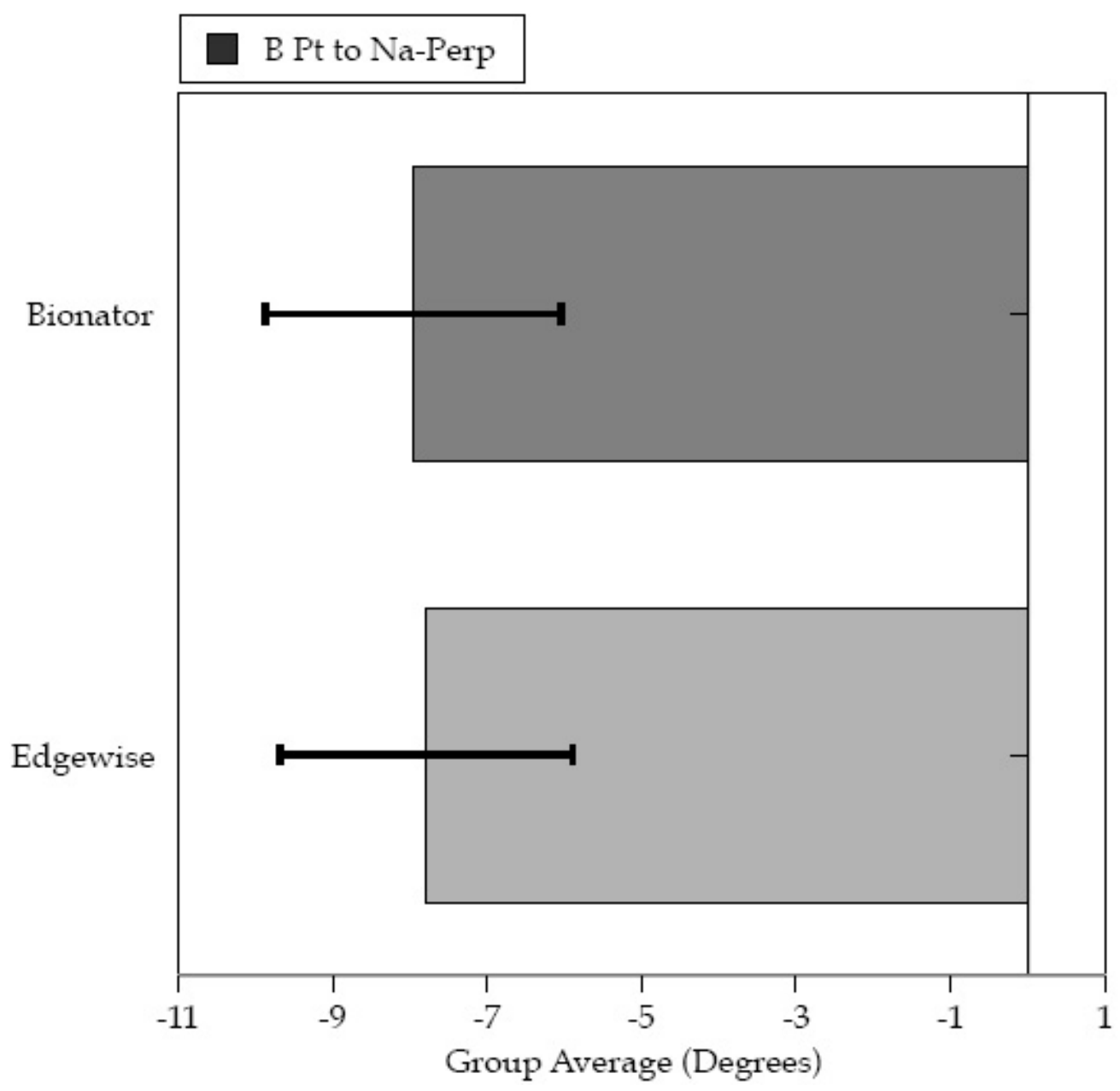

Figure 55. Histogram of group averages for B Point to Nasion-Perpendicular evaluated at the end of treatment. Error bars are 95\% confidence limits. 
group, but this has already been confirmed by the analysis of NAP and ANB.

These previous paragraphs focus attention on the few but critical skeletodental differences between groups (Table 8), but it is at least as valuable to consider the several variables for which both treatment protocols achieve the same end points.

In lateral view, the two integumental variables (E Plane, $\mathrm{Z}$ angle) achieved the same end point (Figures 56, 57). It would, of course, be valuable to know how these two variables progress as the adolescents age, and the nose and chin grow forward.

Average SNA and SNB angles both arrived at the same points statistically (Figures 58, 59). Again, though, these two angles obscure the statistically significant difference of about $1^{\circ}$ in the ANB angle, where the two phases of treatment more fully resolved the inter-arch discrepancy.

Dentally, highly significant differences are seen for $\mathrm{U} 1$ relative to the Nasion-A Point line, because U1 was significantly behind (more distal to the Nasion-A Point line) in the two-phase sample (Figures 60, 61). This, however, is a consequence of operator preference.

Several key dental variables were treated to the same end points. Of note here, the group measures for IMPA (Figure 62), FMIA (Figure 63), and the interincisal angle (Figure 64) were virtually identical in the two groups at the end of treatment. Both treatments also achieved statistically the same positions 


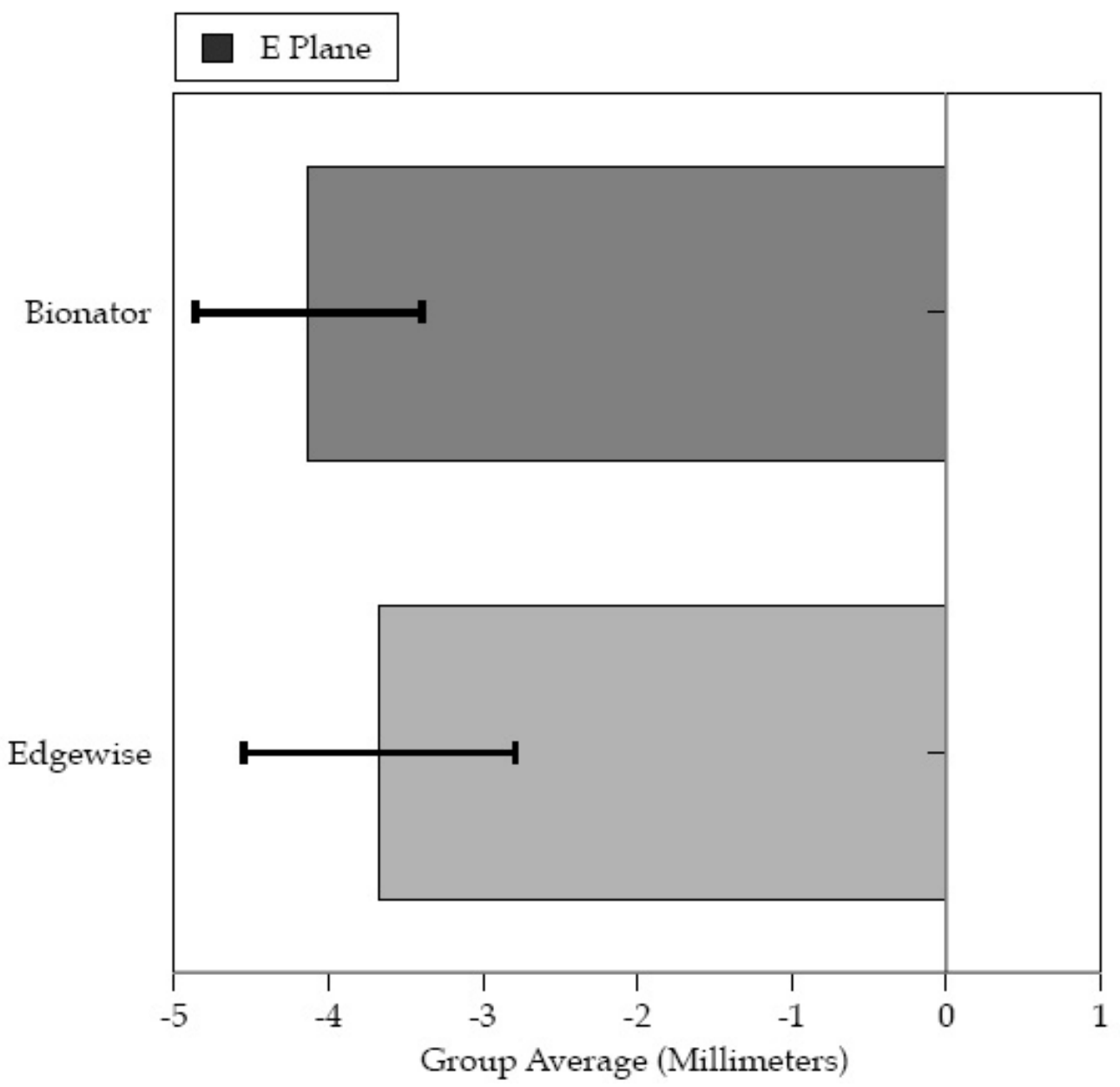

Figure 56. Histogram of group averages for E Plane evaluated at the end of treatment. Error bars are 95\% confidence limits. 


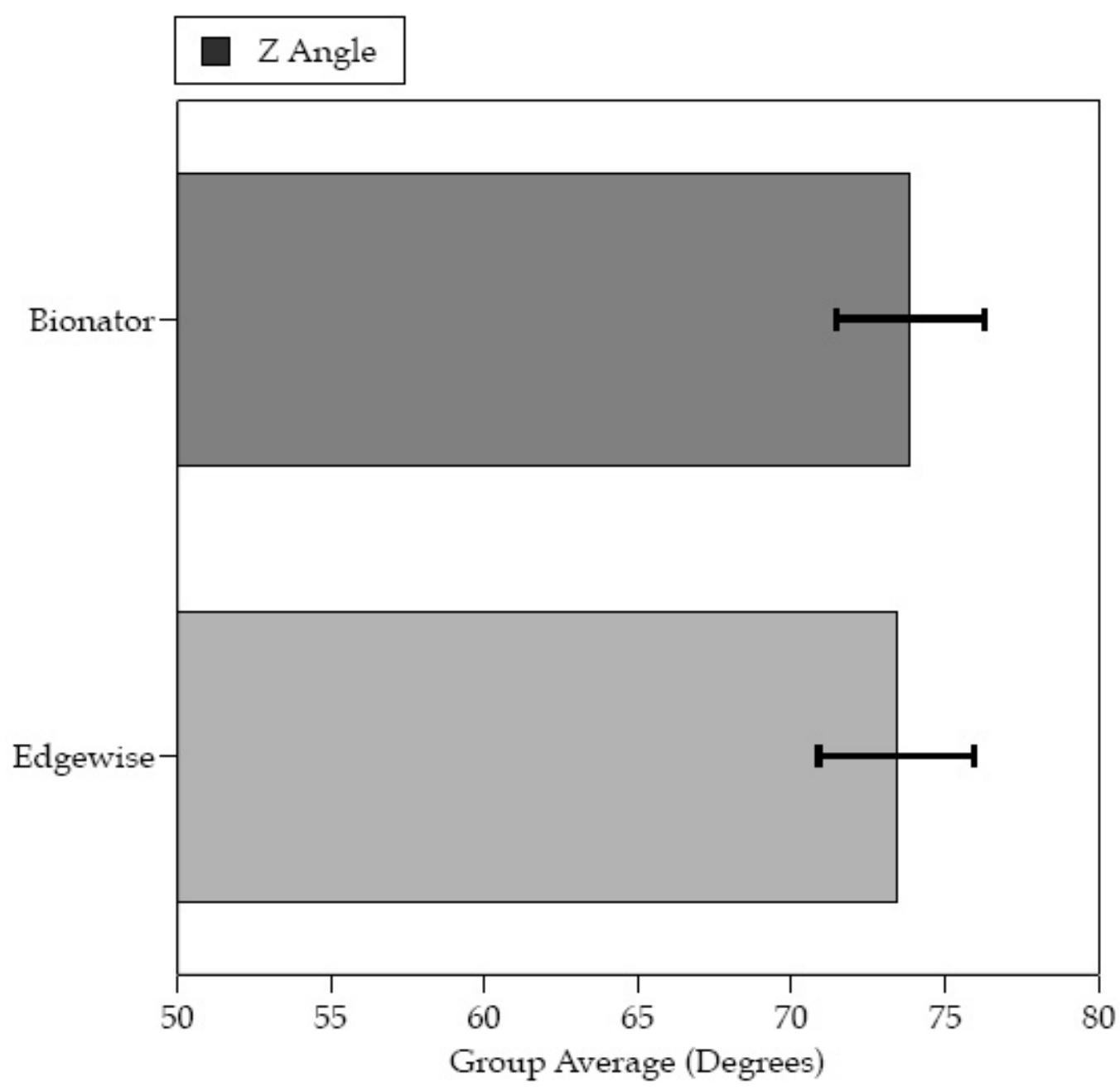

Figure 57. Histogram of group averages for $\mathrm{Z}$ angle evaluated at the end of treatment. Error bars are $95 \%$ confidence limits. 


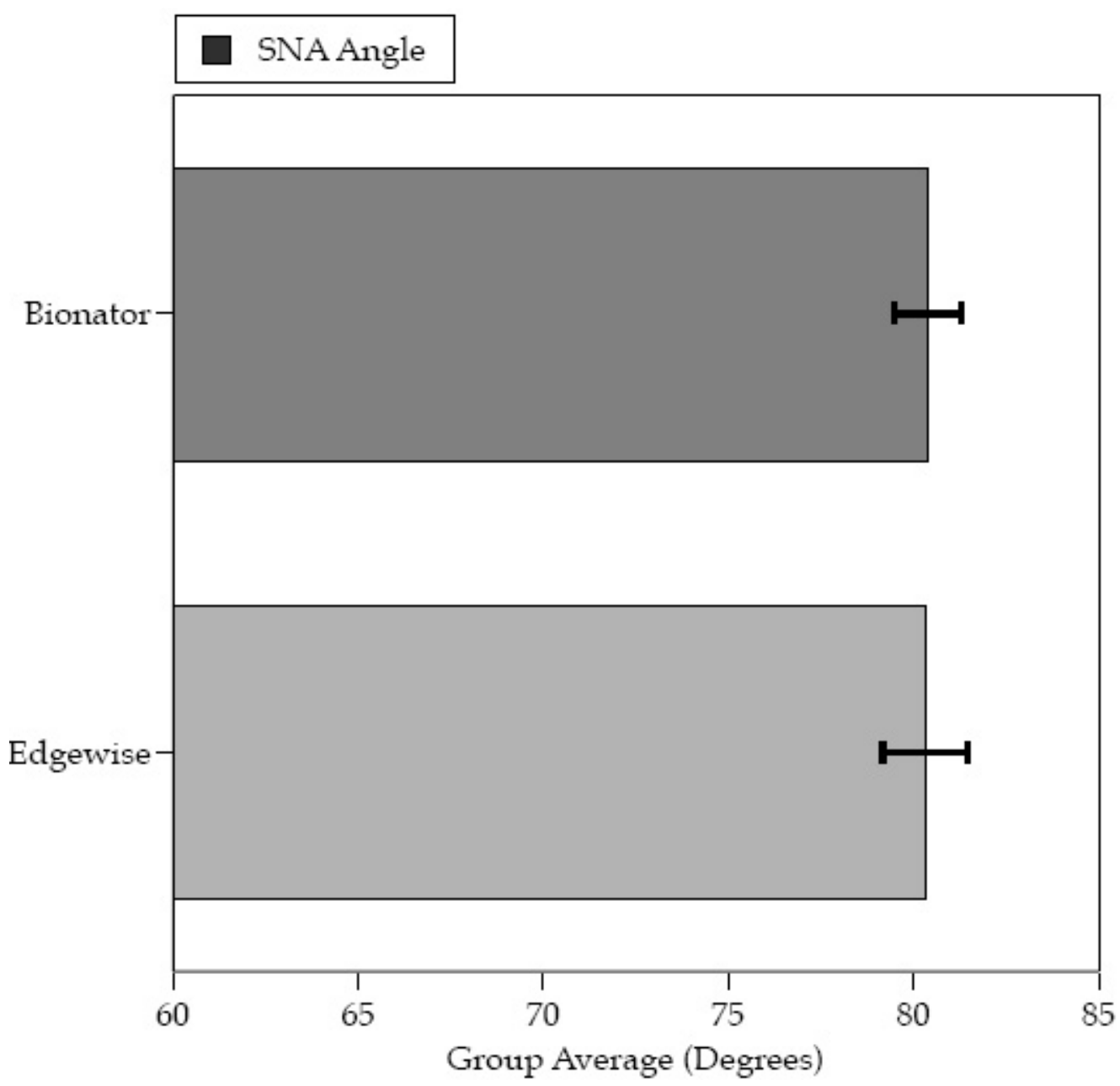

Figure 58. Histogram of group averages for SNA evaluated at the end of treatment. Error bars are $95 \%$ confidence limits. 


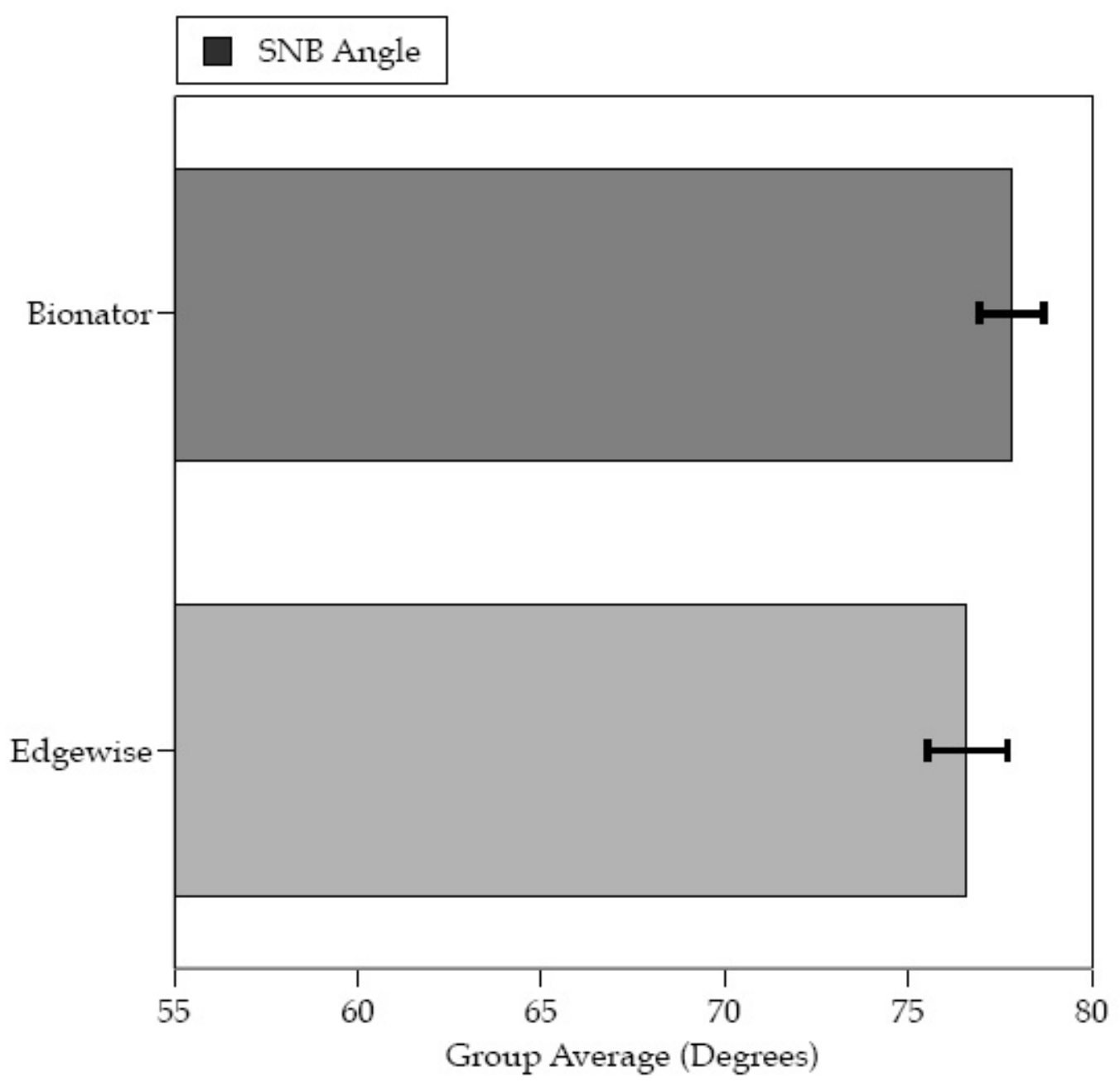

Figure 59. Histogram of group averages for SNB angle evaluated at the end of treatment. Error bars are 95\% confidence limits. 


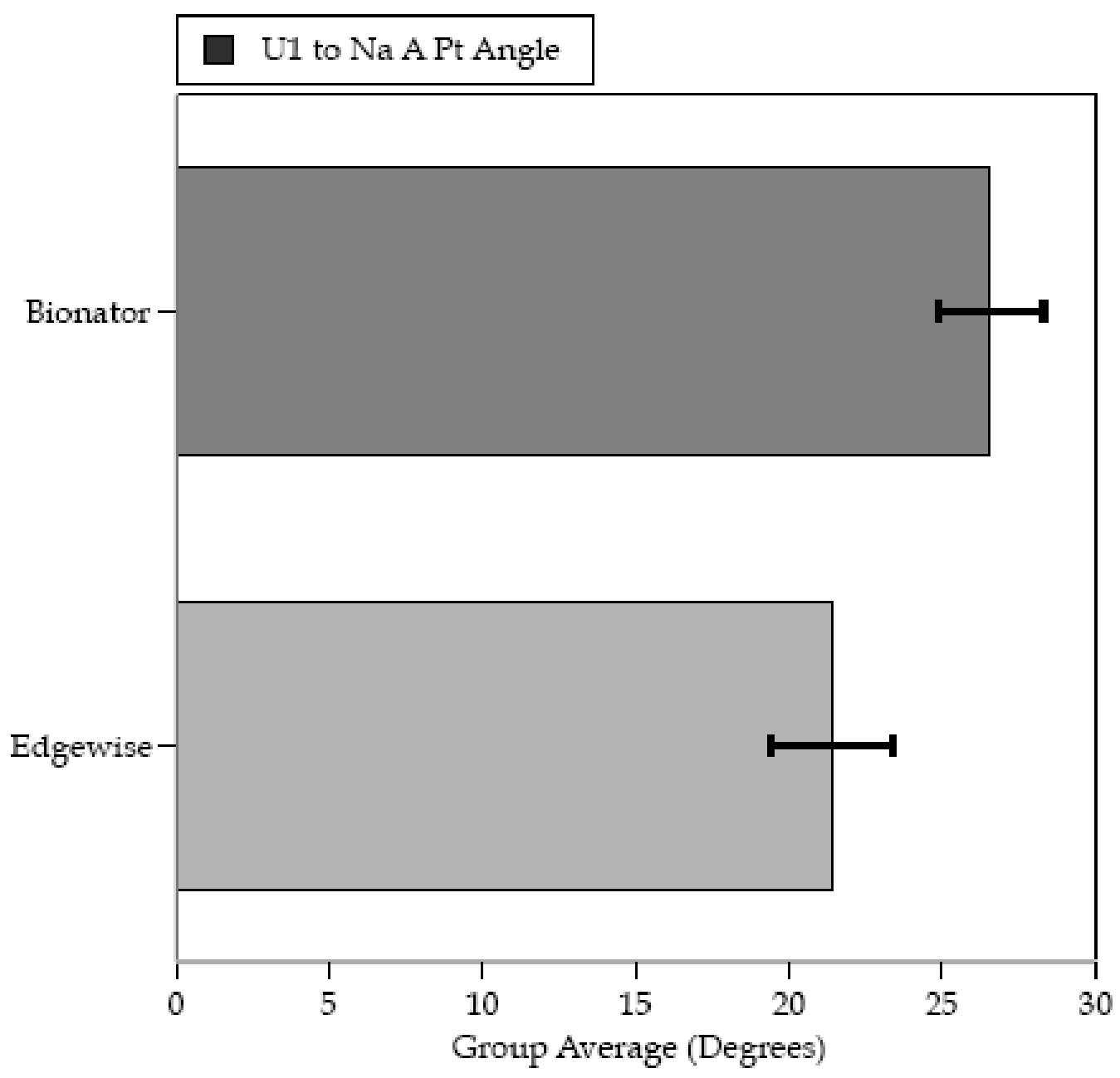

Figure 60. Histogram of group averages for U1 to Nasion-A Point angle evaluated at the end of treatment. Error bars are $95 \%$ confidence limits. 


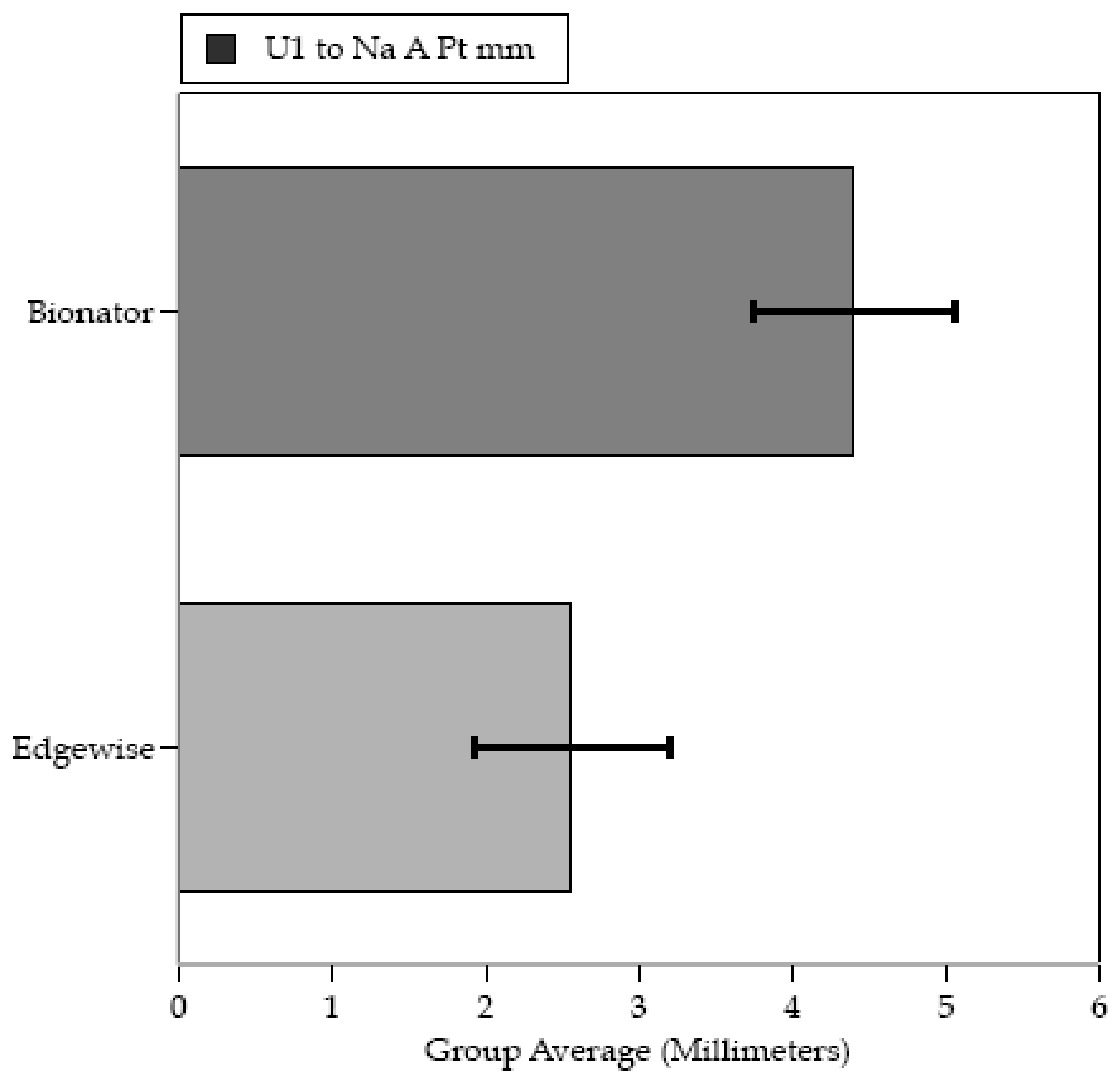

Figure 61. Histogram of group averages for U1 to Nasion-A Point (mm) evaluated at the end of treatment. Error bars are 95\% confidence limits. 


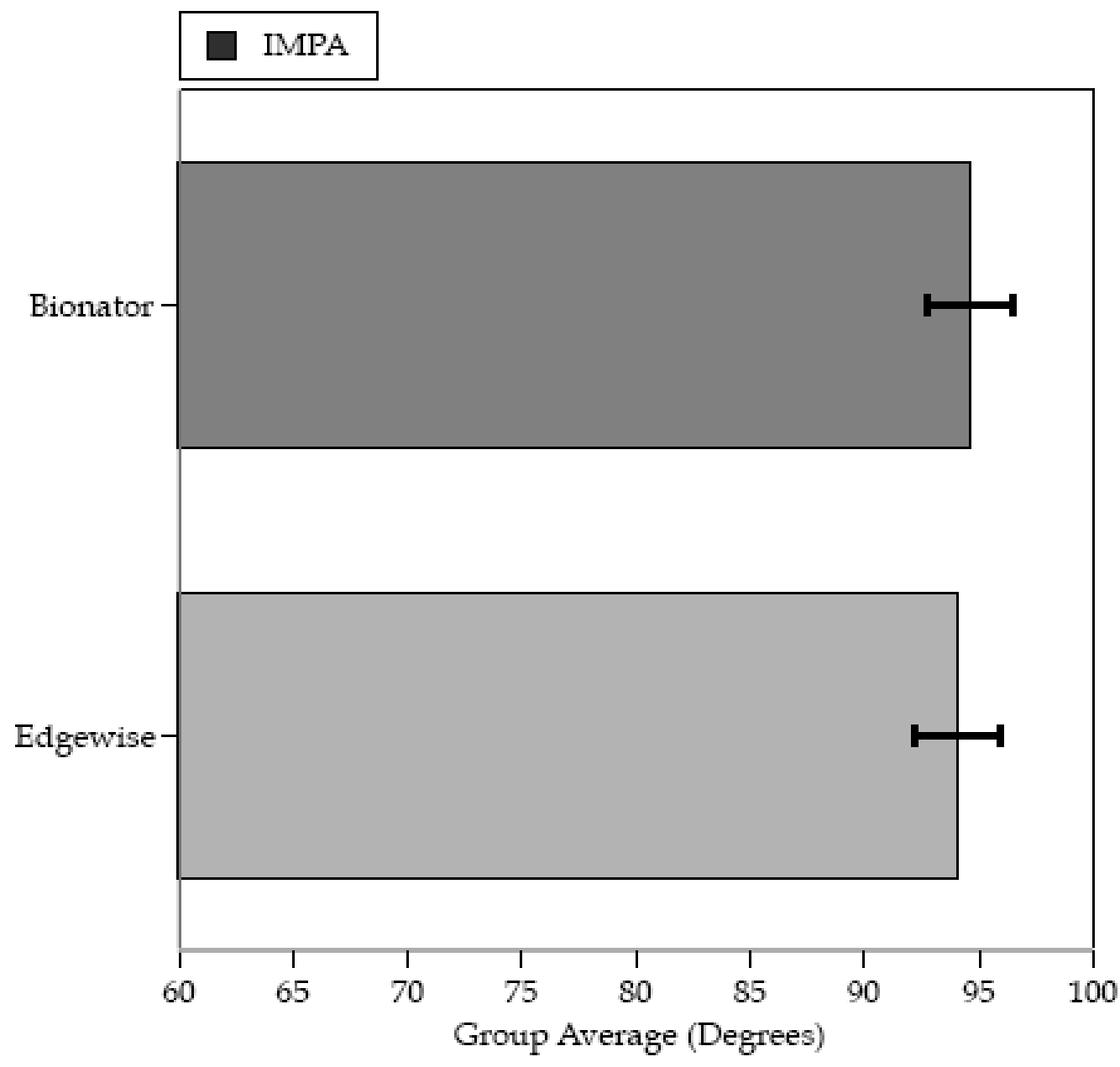

Figure 62. Histogram of group averages for IMPA evaluated at the end of treatment. Error bars are 95\% confidence limits. 


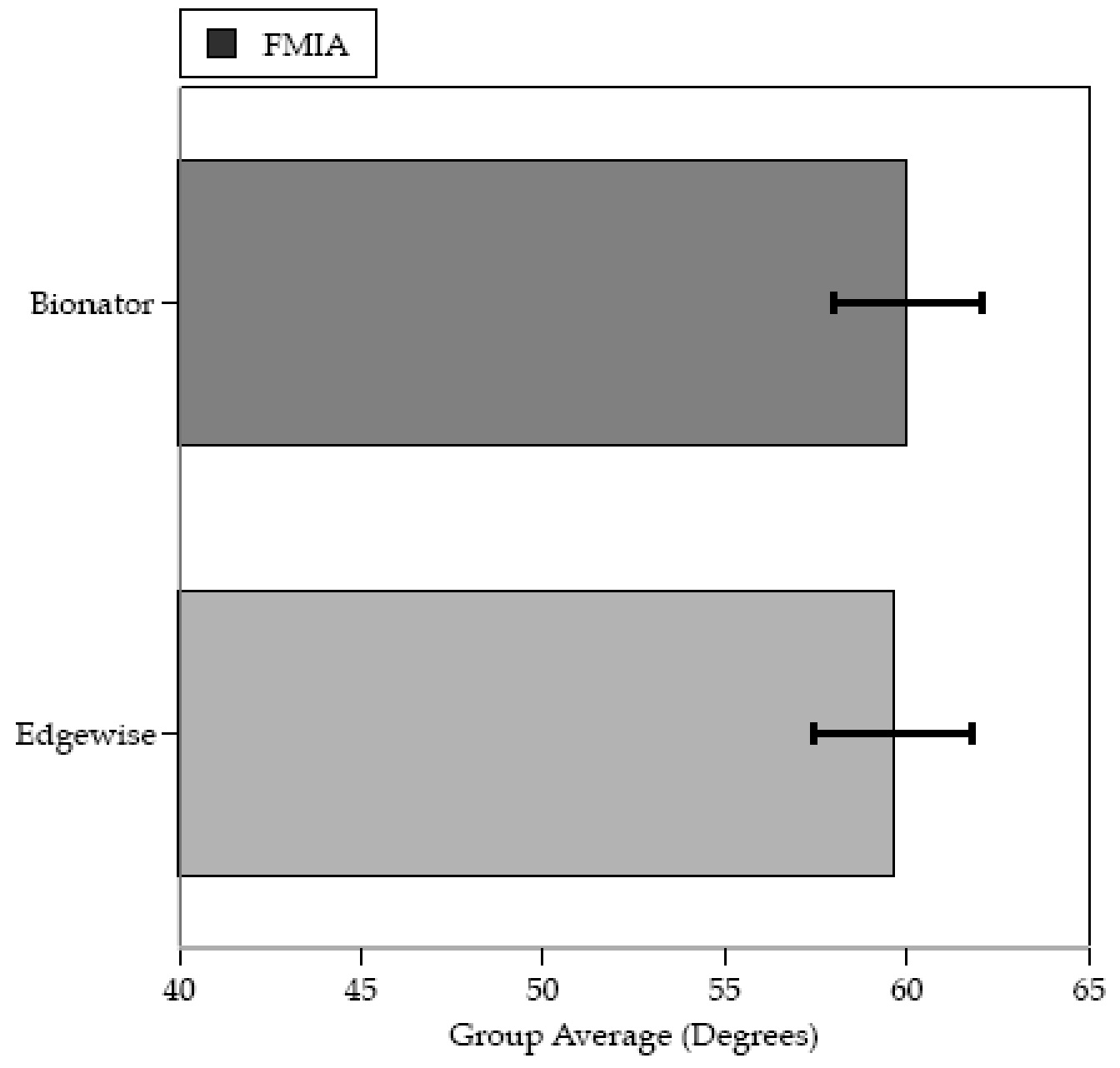

Figure 63. Histogram of group averages for FMIA evaluated at the end of treatment. Error bars are 95\% confidence limits. 


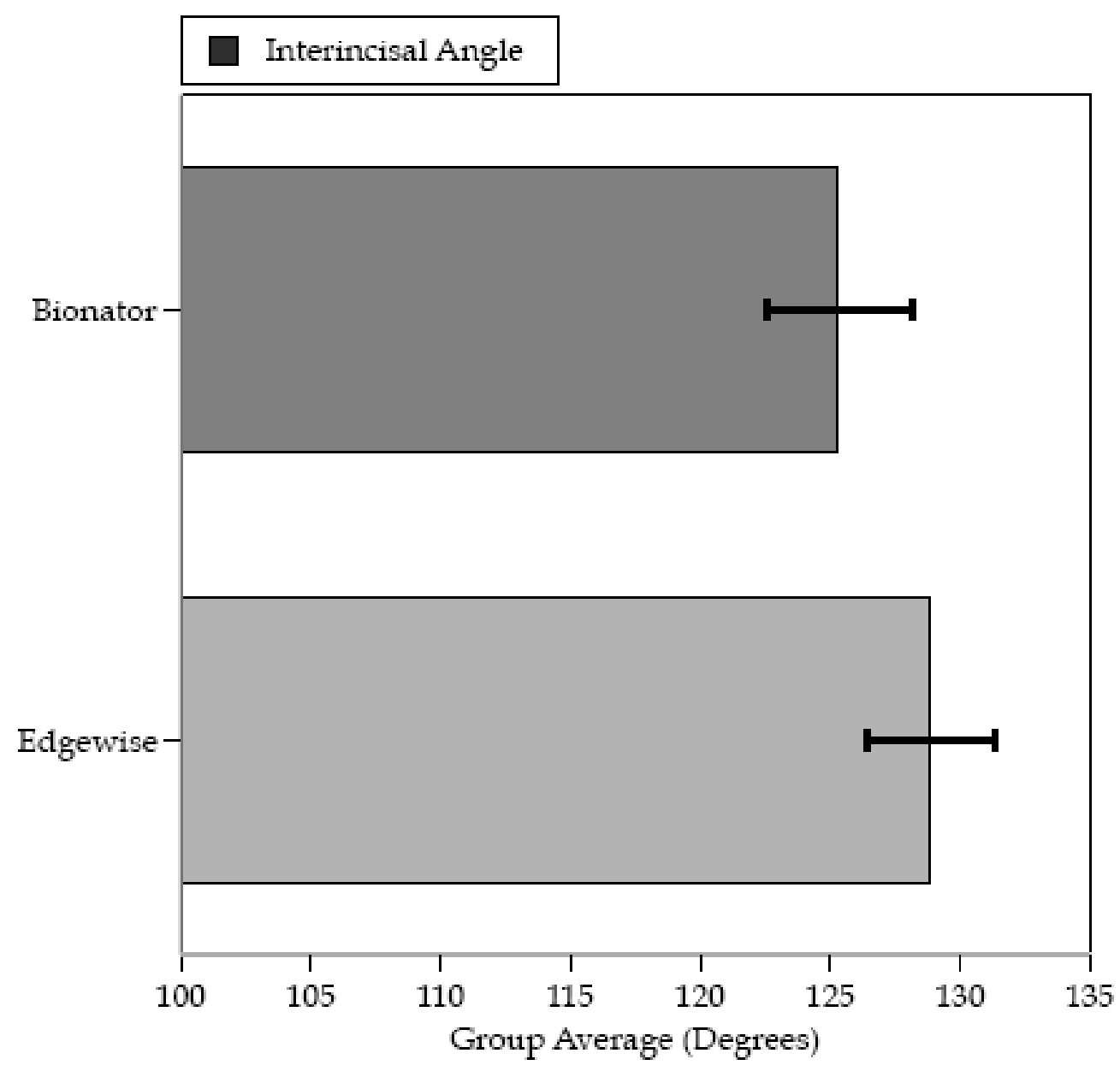

Figure 64. Histogram of group averages for interincisal angle evaluated at the end of treatment. Error bars are 95\% confidence limits. 
were virtually identical in the two groups at the end of treatment. Both treatments also achieved statistically the same positions of the mandibular central incisor as regards to the Nasion-A Point line (Figures 65, 66) and the Nasion-B Point line (Figures 67, 68).

Overjet ended up at just over $3 \mathrm{~mm}$ in both treatments (Figure 69), but overbite was significantly different (Figure 70). Overbite averaged $1.2 \mathrm{~mm}$ in the one-phase series but was significantly deeper $(\mathrm{P}=0.002)$ at an average of $1.8 \mathrm{~mm}$ in the two-phase series. Again, though, this difference is due to operator preference; the minor differences in incisor relationships were intended to provide better disclusion in the two-phase group. 


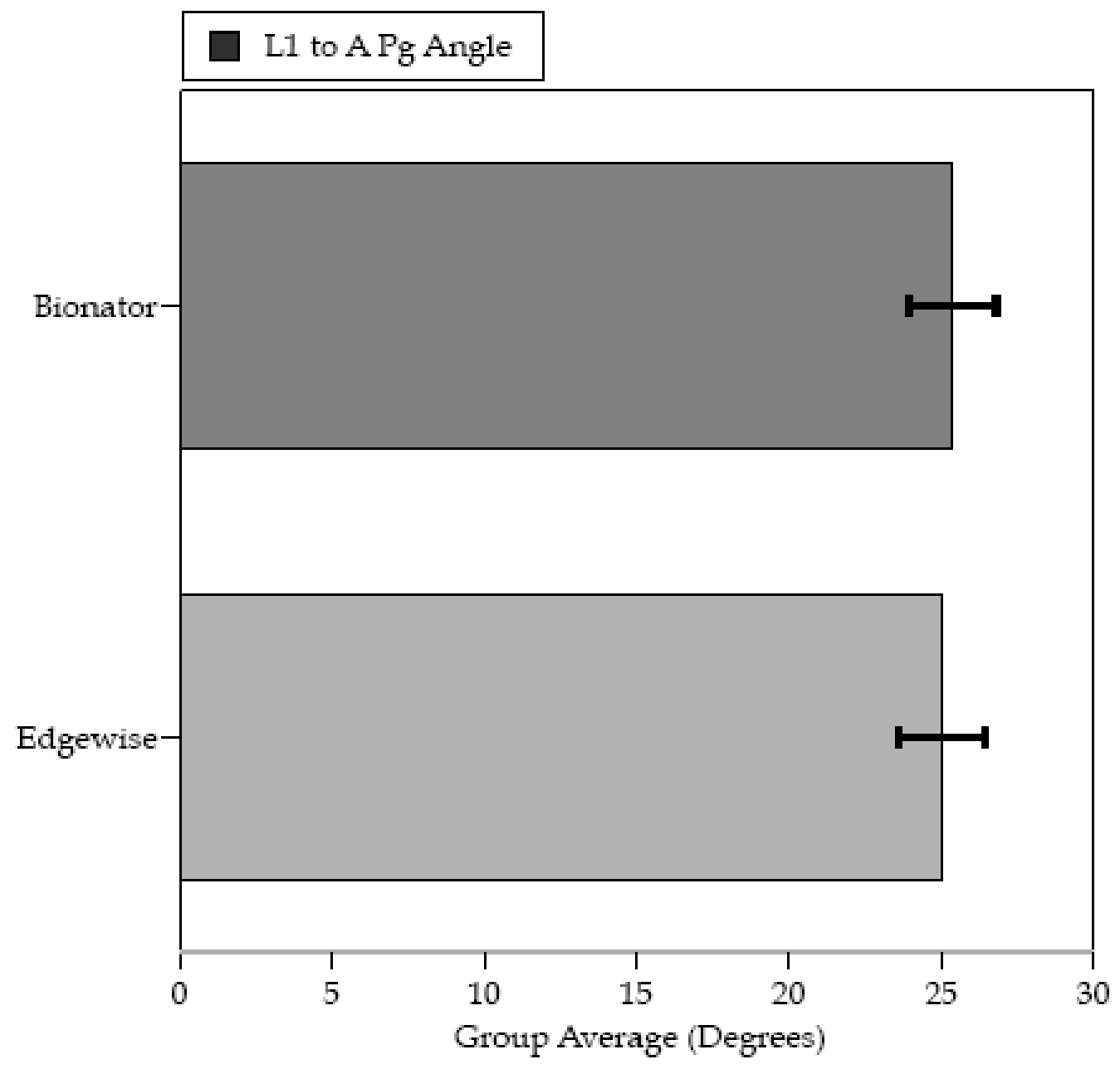

Figure 65. Histogram of group averages for L1 to A-Pogonion angle evaluated at the end of treatment. Error bars are 95\% confidence limits. 


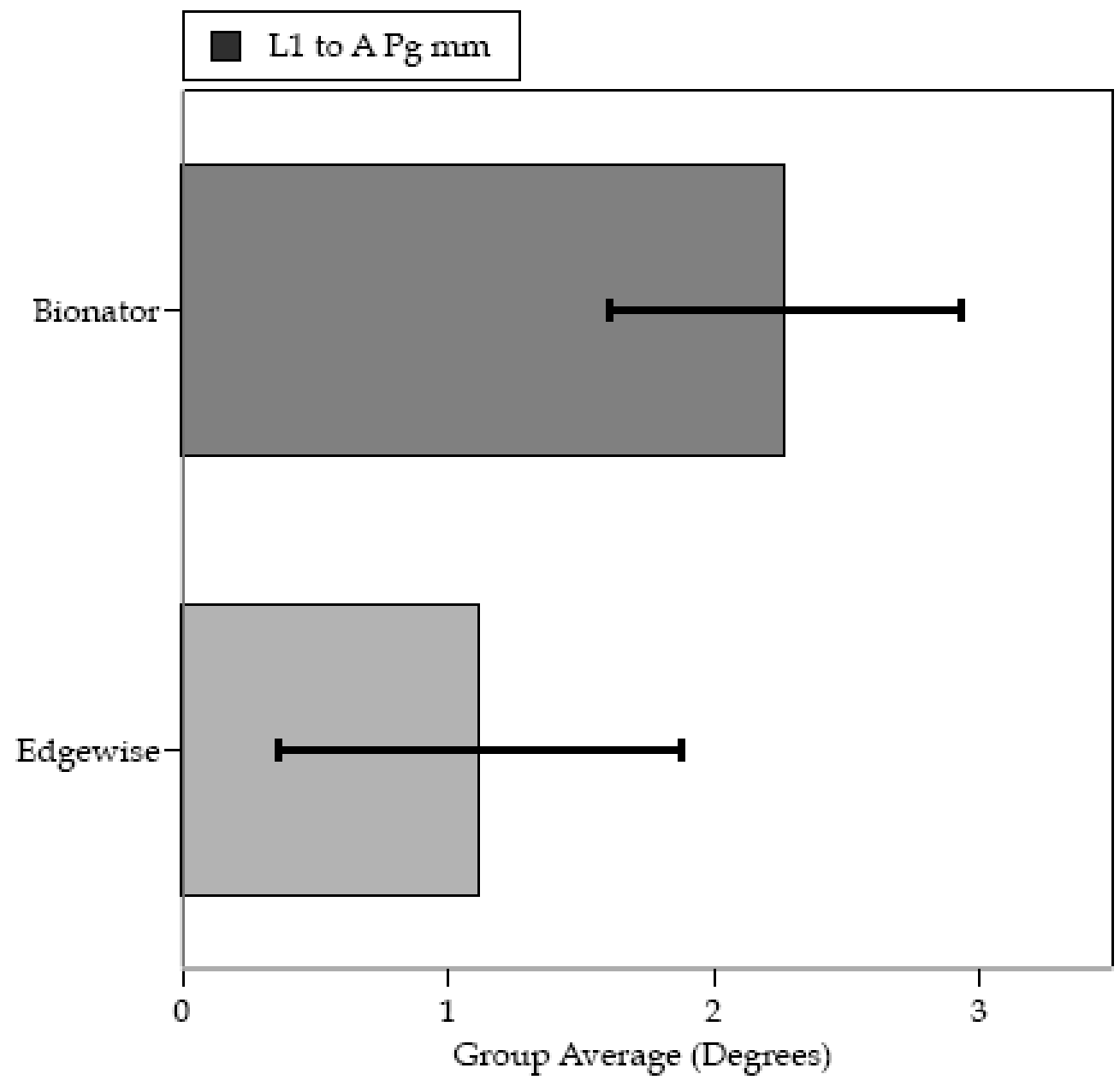

Figure 66. Histogram of group averages for L1 to A-Pogonion (mm) evaluated at the end of treatment. Error bars are 95\% confidence limits. 


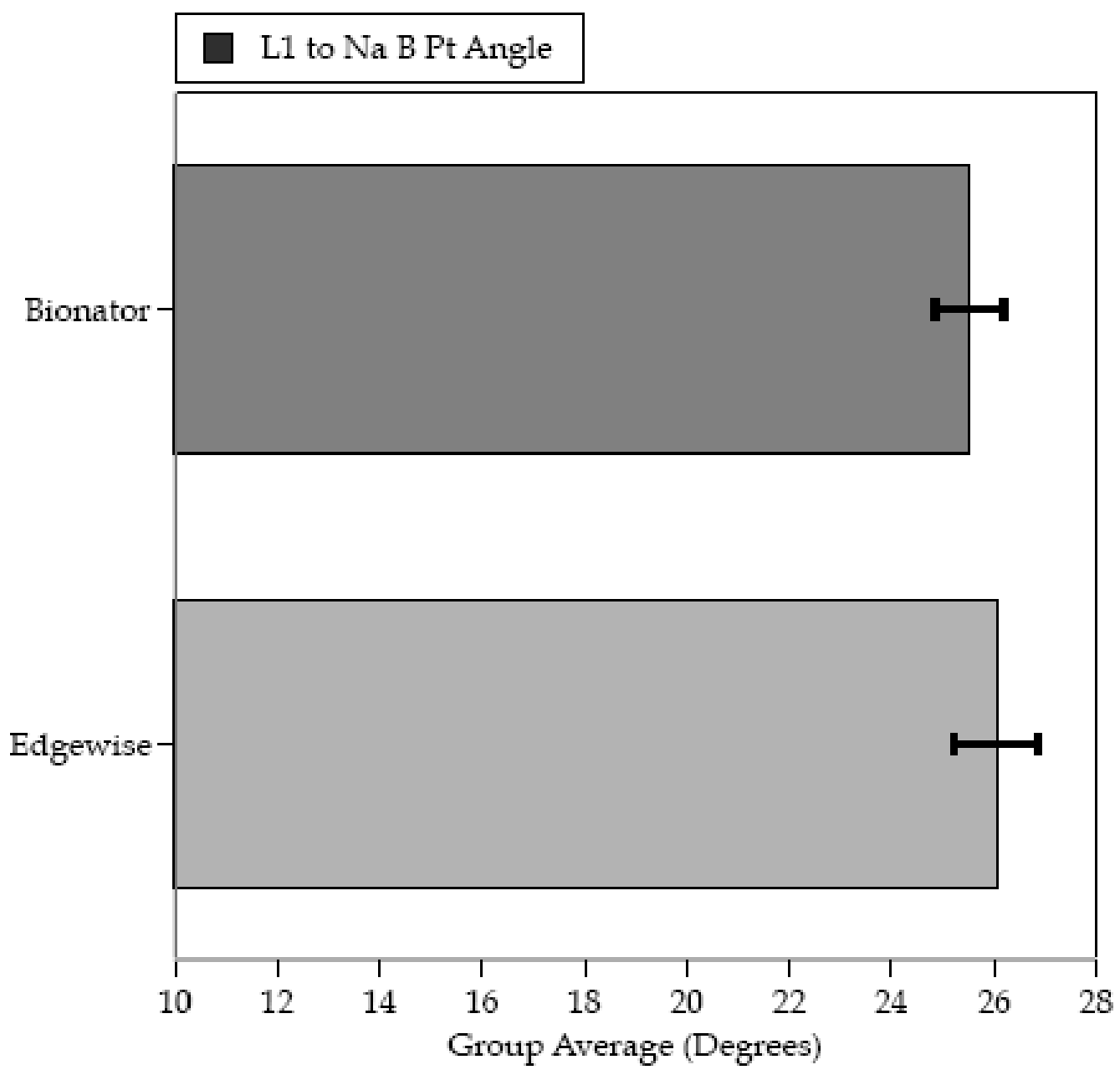

Figure 67. Histogram of group averages for L1 to Nasion-B Point angle evaluated at the end of treatment. Error bars are $95 \%$ confidence limits. 


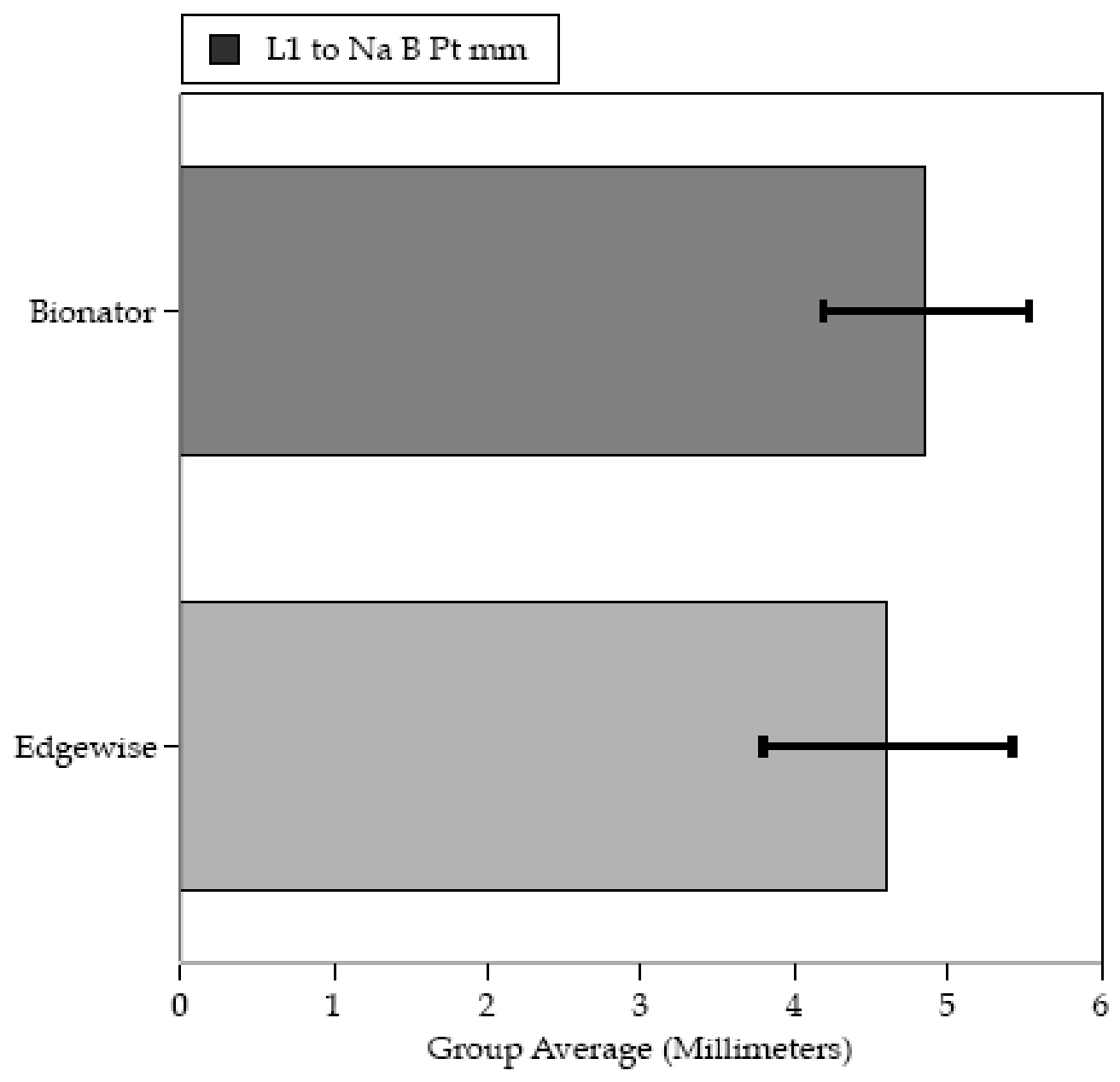

Figure 68. Histogram of group averages for L1 to Nasion-B Point (mm) evaluated at the end of treatment. Error bars are $95 \%$ confidence limits. 


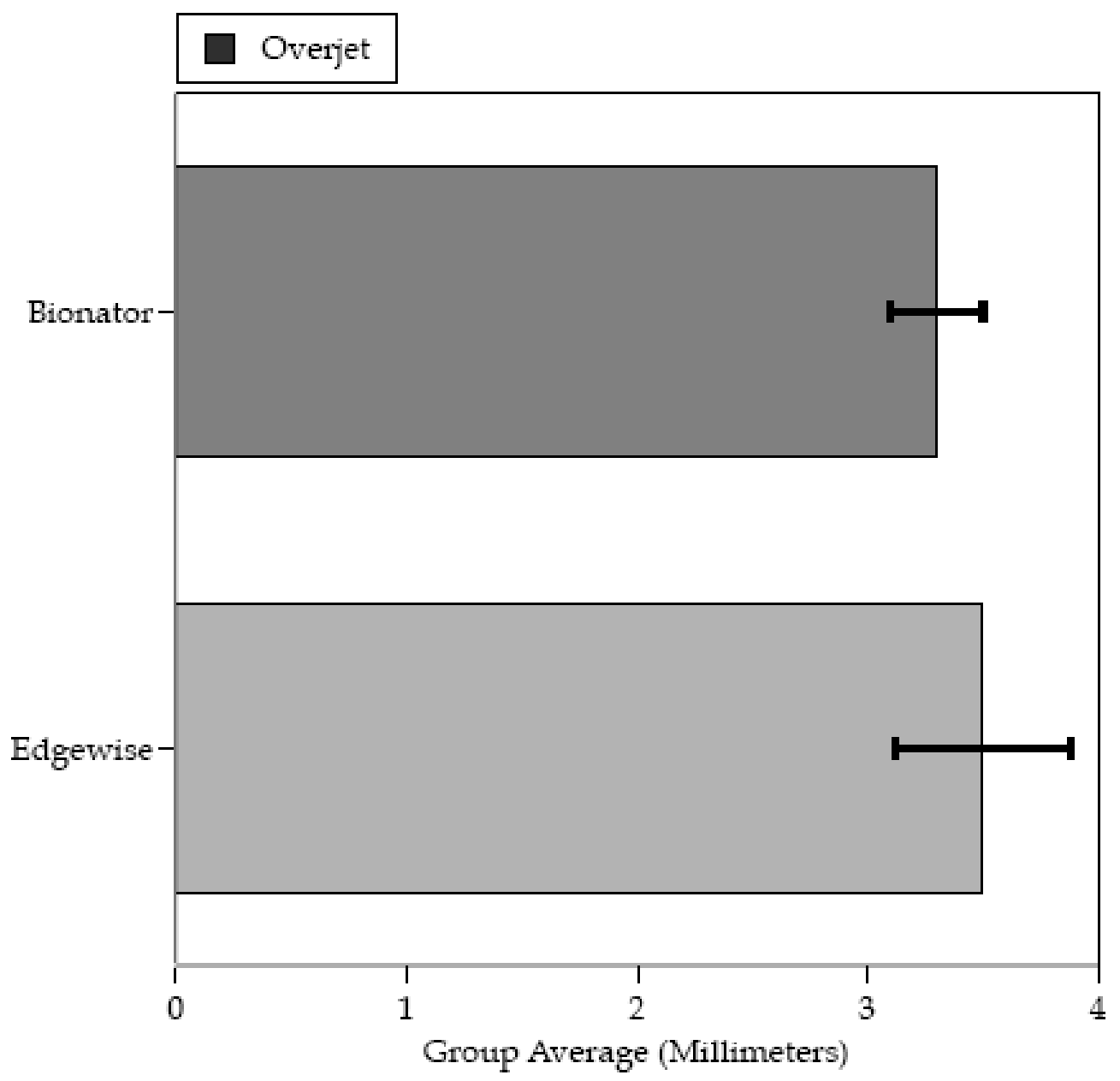

Figure 69. Histogram of group averages for incisor overjet evaluated at the end of treatment. Error bars are 95\% confidence limits. 


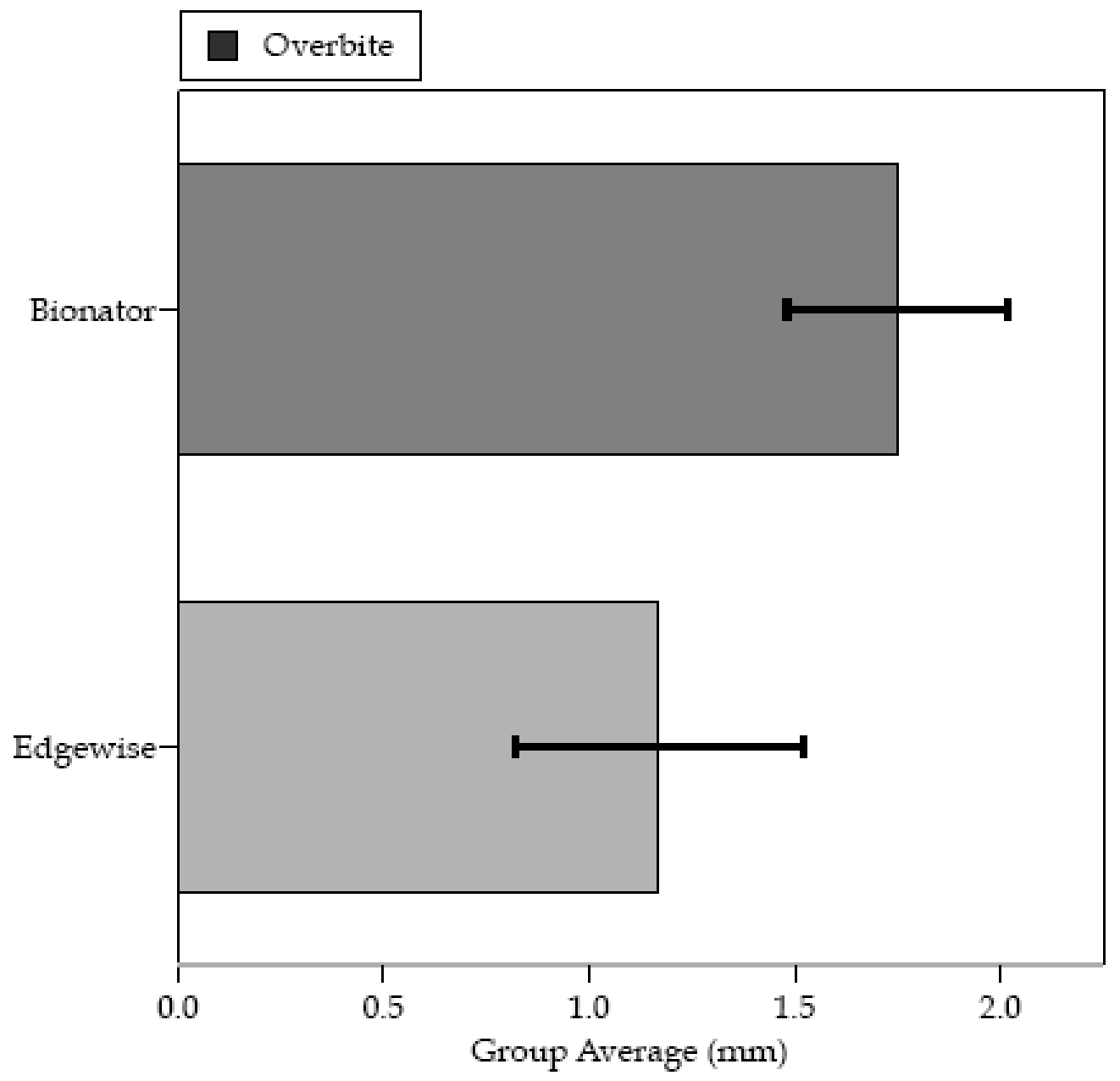

Figure 70. Histogram of group averages for incisor Overbite evaluated at the end of treatment. Error bars are 95\% confidence limits. 


\section{CHAPTER V}

\section{DISCUSSION}

The prevalence of Class II malocclusions among children in the United States is approximately 15\% (Kelly and Harvey 1977), and there are, broadly, two approaches in use today to treat these cases. The conventional approach is to wait until the early permanent dentition has emerged, then use full-bonded fixed appliances to treat the malocclusion in a single phase. Alternatively, the child can be treated with a functional appliance in the mixed dentition, followed by a second phase of fixed treatment. There are proponents (and vocal opponents) to each of these approaches. Results of influential randomized clinical trials (O'Brien 2003; Tulloch et al. 2004; King et al. 2003) uniformly show that there is no benefit from two-phase treatment, yet other publications tout the merits of early intervention rather convincingly. The purpose of this study was to compare the skeletodental outcomes of two samples matched for difficulty of the Class II malocclusion, one sample treated with a Bionator in the mixed dentition (followed by Edgewise treatment) and the other treated with Edgewise mechanics alone in the permanent dentition. 


\section{$\underline{\text { Time in Treatment }}$}

The present study found that the one-phase group had a mean time in treatment of 2.8 years compared to 4.2 years for the two-phase group. Overall clock time, then, was 1.4 years longer for the two-phase group. These findings agree with those of Bremen and Pancherz (2002) who studied the efficiency of early and late Class II division 1 treatment. They noted that patients treated exclusively with fixed appliance had a shorter duration than did patients treated with functional appliances or a combination of appliances (38 months for functional appliances and 49 months for a combination). These data also agree with those of Livieratos and Johnston (1995) who studied a two-stage Bionator-Edgewise regimen in comparison to a conventional one-stage Edgewise treatment in a "matched" Class II sample. This argument, that there is a difference in clock time, hardly warrants intense investigation since it is obvious. Intuitively, two phases of treatment with an earlier starting age ought to take a longer time than a single phase of treatment. Livieratos and Johnston (1995) observed that in addition to taking 18 months longer, the two-stage treatments averaged 10 more appointments. However, the overall appointment rate (13 per year) was lower than for the single-phase Edgewise group (17 per year). This difference was attributed to fewer appointments, with a more "leisurely" pace during the functional phase of treatment. Similar findings also were noted by Tulloch et al. (2004). In the current study, Bionator 
patients were seen every 2 months with minor chair time per appointment during phase 1. It was the opinion of the specialist who treated these cases that (1) appointments required very little of his time during the Bionator phase and (2) the younger children were well motivated and compliant.

It is logical that if early treatment reduced the complexity of later treatment, there should be a reduction in phase 2 treatment time and, as well, a reduction in the need for premolar extractions. In a study of early Class II treatment outcomes, Tulloch et al. (2004) noted that comprehensive treatment took as long in children who had early treatment than in those who did not. They also noted that early treatment did not reduce the need for extractions or surgery. In the present study, overall time spent in Edgewise appliances for the two-phase group was 7 months shorter than in the one-phase treatment. In addition, the two-phase group had a much lower percentage of extractions, $12 \%$ for the two-phase group compared to $84 \%$ for the one-phase group. Complexity of treatment was reduced in the two-phase subjects in so far as there were far fewer extractions and the second phase of treatment was shorter.

Complaints from opponents about longer time in treatment may, however, be a red herring. It seems at least as pertinent to ask (1) when (at what age) does the bulk of the skeletodental correction occur (because this affects the child's perception of his esthetics) and (2) is the final result demonstrably better? These two issues are discussed below. 


\section{$\underline{\text { Extraction Treatment }}$}

It is not clear how to value the preservation of all eight premolars in the two-phase sample. Two-phase treatment substantially decreases chances that teeth need to be extracted, but so what? Preservation of a full dentition does not seem to affect masticatory efficiency. Other than the perception that extractions are "wrong" and parents may want their children to be treated without extractions, there seems to be no scientific basis for preferring the retention of all premolars. There may be gnathological reasons to prefer an intact dentition, but this does not seem to be documented. There is, however, another consideration that often seems to be under-valued by the orthodontist. The compelling reason most adolescents (and their parents) seek treatment is to improve their dental esthetics (e.g., Shaw et al. 1980, 1981; O'Brien et al. 1996). There are at least two interrelated issues here. One, early intervention improves the esthetic aspects of the malocclusion at an earlier age. As discussed previously, much of the skeletal and dental improvement occurs during the Bionator phase of treatment (Figure 50). In the present situation, this means that much of the over-all improvement has been achieved by 11 to 12 years of age versus about 16 years of age with conventional treatment (Figure 71). Two-phase treatment is a means of reducing the psychosocial stresses associated with malocclusions (Mandall et al. 2005) and promoting self esteem (Birkeland et al. 2000). 


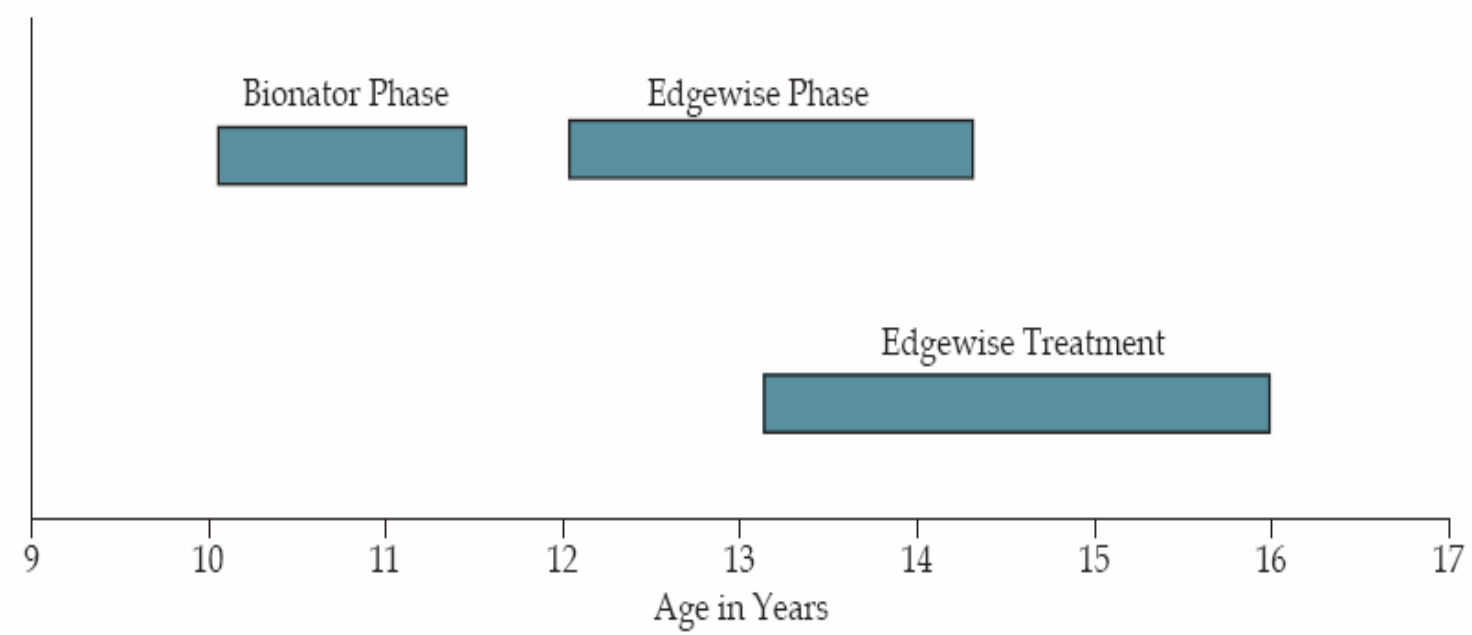

Figure 71. Histogram showing treatment times in years. On the downside, two-phase treatment obviously does take longer, even allowing for the "gap" between the two stages. On the other hand, much of the skeletodental correction is achieved during the Bionator phase, which ought to improve the child's self perception of his facial esthetics earlier. Also, it may be consequential to the patient that treatment is finished around 14 instead of around 16 years of age. This means that, on average, the patient is out of treatment when a freshman in high school instead of a junior. 
In a similar vein, it has been commented that conventional orthodontics, started in the early permanent dentition, may well be the only situation where the health care provider intentionally allows the problem (the malocclusion) to develop to its severest form before initiating treatment. In concept, it would seem easier for the specialist and the patient to resolve the esthetic problem before it is full blown.

This is particularly true for severe problems as noted by Tulloch et al. (2004). Overjet is a case in point. The permanent incisors emerge between about 6 and 8 years of age (van der Linden and Duterloo 1976), which Broadbent (1937) has noted is the beginning of the "ugly duckling" stage of the mixed dentition. Studies show that the greater the overjet, (1) the greater the risk of trauma to these teeth (Schopf 2003; Karaiskos et al. 2005) and (2) the greater the esthetic disability (Brook and Shaw 1989). Additionally, most trauma to the incisors occurs before age 10 , so conventional treatment at 12 or 13 years, occurs after the interval of greatest risk.

\section{Patterns of Skeletodental Change}

During the first phase of treatment in the two-phase group, most of the variables tested $(16 / 28)$ exhibited significant changes. The data suggest that the mandible achieved a less retrognathic relationship during phase 1. NAP decreased on average $3.4^{\circ}$, ANB was reduced on average $1.5^{\circ}$, and AOBO 
decreased on average $2.3 \mathrm{~mm}$. Similar findings have been reported by other studies. Heij and others (1989) investigated the skeletal and dentoalveolar effects of the Bionator on Class II malocclusions and reported an average decrease in ANB of $1.9^{\circ}$. Lange (1995) compared the effects of the Bionator on Class II subjects to an untreated control group. With the Bionator, SNB increased $1.0^{\circ}$ while ANB was reduced $1.9^{\circ}$. Almeida et al. (2002) investigated the treatment effects of the Fränkel and Bionator appliances on Class II malocclusions and compared them to an untreated control group. The Bionator group exhibited an average increase in SNB of $1.4^{\circ}$ and an average reduction in ANB of $1.4^{\circ}$. NAP was reduced by $2.8^{\circ}$. Tulloch et al. (2004) observed a mean annualized reduction in ANB of $0.9^{\circ}$ for early Class II treatment with the Bionator. Jena et al. (2006) in a comparative study of the treatment effects of the Twin-block and Bionator appliances on Class II malocclusions reported an average reduction of $1.5^{\circ}$ in ANB for the individuals treated with the Bionator.

It has been argued that functional appliance therapy corrects skeletal disharmonies mainly through an improvement in mandibular position in relation to the cranial base instead of the restriction of maxillary growth. Indeed, in the current study, the reduction in ANB was achieved mainly during the Bionator phase due to an increase in SNB $\left(1.7^{\circ}\right)$. There was a subsequent decrease in SNA $\left(1.2^{\circ}\right)$ during fixed appliance treatment. ANB improved 
(decreased) significantly more in phase $1\left(\bar{x}=-1.9^{\circ}\right)$ than in phase $2\left(\bar{x}=-1.1^{\circ}\right)$. Janson (1987) reported similar findings with a group of 35 individuals treated with Bionator therapy compared to 99 individuals treated with fixed appliances. The Bionator group achieved a reduction in ANB mainly through an increase in SNB. The fixed appliance group achieved ANB correction mainly through a reduction in SNA. Tulloch et al. (2004) also found an initial reduction in ANB $\left(-0.9^{\circ}\right)$ after phase 1 mainly through an increase in SNB $\left(1.1^{\circ}\right)$ in their Bionator sample.

We also measured $\mathrm{A}$ and $\mathrm{B}$ points relative to Nasion-Perpendicular. A Point changed almost not at all during Bionator treatment, but B Point grew forward an average of $2.7 \mathrm{~mm}$ during the first phase of treatment. These findings are similar to Tulloch and others (2004) who reported a change of 0.1 $\mathrm{mm}$ for A Point relative to Nasion-Perpendicular and $1.1 \mathrm{~mm}$ for Pogonion relative to Nasion-Perpendicular after an initial phase of Bionator treatment.

\section{Patterns of Dentoalveolar Change}

Most of the 10 dental variables changed significantly more during the Bionator treatment even though one would suppose that there is greater control over tooth movement with fixed appliances. Overjet improved an average of $3.1 \mathrm{~mm}$ during the course of treatment, but $3.0 \mathrm{~mm}$ or $97 \%$ of this occurred during the initial phase of treatment. Overbite changed from $3.7 \mathrm{~mm}$ 
to $1.7 \mathrm{~mm}$ with $95 \%$ of the change occurring again during Bionator treatment. Bolmgren (1986), Lange (1995), Illing (1998), and Tulloch et al. (2004) all showed a similar pattern of overbite and overjet reduction after an initial phase of Bionator therapy in Class II malocclusions. It should again be noted that in the current study, most of the overjet and overbite correction was achieved at an early age during the first phase of treatment. It should not be overlooked that for children with unaesthetic incisor relationships, these early corrections should be seen as beneficial.

Numerous studies have shown that functional appliances produce excessive labial tipping of the lower incisors as well as an uprighting of the upper incisors (Illing 1998; Almeida et al. 2002, 2004). The upper incisor was proclined from a mean of $23^{\circ}$ to $27^{\circ}$ during treatment (U1 to Nasion-A Point angle) in the present study. However, there was no difference in the amount of change between the two phases. IMPA changed very little $\left(c a .1^{\circ}\right)$ during the initial phase of treatment. These findings agree with those reported by Bolmgren et al. (1986) and Lange (1995) who found virtually no change in lower incisor position after Bionator treatment. However, Illing (1998) and Almeida et al. (2002) measured an average of 2 to $4^{\circ}$ of labial tipping of the lower incisors after an initial phase of Bionator treatment. As previously mentioned, in the present study the lower incisors were not significantly proclined during the 
first phase of treatment, and were uprighted roughly $4^{\circ}$ during the Edgewise phase.

\section{End of Treatment}

The most informative aspect of this study is the comparison between the cephalometric results of the two treated methods at the end of treatment.

One strong motivation to "bother" with an early functional phase of treatment is to improve the Class II, convex facial profile using some sort of hyperpropulsion device. The present study was not designed to address the question of whether the Bionator increased mandibular length over that expected from growth alone (Meikle 2007). Instead, we simply evaluated the bony facial profile at the end of treatment in the two groups. We focus here on five measures of the bony profile, namely (1) NAP angle, (2) SNA angle, (3) SNB angle, (4) ANB angle, and (5) the AOBO discrepancy. We matched the 50 two-and one-phase cases on the first four of these, and include AOBO here for completeness. Figures 72 through 76 are plots of the group means for these variables at the start and end of treatment, and the figure legends list the results of paired t-tests.

Facial convexity (the NAP angle) is significantly smaller in the twophase sample (Figure 72). Neither SNA nor SNB is statistically different between the samples at the end of treatment, but there was a significantly 


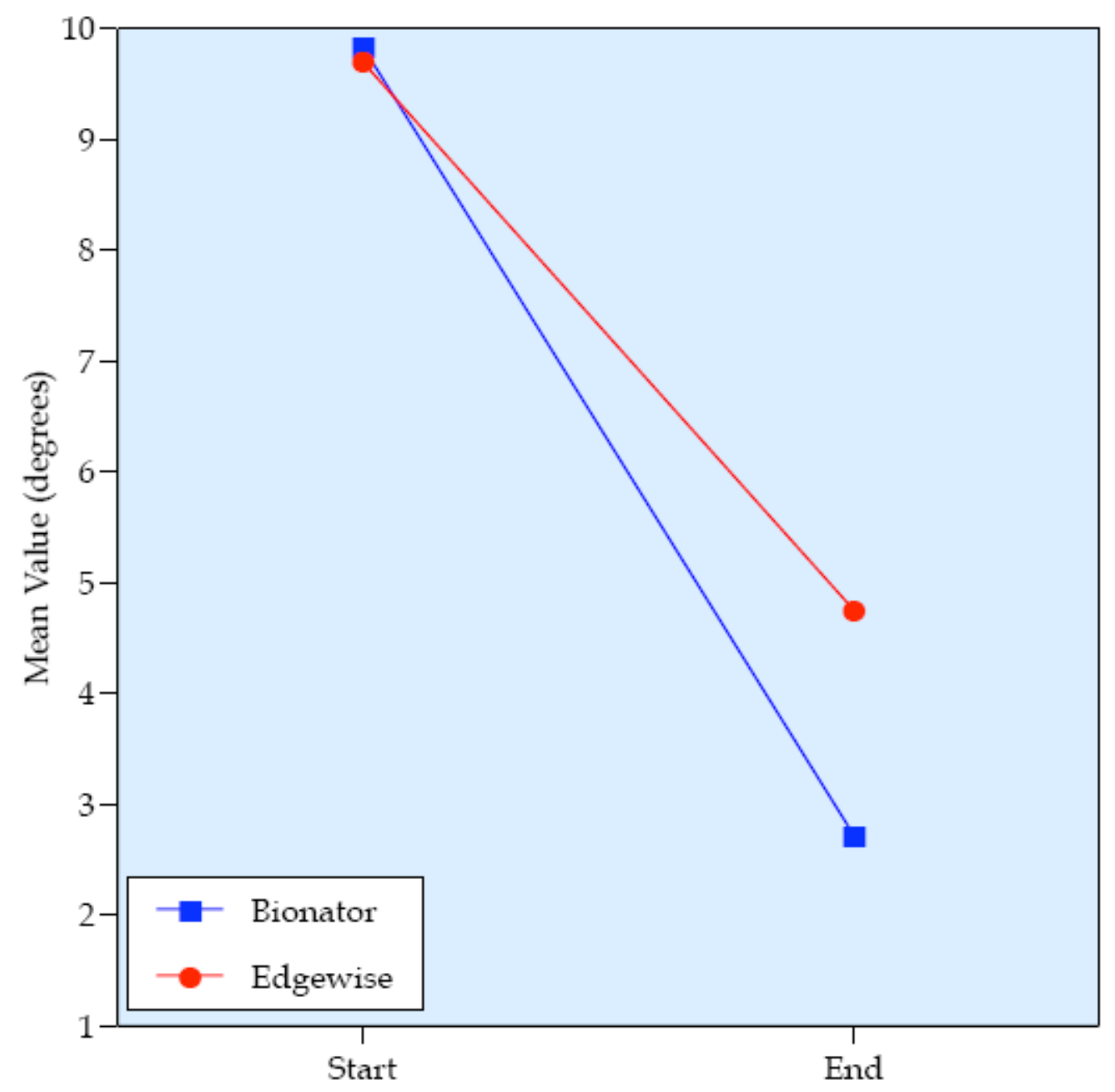

Figure 72. Plot of mean NAP angle at the start and end of treatment in the two groups (sexes pooled). By paired t-test, there was no difference between the two groups at the start of treatment $(\mathrm{t}=0.25 ; \mathrm{P}=0.8012)$, but the difference was significant at the end of treatment $(\mathrm{t}=2.46 ; \mathrm{P}=0.0173)$.

Mean Value (degrees) 


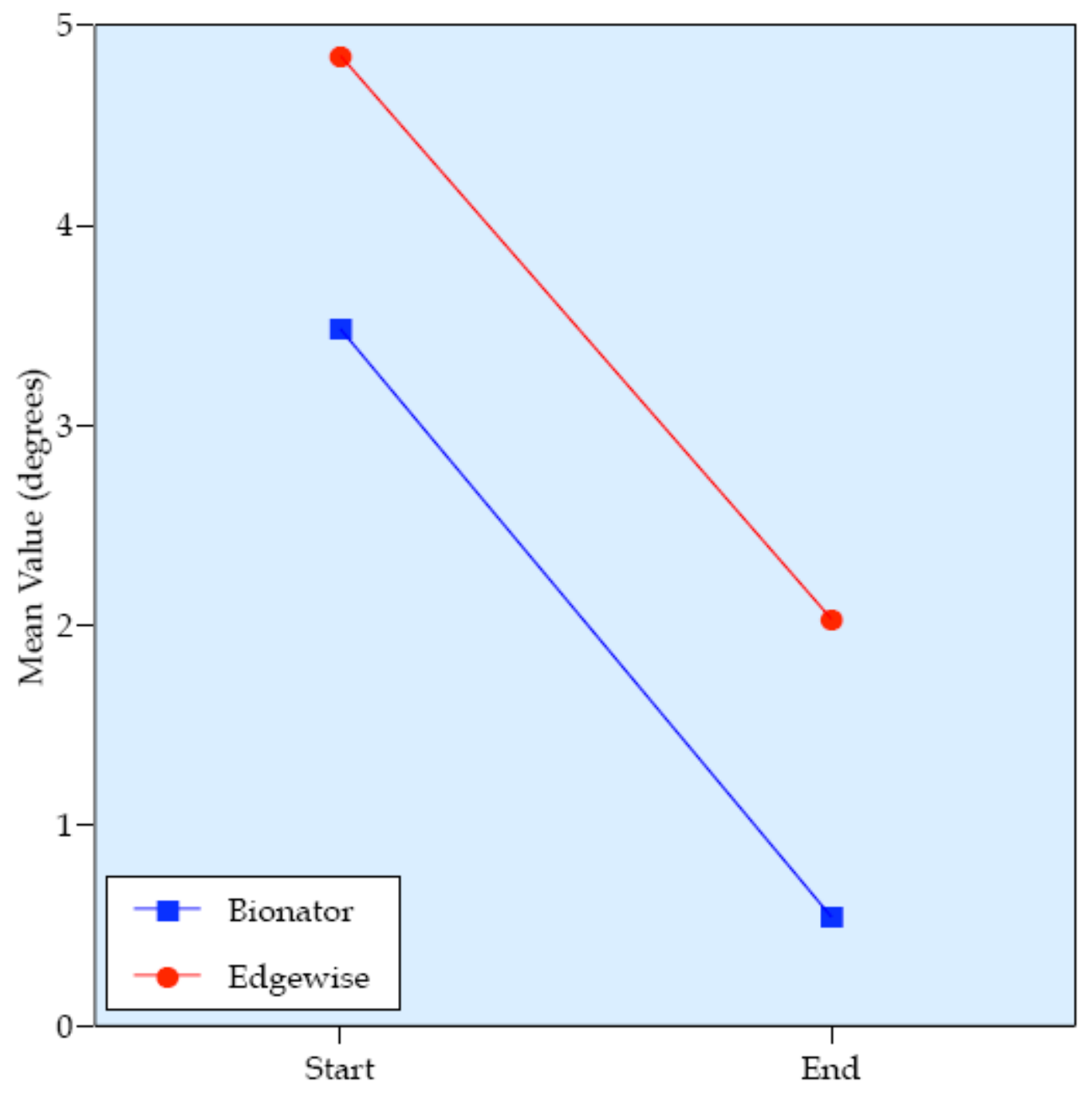

Figure 73. Plot of mean AOBO discrepancy at the start and end of treatment in the two groups (sexes pooled). By paired $t$-test, there was a significant difference between the two groups at the start of treatment $(t=3.35 ; \mathrm{P}=0.0016)$ and at the end of treatment $(t=3.46 ; P=0.0011)$. The samples were not matched on this variable. 


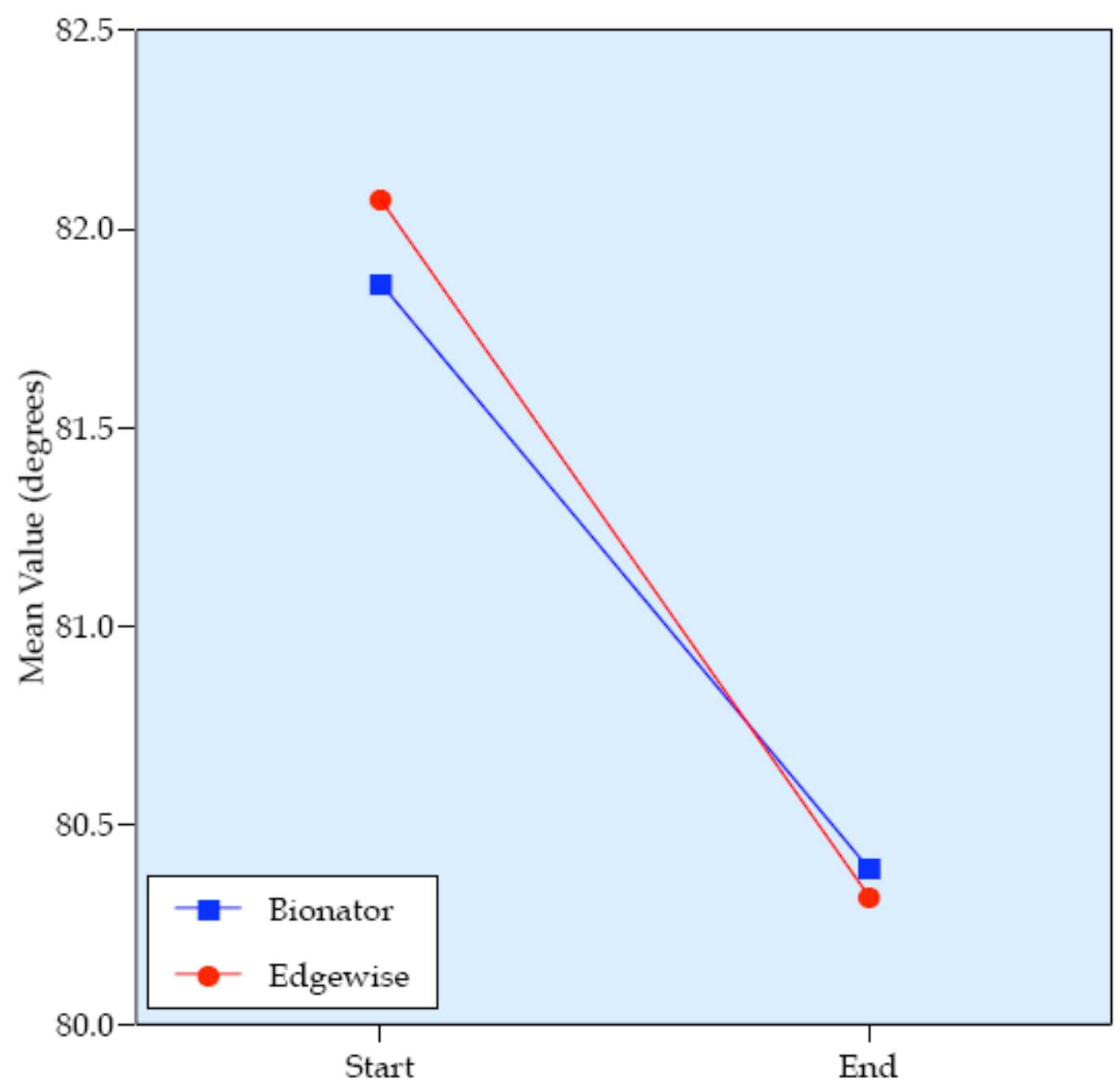

Figure 74. Plot of mean SNA angle at the start and end of treatment in the two groups (sexes pooled). By paired t-test, there was no difference between the two groups at the start of treatment $(\mathrm{t}=0.33 ; \mathrm{P}=0.7454)$ nor at the end of treatment $(t=0.09 ; P=0.9265)$. 


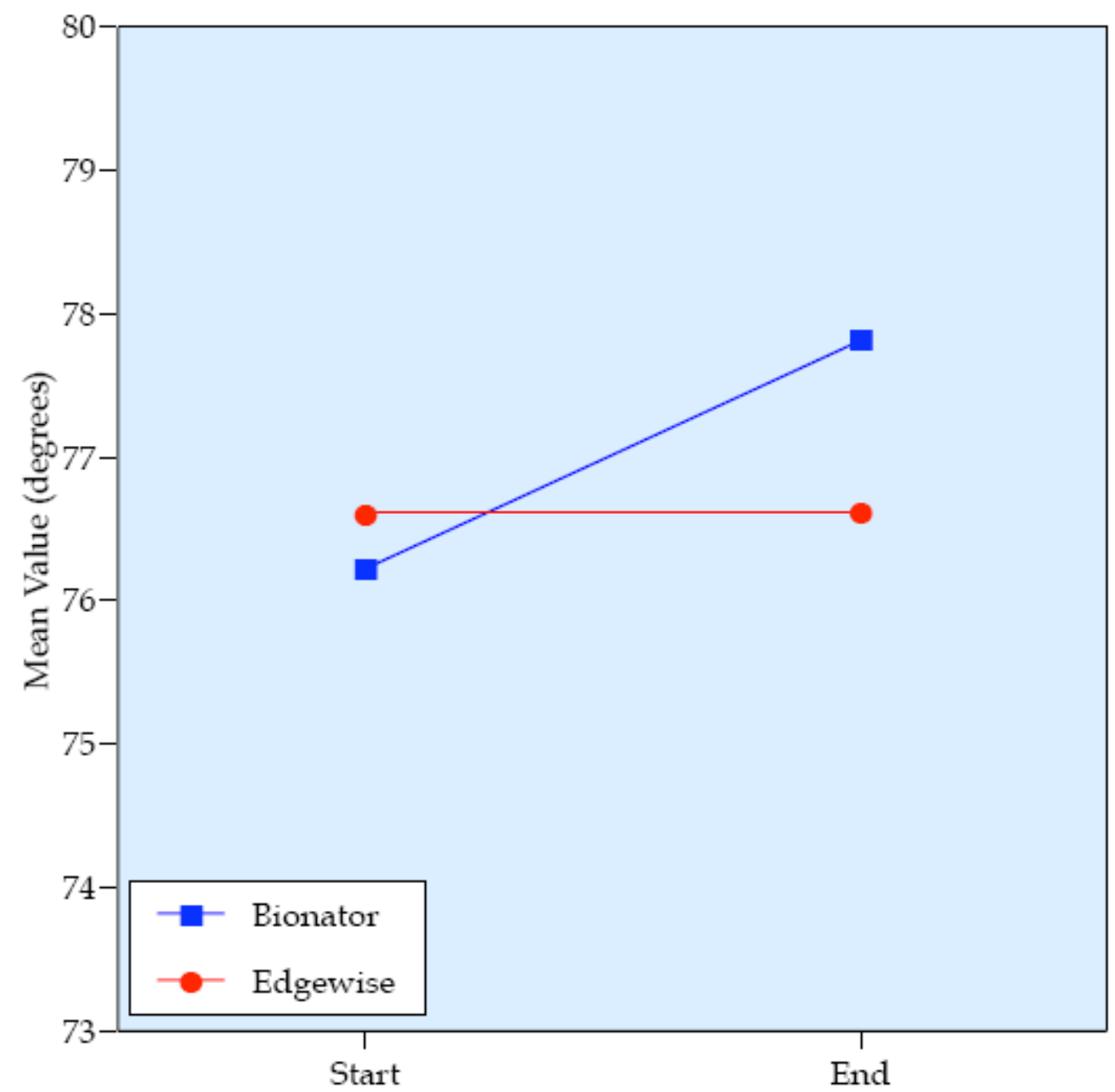

Figure 75. Plot of mean SNB angle at the start and end of treatment in the two groups (sexes pooled). By paired t-test, there was no difference between the two groups at the start of treatment $(\mathrm{t}=0.58 ; \mathrm{P}=0.5652)$ nor at the end of treatment $(\mathrm{t}=1.63 ; \mathrm{P}=0.1088)$. 


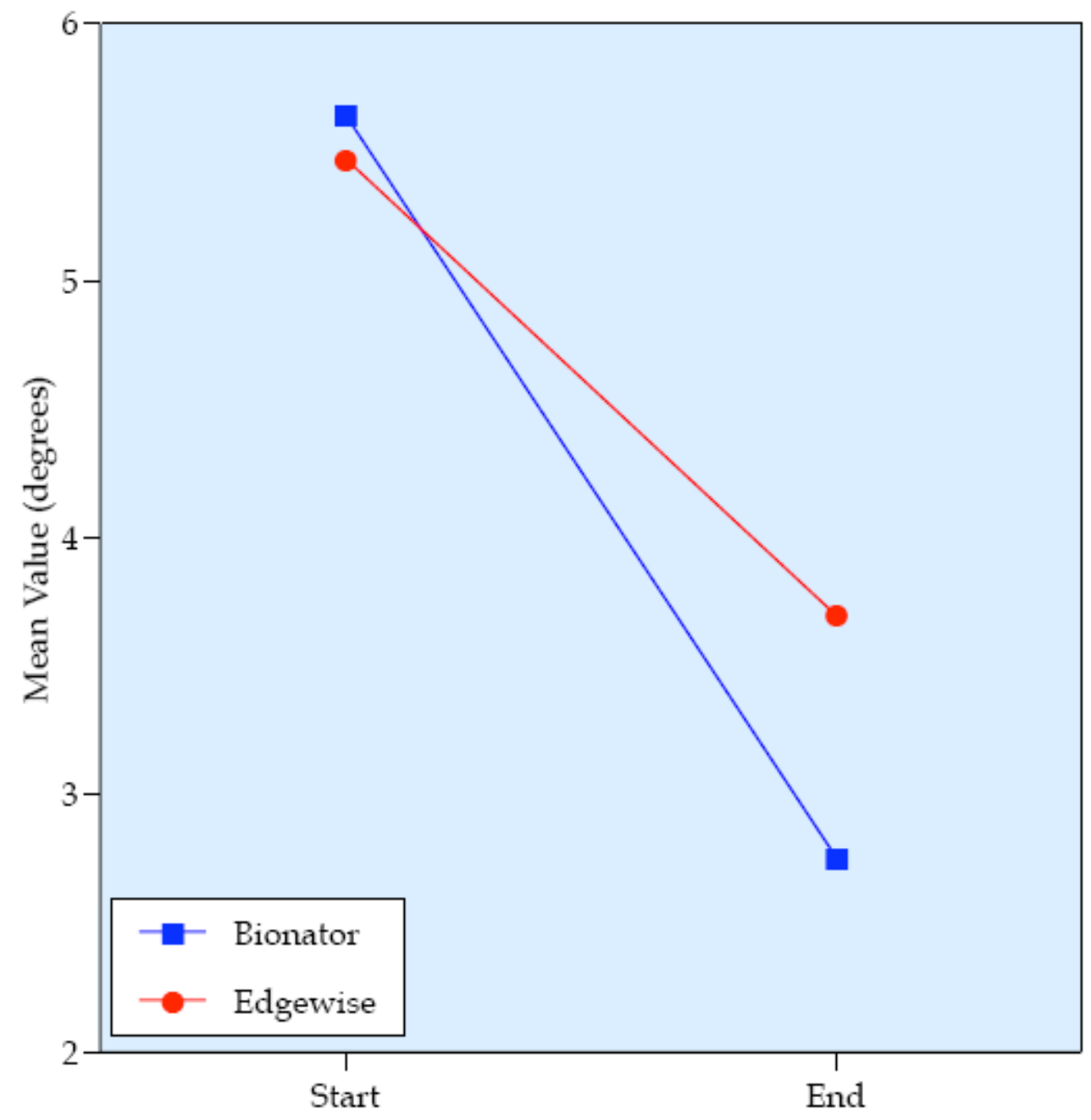

Figure 76. Plot of mean ANB angle at the start and end of treatment in the two groups (sexes pooled). By paired t-test, there was no difference between the two groups at the start of treatment $(t=1.13 ; P=0.2601)$, but the difference was highly significant at the end of treatment $(t=3.73 ; P=0.0005)$. 
greater improvement (reduction) of the ANB angle in the two-phase sample (Figure 75). The AOBO discrepancy improved (was reduced) in both samples, but to equivalent degrees (Figure 76).

We do not know yet whether these improvements in the facial profile are perceptible upon viewing the patients, but these cephalometric values suggest that the Bionator phase of treatment produced a statistically significant improvement at the end of treatment compared to Edgewise treatment alone.

We cannot ascertain from this study how the greater improvement in NAP and ANB were achieved. That is, it is clear that the change was due to a mesial movement of the mandible (B Point) rather than retraction of the maxilla (A Point), because SNA was reduced about $1.5^{\circ}$ in both groups $(\mathrm{P}=0.4896)$. We cannot specify how the forward movement of B Point occurred. It could have resulted from (A) mandibular growth, (B) forward remodeling of the glenoid fossa, (C) counterclockwise rotation of the temporal bone (hard to conceive but speculated in the literature), (D) restriction of growth in the spheno-occipital synchondrosis in the Bionator group, or (E) some undefined combination of these several effects. Statistical analysis shows that, whatever the mechanism, significantly more of the change in SNB, the facial angle (NaPg to FH), and B Point relative to Nasion-Perpendicular occurred during the Bionator phase of treatment. 
It might be argued that improvement in the facial profile is just postural, that the Bionator simply caused the subjects to "bite forward." This is most unlikely because (1) the improvement occurred primarily in phase $1,(2)$ the Bionator was removed before phase 2, and (3) the subjects had an average of 2.2 years (27 months) of Edgewise treatment during which relapse could have occurred. Instead of any relapse, ANB and the facial angle continued to improve (decrease) during the Edgewise phase of treatment. Inspection suggests (Figure 50) that, in fact, B Point came forward a little bit more during the Edgewise phase, and that most of the continued improvement was accomplished by a reduction (restraint) of A Point, which is characteristic of fixed appliance therapy. The net result, however, is that the 2.2 years of fixed appliance treatment afforded ample opportunity for any mandibular relapse to occur, and none was seen.

\section{$\underline{\text { Dentition and Integument }}$}

Of the dental variables measured, upper incisor position differed between the two groups at the end of treatment. The interincisal angle was slightly more acute in the two-phase group and the upper incisor was more proclined by an average of $5^{\circ}$ (U1 to Nasion-A Point angle). The explanation for this difference is operator preference. The orthodontist who treated the two-phase cases prefers to finish them with this type of incisor angulation for 
improved anterior guidance and posterior disclusion in anterior excursive movements. Interestingly, the interincisal angle and U1 to Nasion-A Point angle for the two-phase sample are virtually the same when compared to the values reported by Riolo et al. (1974) for normal untreated children-moreso than for the Edgewise sample.

Both treatments achieved the same positions of the mandibular central incisor in regards to the Nasion-B Point line and IMPA. The two integumental variables (E Plane, $\mathrm{Z}$ angle) achieved the same end points as well.

What can be concluded is that both groups achieved similar dental measurements (with the exception of upper incisor position). However, the two-phase group exhibited a more orthognathic bony facial profile at the end of treatment as shown by the changes in key skeletal variables (NAP, ANB, and AOBO). 


\section{CHAPTER VI}

\section{SUMMARY AND CONCLUSIONS}

There has been considerable discussion in the orthodontic literature regarding the biological and clinical advantages and disadvantages of "early" orthodontic intervention, specifically for Class II malocclusions. Of primary concern is the benefit, or lack thereof, gained with regard to treatment outcomes when using a two-phase treatment protocol compared to one phase of treatment. The present study compared two groups of patients, one treated with the Bionator appliance in the mixed dentition followed by full appliance treatment. The second group was treated in a single phase with conventional Edgewise mechanics. The question was whether the cephalometric results at the end of treatment were comparable.

This study was a retrospective analysis of cephalograms from patients with Class II, division 1 malocclusions at the start of treatment. These were 50 consecutively treated youths who received phase 1 (early) treatment with a Bionator appliance and later treatment with full appliances (all treated by a single clinician). The control group consisted of a sample of subjects with similar Class II, division 1 malocclusions who were treated in a single phase with fixed Edgewise appliances alone. Importantly, the subjects in the two samples were matched, on a one-to-one basis, for demographic and 
cephalometric variables to ensure comparability in the nature and severity of the malocclusions. Major findings are:

- Both groups achieved roughly the same endpoints with regard to the dentition and the soft tissues (e.g., E Plane). The upper incisors, however, were slightly more proclined in the two-phase group, but this was intentional due to operator preference.

- The two-phase group achieved a better bony facial profile compared to the control group. NAP (angle of convexity) averaged $2.7^{\circ}$ in the two-phase group compared to a significantly greater mean of $4.8^{\circ}$ in the one-phase group. Comparably, ANB was significantly smaller $\left(\bar{x}=2.6^{\circ}\right)$ in the twophase group than in the one-phase group $\left(\bar{x}=3.7^{\circ}\right)$.

- The majority of the skeletal and dental correction in the Bionator group was achieved during the first phase of treatment. Almost all of the correction in the lower facial profile (i.e., increase in SNB, decrease in ANB, decrease in $\mathrm{AOBO}$, increase in facial angle, and forward growth of $\mathrm{B}$ Point) occurred during the Bionator phase of treatment. Dentally, most of the overall correction also was achieved prior to treatment with fixed appliances. This made the fixed-phase of treatment easier, and it may also have enhanced these adolescents' self-perceptions of their dental esthetics at a substantially earlier age. 
- The frequency of extractions was $12 \%$ (6/50 cases) in the two-phase group compared to $84 \%(42 / 50)$ in the one-phase group. The long-term advantages of an intact dentition - if any - are unknown, but parents often have the perception that extractions are "bad" and unwarranted.

- The skeletal discrepancy was resolved in the two-phase group mainly through the advancement of B Point. Indeed, the reduction in ANB for the two phase group was achieved mainly during the Bionator phase with an increase in SNB $\left(1.7^{\circ}\right)$. Conversely, the reduction in ANB in the one-phase group was achieved through the restriction of maxillary growth.

- No relapse was evident after the first phase of treatment. The Bionator was removed before phase 2 , so subjects had an average of 2.2 years ( 27 months) of Edgewise treatment during which relapse could have occurred, but none was seen. This argues for the long-term stability of the skeletal improvement in facial harmony. 


\section{LIST OF REFERENCES}


Ahn SJ, Kim JT, Nahm DS. Cephalometric markers to consider in the treatment of Class II Division I malocclusions with the Bionator. Am J Orthod Dentofacial Orthop 2001;119:578-86.

Almeida MR, Henriques JF, Ursi W. Comparative study of the Fränkel (FR-2) and bionator appliances in the treatment of Class II malocclusion. Am J Orthod Dentofacial Orthop 2002;121:458-66.

Almeida MR, Henriques JF, Almeida RR, Almeida-Pedrin RR, Ursi W. treatment effects produced by the bionator appliance: comparison with an untreated Class II sample. Eur J Orthod 2004;26:65-72.

Araujo AM, Buschang PH, Melo AC. Adaptive condylar growth and mandibular remodelling changes with bionator therapy - an implant study. Eur J Orthod 2004;26:515-22.

Arya BS, Savara BS, Thomas DR. Prediction of first molar occlusion. Am J Orthod 1973;63:610-21.

Baccetti T, Franchi L, McNamara JA Jr, Tollaro I. Early dentofacial features of Class II malocclusion: a longitudinal study from the deciduous through the mixed dentition. Am J Orthod Dentofacial Orthop 1997;111:502-9.

Behrents RG. An atlas of growth in the aging craniofacial skeleton, Monograph number 18. Craniofacial Growth Series. Ann Arbor: Center for Human Growth and Development, The University of Michigan, 1985. 
Berg R. Post-retention analysis of treatment problems and failures in 264 consecutively treated cases. Eur J Orthod 1979;1:55-68.

Birkeland K, Boe OE, Wisth PJ. Relationship between occlusion and satisfaction with dental appearance in orthodontically treated and untreated groups: a longitudinal study. Eur J Orthod 2000;22:509-18.

Bishara SE, Hoppens BJ, Jakobsen JR, Kohout FJ. Changes in the molar relationship between the deciduous and permanent dentitions: a longitudinal study. Am J Orthod Dentofacial Orthop 1988;93:19-28.

Bishara SE. Mandibular changes in persons with untreated and treated Class II Division 1 malocclusions. Am J Orthod Dentofacial Orthop 1988;113:661-73.

Bishara SE, Justus R, Graber TM. Proceedings of the workshop discussions on early treatment. Am J Orthod Dentofacial Orthop 1998;113:5-6.

Björk A. The principle of the Andresen method of orthodontic treatment: a discussion based upon cephalometric X-ray analysis of treated cases. Am J Orthod 1951;37:437-58.

Bolmgren GA, Moshiri F. Bionator treatment in Class II, division 1. angle Orthod 1986;56:255-62.

Bremen J, Pancherz H. Efficiency of early and late Class II division 1 treatment. Am J Orthod Dentofacial Orthop 2002;121:31-7.

Broadbent BH. The face of the normal child. angle Orthod 1937;7:183-208. 
Brook PH, Shaw WC. The development of an index of orthodontic treatment priority. Eur J Orthod 1989;11:309-20.

Chadwick SM, Aird JC, Taylor PJ. Functional regulator treatment of Class II division 1 malocclusions. Eur J Orthod 2001;23:495-505. Am J Orthod 1969;55:71-74.

Charlier JP, Petrovic A, Harrmann-Stutzmann J. Effects of mandibular hyperpropulsion on the prechondroblastic zone of young rat condyle. Am J Orthod 1969;55:71-74.

Dann C IV, Phillips C, Broder H, Tulloch C. Self-concept, Class II malocclusion, and early treatment. angle Orthod 1995;65:411-16.

Deicke M, Pancherz P. Is radius-union an indicator for completed facial growth? angle Orthod 2005;75:287-91.

Dolce C, Schader RE, McGorray SP, Wheeler TT. Centrographic analysis of 1phase versus 2-phase treatment for Class II malocclusion. Am J Orthod Dentofacial Orthop 2005;128:195-200.

Dugoni SA. Comprehensive mixed dentition treatment. Am J Orthod Dentofacial Orthop 1998;113:75-84.

Dugoni SA, Aubert M, Baumrind S. Differential diagnosis and treatment planning for early mixed dentition malocclusions. Am J Orthod Dentofacial Orthop 2006;129:S80-1.

Durkin JF. Secondary cartilage: a misnomer? Am J Orthod 1972;62:15-41. 
Eirew HL. The Bionator. Br J Orthod 1981;8:33-36

Elsasser WA, Wylie WL. The craniofacial morphology of mandibular retrusion. J Anthrop 1943;6:461-73.

Enlow DH. The human force: an account of the postnatal growth and development of the craniofacial skeleton. New York: Harper and Row, 1968.

Faltin K, Faltin RM, Baccetti T, Franchi L, Ghiozzi B, McNamara JA. Long-term effectiveness and treatment timing for Bionator therapy. angle Orthod 2003;73:221-30.

Fröhlic FJ. A longitudinal study of untreated Class II type malocclusions. Trans Eur Orthod Soc 1961;37:137-59.

Ghafari J, Shofer FS, Hunt J, Markowitz DL, Laster LL. Headgear versus function regulator in the early treatment of Class II, Division 1 malocclusion: a randomized clinical trial. Am J Orthod Dentofacial Orthop 1998;113:51-61.

Gianelly AA. One-phase versus two-phase treatment. Am J Orthod Dentofacial Orthop 1995;108.

Graber TM. Functional appliances. In: Graber TM, Vanarsdall RL, Vig KWL, editors. Orthodontics: current principles and techniques. St. Louis: CV Mosby; 2005. p. 493-542. 
Graber TM, Neumann B. Removable orthodontic appliances. Philadelphia:

WB Saunders; 1984. p. 85-90.

Harris EF. Effects of patient age and sex on treatment: correction of Class II malocclusion with the Begg technique. angle Orthod 2001 Dec;71:43341.

Heij DGO, Callaert H, Opdebeeck HM. The effect of the amount of protrusion built into the Bionator on condylar growth and displacement: A clinical study. Orthod Dentofac Orthop 1989;95:401-9.

Henry RG. A classification of Class II, division 1 malocclusions. angle Orthod 1957;27:83-92.

Hunter WS. The vertical dimensions of the face and skeletodental retrognathism. Am J Orthod Dentofacial Orthop 1967;53:586-95.

Illing HM, Morris DO, Lee RT. A prospective evaluation of Bass, Bionator and Twin Block appliances. Part I. The hard tissues. Eur J Orthod 1998;20:501-16.

Jacobson A. The 'Wits' appraisal of jaw disharmony. Am J Orthod 1975;67:12538.

Jacobson A. Application of the 'Wits' appraisal. Am J Orthod 1976;70:179-89.

Jacobson A. Update on the Wits appraisal. angle Orthod 1988;58:205-19.

Janson I. A cephalometric study of the efficiency of the Bionator. Trans Eur Orthod Soc 1977;53:283-93. 
Janson I. Skeletal and dentoalveolar changes in patients treated with a Bionator during puberty and prepuberty. In: McNamara JA Jr et al., editors. The Clinical Alterations of the Growing Face. Ann Arbor: Center for Human Growth and Development, The University of Michigan; 1983:131-54.

Jena AK, Duggal R, Parkash H. Skeletal and dentoalveolar effects of Twinblock and Bionator appliances in the treatment of Class II malocclusion: a comparative study. Am J Orthod Dentofacial Orthop 2006;130:594-602.

Karaiskos N, Wiltshire WA, Odlum O, Brothwell D, Hassard TH. Preventive and interceptive orthodontic treatment needs of an inner-city group of 6and 9-year-old Canadian children. J Can Dent Assoc 2005;71:649.

Katsavrias EG. The effect of mandibular protrusive (Activator) appliances on articular eminence morphology. angle Orthod 2003;73:647-53.

Keeling SD, King GJ, Wheeler TT, McGorry S. Timing of Class II treatment: rationale, methods, and early results of an ongoing randomized clinical trail. In: Trotman C, McNamara JA Jr., editors. Orthodontic treatment: outcome and effectiveness, Monograph 30. Craniofacial Growth Series. Ann Arbor: Center for Human Growth and Development, The University of Michigan, 1995, p 1-112.

Kelly J, Harvey C. An assessment of the teeth of youth 12-17 years. United States. DHEW publication no. (HRA) 77-1644. Washington, DC: National Center for Health Statistics, 1977, p 1-18 
King GJ, Wheeler TT, McGorray SP, Aiosa LS, bloom RM, Taylor MG.

Orthodontists' perceptions of the impact of phase 1 treatment for Class II malocclusion on phase 2 needs. J Dent Res 1999;78:1745-53.

King GJ, McGorray SP, Wheeler TT, Dolce C, Taylor M. Comparison of peer assessment ratings (PAR) from 1-phase and 2-phase treatment for Class II malocclusions. Am J Orthod Dentofacial Orthop 2003;123:489-96.

Koski K. The role of the craniofacial cartilages in the postnatal growth of the craniofacial skeleton. In: Dahlberg AA, Graber TM, editors. Orofacial growth and development. Philadelphia: The Hague, 1977, p 9-34.

Lange DW, Kalra V, Broadbent BH, Powers M, Nelson S. Changes in soft tissue profile following treatment with the bionator. angle Orthod 1995;65:42330.

Livieratos FA, Johnston LE Jr. A comparison of one-stage and two-stage nonextraction alternatives in matched Class II samples. Am J Orthod Dentofacial Orthop 1995;108:118-31.

Mamandras AH, Allen LP. Mandibular response to orthodontic treatment with the Bionator appliance. Am J Orthod Dentofacial Orthop 1990;97:113-20.

Mandall NA, Wright J, Conboy F, Kay E, Harvey L, O’Brien KD. Index of orthodontic treatment need as a predictor of orthodontic treatment uptake. Am J Orthod Dentofacial Orthop 2005;128:703-7. 
McNamara JA Jr, Connelly TG, McBride MC. Histological studies of temporomandibular joint adaptations. In: McNamara JA Jr., editor. Control mechanisms in craniofacial growth, Monograph 3. Craniofacial Growth Series. Ann Arbor: Center for Human Growth and Development, The University of Michigan, 1975.

McNamara JA. Components of Class II malocclusion in children 8-10 years of age. angle Orthod 1981;51:177-202.

McNamara JA. Long-term mandibular adaptations to protrusive function: an experimental study in Macaca mulatta. Am J Orthod Dentofacial Orthop $1987 ; 92: 98-108$

McNamara JA Jr, Brudon WL. Orthodontic and orthopedic treatment in the mixed dentition. Ann Arbor: Needham Press 1993;3-6.

Meikle MC. Remodeling the dentofacial skeleton: the biological basis of orthodontics and dentofacial orthopedics. J Dent Res 2007;86:12-24.

Mills JR. The effect of functional appliances on the skeletal pattern. J Orthod 1991;18:267-8.

Morris DO, Illing HM, Lee RT. A prospective evaluation of Bass, Bionator and Twin Block appliances. Part II-The soft tissues. Eur J Orthod 1998;20:663-84. 
Moyers RE, Riolo ML, Guire KE, Wainright RL, Bookstein FL. Differential diagnosis of class II malocclusions. Part 1. Facial types associated with class II malocclusions. Am J Orthod 1980;78:477-94.

Moyers RE. Handbook of orthodontics, 4th ed. Chicago: Year Book Medical Publishers, 1988, p 346-7, p 433-4.

Nelson C, Harkness M, Herbisson P. Mandibular changes during functional appliance treatment. Am J Orthod Dentofacial Orthop 1993;104:153-61.

Ngan PW, Byczek E, Scheick J. Longitudinal evaluation of growth changes in Class II division 1 subjects. Semin Orthod 1997;3:222-31.

O’Brien K, McComb JL, Fox N, Wright J. Factors influencing the uptake of orthodontic treatment. Br J Orthod 1996 23:331-4.

O'Brien K, Wright J, Conboy F. Effectiveness of treatment for Class II malocclusion with the Herbst or Twin-block appliances: a randomized, controlled trial. Am J Orthod Dentofacial Orthop 2003;124:128-37.

O'Brien K, Wright J, Conboy F. Effectiveness of early orthodontic treatment with the Twin-block appliance: a multicenter, randomized, controlled trial. Part 2: Psychosocial effects. Am J Orthod Dentofacial Orthop 2003;124:488-95.

O'Reilly MT, Yaniniello GJ. Mandibular growth changes and maturation of cervical vertebrae - a longitudinal cephalometric study. angle Orthod 1988;58:179-84. 
Oudet C, Petrovic A, Stutzmann J. Time-dependent effects of a 'functional'type orthopedic appliance on the rat mandible growth. Chronobiol Int $1984 ; 1: 51-7$.

Petrovic A, Gasson N, Oudet C. Growth Hormone, postural hyperpropulsion and condylar prechondroblast proliferation in rats. J Dent Res 1974:53;745-6.

Petrovic AG. Control processes in the postnatal growth of the condylar cartilage of the mandible. In: Johnston LE Jr., editor. Control mechanisms in craniofacial growth. Craniofacial Growth Series. Ann Arbor: Center for Human Growth and Development, The University of Michigan, 1975:101-54.

Petrovic AG, Stutzmann JJ, Gasson N: The final length of the mandible: is it genetically predetermined? Is the functional maxipropulsion involving periodic forward repositioning the best procedure to elicit over lengthening? In: Carlson DS, editor. Craniofacial biology, Monograph 10. Craniofacial Growth Series. Ann Arbor: Center for Human Growth and Development, The University of Michigan, 1981.

Proffit WR, Fields HW, Moray LJ. Prevalence of malocclusion and orthodontic treatment need in the United States: estimates from the NHANES III survey. Int J Adult Orthognath Surg 1998;13:97-106. 
Rabie AB, She TT, Hägg U. Functional appliance therapy accelerates and enhances condylar growth. Am J Orthod Dentofacial Orthop 2003;123:40-8.

Renfroe EW. A study of the facial patterns associated with Class I, Class II, and division 1, and Class II, division 2 malocclusions. angle Orthod $1948 ; 19: 12-5$.

Riedel RA. The relation of maxillary structures to the cranium in malocclusion and normal occlusion. angle Orthod 1952;22:142-5.

Riedel RA. A review of the retention problem. angle Orthod 1960;30:179-99.

Riolo ML, Moyers RE, McNamara JA Jr, Hunter WS. An atlas of craniofacial growth: cephalometric standards from the University School Growth Study, the University of Michigan. Monograph 2, Craniofacial Growth Series. Ann Arbor: Center for Human Growth and Development, University of Michigan; 1974.

Rodrigues de Almeida M, Henriques JFC, Ursi W. Comparative study of the Frankel (FR-2) and bionator appliances in the treatment of Class II malocclusion. Am J Orthod Dentofacial Orthop 2002;121:458-66.

Ruf S, Pancherz H. Temporomandibular joint remodeling in adolescents and young adults during Herbst treatment: A prospective longitudinal magnetic resonance imaging and cephalometric radiographic investigation. Am J Orthod Dentofacial Orthop 1999;115:607-18. 
Sampson W, Fazzalari N, Wilson D, Wiebkin O. Induced mandibular condylar growth in a sheep model after functional appliance treatment. Aust Orthod J 2001;17:81-8.

Sandusky WC, Gramling JF. The Edgewise technique. In: Hardin JF, ed. Clark's clinical dentistry, chapter 28. Philadelphia: JB Lippincott; 1988, vol 2, p. 1-20.

Schopf P. Indication for and frequency of early orthodontic therapy or interceptive measures. J Orofac Orthop 2003;64:186-200.

Shaw WC, Addy M, Ray C. Dental and social effects of malocclusion and effectiveness of orthodontic treatment: a review. Community Dent Oral Epidemiol 1980;8:36-45.

Shaw WC. Factors influencing the desire for orthodontic treatment. Eur J Orthod 1981;3:151-62.

Sokal RR, Rohlf FJ. Biometry: the principles and practice of statistics in biological research, 3rd ed. San Francisco: WH Freeman and Company; 1995.

Stockli PW, Willert HG. Tissue reactions in the temporomandibular joint resulting from anterior displacement of the mandible in the monkey. Am J Orthod 1973;60:142-154.

Tanner JM. Growth at adolescence, 2nd ed. Oxford: Blackwell Scientific Publications, 1962. 
Tukey JW. Exploratory data analysis. Massachusetts: Addison-Wesley, Reading; 1977.

Tulloch JF, Proffit WR, Phillips C. Outcomes in a 2-phase randomized clinical trial of early Class II treatment. Am J Orthod Dentofacial Orthop 2004;125:657-67.

van der Linden FPGM, Duterloo HS. Development of the human dentition: an atlas. Hagerstown, MD: Harper and Row; 1976.

White RC. The role of mandibular growth in occlusal development. Unpublished M.S. Thesis. St Louis University, St. Louis, Missouri, 1983. Whitney DJ. A longitudinal morphometric analysis and classification of the Class II individual. Unpublished M.S. Thesis. Case Western Reserve University, Cleveland, Ohio, 1983.

Winer BJ, Brown DR, Michels KM. Statistical principles in experimental design, 3rd ed. New York: McGraw-Hill Book Company; 1991.

Woolf CM. Principles of biometry. Princeton, NJ: D Van Nostrand Company Inc; 1968.

Zhao Z, Hägg U, Rabie AB, Shen G. Quantitative evaluation of new bone formation in the posterior zone of the glenoid fossa in response to the mandibular advancement in rats. West China J Stomatology 1999;17:152-4. 
APPENDIX A

DESCRIPTIVE STATISTICS 
Table A-1. Descriptive statistics for the two-phase sample evaluated at the start of treatment.

\begin{tabular}{lrrrrrr}
\hline \multicolumn{1}{c}{ Variable } & $\overline{\mathrm{x}}$ & $\mathrm{sd}$ & $\mathrm{sem}$ & $\mathrm{L} 2$ & $\mathrm{~L} 1$ & $\mathrm{n}$ \\
\hline & & & & & & \\
Z angle & 66.39 & 10.20 & 1.44 & 69.28 & 63.49 & 50 \\
E Plane & -0.85 & 2.82 & 0.40 & -0.04 & -1.65 & 50 \\
Y-Axis angle & 66.90 & 3.43 & 0.49 & 67.87 & 65.92 & 50 \\
NAP angle & 9.84 & 4.09 & 0.58 & 11.00 & 8.67 & 50 \\
SNA angle & 81.86 & 3.23 & 0.46 & 82.78 & 80.95 & 50 \\
SNB angle & 76.23 & 3.30 & 0.47 & 77.16 & 75.29 & 50 \\
ANB angle & 5.64 & 1.55 & 0.22 & 6.08 & 5.20 & 50 \\
AOBO & 3.48 & 1.93 & 0.27 & 4.03 & 2.94 & 50 \\
FMA angle & 26.02 & 5.75 & 0.81 & 27.65 & 24.39 & 50 \\
IMPA & 94.46 & 6.60 & 0.93 & 96.33 & 92.58 & 50 \\
FMIA & 59.52 & 8.08 & 1.14 & 61.82 & 57.23 & 50 \\
OP to FH angle & 9.22 & 4.62 & 0.65 & 10.53 & 7.90 & 50 \\
U1 to SeNa angle & 104.61 & 8.73 & 1.23 & 107.09 & 102.13 & 50 \\
Interincisal angle & 127.76 & 13.08 & 1.85 & 131.48 & 124.05 & 50 \\
U1 to Na A Pt angle & 22.75 & 8.20 & 1.16 & 25.08 & 20.42 & 50 \\
U1 to Na A Pt mm & 3.01 & 3.17 & 0.45 & 3.91 & 2.10 & 50 \\
L1 to A Pg mm & 0.32 & 2.65 & 0.37 & 1.07 & -0.43 & 50 \\
L1 to A Pg angle & 19.65 & 6.12 & 0.87 & 21.39 & 17.91 & 50 \\
L1 to Na B Pt angle & 23.84 & 7.26 & 1.03 & 25.90 & 21.78 & 50 \\
L1 to Na B Pt mm & 4.19 & 2.39 & 0.34 & 4.87 & 3.51 & 50 \\
Facial angle & 84.37 & 4.15 & 0.59 & 85.54 & 83.19 & 50 \\
Mand Length & 102.01 & 5.97 & 0.84 & 103.71 & 100.31 & 50 \\
Sella-to-A Point & 82.47 & 4.45 & 0.63 & 83.74 & 81.21 & 50 \\
Sella-to-B Point & 101.37 & 5.48 & 0.78 & 102.93 & 99.81 & 50 \\
Overbite & 3.67 & 2.17 & 0.31 & 4.29 & 3.05 & 50 \\
Overjet & 6.43 & 2.03 & 0.29 & 7.01 & 5.85 & 50 \\
A Pt to Na-Perp & -0.92 & 3.64 & 0.51 & 0.12 & -1.95 & 50 \\
B Pt to Na-Perp & -10.54 & 6.41 & 0.91 & -8.72 & -12.36 & 50 \\
\hline
\end{tabular}


Table A-2. Descriptive statistics for the two-phase sample evaluated at the end of the Bionator phase of treatment.

\begin{tabular}{|c|c|c|c|c|c|c|}
\hline Variable & $\bar{x}$ & $\mathrm{sd}$ & sem & $\mathrm{L}_{2}$ & $\mathrm{~L}_{1}$ & $\mathrm{n}$ \\
\hline$Z$ angle & 71.31 & 8.94 & 1.28 & 73.88 & 68.74 & 49 \\
\hline E Plane & -2.48 & 2.33 & 0.33 & -1.81 & -3.15 & 49 \\
\hline Y-Axis angle & 66.90 & 3.43 & 0.49 & 67.89 & 65.92 & 49 \\
\hline NAP angle & 6.09 & 4.00 & 0.57 & 7.23 & 4.94 & 49 \\
\hline SNA angle & 81.54 & 3.02 & 0.43 & 82.40 & 80.67 & 49 \\
\hline SNB angle & 77.83 & 2.98 & 0.43 & 78.69 & 76.98 & 49 \\
\hline ANB angle & 3.70 & 1.49 & 0.21 & 4.13 & 3.27 & 49 \\
\hline AOBO & 0.92 & 2.10 & 0.30 & 1.53 & 0.32 & 49 \\
\hline FMA angle & 25.85 & 5.52 & 0.79 & 27.43 & 24.26 & 49 \\
\hline IMPA & 95.83 & 6.66 & 0.95 & 97.74 & 93.92 & 49 \\
\hline FMIA & 58.33 & 7.64 & 1.09 & 60.52 & 56.13 & 49 \\
\hline $\mathrm{OP}$ to $\mathrm{FH}$ angle & 8.32 & 4.05 & 0.58 & 9.48 & 7.15 & 49 \\
\hline U1 to SeNa angle & 104.70 & 6.08 & 0.87 & 106.45 & 102.95 & 49 \\
\hline Interincisal angle & 125.96 & 9.88 & 1.41 & 128.79 & 123.12 & 49 \\
\hline $\mathrm{U} 1$ to $\mathrm{Na} \mathrm{A} \mathrm{Pt} \mathrm{angle}$ & 23.16 & 5.16 & 0.74 & 24.64 & 21.68 & 49 \\
\hline $\mathrm{U} 1$ to $\mathrm{Na} \mathrm{A} \mathrm{Pt} \mathrm{mm}$ & 3.65 & 2.01 & 0.29 & 4.23 & 3.07 & 49 \\
\hline L1 to A Pg mm & 2.72 & 2.38 & 0.34 & 3.40 & 2.03 & 49 \\
\hline L1 to A Pg angle & 24.79 & 5.21 & 0.74 & 26.29 & 23.29 & 49 \\
\hline L1 to $\mathrm{Na} \mathrm{B} \mathrm{Pt} \mathrm{angle}$ & 27.18 & 6.62 & 0.95 & 29.08 & 25.27 & 49 \\
\hline L1 to $\mathrm{Na} \mathrm{B} \mathrm{Pt} \mathrm{mm}$ & 5.49 & 2.29 & 0.33 & 6.15 & 4.84 & 49 \\
\hline Facial angle & 86.31 & 3.80 & 0.54 & 87.40 & 85.22 & 49 \\
\hline Mand Length & 110.50 & 5.80 & 0.83 & 112.17 & 108.83 & 49 \\
\hline Sella-to-A Point & 85.65 & 4.04 & 0.58 & 86.81 & 84.49 & 49 \\
\hline Sella-to-B Point & 109.11 & 5.35 & 0.76 & 110.64 & 107.57 & 49 \\
\hline Overbite & 1.86 & 1.64 & 0.23 & 2.33 & 1.39 & 49 \\
\hline Overjet & 3.43 & 1.32 & 0.19 & 3.81 & 3.05 & 49 \\
\hline A Pt to Na-Perp & -0.82 & 3.34 & 0.48 & 0.14 & -1.78 & 49 \\
\hline B Pt to Na-Perp & -7.66 & 6.21 & 0.89 & -5.88 & -9.45 & 49 \\
\hline
\end{tabular}


Table A-3. Descriptive statistics for the two-phase sample evaluated at the end of the fixed phase of treatment.

\begin{tabular}{|c|c|c|c|c|c|c|}
\hline Variable & $\bar{x}$ & sd & sem & $\mathrm{L}_{2}$ & $\mathrm{~L}_{1}$ & $\mathrm{n}$ \\
\hline $\mathrm{Z}$ angle & 73.85 & 8.50 & 1.20 & 76.26 & 71.43 & 50 \\
\hline E Plane & -4.13 & 2.55 & 0.36 & -3.40 & -4.85 & 50 \\
\hline Y-Axis angle & 67.31 & 3.37 & 0.48 & 68.27 & 66.36 & 50 \\
\hline NAP angle & 2.72 & 4.28 & 0.60 & 3.94 & 1.50 & 50 \\
\hline SNA angle & 80.39 & 3.19 & 0.45 & 81.30 & 79.49 & 50 \\
\hline SNB angle & 77.82 & 3.16 & 0.45 & 78.71 & 76.92 & 50 \\
\hline ANB angle & 2.58 & 1.59 & 0.23 & 3.03 & 2.13 & 50 \\
\hline AOBO & 0.55 & 2.39 & 0.34 & 1.22 & -0.13 & 50 \\
\hline FMA angle & 25.39 & 5.64 & 0.80 & 27.00 & 23.79 & 50 \\
\hline IMPA & 94.62 & 6.47 & 0.92 & 96.46 & 92.78 & 50 \\
\hline FMIA & 59.99 & 7.22 & 1.02 & 62.04 & 57.94 & 50 \\
\hline $\mathrm{OP}$ to $\mathrm{FH}$ angle & 7.37 & 4.40 & 0.62 & 8.62 & 6.12 & 50 \\
\hline U1 to SeNa angle & 106.98 & 6.61 & 0.93 & 108.86 & 105.10 & 50 \\
\hline Interincisal angle & 125.31 & 9.82 & 1.39 & 128.10 & 122.52 & 50 \\
\hline U1 to $\mathrm{Na} \mathrm{A} \mathrm{Pt} \mathrm{angle}$ & 26.58 & 6.07 & 0.86 & 28.31 & 24.86 & 50 \\
\hline $\mathrm{U} 1$ to $\mathrm{Na} \mathrm{A} \mathrm{Pt} \mathrm{mm}$ & 4.40 & 2.29 & 0.32 & 5.05 & 3.75 & 50 \\
\hline L1 to A Pg mm & 2.27 & 2.34 & 0.33 & 2.93 & 1.60 & 50 \\
\hline L1 to A Pg angle & 25.37 & 5.01 & 0.71 & 26.79 & 23.95 & 50 \\
\hline L1 to Na B Pt angle & 25.52 & 5.81 & 0.82 & 27.17 & 23.87 & 50 \\
\hline L1 to $\mathrm{Na} \mathrm{B} \mathrm{Pt} \mathrm{mm}$ & 4.86 & 2.36 & 0.33 & 5.53 & 4.19 & 50 \\
\hline Facial angle & 86.78 & 3.97 & 0.56 & 87.91 & 85.65 & 50 \\
\hline Mand Length & 114.46 & 6.77 & 0.96 & 116.38 & 112.54 & 50 \\
\hline Sella-to-A Point & 87.08 & 4.52 & 0.64 & 88.37 & 85.80 & 50 \\
\hline Sella-to-B Point & 112.30 & 6.16 & 0.87 & 114.05 & 110.55 & 50 \\
\hline Overbite & 1.75 & 0.94 & 0.13 & 2.02 & 1.49 & 50 \\
\hline Overjet & 3.30 & 0.71 & 0.10 & 3.50 & 3.10 & 50 \\
\hline A Pt to Na-Perp & -2.01 & 3.99 & 0.56 & -0.87 & -3.14 & 50 \\
\hline B Pt to Na-Perp & -7.96 & 6.73 & 0.95 & -6.04 & -9.87 & 50 \\
\hline
\end{tabular}


Table A-4. Descriptive statistics for the changes during the Bionator (phase 1) of treatment.

\begin{tabular}{lrccrrrrr}
\hline \multicolumn{1}{c}{ Variable } & $\overline{\mathrm{x}}$ & $\mathrm{sd}$ & $\mathrm{sem}$ & \multicolumn{1}{c}{$\mathrm{L}_{2}$} & \multicolumn{1}{c}{$\mathrm{L}_{1}$} & $\mathrm{n}$ & $\mathrm{t}$-test & P-Value \\
\hline Z angle & 4.98 & 5.50 & 0.79 & 6.56 & 3.40 & 49 & 6.34 & $<0.0001$ \\
E Plane & -1.71 & 2.12 & 0.30 & -1.10 & -2.32 & 49 & -5.66 & $<0.0001$ \\
Y-Axis angle & -0.05 & 1.18 & 0.17 & 0.28 & -0.39 & 49 & -0.32 & 0.7535 \\
NAP angle & -3.72 & 2.45 & 0.35 & -3.01 & -4.42 & 49 & -10.62 & $<0.0001$ \\
SNA angle & -0.24 & 1.36 & 0.19 & 0.15 & -0.63 & 49 & -1.24 & 0.2219 \\
SNB angle & 1.69 & 1.40 & 0.20 & 2.09 & 1.28 & 49 & 8.40 & $<0.0001$ \\
ANB angle & -1.93 & 1.23 & 0.18 & -1.58 & -2.29 & 49 & -11.04 & $<0.0001$ \\
AOBO & -2.53 & 2.44 & 0.35 & -1.83 & -3.23 & 49 & -7.27 & $<0.0001$ \\
FMA angle & -0.18 & 1.46 & 0.21 & 0.25 & -0.60 & 49 & -0.84 & 0.4056 \\
IMPA & 1.39 & 3.79 & 0.54 & 2.48 & 0.30 & 49 & 2.57 & 0.0133 \\
FMIA & -1.21 & 3.84 & 0.55 & -0.11 & -2.31 & 49 & -2.21 & 0.0323 \\
OP to FH angle & -0.83 & 2.44 & 0.35 & -0.14 & -1.53 & 49 & -2.40 & 0.0204 \\
U1 to SeNa angle & -0.05 & 6.20 & 0.89 & 1.73 & -1.83 & 49 & -0.05 & 0.9579 \\
Interincisal angle & -1.52 & 8.09 & 1.16 & 0.80 & -3.85 & 49 & -1.32 & 0.1936 \\
U1 to Na A Pt angle & 0.18 & 6.12 & 0.87 & 1.94 & -1.58 & 49 & 0.21 & 0.8382 \\
U1 to Na A Pt mm & 0.57 & 2.06 & 0.29 & 1.16 & -0.02 & 49 & 1.94 & 0.0577 \\
L1 to A Pg mm & 2.32 & 1.56 & 0.22 & 2.77 & 1.87 & 49 & 10.43 & $<0.0001$ \\
L1 to A Pg angle & 5.05 & 4.10 & 0.59 & 6.23 & 3.88 & 49 & 8.63 & $<0.0001$ \\
L1 to Na B Pt angle & 3.27 & 3.79 & 0.54 & 4.36 & 2.18 & 49 & 6.05 & $<0.0001$ \\
L1 to Na B Pt mm & 1.25 & 1.38 & 0.20 & 1.65 & 0.86 & 49 & 6.35 & $<0.0001$ \\
Facial angle & 1.87 & 1.40 & 0.20 & 2.27 & 1.47 & 49 & 9.36 & $<0.0001$ \\
Mand Length & 8.68 & 3.60 & 0.51 & 9.71 & 7.64 & 49 & 16.89 & $<0.0001$ \\
Sella-to-A Point & 3.37 & 1.82 & 0.26 & 3.89 & 2.84 & 49 & 12.95 & $<0.0001$ \\
Sella-to-B Point & 7.92 & 2.79 & 0.40 & 8.72 & 7.12 & 49 & 19.86 & $<0.0001$ \\
Overbite & -1.75 & 1.98 & 0.28 & -1.18 & -2.32 & 49 & -6.19 & $<0.0001$ \\
Overjet & -3.00 & 2.30 & 0.33 & -2.34 & -3.66 & 49 & -9.12 & $<0.0001$ \\
A Pt to Na-Perp & 0.03 & 1.26 & 0.18 & 0.39 & -0.33 & 49 & 0.16 & 0.8747 \\
B Pt to Na-Perp & 2.74 & 2.15 & 0.31 & 3.35 & 2.12 & 49 & 8.94 & $<0.0001$ \\
\hline
\end{tabular}


Table A-5. Descriptive statistics for the changes during the second, fixed (Edgewise) phase of treatment.

\begin{tabular}{lrrrrrrrr}
\hline \multicolumn{1}{c}{ Variable } & $\overline{\mathrm{x}}$ & $\mathrm{sd}$ & $\mathrm{sem}$ & $\mathrm{L}_{2}$ & $\mathrm{~L} 1$ & $\mathrm{n}$ & t-test & P-Value \\
\hline Z angle & & & & & & & & \\
E Plane & -1.61 & 4.39 & 0.63 & 3.87 & 1.34 & 49 & 4.15 & 0.0001 \\
Y-Axis angle & 0.51 & 1.70 & 0.24 & -1.17 & -2.15 & 49 & -6.84 & $<0.0001$ \\
NAP angle & -3.29 & 2.87 & 0.41 & -2.47 & -4.12 & 49 & -8.03 & $<0.0001$ \\
SNA angle & -1.20 & 1.81 & 0.26 & -0.68 & -1.72 & 49 & -4.63 & $<0.0001$ \\
SNB angle & -0.11 & 1.76 & 0.25 & 0.40 & -0.61 & 49 & -0.43 & 0.6698 \\
ANB angle & -1.09 & 1.25 & 0.18 & -0.73 & -1.45 & 49 & -6.09 & $<0.0001$ \\
AOBO & -0.35 & 2.40 & 0.34 & 0.34 & -1.04 & 49 & -1.02 & 0.3135 \\
FMA angle & -0.43 & 1.88 & 0.27 & 0.11 & -0.97 & 49 & -1.61 & 0.1150 \\
IMPA & -1.31 & 5.26 & 0.75 & 0.20 & -2.82 & 49 & -1.74 & 0.0882 \\
FMIA & 1.73 & 5.22 & 0.75 & 3.23 & 0.23 & 49 & 2.31 & 0.0250 \\
OP to FH angle & -1.01 & 2.92 & 0.42 & -0.17 & -1.85 & 49 & -2.42 & 0.0194 \\
U1 to SeNa angle & 2.00 & 6.04 & 0.86 & 3.73 & 0.27 & 49 & 2.32 & 0.0247 \\
Interincisal angle & -0.45 & 8.58 & 1.23 & 2.01 & -2.92 & 49 & -0.37 & 0.7145 \\
U1 to Na A Pt angle & 3.20 & 6.20 & 0.89 & 4.98 & 1.42 & 49 & 3.61 & 0.0007 \\
U1 to Na A Pt mm & 0.74 & 2.28 & 0.33 & 1.40 & 0.09 & 49 & 2.28 & 0.0269 \\
L1 to A Pg mm & -0.43 & 1.97 & 0.28 & 0.14 & -0.99 & 49 & -1.52 & 0.1347 \\
L1 to A Pg angle & 0.53 & 4.99 & 0.71 & 1.97 & -0.90 & 49 & 0.75 & 0.4567 \\
L1 to Na B Pt angle & -1.66 & 4.85 & 0.69 & -0.27 & -3.06 & 49 & -2.40 & 0.0203 \\
L1 to Na B Pt mm & -0.60 & 1.77 & 0.25 & -0.09 & -1.10 & 49 & -2.36 & 0.0225 \\
Facial angle & 0.53 & 1.78 & 0.25 & 1.04 & 0.02 & 49 & 2.09 & 0.0421 \\
Mand Length & 3.84 & 4.11 & 0.59 & 5.02 & 2.66 & 49 & 6.54 & $<0.0001$ \\
Sella-to-A Point & 1.29 & 2.43 & 0.35 & 1.99 & 0.59 & 49 & 3.71 & 0.0005 \\
Sella-to-B Point & 3.07 & 3.51 & 0.50 & 4.08 & 2.07 & 49 & 6.13 & $<0.0001$ \\
Overbite & -0.10 & 1.69 & 0.24 & 0.38 & -0.58 & 49 & -0.41 & 0.6802 \\
Overjet & -0.12 & 1.53 & 0.22 & 0.32 & -0.56 & 49 & -0.54 & 0.5910 \\
A Pt to Na-Perp & -1.08 & 1.88 & 0.27 & -0.54 & -1.62 & 49 & -4.03 & 0.0002 \\
B Pt to Na-Perp & -0.19 & 3.11 & 0.44 & 0.71 & -1.08 & 49 & -0.42 & 0.6774 \\
\hline
\end{tabular}


Table A-6. Descriptive statistics for the changes during treatment in the Edgewise (one phase) sample.

\begin{tabular}{lrrrrrrrr}
\hline \multicolumn{1}{c}{ Variable } & $\overline{\mathrm{x}}$ & $\mathrm{sd}$ & $\mathrm{sem}$ & \multicolumn{1}{c}{$\mathrm{L}_{2}$} & $\mathrm{~L}_{1}$ & $\mathrm{n}$ & $\mathrm{t}$-test & P-Value \\
\hline Z angle & & & & & & & & \\
E Plane & -3.46 & 6.48 & 0.92 & 9.30 & 5.62 & 50 & 8.14 & $<0.0001$ \\
Y-Axis angle & 2.42 & 0.34 & -2.59 & -3.97 & 50 & -9.57 & $<0.0001$ \\
NAP angle & 0.42 & 1.72 & 0.24 & 0.91 & -0.07 & 50 & 1.71 & 0.0929 \\
SNA angle & -7.12 & 3.32 & 0.47 & -6.17 & -8.06 & 50 & -15.14 & $<0.0001$ \\
SNB angle & -1.47 & 2.10 & 0.30 & -0.88 & -2.07 & 50 & -4.97 & $<0.0001$ \\
ANB angle & 1.59 & 1.68 & 0.24 & 2.07 & 1.11 & 50 & 6.68 & $<0.0001$ \\
AOBO & -3.07 & 1.49 & 0.21 & -2.64 & -3.49 & 50 & -14.58 & $<0.0001$ \\
FMA angle & -2.94 & 2.89 & 0.41 & -2.12 & -3.76 & 50 & -7.18 & $<0.0001$ \\
IMPA & -0.63 & 2.18 & 0.31 & -0.01 & -1.24 & 50 & -2.03 & 0.0474 \\
FMIA & 0.16 & 6.04 & 0.85 & 1.88 & -1.56 & 50 & 0.19 & 0.8521 \\
OP to FH angle & 0.46 & 5.96 & 0.84 & 2.16 & -1.23 & 50 & 0.55 & 0.5860 \\
U1 to SeNa angle & -1.85 & 3.23 & 0.46 & -0.93 & -2.77 & 50 & -4.04 & 0.0002 \\
Interincisal angle & 2.37 & 9.73 & 1.38 & 5.14 & -0.39 & 50 & 1.73 & 0.0907 \\
U1 to Na A Pt angle & -2.46 & 13.00 & 1.84 & 1.24 & -6.15 & 50 & -1.34 & 0.1877 \\
U1 to Na A Pt mm & 1.39 & 3.78 & 1.38 & 6.61 & 1.05 & 50 & 2.77 & 0.0079 \\
L1 to A Pg mm & 1.95 & 2.27 & 0.47 & 2.34 & 0.44 & 50 & 2.95 & 0.0048 \\
L1 to A Pg angle & 5.72 & 5.94 & 0.84 & 7.41 & 4.04 & 50 & 6.82 & $<0.0001$ \\
to Na B Pt angle & 1.68 & 5.62 & 0.79 & 3.28 & 0.08 & 50 & 2.11 & 0.0399 \\
L1 to Na B Pt mm & 0.67 & 2.06 & 0.29 & 1.26 & 0.09 & 50 & 2.31 & 0.0253 \\
Facial angle & 2.41 & 1.54 & 0.22 & 2.85 & 1.97 & 50 & 11.07 & $<0.0001$ \\
Mand Length & 12.45 & 3.80 & 0.54 & 13.53 & 11.37 & 50 & 23.17 & $<0.0001$ \\
Sella-to-A Point & 4.61 & 2.73 & 0.39 & 5.39 & 3.83 & 50 & 11.93 & $<0.0001$ \\
Sella-to-B Point & 10.93 & 3.65 & 0.52 & 11.97 & 9.89 & 50 & 21.16 & $<0.0001$ \\
Overbite & -1.92 & 2.02 & 0.29 & -1.34 & -2.49 & 50 & -6.71 & $<0.0001$ \\
Overjet & -3.13 & 1.98 & 0.28 & -2.57 & -3.69 & 50 & -11.17 & $<0.0001$ \\
A Pt to Na-Perp & -1.09 & 1.97 & 0.28 & -0.53 & -1.65 & 50 & -3.91 & 0.0003 \\
B Pt to Na-Perp & 2.58 & 2.73 & 0.39 & 3.36 & 1.81 & 50 & 6.68 & $<0.0001$ \\
\hline
\end{tabular}


Table A-7. Descriptive statistics for the Edgewise (one phase) sample at the start of treatment.

\begin{tabular}{lrrrrrr}
\hline \multicolumn{1}{c}{ Variable } & $\overline{\mathrm{x}}$ & $\mathrm{sd}$ & $\mathrm{sem}$ & $\mathrm{L} 2$ & $\mathrm{~L} 1$ & $\mathrm{n}$ \\
\hline Z angle & & & & & & \\
E Plane & 67.39 & 8.17 & 1.16 & 69.71 & 65.07 & 50 \\
Y-Axis angle & -0.87 & 2.78 & 0.39 & -0.08 & -1.66 & 50 \\
NAP angle & 68.41 & 3.71 & 0.52 & 69.47 & 67.36 & 50 \\
SNA angle & 9.70 & 4.61 & 0.65 & 11.01 & 8.39 & 50 \\
SNB angle & 82.08 & 3.72 & 0.53 & 83.13 & 81.02 & 50 \\
ANB angle & 76.61 & 3.31 & 0.47 & 77.55 & 75.67 & 50 \\
AOBO & 5.47 & 1.62 & 0.23 & 5.93 & 5.01 & 50 \\
FMA angle & 4.85 & 2.34 & 0.33 & 5.51 & 4.18 & 50 \\
IMPA & 26.71 & 6.49 & 0.92 & 28.56 & 24.87 & 50 \\
FMIA & 94.99 & 7.02 & 0.99 & 96.99 & 93.00 & 50 \\
OP to FH angle & 58.29 & 6.19 & 0.87 & 60.04 & 56.53 & 50 \\
U1 to SeNa angle & 5.56 & 4.70 & 0.66 & 6.89 & 4.22 & 50 \\
Interincisal angle & 106.23 & 8.69 & 1.23 & 108.70 & 103.76 & 50 \\
U1 to Na A Pt angle & 123.29 & 10.77 & 1.52 & 126.35 & 120.23 & 50 \\
U1 to Na A Pt mm & 24.14 & 8.19 & 1.16 & 26.47 & 21.81 & 50 \\
L1 to A Pg mm & 1.33 & 3.28 & 0.46 & 5.26 & 3.40 & 50 \\
L1 to A Pg angle & 22.86 & 2.81 & 0.40 & 2.30 & 0.70 & 50 \\
L1 to Na B Pt angle & 27.09 & 6.13 & 0.73 & 24.32 & 21.40 & 50 \\
L1 to Na B Pt mm & 5.45 & 2.68 & 0.85 & 28.81 & 25.38 & 50 \\
Facial angle & 86.14 & 3.92 & 0.38 & 6.21 & 4.69 & 50 \\
Mand Length & 113.02 & 6.14 & 0.87 & 87.25 & 85.02 & 50 \\
Sella-to-A Point & 87.83 & 4.42 & 0.63 & 89.09 & 86.57 & 50 \\
Sella-to-B Point & 109.59 & 6.10 & 0.86 & 111.32 & 107.86 & 50 \\
Overbite & 2.99 & 2.61 & 0.37 & 3.73 & 2.25 & 50 \\
Overjet & 6.94 & 2.53 & 0.36 & 7.66 & 6.22 & 50 \\
A Pt to Na-Perp & 1.18 & 3.86 & 0.55 & 2.28 & 0.08 & 50 \\
B Pt to Na-Perp & -7.60 & 6.46 & 0.91 & -5.77 & -9.44 & 50 \\
\hline
\end{tabular}


Table A-8. Descriptive statistics for the Edgewise (one phase) sample at the end of treatment.

\begin{tabular}{lrllrrl}
\hline \multicolumn{1}{c}{ Variable } & $\overline{\mathrm{x}}$ & $\mathrm{sd}$ & $\mathrm{sem}$ & $\mathrm{L}_{2}$ & $\mathrm{~L} 1$ & $\mathrm{n}$ \\
\hline Z angle & & & & & & \\
E Plane & 73.41 & 8.90 & 1.26 & 75.94 & 70.88 & 50 \\
Y-Axis angle & -3.67 & 3.10 & 0.44 & -2.79 & -4.55 & 50 \\
NAP angle & 68.68 & 4.31 & 0.61 & 69.91 & 67.46 & 50 \\
SNA angle & 4.75 & 5.12 & 0.72 & 6.20 & 3.30 & 50 \\
SNB angle & 80.32 & 4.01 & 0.57 & 81.46 & 79.18 & 50 \\
ANB angle & 76.61 & 3.85 & 0.54 & 77.71 & 75.52 & 50 \\
AOBO & 3.70 & 1.87 & 0.26 & 4.23 & 3.17 & 50 \\
FMA angle & 2.03 & 2.47 & 0.35 & 2.74 & 1.33 & 50 \\
IMPA & 26.35 & 6.19 & 0.88 & 28.11 & 24.59 & 50 \\
FMIA & 94.04 & 6.55 & 0.93 & 95.90 & 92.18 & 50 \\
OP to FH angle & 59.61 & 7.63 & 1.08 & 61.78 & 57.44 & 50 \\
U1 to SeNa angle & 6.88 & 4.97 & 0.70 & 8.29 & 5.47 & 50 \\
Interincisal angle & 101.72 & 7.73 & 1.09 & 103.92 & 99.52 & 50 \\
U1 to Na A Pt angle & 128.85 & 8.65 & 1.22 & 131.31 & 126.40 & 50 \\
U1 to Na A Pt mm & 21.40 & 7.00 & 0.99 & 23.39 & 19.41 & 50 \\
L1 to A Pg mm & 1.12 & 2.26 & 0.32 & 3.20 & 1.92 & 50 \\
L1 to A Pg angle & 24.99 & 4.98 & 0.38 & 1.88 & 0.35 & 50 \\
L1 to Na B Pt angle & 26.04 & 6.52 & 0.70 & 26.40 & 23.57 & 50 \\
L1 to Na B Pt mm & 4.61 & 2.85 & 0.42 & 27.89 & 24.19 & 50 \\
Facial angle & 87.06 & 3.92 & 0.55 & 88.42 & 3.80 & 50 \\
Mand Length & 118.80 & 7.38 & 1.04 & 120.90 & 116.70 & 50 \\
Sella-to-A Point & 89.05 & 5.30 & 0.75 & 90.56 & 87.54 & 50 \\
Sella-to-B Point & 113.28 & 6.53 & 0.92 & 115.14 & 111.43 & 50 \\
Overbite & 1.17 & 1.24 & 0.18 & 1.52 & 0.82 & 50 \\
Overjet & 3.50 & 1.35 & 0.19 & 3.88 & 3.11 & 50 \\
A Pt to Na-Perp & -0.60 & 4.05 & 0.57 & 0.55 & -1.76 & 50 \\
B Pt to Na-Perp & -7.79 & 6.65 & 0.94 & -5.90 & -9.68 & 50 \\
\hline
\end{tabular}


Table A-9. Descriptive statistics for the changes during treatment in the Edgewise (one phase) sample.

\begin{tabular}{lrrrrrrrr}
\hline \multicolumn{1}{c}{ Variable } & $\overline{\mathrm{x}}$ & $\mathrm{sd}$ & $\mathrm{sem}$ & $\mathrm{L}_{2}$ & $\mathrm{~L} 1$ & $\mathrm{n}$ & t-test & P-Value \\
\hline Z angle & & & & & & & & \\
E Plane & -2.01 & 7.41 & 1.05 & 8.12 & 3.91 & 50 & 5.74 & $<0.0001$ \\
Y-Axis angle & 2.44 & 0.34 & -2.10 & -3.48 & 50 & -8.10 & $<0.0001$ \\
NAP angle & 0.27 & 1.72 & 0.24 & 0.76 & -0.22 & 50 & 1.11 & 0.2716 \\
SNA angle & -4.95 & 4.11 & 0.58 & -3.78 & -6.12 & 50 & -8.51 & $<0.0001$ \\
SNB angle & -1.75 & 2.19 & 0.31 & -1.13 & -2.38 & 50 & -5.65 & $<0.0001$ \\
ANB angle & 0.00 & 1.83 & 0.26 & 0.52 & -0.52 & 50 & 0.01 & 0.9939 \\
AOBO & -1.78 & 1.60 & 0.23 & -1.32 & -2.23 & 50 & -7.87 & $<0.0001$ \\
FMA angle & -2.81 & 3.29 & 0.47 & -1.88 & -3.75 & 50 & -6.05 & $<0.0001$ \\
IMPA & -0.36 & 2.38 & 0.34 & 0.31 & -1.04 & 50 & -1.07 & 0.2877 \\
FMIA & -0.95 & 7.04 & 1.00 & 1.05 & -2.95 & 50 & -0.95 & 0.3448 \\
OP to FH angle & 1.32 & 6.21 & 0.88 & 3.09 & -0.44 & 50 & 1.51 & 0.1385 \\
U1 to SeNa angle & 1.32 & 3.95 & 0.56 & 2.44 & 0.20 & 50 & 2.37 & 0.0219 \\
Interincisal angle & -4.51 & 10.02 & 1.42 & -1.66 & -7.36 & 50 & -3.18 & 0.0025 \\
U1 to Na A Pt angle & -2.74 & 12.72 & 1.80 & 9.18 & 1.95 & 50 & 3.09 & 0.0033 \\
U1 to Na A Pt mm & -1.77 & 3.15 & 1.35 & -0.03 & -5.46 & 50 & -2.03 & 0.0474 \\
L1 to A Pg mm & -0.39 & 2.42 & 0.34 & -0.88 & -2.67 & 50 & -3.99 & 0.0002 \\
L1 to A Pg angle & 2.13 & 6.67 & 0.94 & 4.02 & -1.08 & 50 & -1.13 & 0.2625 \\
L1 to Na B Pt angle & -1.05 & 6.28 & 0.89 & 0.73 & -2.84 & 50 & 2.26 & 0.0285 \\
L1 to Na B Pt mm & -0.84 & 2.54 & 0.36 & -0.12 & -1.56 & 50 & -1.19 & 0.2410 \\
Facial angle & 0.92 & 2.01 & 0.28 & 1.49 & 0.35 & 50 & 3.23 & 0.0238 \\
Mand Length & 5.78 & 6.14 & 0.87 & 7.52 & 4.03 & 50 & 6.65 & $<0.00001$ \\
Sella-to-A Point & 1.22 & 4.40 & 0.62 & 2.47 & -0.03 & 50 & 1.96 & 0.0562 \\
Sella-to-B Point & 3.69 & 5.54 & 0.78 & 5.26 & 2.12 & 50 & 4.71 & $<0.0001$ \\
Overbite & -1.82 & 2.42 & 0.34 & -1.13 & -2.51 & 50 & -5.32 & $<0.0001$ \\
Overjet & -3.44 & 2.76 & 0.39 & -2.66 & -4.23 & 50 & -8.82 & $<0.0001$ \\
A Pt to Na-Perp & -1.78 & 2.44 & 0.34 & -1.09 & -2.48 & 50 & -5.17 & $<0.0001$ \\
B Pt to Na-Perp & -0.19 & 3.49 & 0.49 & 0.80 & -1.18 & 50 & -0.38 & 0.7048 \\
\hline
\end{tabular}




\section{APPENDIX B}

\section{CEPHALOMETRIC TRACINGS}

Prints of the cephalometric tracings (superimpositions) of the subjects used in the present study. For the Bionator-treated group, the black lines are at pretreatment, the green lines are at the beginning of fixed appliances, and the red lines are at the end of treatment. For the Edgewise cases, the black lines are at the start of treatment, and the red lines are at the end of treatment. Each superimposition is followed by its matched counterpart superimposition. All tracings are printed at true size (i.e., 1:1 with the original radiograph). 


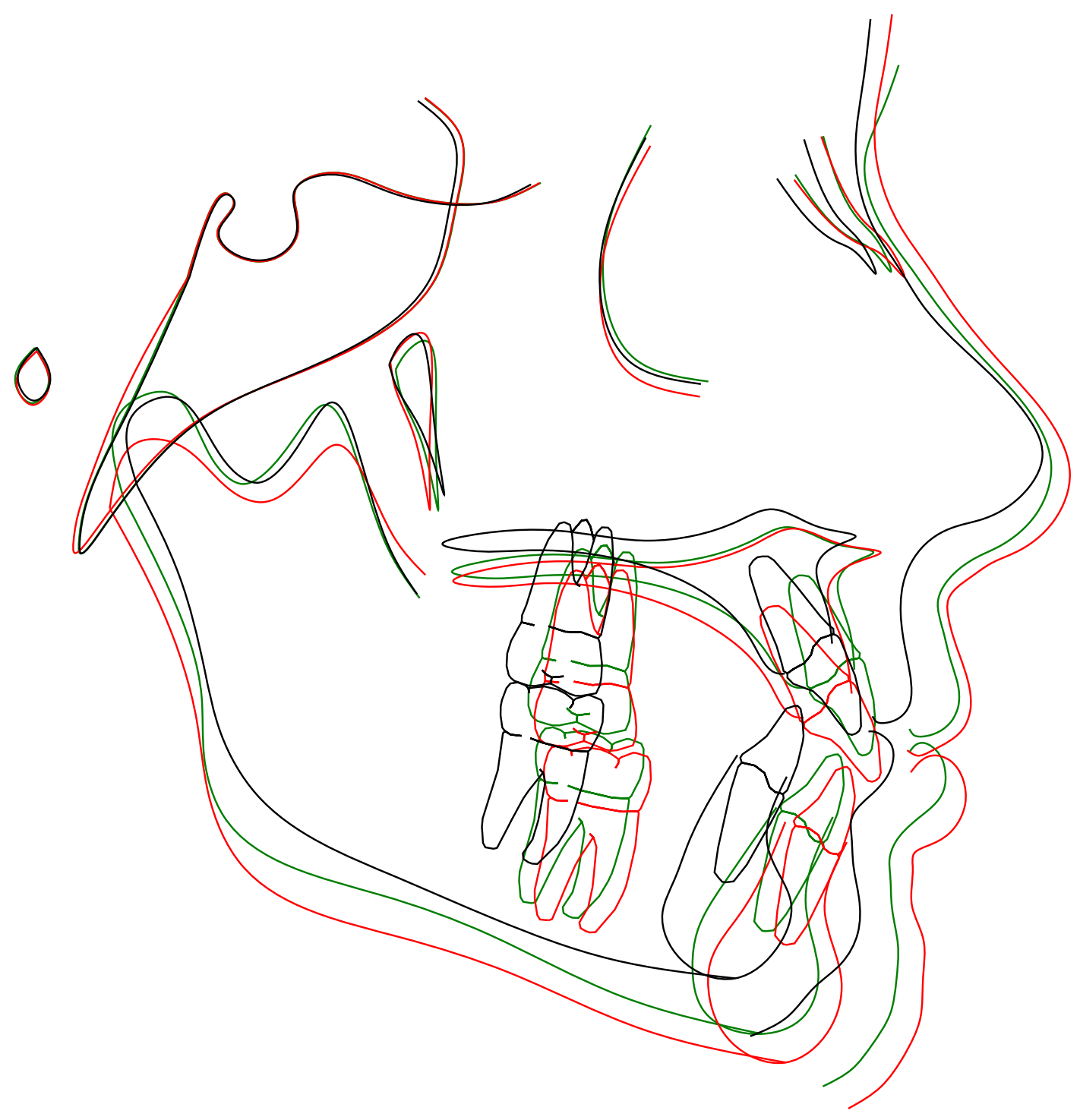

Figure B-1. Print of cephalometric tracing superimposition subject 01: (tracing is $1: 1$ with original radiograph). 


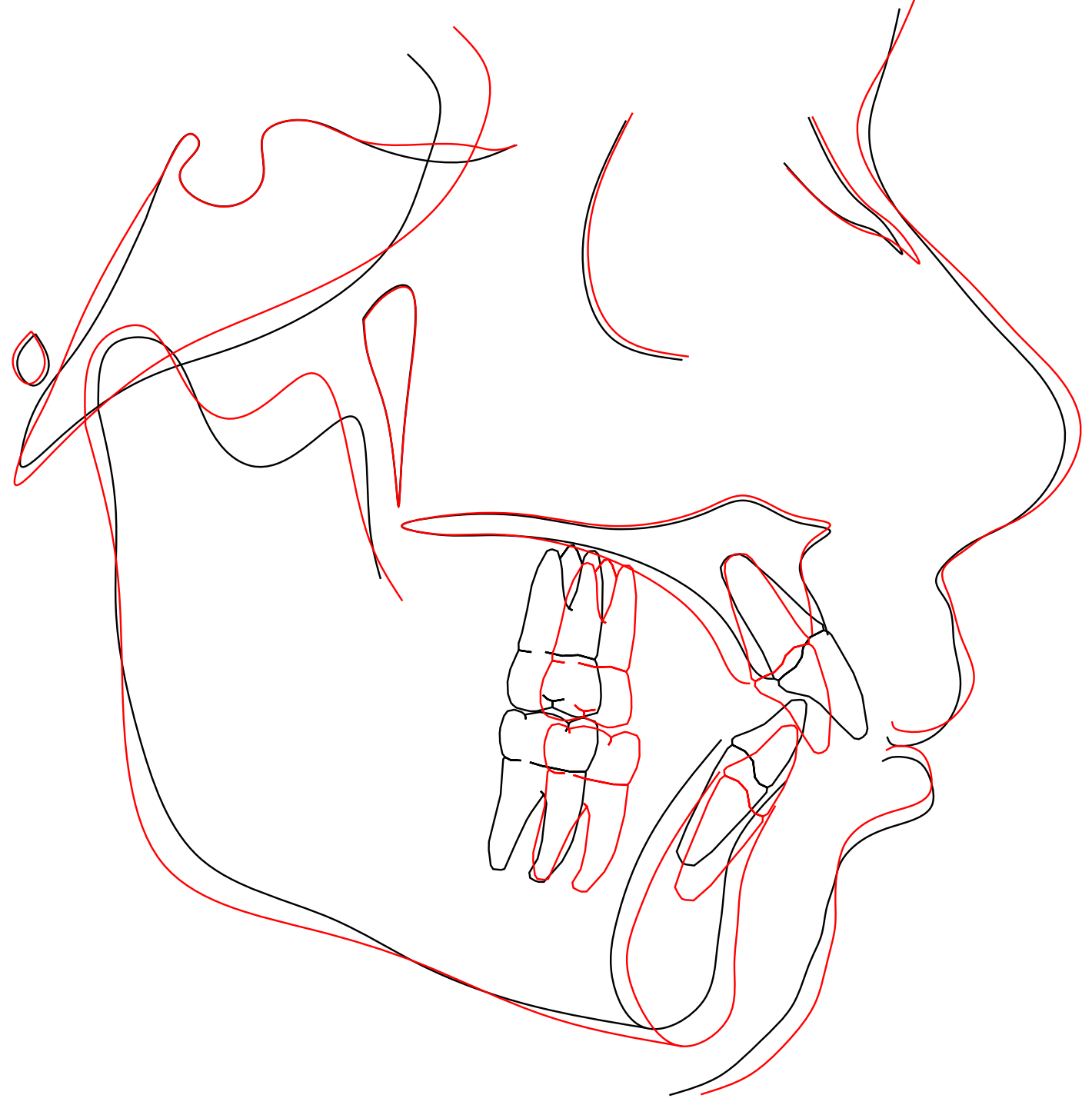

Figure B-2. Print of cephalometric tracing superimposition subject 02: (tracing is 1:1 with original radiograph). 


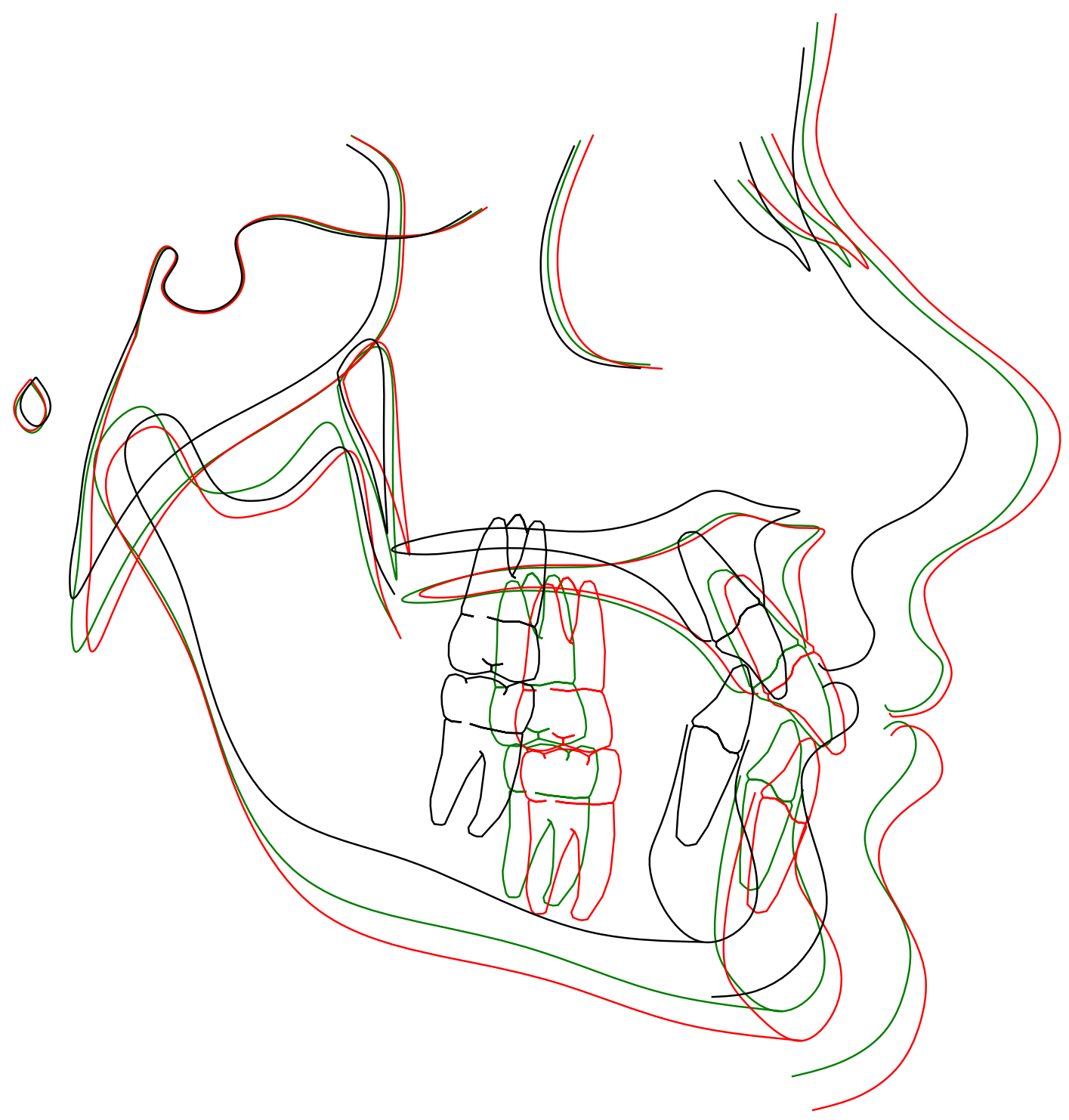

Figure B-3. Print of cephalometric tracing superimposition subject 03: (tracing is $1: 1$ with original radiograph). 


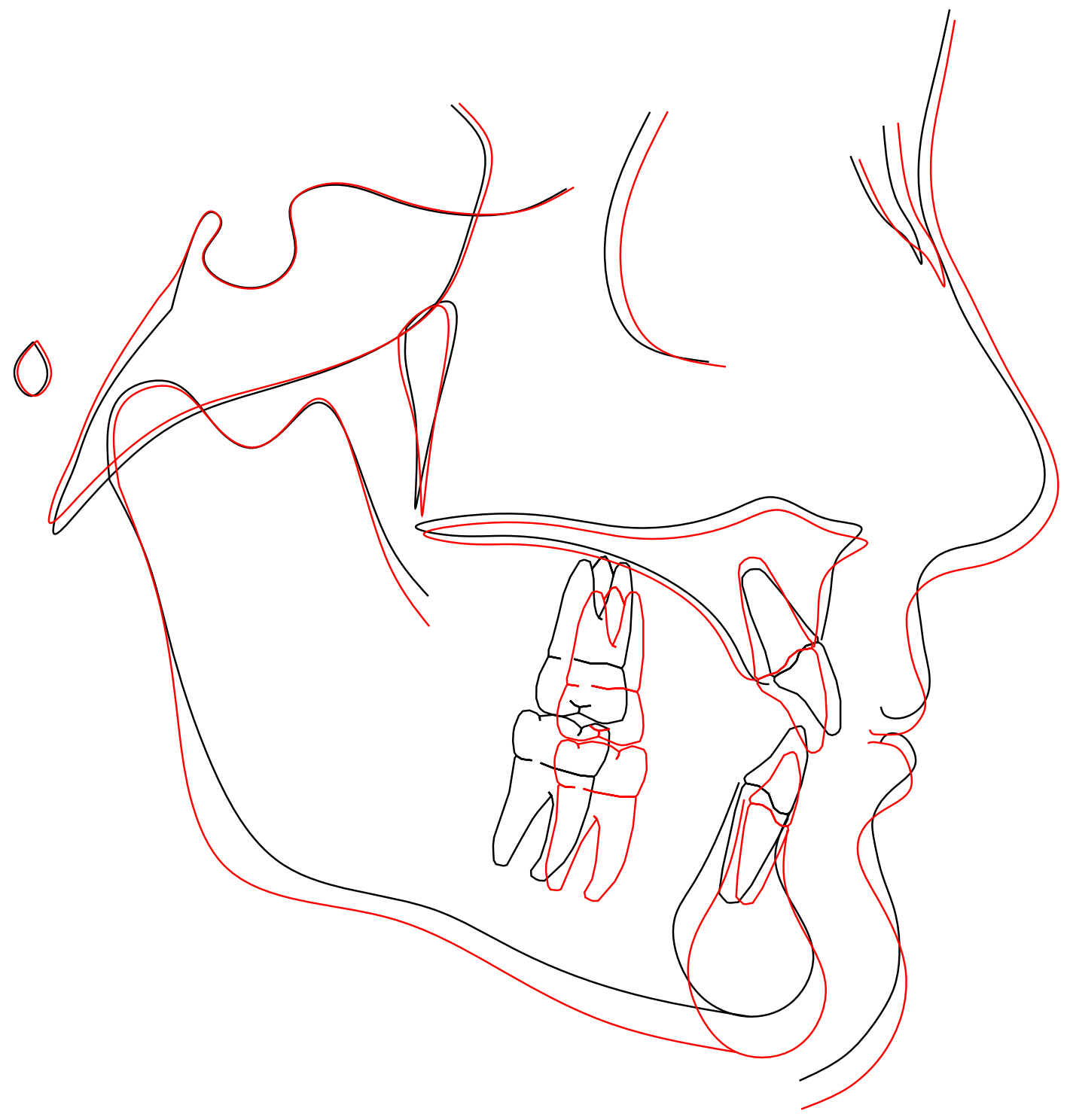

Figure B-4. Print of cephalometric tracing superimposition subject 04: (tracing is $1: 1$ with original radiograph). 


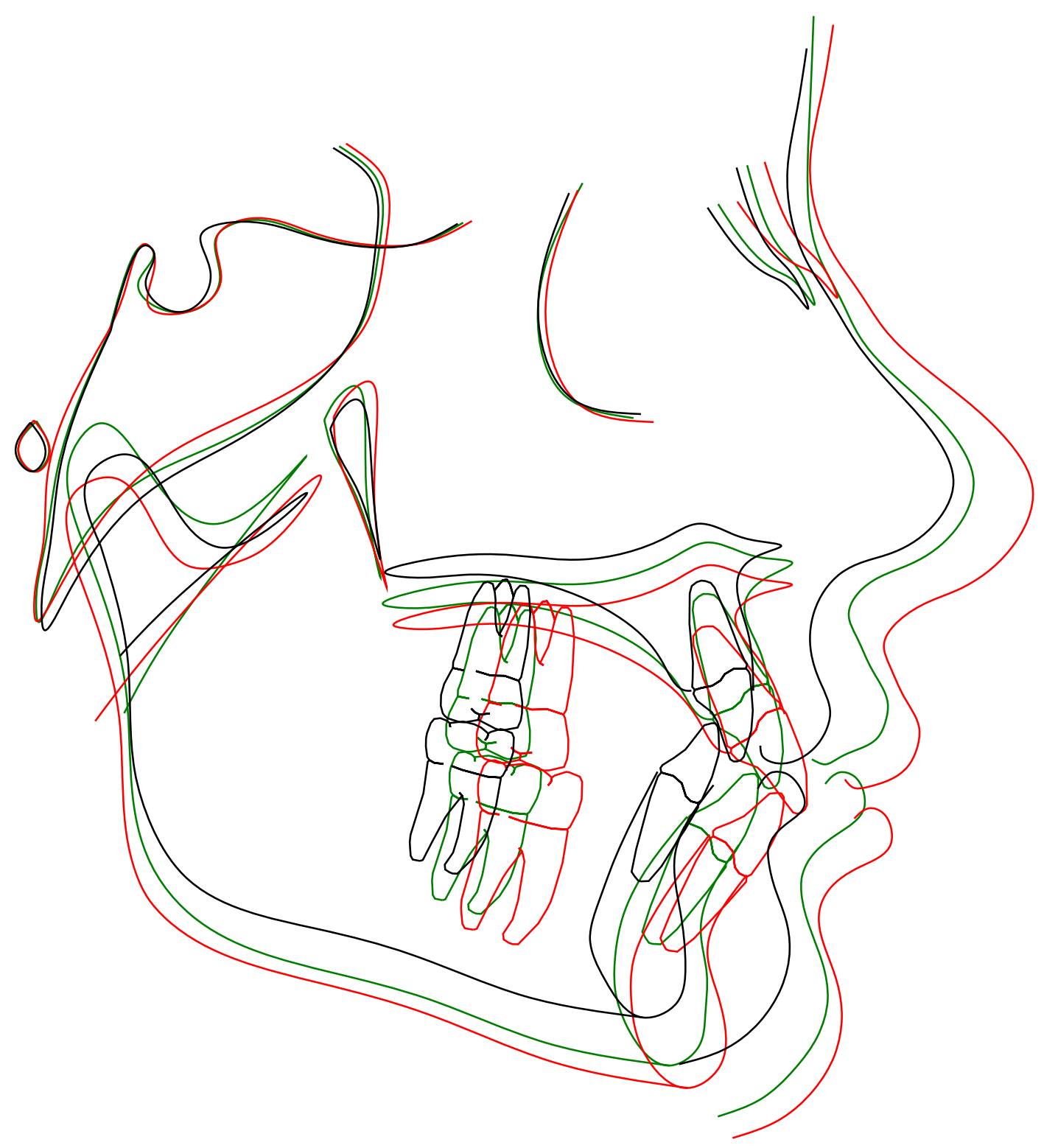

Figure B-5. Print of cephalometric tracing superimposition subject 05: (tracing is $1: 1$ with original radiograph). 


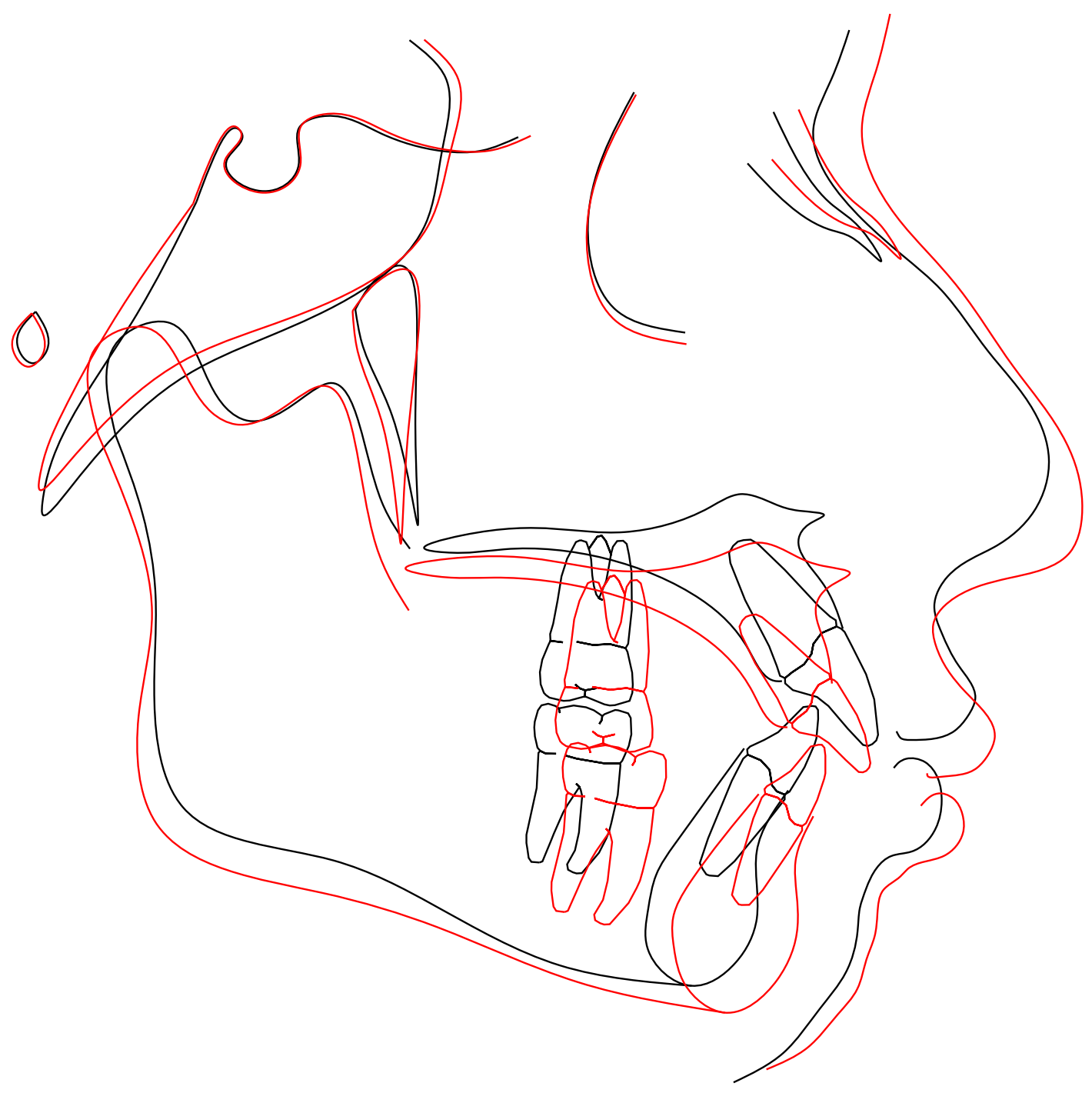

Figure B-6. Print of cephalometric tracing superimposition subject 06: (tracing is $1: 1$ with original radiograph). 


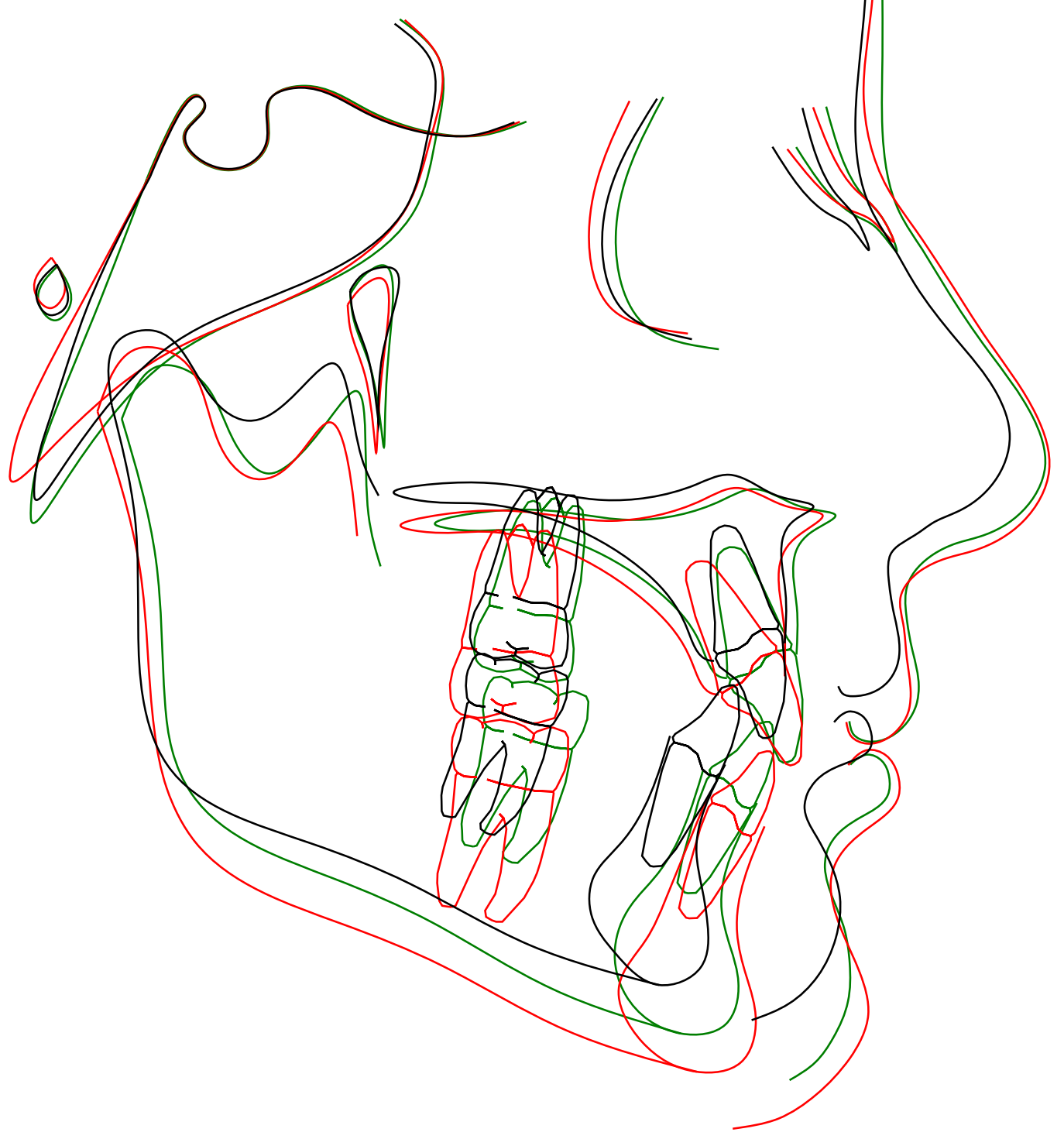

Figure B-7. Print of cephalometric tracing superimposition subject 07: (tracing is $1: 1$ with original radiograph). 


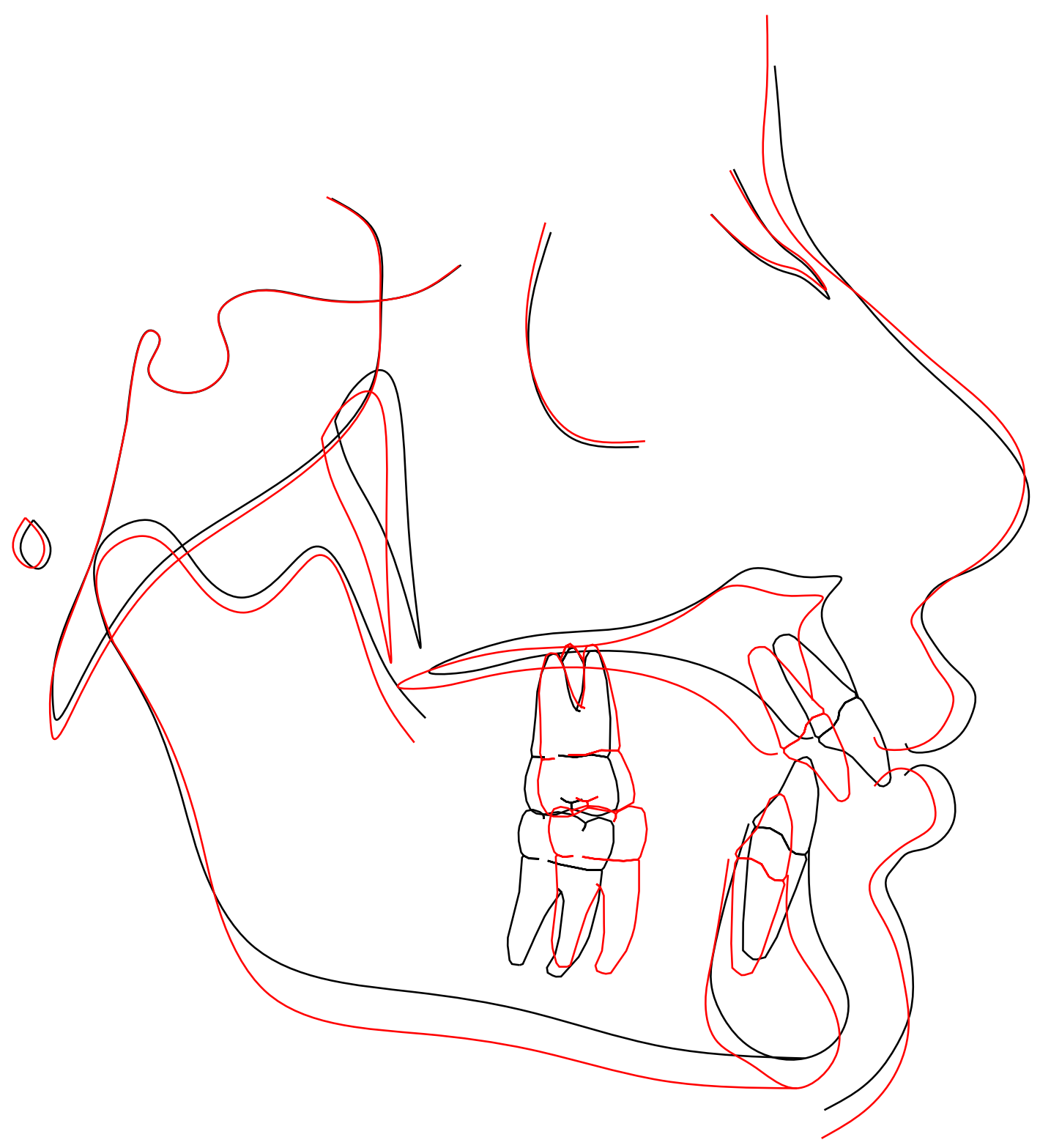

Figure B-8. Print of cephalometric tracing superimposition subject 08: (tracing is $1: 1$ with original radiograph). 


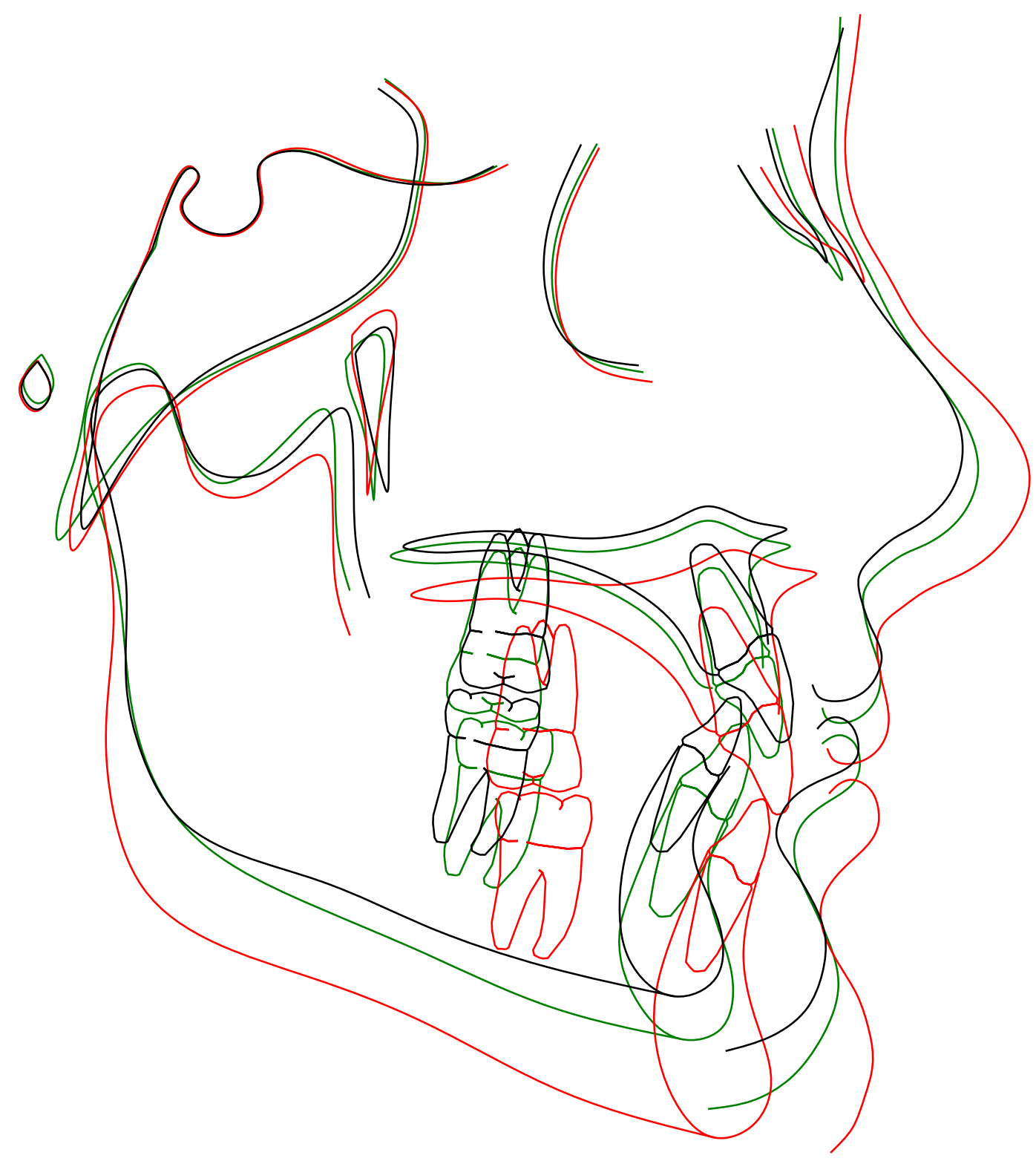

Figure B-9. Print of cephalometric tracing superimposition subject 09: (tracing is 1:1 with original radiograph). 


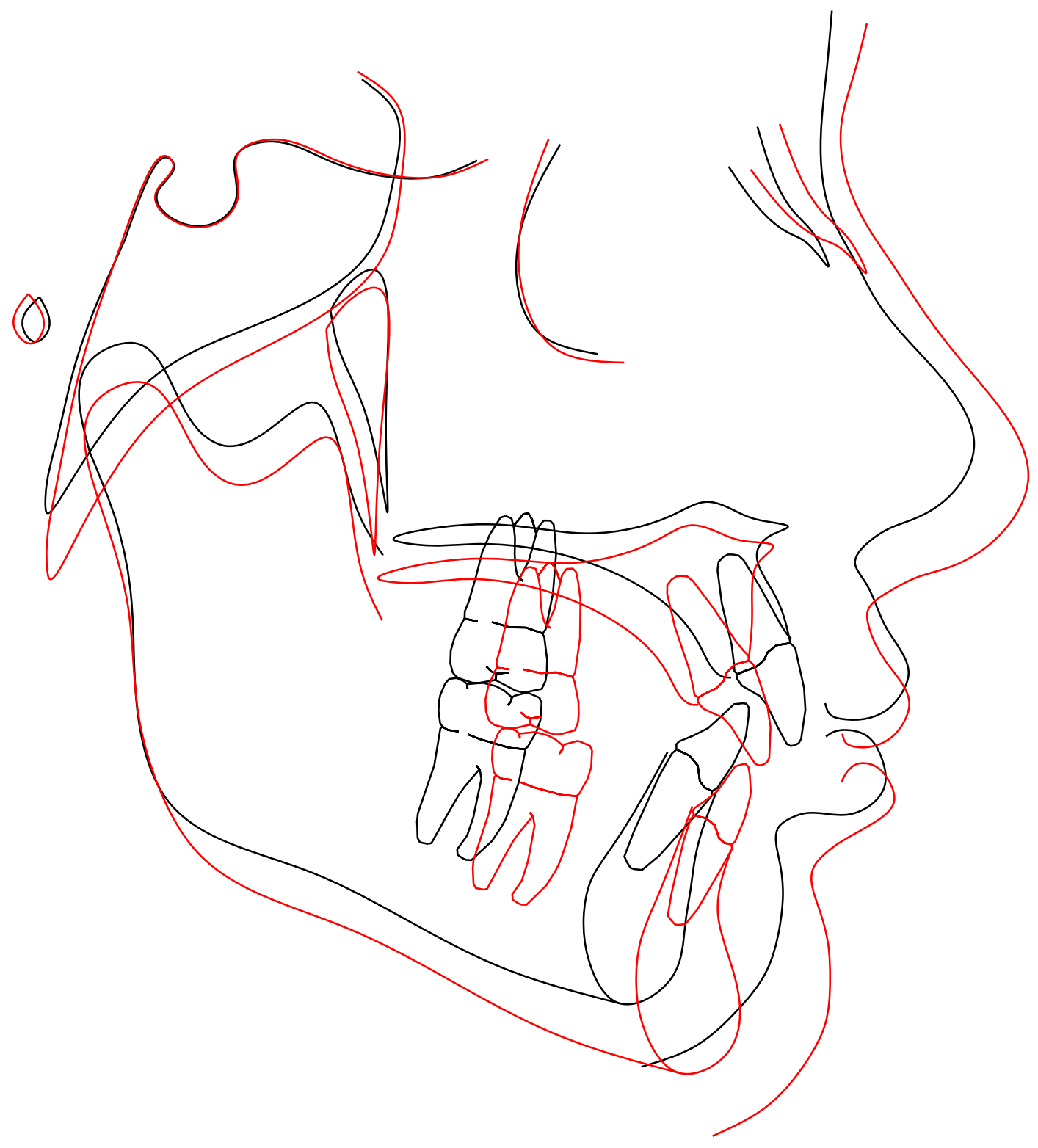

Figure B-10. Print of cephalometric tracing superimposition subject 10: (tracing is 1:1 with original radiograph). 


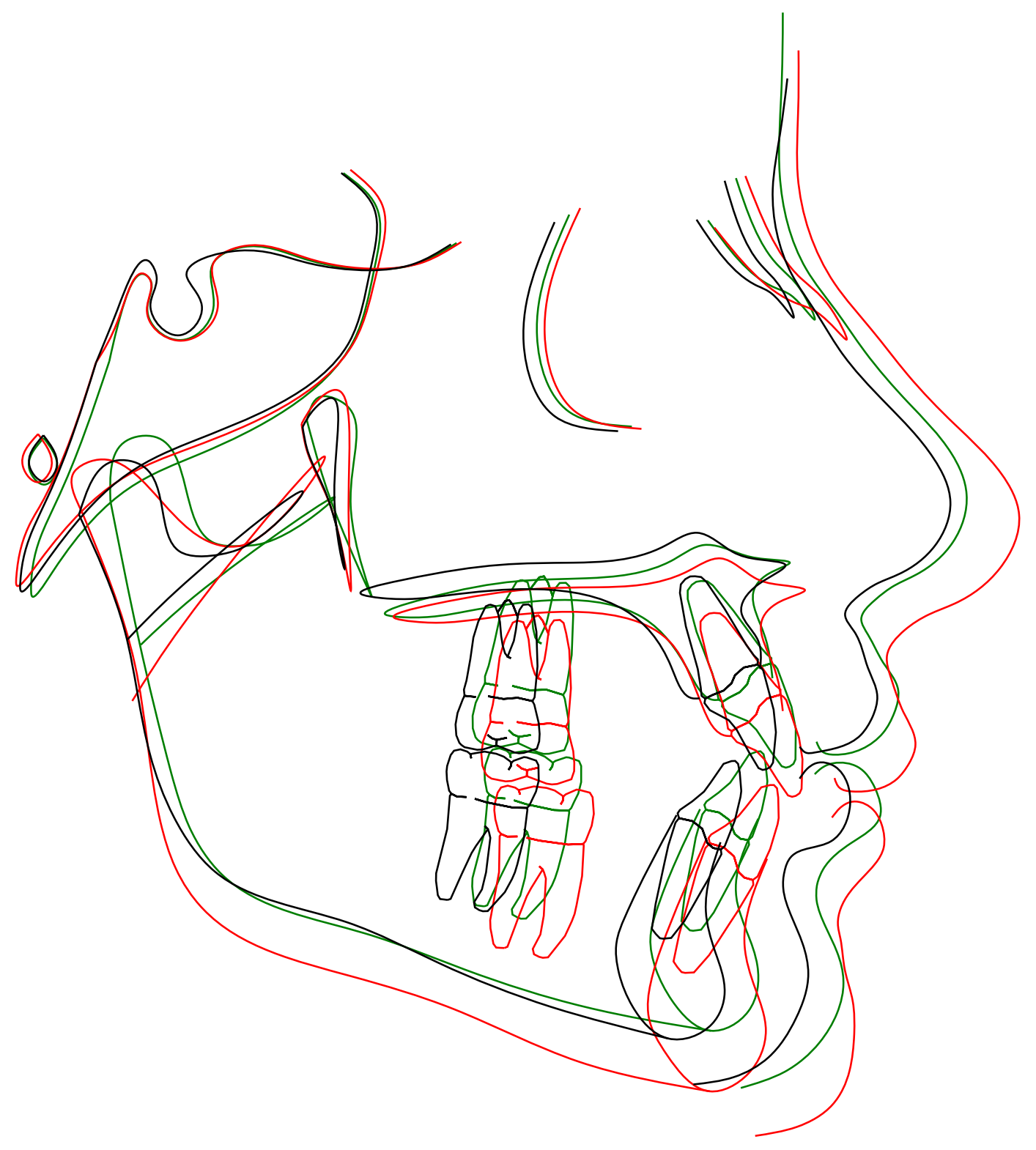

Figure B-11. Print of cephalometric tracing superimposition subject 11: (tracing is $1: 1$ with original radiograph). 


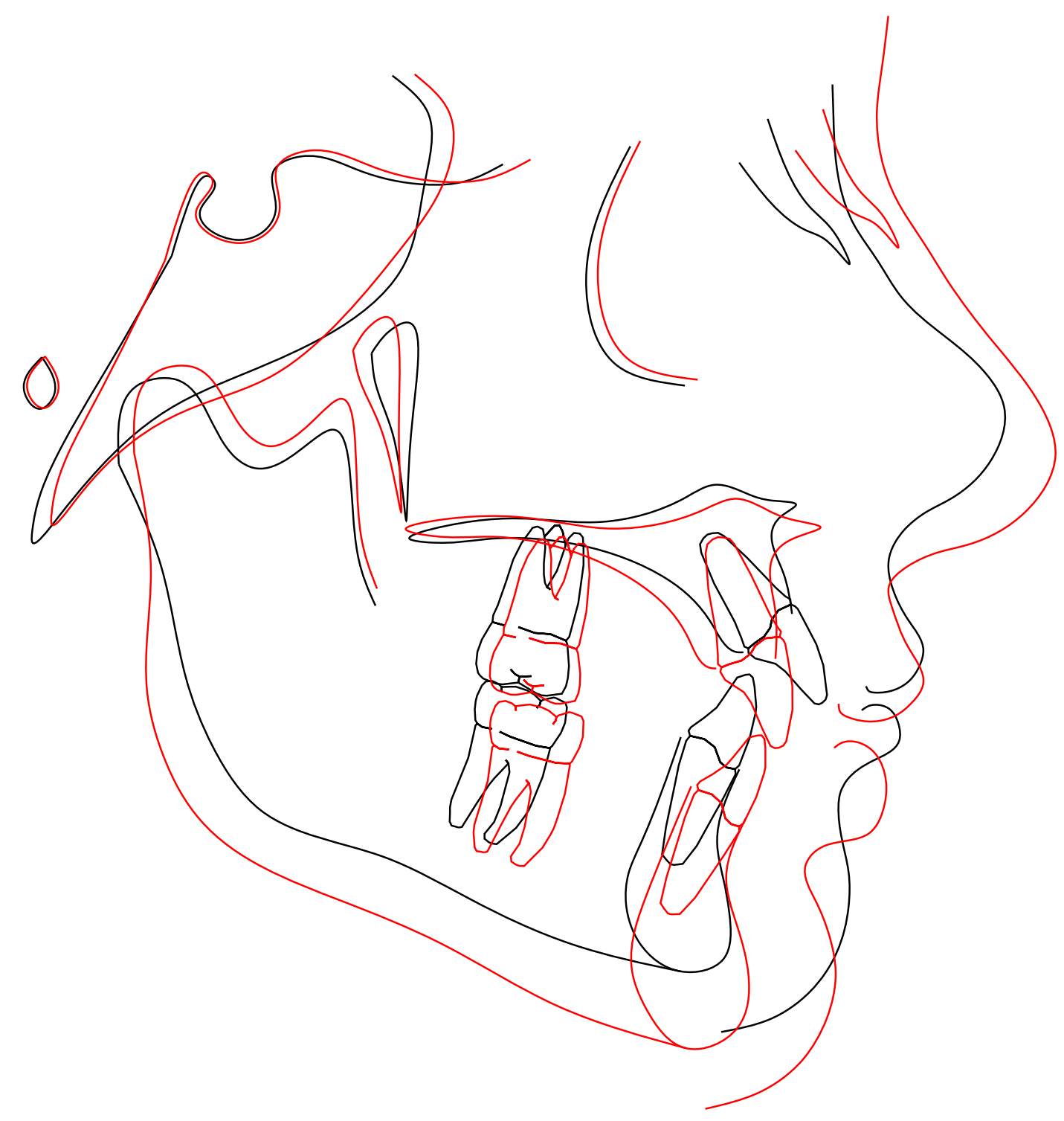

Figure B-12. Print of cephalometric tracing superimposition subject 12: (tracing is 1:1 with original radiograph). 


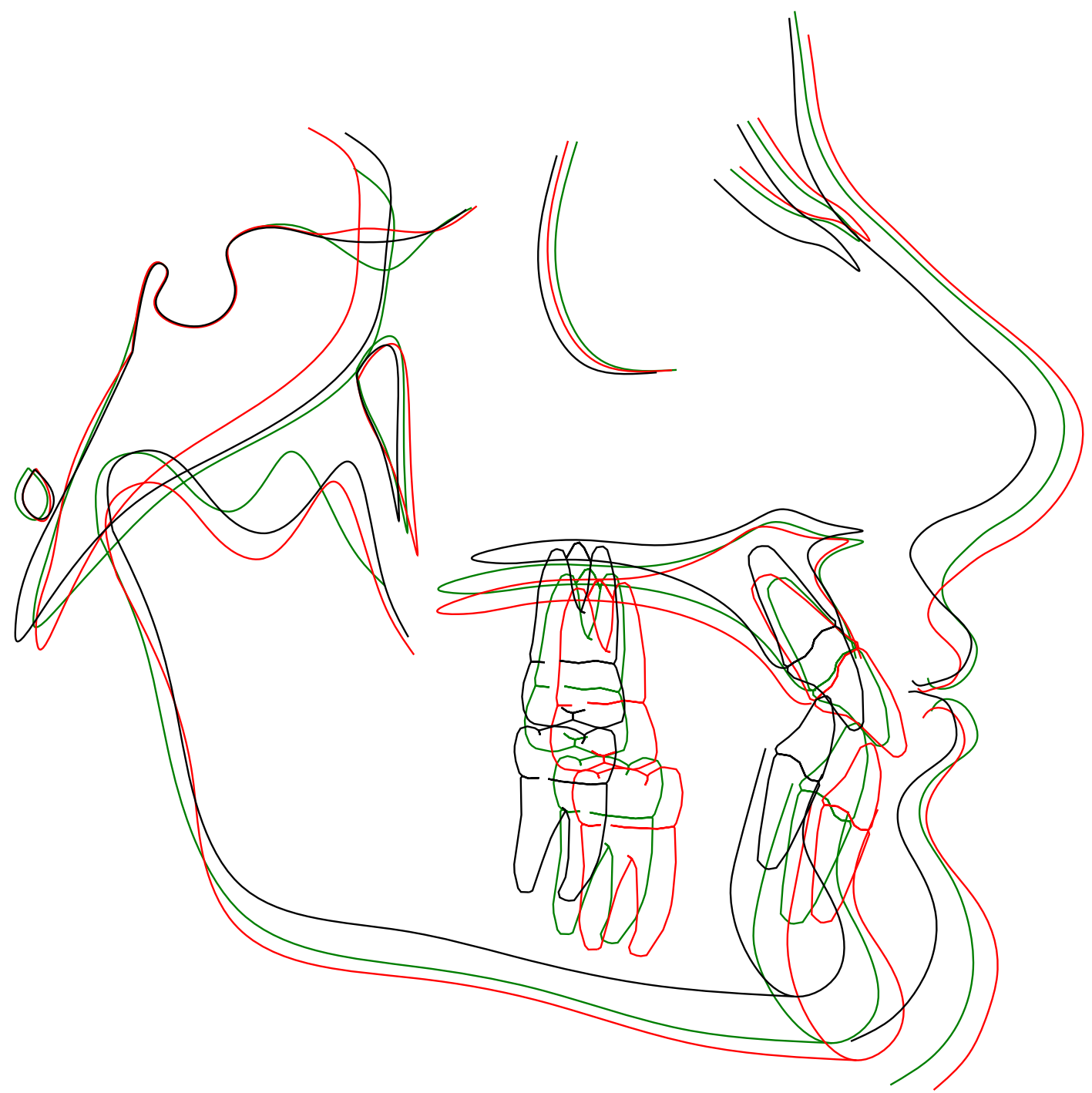

Figure B-13. Print of cephalometric tracing superimposition subject 13: (tracing is $1: 1$ with original radiograph). 


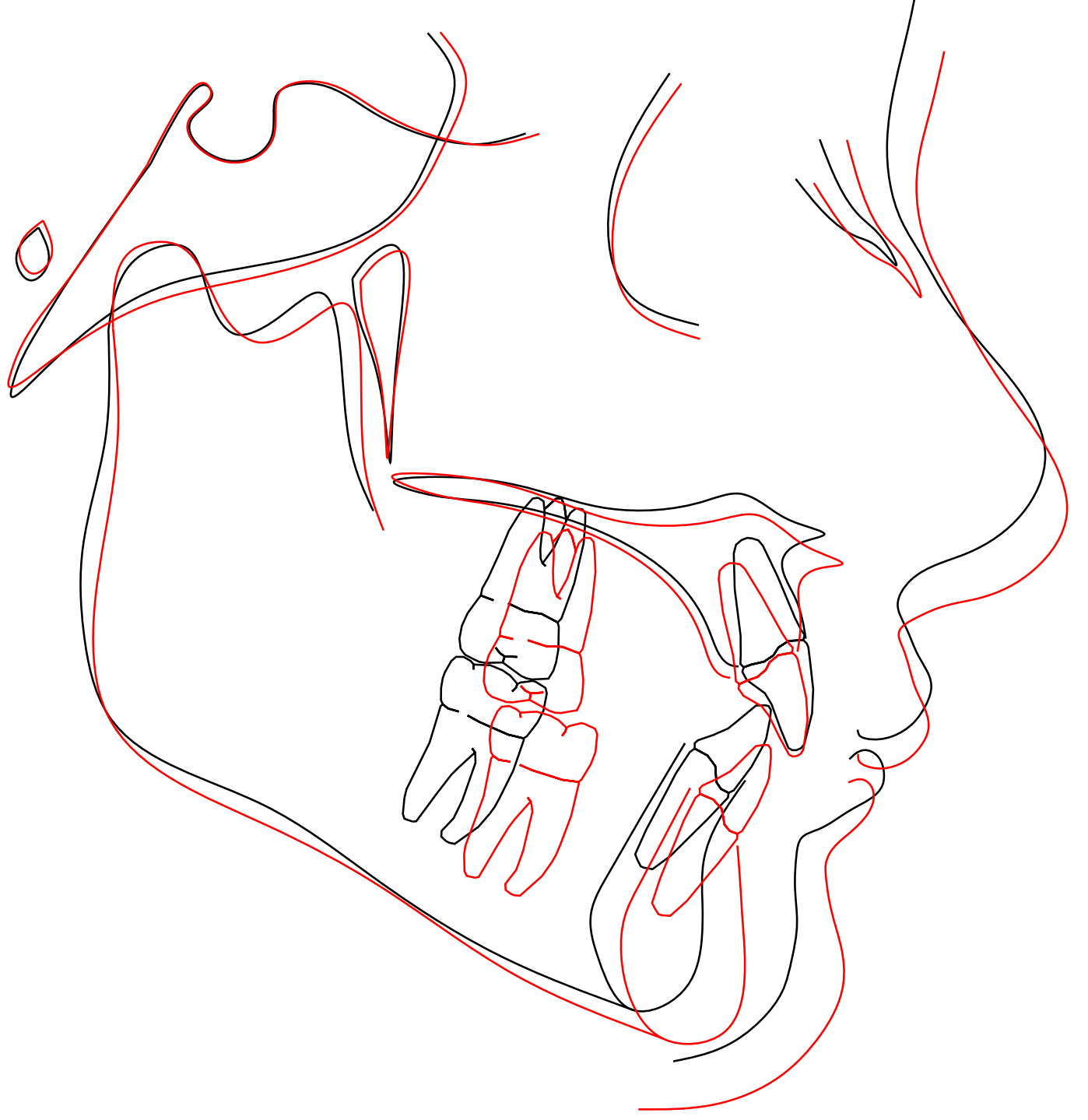

Figure B-14. Print of cephalometric tracing superimposition subject 14: (tracing is 1:1 with original radiograph). 


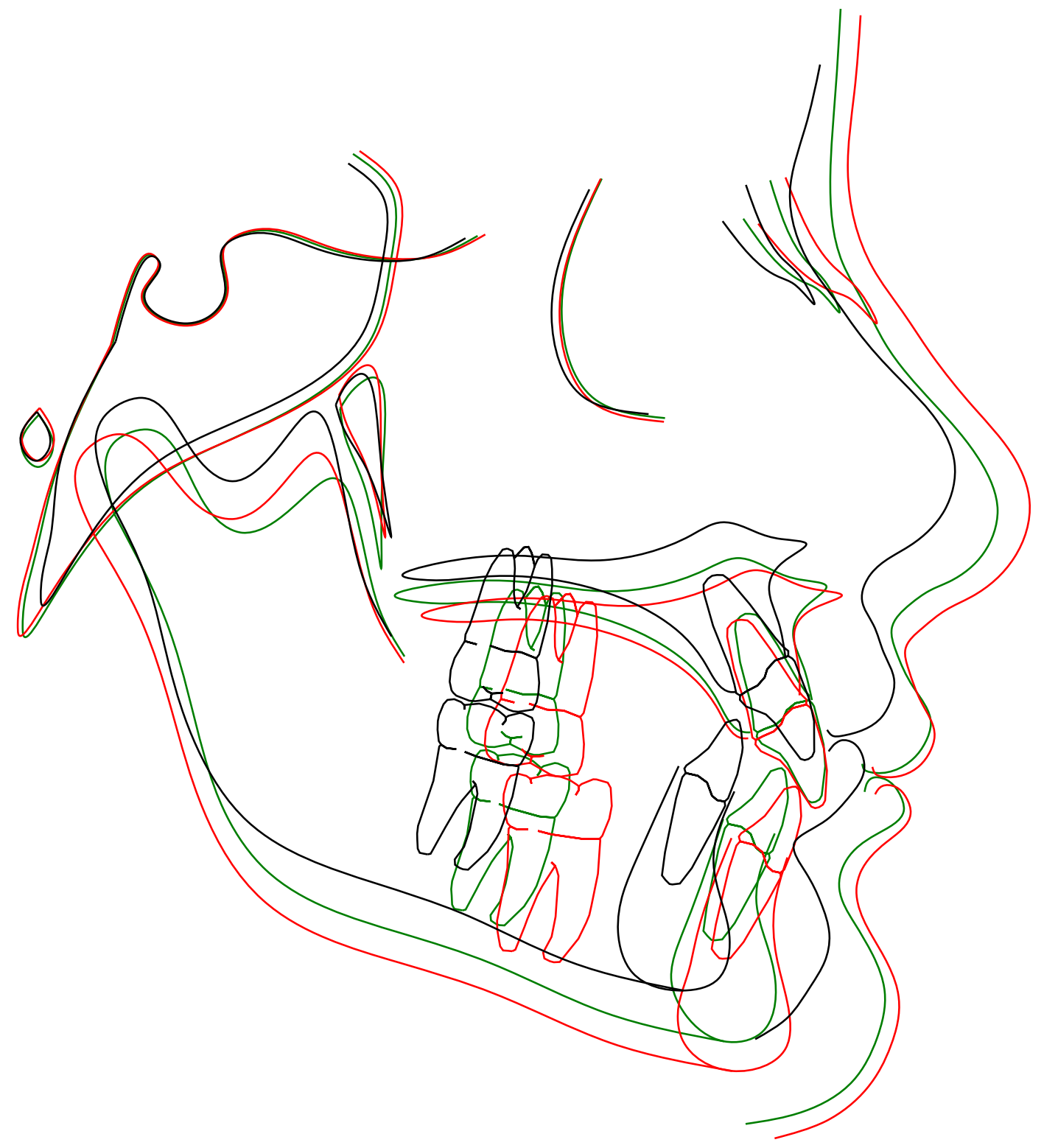

Figure B-15. Print of cephalometric tracing superimposition subject 15: (tracing is 1:1 with original radiograph). 


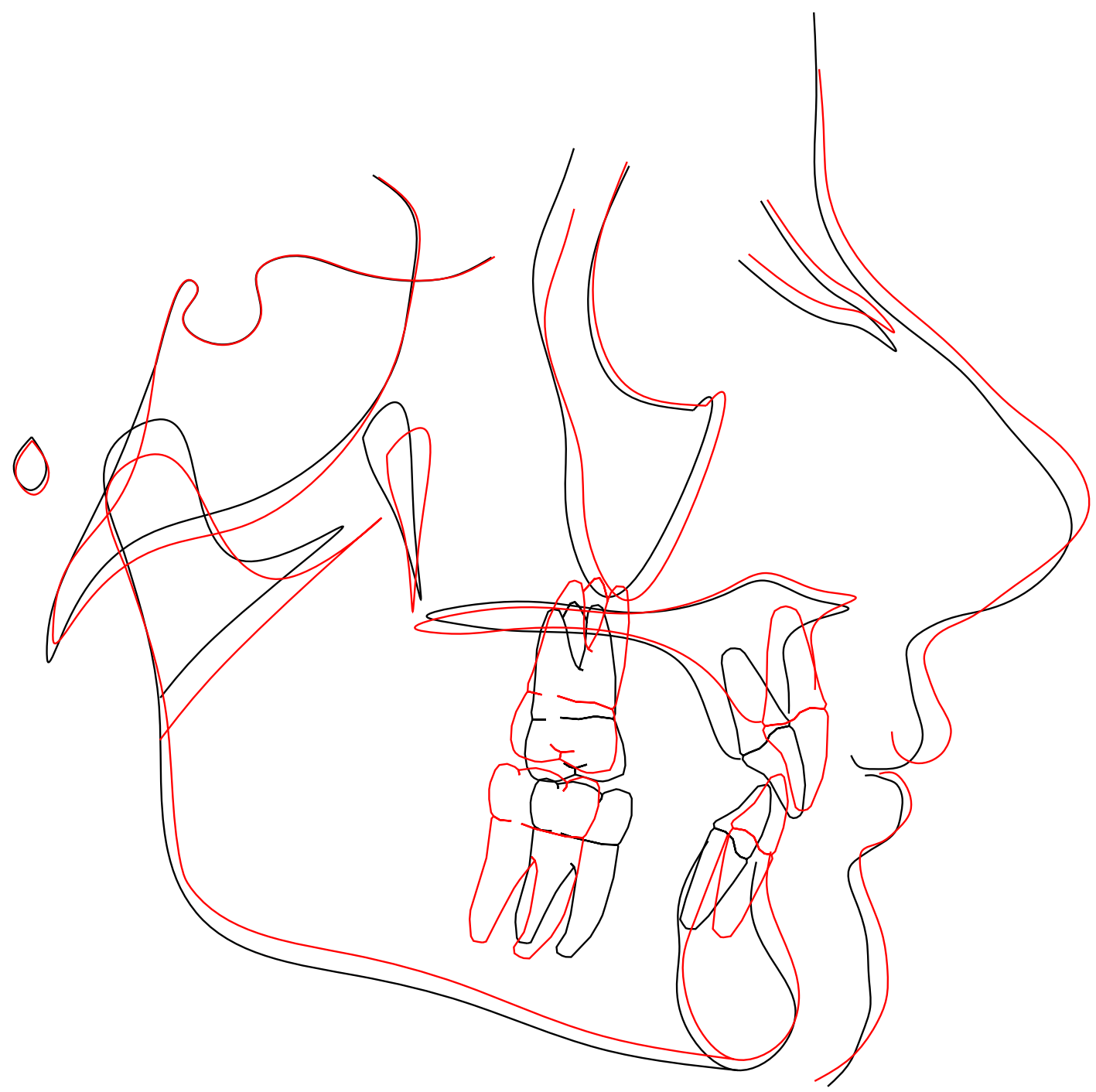

Figure B-16. Print of cephalometric tracing superimposition subject 16: (tracing is 1:1 with original radiograph). 


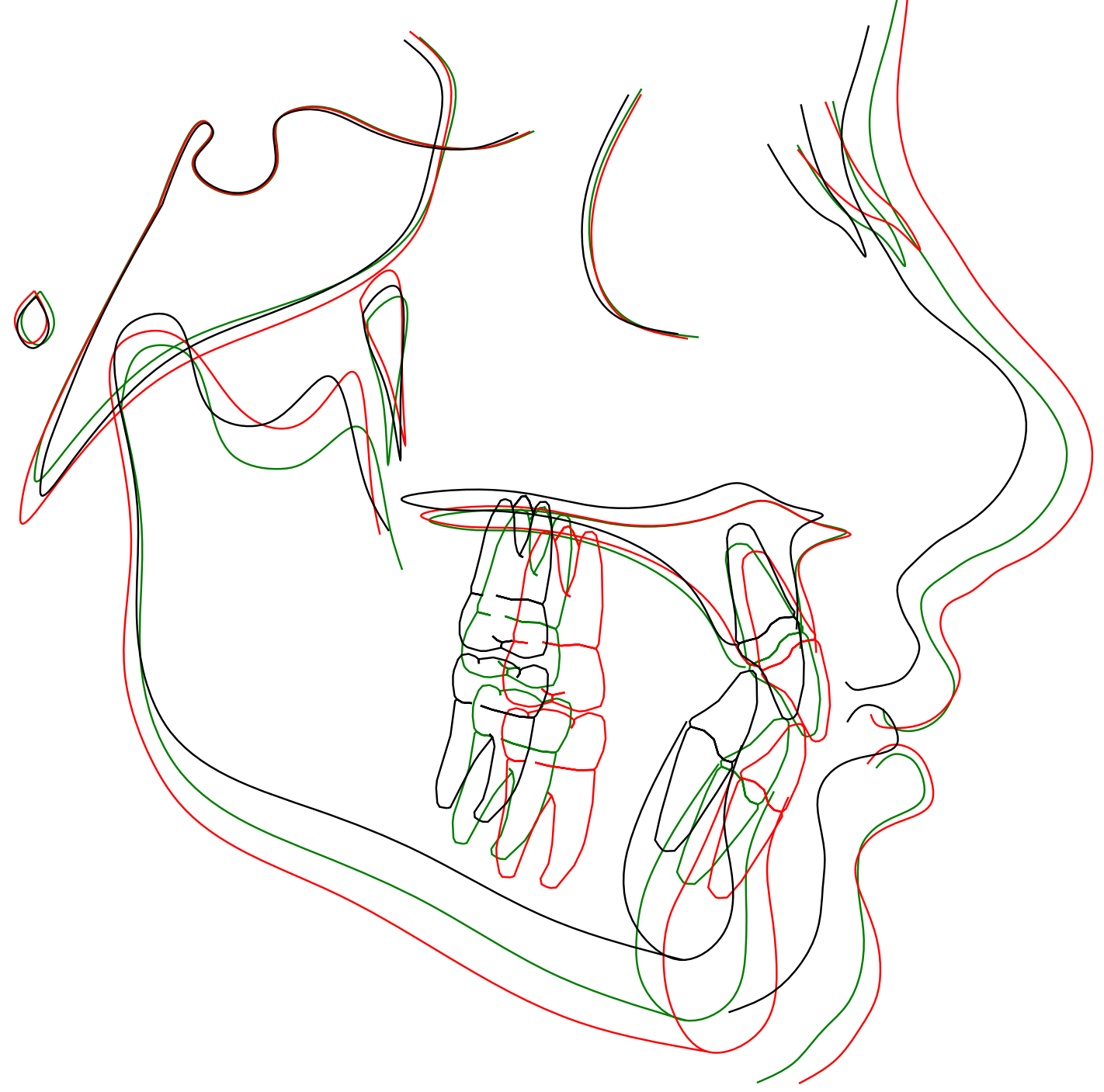

Figure B-17. Print of cephalometric tracing superimposition subject 17: (tracing is 1:1 with original radiograph). 


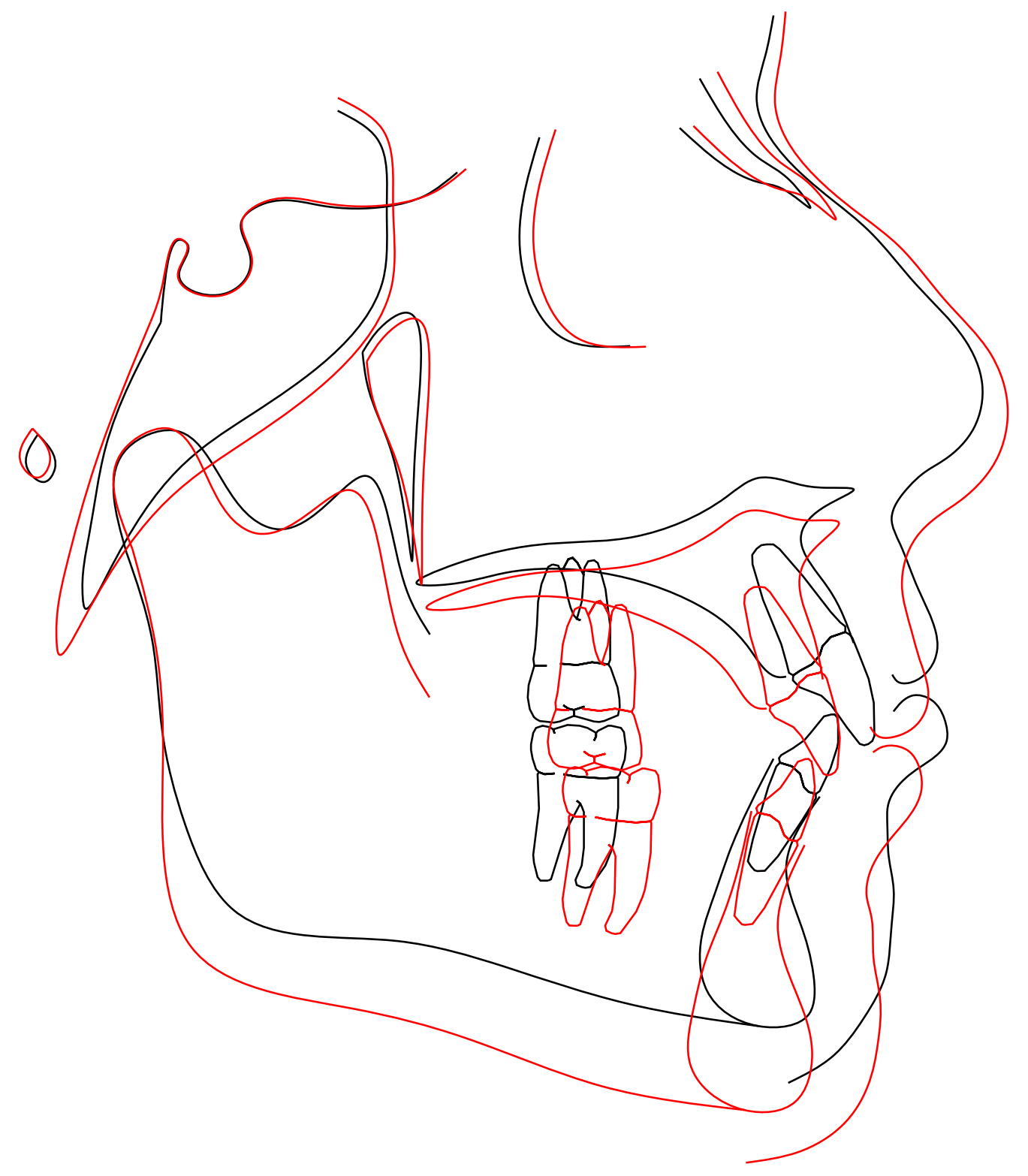

Figure B-18. Print of cephalometric tracing superimposition subject 18: (tracing is 1:1 with original radiograph). 


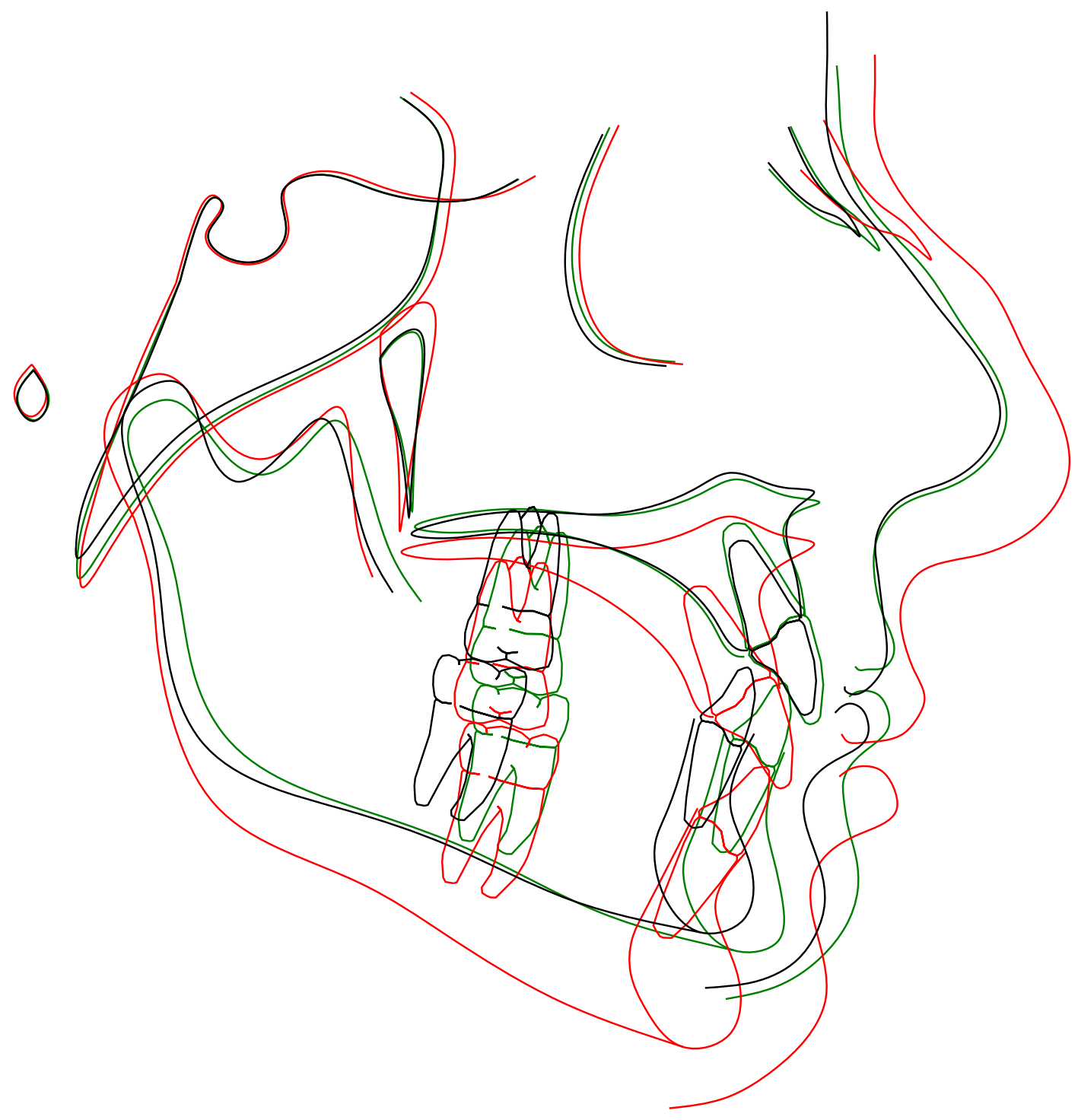

Figure B-19. Print of cephalometric tracing superimposition subject 19: (tracing is 1:1 with original radiograph). 


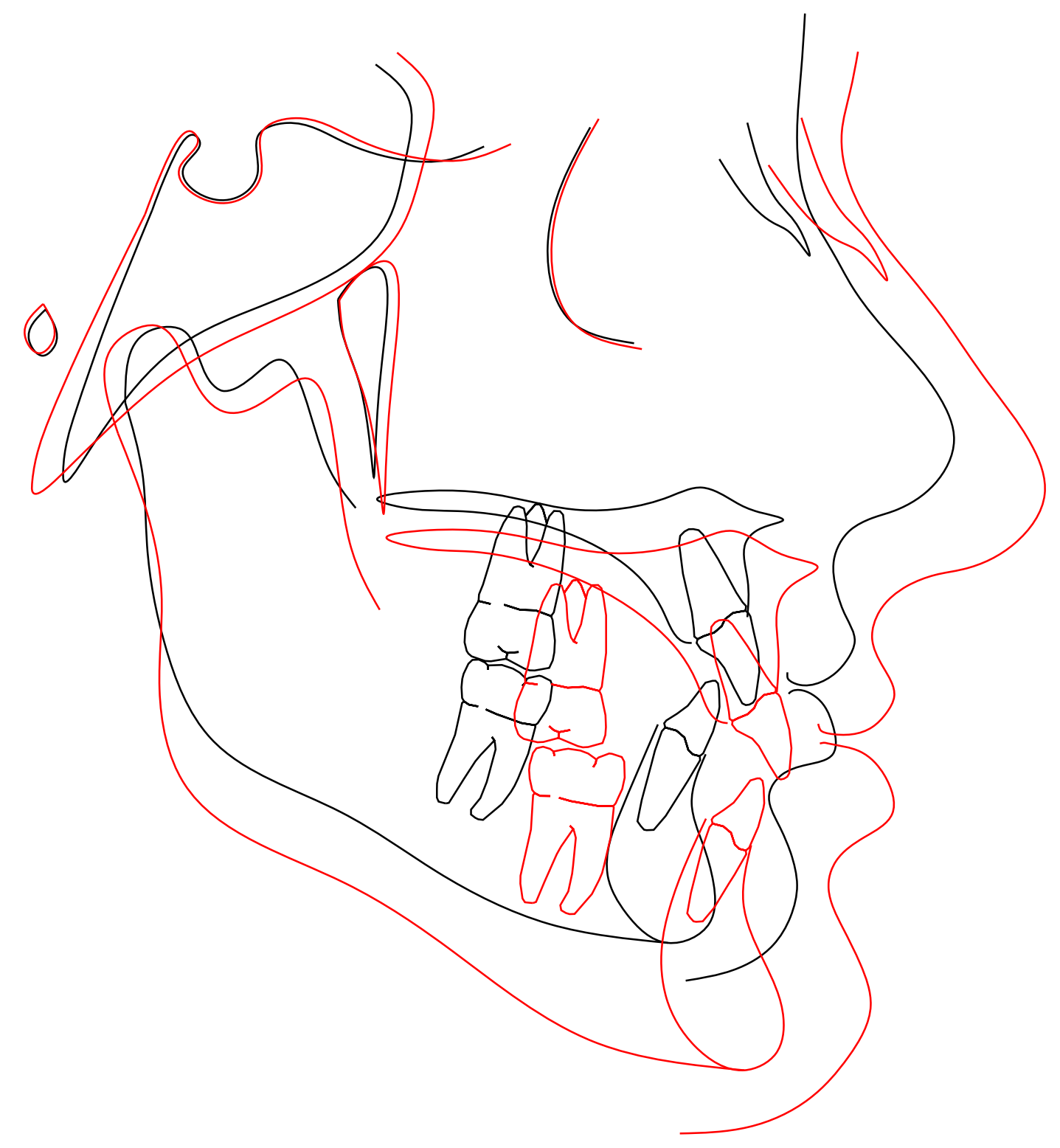

Figure B-20. Print of cephalometric tracing superimposition subject 20: (tracing is $1: 1$ with original radiograph). 


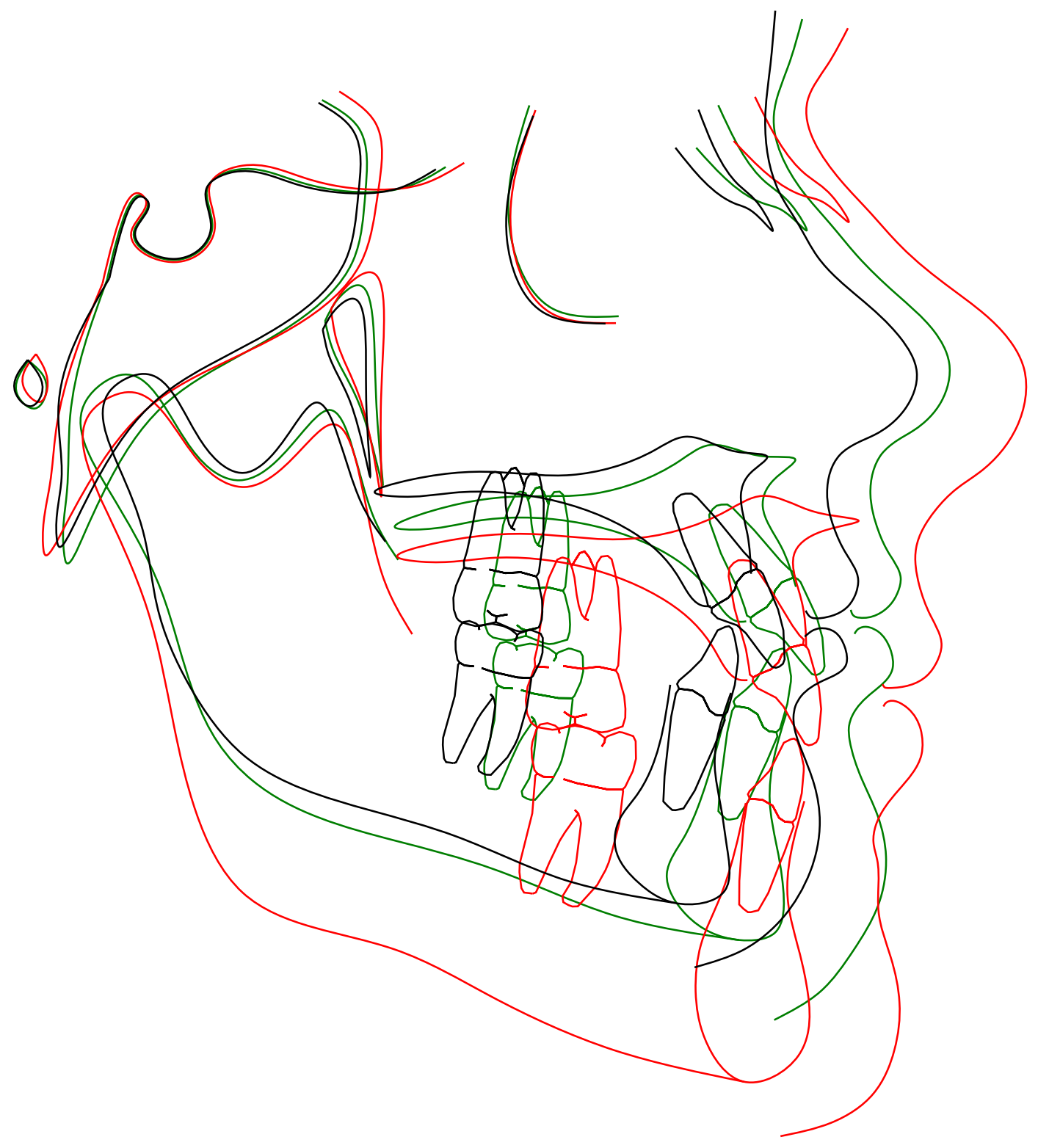

Figure B-21. Print of cephalometric tracing superimposition subject 21: (tracing is 1:1 with original radiograph). 


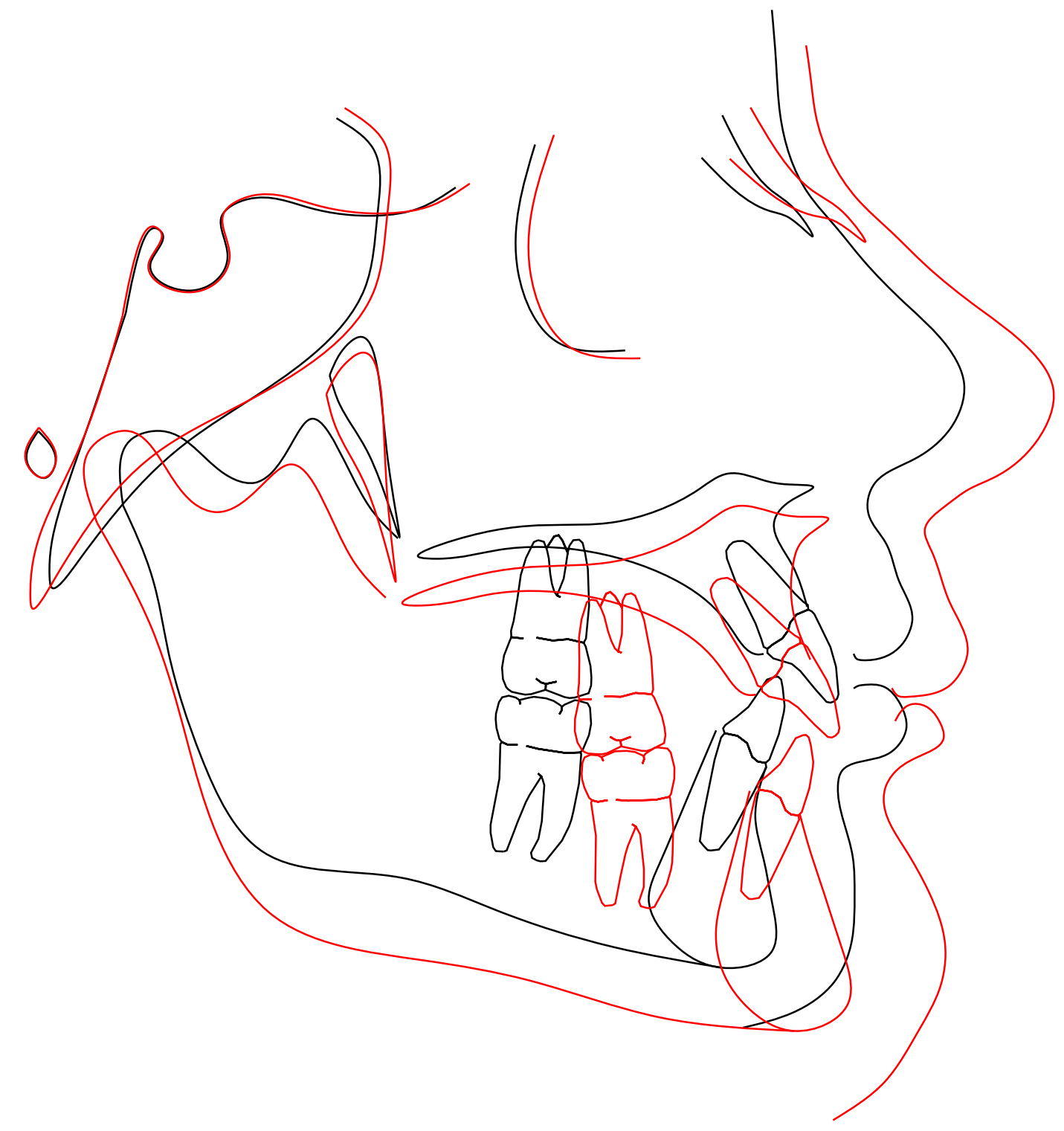

Figure B-22. Print of cephalometric tracing superimposition subject 22: (tracing is 1:1 with original radiograph). 


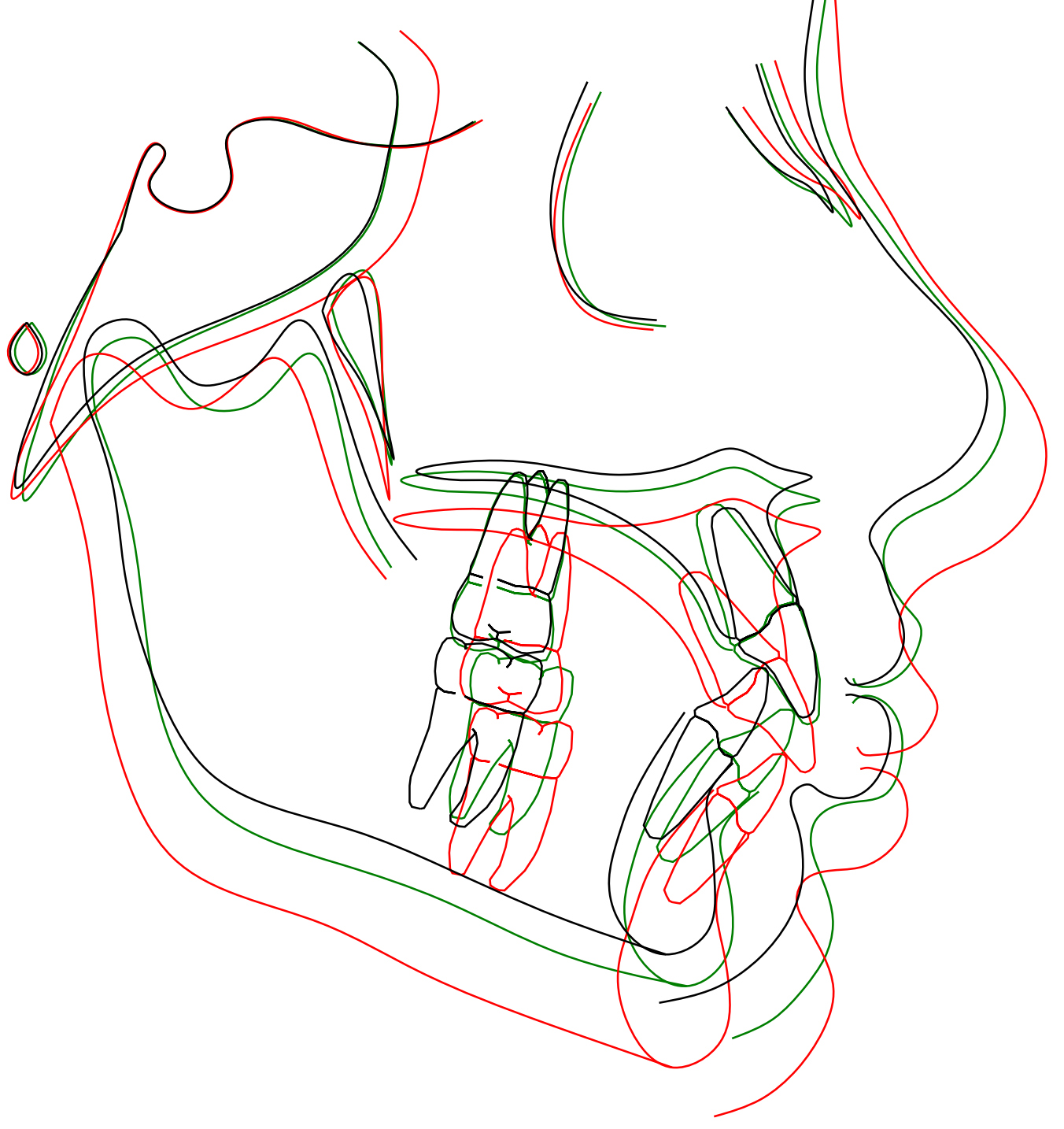

Figure B-23. Print of cephalometric tracing superimposition subject 23: (tracing is $1: 1$ with original radiograph). 


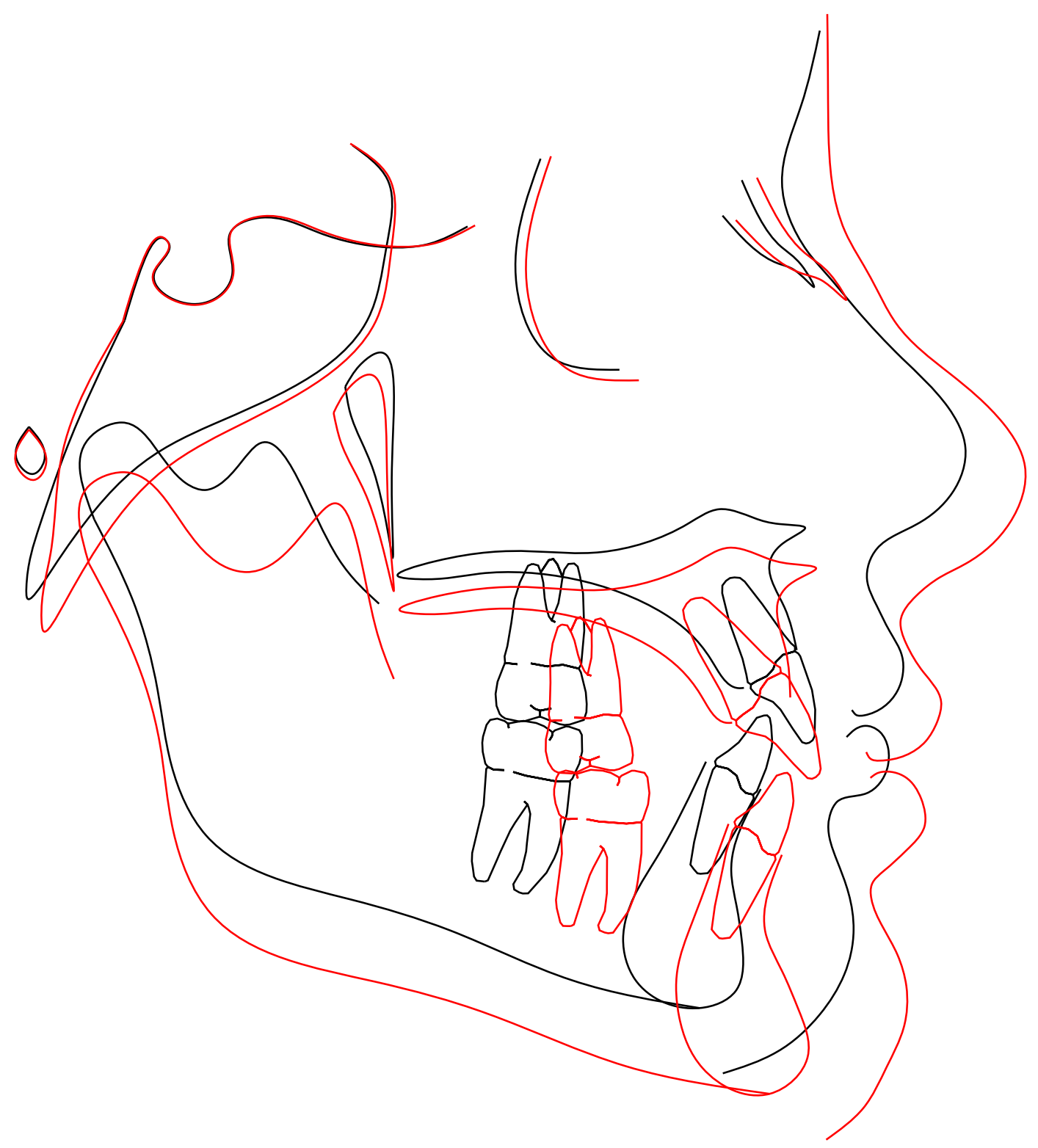

Figure B-24. Print of cephalometric tracing superimposition subject 24: (tracing is 1:1 with original radiograph). 


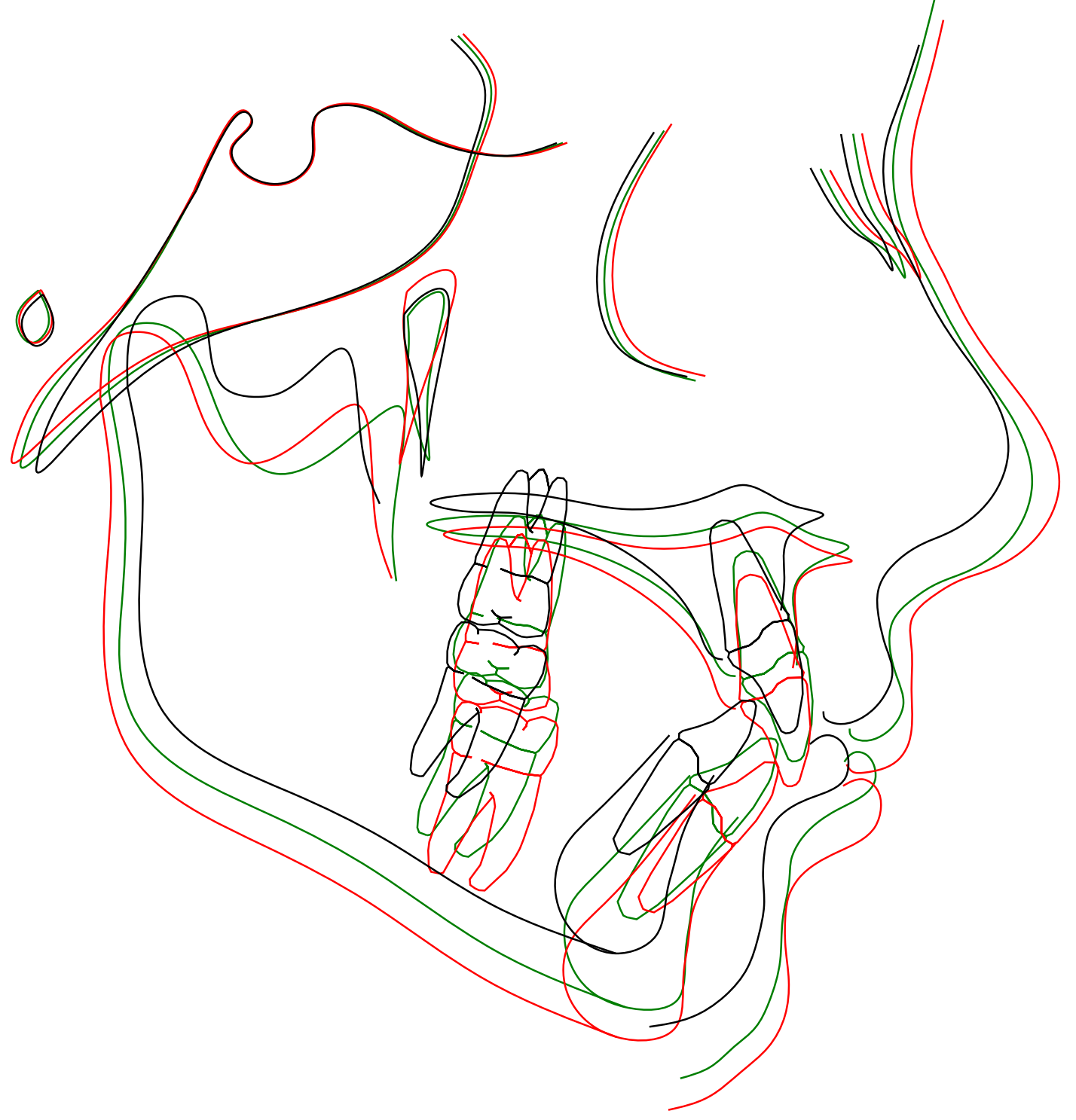

Figure B-25. Print of cephalometric tracing superimposition subject 25: (tracing is 1:1 with original radiograph). 


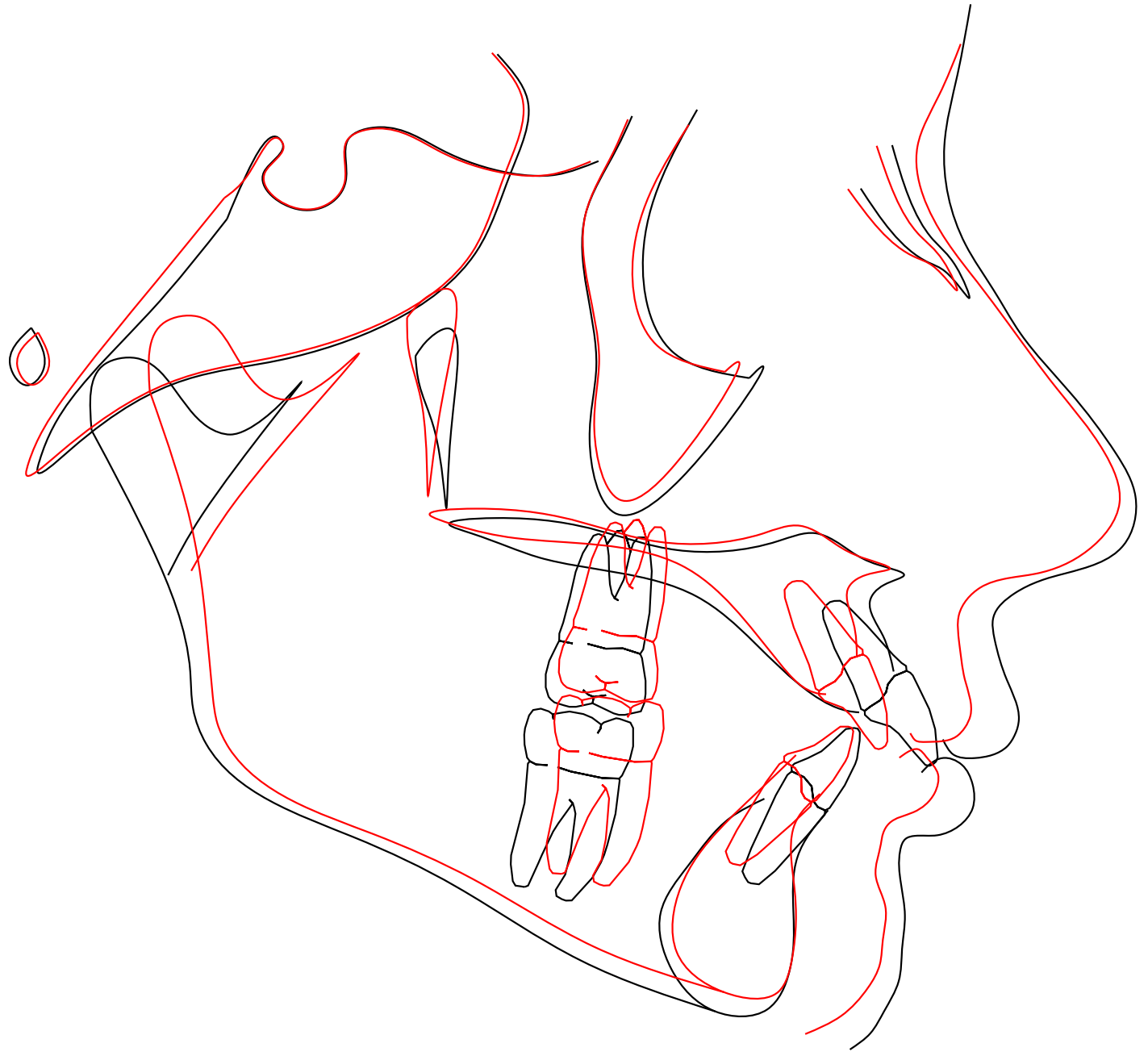

Figure B-26. Print of cephalometric tracing superimposition subject 26: (tracing is 1:1 with original radiograph). 


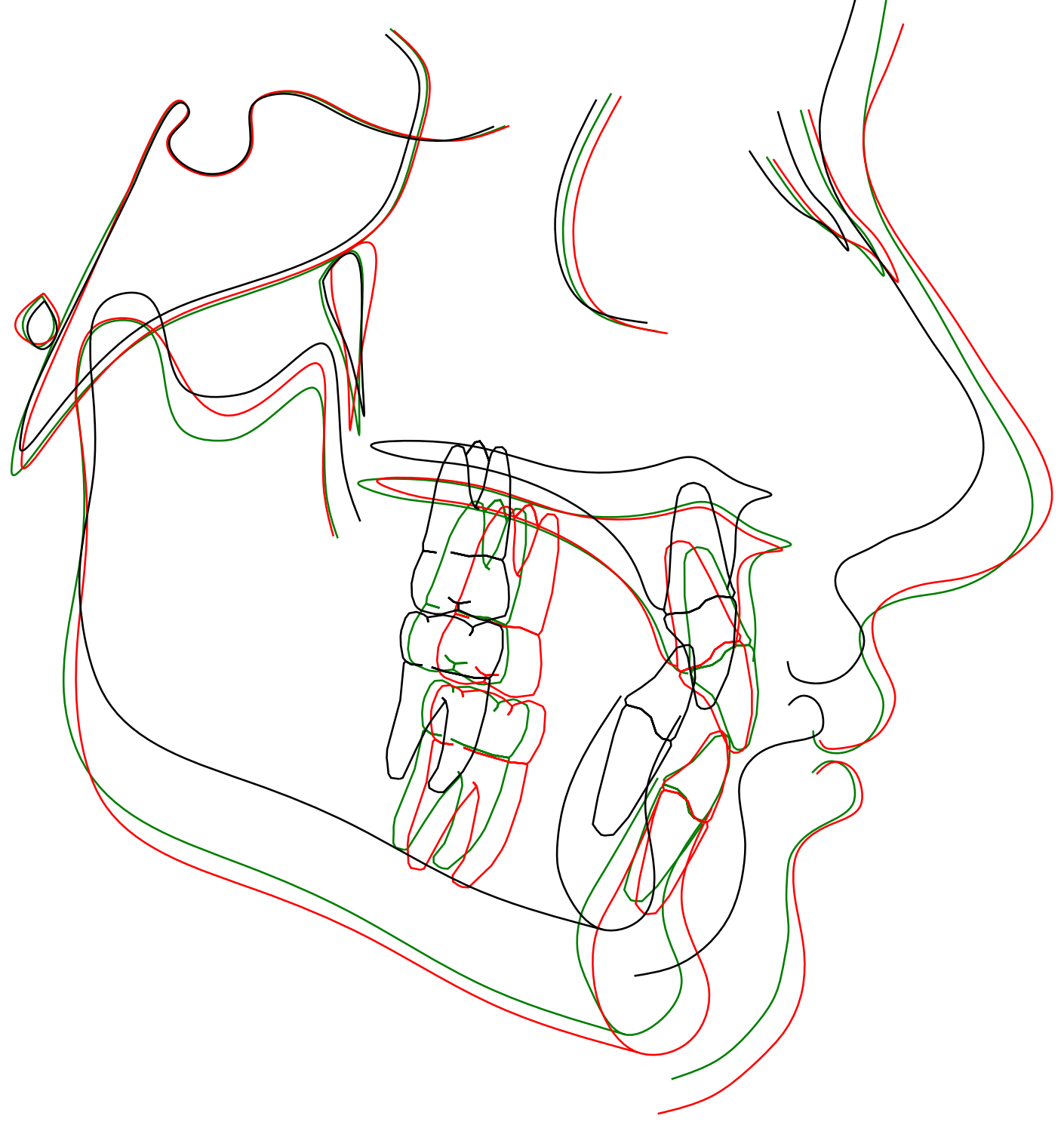

Figure B-27. Print of cephalometric tracing superimposition subject 27: (tracing is $1: 1$ with original radiograph). 


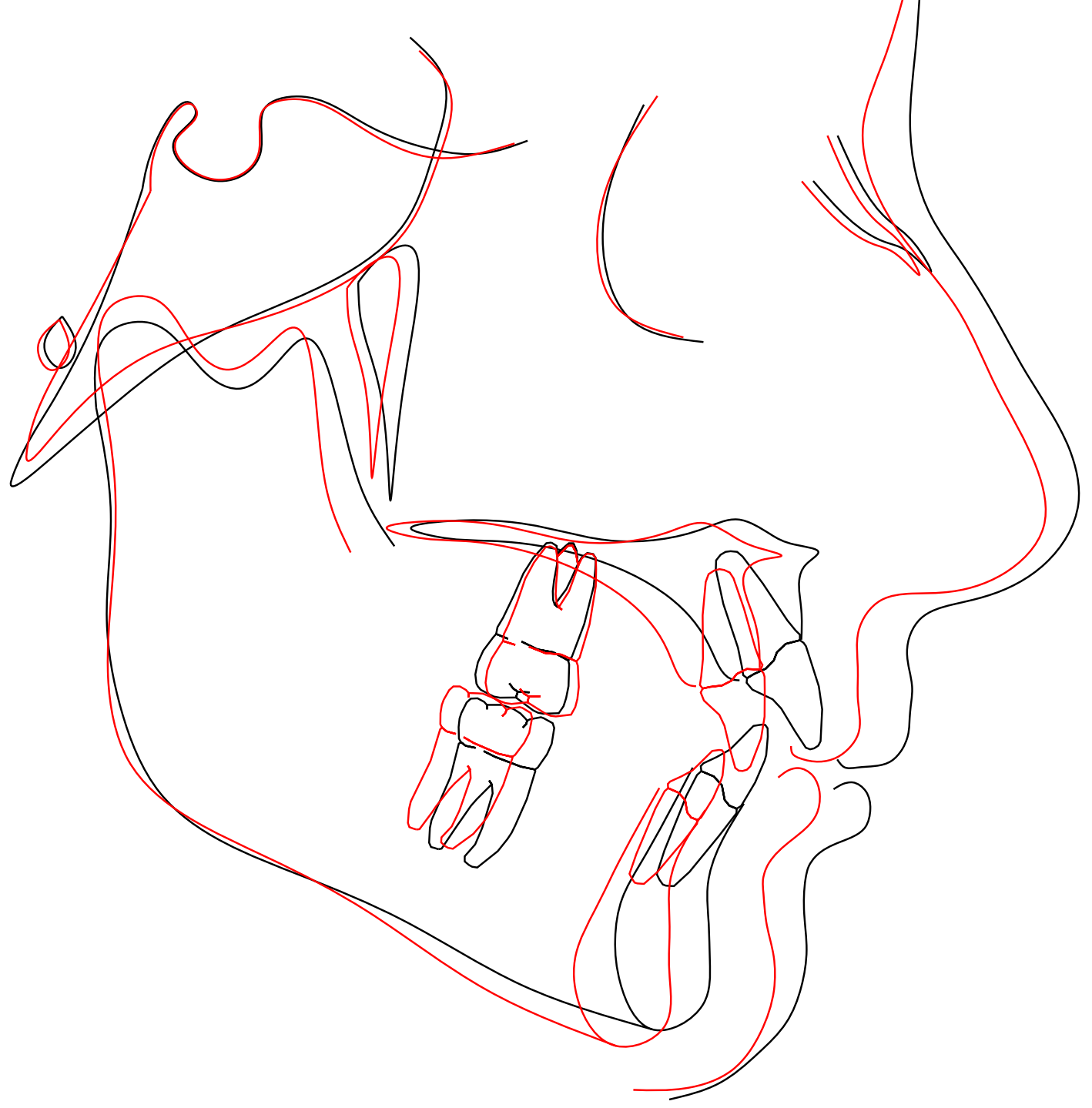

Figure B-28. Print of cephalometric tracing superimposition subject 28: (tracing is 1:1 with original radiograph). 


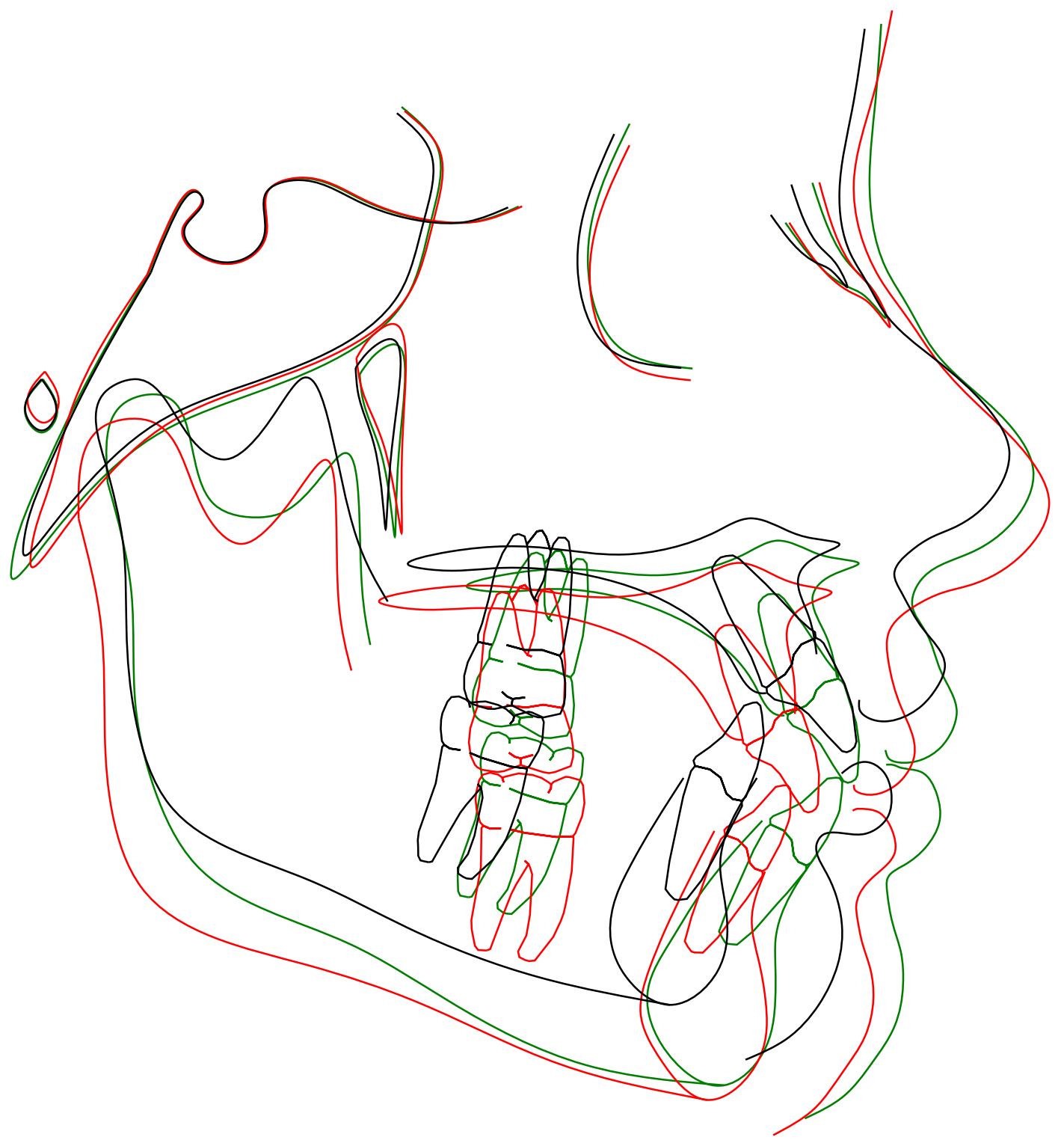

Figure B-29. Print of cephalometric tracing superimposition subject 29: (tracing is $1: 1$ with original radiograph). 


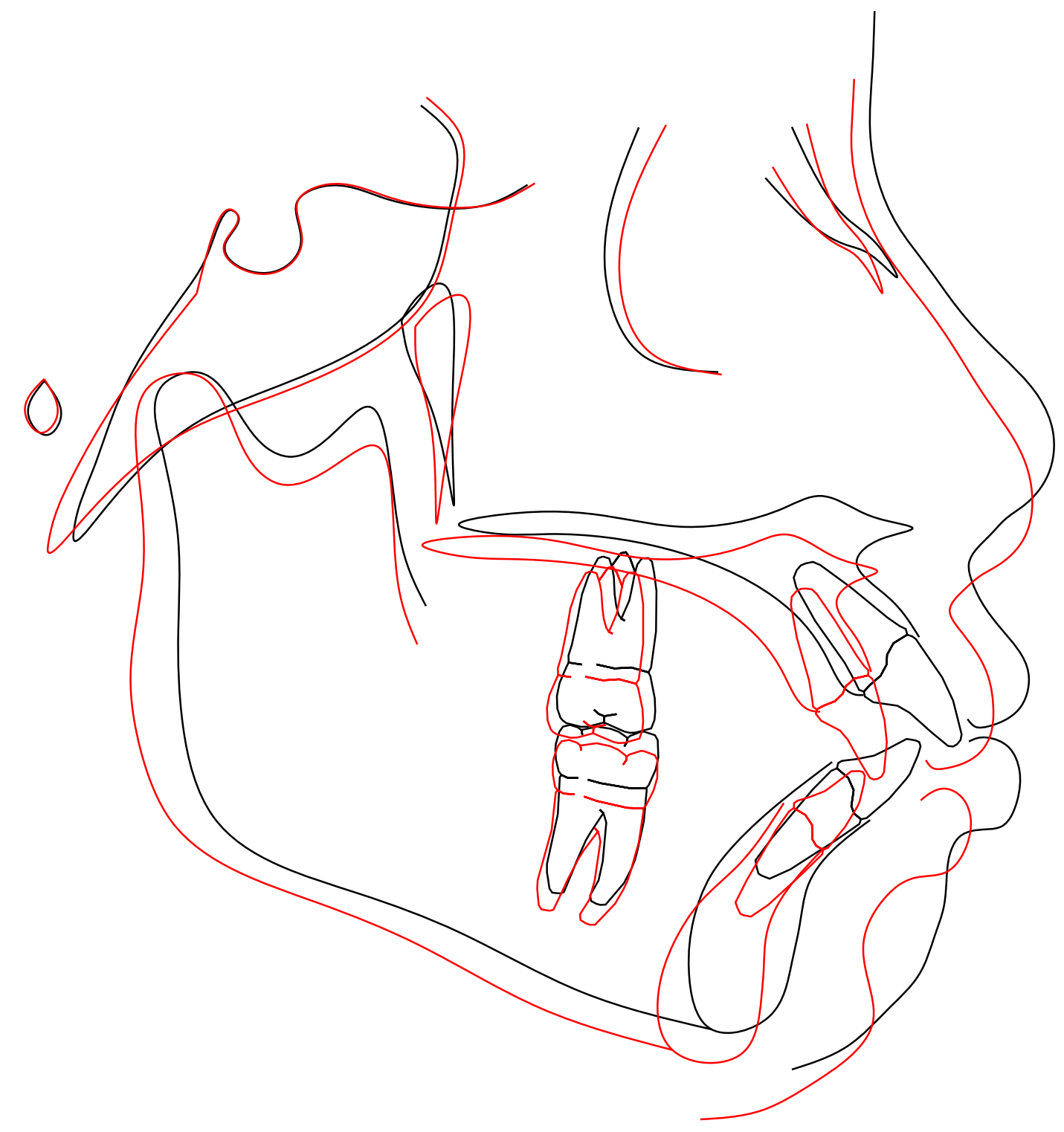

Figure B-30. Print of cephalometric tracing superimposition subject 30: (tracing is 1:1 with original radiograph). 


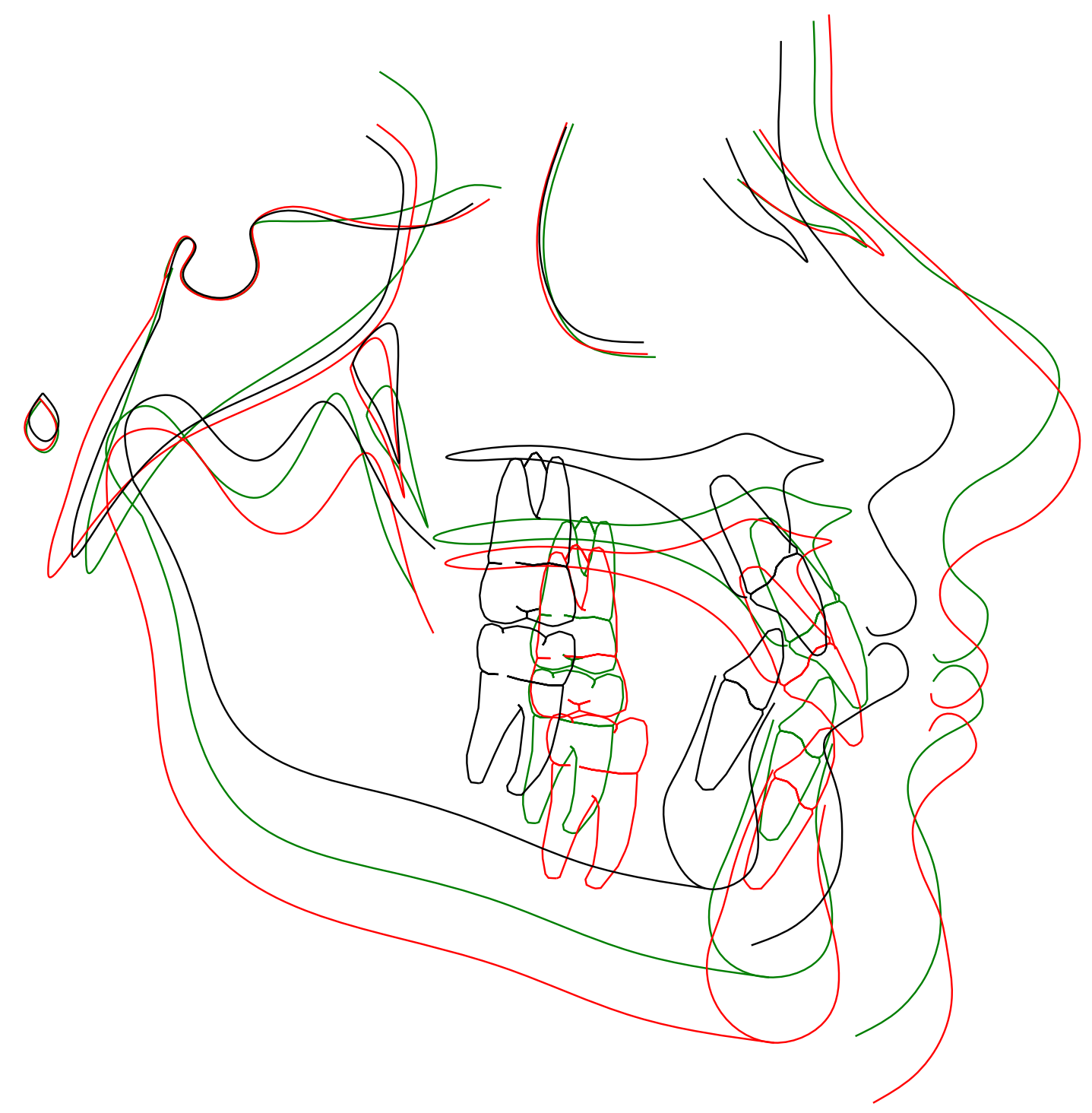

Figure B-31. Print of cephalometric tracing superimposition subject 31: (tracing is $1: 1$ with original radiograph). 


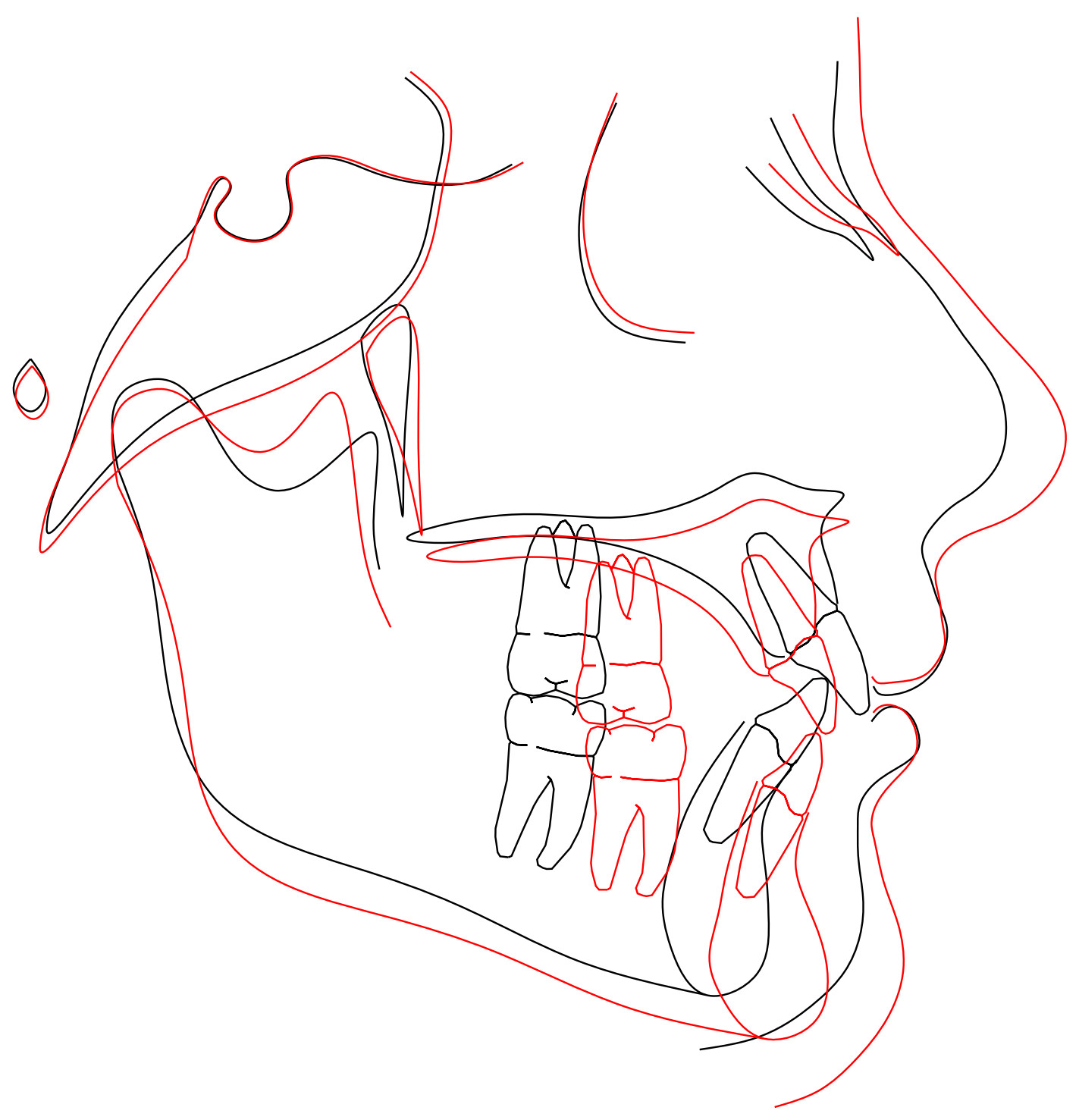

Figure B-32. Print of cephalometric tracing superimposition subject 32: (tracing is $1: 1$ with original radiograph). 


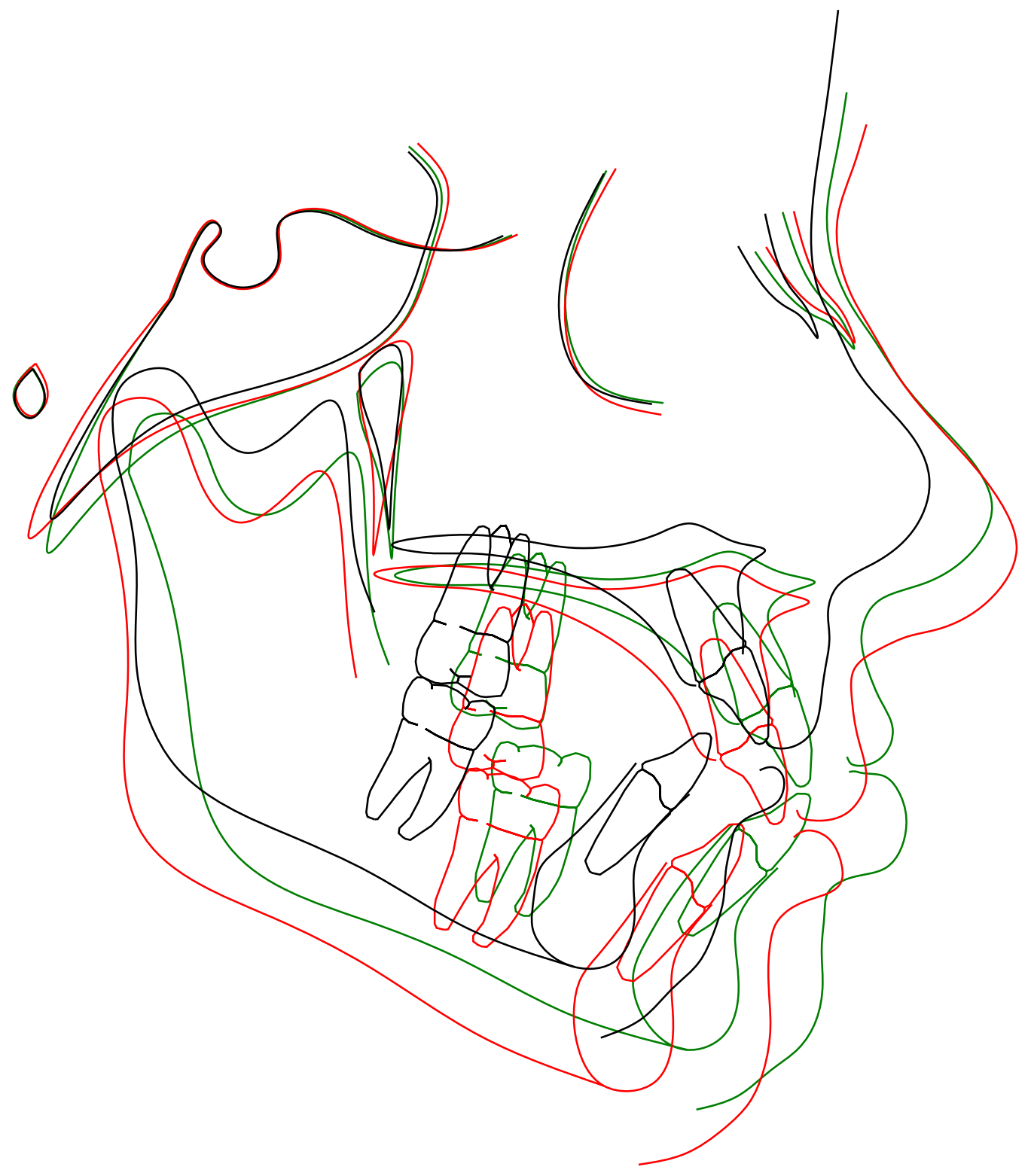

Figure B-33. Print of cephalometric tracing superimposition subject 33: (tracing is 1:1 with original radiograph). 


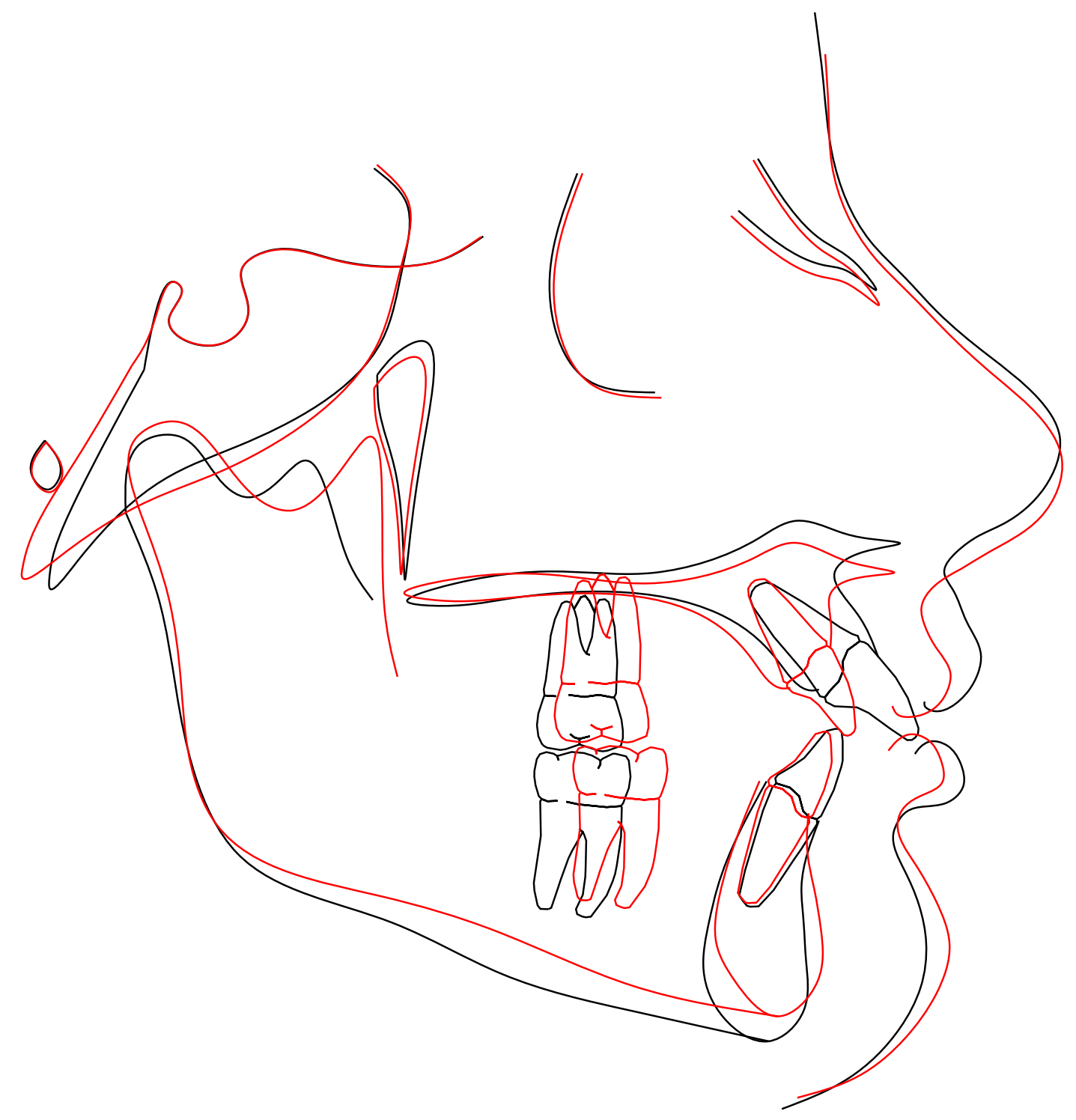

Figure B-34. Print of cephalometric tracing superimposition subject 34: (tracing is 1:1 with original radiograph). 


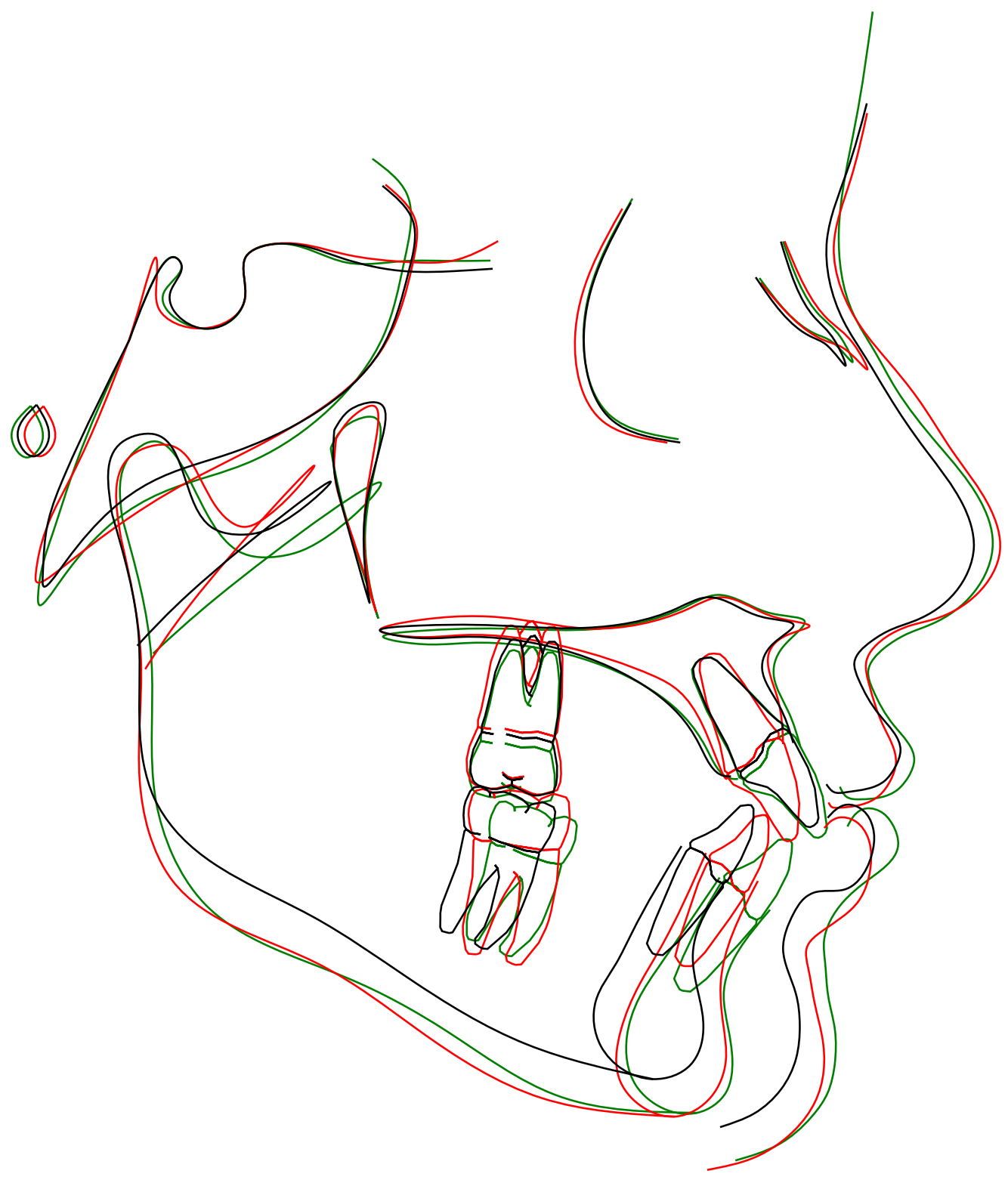

Figure B-35. Print of cephalometric tracing superimposition subject 35: (tracing is $1: 1$ with original radiograph). 


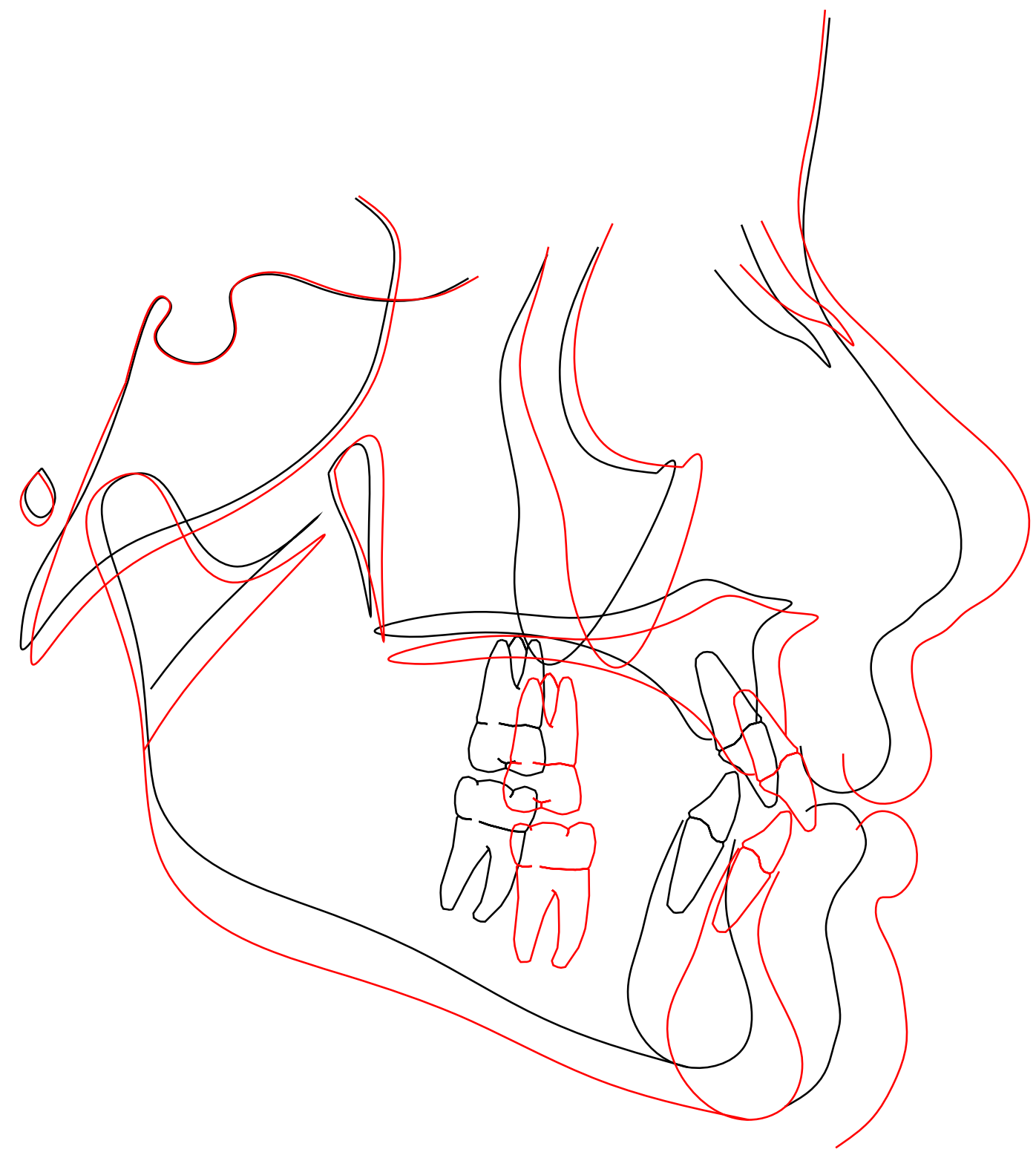

Figure B-36. Print of cephalometric tracing superimposition subject 36: (tracing is 1:1 with original radiograph). 


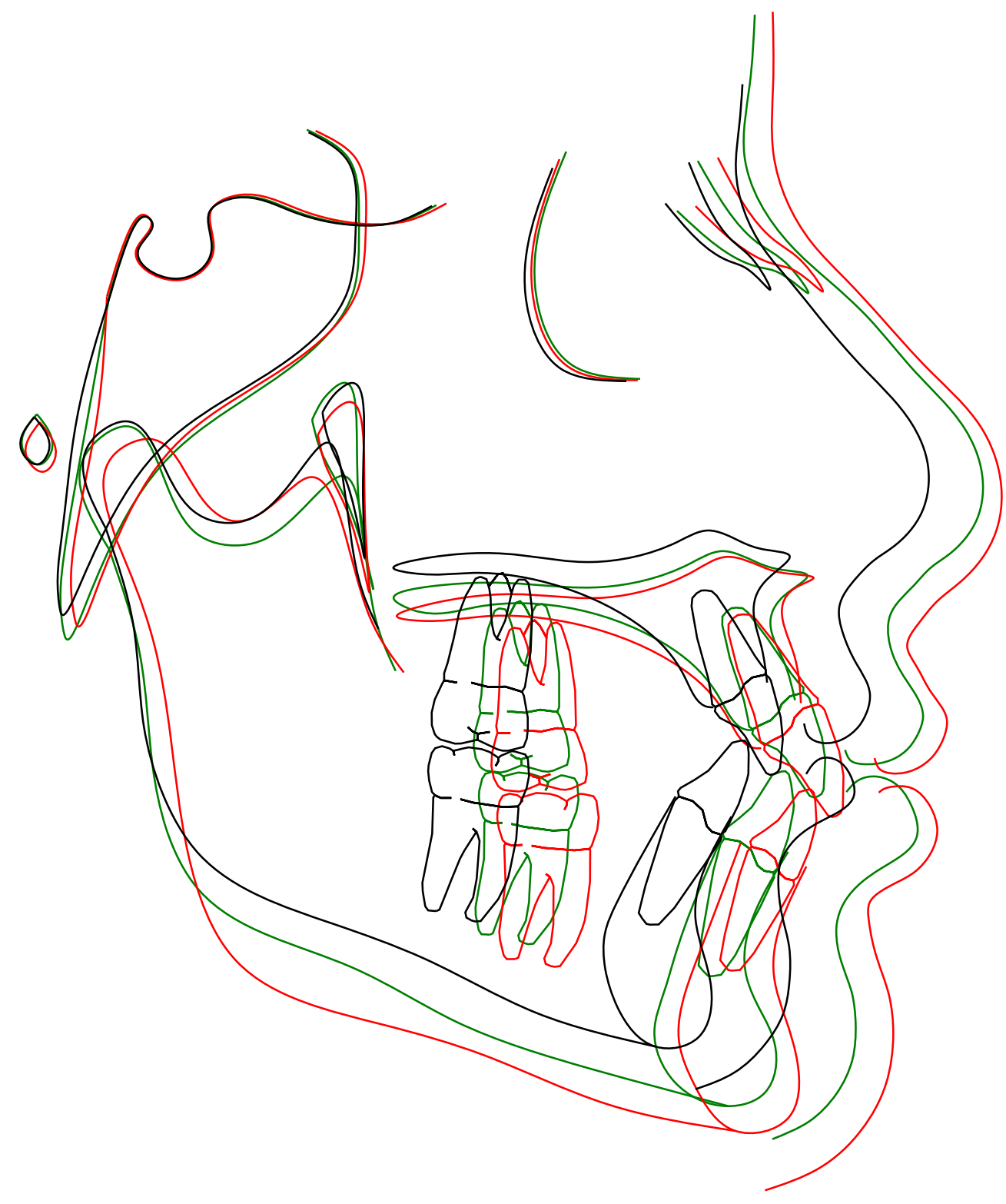

Figure B-37. Print of cephalometric tracing superimposition subject 37: (tracing is 1:1 with original radiograph). 


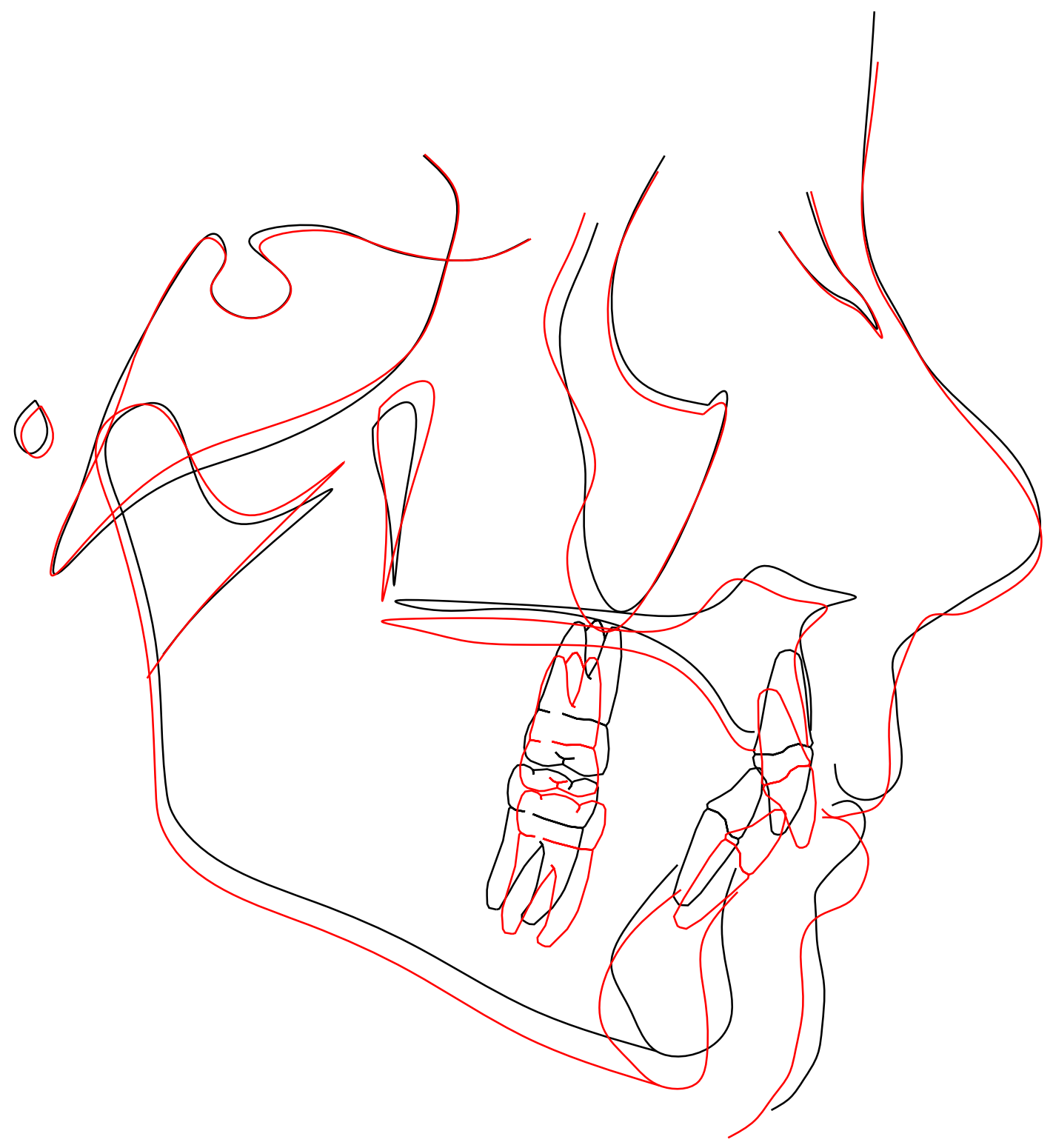

Figure B-38. Print of cephalometric tracing superimposition subject 38: (tracing is 1:1 with original radiograph). 


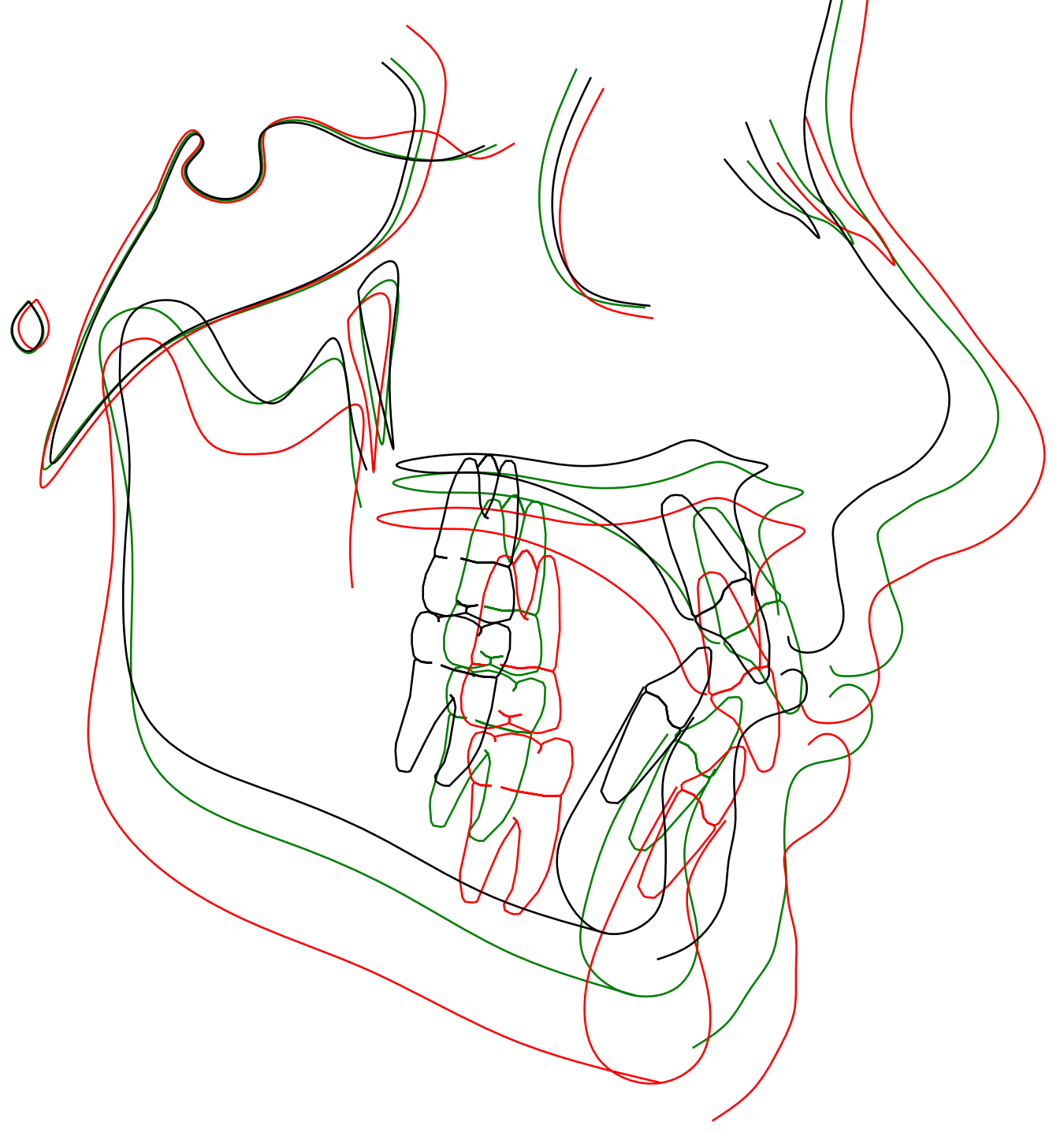

Figure B-39. Print of cephalometric tracing superimposition subject 39: (tracing is 1:1 with original radiograph). 


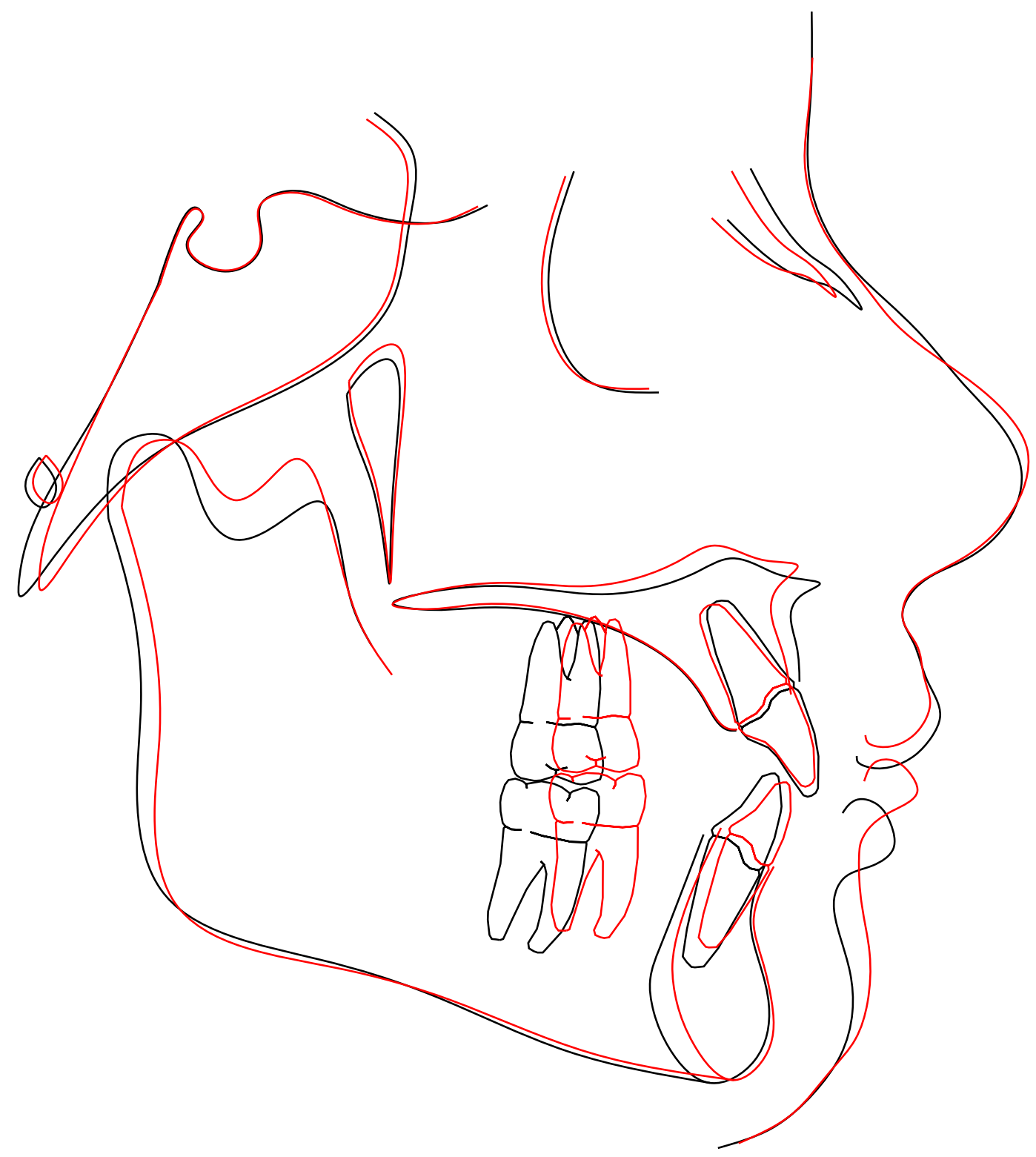

Figure B-40. Print of cephalometric tracing superimposition subject 40: (tracing is 1:1 with original radiograph). 


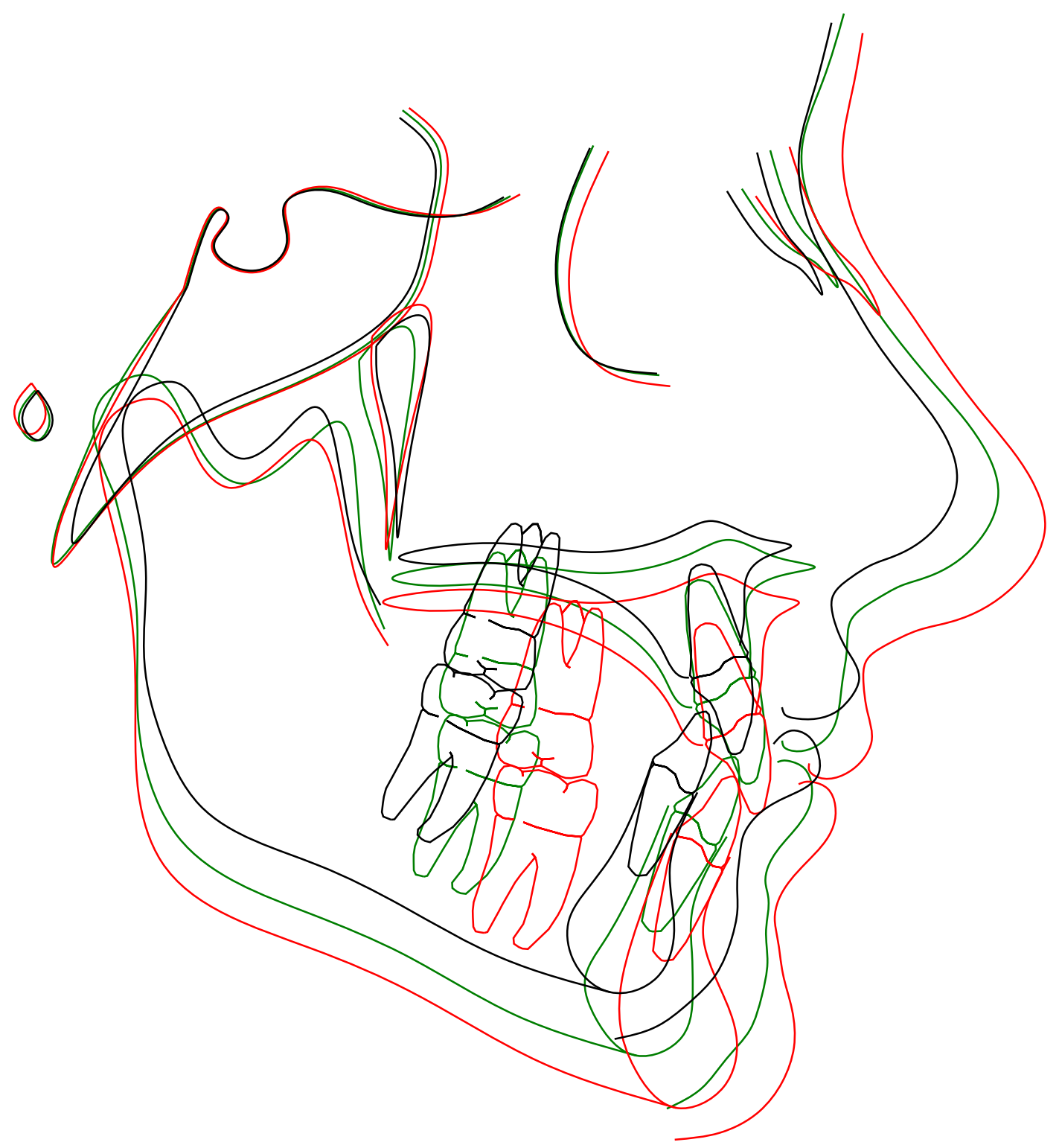

Figure B-41. Print of cephalometric tracing superimposition subject 41: (tracing is 1:1 with original radiograph). 


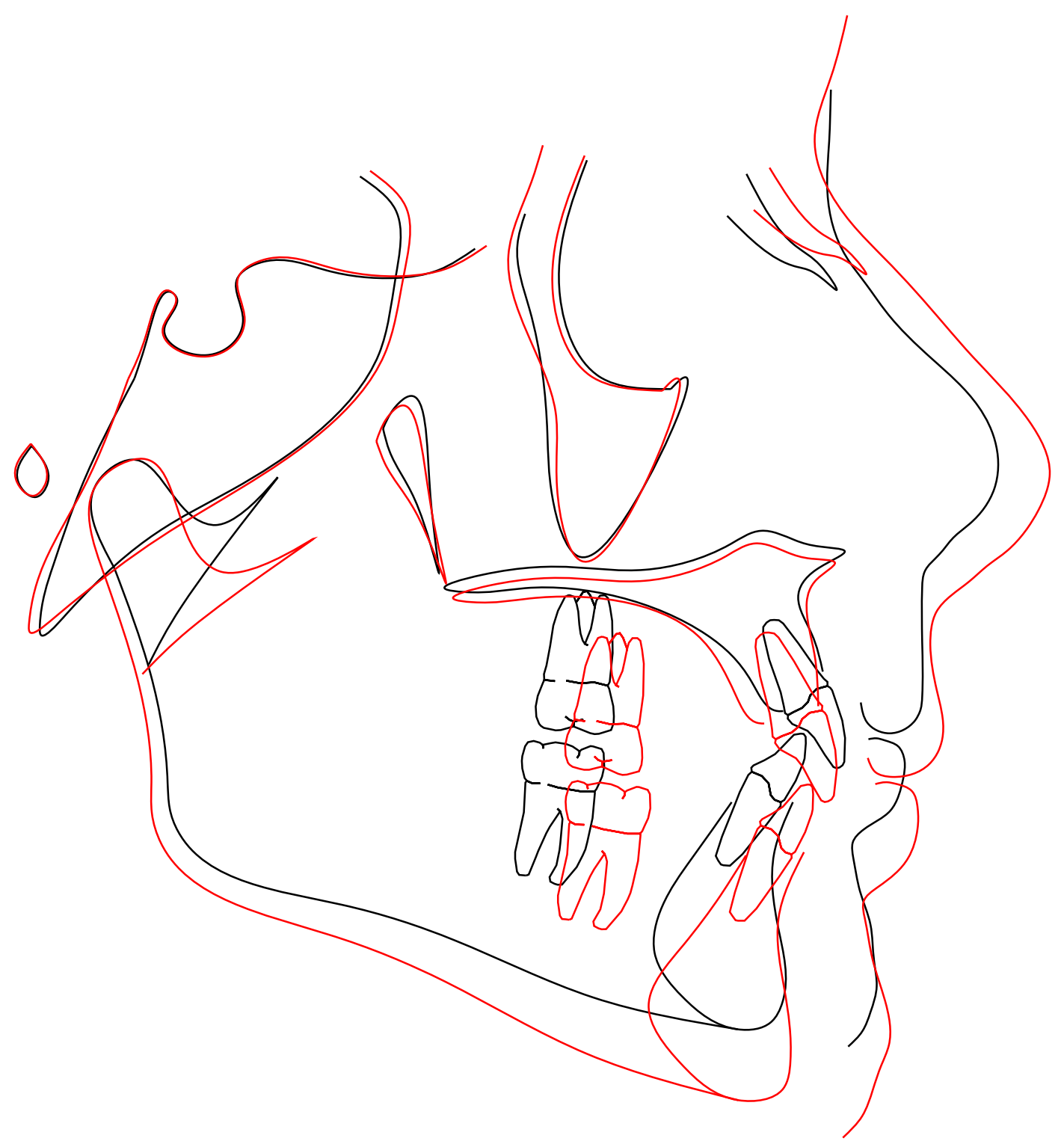

Figure B-42. Print of cephalometric tracing superimposition subject 42: (tracing is $1: 1$ with original radiograph). 


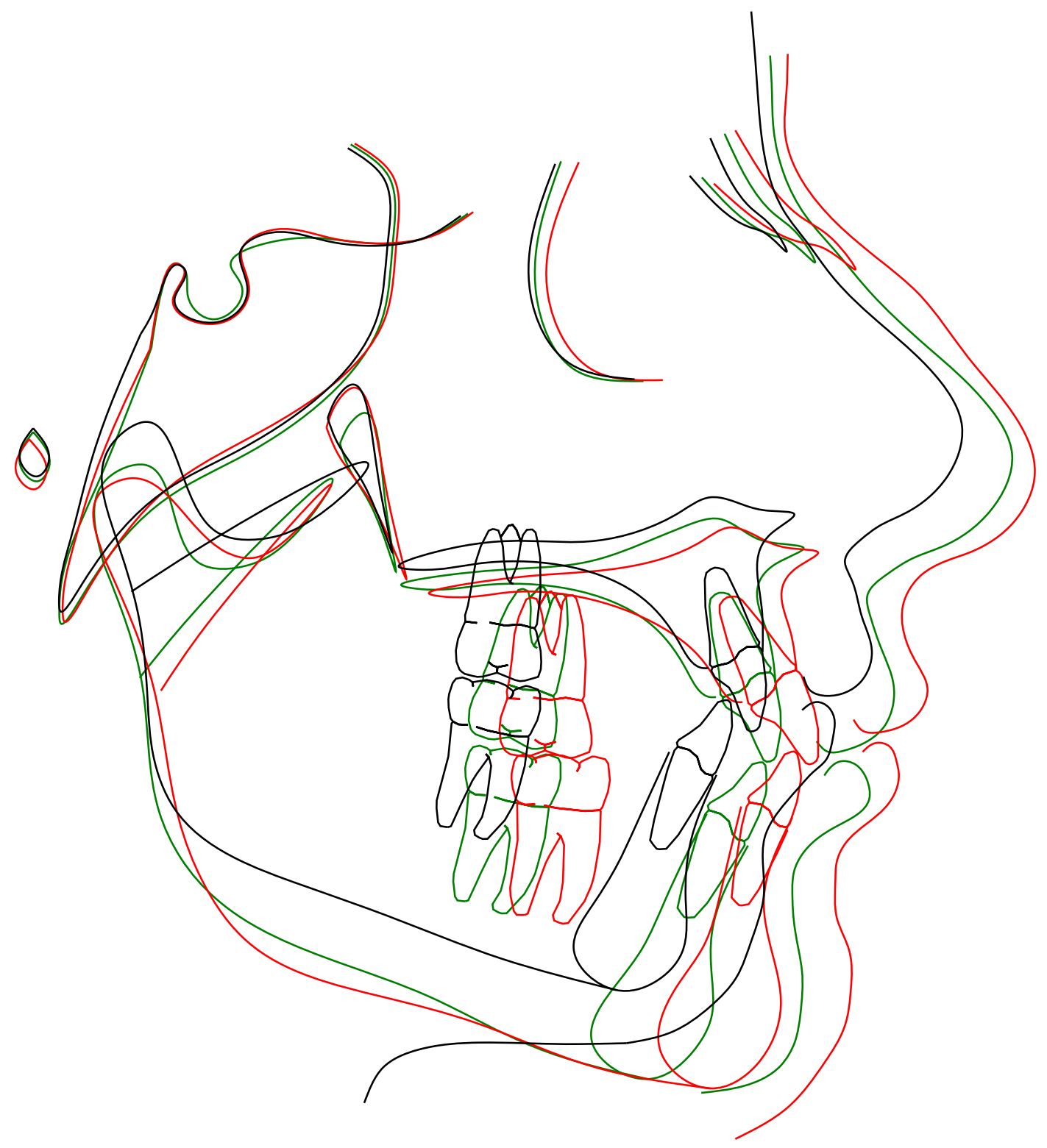

Figure B-43. Print of cephalometric tracing superimposition subject 43: (tracing is 1:1 with original radiograph). 


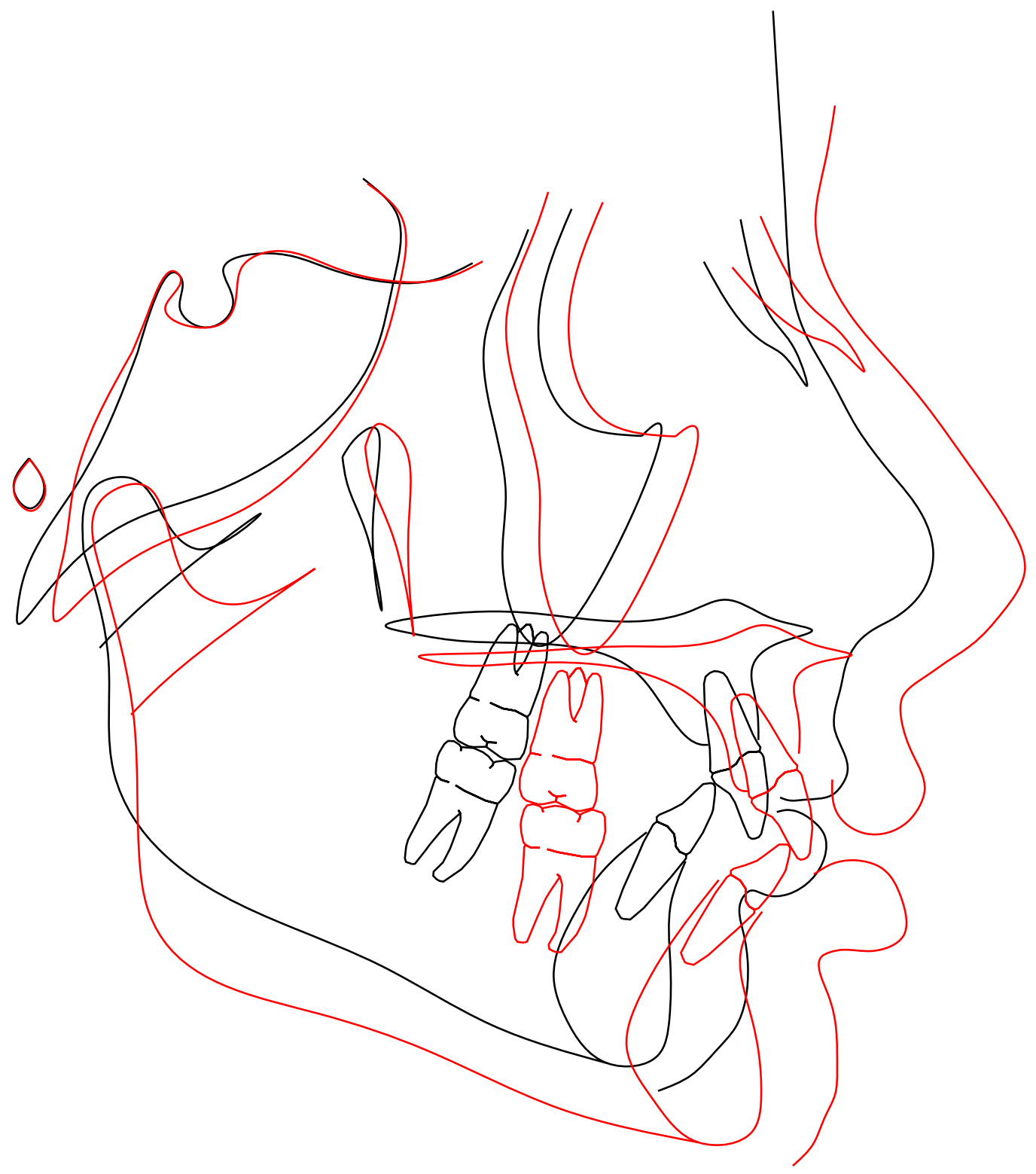

Figure B-44. Print of cephalometric tracing superimposition subject 44: (tracing is 1:1 with original radiograph). 


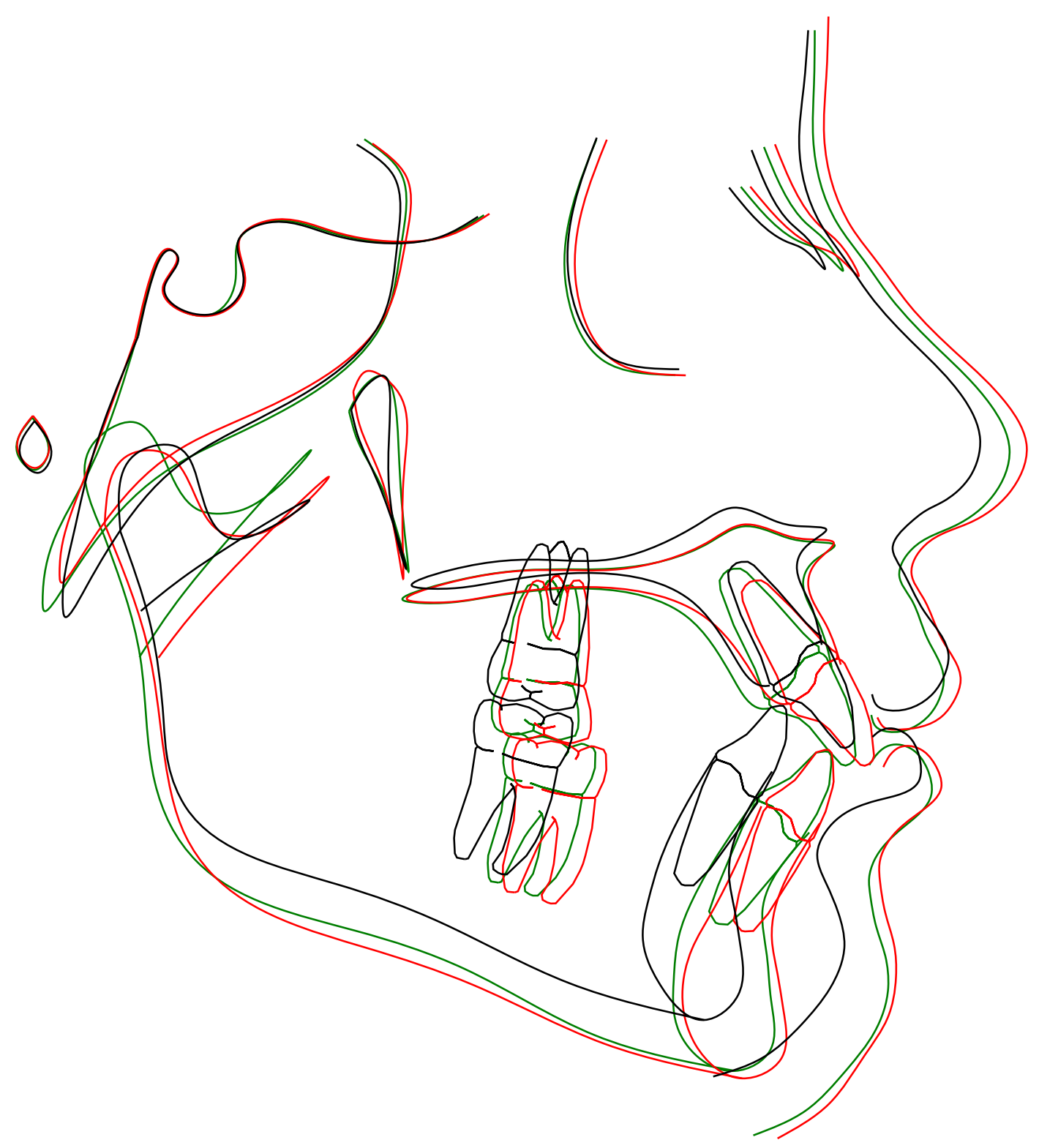

Figure B-45. Print of cephalometric tracing superimposition subject 45: (tracing is 1:1 with original radiograph). 


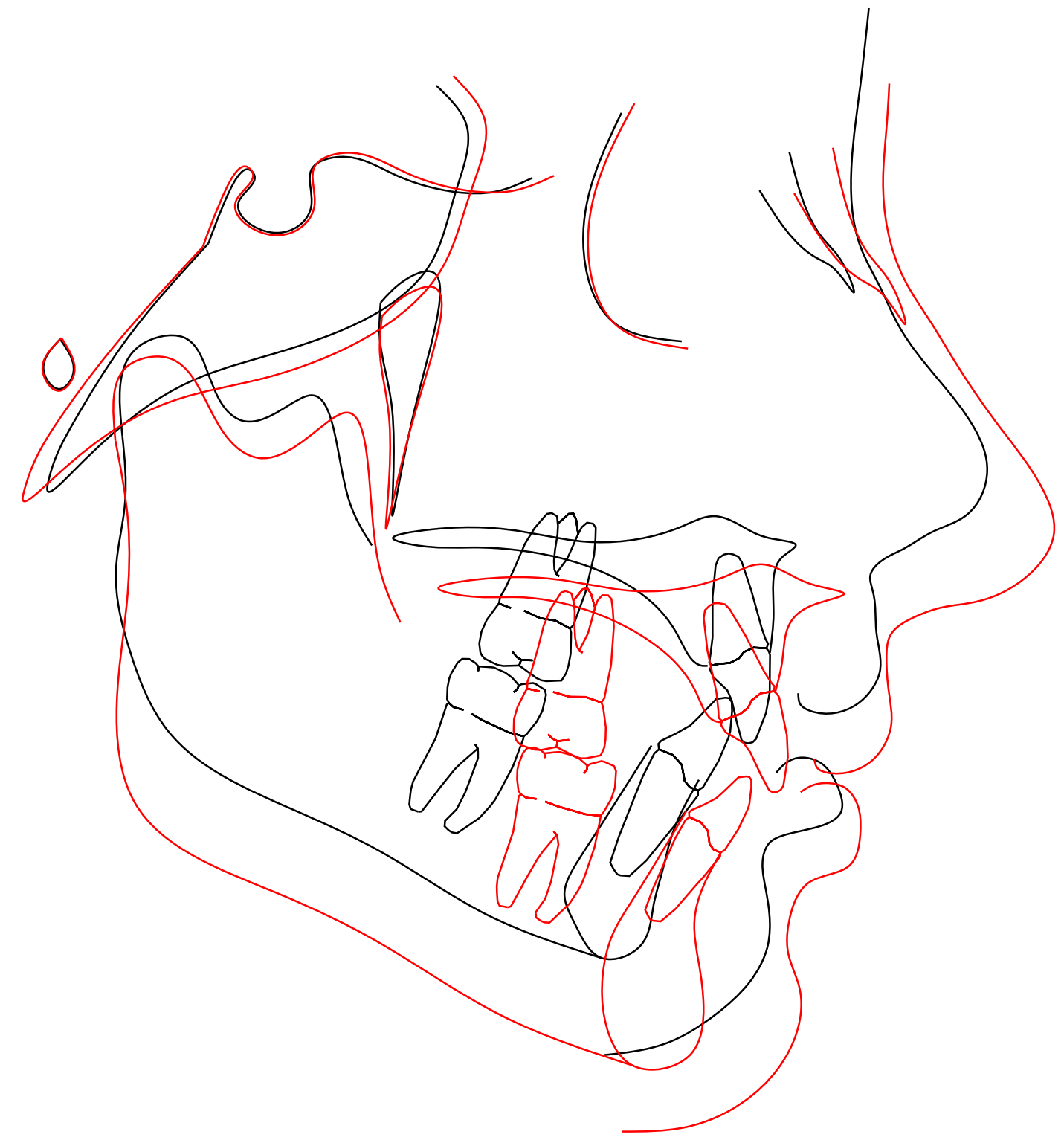

Figure B-46. Print of cephalometric tracing superimposition subject 46: (tracing is 1:1 with original radiograph). 


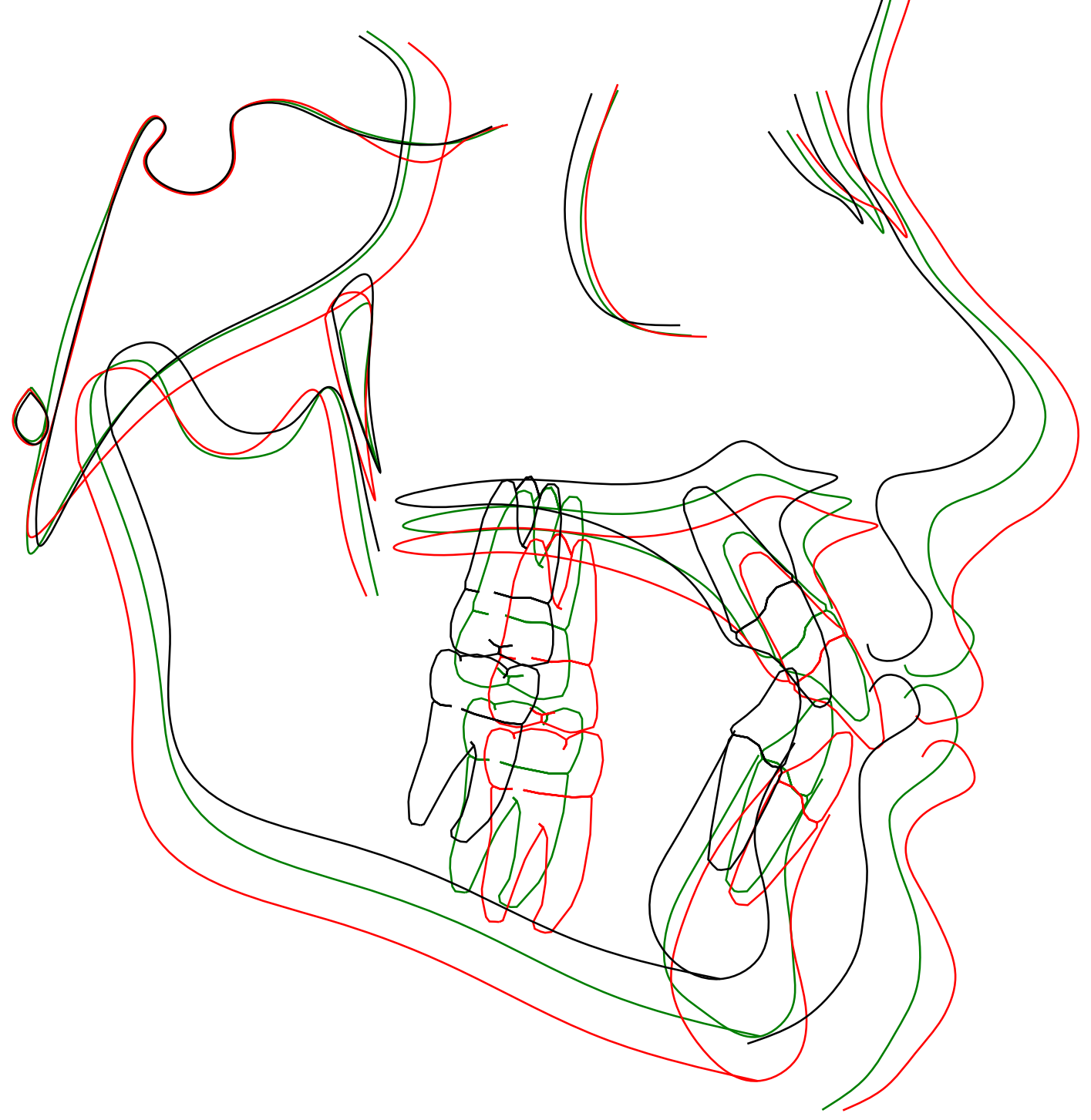

Figure B-47. Print of cephalometric tracing superimposition subject 47: (tracing is 1:1 with original radiograph). 


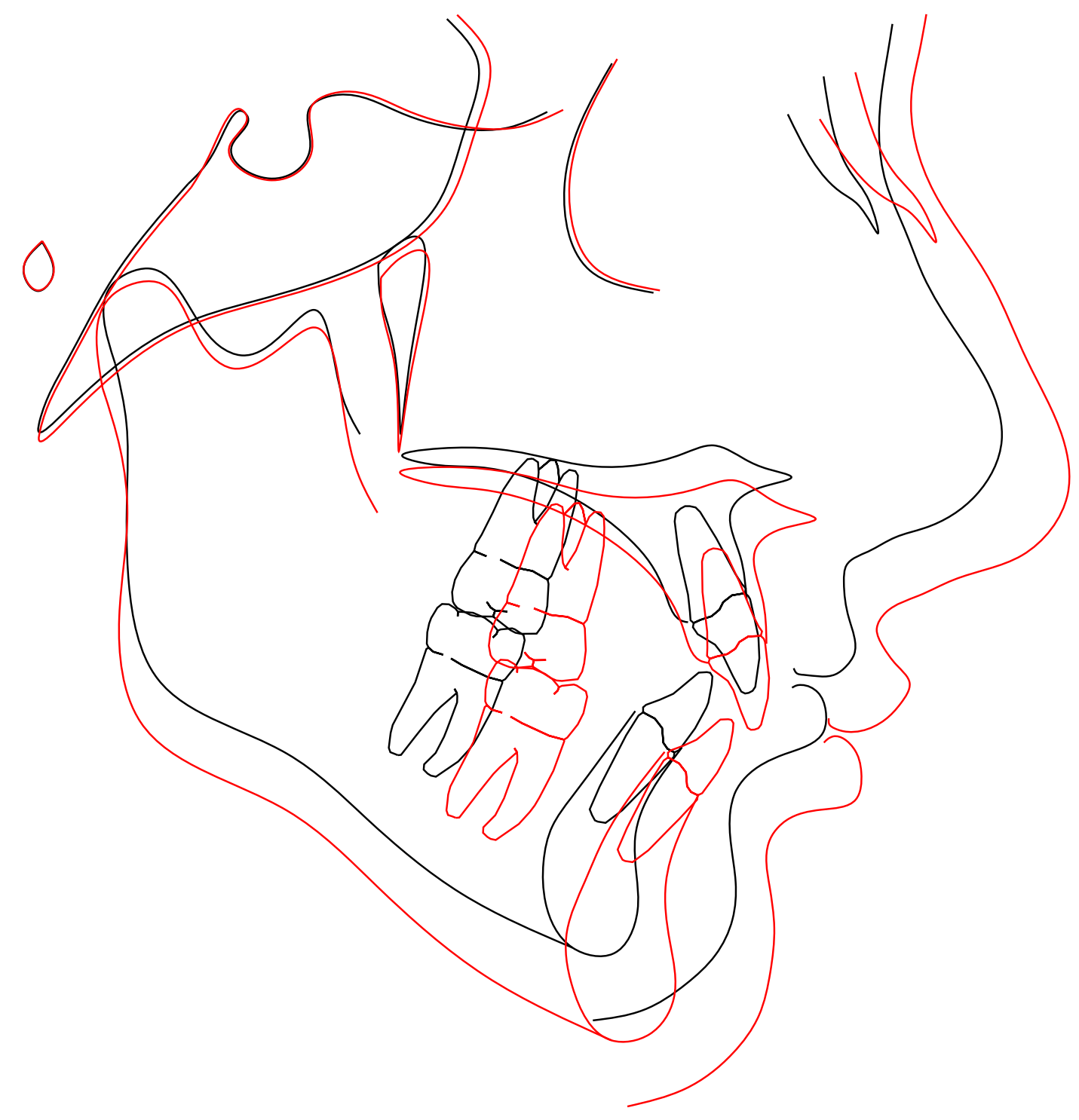

Figure B-48. Print of cephalometric tracing superimposition subject 48: (tracing is 1:1 with original radiograph). 


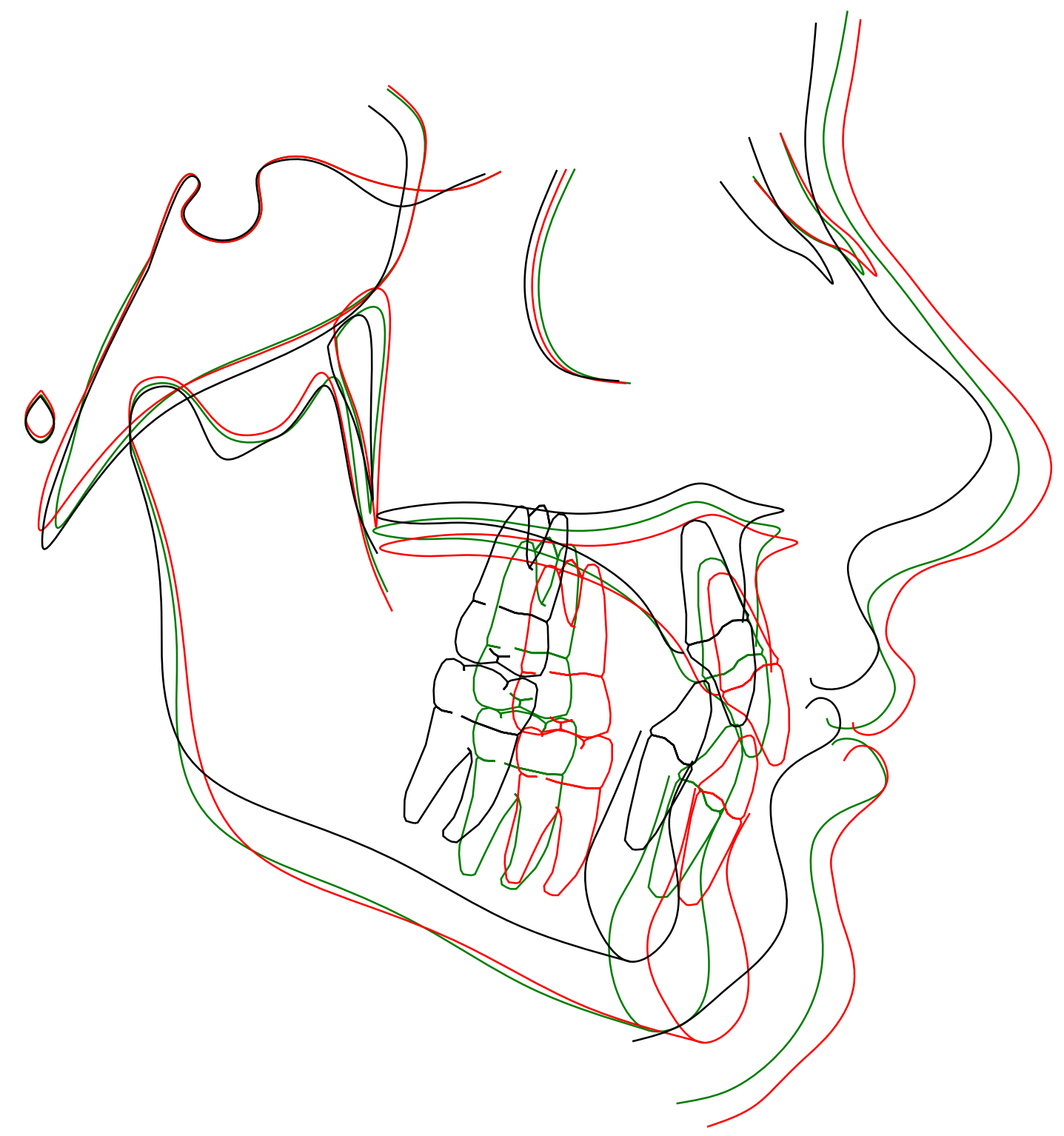

Figure B-49. Print of cephalometric tracing superimposition subject 49: (tracing is 1:1 with original radiograph). 


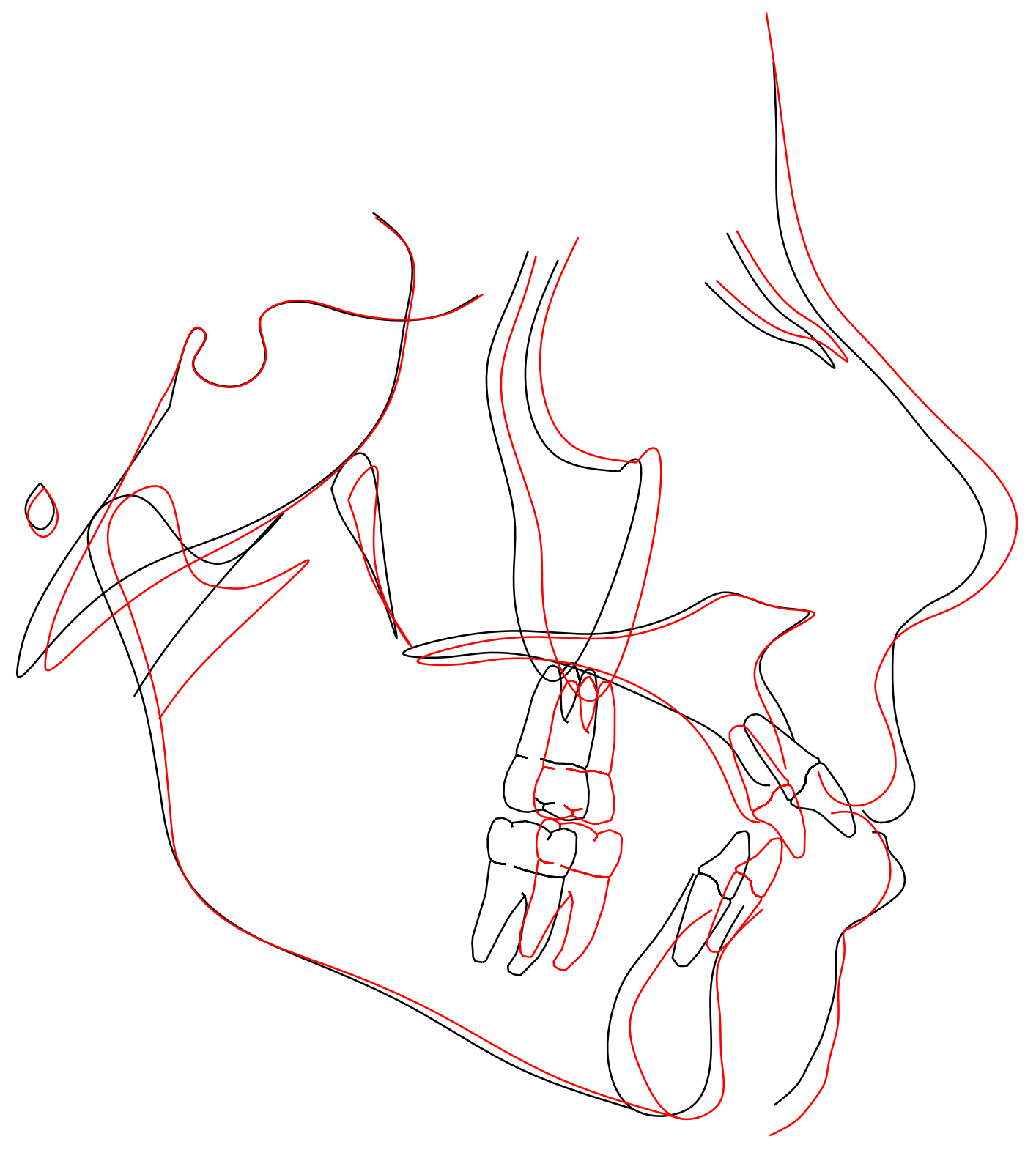

Figure B-50. Print of cephalometric tracing superimposition subject 50: (tracing is 1:1 with original radiograph). 


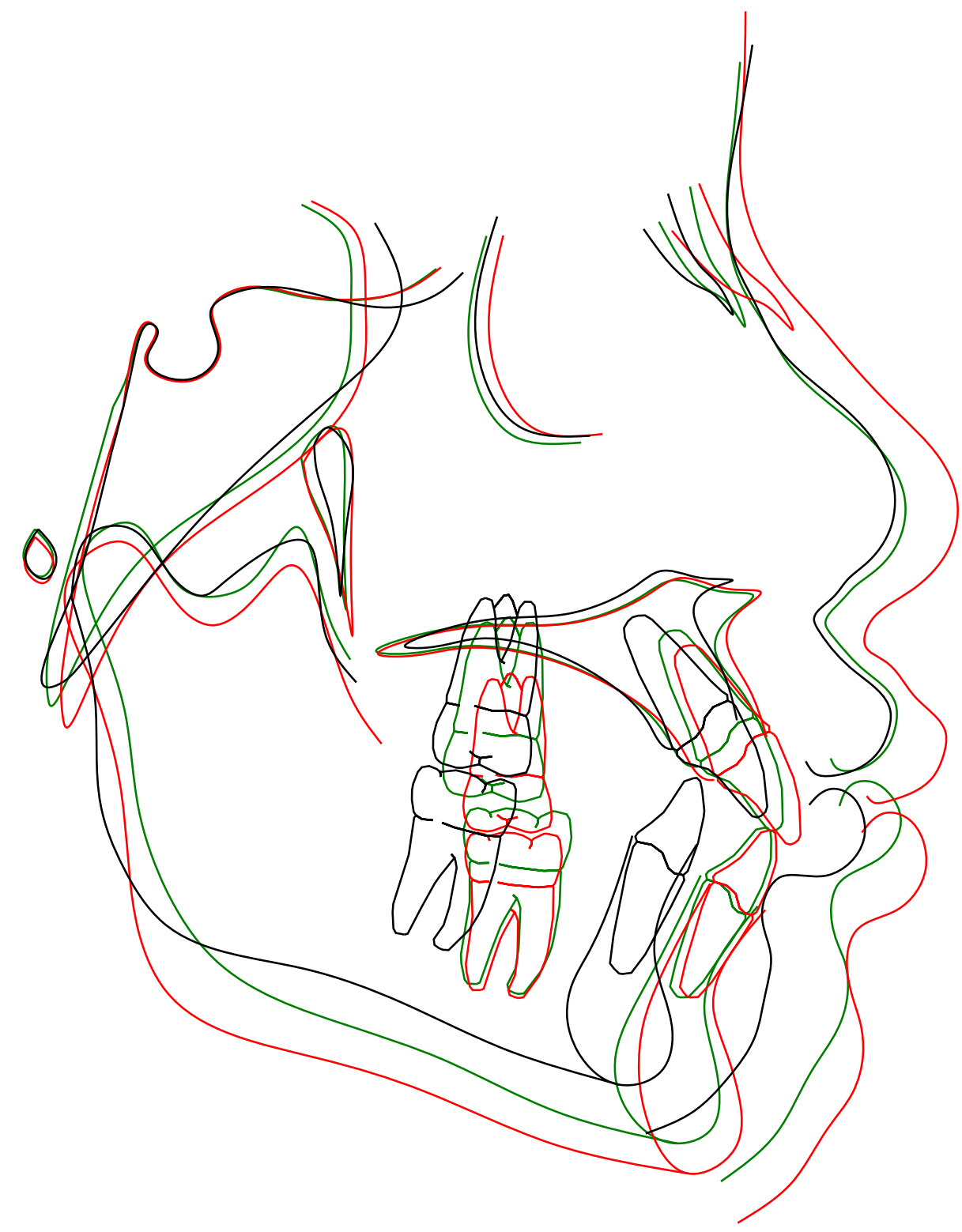

Figure B-51. Print of cephalometric tracing superimposition subject 51: (tracing is $1: 1$ with original radiograph). 


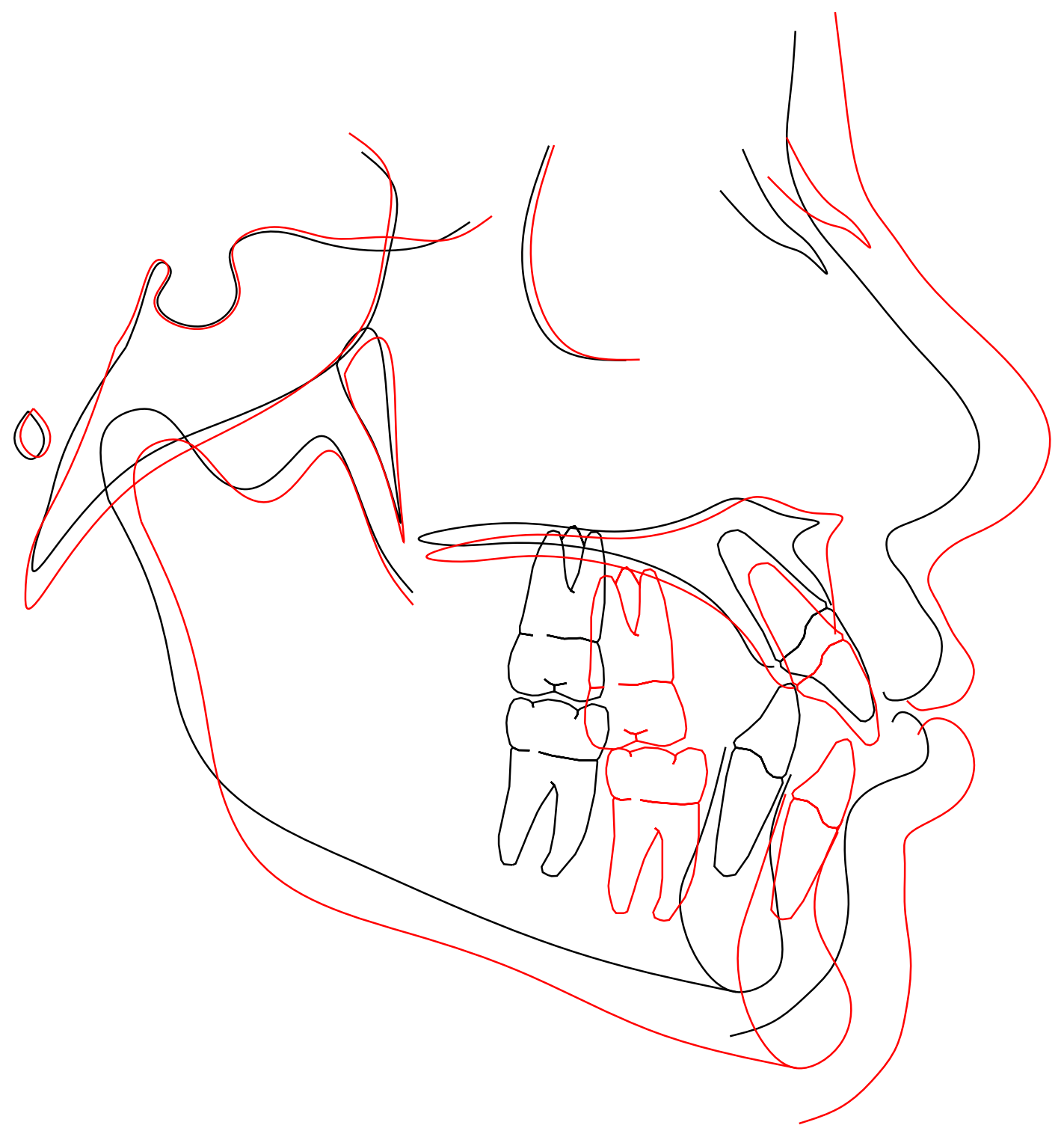

Figure B-52. Print of cephalometric tracing superimposition subject 52: (tracing is 1:1 with original radiograph). 


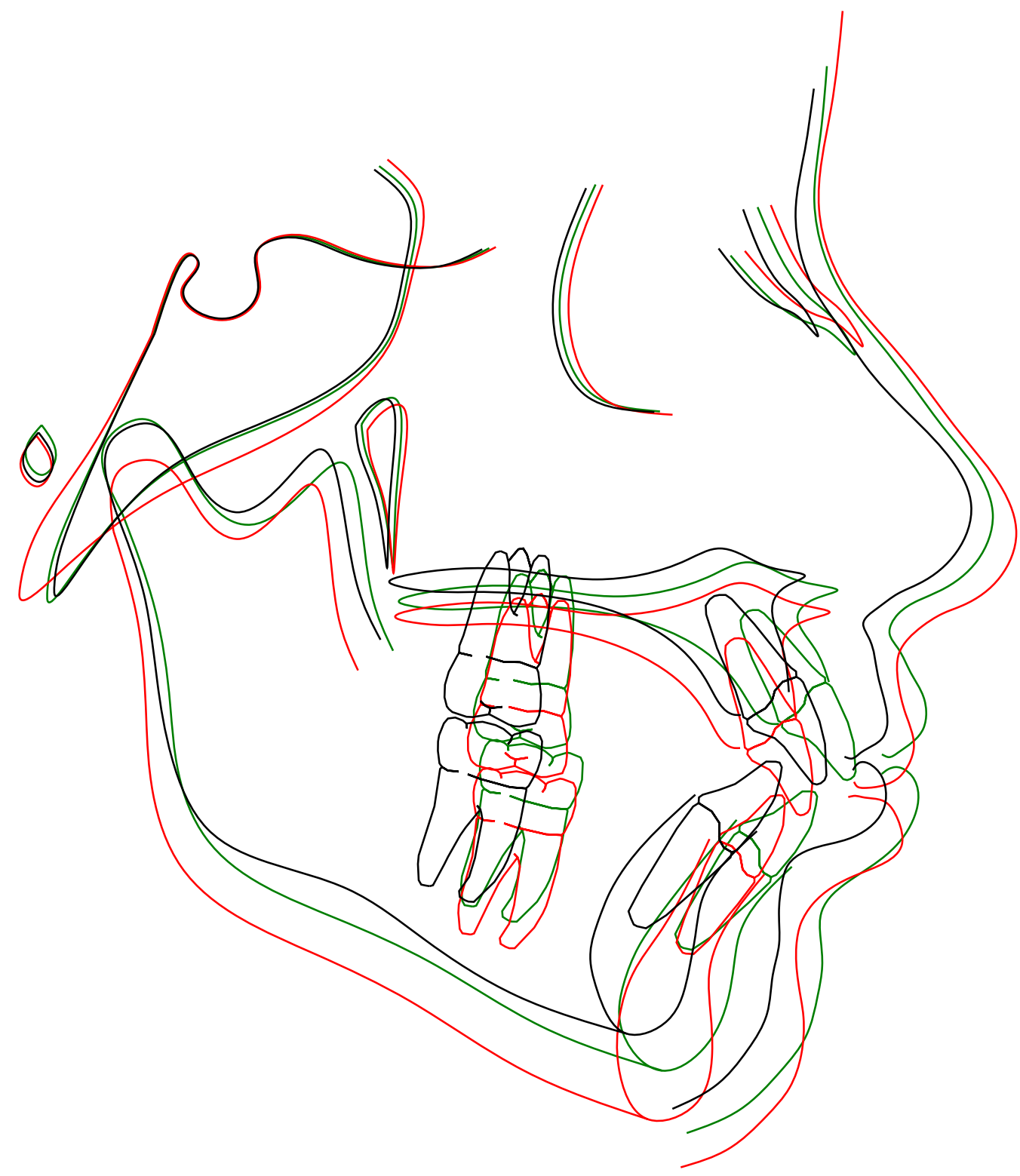

Figure B-53. Print of cephalometric tracing superimposition subject 53: (tracing is 1:1 with original radiograph). 


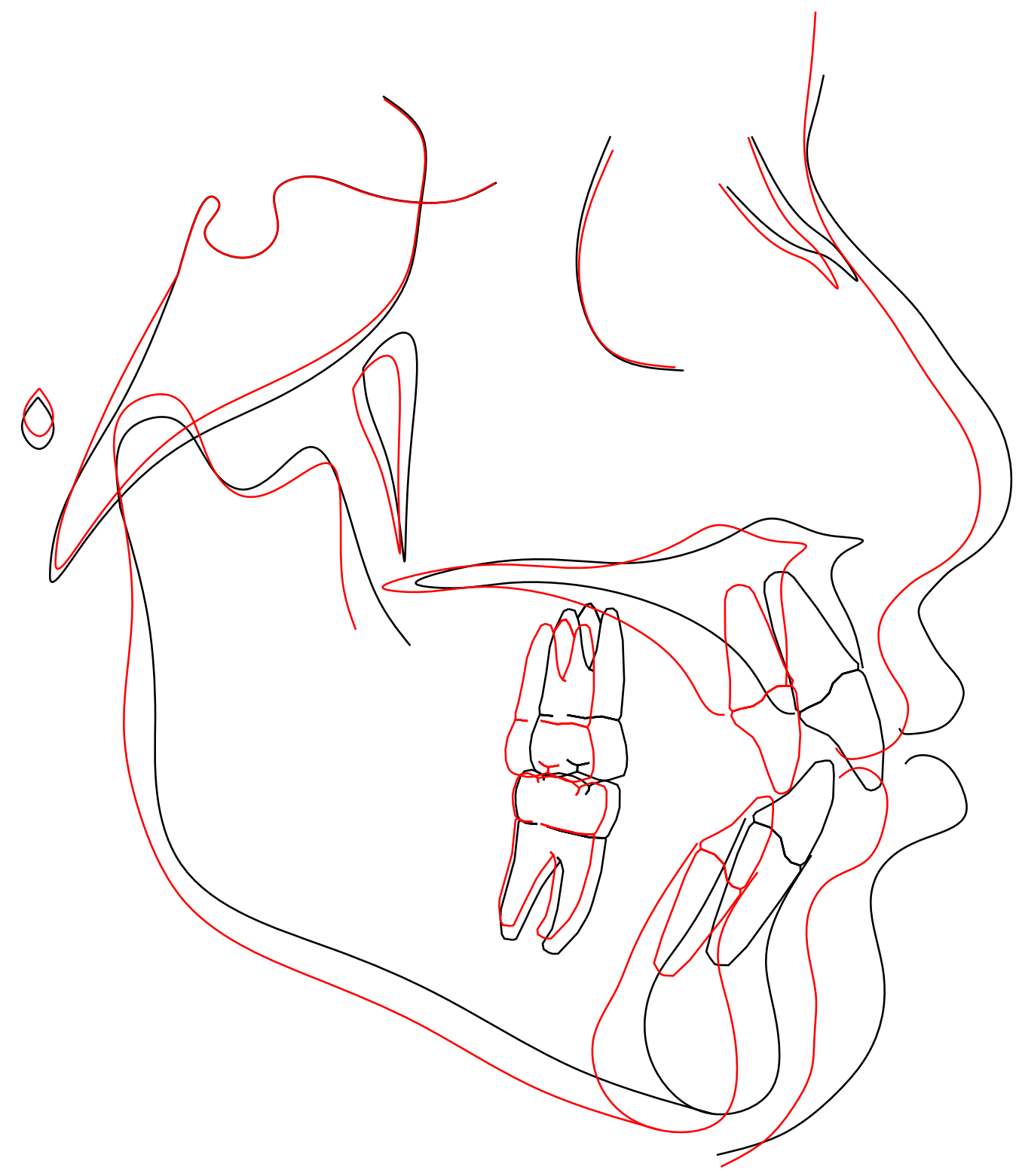

Figure B-54. Print of cephalometric tracing superimposition subject 54: (tracing is 1:1 with original radiograph). 


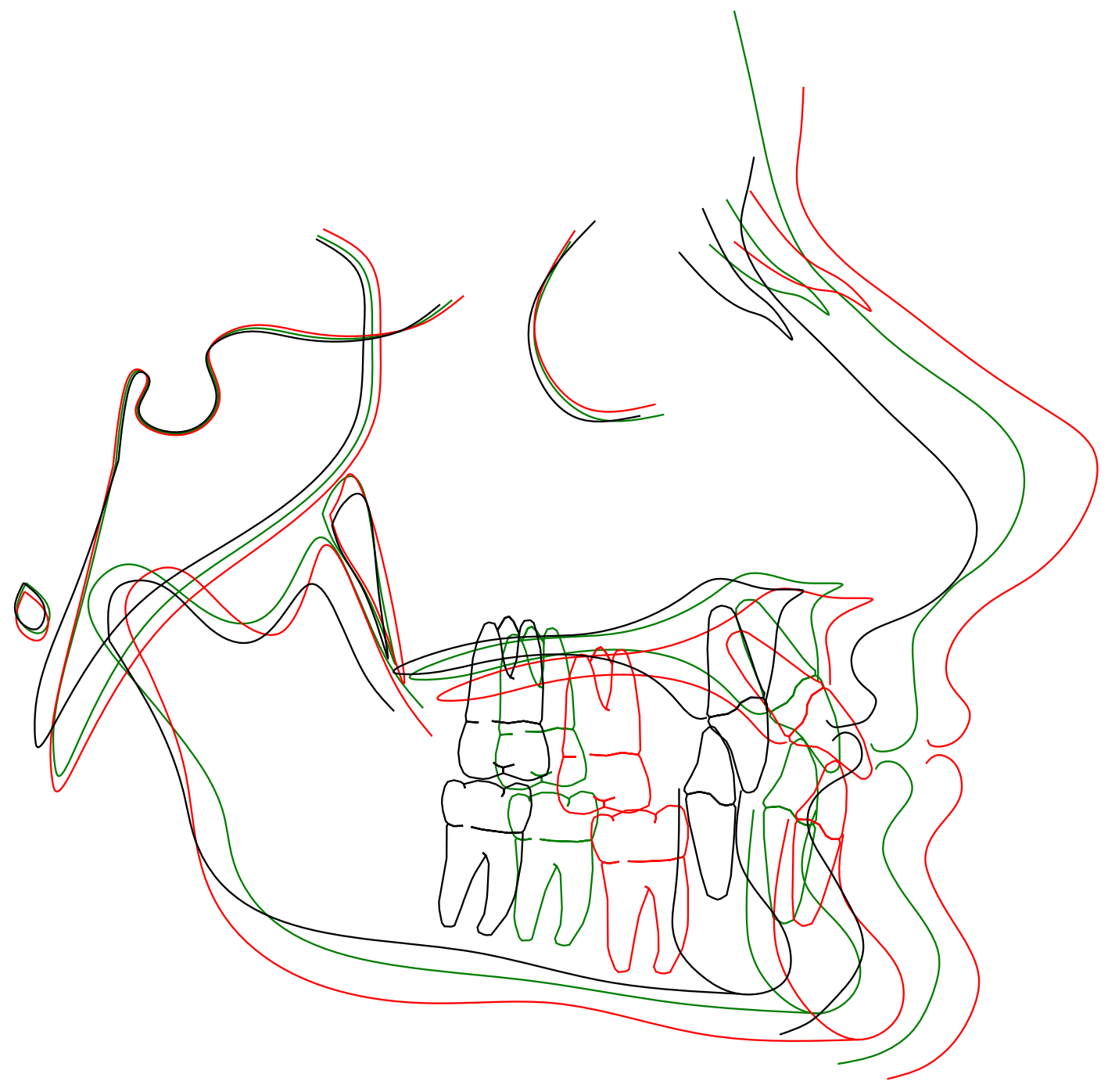

Figure B-55. Print of cephalometric tracing superimposition subject 55: (tracing is $1: 1$ with original radiograph). 


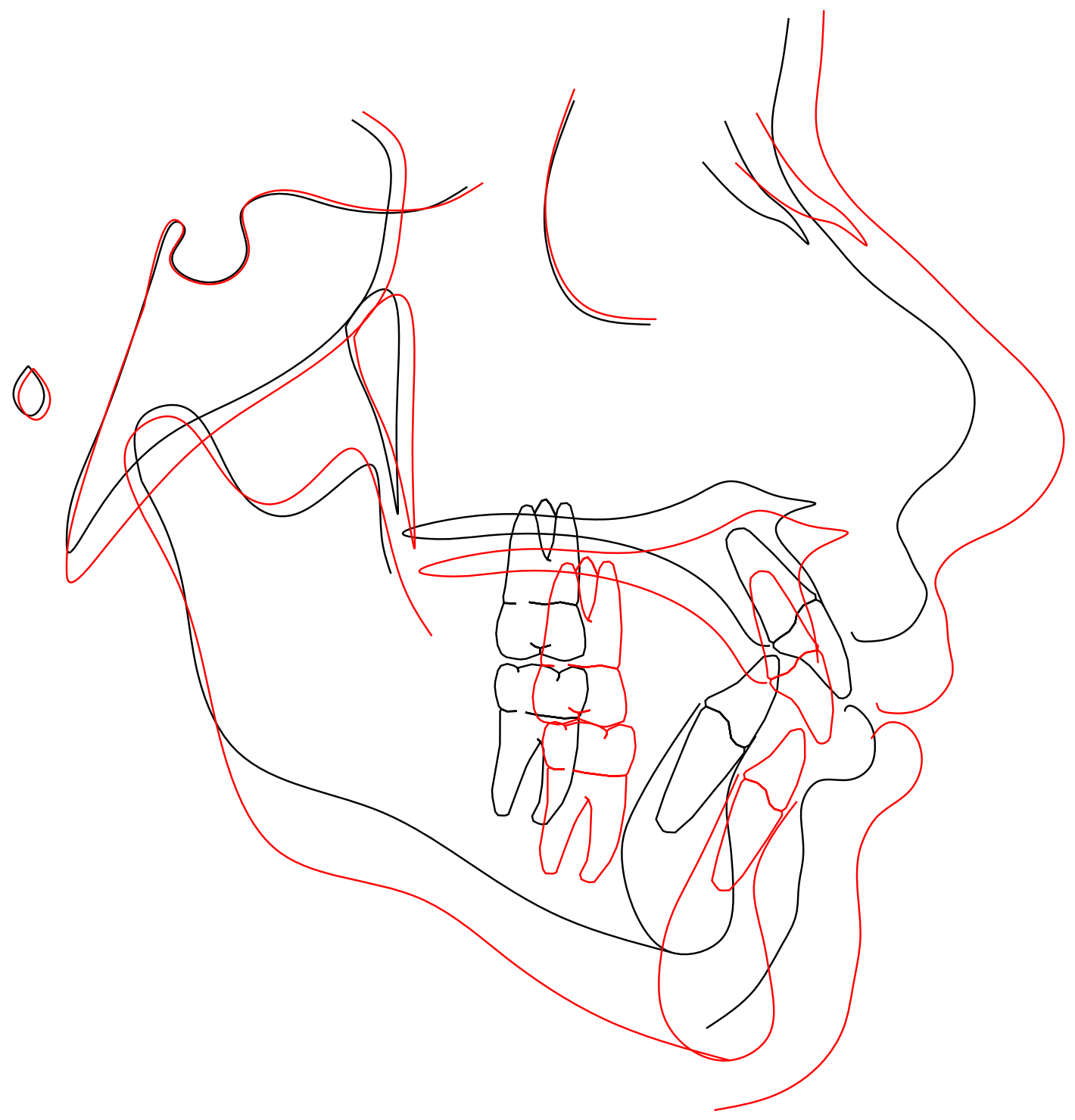

Figure B-56. Print of cephalometric tracing superimposition subject 56: (tracing is $1: 1$ with original radiograph). 


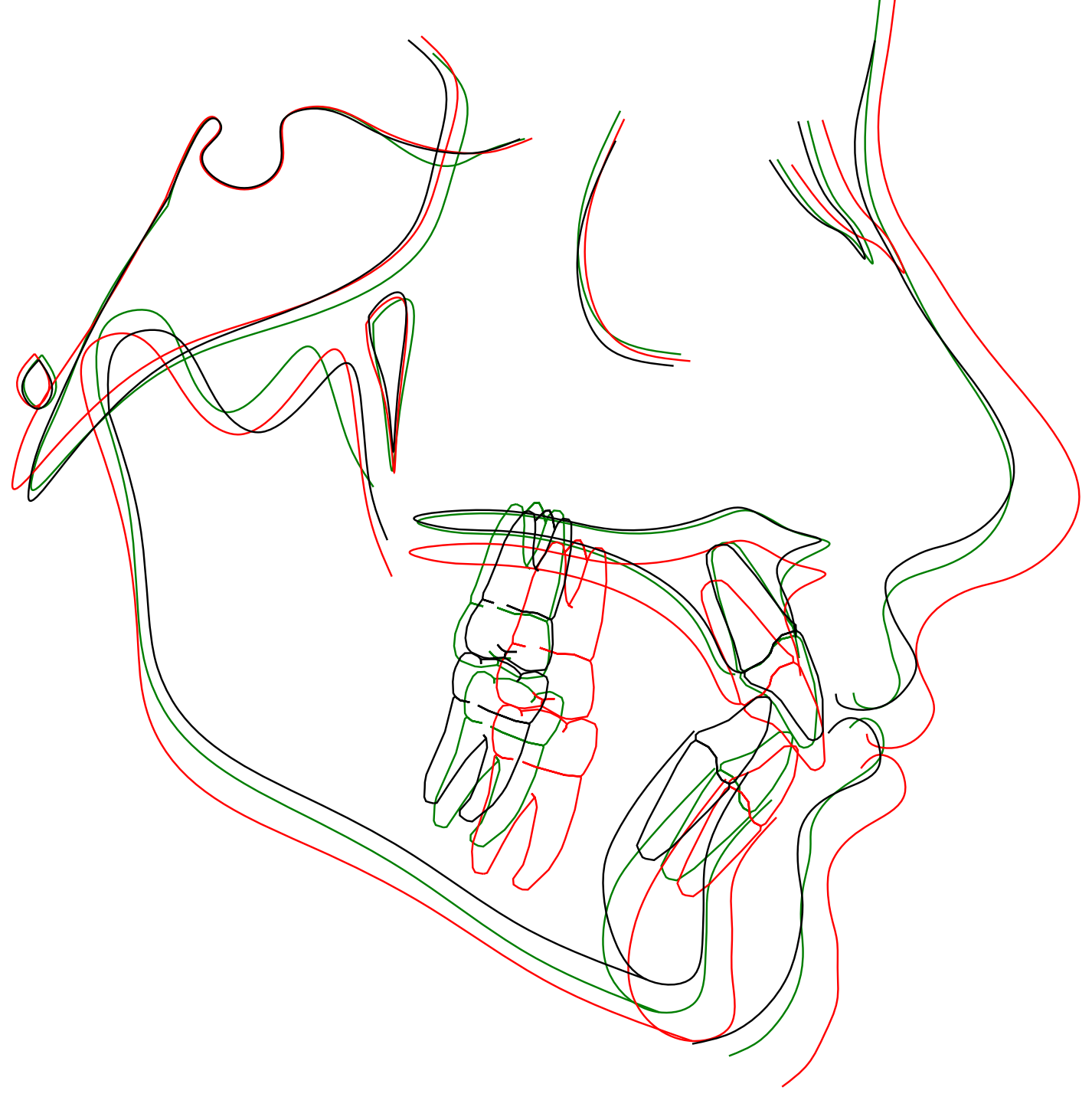

Figure B-57. Print of cephalometric tracing superimposition subject 57: (tracing is 1:1 with original radiograph). 


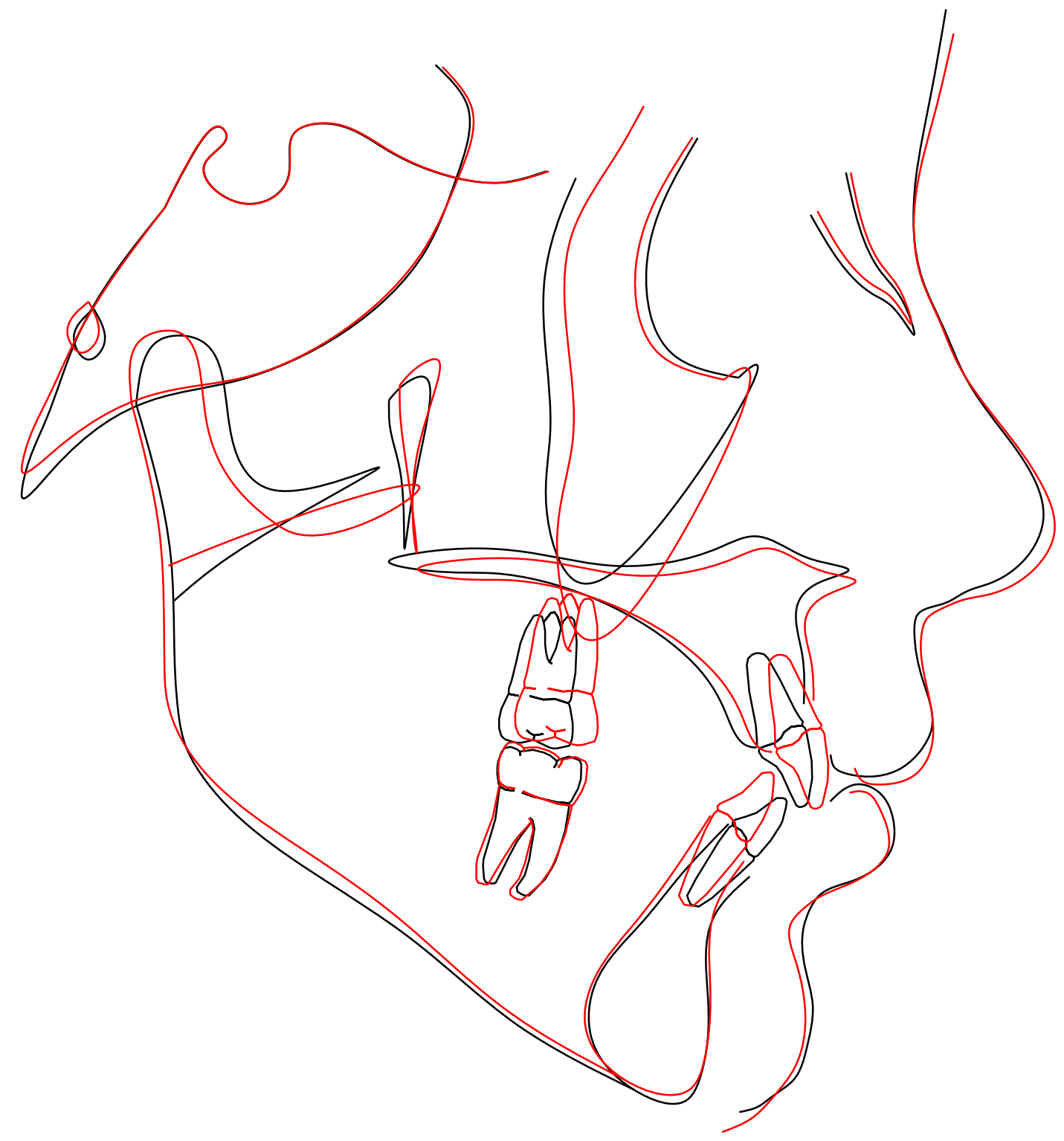

Figure B-58. Print of cephalometric tracing superimposition subject 58: (tracing is 1:1 with original radiograph). 


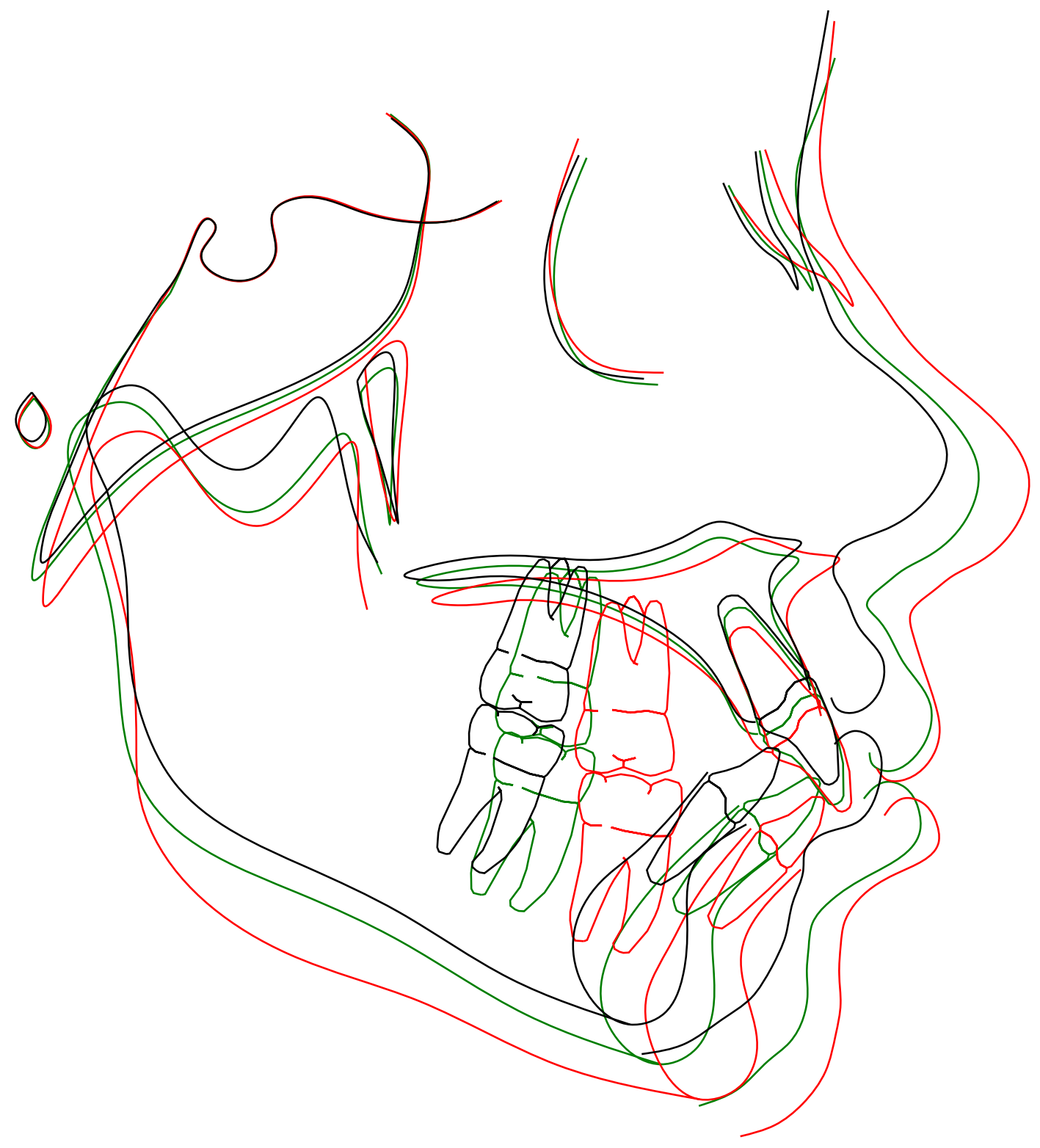

Figure B-59. Print of cephalometric tracing superimposition subject 59: (tracing is 1:1 with original radiograph). 


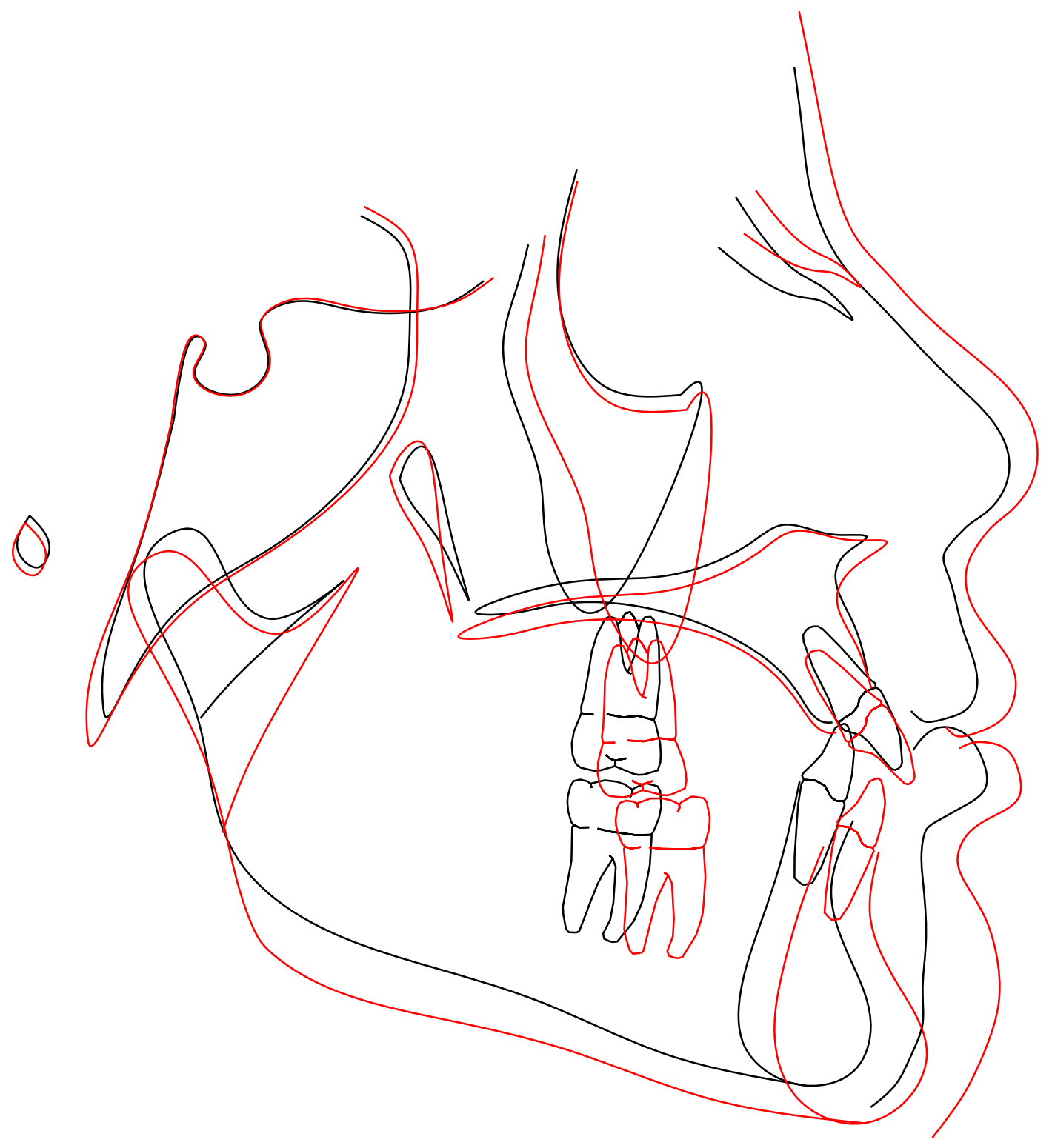

Figure B-60. Print of cephalometric tracing superimposition subject 60: (tracing is $1: 1$ with original radiograph). 


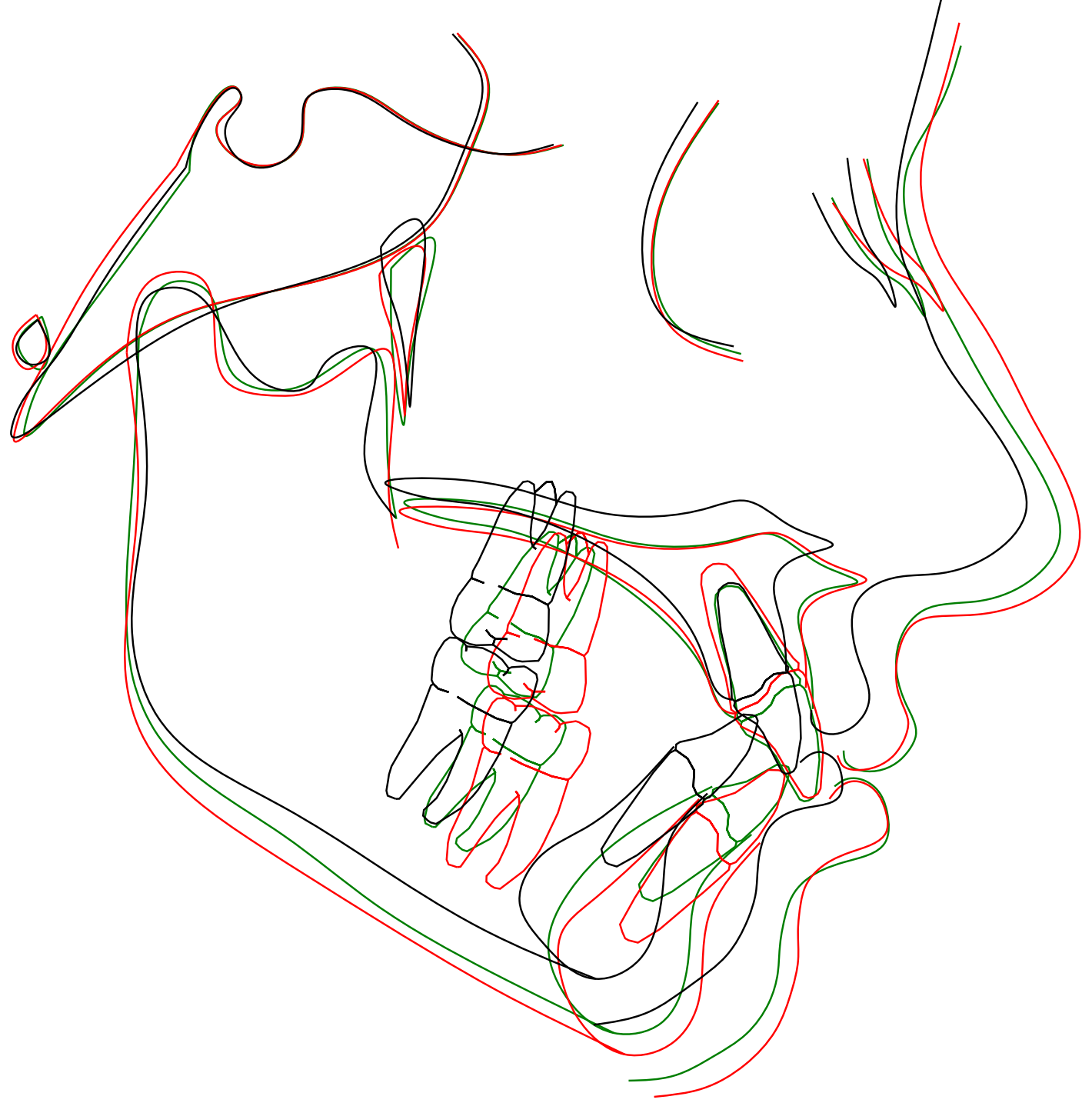

Figure B-61. Print of cephalometric tracing superimposition subject 61: (tracing is $1: 1$ with original radiograph). 


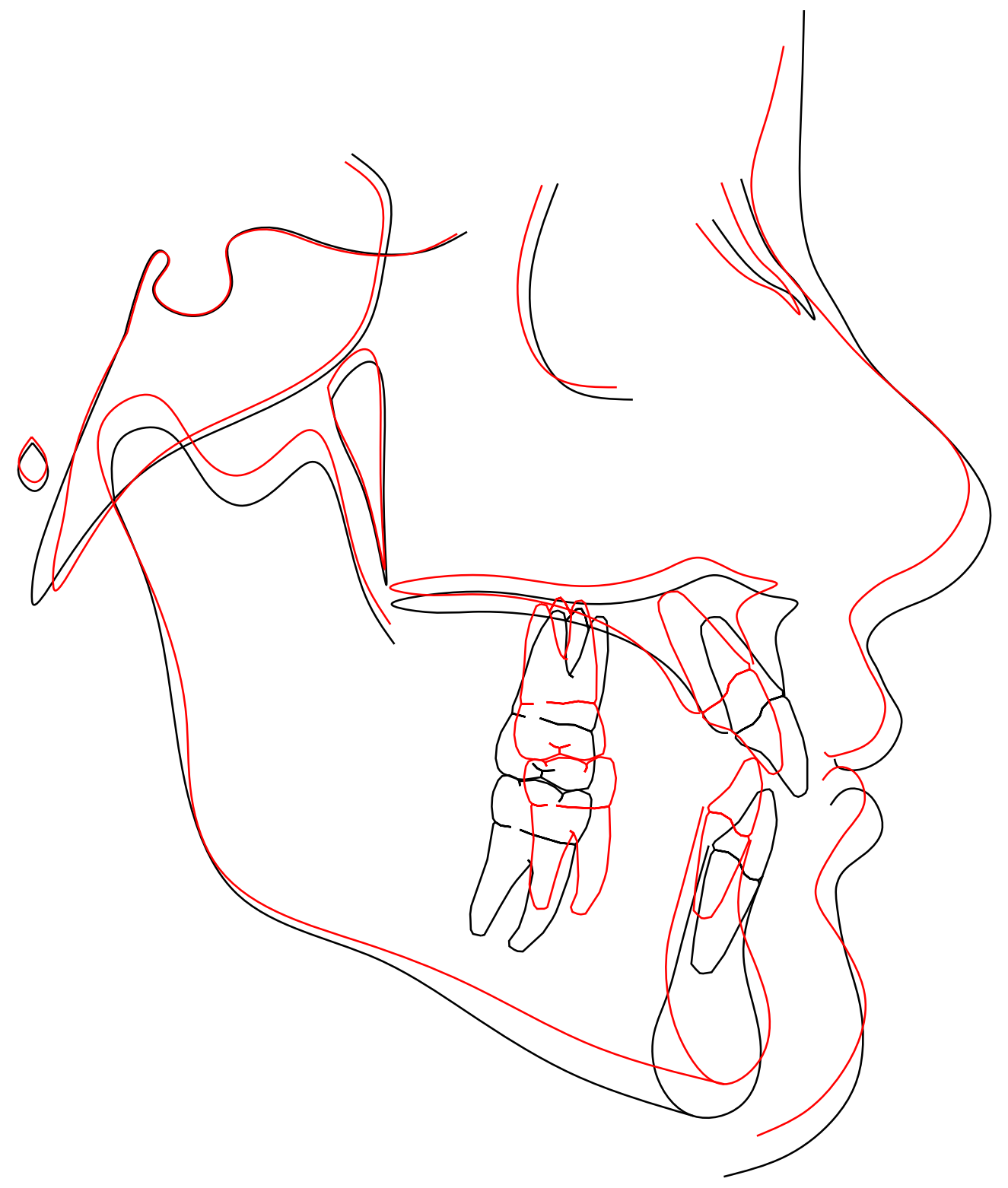

Figure B-62. Print of cephalometric tracing superimposition subject 62: (tracing is 1:1 with original radiograph). 


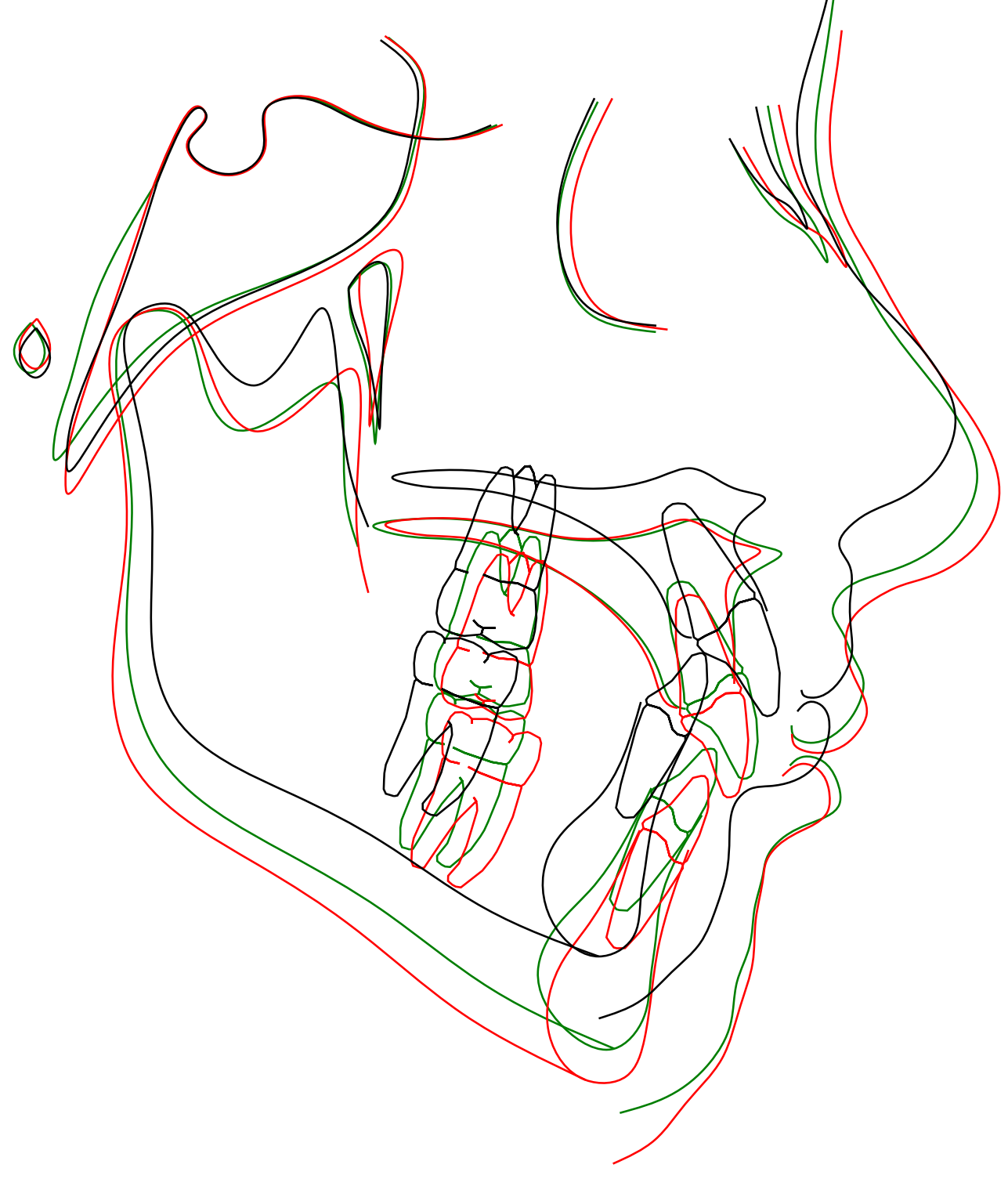

Figure B-63. Print of cephalometric tracing superimposition subject 63: (tracing is 1:1 with original radiograph). 


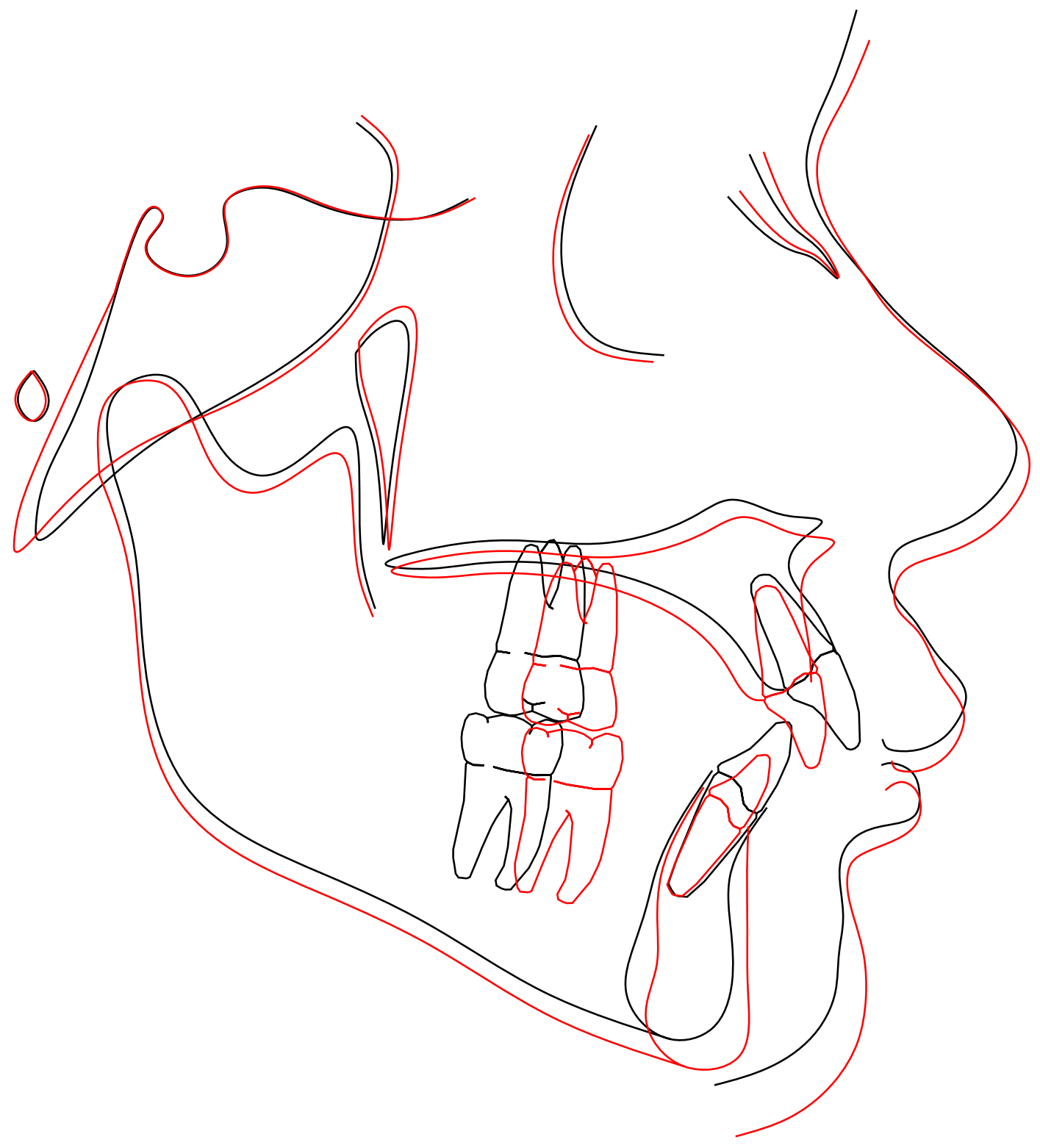

Figure B-64. Print of cephalometric tracing superimposition subject 64: (tracing is 1:1 with original radiograph). 


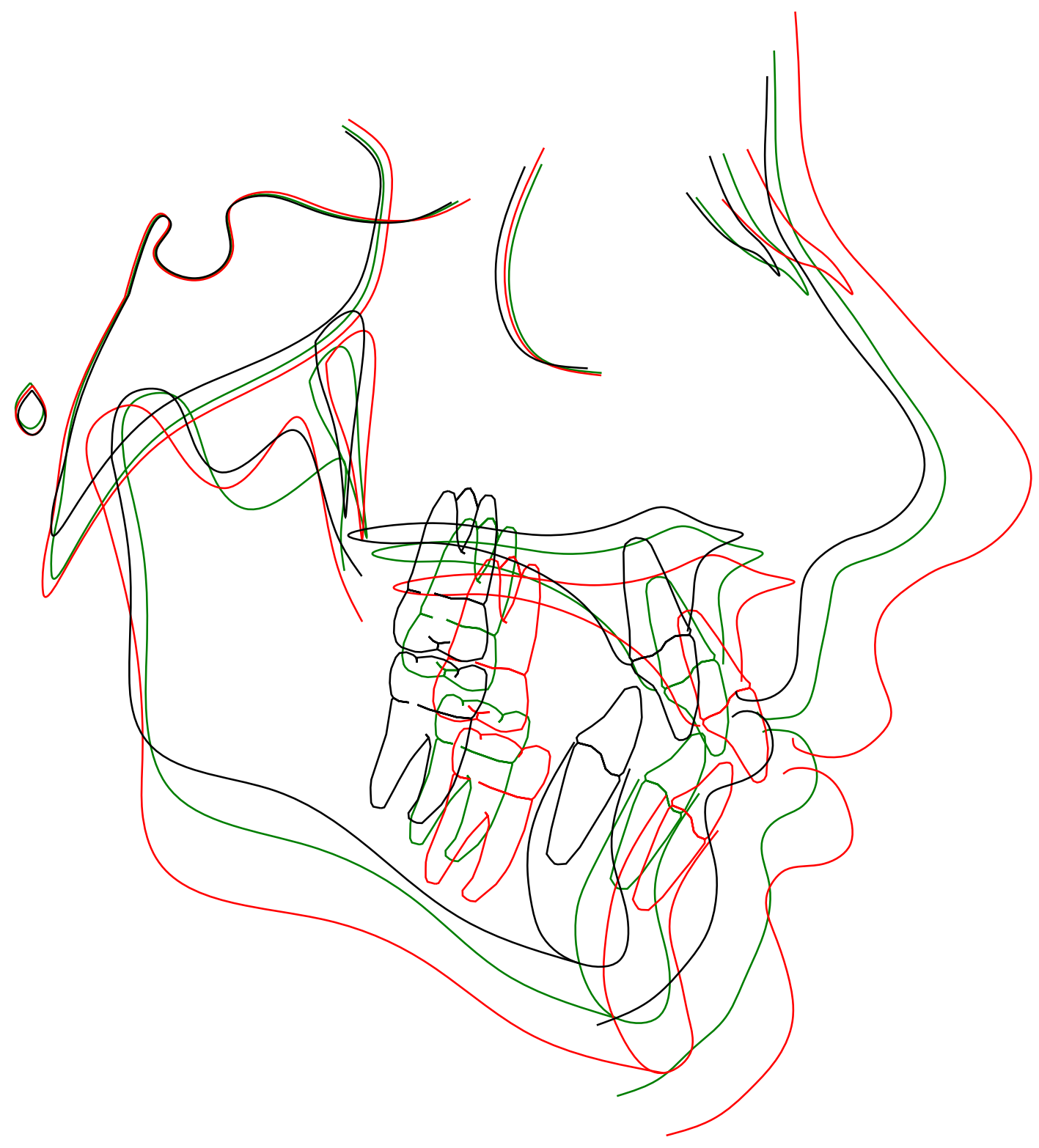

Figure B-65. Print of cephalometric tracing superimposition subject 65: (tracing is 1:1 with original radiograph). 


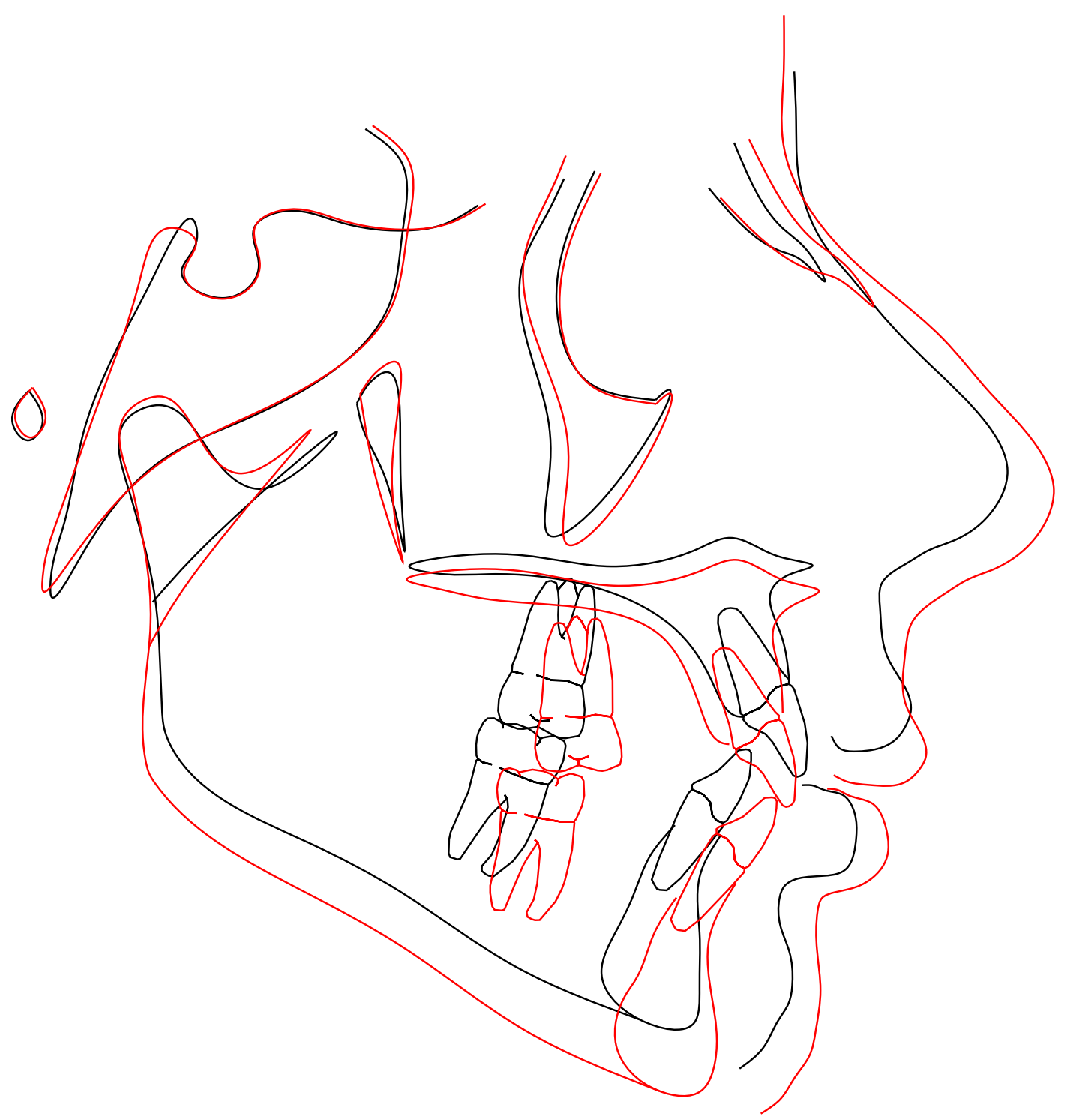

Figure B-66. Print of cephalometric tracing superimposition subject 66: (tracing is $1: 1$ with original radiograph). 


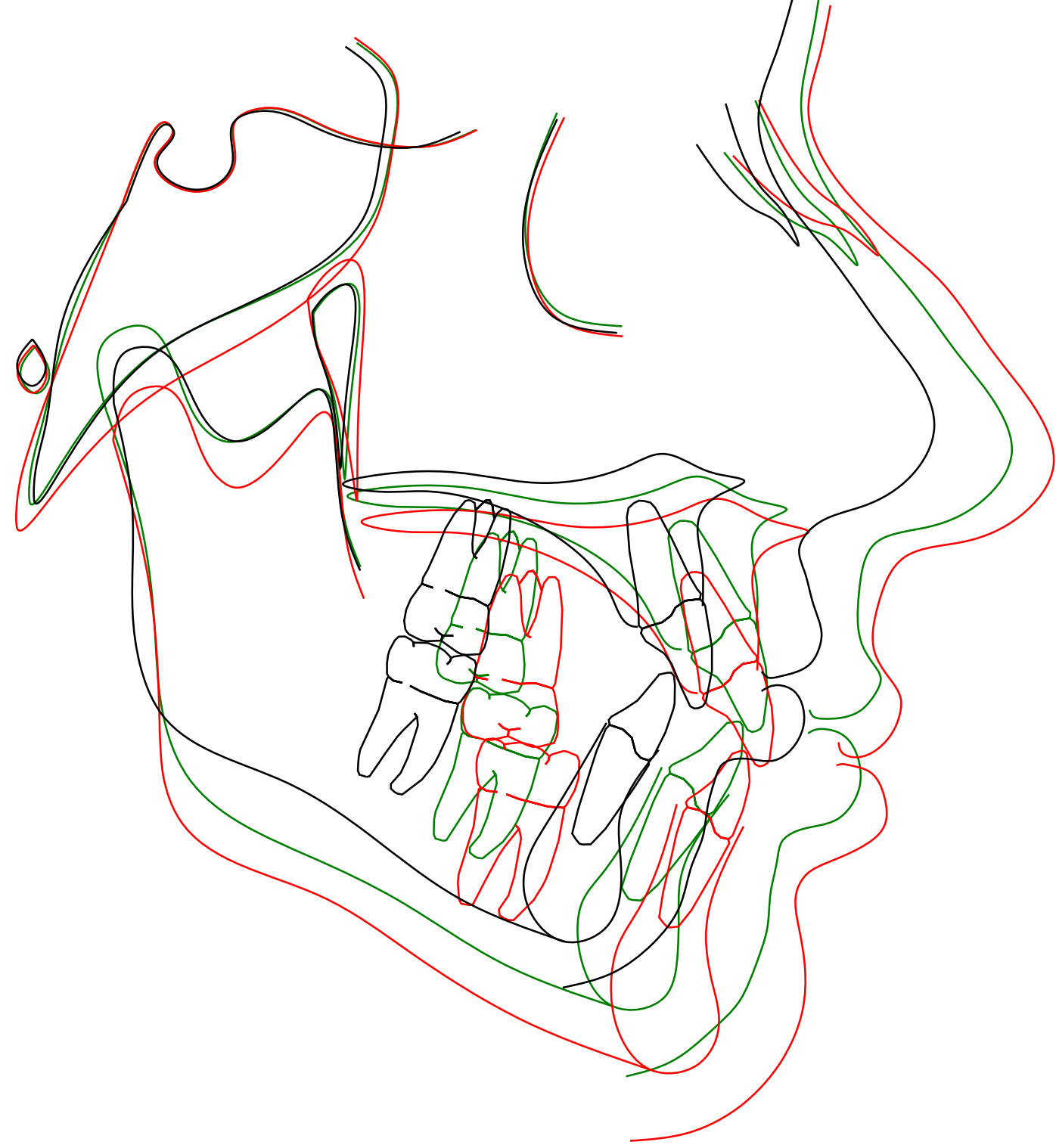

Figure B-67. Print of cephalometric tracing superimposition subject 67: (tracing is 1:1 with original radiograph). 


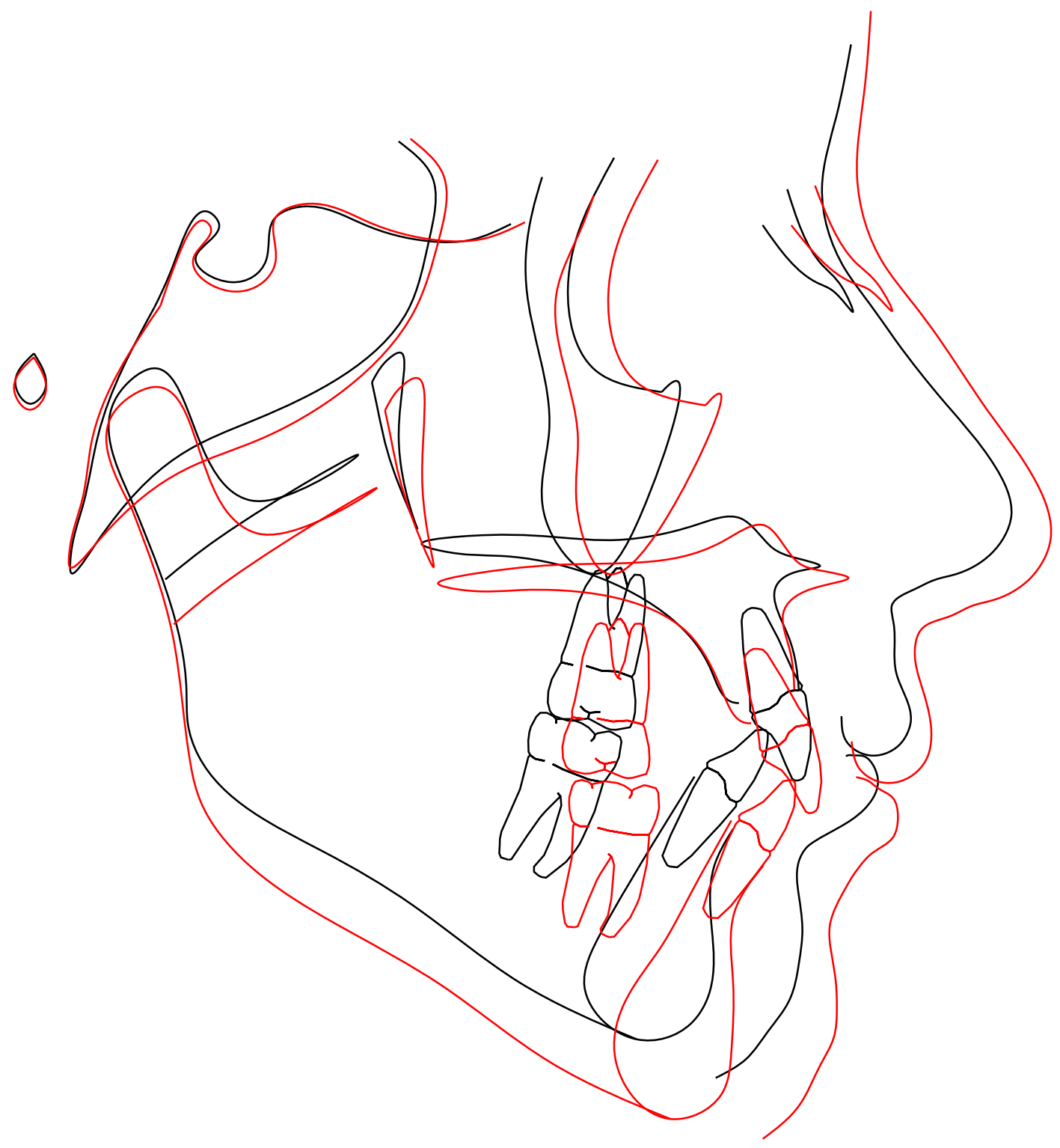

Figure B-68. Print of cephalometric tracing superimposition subject 68: (tracing is 1:1 with original radiograph). 


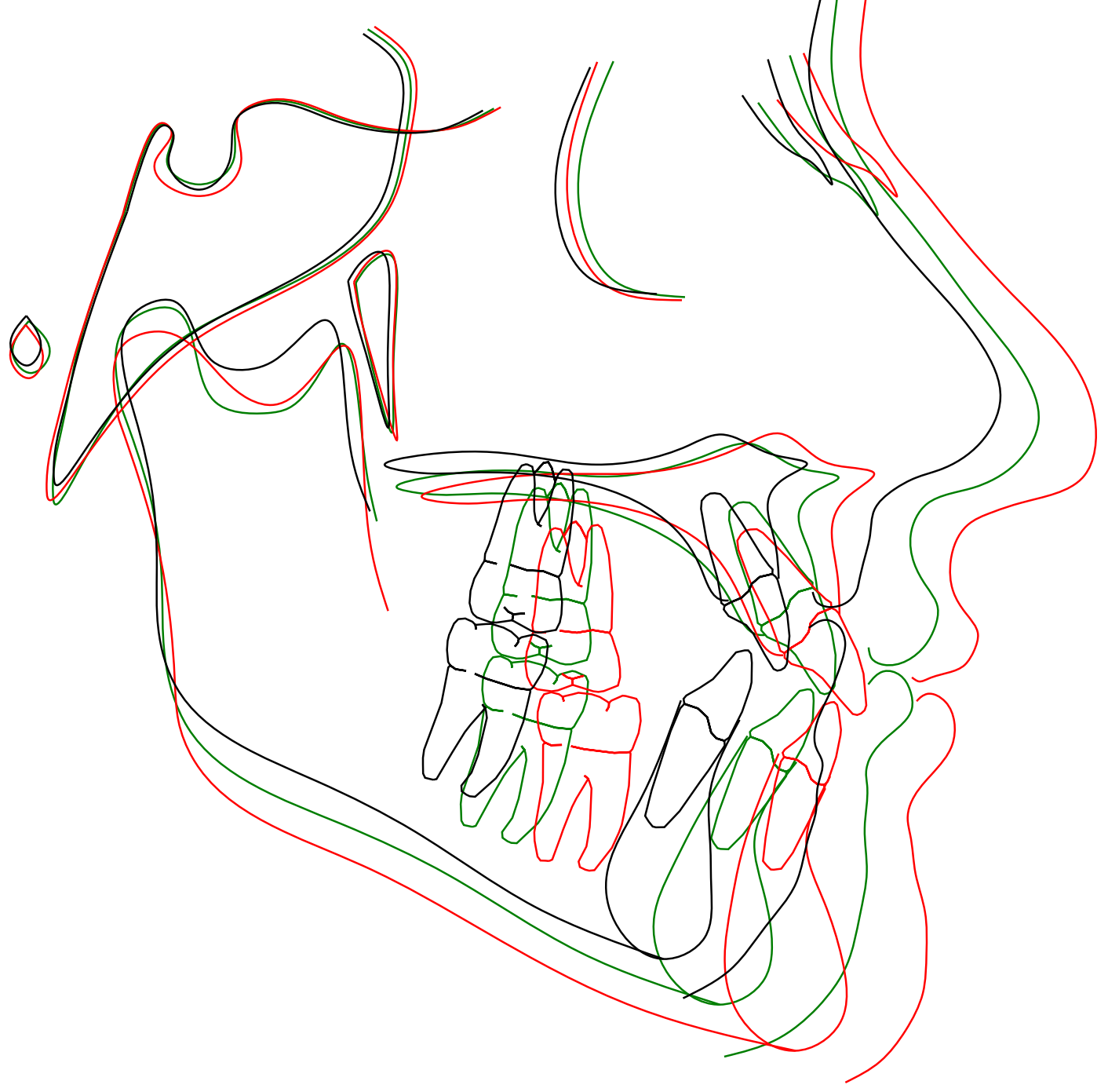

Figure B-69. Print of cephalometric tracing superimposition subject 69: (tracing is 1:1 with original radiograph). 


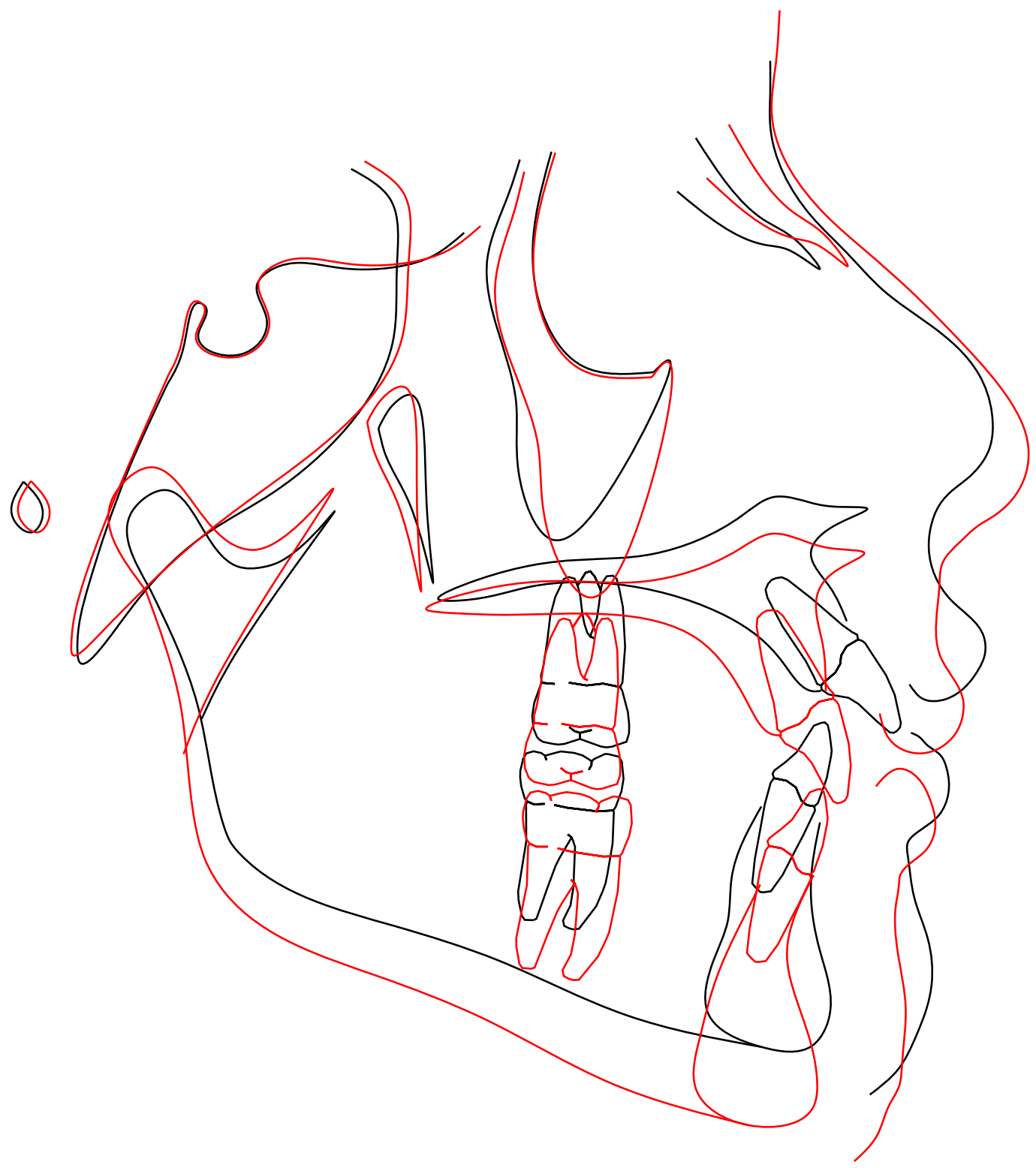

Figure B-70. Print of cephalometric tracing superimposition subject 70: (tracing is 1:1 with original radiograph). 


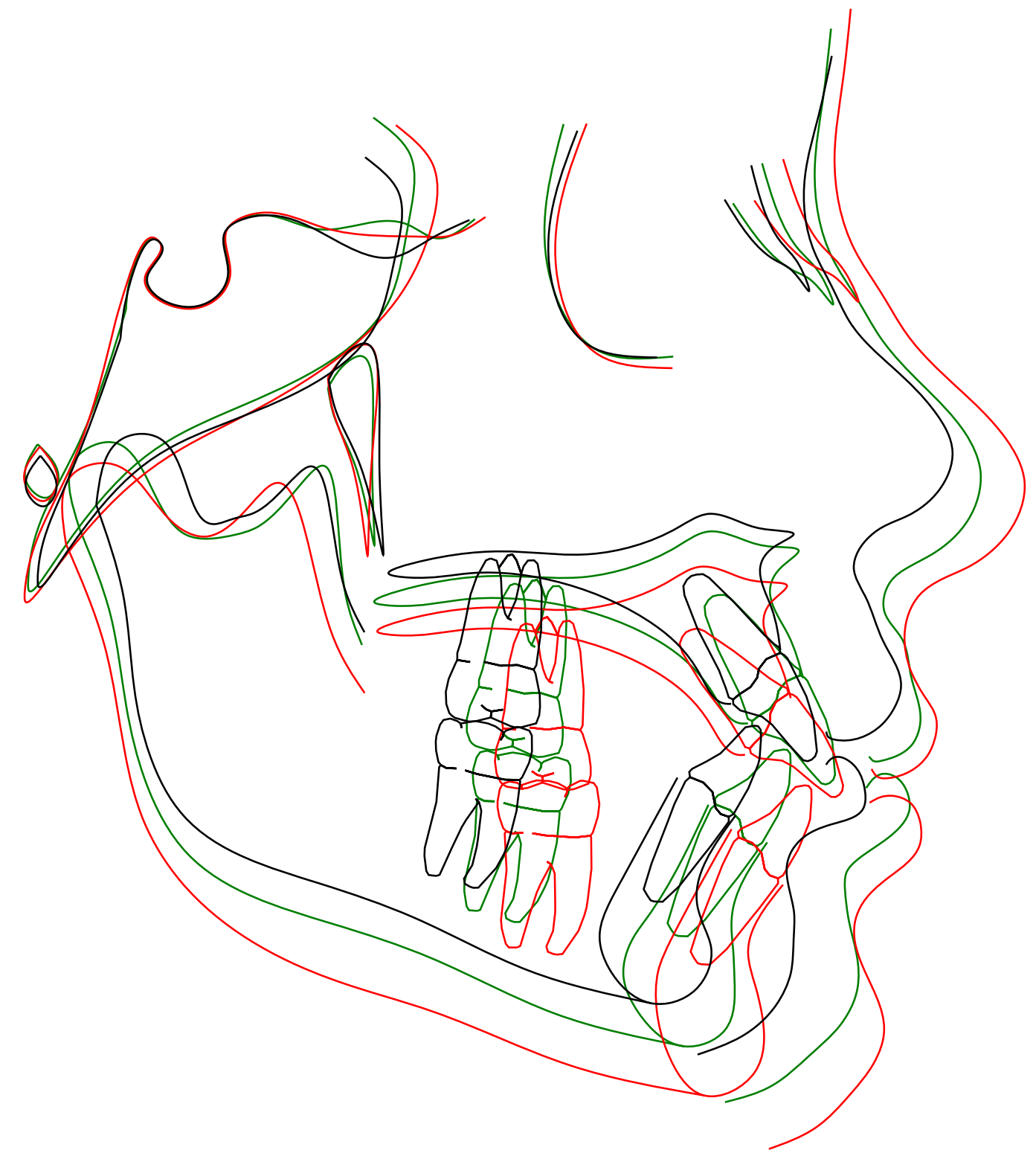

Figure B-71. Print of cephalometric tracing superimposition subject 71: (tracing is 1:1 with original radiograph). 


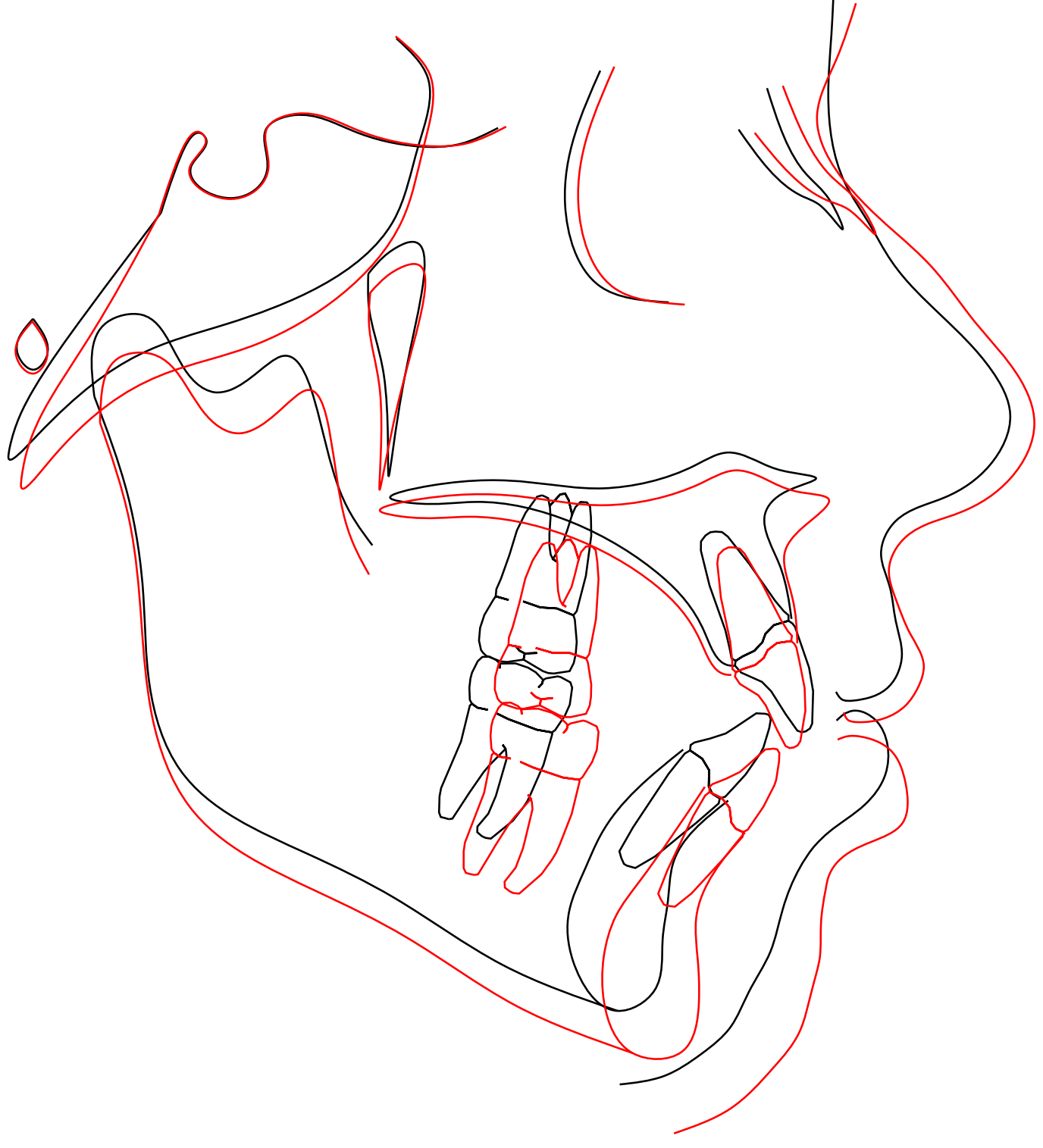

Figure B-72. Print of cephalometric tracing superimposition subject 72: (tracing is 1:1 with original radiograph). 


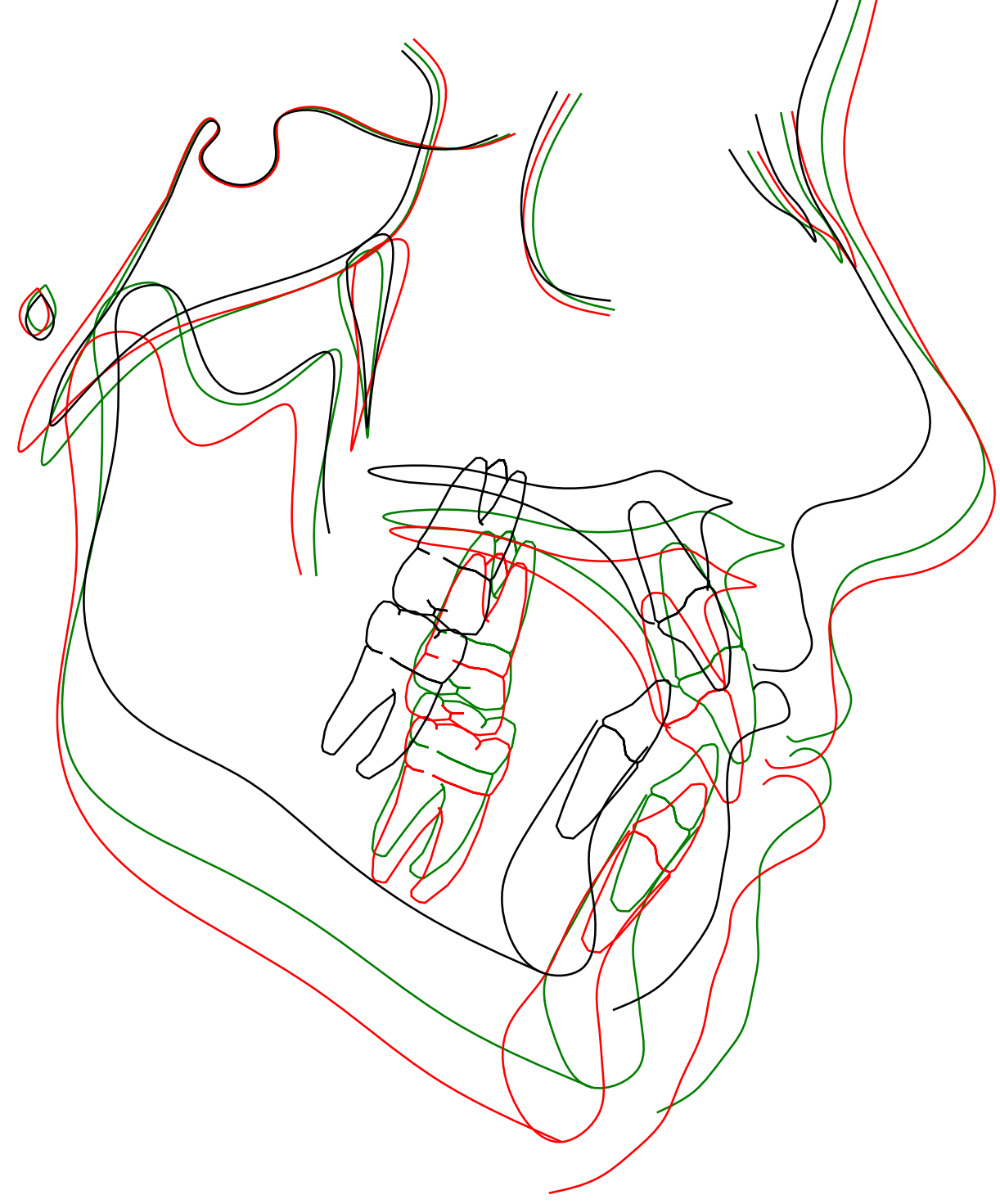

Figure B-73. Print of cephalometric tracing superimposition subject 73: (tracing is 1:1 with original radiograph). 


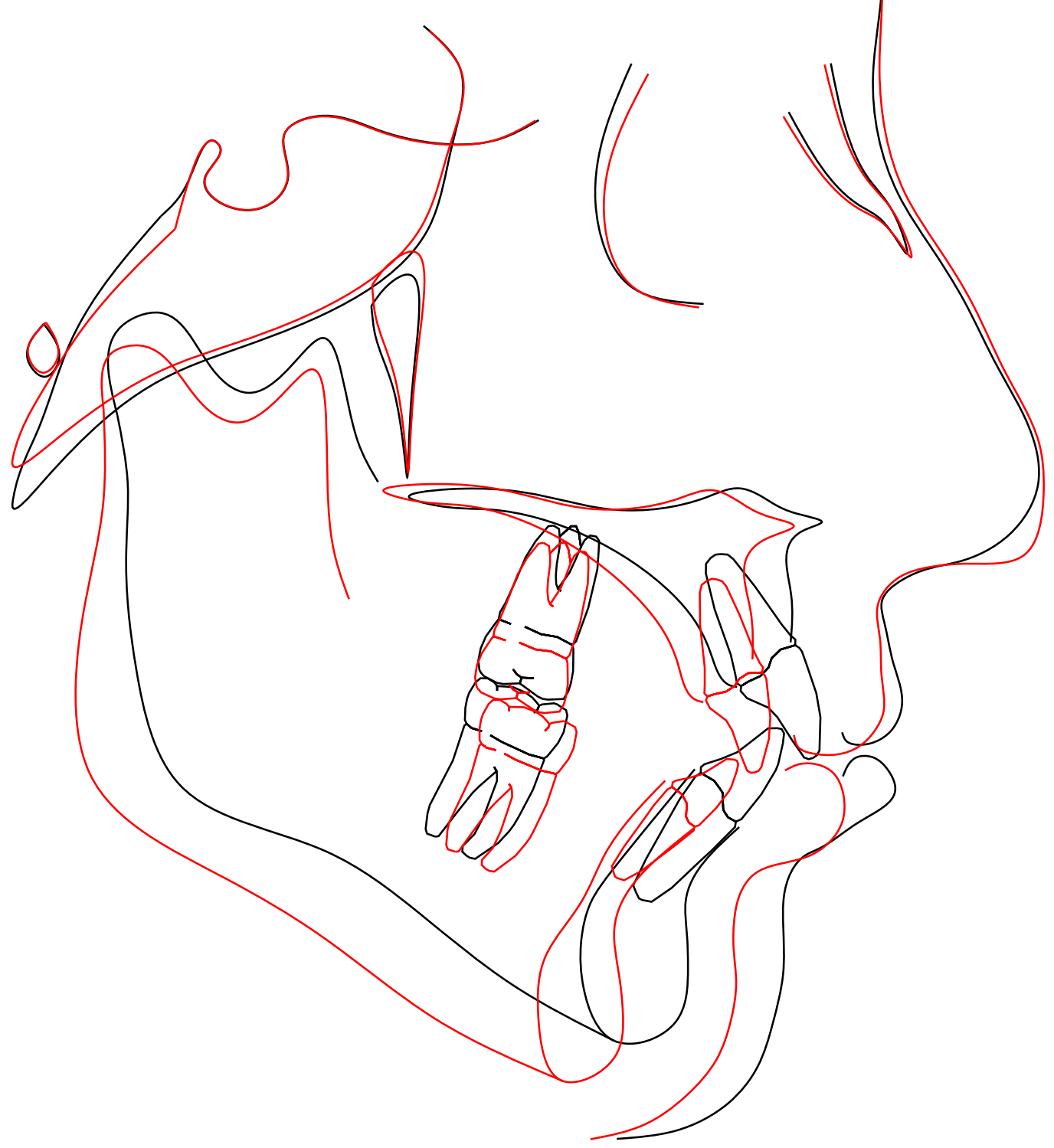

Figure B-74. Print of cephalometric tracing superimposition subject 74: (tracing is 1:1 with original radiograph). 


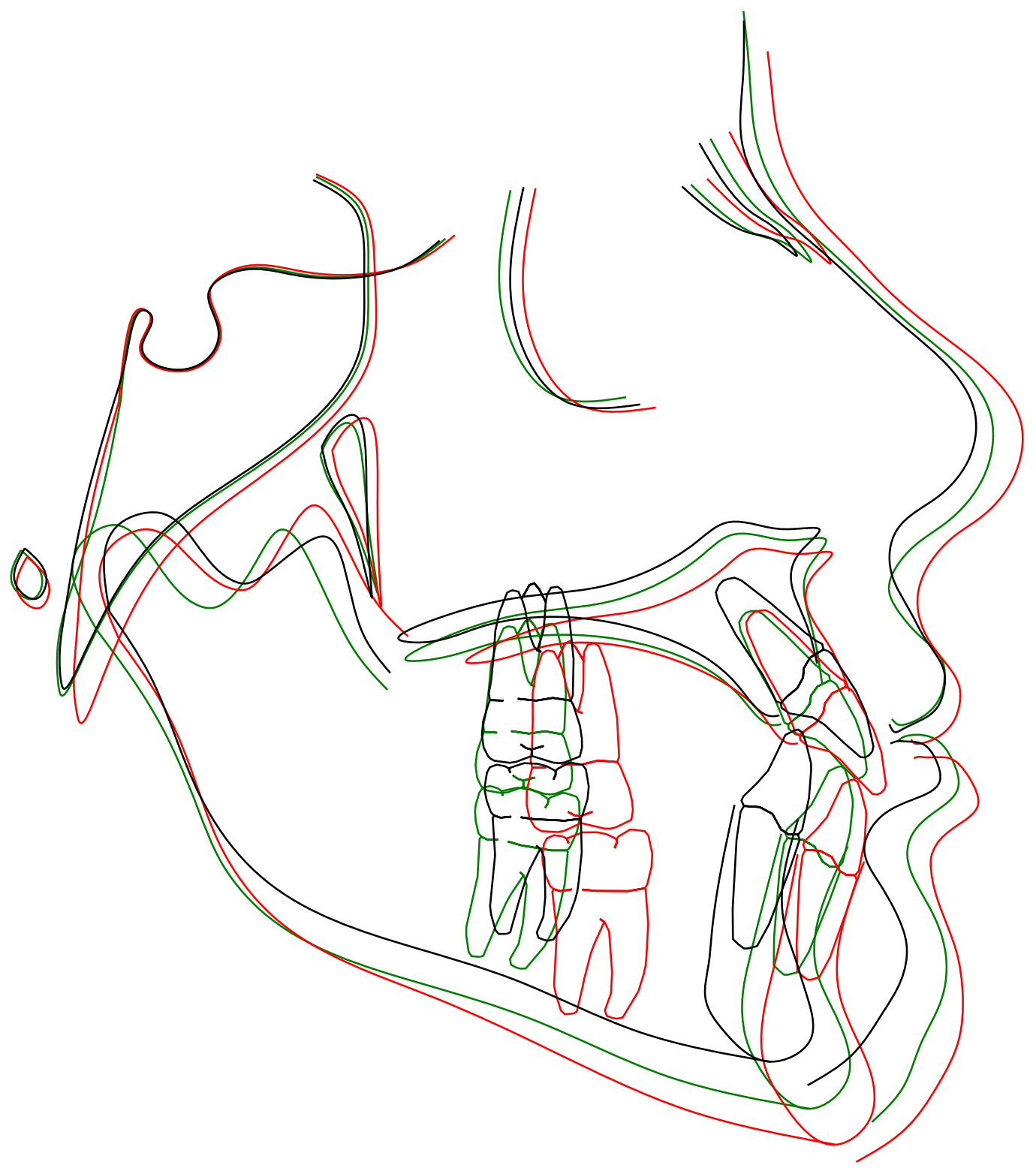

Figure B-75. Print of cephalometric tracing superimposition subject 75: (tracing is 1:1 with original radiograph). 


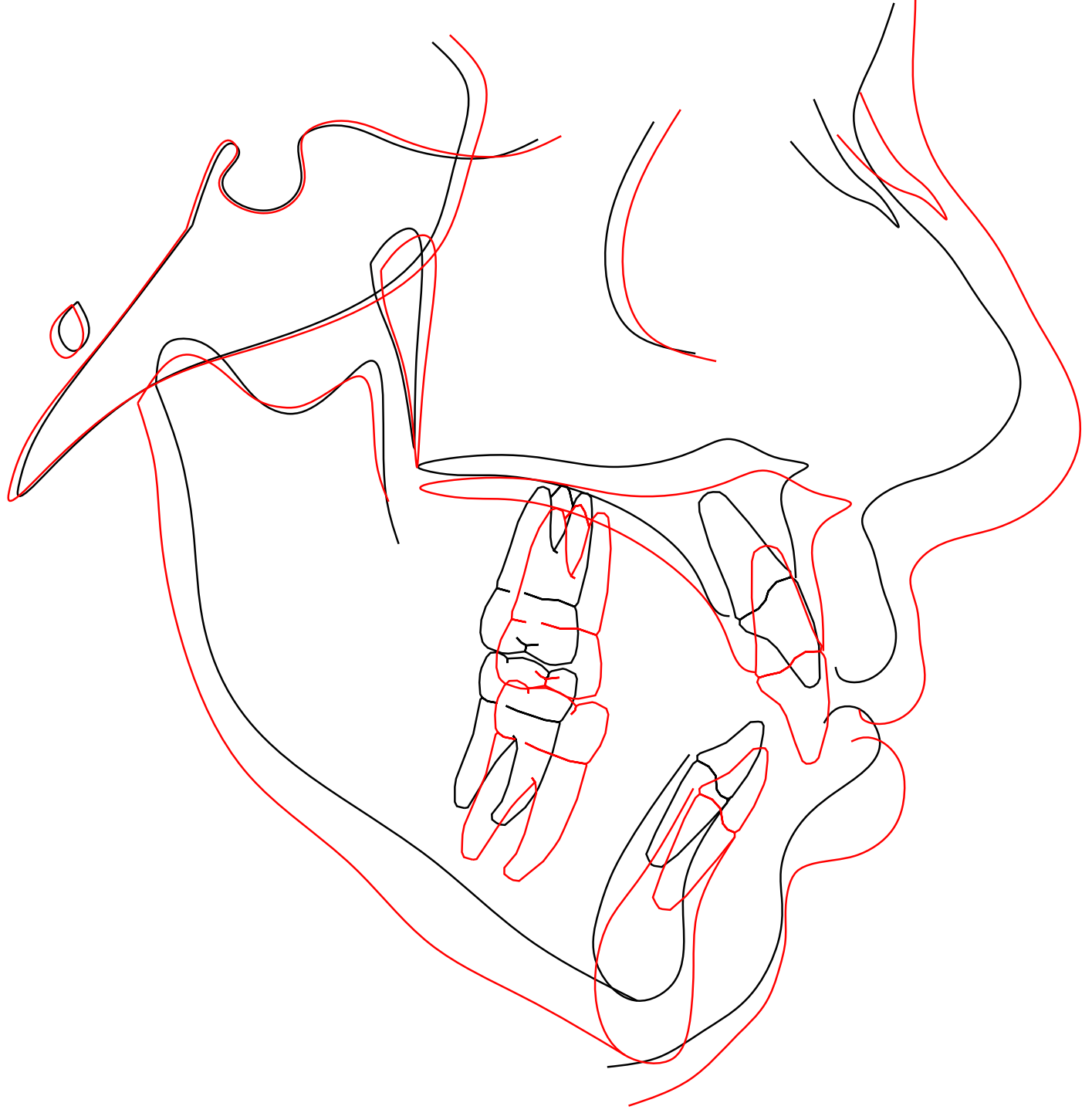

Figure B-76. Print of cephalometric tracing superimposition subject 76: (tracing is $1: 1$ with original radiograph). 


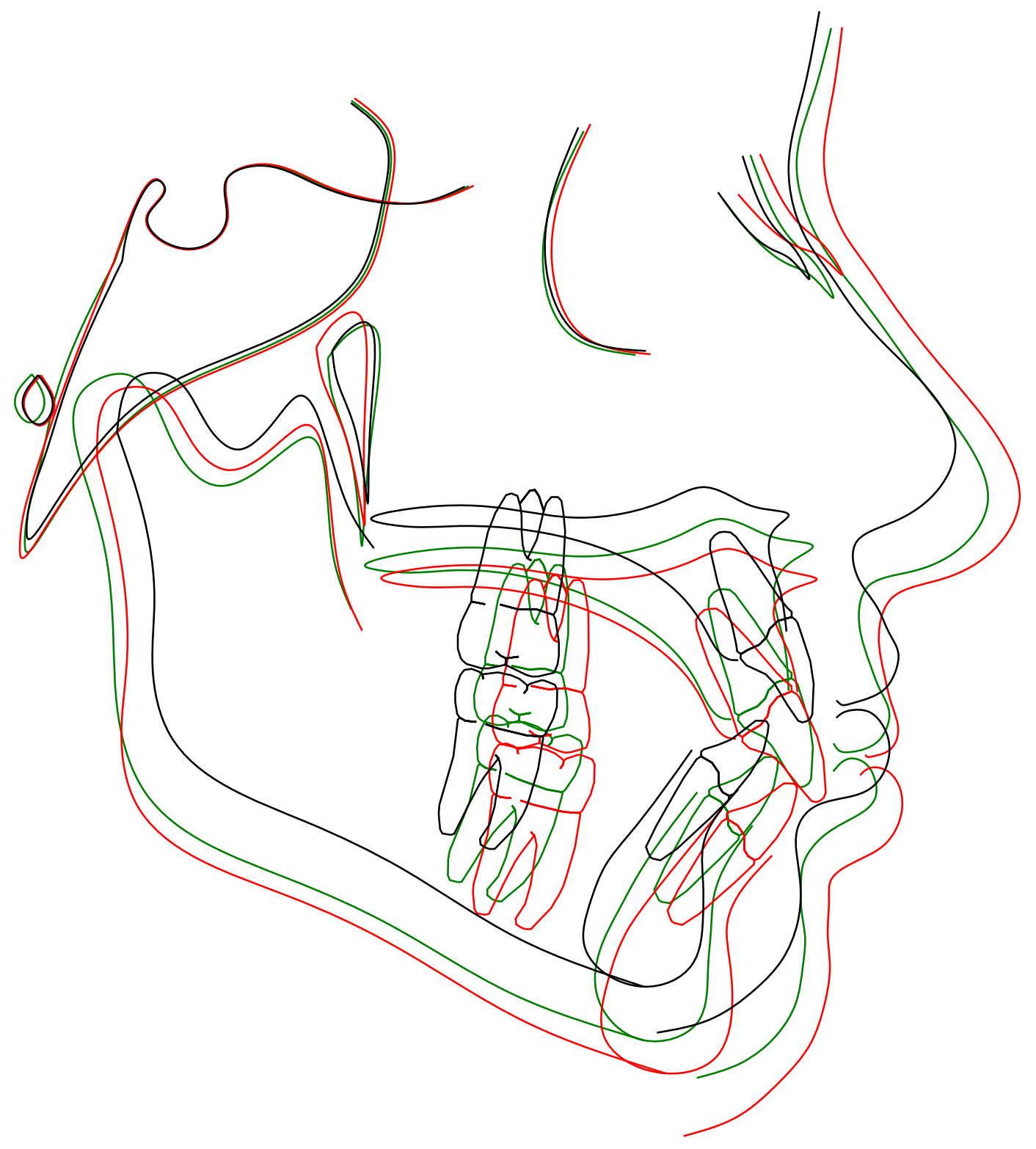

Figure B-77. Print of cephalometric tracing superimposition subject 77: (tracing is 1:1 with original radiograph). 


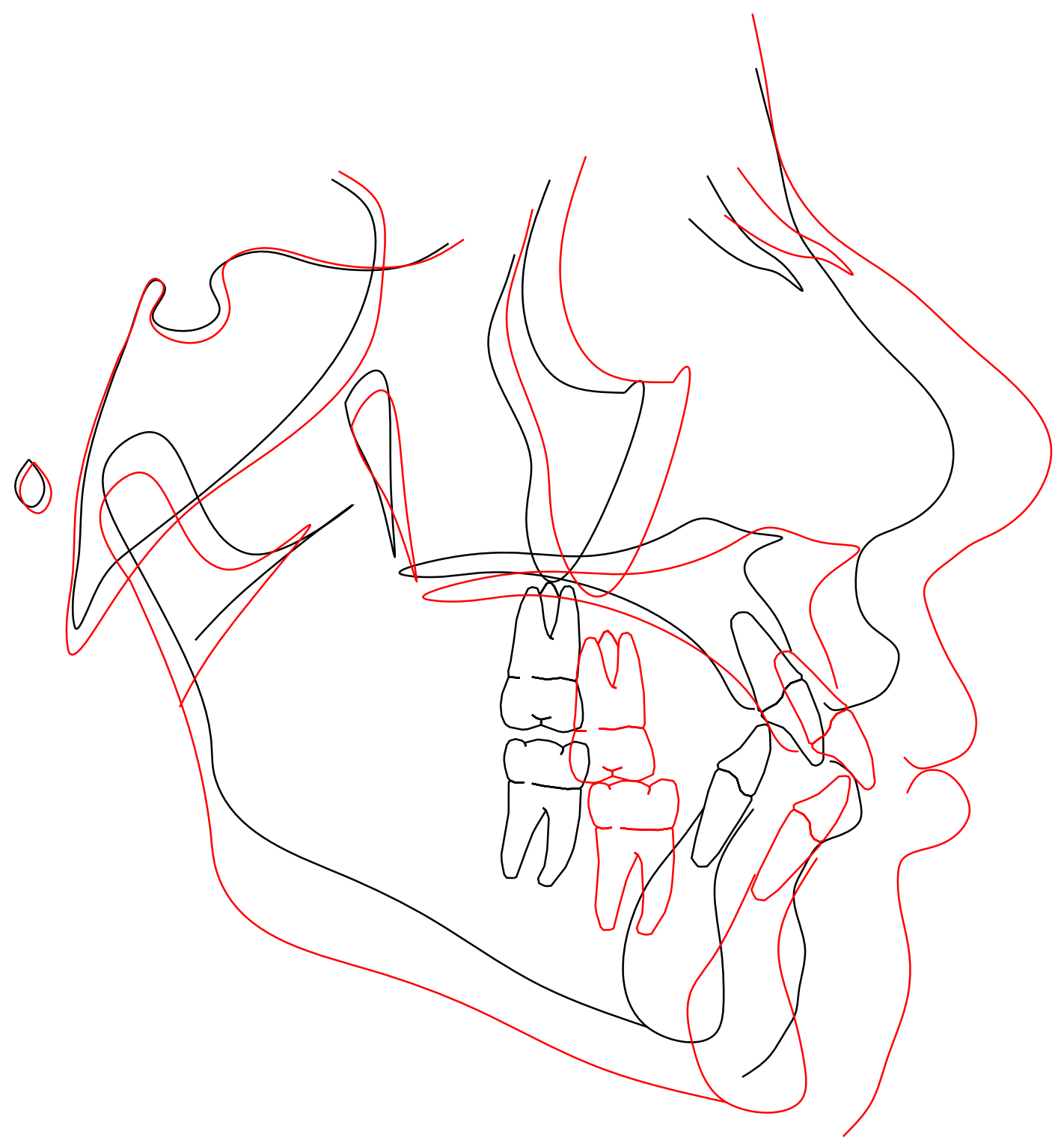

Figure B-78. Print of cephalometric tracing superimposition subject 78: (tracing is $1: 1$ with original radiograph). 


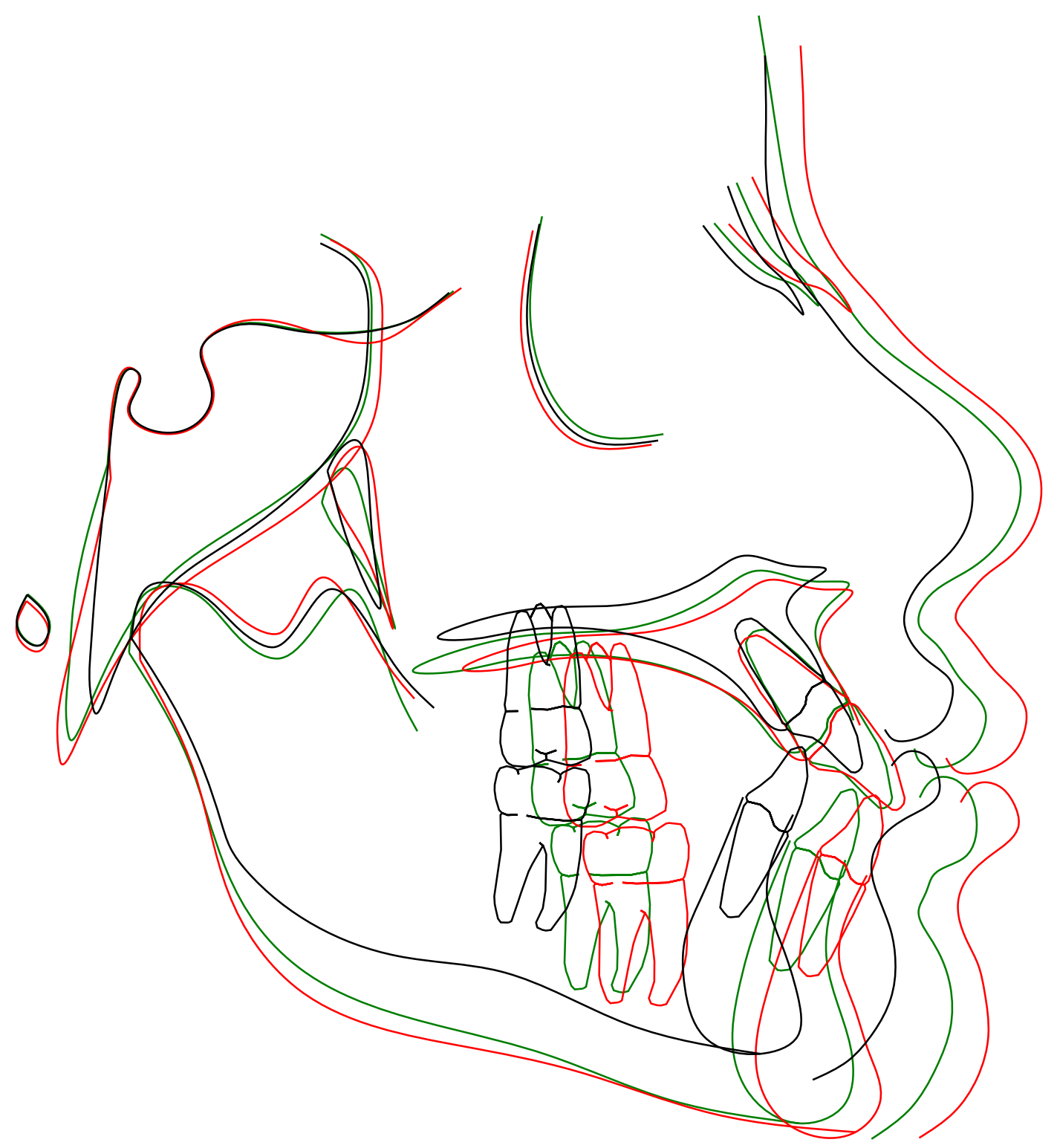

Figure B-79. Print of cephalometric tracing superimposition subject 79: (tracing is 1:1 with original radiograph). 


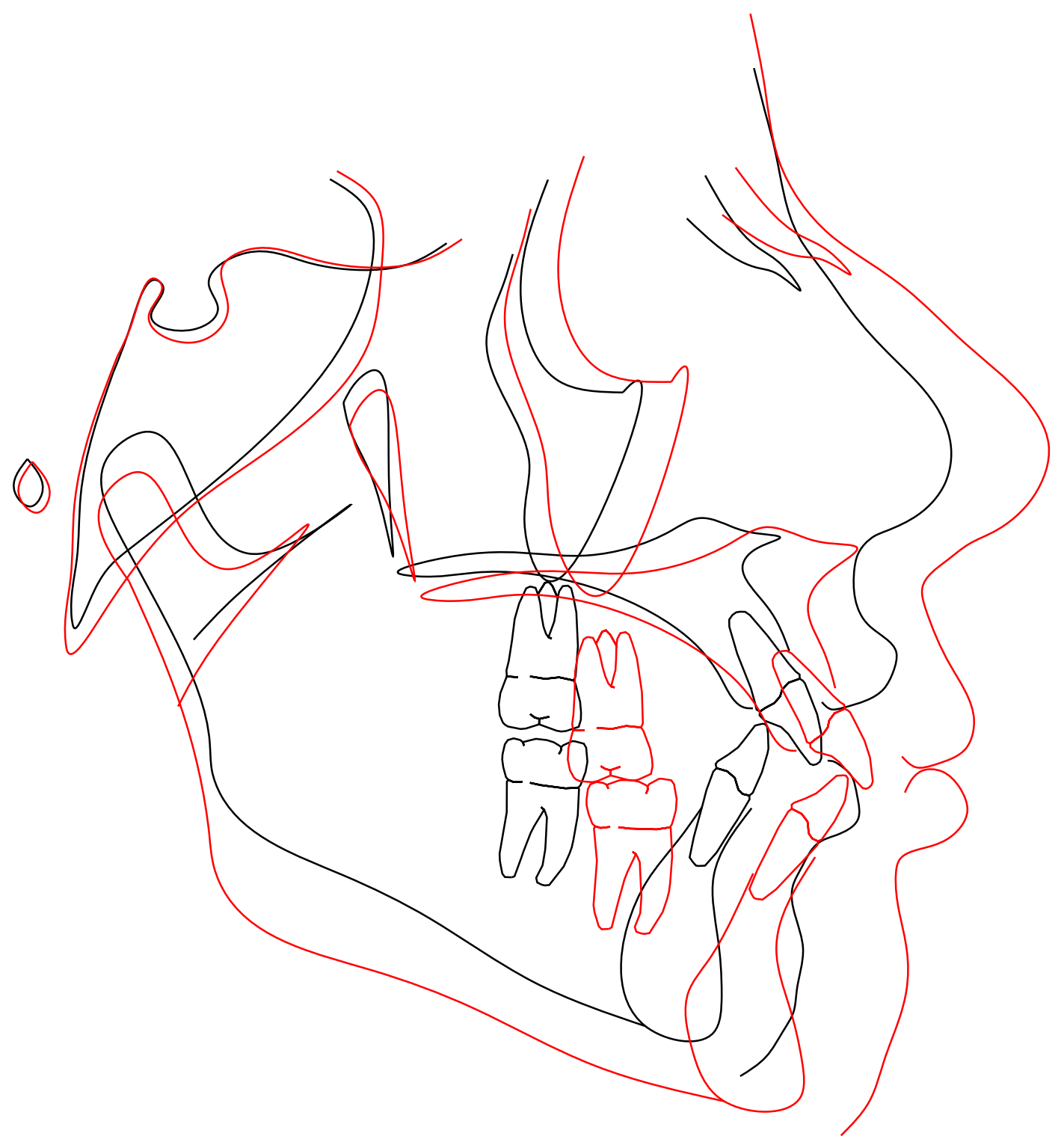

Figure B-80. Print of cephalometric tracing superimposition subject 80: (tracing is $1: 1$ with original radiograph). 


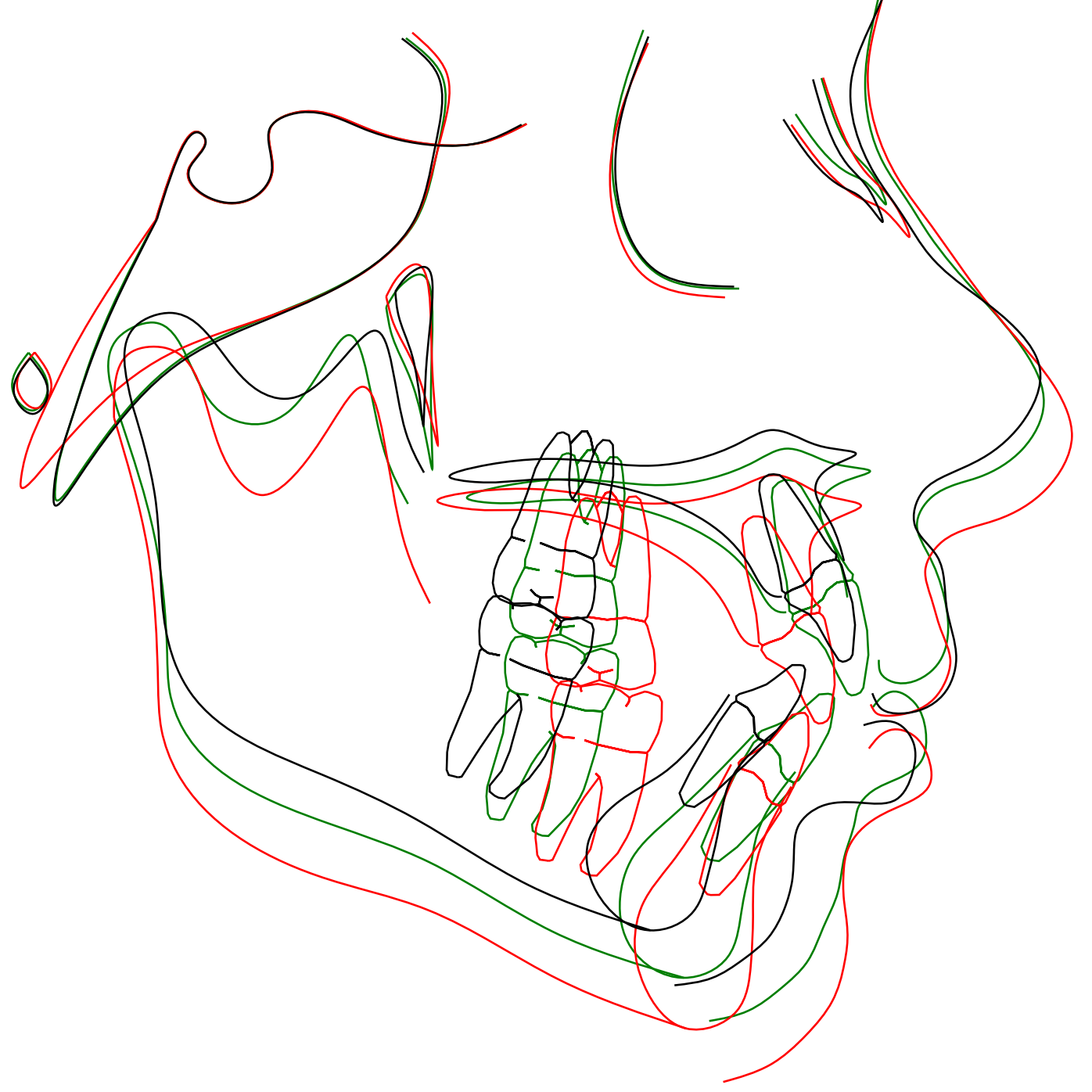

Figure B-81. Print of cephalometric tracing superimposition subject 81: (tracing is 1:1 with original radiograph). 


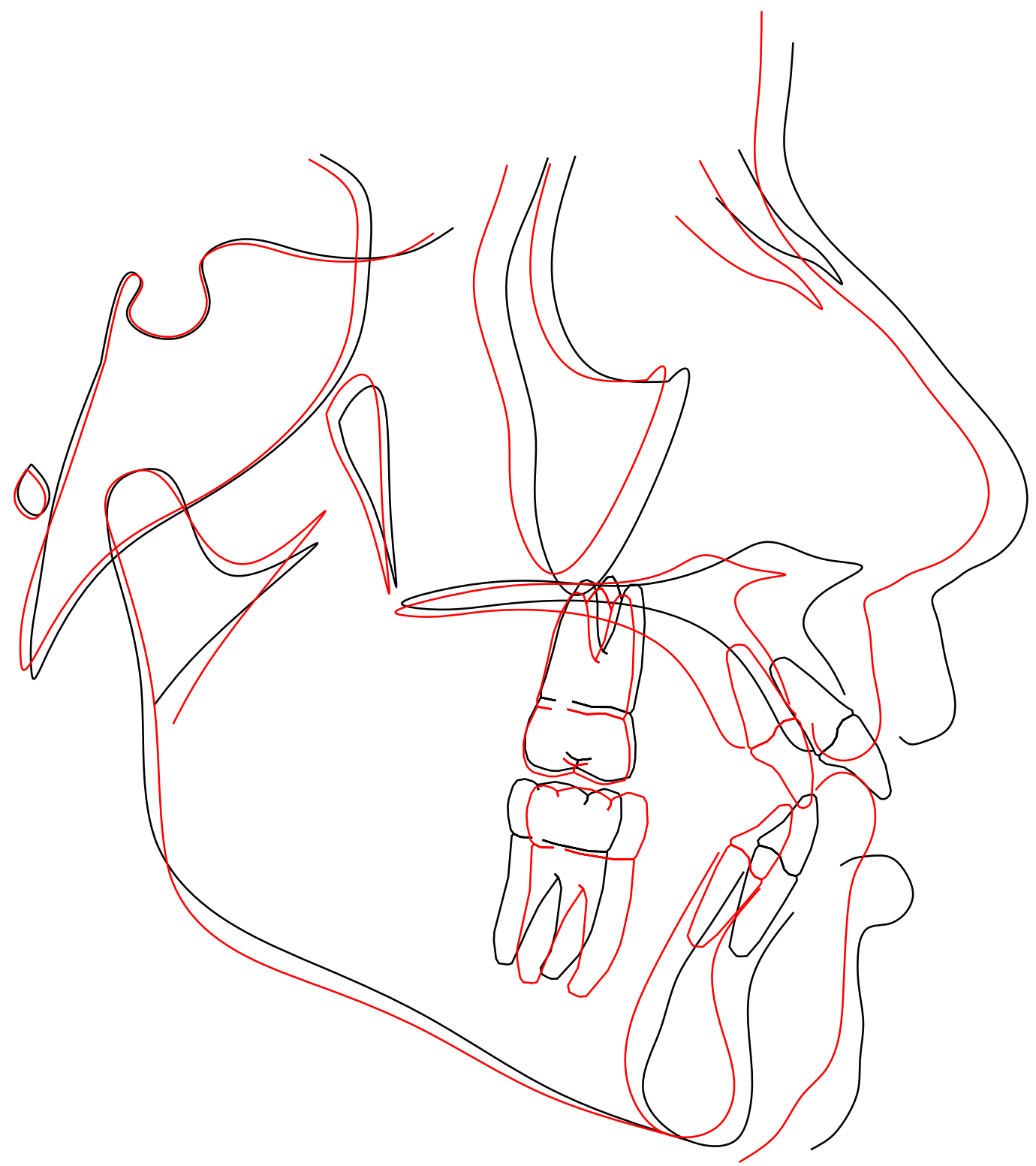

Figure B-82. Print of cephalometric tracing superimposition subject 82: (tracing is 1:1 with original radiograph). 


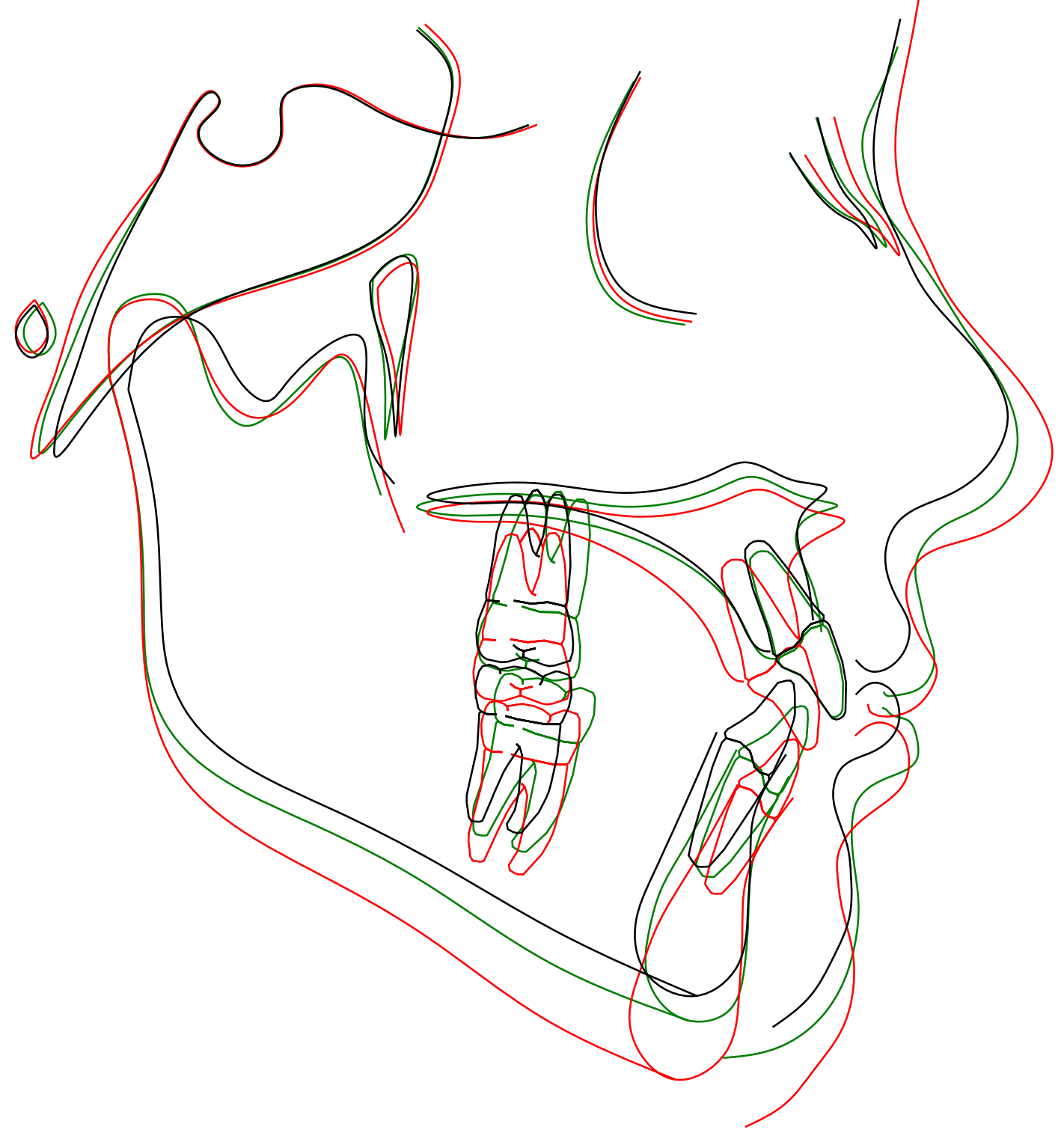

Figure B-83. Print of cephalometric tracing superimposition subject 83: (tracing is 1:1 with original radiograph). 


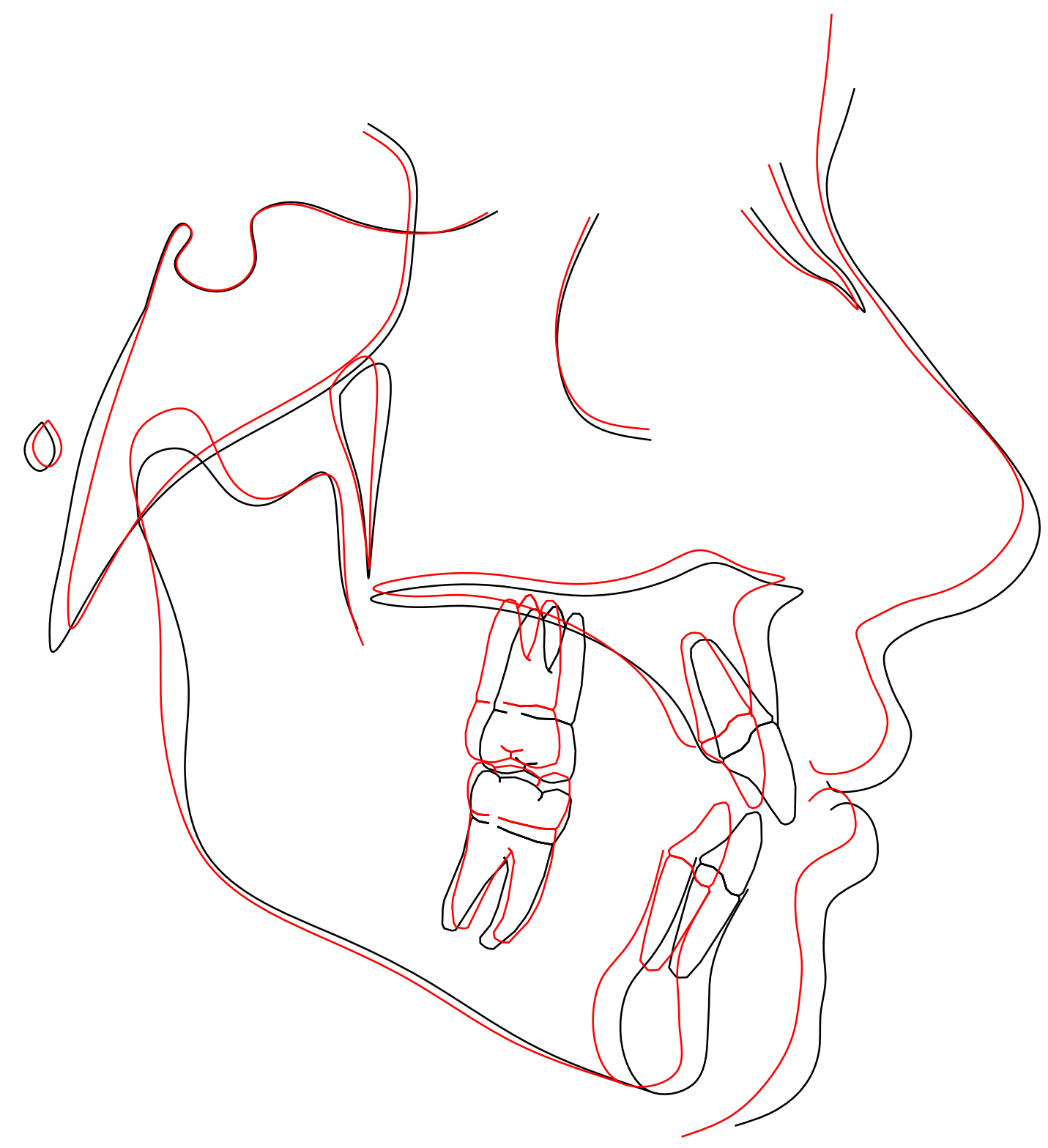

Figure B-84. Print of cephalometric tracing superimposition subject 84: (tracing is 1:1 with original radiograph). 


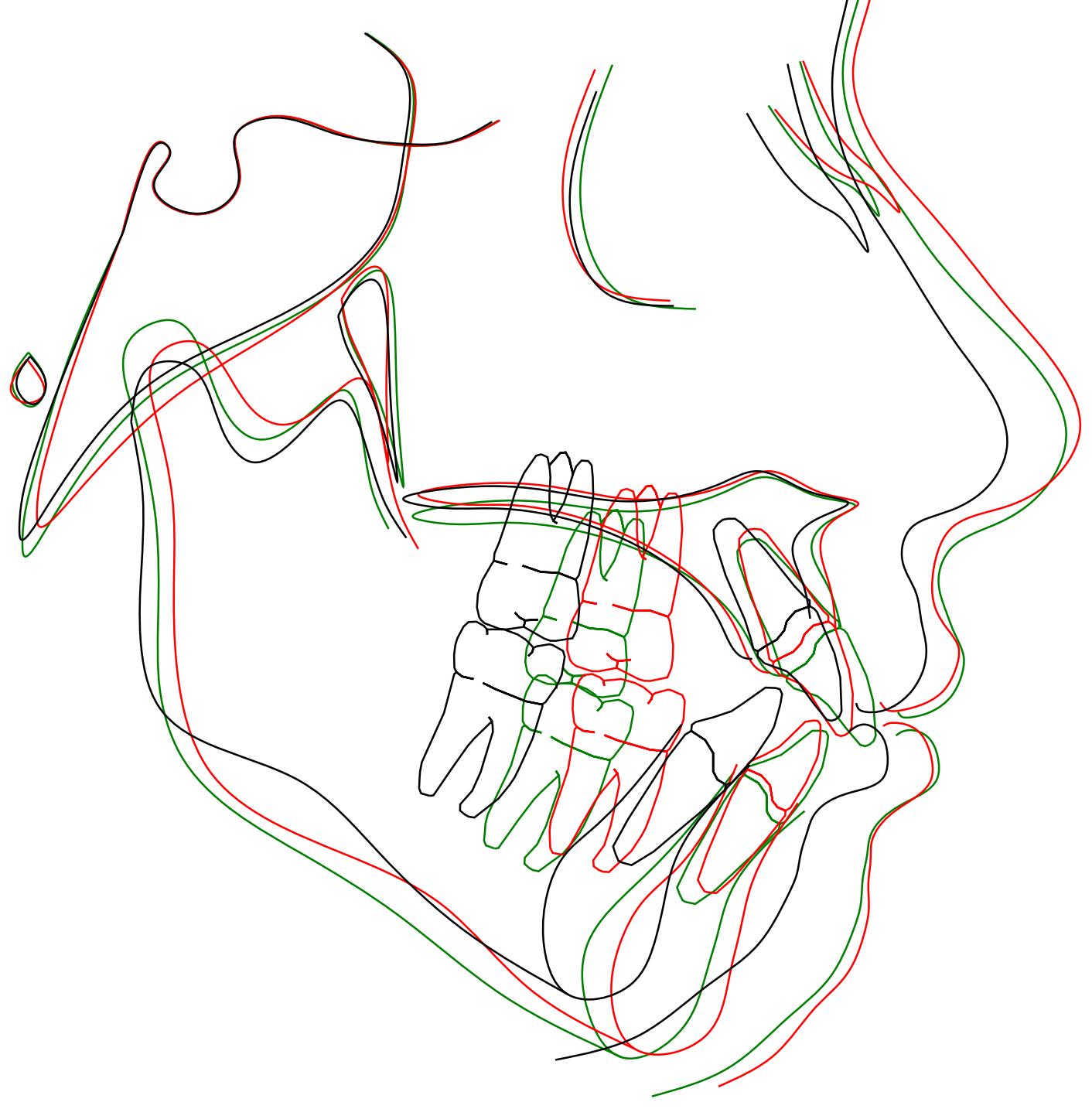

Figure B-85. Print of cephalometric tracing superimposition subject 85: (tracing is 1:1 with original radiograph). 


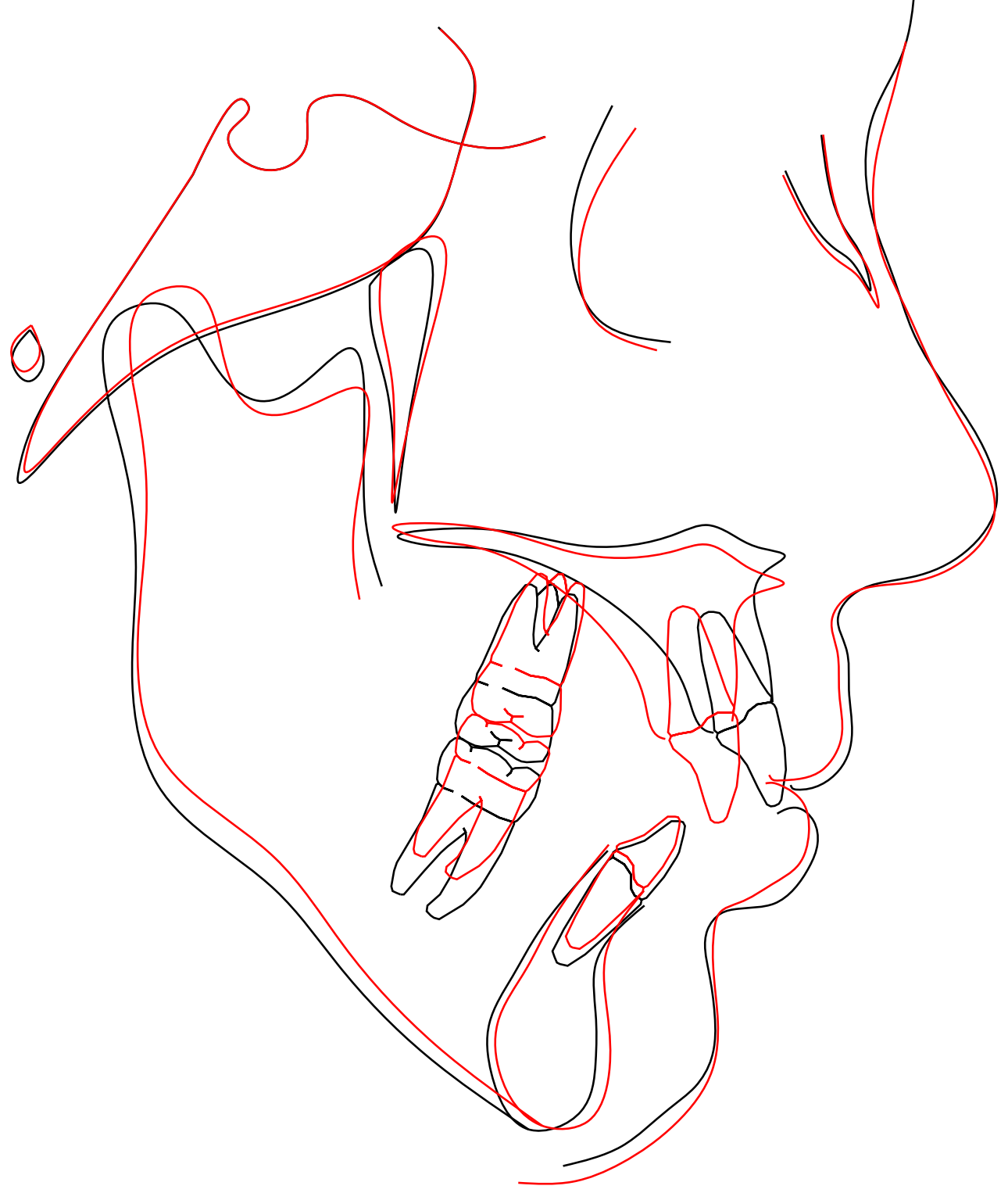

Figure B-86. Print of cephalometric tracing superimposition subject 86: (tracing is $1: 1$ with original radiograph). 


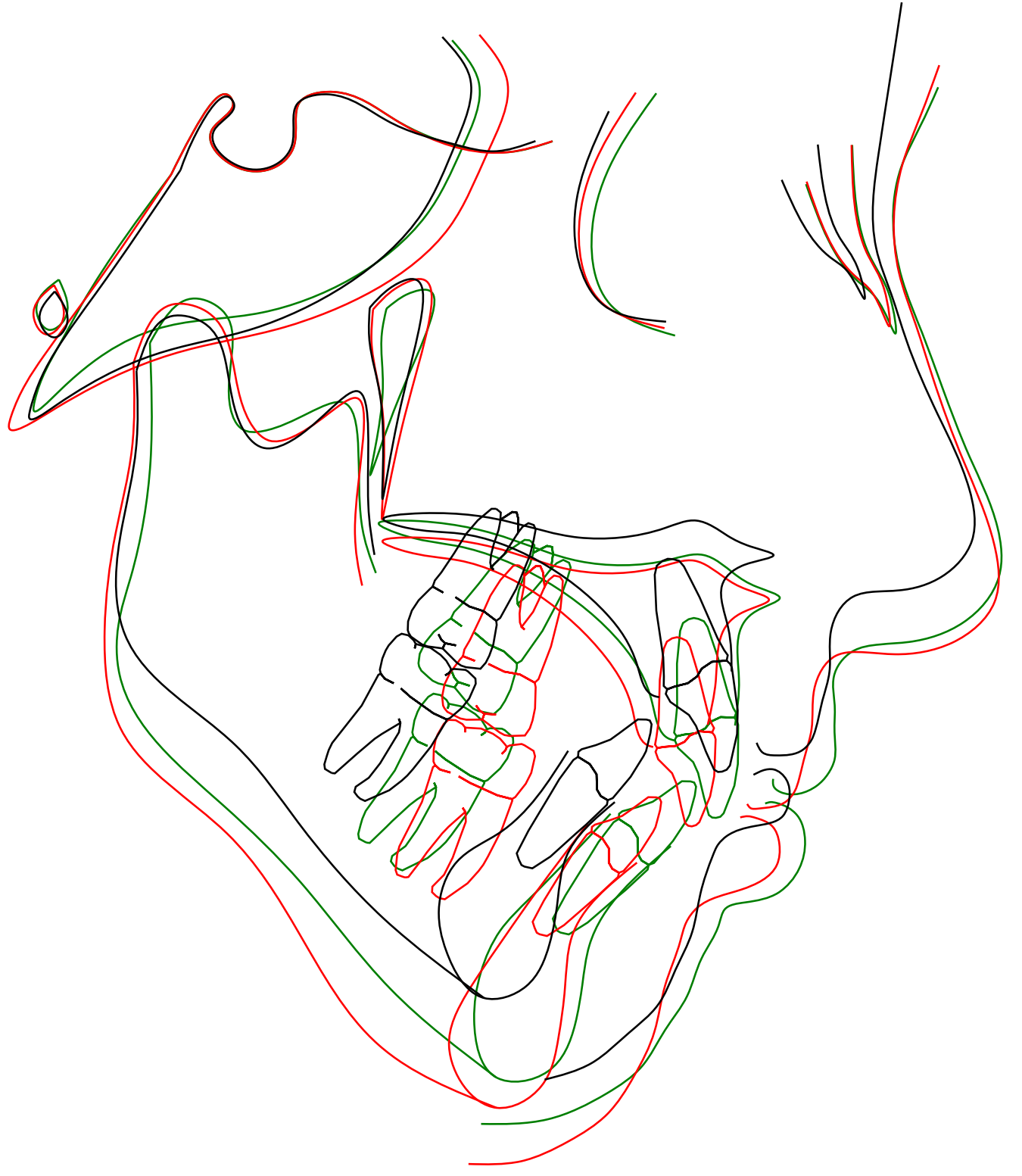

Figure B-87. Print of cephalometric tracing superimposition subject 87: (tracing is $1: 1$ with original radiograph). 


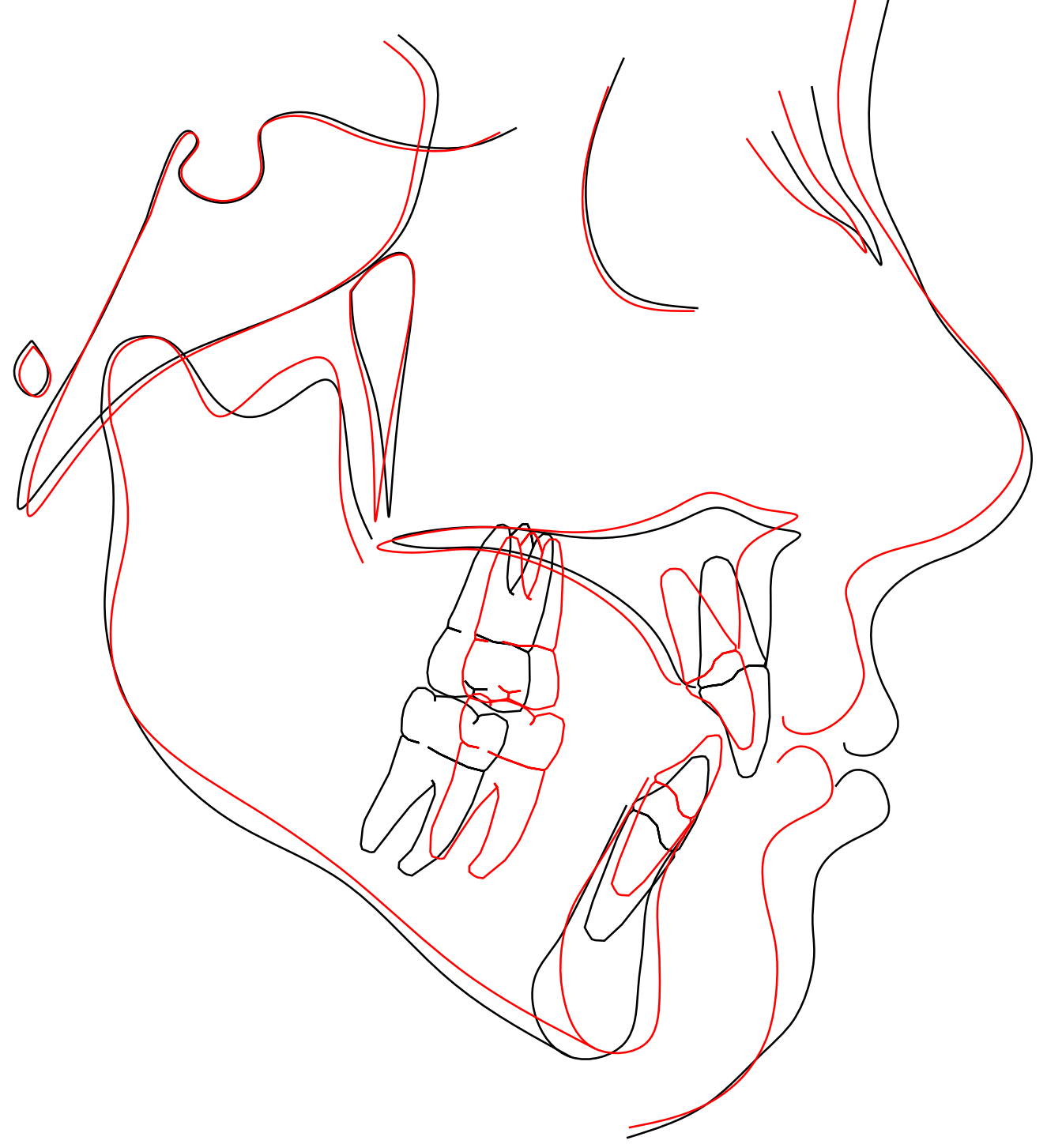

Figure B-88. Print of cephalometric tracing superimposition subject 88: (tracing is $1: 1$ with original radiograph). 


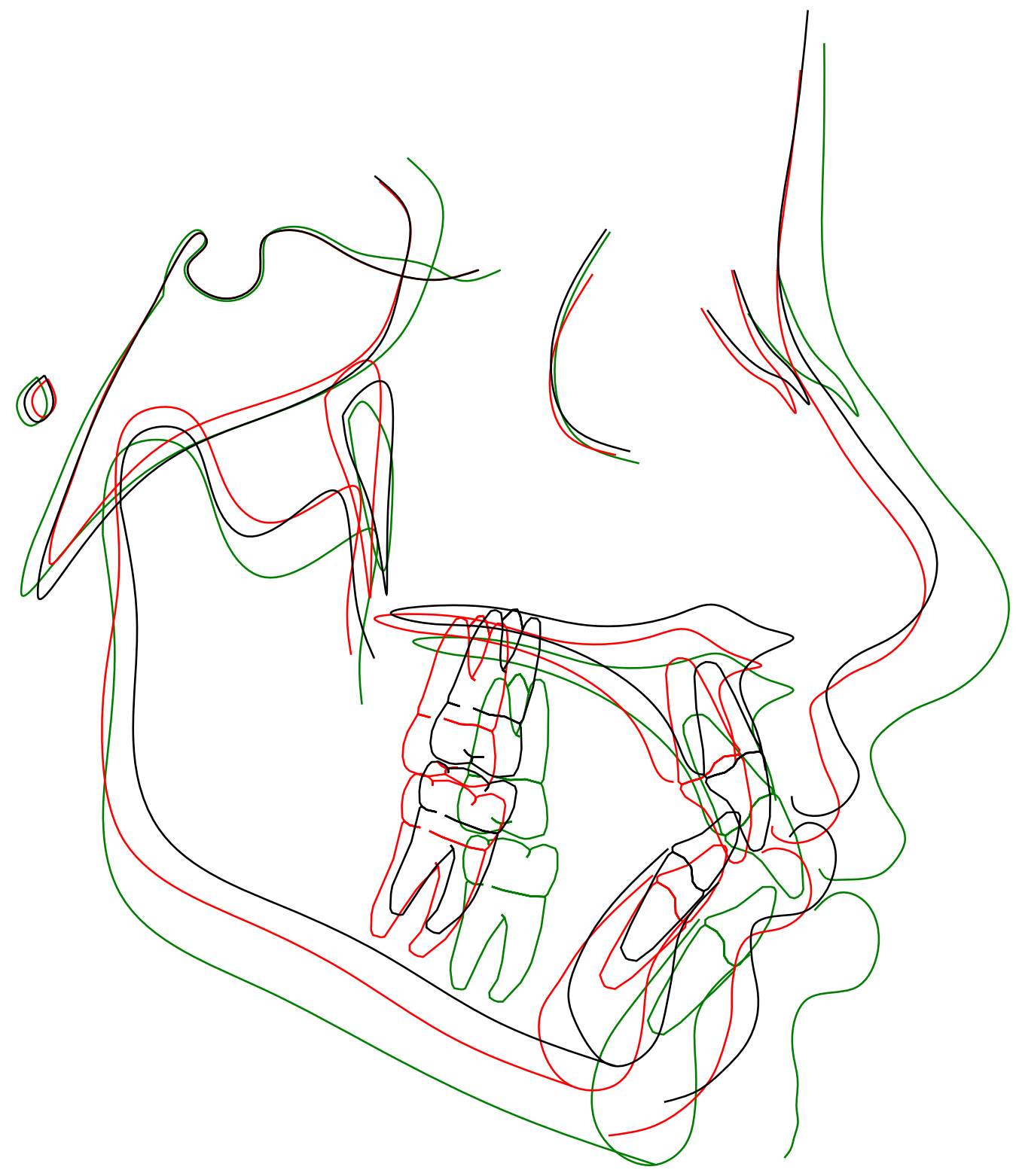

Figure B-89. Print of cephalometric tracing superimposition subject 89: (tracing is $1: 1$ with original radiograph). 


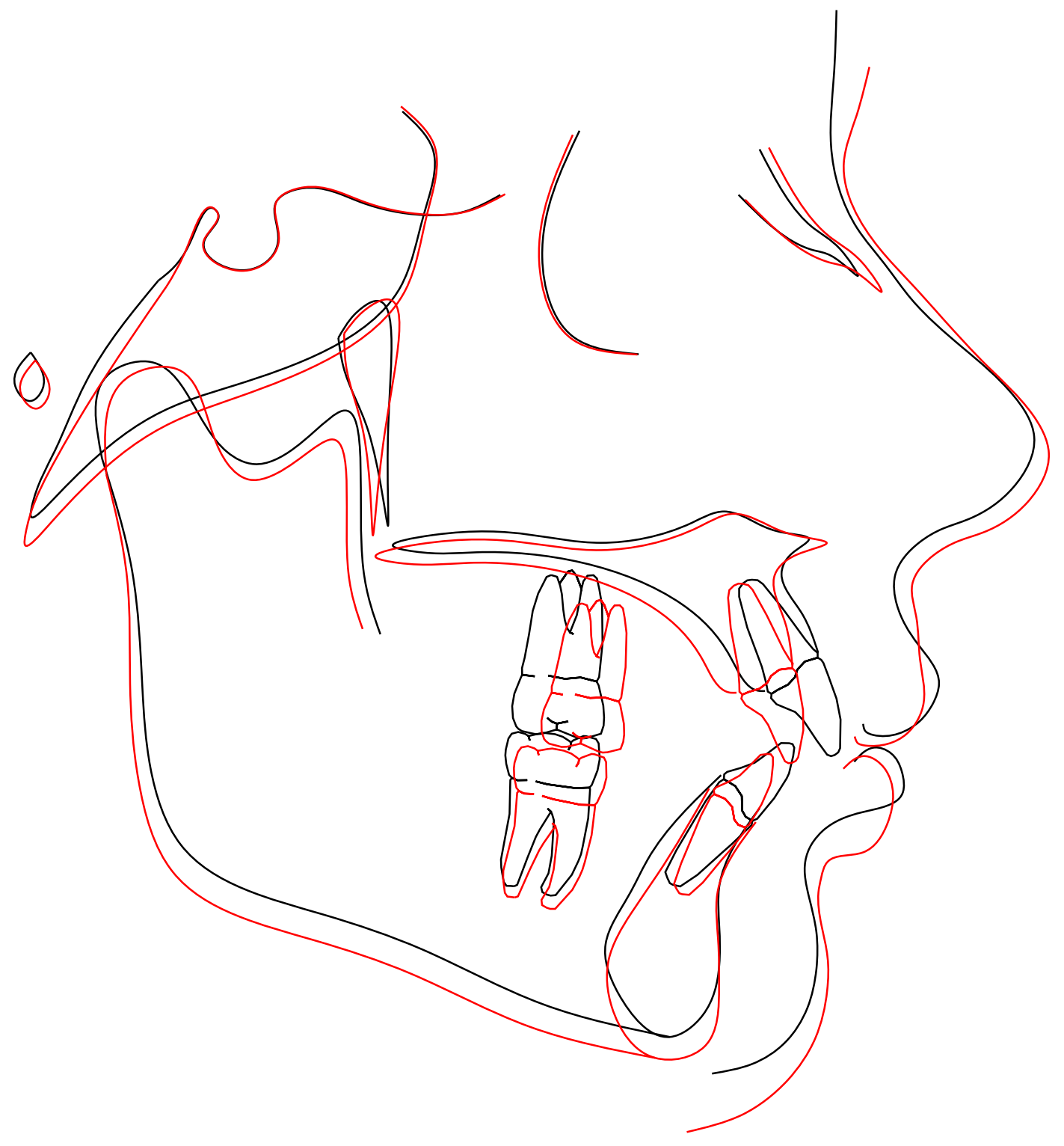

Figure B-90. Print of cephalometric tracing superimposition subject 90: (tracing is $1: 1$ with original radiograph). 


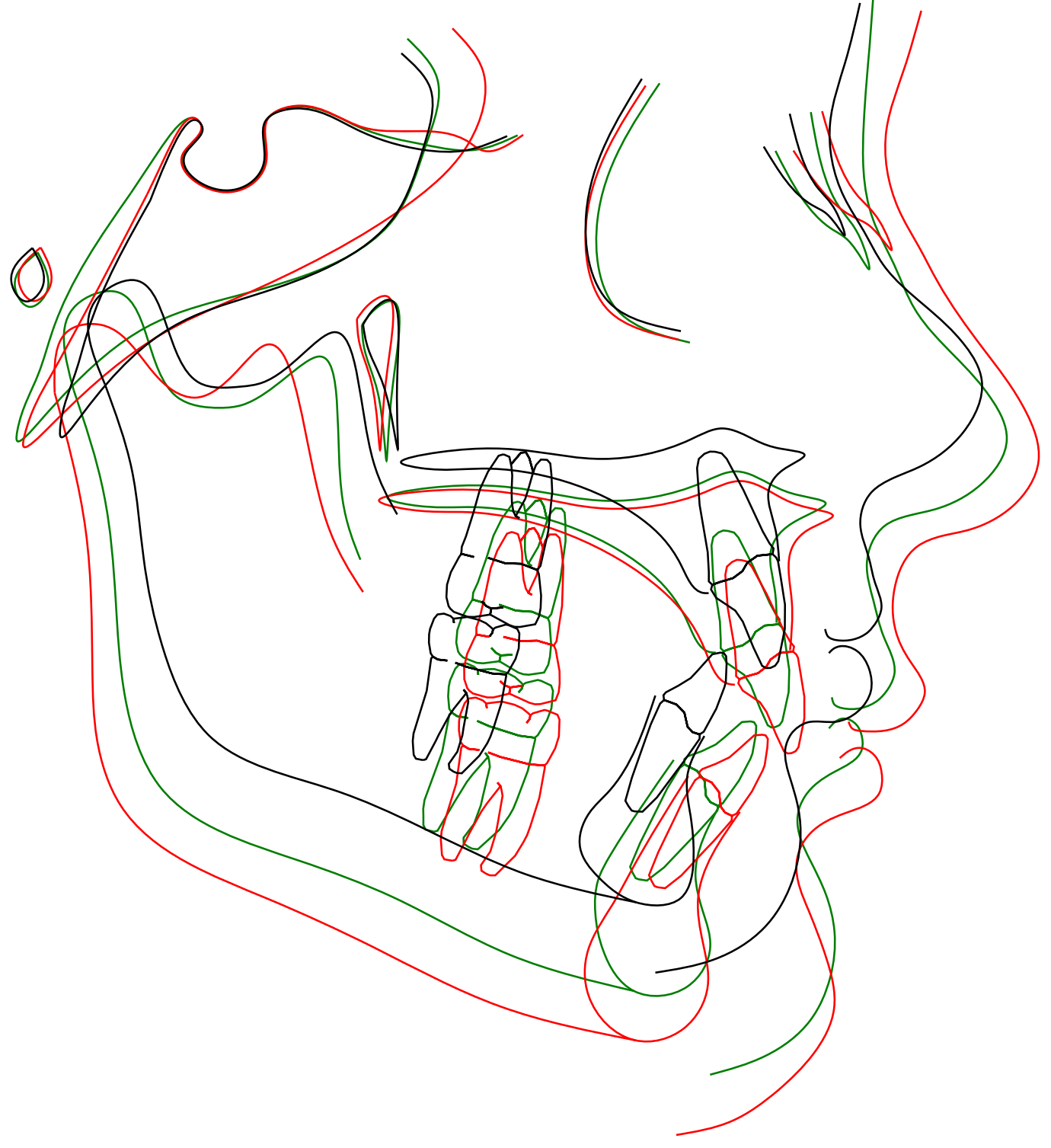

Figure B-91. Print of cephalometric tracing superimposition subject 91: (tracing is 1:1 with original radiograph). 


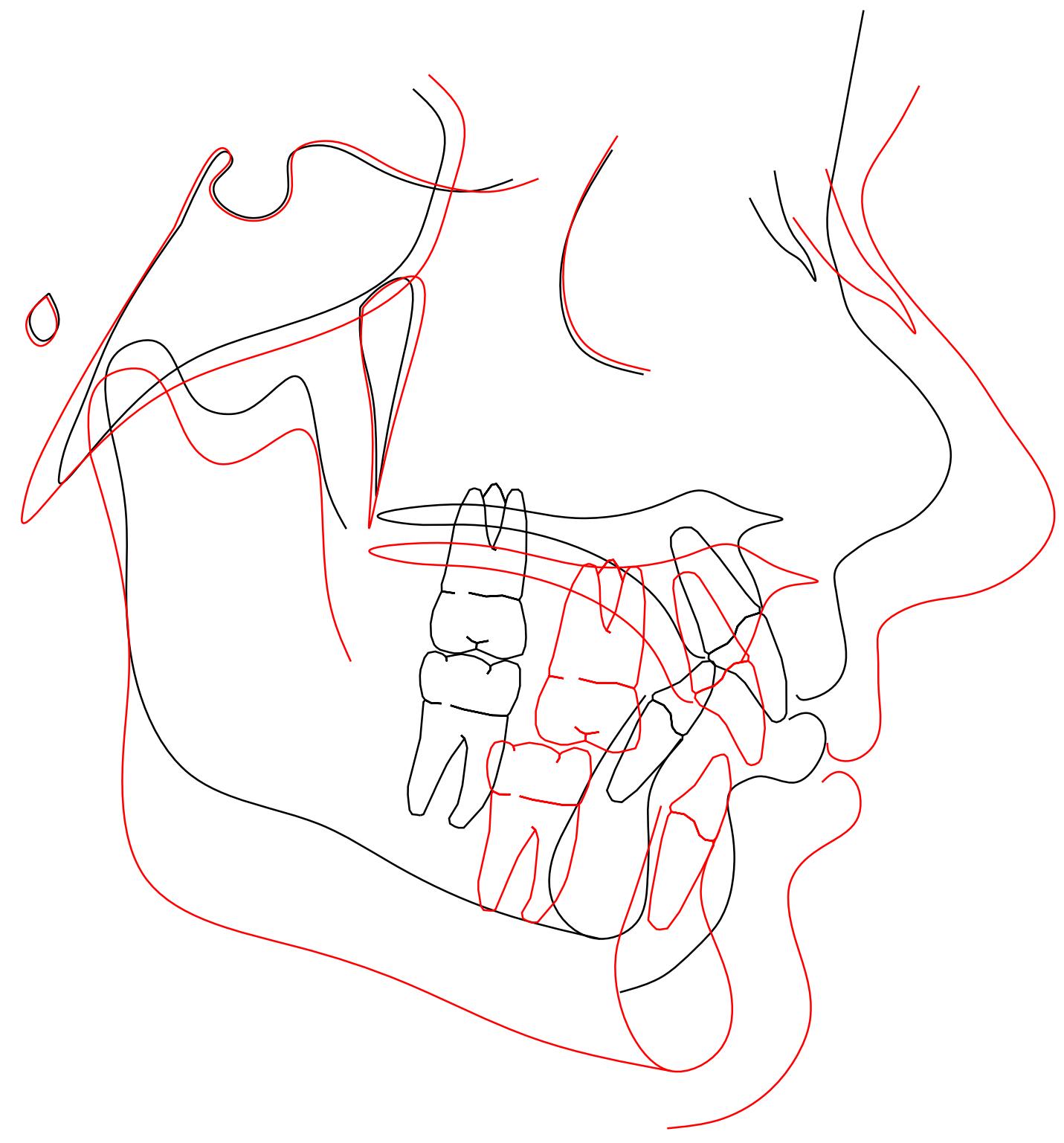

Figure B-92. Print of cephalometric tracing superimposition subject 92: (tracing is $1: 1$ with original radiograph). 


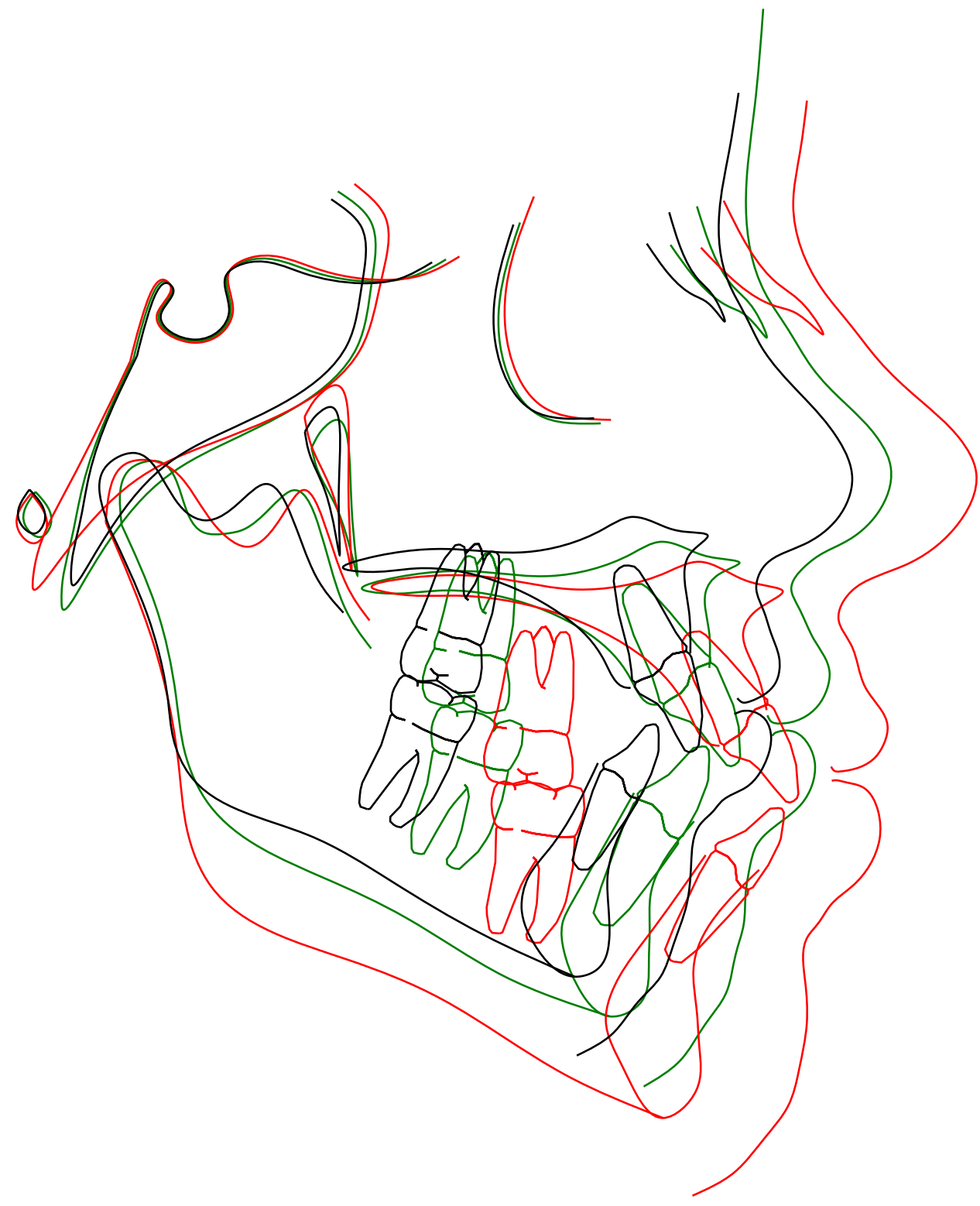

Figure B-93. Print of cephalometric tracing superimposition subject 93: (tracing is 1:1 with original radiograph). 


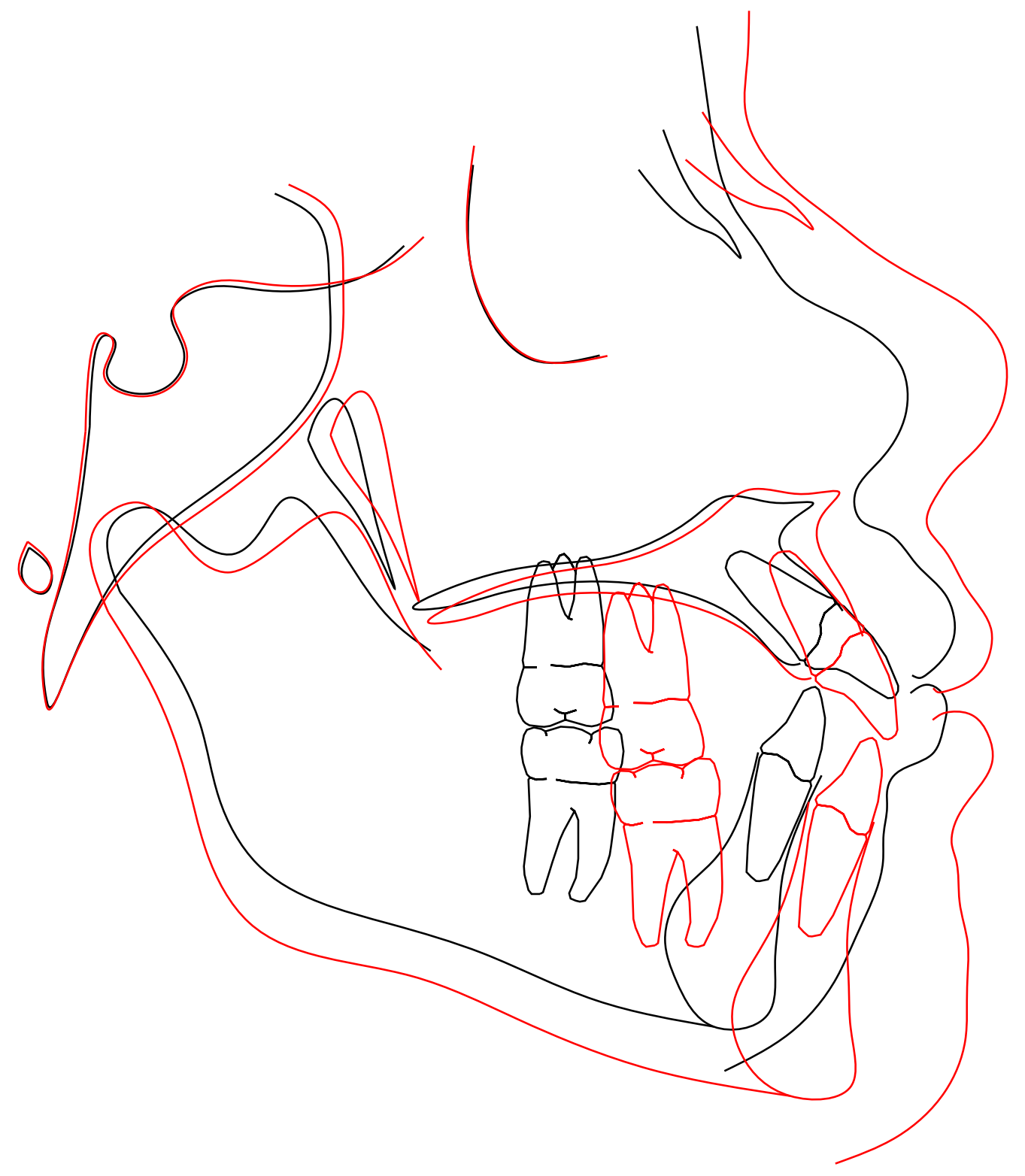

Figure B-94. Print of cephalometric tracing superimposition subject 94: (tracing is 1:1 with original radiograph). 


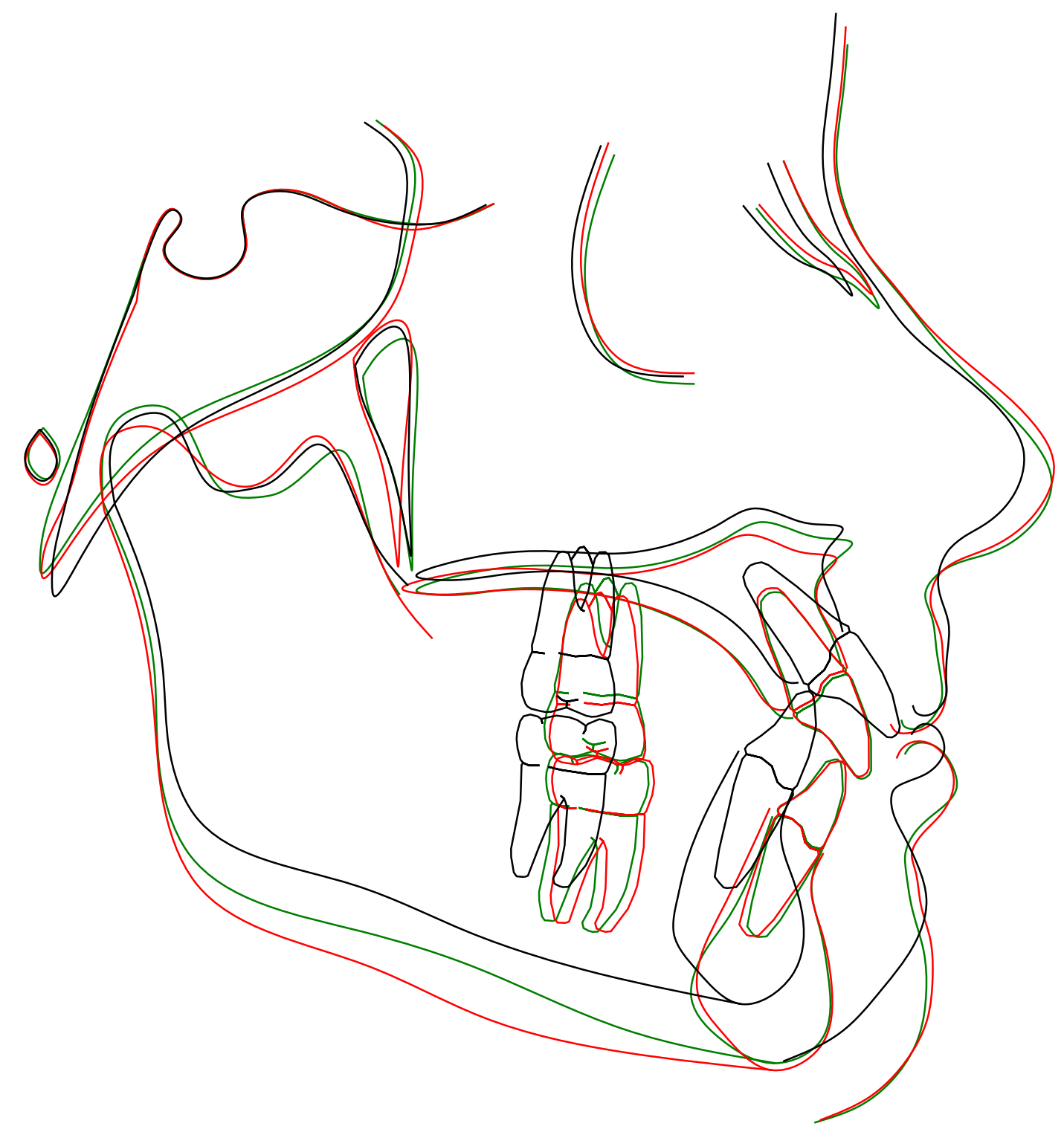

Figure B-95. Print of cephalometric tracing superimposition subject 95 (tracing is 1:1 with original radiograph). 


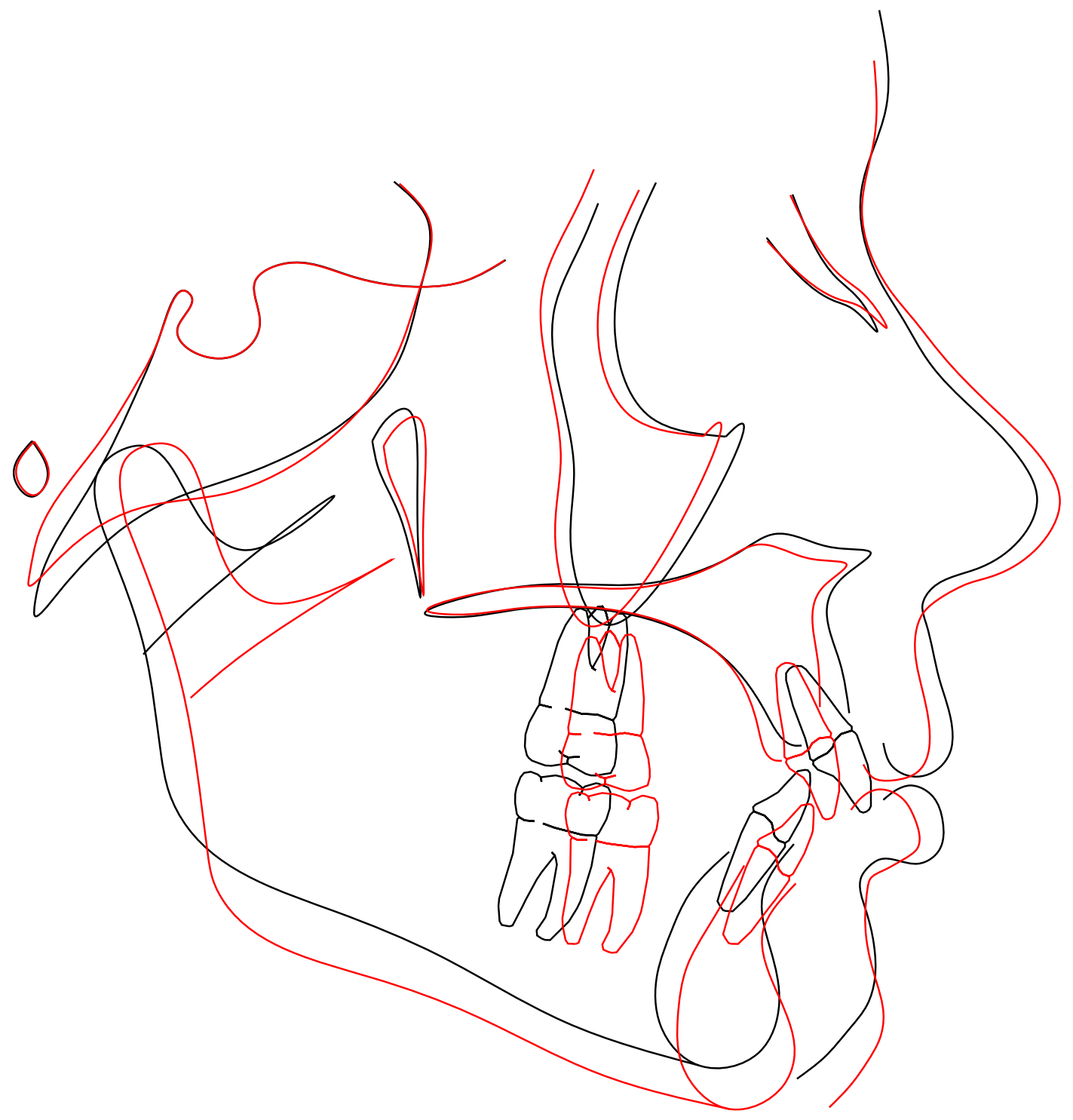

Figure B-96. Print of cephalometric tracing superimposition subject 96: (tracing is 1:1 with original radiograph). 


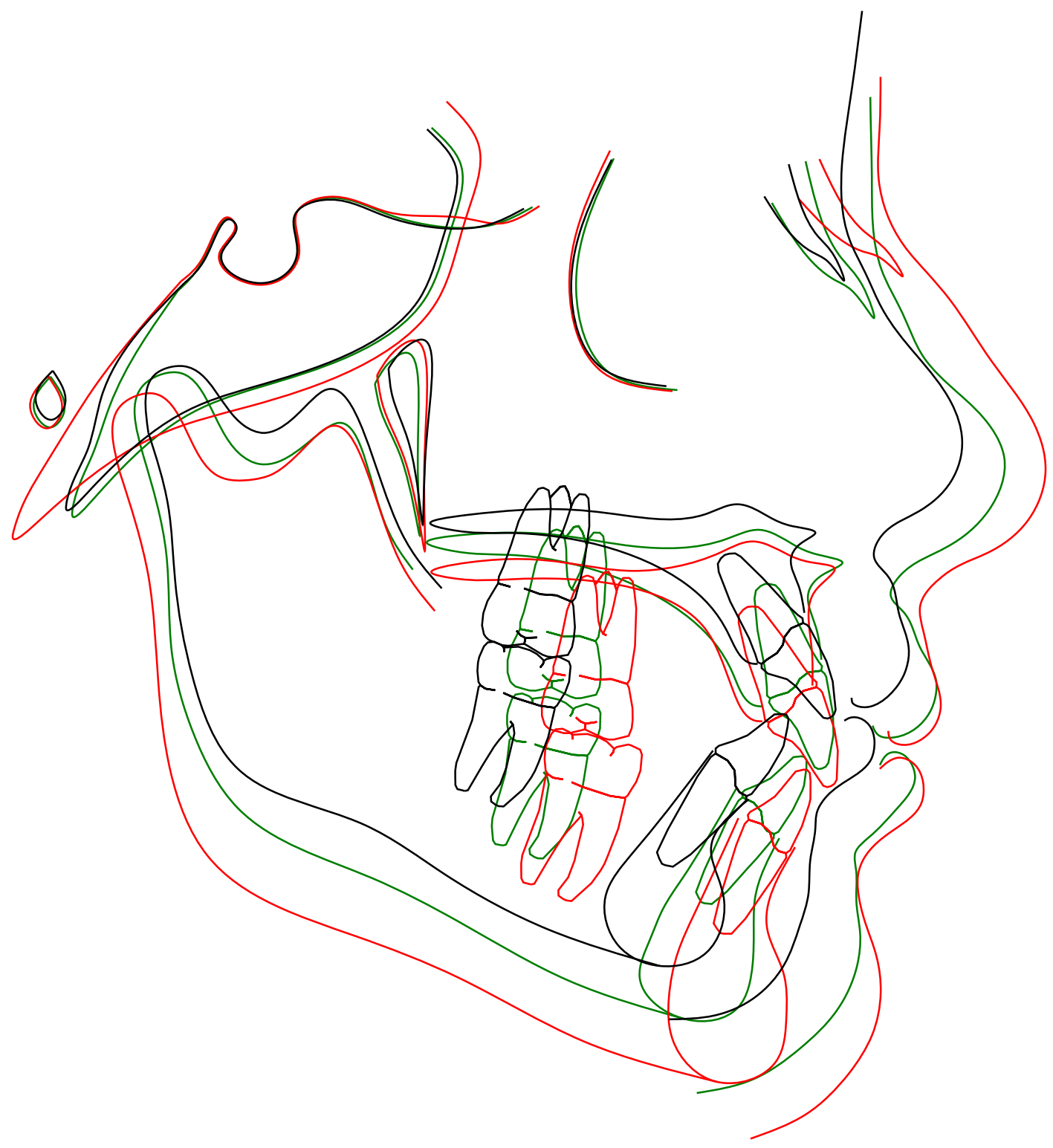

Figure B-97. Print of cephalometric tracing superimposition subject 97: (tracing is $1: 1$ with original radiograph). 


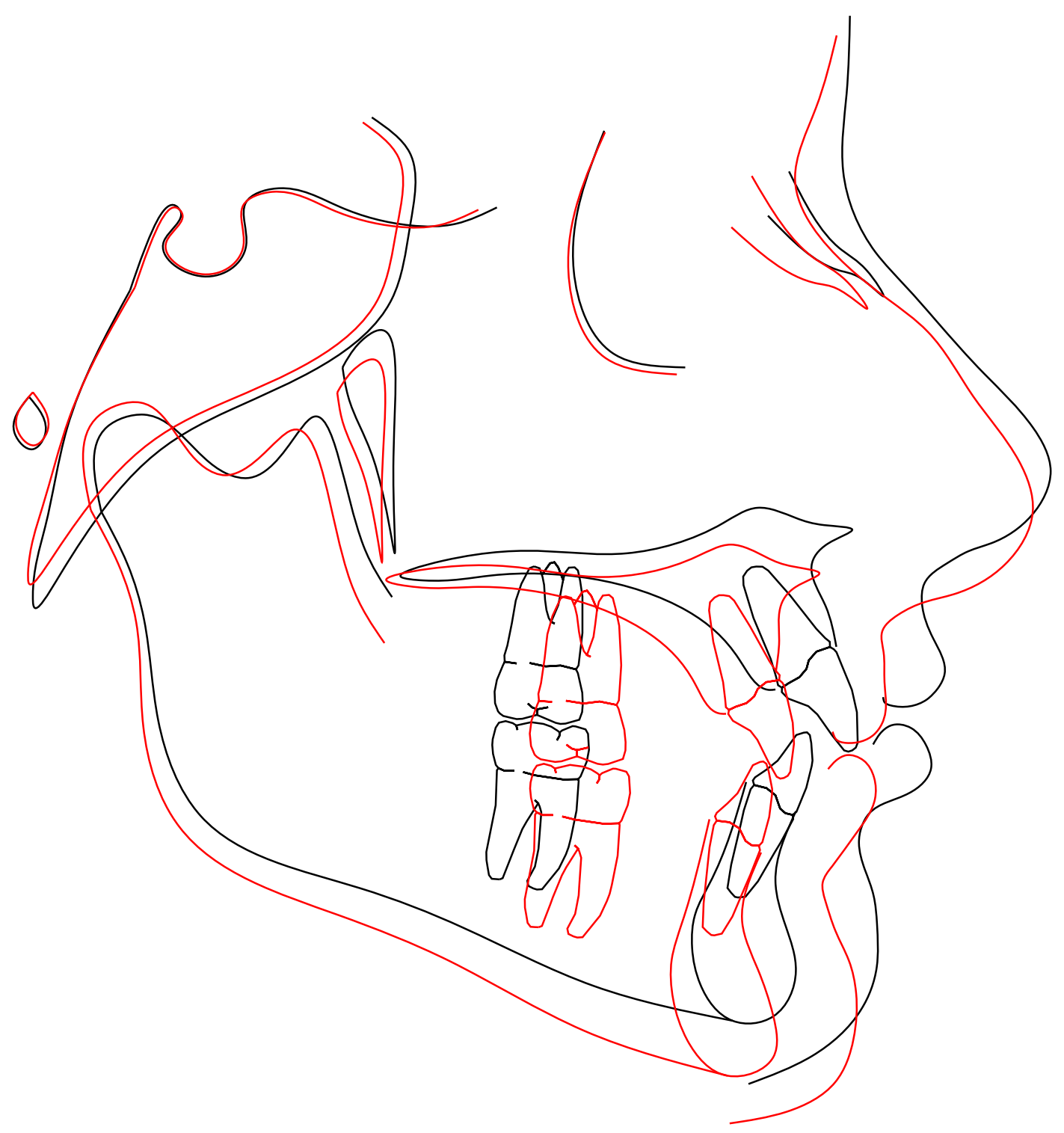

Figure B-98. Print of cephalometric tracing superimposition subject 98: (tracing is $1: 1$ with original radiograph). 


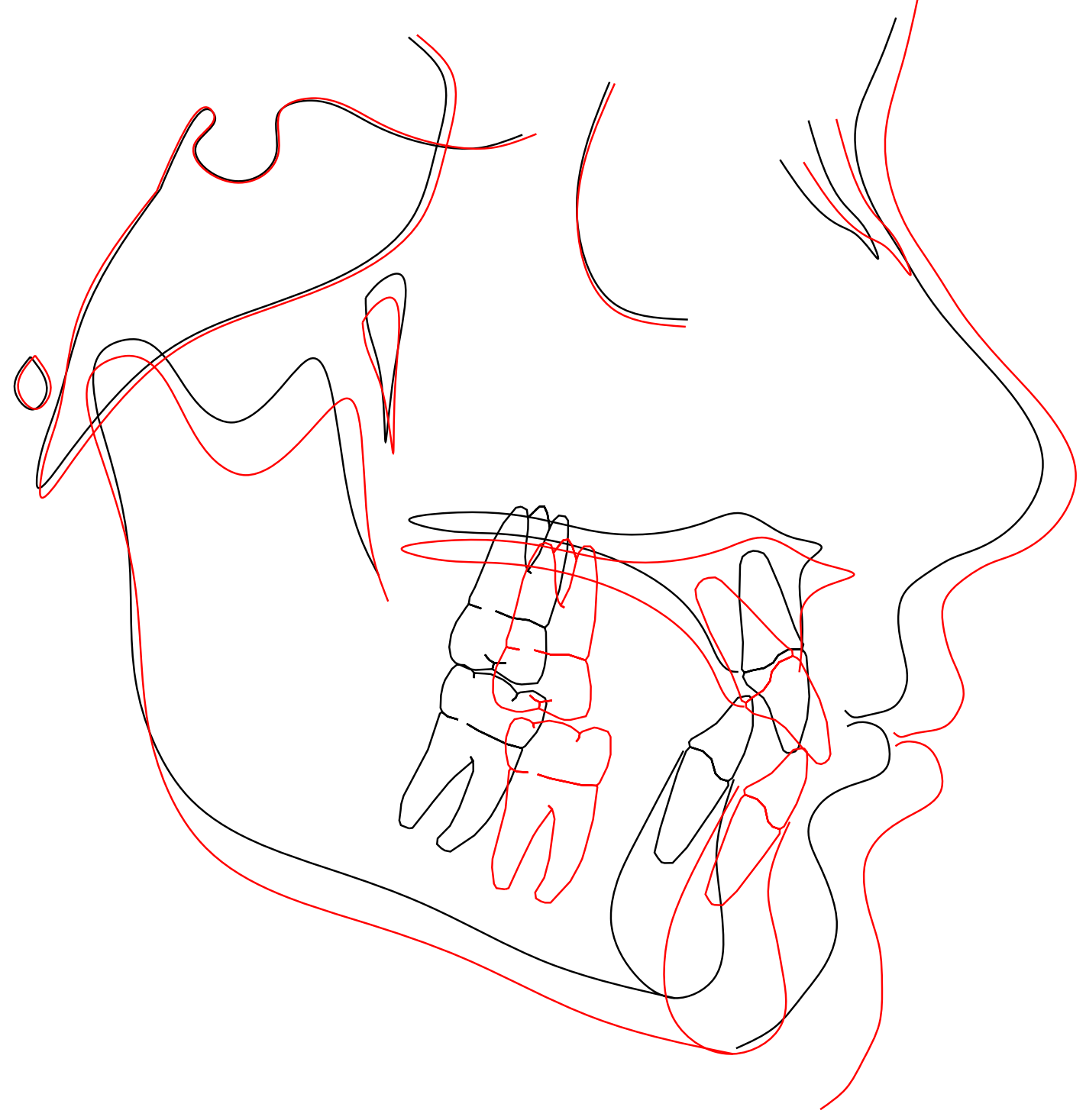

Figure B-99. Print of cephalometric tracing superimposition subject 99: (tracing is 1:1 with original radiograph). 


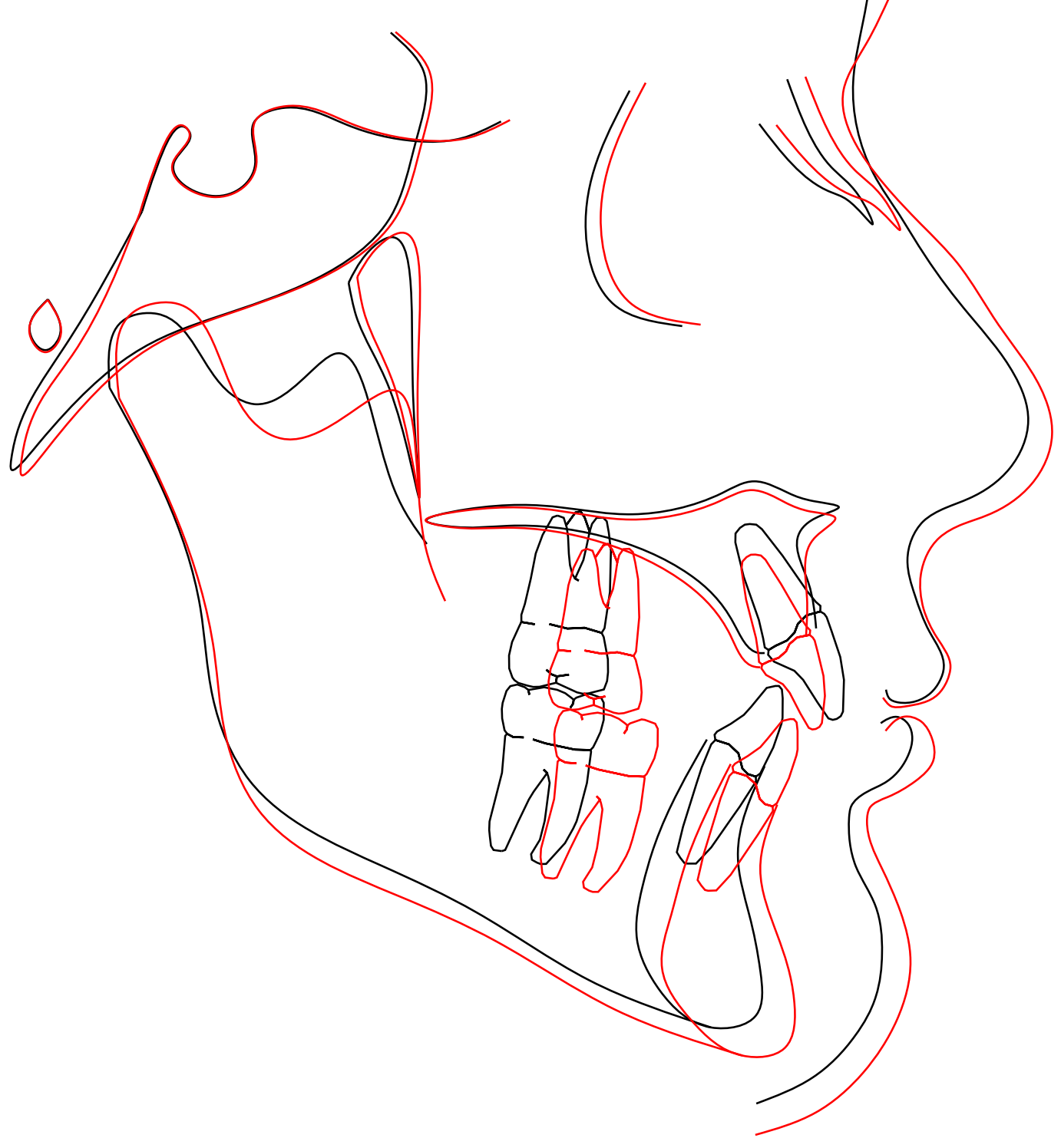

Figure B-100. Print of cephalometric tracing superimposition subject 100: (tracing is 1:1 with original radiograph). 


\section{VITA}

Daniel Corey Sawrie was born in Chattanooga, Tennessee on June 8, 1973. Daniel graduated from Baylor High School in 1991 then attended The University of Tennessee, Knoxville where he received a Bachelor of Arts in English. After The University of Tennessee, Knoxville he attended The University of Tennessee Health Science Center, College of Dentistry and was awarded the Doctor of Dental Surgery degree in May 2005. In August of 2005, he became a graduate student in the Department of Orthodontics, The University of Tennessee Health Science Center and is expected to receive his Master of Dental Science in May 2008. Daniel and his wife Lucy plan to live in Chattanooga, Tennessee upon graduation. 